\title{
Ester-Functionalized Imidazolium- and Pyridinium-Based Ionic Liquids: Design, Synthesis and Cytotoxicity Evaluation
}

\author{
Diana Hodyna 1,*(D), Vasyl Kovalishyn ${ }^{1}$, Ivan Semenyuta ${ }^{1}$, Sergiy Rogalsky ${ }^{2}$, Olena Trokhimenko ${ }^{3}$, \\ Anastasiia Gryniukova ${ }^{1}$, Larysa Metelytsia ${ }^{1}$ \\ 1 Department of Medical and Biological Researches, V.P. Kukhar Institute of Bioorganic Chemistry and Petrochemistry, \\ National Academy of Science of Ukraine, Kyiv, 02094, Ukraine \\ 2 Laboratory of Modification of Polymers, V.P. Kukhar Institute of Bioorganic Chemistry and Petrochemistry, National \\ Academy of Science of Ukraine, Kyiv, 02094, Ukraine \\ 3 Department of Virology, Shupyk National Healthcare University of Ukraine, Kyiv, 04112, Ukraine \\ * Correspondence: dianahodyna@gmail.com (D.H.);
}

Scopus Author ID 56897133500

Received: 9.06.2021; Revised: 15.07.2021; Accepted: 20.07.2021; Published: 8.08.2021

\begin{abstract}
The QSAR model for the prediction of cytotoxicity of Ionic liquids (ILs) was developed using a data set of 1195 compounds. The Artificial Neural Networks learning technique was used. The predictive ability of the models was tested by means of cross-validation; the $\mathrm{q}^{2}$ value was 0.76 for the regression model. The prediction for the external evaluation set afforded high predictive power $\left(\mathrm{q}^{2}\right.$ $=0.75$ for 239 compounds). The developed QSAR models evaluated the anticancer activity of a small set of virtual compounds and 6 compounds were selected for synthesis and biological testing. It was found that imidazolium and pyridinium ILs with $\mathrm{C}_{12}$ and $\mathrm{C}_{10}$ alkyl chain length exhibited significant cytotoxicity, particularly, compounds 3 and 6 were identified as the most potent anticancer agents with $\mathrm{IC}_{50}$ values $0.18 \mu \mathrm{M}$ and $5.75 \mu \mathrm{M}$ against Hep-2 cell line and different acute toxicity levels to cladoceran Daphnia magna. Molecular docking showed that the high cytotoxic activity of imidazolium and pyridinium ILs with $\mathrm{C}_{12}$ alkyl chain length may be associated with specific DNA binding in the region of CG nucleotides.
\end{abstract}

Keywords: ionic liquids; QSAR; cytotoxicity; molecular docking; acute toxicity.

(C) 2021 by the authors. This article is an open-access article distributed under the terms and conditions of the Creative Commons Attribution (CC BY) license (https://creativecommons.org/licenses/by/4.0/).

\section{Introduction}

It is known that the increase in the incidence of various types of cancer creates a constant need to develop new anticancer drugs, including synthetic compounds of different chemical classes, possessing cytotoxic properties [1-5].

Today the Ionic liquids (ILs) are the actual topics of study by researchers in the pharmaceutical industry in their search for new therapeutic agents [6-8]. Apart from the physicochemical features of ILs, their high biological activity attracts significant attention from medical scientists, biochemists, and ecologists. It is now clearly established that ILs have an impact on different levels of life, from single proteins to higher multicellular organisms. In addition to the use of ILs as antibacterial and antifungal agents [9-11], several studies have reported using different ILs in biomedical applications as therapeutic agents, namely as antitumor cytotoxic agents $[8,12,13]$. The cancerous cells of invertebrate and vertebrate species were investigated, including insects Drosophila melanogaster S2 cell culture [13], channel 
catfish ovary cell line CCO [14], mouse fibroblasts 3T3 [15], rat glial cells C6 [16], promyelocytic leukemia IPC-81 [17], pheochromocytoma PC12 [18], lung carcinoma A431 [19], squamous carcinoma CaCo-2 [20], colon carcinoma HaCaT [21], embryonic kidney HeLa [16], cervical cancer HepG2 [22], hepatocyte carcinoma HT-29 [23], colon carcinoma MCF-7 [20], breast cancer T98G [24], brain cancer U937 [25] and others.

It is established that the cytotoxicity of ILs depends on their structure and has a wide range of values from micromolar to millimolar. The preliminary structure-activity relationship (SAR) showed that the chain length of alkyl chains on the cations plays a crucial role in the anti-tumor activity and cytotoxicity of ILs [20]. The nature of the cation also affects the activity of ILs. So, cholinium ILs demonstrated lower cytotoxicity than imidazolium- and pyridiniumbased ILs. A number of potential anticancer agents with high cytostatic and low cytotoxic activity among imidazolium, phosphonium, and ammonium ILs was identified in 60 human tumor cell line tests $[20,26]$. In general, phosphonium-based ILs were identified as more active and less cytotoxic as compared to ammonium and imidazolium salts. Guanidinium tetrafluoroborate with long alkyl chains and the entered natural amino acids showed very high cytotoxicity toward several tumor cell lines [27]. The authors assumed that such an increase in cytotoxicity can be connected with the enhancement of transport of the toxic anion into the cells [28]. Short-chain ILs, triethylammonium sulfate, triethylammonium phosphate, 1methylimidazolium chloride, and 1-butyl-3-methylimidazolium chloride were found to have poor anti-tumor activity and were less toxic toward nonmalignant HEK (human embryonic kidney) cells as compared to T98G brain cancer cells [25].

Accumulated information about mechanisms of ILs cytotoxicity demonstrates that ILs disturb the lipid bilayers of the cell membrane, and cation hydrophobicity and lipophilicity correlate with the cytotoxic effect [29-32]. 1-alkylquinolinium ILs caused cell membrane disruption in the 3T3 cells culture [33]. Imidazolium ILs induced mitochondrial failure, oxidative stress, and apoptosis in malignant and normal cells [20,34-36]. The expression of cytochrome $\mathrm{P} 450$ genes, which products were involved in the metabolism of drugs and other exogenous substances, were induced by1-octyl-3-methylimidazolium chloride in the mammary carcinoma cells EMT6 of mouse [37]. A number of studies of the anticancer activity of ionic liquids based on imidazolium and pyridinium deserve special attention [38-40]. The authors associate this activity with their specific interaction with the cellular DNA.

It is worth noting that the long-chain ILs commonly demonstrate cytotoxicity to both normal and cancer cell lines that is one of the main problems limiting their practical applications $[19,20,41]$. However, the major advantage of ILs are in tuning their toxicity while tailoring pharmacological properties necessary for desired therapeutic applications [26,42]. The hydrophobic interaction between the lipophilic tails of cationic biocides and phospholipids of eukaryote cell membranes plays a major role in forming biocide-cell binding and consequently toxic impact [43]. From this point of view, introducing polar functional groups into the hydrocarbon chains of ILs may retard their penetration through the cell membrane due to reduced lipophilicity [44]. Thus, long-chain pyridinium-based ILs, comprising the polar ester group in the hydrocarbon radical, were found to have dramatically reduced acute toxicity towards Danio rerio model freshwater hydrobiont as compared to common long-chain ILs [45]. However, the anti-tumor activity of ester-functionalized ILs has not yet been studied.

Several attempts to create a prediction model for assessing ILs cytotoxicity in silico have been made [46-50] however, at the moment, there is no possibility to distinguish the cytotoxicity of ILs only by their structure because it also strongly depends on the external 
factors, such as the cell type. Despite active investigations in the ILs anticancer properties area, this problem remains open for further scientific finds.

The cytotoxic activity and acute toxicity of a number of ionic liquids are presented in this study using the created QSAR models, biological testing, and docking analysis.

\section{Materials and Methods}

\subsection{QSAR modeling.}

2.1.1. Composition of training and test sets and calculation of molecular descriptors.

The data for our analysis consisted of 1195 compounds cytotoxic against Hep-2 cells that were obtained from the ChEMBL database [51]. The activity of compounds was expressed as $\mathrm{IC}_{50}$ ranging from 0.0002 to $5000 \mu \mathrm{M}$ (Supplementary material).

Biological data obtained as inhibitory concentration ( $\left.\mathrm{IC}_{50}\right)$ were converted to $\lg \left(1 / \mathrm{IC}_{50}\right)$ and used as a dependent variable in the subsequent QSAR studies. In this work, external testing was performed using a test set that was formed using the method of Kennard-Stone Design (KSD) [52]. In this case, 20-25\% of compounds were selected in the test set, while the remaining compounds from the general data set were used to build QSAR models [53]. Thus, the initial sample was divided into a test set that included 239 (20\%) compounds, while the remaining 956 compounds were used as a training data sample.

Each molecule was modeled using ChemAxon Standardizer [54]. The 2D coordinates of the atoms were recalculated, ions and salts were removed from the molecular structure, the molecules were reduced to neutral, 3D structures were optimized and saved in SDF format. The molecular descriptors' calculation was done using the DRAGON package [55] as the original variables, including molecular properties topological, geometrical, constitutional descriptors, atom-centered fragments, and many others [56]. The descriptors with coefficients with variance less than $5 \%$ and constants were deleted. In addition, if any of the two descriptors were at least $99 \%$ correlated, one of them was deleted.

Detailed information about the descriptors can be found on the Talete website [55]. As a result, the 1016 descriptors were selected.

\subsubsection{Machine learning methods.}

In this work, data analysis and selection of descriptors were performed using Batch Pruning Algorithm (BPA) [57]. This algorithm is a combination of the Associative Neural Network (ASNN) method [58], Self-Organized Map (SOM) of Kohonen [59], and the descriptor selection methods $[60,61]$, used together. The Kohonen network was used to analyze the similarity of molecular descriptors, while ASNN was used to construct predictive models.

ASNN consists of an ensemble of Back Propagation Neural Networks (BPNN) and the method of k-nearest neighbors (k-NN) [58]. Traditional BPNNs are memoryless networks, i.e., after learning neural networks, the input data is no longer needed, and all the information needed to predict the properties of the new data is stored using weights. On the contrary, the kNearest Neighbours (k-NN) method maintains a memory of all training samples, and predictions are based on a local approximation of the stored data [62]. ASNN uses the k-NN method in the space of ensemble residuals. After learning ASNN, all compounds are represented as vectors of neural network predictions by the neural network ensemble. The nearest neighbor method uses the correlation between these vectors to measure the distance 
between the analyzed cases. Thus, this approach increases the accuracy of the forecast by adjusting the source data based on information about the input data [58].

For data analysis, we used neural networks consisting of three levels. The number of input neurons was equal to the number of descriptors. One hidden layer with five neurons was used. Weights were initialized with random numbers within $[-0.5 ;+0.5]$ for each of the 200 networks in the ensemble. This initialization allows the ASNN to avoid local minima and speeds up the ensemble learning procedure [63]. A bias neuron was also presented in both the input and hidden layers. One output neuron was used for regression tasks, and the output activity values $\lg \left(1 / \mathrm{IC}_{50}\right)$ were linearly scaled between 0.1 and 0.9 [63]. An algorithm known as the SuperSAB algorithm [64] was used for training. All the networks had the same architecture. Cross-validation techniques were used to strictly control the possibility of overfitting the data known as the Early Stopping over Ensemble (ESE) [63]. Research manuscripts reporting large datasets that are deposited in a publicly available database should specify where the data have been deposited and provide the relevant accession numbers. If the accession numbers have not yet been obtained at the time of submission, please state that they will be provided during review. They must be provided prior to publication.

\subsubsection{Statistical coefficients.}

The prediction statistics for the models are given in Table 1 (section Results and Discussion). The generally used measures of a regression QSAR model performance are the root mean square error (RMSE), the mean absolute error (MAE), the squared correlation coefficient $\mathrm{R}^{2}$ [65], and cross-validated coefficient $\mathrm{q}^{2}$ [66]. ASNN calculates these statistical parameters for both the training and the validation sets.

\subsection{Synthesis of ionic liquids.}

Following chemicals were used for the synthesis of ionic liquids: pyridine (99\%), chloroacetyl chloride (98\%), 1-octanol, 1-decanol, 1-dodecanol (98\%), 1-methylimidazole (for synthesis), benzene, hexane, ethyl acetate (98\%). All chemicals were purchased from SigmaAldrich and used without further purification.

Proton Nuclear Magnetic Resonance (1H NMR) spectroscopy was used to confirm the structure of synthesized compounds. The spectra were recorded on a Varian Gemini-2000 (400 $\mathrm{MHz}$ ) NMR spectrometer.

\subsubsection{Synthesis of long-chain alkyl esters of chloroacetic acid.}

Alkyl esters of chloroacetic acid (1-octyl chloroacetate, 1-decyl chloroacetate, and 1dodecyl chloroacetate) were synthesized as follows (Scheme 1).

Chloroacetyl chloride $(13.5 \mathrm{~g}, 0.12 \mathrm{~mol})$ was added dropwise to the stirred mixture of corresponding alcohol $(0.1 \mathrm{~mol})$ and potassium carbonate $(14 \mathrm{~g}, 0.1 \mathrm{~mol})$ in dry benzene $(100$ $\mathrm{mL}$ ). The reaction was carried out for $6 \mathrm{~h}$ at room temperature. After completion of the reaction, the reactionary mixture was washed with water $(3 \times 200 \mathrm{~mL})$. The organic layer was separated and dried overnight over calcium chloride. Benzene was distilled, the residual solvent was removed in a vacuum $10 \mathrm{mbar}$ at $60^{\circ} \mathrm{C}$.

$$
\mathrm{ClCH}_{2} \mathrm{COCl}+\mathrm{ROH} \underset{\mathrm{K}_{2} \mathrm{CO}_{3}}{\stackrel{\mathrm{C}_{6} \mathrm{H}_{6}}{\longrightarrow}} \mathrm{ClCH}_{2} \mathrm{COOR} \quad \mathrm{R}=\mathrm{C}_{8} \mathrm{H}_{17}, \mathrm{C}_{10} \mathrm{H}_{21}, \mathrm{C}_{12} \mathrm{H}_{25}
$$

Scheme 1. Synthesis of long-chain esters of chloroacetic acid. 
Octyl chloroacetate - 1H NMR (400 MHz, $\left.\mathrm{CDCl}_{3}\right): \delta=0.88\left(\mathrm{t}, 3 \mathrm{H}, \mathrm{CH}_{3}\right), 1.27(\mathrm{~m}, 10 \mathrm{H}$, $\left.\mathrm{CH}_{3}\left(\mathrm{CH}_{2}\right)_{5}\right), 1.66\left(\mathrm{~m}, 2 \mathrm{H}, \mathrm{OCH}_{2} \mathrm{CH}_{2}\right), 4.05$ (s, 2H, $\left.\mathrm{COCH}_{2}\right), 4.18$ (t, 2H, $\left.\mathrm{OCH}_{2}\right)$;

Decyl chloroacetate - 1H NMR (400 $\left.\mathrm{MHz}_{2} \mathrm{CDCl}_{3}\right): \delta=0.89\left(\mathrm{t}, 3 \mathrm{H}, \mathrm{CH}_{3}\right), 1.27(\mathrm{~m}$, 14H, $\left.\mathrm{CH}_{3}\left(\mathrm{CH}_{2}\right)_{7}\right), 1.67$ (m, 2H, $\left.\mathrm{OCH}_{2} \mathrm{CH}_{2}\right), 4.07$ (s, 2H, $\left.\mathrm{COCH}_{2}\right), 4.19$ (t, 2H, $\mathrm{OCH}_{2}$ );

Dodecyl chloroacetate - 1H NMR (400 MHz, $\left.\mathrm{CDCl}_{3}\right): \delta=0.89\left(\mathrm{t}, 3 \mathrm{H}, \mathrm{CH}_{3}\right), 1.28(\mathrm{~m}$, $\left.18 \mathrm{H}, \mathrm{CH}_{3}\left(\mathrm{CH}_{2}\right) 9\right), 1.66\left(\mathrm{~m}, 2 \mathrm{H}, \mathrm{OCH}_{2} \mathrm{CH}_{2}\right), 4.06\left(\mathrm{~s}, 2 \mathrm{H}, \mathrm{COCH}_{2}\right), 4.18\left(\mathrm{t}, 2 \mathrm{H}, \mathrm{OCH}_{2}\right)$;

2.2.2. Synthesis of ester-functionalized pyridinium ILs.

Long-chain ester-functionalized pyridinium ILs were synthesized by the following method (Scheme 2). The mixture of pyridine and corresponding alkyl chloroacetate (10\% molar excess) was stirred at $120{ }^{\circ} \mathrm{C}$ for $2 \mathrm{~h}$. After cooling to room temperature, the solid products were obtained. They were purified by recrystallizing from ethyl acetate $\left(\mathrm{PyrCH}_{2} \mathrm{COOC}_{12}-\mathrm{Cl}\right)$ or washed with a hexane-ethyl acetate mixture $(3: 1, \mathrm{v} / \mathrm{v})$.

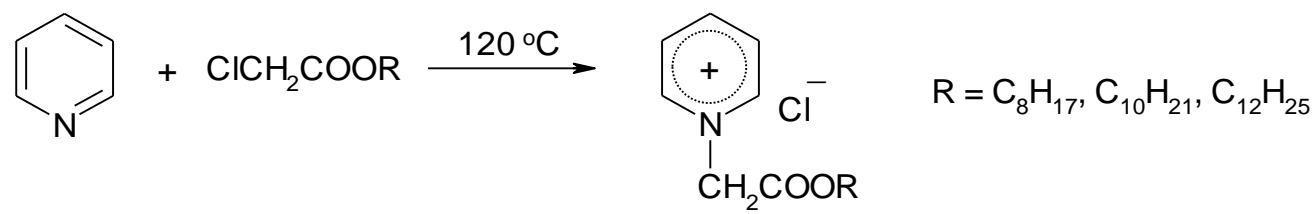

Scheme 2. Synthesis of ester-functionalized pyridinium ILs.

1-octyloxycarbonylmethylpyridinium chloride $\left(\mathrm{PyrCH}_{2} \mathrm{COOC}_{8}-\mathrm{Cl}\right)$ (1)

Amorphous solid of light brown color (m. p. $59^{\circ} \mathrm{C}$ )

1H NMR (400 MHz, $\left.\mathrm{CDCl}_{3}\right): \delta=0.82\left(\mathrm{t}, 3 \mathrm{H}, \mathrm{CH}_{3}\right), 1.21\left(\mathrm{~m}, 14 \mathrm{H}, \mathrm{CH}_{3}\left(\mathrm{CH}_{2}\right)_{7}\right), 1.58$ (m, 2H, $\left.\mathrm{OCH}_{2} \mathrm{CH}_{2}\right), 4.12\left(\mathrm{t}, 2 \mathrm{H}, \mathrm{OCH}_{2}\right), 6.26\left(\mathrm{~s}, 2 \mathrm{H}, \mathrm{NCH}_{2}\right), 8.05$ (t, 2H, $\left.\mathrm{C}_{3}-\mathrm{H}, \mathrm{C}_{5}-\mathrm{H}\right), 8.49$ (t, $\left.1 \mathrm{H}, \mathrm{C}_{4}-\mathrm{H}\right), 9.46\left(\mathrm{~d}, 2 \mathrm{H}, \mathrm{C}_{2}-\mathrm{H}, \mathrm{C}_{6}-\mathrm{H}\right)$.

1-decyloxycarbonylmethylpyridinium chloride $\left(\mathrm{PyrCH}_{2} \mathrm{COOC}_{10-} \mathrm{Cl}\right)$ (2)

White solid (m. p. 89-91 ${ }^{\circ} \mathrm{C}$ )

1H NMR (400 MHz, DMSO-D6): $\delta=0.86\left(\mathrm{t}, 3 \mathrm{H}, \mathrm{CH}_{3}\right), 1.27\left(\mathrm{~m}, 14 \mathrm{H}, \mathrm{CH}_{3}\left(\mathrm{CH}_{2}\right) 7\right.$ ), $1.61\left(\mathrm{~m}, 2 \mathrm{H}, \mathrm{OCH}_{2} \mathrm{CH}_{2}\right), 4.17\left(\mathrm{t}, 2 \mathrm{H}, \mathrm{OCH}_{2}\right), 5.8\left(\mathrm{~s}, 2 \mathrm{H}, \mathrm{NCH}_{2}\right), 8.26$ (t, 2H, $\left.\mathrm{C}_{3}-\mathrm{H}, \mathrm{C}_{5}-\mathrm{H}\right), 8.72$ (t, 1H, C4-H), 9.18 (d, 2H, C2-H, C6-H).

1-dodecyloxycarbonylmethylpyridinium chloride $\left(\mathrm{PyrCH}_{2} \mathrm{COOC}_{12}-\mathrm{Cl}\right)(3)$

White solid (m. p. $70-73{ }^{\circ} \mathrm{C}$ )

1H NMR (400 MHz, DMSO-D $): ~ \delta=0.85\left(\mathrm{t}, 3 \mathrm{H}, \mathrm{CH}_{3}\right), 1.25\left(\mathrm{~m}, 18 \mathrm{H}, \mathrm{CH}_{3}\left(\mathrm{CH}_{2}\right) 9\right)$, $1.61\left(\mathrm{~m}, 2 \mathrm{H}, \mathrm{OCH}_{2} \mathrm{CH}_{2}\right), 4.17\left(\mathrm{t}, 2 \mathrm{H}, \mathrm{OCH}_{2}\right), 5.9\left(\mathrm{~s}, 2 \mathrm{H}, \mathrm{NCH}_{2}\right), 8.26\left(\mathrm{t}, 2 \mathrm{H}, \mathrm{C}_{3}-\mathrm{H}, \mathrm{C}_{5}-\mathrm{H}\right), 8.74$ $\left(\mathrm{t}, 1 \mathrm{H}, \mathrm{C}_{4}-\mathrm{H}\right), 9.27\left(\mathrm{~d}, 2 \mathrm{H}, \mathrm{C}_{2}-\mathrm{H}, \mathrm{C}_{6}-\mathrm{H}\right)$.

\subsubsection{Synthesis of ester-functionalized imidazolium ILs.}

Long-chain ester-functionalized imidazolium ILs were synthesized according to Scheme 3.

The mixture of 1-methylimidazole and alkyl chloroacetate (10\% molar excess) was stirred at $120{ }^{\circ} \mathrm{C}$ for $2 \mathrm{~h}$. After cooling, the solid products were obtained. They were purified by recrystallizing from ethyl acetate $\left(\mathrm{IMC}_{1} \mathrm{CH}_{2} \mathrm{COOC}_{12}-\mathrm{Cl}\right)$ or washed with a hexane-ethyl acetate mixture $(3: 1, \mathrm{v} / \mathrm{v})$. 


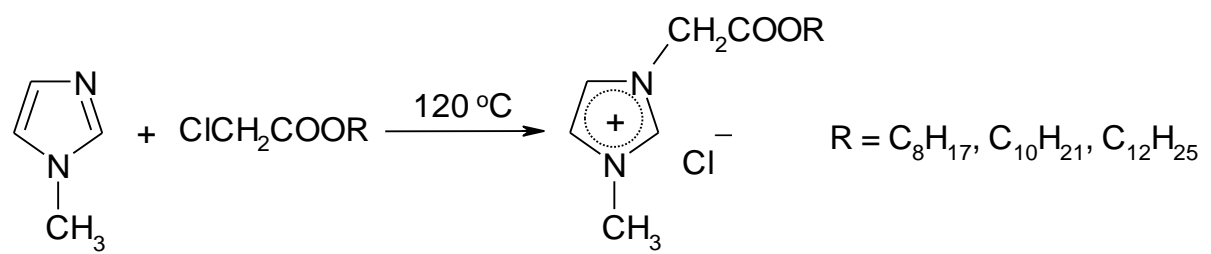

Scheme 3. Synthesis of ester-functionalized imidazolium ILs.

1-octyl-3-methylimidazolium chloride ( $\left.\mathrm{IMC}_{1} \mathrm{CH}_{2} \mathrm{COOC}_{8}-\mathrm{Cl}\right)$ (4)

$\left.1 \mathrm{H} \mathrm{NMR} \mathrm{(400} \mathrm{MHz,} \mathrm{CDCl}_{3}\right): \delta=0.83\left(\mathrm{t}, 3 \mathrm{H}, \mathrm{CH}_{3}\right), 1.22\left(\mathrm{~m}, 14 \mathrm{H},\left(\mathrm{CH}_{2}\right)_{7}\right), 1.6(\mathrm{~m}, 2 \mathrm{H}$, $\mathrm{COOCH}_{2} \mathrm{CH}_{2}$ ), 4.04 (s, 3H, NCH 3$), 4.13$ (t, 2H, $\left.\mathrm{COOCH}_{2}\right), 5.44$ (s, 2H, $\left.\mathrm{NCH}_{2} \mathrm{CO}\right), 7.52$ (d, $\left.1 \mathrm{H}, \mathrm{C}_{4}-\mathrm{H}\right), 7.59\left(\mathrm{~d}, 1 \mathrm{H}, \mathrm{C}_{5}-\mathrm{H}\right), 10.35\left(\mathrm{~s}, 1 \mathrm{H}, \mathrm{C}_{2}-\mathrm{H}\right)$.

White solid (m. p. $42-45^{\circ} \mathrm{C}$ )

1-decyl-3-methylimidazolium chloride $\left(\mathrm{IMC}_{1} \mathrm{CH}_{2} \mathrm{COOC}_{10}-\mathrm{Cl}\right)(\mathbf{5})$

1H NMR (400 MHz, $\left.\mathrm{CDCl}_{3}\right): \delta=0.83\left(\mathrm{t}, 3 \mathrm{H}, \mathrm{CH}_{3}\right), 1.22\left(\mathrm{~m}, 14 \mathrm{H},\left(\mathrm{CH}_{2}\right)_{7}\right), 1.61(\mathrm{~m}$, $\left.2 \mathrm{H}, \mathrm{COOCH}_{2} \mathrm{CH}_{2}\right), 4.04$ (s, 3H, NCH 3$), 4.13\left(\mathrm{t}, 2 \mathrm{H}, \mathrm{COOCH}_{2}\right), 5.43$ (s, 2H, $\left.\mathrm{NCH}_{2} \mathrm{CO}\right), 7.53$ $\left(\mathrm{d}, 1 \mathrm{H}, \mathrm{C}_{4}-\mathrm{H}\right), 7.58\left(\mathrm{~d}, 1 \mathrm{H}, \mathrm{C}_{5}-\mathrm{H}\right), 10.18\left(\mathrm{~s}, 1 \mathrm{H}, \mathrm{C}_{2}-\mathrm{H}\right)$.

White solid (m. p. $74-77^{\circ} \mathrm{C}$ )

1-dodecyl-3-methylimidazolium chloride $\left(\mathrm{IMC}_{1} \mathrm{CH}_{2} \mathrm{COOC}_{12}-\mathrm{Cl}\right)(\mathbf{6})$

White solid (m. p. $56-58^{\circ} \mathrm{C}$ )

1H NMR (400 MHz, $\left.\mathrm{CDCl}_{3}\right): \delta=0.86\left(\mathrm{t}, 3 \mathrm{H}, \mathrm{CH}_{3}\right), 1.24\left(\mathrm{~m}, 18 \mathrm{H},\left(\mathrm{CH}_{2}\right)\right.$ ) $), 1.63(\mathrm{~m}$, $\left.2 \mathrm{H}, \mathrm{COOCH}_{2} \mathrm{CH}_{2}\right), 4.06$ (s, 3H, NCH 3$), 4.16$ (t, 2H, $\left.\mathrm{COOCH}_{2}\right), 5.45$ (s, 2H, $\left.\mathrm{NCH}_{2} \mathrm{CO}\right), 7.47$ $\left(\mathrm{d}, 1 \mathrm{H}, \mathrm{C}_{4}-\mathrm{H}\right), 7.54\left(\mathrm{~d}, 1 \mathrm{H}, \mathrm{C}_{5}-\mathrm{H}\right), 10.24\left(\mathrm{~s}, 1 \mathrm{H}, \mathrm{C}_{2}-\mathrm{H}\right)$.

\subsection{Biological evaluation.}

\subsubsection{In vitro cytotoxic activity study.}

The cytotoxic activity of studied compounds was evaluated in vitro using the throat cancer Hep-2 cell lines as a test object. The cells were cultivated in RPMI-1640 medium (Sigma, USA) supplemented with cow embryos serum (5\%) and antibiotics at $37{ }^{\circ} \mathrm{C}$ for $72 \mathrm{~h}$. The cytotoxic activity test was carried out in polystyrene 96-well culture plates (Sarstedt, Germany). $100 \mu \mathrm{L}$ of cell suspension in a growth medium at cell density ranging $5 \times 10^{5}$ cells $/ \mathrm{mL}$ was introduced into wells of culture plates and cultured at $37{ }^{\circ} \mathrm{C}$ in an atmosphere of $5 \% \mathrm{CO}_{2}$ for $24 \mathrm{~h}$ until complete cellular monolayer formation.

Studied compounds dissolved in distilled water or $1.5 \%$ DMSO to the final concentration of $2000 \mu \mathrm{g} / \mathrm{mL}$. In a separate plate, serial two-fold and ten-fold dilutions of the studied compounds were prepared. The content of compounds was equal to the concentrations for two-fold dilutions: $1000 ; 500 ; 250 ; 125 ; 62.5 ; 32.25 ; 15.63 ; 7.81 ; 3.91 ; 1.95 ; \mu \mathrm{g} / \mathrm{mL}$ and for ten-fold dilutions: 200.00, 20.00, 2.00, 0.20 , and $0.02 \mu \mathrm{g} / \mathrm{mL}$. The studied compounds were brought into cultural plate holes with the cell culture in a volume of $100 \mu \mathrm{L}$. Cytotoxic effect of compounds was conducted after 24,48 , and $72 \mathrm{~h}$ of incubation with the cellular culture at $37{ }^{\circ} \mathrm{C}$ under $5 \% \mathrm{CO}_{2}$ using the inverted microscope. The cytotoxic parameter, that is, $\mathrm{IC}_{50}$ expressed as the amount of compound $(\mu \mathrm{M})$ which causes cell degeneration in half of the test objects. The cellular degeneration was determined with reference to the control values (without test compound). Cisplatin [67], as a chemotherapy medication used to treat a number of cancers, was tested as the positive control. DMSO (1.5\%) used in our study as a known solvent was tested as the negative control. 


\subsubsection{In vivo acute toxicity assessment.}

The acute toxicity test of the investigated compounds to freshwater organism Daphnia magna was estimated by calculating the lethal concentration ( $\mathrm{LC}_{50}$ ), which is the concentration of a chemical that kills $50 \%$ of the tested organisms. The D. magna tests were performed according to the procedures set out in the Organization for Economic Co-operation and Development Guideline 202 [68] in a light incubator with the $22 \pm 1^{\circ} \mathrm{C}$ temperature and $16: 8-\mathrm{h}$ light:dark photoperiod. D. magna neonates (6-24 h-old) were used for the controls and for the geometric series of concentrations of each test compound according to the 48-h acute toxicity test procedure without food or organic extract. Five neonates were randomly taken and placed in a glass beaker $(50 \mathrm{~mL})$ containing the test solution $(30 \mathrm{~mL})$. The mortality of the neonates in each beaker was observed after $48 \mathrm{~h}$ that is the endpoint for effect calculation. Animals that were not able to swim within $15 \mathrm{~s}$ after gentle agitation of the test vessel were considered to be immobilized. The sensitivity of the D. magna to the reference toxicant potassium dichromate $\left(\mathrm{K}_{2} \mathrm{Cr}_{2} \mathrm{O}_{7}\right)$ was determined as well.

All experiments were run in triplicate. The $\mathrm{LC}_{50}$ values in $\mathrm{mg} / \mathrm{L}$ with their $95 \%$ confidence intervals (CI) were determined using Statistica 7 program. The degree of toxicity of the studied compounds was determined by D.R. Passino with co-authors classification [69].

\subsection{Molecular docking.}

IL docking was performed using the DNA dodecamer (PDB ID: 1BNA) [70] as a known model structure for studying DNA-oriented biologically active compounds. AutoDock Tools (ADT) 1.5.6 [71] was used to prepare DNA fragments and studied ligands for docking. All polar hydrogens were added to the DNA oligomer by ADT. The Gasteiger method was used for the calculation and addition of partial charges. The prepared DNA-dodecamer was saved in PDBQT format. The ChemAxon Marvin Sketch 5.3.735 program [54] was used to make, optimize, and save the ligand structures in Mol2 format. The ligands energy minimization was conducted by the MOPAC 2016 program [72]. The prepared DNA dodecamer and ligands were used for molecular docking using AutoDock Vina 1.1.2 [73]. A grid box of $30 * 30 * 30$ points was used with a grid spacing of $1 \AA$. The analysis and visualization of DNA-ligand interactions were performed by Accelrys DS [74].

\section{Results and Discussion}

\subsection{Results of QSAR modeling.}

The initial set of 1196 compounds was split into training (956 compounds) and test (239 compounds) sets following the Kennard-Stone algorithm described in Materials and Methods. The initial number of descriptors was submitted to an additional reduction procedure, as follows: descriptors with constant values were removed, and a pairwise correlation analysis was then performed, whereby a given descriptor was eliminated if its correlation coefficient with another descriptor was equal to or greater than 0.99. As a result of this process, 1016 descriptors were selected.

In the second stage, the descriptors' contribution to the analyzed activity was evaluated, and the number of descriptors was pruned using our Batch Pruning Algorithm [57]. As a result of descriptors pruning, the BPA selected 66 best fitting descriptors out of 1016. 
Table 1 summarizes the statistical parameters obtained for the final QSAR model. The full computational details of the proposed approach, including the methods used to analyze the results, can be found elsewhere (section Materials and methods) [57].

QSAR model was first developed based on the training set only, and their accuracy was estimated using the cross-validation techniques (Materials and methods). The $\mathrm{q}^{2}$ coefficient for the training set was $0.76, \mathrm{RMSE}=0.42$, MAE $=32$. In the external test set the compounds were predicted with the accuracy, $\mathrm{q}^{2}=0.75, \mathrm{RMSE}=0.42, \mathrm{MAE}=0.32$ (Table 1). Deviation of coefficients ensemble did not exceed 0.01 .

Table 1. Statistical coefficients calculated using ASNN for series of compounds possessing cytotoxicity against throat cancer Hep-2 cell lines.

\begin{tabular}{|c|c|c|c|c|c|}
\hline $\begin{array}{l}\text { Molecule } \\
\text { numbers }\end{array}$ & $\begin{array}{l}\text { Amount of } \\
\text { descriptors }\end{array}$ & Data set & $\mathbf{q}^{2}$ & $\mathbf{R M S E}^{\mathbf{a}}$ & $\mathbf{M A E}^{\mathbf{b}}$ \\
\hline 956 & \multirow{2}{*}{66} & Training set & $0.76 \pm 0.01$ & 0.42 & 0.32 \\
\hline 239 & & Test set & $0.75 \pm 0.01$ & 0.42 & 0.32 \\
\hline
\end{tabular}

The results of predictions for ASNN for the training and test set are given in Figure 1. A good correlation between the predicted and the observed values for the test set indicates that the obtained model can be used for predicting the $\lg \left(1 / \mathrm{IC}_{50}\right)$ within the applicability domain for compounds similar to those in the training and the test sets. In the training set, $97 \%$ of predicted values do not differ from the experimental values by more than $1 \mathrm{lg}$ unit. In the test set, also $98 \%$ of chemicals have residuals between the experimental and predicted $\lg \left(1 / \mathrm{IC}_{50}\right)$ lower than $1 \mathrm{lg}$ unit. This result supports the robustness of the proposed model (Figure 1, Table 1).

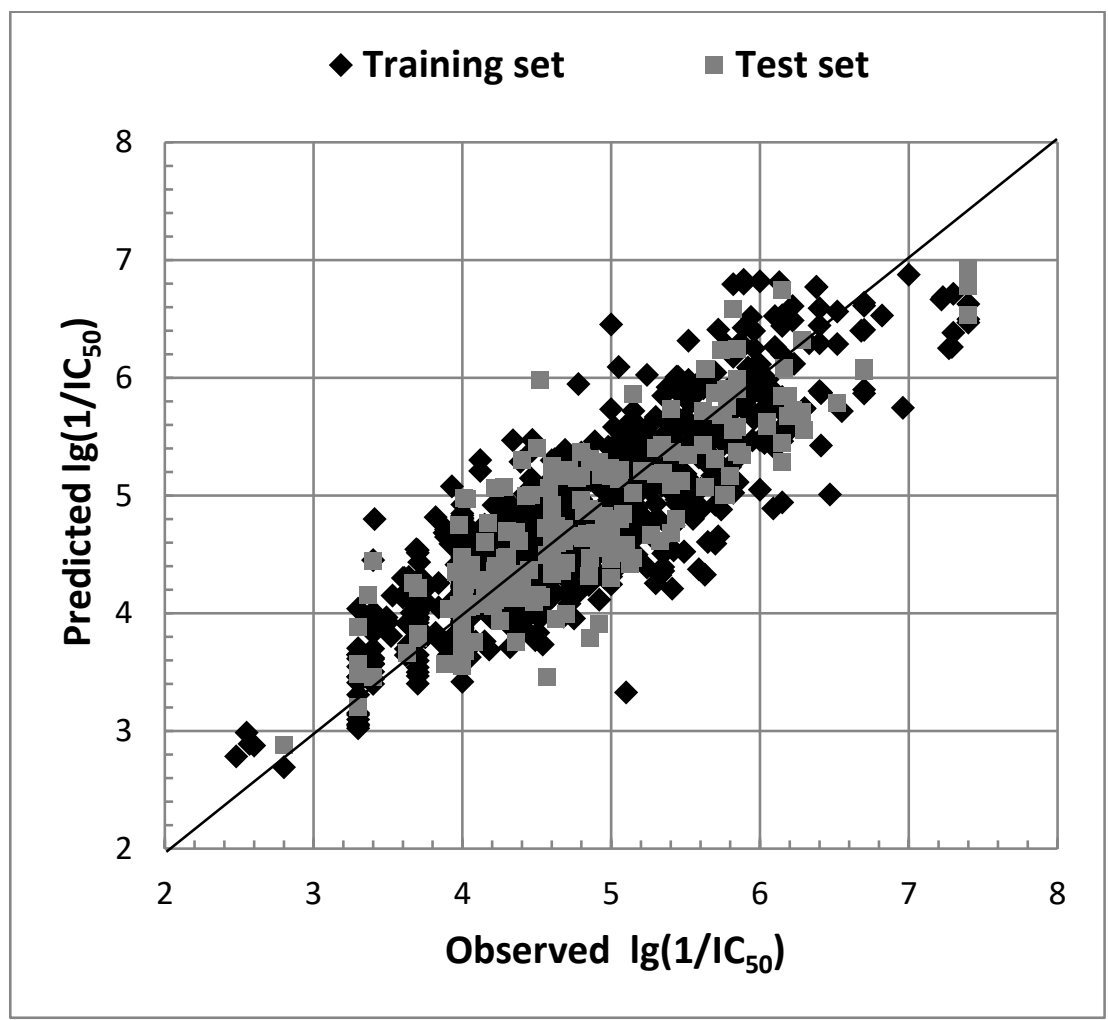

Figure 1. Plots of experimental versus predicted values for the QSAR model data set.

In the present study, we generated virtual sets of drug-like molecules in order to screen potential inhibitors of throat cancer Hep-2 cells. The activity of 12 virtual compounds was predicted using the proposed QSAR model (Supplementary material). The cytotoxic activity 
of 6 compounds was predicted lower than $50 \mu \mathrm{M}$, and the rest compounds were predicted in the range 50 and $100 \mu \mathrm{M}$. The 6 compounds predicted as more active were selected for synthesis and testing. The results of the biological testing confirmed the most QSAR predictions (Table 2).

\subsection{Biological evaluation.}

3.2.1. In vitro evaluating of cytotoxic activity.

Synthesized compounds 1-6 were evaluated in vitro toward the throat cancer Hep-2 cell lines. The results of cytotoxic activity of each water-soluble and DMSO-soluble compound are presented in Table 2.

Table 2. Predicted and experimental cytotoxic activity of studied compounds.

\begin{tabular}{|c|c|c|c|c|c|c|}
\hline \multirow[t]{2}{*}{ № } & \multirow[t]{2}{*}{ Compounds } & \multicolumn{2}{|c|}{ Predicted activity } & \multicolumn{3}{|c|}{$\begin{array}{c}\text { Biological testing of cytotoxic activity by } \mathrm{IC}_{5} \\
(\mu \mathrm{M}) \text { values after } \\
\text { incubation time (hours) }\end{array}$} \\
\hline & & $\lg \left(1 / \mathrm{IC}_{50}\right)$ & $\mathrm{IC}_{50}(\mu \mathrm{M})$ & 24 & 48 & 72 \\
\hline \multirow{2}{*}{1} & \multirow{2}{*}{$\mathrm{PyrCH}_{2} \mathrm{COOC}_{8}-\mathrm{Cl}$} & \multirow{2}{*}{4.31} & \multirow{2}{*}{48.9} & $3898.85 *$ & 1949.4 & 734.37 \\
\hline & & & & $359.43 * *$ & 139.54 & 139.54 \\
\hline \multirow{2}{*}{2} & \multirow{2}{*}{$\mathrm{PyrCH}_{2} \mathrm{COOC}_{10}-\mathrm{Cl}$} & \multirow{2}{*}{4.78} & \multirow{2}{*}{16.6} & 739.14 & 297.48 & 34.13 \\
\hline & & & & 122.82 & 20.54 & 20.54 \\
\hline \multirow{2}{*}{3} & \multirow{2}{*}{$\mathrm{PyrCH}_{2} \mathrm{COOC}_{12}-\mathrm{Cl}$} & \multirow{2}{*}{4.88} & \multirow{2}{*}{13.2} & $\mathrm{ND}^{\mathrm{a}}$ & ND & ND \\
\hline & & & & 0.181 & 0.181 & 0.181 \\
\hline \multirow{2}{*}{4} & \multirow{2}{*}{$\mathrm{IMC}_{1} \mathrm{CH}_{2} \mathrm{COOC}_{8}-\mathrm{Cl}$} & \multirow{2}{*}{4.55} & \multirow{2}{*}{28.2} & 472.79 & 302.22 & 302.22 \\
\hline & & & & 411.58 & 263.64 & 263.64 \\
\hline \multirow{2}{*}{5} & \multirow{2}{*}{$\mathrm{IMC}_{1} \mathrm{CH}_{2} \mathrm{COOC}_{10-} \mathrm{Cl}$} & \multirow{2}{*}{4.43} & \multirow{2}{*}{37.1} & 502.65 & 412.67 & 263.5 \\
\hline & & & & 80.98 & 67.93 & 67.93 \\
\hline \multirow[b]{2}{*}{6} & \multirow{2}{*}{$\mathrm{IMC}_{1} \mathrm{CH}_{2} \mathrm{COOC}_{12}-\mathrm{Cl}$} & \multirow{2}{*}{4.45} & \multirow{2}{*}{35.5} & ND & ND & ND \\
\hline & & & & 5.75 & 5.75 & 5.75 \\
\hline \multirow{2}{*}{7} & \multirow{2}{*}{ Cisplatin } & \multirow{2}{*}{ ND } & \multirow{2}{*}{ ND } & 44.8 & 44.8 & 44.8 \\
\hline & & & & 39.77 & 39.77 & 39.77 \\
\hline
\end{tabular}

The obtained results indicated the highest cytotoxic activity of ester-functionalized pyridinium- and imidazolium ILs with $\mathrm{C}_{12}$ alkyl chains. Compounds $\mathbf{3}$ and $\mathbf{6}$ dissolved in DMSO have the highest cytotoxic activity with $\mathrm{IC}_{50}$ of 0.181 and $5.75 \mu \mathrm{M}$ for $72 \mathrm{~h}$, respectively, compared with standard anticancer agent cisplatin $\left(\mathrm{IC}_{50}=39.77 \mu \mathrm{M}\right)$. Moreover, these effects persisted for 24, 48, and 72 hours of the observed experiment.

It is evident from the dose screening data that compound $\mathbf{5}$ dissolved in DMSO with alkyl chain length $\mathrm{C}_{10}$ was more active with imidazolium cation ( $\mathrm{IC}_{50}$ value of $67.93 \mu \mathrm{M}$ ) than the same compound 4 with $\mathrm{C}_{8}$ alkyl chain length ( $\mathrm{IC}_{50}$ value of $263.64 \mu \mathrm{M}$ ).

The same pattern is observed in cytotoxic effects of the pyridinium salts dissolved in DMSO with alkyl chain lengths $\mathrm{C}_{8}$ and $\mathrm{C}_{10}$. The $\mathrm{IC}_{50}$ value of pyridinium salt 1 with $\mathrm{C}_{8}$ alkyl chain length was $139.54 \mu \mathrm{M}$, and the $\mathrm{IC}_{50}$ value of pyridinium salt with $\mathrm{C}_{10}$ alkyl chain length was $20.54 \mu \mathrm{M}$.

Also, it is worth mentioning that compound 2 with alkyl chain length $\mathrm{C}_{10}$ and pyridinium cation dissolved in $\mathrm{H}_{2} \mathrm{O}$ showed cytotoxicity for $72 \mathrm{~h}$ indicated by $\mathrm{IC}_{50}=34.13 \mu \mathrm{M}$. All other compounds dissolved in $\mathrm{H}_{2} \mathrm{O}$ do not show any improvement in cytotoxic activity. 


\subsubsection{In vivo acute toxicity tests.}

A series of toxicity tests of the studied compounds was carried out, taking into account the promising results of their biological activity.

The results of toxicological effects of studied compounds on D. magna assessed by measuring their lethal effect after 48 hours are presented in Table 3.

Table 3. Acute toxicity of the studied compounds on the mortality of D. magna.

\begin{tabular}{l|l|l|l} 
№ & Compounds & $\begin{array}{l}\text { LC } \\
(48 \mathrm{~h}, \mathrm{mg} / \mathrm{L})\end{array}$ & $95 \% \mathrm{CI}$ \\
\hline 1 & $\mathrm{PyrCH}_{2} \mathrm{COOC}_{8}-\mathrm{Cl}$ & $13.02 \pm 01.71$ & $9.49-16.55$ \\
\hline 2 & $\mathrm{PyrCH}_{2} \mathrm{COOC}_{10}-\mathrm{Cl}$ & $5.82 \pm 0.63$ & $4.51-7.14$ \\
\hline 3 & $\mathrm{PyrCH}_{2} \mathrm{COOC}_{12}-\mathrm{Cl}$ & $0.57 \pm 0.11$ & $0.33-0.79$ \\
\hline 4 & $\mathrm{IMC}_{1} \mathrm{CH}_{2} \mathrm{COOC}_{8}-\mathrm{Cl}$ & $2.13 \pm 0.63$ & $0.85-3.40$ \\
\hline 5 & $\mathrm{IMC}_{1} \mathrm{CH}_{2} \mathrm{COOC}_{10}-\mathrm{Cl}$ & $0.83 \pm 0.20$ & $0.42-1.24$ \\
\hline 6 & $\mathrm{IMC}_{1} \mathrm{CH}_{2} \mathrm{COOC}_{12}-\mathrm{Cl}$ & $0.074 \pm 0.018$ & $0.037-0.11$ \\
\hline 7 & isplatin & $0.15 \pm 0.03$ & $0.082-0.22$
\end{tabular}

The acute toxicity results of tested ILs toward D. magna indicated the dependence of toxicity level on ILs structure (Table 2). The increase of the alkyl chain length from $\mathrm{C}_{8}$ to $\mathrm{C}_{12}$ of all compounds led to the gradual increase of the toxicity level. Thus, the acute toxicity of compounds with $\mathrm{C}_{8}$ species increased from $\mathrm{LC}_{50}$ values 13.02 and $2.13 \mathrm{mg} / \mathrm{L}$ to $\mathrm{LC}_{50}$ values 0.57 and $0.074 \mathrm{mg} / \mathrm{L}$ of compounds with $\mathrm{C}_{12}$ species, respectively. It should be noted that ILs based on pyridinium cation turned out to be the least toxic in contrast with ILs based on imidazolium cation. Generally, according to classification D.R. Passino and S.B. Smith studied ILs in the $D$. magna tests were slightly toxic with $\mathrm{LC}_{50}=13.02 \mathrm{mg} / \mathrm{L}$ (IL 1), moderately toxic with the range of $\mathrm{LC}_{50}$ from 2.13 to $5.82 \mathrm{mg} / \mathrm{L}$ (IL 2 and IL 4), highly toxic with $\mathrm{LC}_{50}=0.57$ $0.83 \mathrm{mg} / \mathrm{L}$ (IL 3 and IL 5) and extremely toxic with LC50 $0.074 \mathrm{mg} / \mathrm{L}$ (IL 6) compared to highly toxic cisplatin $\left(\mathrm{LC}_{50}=0.15 \mathrm{mg} / \mathrm{L}\right)$.

\subsection{Docking study.}

To analyze the potential interaction mechanisms of the most active ligands $\mathbf{3}$ and $\mathbf{6}$, molecular docking was performed in two different regions of the DNA-oligomer 1BNA (Figure 2,3). The features of the formed DNA ligand complexes are presented in Table 4.

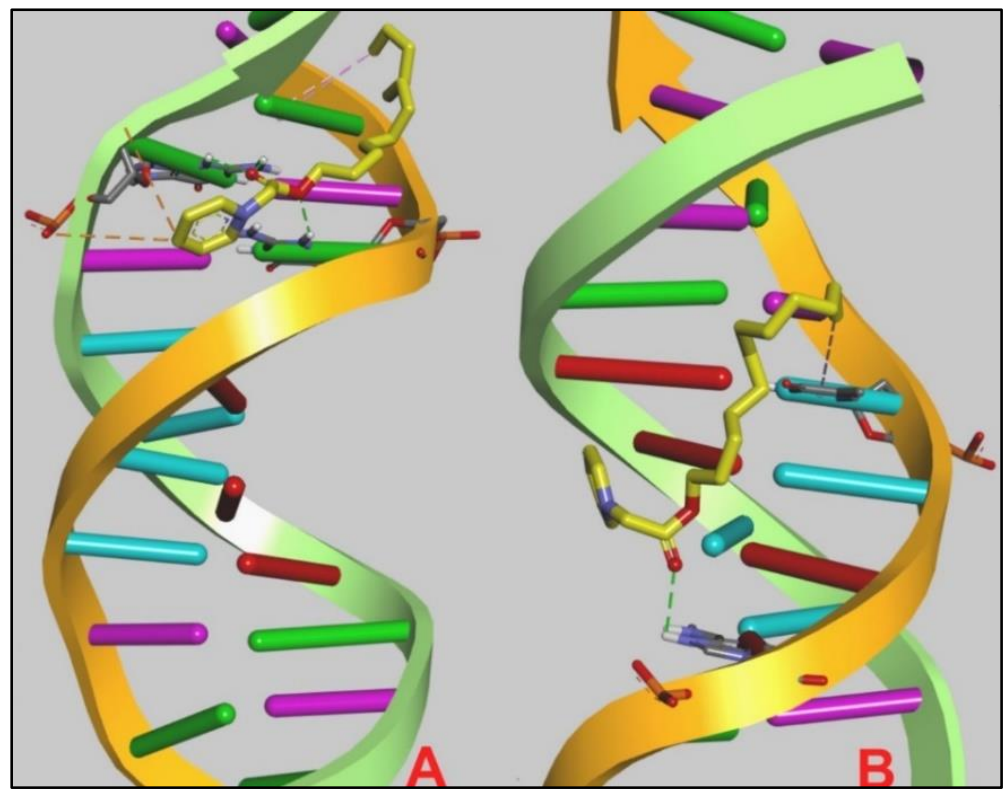

Figure 2. Docking of ligand 3 into the (A) CG; (B) AT base pair regions of DNA oligonucleotide. 


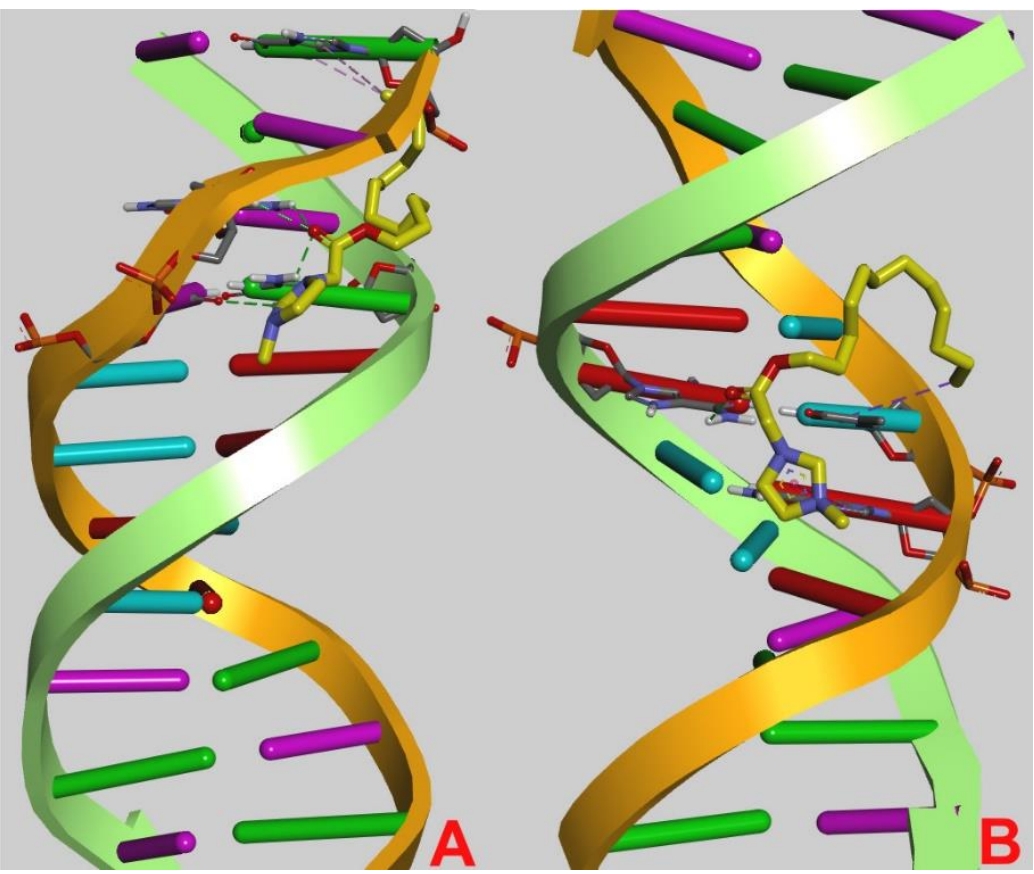

Figure 3. Docking ligand 6 into the (A) CG; (B) AT base pair regions of DNA oligonucleotide.

Table 4. Comparative characteristics of the ligands' interactions with different DNA base pairs.

\begin{tabular}{|c|c|c|c|c|}
\hline $\begin{array}{c}\text { Compound /region } \\
\text { DNA }\end{array}$ & $\begin{array}{c}\Delta \mathrm{G}, \\
(\mathrm{kcal} / \mathrm{mol})\end{array}$ & $\begin{array}{l}\text { Hydrogen } \\
\text { bonds }\end{array}$ & $\begin{array}{l}\text { Electrostatic } \\
\text { interaction }\end{array}$ & $\begin{array}{l}\text { Hydrophobic } \\
\text { interactions }\end{array}$ \\
\hline $3 / \mathrm{A}$ & -6.8 & $\begin{array}{l}\text { DG10 }(2.46 \AA \AA) \\
\text { DG10 }(2.07 \AA \AA) \\
\text { DG16 }(2.25 \AA \AA)\end{array}$ & 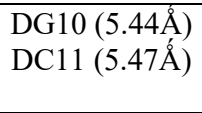 & 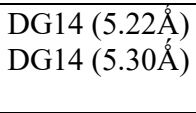 \\
\hline $3 / \mathrm{B}$ & -5.1 & 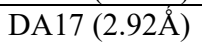 & & DT20 (5.48Å) \\
\hline $6 / \mathrm{A}$ & -6.7 & $\begin{array}{c}\text { DG10 }(2.21 \AA \AA) \\
\text { DG10 }(2.68 \AA \AA) \\
\text { DG16 }(2.24 \AA \AA) \\
\text { DG9 }(3.54 \AA \AA)\end{array}$ & & 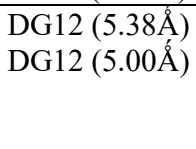 \\
\hline $6 / \mathrm{B}$ & -5.0 & DA6 (2.38Å) & & 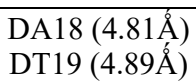 \\
\hline
\end{tabular}

The docking results indicate that the CG region of the DNA-oligonucleotide is the most energetically favorable for the complexation of the studied ligands (Figure 2,3 and Table 4). The formation of ligand-DNA complexes is accompanied by estimated binding energies in the ranges from -6.7 to $-6.8 \mathrm{kcal} / \mathrm{mol}$ (CG base pair region) and from -5.0 to $-5.1 \mathrm{kcal} / \mathrm{mol}$ (AT base pair region). Also, the ligand-DNA complexes in the CG region stabilize by multiple

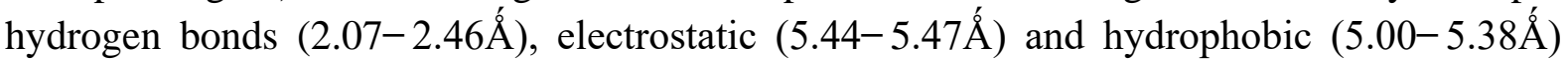
interactions. At the same time, the ligand-DNA complexes in the AT region were stabilized by

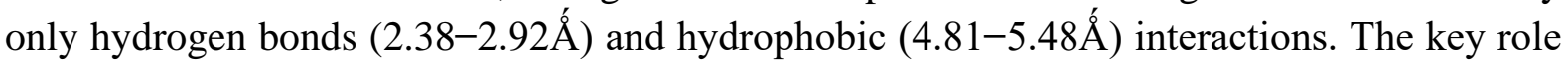
in complexation in the CG base pair region belongs to DG9, DG10, DC11, DG12, DG14, DG16 nucleotide bases of DNA.

\section{Conclusions}

In this study, long-chain pyridinium- and imidazolium-based ionic liquids, comprising polar ester groups in the alkyl substituents, have been designed, synthesized, and evaluated in vitro and in vivo as potential anticancer agents. The preliminary prediction of cytotoxic activity of ionic liquids by constructed regression QSAR model revealed the most active compounds from a small set of virtual compounds for synthesis and in vitro testing. The obtained results indicated that most studied compounds showed a significant cytotoxic effect on the human 
Hep-2 cell line. Ester-functionalized ILs based on imidazolium and pyridinium cation with $\mathrm{C}_{12}$ and $\mathrm{C}_{10}$ alkyl chain length demonstrated high cytotoxic potential on Hep-2 cell line and different acute toxicity levels to aquatic commonly used model organism $D$. magna. It should be noted that salts 3 and 6 ( $\mathrm{IC}_{50}=0.181 \mu \mathrm{M}$ and $5.75 \mu \mathrm{M}$ ) showed extremely potent cytotoxicity compared to cisplatin $\left(\mathrm{IC}_{50}=39.77 \mu \mathrm{M}\right)$. The acute toxicity results of tested ILs displayed that in $D$. magna tests in contrast to highly toxic cisplatin, compounds $\mathbf{1 - 5}$ were less toxic (slightly toxic, moderately toxic, and highly toxic) except extremely toxic compound 6 according to classification D.R. Passino and S.B. Smith. The established ILs properties are associated with their structural features. The degree of hydrophobicity of the alkyl chain and cationic type of functionalized salts are important in their activity potential as highly active and low toxic anticancer agents with specific DNA binding mechanisms associated with the CG base pair region.

\section{Funding}

This research received no external funding.

\section{Acknowledgments}

This research has no acknowledgment.

\section{Conflicts of Interest}

The authors declare no conflict of interest

\section{References}

1. Pucci, C.; Martinelli, C.; Ciofani, G. Innovative approaches for cancer treatment: Current perspectives and new challenges. Ecancermedicalscience 2019, 13, 961, https://doi.org/10.3332/ecancer.2019.961.

2. Chikara, S.; Nagaprashantha, L.D.; Singhal, J.; Horne, D.; Awasthi, S.; Singhal, S.S. Oxidative stress and dietaryphytochemicals: Role in cancer chemoprevention and treatment. Cancer Lett. 2018, 413, 122-134, https://doi.org/10.1016/j.canlet.2017.11.002.

3. Kurzrock, R.; Kantarjian, H.M.; Kesselheim, A.S.; Sigal, E.V. New drug approvals in oncology. Nature Reviews Clinical Oncology 2020, 17, 140-146, https://doi.org/10.1038/s41571-019-0313-2.

4. Wan, M.; Zhang, L.; Chen, Y.; Li, Q.; Fan, W.; Xue, Q.; Yan, F.; Song, W. Synthesis and Anticancer Activity Evaluation of Novel Phenanthridine Derivatives. Front. Oncol. 2019, 9, 274, https://doi.org/10.3389/fonc.2019.00274.

5. Haq, S.H.; Al-Ruwaished, G.; Al-Mutlaq, M.A.; Naji, S.A.; Al-Mogren, M.; Al-Rashed, S.; Ain, Q.T.; AlAmro, A.A.; Al-Mussallam, A. Antioxidant, Anticancer Activity and Phytochemical Analysis of Green Algae, Chaetomorpha Collected from the Arabian Gulf. Sci. Rep. 2019, 9, 18906, https://doi.org/10.1038/s41598-019-55309-1.

6. Pedro, S.N.; Freire, C.S.R.; Silvestreand, AJ.D.1 Freire M.G. The Role of Ionic Liquids in the Pharmaceutical Field:An Overview of Relevant Applications. Int. J. Mol. Sci. 2020, 21, 8298, https://doi.org/10.3390/ijms21218298.

7. Shamshina, J.L.; Berton, P.; Wang, H.; Zhou, X.; Gurau, G.; Rogers, RD. Ionic Liquids in Pharmaceutical Industry. Green Techniques for Organic Synthesis and Medicinal Chemistry 2018, https://doi.org/10.1002/9781119288152.ch20.

8. Amaral, M.; Pereiro, A.B.; Gaspar, M.M;. Reis, C.P. Recent advances in ionic liquids and nanotechnology for drug delivery. Nanomedicine (Lond) 2021, 16, 63-80, https://doi.org/10.2217/nnm-2020-0340.

9. Ferraz, R.; Silva, D.; Dias, A.; Dias, V.; Santos, M.M.; Pinheiro, L.; Prudêncio, C.; Noronha, J.; Petrovski, Ž.; Branco, L. Synthesis and Antibacterial Activity of Ionic Liquids and Organic Salts Based on Penicillin G and Amoxicillin hydrolysate Derivatives against Resistant Bacteria. Pharmaceutics 2020, 12, https://doi.org/10.3390/pharmaceutics12030221. 
10. Karaman, M.;Vraneš, M.; Tot, A.; Papović, S.; Miljaković, D.; Gadžurić, S.; Ignjatov, M. Ionic liquids as potentially new antifungal agents against Alternaria species. RSC Advances 2020, 10, 22318-22323, https://doi.org/10.1039/D0RA02475A.

11. Kiran Kumar Reddy, G.; Nancharaiah, Y.V. Alkylimidazolium Ionic Liquids as Antifungal Alternatives: Antibiofilm Activity Against Candida albicans and Underlying Mechanism of Action. Frontiers in Microbiology 2020, 11, https://doi.org/10.3389/fmicb.2020.00730.

12. Jovanović-Šanta, S. Anticancer and antimicrobial properties of imidazolium based ionic liquids with salicylate anion. J. Serb. Chem. Soc. 2020, 85, 291-303, https://doi.org/10.2298/JSC190717098J.

13. Rahman, M.; O’Donnell, J.M.; Brazel, C.S. Cytotoxicity of Plasticizers and Ionic Liquids Using Drosophila melanogaster S2 Cell Culture. Chem. Eng. Technol. 2011, 34, 429-438, https://doi.org/10.1002/ceat.201000199.

14. Radošević, K.; Železnjak, J.; Cvjetko Bubalo, M.; Radojčić Redovniković, I.; Slivac, I.; Gaurina Srček, V. Comparative In Vitro Study of Cholinium-Based Ionic Liquids and Deep Eutectic Solvents toward Fish Cell Line. Ecotoxicol. Environ. Saf. 2016, 131, 30-36, https://doi.org/10.1016/j.ecoenv.2016.05.005.

15. Egorova, K.S.; Seitkalieva, M.M.; Posvyatenko, A.V.; Ananikov, V.P. An Unexpected Increase of Toxicity of Amino Acid-Containing Ionic Liquids. Toxicol. Res. 2015, 4, 152-159, https://doi.org/10.1039/C4TX00079J.

16. Yu, J.; Zhang, S.; Dai, Y.; Lu, X.; Lei, Q.; Fang, W. Antimicrobial Activity and Cytotoxicity of Piperaziniumand Guanidinium-Based Ionic Liquids J. Hazard. Mater. 2016, 307, 73-81, https://doi.org/10.1016/j.jhazmat.2015.12.028.

17. Steudte, S.; Stepnowski, P.; Cho, C.W.; Thöming, J.; Stolte, S. (Eco)toxicity of Fuoro-Organic and CyanoBased Ionic Liquid Anions. Chem. Commun. 2012, 48, 9382-9384, https://doi.org/10.1039/c2cc34955h.

18. Li, X.Y.; Jing, C.Q.; Zang, X.Y.; Yang, S.; Wang, J.J. Toxic Cytological Alteration and Mitochondrial Dysfunction in PC12 Cells Induced by 1-octyl-3-methylimidazolium Chloride. Toxicol. In Vitro. 2012, 26, 1087-1092, https://doi.org/10.1016/j.tiv.2012.07.006.

19. Malhotra, S.V.; Kumar, V.A. Profile of the In vitro Anti-Tumor Activity of Imidazolium-Based Ionic Liquids. Bioorg. Med. Chem. Lett. 2010, 20, 581-585, https://doi.org/10.1016/j.bmcl.2009.11.085.

20. Malhotra, S.V.; Kumar, V.; Velez, C.; Zayas, B. Imidazolium-Derived Ionic Salts Induce Inhibition of Cancerous Cell Growth through Apoptosis. MedChemComm. 2014, 5, 1404-1409, https://doi.org/10.1039/C4MD00161C.

21. Egorova, K.S.; Seitkalieva, M.M.; Posvyatenko, A.V.; Khrustalev, V.N.; Ananikov, V.P. Cytotoxic Activity of Salicylic Acid-Containing Drug Models with Ionic and Covalent Binding ACS Med. Chem. Lett. 2015, 6, 1099-1104, https://doi.org/10.1021/acsmedchemlett.5b00258.

22. Gouveia, W.; Jorge, T.F.; Martins, S.; Meireles, M.; Carolino, M.; Cruz, C.; Almeida, T.V.; Araújo, M.E.M. Toxicity of ionic liquids prepared from biomaterials. Chemosphere 2014, 104, 51-56, https://doi.org/10.1016/j.chemosphere.2013.10.055.

23. Messali, M.; Almtiri, M.N.; Abderrahman, B.; Salghi, R.; Aouad, M.R.; Alshahateet, S.F.; Ali, A.A.S. New pyridazinium-based ionic liquids: An eco-friendly ultrasound-assisted synthesis, characterization and biological activity. S. Afr. J. Chem. 2015, 68, 219-225, https://doi.org/10.17159/0379-4350/2015/v68a30.

24. Wang, C.L.; Zhu, X.W.; Liu, S.S. Toxicity Studies of Ionic Liquids and Heavy Metal Compounds to MCF-7 and Photobacteria Q67. Adv. Mater. Res. 2013, 610-613, 721-724, https://doi.org/10.4028/www.scientific.net/AMR.610-613.721.

25. Kaushik, N.K.; Attri, P.; Kaushik, N.; Choi, E.H. Synthesis and Antiproliferative Activity of Ammonium and Imidazolium Ionic Liquids against T98G Brain Cancer Cells. Molecules 2012, 17, 13727-13739, https://doi.org/10.3390/molecules171213727.

26. Kumar, V.; Malhotra, S.V. Study on the Potential Anti-Cancer Activity of Phosphonium and AmmoniumBased Ionic Liquids. Bioorg. Med. Chem. Lett. 2009, 19, 4643-4646, https://doi.org/10.1016/j.bmcl.2009.06.086.

27. Flieger, J.; Flieger, M. Ionic Liquids Toxicity-Benefits and Threats. Int J Mol Sci. 2020, 21, 6267, https://doi.org/10.3390/ijms21176267.

28. Egorova, K.S.; Seitkalieva, M.M.; Posvyatenko, A.V.; Ananikov, V.P. An Unexpected Increase of Toxicity of Amino Acid-Containing Ionic Liquids. Toxicol. Res. 2015, 4, 152-159, https://doi.org/10.1039/C4TX00079J.

29. Pérez, S.A.; Montalbán, M.G.; Carissimi, G.; Licence, P.; Víllora, G. In vitro cytotoxicity assessment of monocationic and dicationic pyridinium-based ionic liquids on HeLa, MCF-7, BGM and EA.hy926 cell lines. Journal of Hazardous Materials 2020, 385, 121513, https://doi.org/10.1016/j.jhazmat.2019.121513.

30. Gal, N.; Malferrari, D.; Kolusheva, S.; Galletti, P.; Tagliavini, E.; Jelinek, R. Membrane Interactions of Ionic Liquids: Possible Determinants for Biological Activity and Toxicity. Biochim. Biophys. Acta 2012, 1818, 2967-2974, https://doi.org/10.1016/j.bbamem.2012.07.025. 
31. Jing, B.; Lan, N.; Qiu, J.; Zhu, Y. Interaction of Ionic Liquids with a Lipid Bilayer: A Biophysical Study of Ionic Liquid Cytotoxicity. J. Phys. Chem. B 2016, 120, 2781-2789, https://doi.org/10.1021/acs.jpcb.6b00362.

32. Benedetto, A.; Ballone, P. Room Temperature Ionic Liquids Interacting with Bio-Molecules: An Overview of Experimental and Computational Studies. Philos. Mag. 2016, 96, 870-894, https://doi.org/10.1080/14786435.2015.1119323.

33. McLaughlin, M.; Earle, M.J.; Gîlea, M.A.; Gilmore, B.F.; Gorman, S.P.; Seddon, K.R. Cytotoxicity of 1Alkylquinolinium Bromide Ionic Liquids in Murine Fibroblast NIH 3T3 Cells. Green Chem. 2011, 13, 27942800, https://doi.org/10.1039/c0gc00813c.

34. Li, X.Y.; Jing, C.Q.; Lei, W.L.; Li, J.; Wang, J.J. Apoptosis Caused by Imidazolium-Based Ionic Liquids in PC12 Cells. Ecotoxicol. Environ. Saf. 2012, 83, 102-107, https://doi.org/10.1016/j.ecoenv.2012.06.013.

35. Wang, X.; Ohlin, C.A.; Lu, Q.; Fei, Z.; Hu, J.; Dyson, P.J. Cytotoxicity of Ionic Liquids and Precursor Compounds towards Human Cell Line HeLa. Green Chem. 2007, 9, 1191-1197, https://doi.org/10.1039/b704503d.

36. Li, X.; Ma, J.; Wang, J. Cytotoxicity, Oxidative Stress, and Apoptosis in HepG2 Cells Induced by Ionic Liquid 1-methyl-3-octylimidazolium Bromide. Ecotoxicol. Environ. Saf. 2015, 120, 342-348, https://doi.org/10.1016/j.ecoenv.2015.06.018.

37. Li, X.; Ma, J.; Jing, C.; Wang, J. Expression Alterations of Cytochromes P4501A1, 2E1 and 3A, and Their Receptors AhR and PXR Caused by 1-Octyl-3-methylimidazolium Chloride in Mouse Mammary Carcinoma Cells. Chemosphere 2013, 93, 2488-2492, https://doi.org/10.1016/j.chemosphere.2013.08.092.

38. Rezki, N.; Al-Blewi, F.F.; Al-Sodies, S.A.; Alnuzha, A.K.; Messali, M.; Ali, I.; Aouad, M.R. Synthesis, Characterization, DNA Binding, Anticancer, and Molecular Docking Studies of Novel Imidazolium-Based Ionic Liquids with Fluorinated Phenylacetamide Tethers. ACS Omega 2020, 10, 4807-4815, https://doi.org/10.1021/acsomega.9b03468.

39. Bonsignore, R.; Notaro, A.; Salvo, A.M.P.; Spinello, A.; Fiasconaro, G.; Terenzi, A.; Giacalone, F.; Keppler, B.K.; Giuliano, M.; Gruttadauria, M.; Barone, G. DNA-Binding and Anticancer Activity of PyreneImidazolium Derivatives. ChemistrySelect 2016, 1, 6755-6761, https://doi.org/10.1002/slct.201601502.

40. Al-Sodies, S.; Aouad, M.; Ihmaid, S.; Aljuhani, A.; Messali, M.; Ali, I.; Rezki, N. Microwave and conventional synthesis of ester based dicationic pyridinium ionic liquids carrying hydrazone linkage: DNA binding, anticancer and docking studies. J. Mol. Struct. 2020, 1207, 127756, https://doi.org/10.1016/j.molstruc.2020.127756.

41. Chen, H-L.; Kao, H-F.; Wang, J-Y.; Wei, G-T. Cytotoxicity of imidazole ionic liquids in human lung carcinoma A549 cell line. J. Chin. Chem. Soc. 2014, 61, 763-769, https://doi.org/10.1002/jccs.201300632.

42. Marrucho, I.M.; Branco, L.C.; Rebelo, L.P.N. Ionic liquids in pharmaceutical applications. Annu. Rev. Chem. Biomol. Eng. 2014, 5, 527-546, https://doi.org/10.1146/annurev-chembioeng-060713-040024.

43. Przestalski, S.; Sarapuk, J.; Kleszczyńska, H.; Gabrielska, J.; Hładyszowski, J.; Trela, Z.; Kuczera, J. Influence of amphiphilic compounds on membranes. Acta Biochim Pol. 2000, 47, 627-638.

44. Stolte, S.; Matzke, M.; Arning, J.; Böschen, A.; Pitner, W.-R.; Welz-Biermann, U.; Jastorff, B.; Ranke, J. Effects of different head groups and functionalised side chains on the aquatic toxicity of ionic liquids. Green Chem. 2007, 9, 1170-1179, https://doi.org/10.1039/B711119C.

45. Trush, M.; Metelytsia, L.; Semenyuta, I.; Kalashnikova, L.; Papeykin, O.; Venger, I.; Tarasyuk, O.; Bodachivska, L.; Blagodatnyi, V.; Rogalsky, S. Reduced ecotoxicity and improved biodegradability of cationic biocides based on ester-functionalized pyridinium ionic liquids. Environmental Science and Pollution Research 2019, 26, 4878-4889, https://doi.org/10.1007/s11356-018-3924-8.

46. Torrecilla, J.S.; Garcia, J.; Rojo, E.; Rodriguez, F. Estimation of Toxicity of Ionic Liquids in Leukemia Rat Cell Line and Acetylcholinesterase Enzyme by Principal Component Analysis, Neural Networks and Multiple Lineal Regressions. J. Hazard. Mater. 2009, 164, 182-194, https://doi.org/10.1016/j.jhazmat.2008.08.022.

47. Fatemi, M.H.; Izadiyan, P. Cytotoxicity Estimation of Ionic Liquids Based on Their Effective Structural Features. Chemosphere 2011, 84, 553-563, https://doi.org/10.1016/j.chemosphere.2011.04.021.

48. Paternò, A.; D’Anna, F.; Musumarra, G.; Noto, R.; Scirè, S. A Multivariate Insight into Ionic Liquids Toxicities. RSC Adv. 2014, 4, 23985-24000, https://doi.org/10.1039/c4ra03230f.

49. Sosnowska, A.; Barycki, M.; Zaborowska, M.; Rybinska, A.; Puzyn, T. Towards Designing Environmentally Safe Ionic Liquids: the Influence of the Cation Structure. Green Chem. 2014, 16, 4749-4757, https://doi.org/10.1039/C4GC00526K.

50. De Melo, E.B. A Structure-Activity Relationship Study of the Toxicity of Ionic Liquids Using an Adapted Ferreira-Kiralj Hydrophobicity Parameter Phys. Chem. Chem. Phys. 2015, 17, 4516-4523, https://doi.org/10.1039/C4CP04142A.

51. ChEMBL. https://www.ebi.ac.uk/chembl/ (accessed on 12 January 2021).

52. Kennard, R.W.; Stone, L.A. Computer aided design of experiments. Technometrics 1969, 11, 137-147, https://doi.org/10.2307/1266770. 
53. Kovalishyn, V.; Kopernyk, I.; Chumachenko, S.; Shablykin, O.; Kondratyuk, K.; Pil'o, S.; Prokopenko, V.; Brovarets, V.; Metelytsia, L. QSAR studies, design, synthesis and antimicrobial evaluation of azole derivatives. Comput. Biol. Bioinfor 2014, 2, 25-32, https://doi.org/10.11648/j.cbb.20140202.12.

54. ChemAxon. https://www.chemaxon.com/ (accessed on 12 January 2021).

55. Talete SRL. http://www.talete.mi.it/products/dragon_description.htm (accessed on 1 April 2020).

56. Todeschini, R.; Consonni, V. Handbook of Molecular Descriptors. Weinheim: Wiley-VCH Verlag GmbH; 2008.

57. Kovalishyn, V.; Poda, G. Efficient Variable Selection Batch Pruning Algorithm for Artificial Neural Networks. Int. Lab. Sys. 2015, 149, 10-16, https://doi.org/10.1016/j.chemolab.2015.10.005.

58. Tetko, I.V. Neural network studies. 4. Introduction to associative neural network. J. Chem. Inf. Comput. Sci. 2002, 42, 717-728, https://doi.org/10.1021/ci010379o.

59. Kohonen, T. Self-Organizing Maps. Berlin: Springer-Verlag; 2001; 501, http://dx.doi.org/10.1007/978-3642-56927-2.

60. Tetko, I.V.; Villa, A.E.P.; Livingstone, D.J. Neural network studies. 2. Variable selection. J. Chem. Inf. Comput. Sci. 1996, 36, 794-803, https://doi.org/10.1021/ci950204c.

61. Kovalishyn, V.V.; Tetko, I.V.; Luik, A.I.; Kholodovych, V.V.; Villa, A.E.P.; Livingstone, D.J. Neural network studies. 3. Variable selection in the cascade-correlation learning architecture. J. Chem. Inf. Comput. Sci. 1998, 38, 651-659, https://doi.org/10.1021/ci980325n.

62. Dasarathy, B.V. Nearest neighbor (NN) norms: NN pattern classification techniques. Washington: IEEE Computer Society Press; 1991, 447.

63. Tetko, I.V.; Livingstone, D.J.; Luik, A.I. Neural Network Studies. 1. Comparison of Overfitting and Overtraining. J. Chem. Inf. Comput. Sci. 1995, 35, 826-833, https://doi.org/10.1021/ci00027a006.

64. Tollenaere, T. Super SAB: Fast adaptive back propagation with good scaling properties. Neural Networks. 1990, 3, 561-573, https://doi.org/10.1016/0893-6080(90)90006-7.

65. Johnson, J.L.; Leone, F.C. Statistic and Experimental Design in Engineering and the Physical Sciences. New York: John Wiley \& Sons; 1977.

66. Cramer, R.D.; Patterson, D.E.; Bunce, J.D. Comparative molecular field analysis (CoMFA). 1. Effect of shape on binding of steroids to carrier proteins. $J$ Am Chem Soc. 1988, 110, 5959-67, https://doi.org/10.1021/ja00226a005.

67. Aldossary S.A. Review on Pharmacology of Cisplatin: Clinical Use, Toxicity and Mechanism of Resistance of Cisplatin. Biomed Pharmacol J 2019, 12, https://dx.doi.org/10.13005/bpj/1608.

68. OECD. OECD Guidelines for the testing of chemicals, Section 2: Effects on Biotic Systems. Test No. 202: Daphnia sp. Acute Immobilisation Test. 2004, https://doi.org/10.1787/20745761.

69. Passino, D.R.; Smith, S.B. Acute bioassays and hazard evaluation of representative contaminants detected in Great Lakes fish. Environ Toxicol Chem. 1987, 6, 901-7, https://doi.org/10.1002/etc.5620061111.

70. Protein Data Bank. http://www.rcsb.org/structure/1BNA (accessed on 1 February 2021).

71. Sanner, M.F. Python: A programming language for software integration and development. J. Mol. Graph. Model. 1999, 17, 57-61.

72. Stewart, J.J.P. MOPAC2016. Stewart Computational Chemistry. Colorado Springs, CO, USA, HTTP://OpenMOPAC.net (2016). (accessed on 1 February 2021).

73. Trott, O.; Olson, A.J. AutoDock Vina: improving the speed and accuracy of docking with a new scoring function, efficient optimization, and multithreading. J. Comput. Chem. 2010, 31, 455-461, https://doi.org/10.1002/jcc.21334.

74. Dassault Systèmes BIOVIA. Discovery Studio Visualizer, v4.0.100.13345. San Diego: Dassault Systèmes; 2020. 


\section{Supplementary materials}

Table S1. Initial data set.

\begin{tabular}{|c|c|c|}
\hline NAME & SMILE & $\lg (1 / \mathbf{I C 5 0})$ \\
\hline 1 & $\mathrm{ONC}(=\mathrm{O}) \mathrm{NC}(\mathrm{C}(=\mathrm{O}) \mathrm{NC}(\mathrm{C} 1=\mathrm{CC}=\mathrm{CC}=\mathrm{C} 1) \mathrm{C} 1=\mathrm{CC}=\mathrm{CC}=\mathrm{C} 1) \mathrm{C} 1=\mathrm{CC}=\mathrm{CC}=\mathrm{C} 1$ & 4,08 \\
\hline 2 & $\mathrm{ONC}(=\mathrm{O}) \mathrm{NC}(\mathrm{CC} 1=\mathrm{CC}=\mathrm{CC}=\mathrm{C} 1) \mathrm{C}(=\mathrm{O}) \mathrm{NC}(\mathrm{C} 1=\mathrm{CC}=\mathrm{CC}=\mathrm{C} 1) \mathrm{C} 1=\mathrm{CC}=\mathrm{CC}=\mathrm{C} 1$ & 4,21 \\
\hline 3 & $\mathrm{CCC}(\mathrm{C}) \mathrm{C}(\mathrm{NC}(=\mathrm{O}) \mathrm{NO}) \mathrm{C}(=\mathrm{O}) \mathrm{NC}(\mathrm{C} 1=\mathrm{CC}=\mathrm{CC}=\mathrm{C} 1) \mathrm{C} 1=\mathrm{CC}=\mathrm{CC}=\mathrm{C} 1$ & 4,45 \\
\hline 4 & $\mathrm{O}=\mathrm{C}(\mathrm{NC} 1 \mathrm{CCCC} 1) \mathrm{C}(\mathrm{CC} 1=\mathrm{CC}=\mathrm{CC}=\mathrm{C} 1) \mathrm{NC}(=\mathrm{O}) \mathrm{N} 1 \mathrm{~N}=\mathrm{NC} 2=\mathrm{CC}=\mathrm{CC}=\mathrm{C} 12$ & 4,02 \\
\hline 5 & $\mathrm{ONC}(=\mathrm{O}) \mathrm{NC}(\mathrm{C}(=\mathrm{O}) \mathrm{NCC} 1=\mathrm{CC}=\mathrm{CC}=\mathrm{C} 1) \mathrm{C} 1=\mathrm{CC}=\mathrm{CC}=\mathrm{C} 1$ & 4,49 \\
\hline 6 & $\mathrm{O}=\mathrm{C}(\mathrm{NC}(\mathrm{C} 1=\mathrm{CC}=\mathrm{CC}=\mathrm{C} 1) \mathrm{C} 1=\mathrm{CC}=\mathrm{CC}=\mathrm{C} 1) \mathrm{C}(\mathrm{NC}(=\mathrm{O}) \mathrm{N} 1 \mathrm{~N}=\mathrm{NC} 2=\mathrm{CC}=\mathrm{CC}=\mathrm{C} 12) \mathrm{C} 1=\mathrm{CC}=\mathrm{CC}=\mathrm{C} 1$ & 4,23 \\
\hline 7 & $\mathrm{CC}(\mathrm{C}) \mathrm{C}(\mathrm{NC}(=\mathrm{O}) \mathrm{NO}) \mathrm{C}(=\mathrm{O}) \mathrm{NC} 1 \mathrm{CCCC} 1$ & 4,48 \\
\hline 8 & $\mathrm{O}=\mathrm{C}(\mathrm{NC}(\mathrm{C}(=\mathrm{O}) \mathrm{N} 1 \mathrm{CCCCC} 1) \mathrm{C} 1=\mathrm{CC}=\mathrm{CC}=\mathrm{C} 1) \mathrm{N} 1 \mathrm{~N}=\mathrm{NC} 2=\mathrm{C} 1 \mathrm{C}=\mathrm{CC}=\mathrm{C} 2$ & 4 \\
\hline 9 & $\mathrm{CC}(\mathrm{C}) \mathrm{C} 1 \mathrm{NC}(=\mathrm{O}) \mathrm{N}(\mathrm{C} 2 \mathrm{CCCC} 2) \mathrm{C} 1=\mathrm{O}$ & 4,01 \\
\hline 10 & $\mathrm{O}=\mathrm{C} 1 \mathrm{NC}(\mathrm{C}(=\mathrm{O}) \mathrm{N} 1 \mathrm{CC} 1=\mathrm{CC}=\mathrm{CC}=\mathrm{C} 1) \mathrm{C} 1=\mathrm{CC}=\mathrm{CC}=\mathrm{C} 1$ & 4,08 \\
\hline 11 & $\mathrm{O}=\mathrm{C}(\mathrm{NC}(\mathrm{CC} 1=\mathrm{CC}=\mathrm{CC}=\mathrm{C} 1) \mathrm{C}(=\mathrm{O}) \mathrm{N} 1 \mathrm{CCCC} 1) \mathrm{N} 1 \mathrm{~N}=\mathrm{NC} 2=\mathrm{CC}=\mathrm{CC}=\mathrm{C} 12$ & 4 \\
\hline 12 & $\mathrm{ONC}(=\mathrm{O}) \mathrm{NC}(\mathrm{C}(=\mathrm{O}) \mathrm{N} 1 \mathrm{CCCCC} 1) \mathrm{C} 1=\mathrm{CC}=\mathrm{CC}=\mathrm{C} 1$ & 4 \\
\hline 13 & $\mathrm{O}=\mathrm{C}(\mathrm{NCC} 1=\mathrm{CC}=\mathrm{CC}=\mathrm{C} 1) \mathrm{C}(\mathrm{CC} 1=\mathrm{CC}=\mathrm{CC}=\mathrm{C} 1) \mathrm{NC}(=\mathrm{O}) \mathrm{N} 1 \mathrm{~N}=\mathrm{NC} 2=\mathrm{CC}=\mathrm{CC}=\mathrm{C} 12$ & 4,09 \\
\hline 14 & $\mathrm{CC}(\mathrm{C}) \mathrm{C}(\mathrm{NC}(=\mathrm{O}) \mathrm{N} 1 \mathrm{~N}=\mathrm{NC} 2=\mathrm{CC}=\mathrm{CC}=\mathrm{C} 12) \mathrm{C}(=\mathrm{O}) \mathrm{NC} 1 \mathrm{CCCCC} 1$ & 4,22 \\
\hline 15 & $\mathrm{O}=\mathrm{C}(\mathrm{NC}(\mathrm{C} 1=\mathrm{CC}=\mathrm{CC}=\mathrm{C} 1) \mathrm{C} 1=\mathrm{CC}=\mathrm{CC}=\mathrm{C} 1) \mathrm{C}(\mathrm{CC} 1=\mathrm{CC}=\mathrm{CC}=\mathrm{C} 1) \mathrm{NC}(=\mathrm{O}) \mathrm{N} 1 \mathrm{~N}=\mathrm{NC} 2=\mathrm{CC}=\mathrm{CC}=\mathrm{C} 12$ & 4,39 \\
\hline 16 & $\mathrm{O}=\mathrm{C} 1 \mathrm{NC}(\mathrm{C}(=\mathrm{O}) \mathrm{N} 1 \mathrm{C} 1 \mathrm{CCCC} 1) \mathrm{C} 1=\mathrm{CC}=\mathrm{CC}=\mathrm{C} 1$ & 4 \\
\hline 17 & $\mathrm{CCC}(\mathrm{C}) \mathrm{C}(\mathrm{NC}(=\mathrm{O}) \mathrm{NO}) \mathrm{C}(=\mathrm{O}) \mathrm{NC} 1 \mathrm{CCCCC} 1$ & 4 \\
\hline 18 & $\mathrm{O}=\mathrm{C}(\mathrm{NC} 1 \mathrm{CCCCC} 1) \mathrm{C}(\mathrm{CC} 1=\mathrm{CC}=\mathrm{CC}=\mathrm{C} 1) \mathrm{NC}(=\mathrm{O}) \mathrm{N} 1 \mathrm{~N}=\mathrm{NC} 2=\mathrm{CC}=\mathrm{CC}=\mathrm{C} 12$ & 4,19 \\
\hline 19 & $\mathrm{ONC}(=\mathrm{O}) \mathrm{NC}(\mathrm{CC} 1=\mathrm{CC}=\mathrm{CC}=\mathrm{C} 1) \mathrm{C}(=\mathrm{O}) \mathrm{NC} 1 \mathrm{CCCCC} 1$ & 4,03 \\
\hline 20 & $\mathrm{CCC}(\mathrm{C}) \mathrm{C}(\mathrm{NC}(=\mathrm{O}) \mathrm{NO}) \mathrm{C}(=\mathrm{O}) \mathrm{NC} 1 \mathrm{CCCC} 1$ & 4 \\
\hline 21 & $\mathrm{ONC}(=\mathrm{O}) \mathrm{NC}(\mathrm{CC} 1=\mathrm{CC}=\mathrm{CC}=\mathrm{C} 1) \mathrm{C}(=\mathrm{O}) \mathrm{N} 1 \mathrm{CCCC} 1$ & 4 \\
\hline 22 & $\mathrm{CCC}(\mathrm{C}) \mathrm{C}(\mathrm{NC}(=\mathrm{O}) \mathrm{N} 1 \mathrm{~N}=\mathrm{NC} 2=\mathrm{CC}=\mathrm{CC}=\mathrm{C} 12) \mathrm{C}(=\mathrm{O}) \mathrm{NC}(\mathrm{C} 1=\mathrm{CC}=\mathrm{CC}=\mathrm{C} 1) \mathrm{C} 1=\mathrm{CC}=\mathrm{CC}=\mathrm{C} 1$ & 4,26 \\
\hline 23 & $\mathrm{O}=\mathrm{C}(\mathrm{NC} 1 \mathrm{CCCCC} 1) \mathrm{C}(\mathrm{NC}(=\mathrm{O}) \mathrm{N} 1 \mathrm{~N}=\mathrm{NC} 2=\mathrm{C} 1 \mathrm{C}=\mathrm{CC}=\mathrm{C} 2) \mathrm{C} 1=\mathrm{CC}=\mathrm{CC}=\mathrm{C} 1$ & 4,16 \\
\hline 24 & $\mathrm{CCC}(\mathrm{C}) \mathrm{C}(\mathrm{NC}(=\mathrm{O}) \mathrm{N} 1 \mathrm{~N}=\mathrm{NC} 2=\mathrm{CC}=\mathrm{CC}=\mathrm{C} 12) \mathrm{C}(=\mathrm{O}) \mathrm{NC} 1 \mathrm{CCCCC} 1$ & 4,24 \\
\hline 25 & $\mathrm{CCC}(\mathrm{C}) \mathrm{C}(\mathrm{NC}(=\mathrm{O}) \mathrm{N} 1 \mathrm{~N}=\mathrm{NC} 2=\mathrm{CC}=\mathrm{CC}=\mathrm{C} 12) \mathrm{C}(=\mathrm{O}) \mathrm{NC} 1 \mathrm{CCCC} 1$ & 4,25 \\
\hline 26 & $\mathrm{CCC}(\mathrm{C}) \mathrm{C} 1 \mathrm{NC}(=\mathrm{O}) \mathrm{N}(\mathrm{C} 2 \mathrm{CCCCC} 2) \mathrm{C} 1=\mathrm{O}$ & 4,16 \\
\hline 27 & $\mathrm{CC}(\mathrm{C}) \mathrm{C}(\mathrm{NC}(=\mathrm{O}) \mathrm{N} 1 \mathrm{~N}=\mathrm{NC} 2=\mathrm{CC}=\mathrm{CC}=\mathrm{C} 12) \mathrm{C}(=\mathrm{O}) \mathrm{NC} 1 \mathrm{CCCC} 1$ & 4,22 \\
\hline 28 & $\mathrm{ONC}(=\mathrm{O}) \mathrm{NC}(\mathrm{CC} 1=\mathrm{CC}=\mathrm{CC}=\mathrm{C} 1) \mathrm{C}(=\mathrm{O}) \mathrm{NC} 1 \mathrm{CCCC} 1$ & 4 \\
\hline 29 & $\mathrm{CC}(\mathrm{C}) \mathrm{C}(\mathrm{NC}(=\mathrm{O}) \mathrm{NO}) \mathrm{C}(=\mathrm{O}) \mathrm{NC} 1 \mathrm{CCCCC} 1$ & 4 \\
\hline 30 & $\mathrm{ONC}(=\mathrm{O}) \mathrm{NC}(\mathrm{CC} 1=\mathrm{CC}=\mathrm{CC}=\mathrm{C} 1) \mathrm{C}(=\mathrm{O}) \mathrm{NCC} 1=\mathrm{CC}=\mathrm{CC}=\mathrm{C} 1$ & 4 \\
\hline 31 & $\mathrm{O}=\mathrm{C}(\mathrm{NCC} 1 \mathrm{CCCCC} 1) \mathrm{C}(\mathrm{NC}(=\mathrm{O}) \mathrm{N} 1 \mathrm{~N}=\mathrm{NC} 2=\mathrm{C} 1 \mathrm{C}=\mathrm{CC}=\mathrm{C} 2) \mathrm{C} 1=\mathrm{CC}=\mathrm{CC}=\mathrm{C} 1$ & 4,16 \\
\hline 32 & $\mathrm{ONC}(=\mathrm{O}) \mathrm{NC}(\mathrm{C}(=\mathrm{O}) \mathrm{NC} 1=\mathrm{CC}=\mathrm{CC}=\mathrm{C} 1) \mathrm{C} 1=\mathrm{CC}=\mathrm{CC}=\mathrm{C} 1$ & 4 \\
\hline 33 & $\mathrm{CCC}(\mathrm{C}) \mathrm{C}(\mathrm{NC}(=\mathrm{O}) \mathrm{NO}) \mathrm{C}(=\mathrm{O}) \mathrm{NCC} 1=\mathrm{CC}=\mathrm{CC}=\mathrm{C} 1$ & 4 \\
\hline 34 & $\mathrm{CCC}(\mathrm{C}) \mathrm{C}(\mathrm{NC}(=\mathrm{O}) \mathrm{N} 1 \mathrm{~N}=\mathrm{NC} 2=\mathrm{CC}=\mathrm{CC}=\mathrm{C} 12) \mathrm{C}(=\mathrm{O}) \mathrm{NCC} 1=\mathrm{CC}=\mathrm{CC}=\mathrm{C} 1$ & 4 \\
\hline 35 & $\mathrm{CC}(\mathrm{C}) \mathrm{C} 1 \mathrm{NC}(=\mathrm{O}) \mathrm{N}(\mathrm{C} 2 \mathrm{CCCCC} 2) \mathrm{C} 1=\mathrm{O}$ & 4 \\
\hline 36 & $\mathrm{CCC}(\mathrm{C}) \mathrm{C} 1 \mathrm{NC}(=\mathrm{O}) \mathrm{N}(\mathrm{C} 2 \mathrm{CCCC} 2) \mathrm{C} 1=\mathrm{O}$ & 4 \\
\hline 37 & $\mathrm{CCC}(\mathrm{C}) \mathrm{C} 1 \mathrm{NC}(=\mathrm{O}) \mathrm{N}(\mathrm{CC} 2=\mathrm{CC}=\mathrm{CC}=\mathrm{C} 2) \mathrm{C} 1=\mathrm{O}$ & 4,33 \\
\hline 38 & $\mathrm{CC}(\mathrm{C}) \backslash \mathrm{N}=\mathrm{C}(\mathrm{N}) \mathrm{C} 1=\mathrm{CC}=\mathrm{C} 2 \mathrm{~N}=\mathrm{C}(\mathrm{O}) \mathrm{C} 3=\mathrm{C}(\mathrm{C} 4=\mathrm{C}(\mathrm{S} 3) \mathrm{C}=\mathrm{C}(\mathrm{C}=\mathrm{C} 4) \mathrm{C} \# \mathrm{~N}) \mathrm{C} 2=\mathrm{C} 1$ & 4 \\
\hline 39 & $\mathrm{CC} 1=\mathrm{CC}=\mathrm{C}(\mathrm{NC}(=\mathrm{O}) \mathrm{C} 2=\mathrm{C}(\mathrm{Cl}) \mathrm{C} 3=\mathrm{CC}=\mathrm{C}(\mathrm{C}=\mathrm{C} 3 \mathrm{~S} 2) \mathrm{C} \# \mathrm{~N}) \mathrm{C}=\mathrm{C} 1$ & 4 \\
\hline 40 & $\mathrm{COC}(=\mathrm{O}) \mathrm{C} 1=\mathrm{CC}=\mathrm{C} 2 \mathrm{~N}=\mathrm{C}(\mathrm{O}) \mathrm{C} 3=\mathrm{C}(\mathrm{C} 4=\mathrm{C}(\mathrm{S} 3) \mathrm{C}=\mathrm{C}(\mathrm{C}=\mathrm{C} 4) \mathrm{C}(=\mathrm{N}) \mathrm{NC}(\mathrm{C}) \mathrm{C}) \mathrm{C} 2=\mathrm{C} 1$ & 4,01 \\
\hline 41 & $\mathrm{CC}(\mathrm{C}) \backslash \mathrm{N}=\mathrm{C}(\mathrm{N}) \mathrm{C} 1=\mathrm{CC}=\mathrm{C}(\mathrm{NC}(=\mathrm{O}) \mathrm{C} 2=\mathrm{C}(\mathrm{Cl}) \mathrm{C} 3=\mathrm{CC}=\mathrm{CC}=\mathrm{C} 3 \mathrm{~S} 2) \mathrm{C}=\mathrm{C} 1$ & 4,82 \\
\hline 42 & $\mathrm{ClC} 1=\mathrm{C}(\mathrm{SC} 2=\mathrm{CC}=\mathrm{CC}=\mathrm{C} 12) \mathrm{C}(=\mathrm{O}) \mathrm{NC} 1=\mathrm{CC}=\mathrm{C}(\mathrm{C}=\mathrm{C} 1) \mathrm{C} \# \mathrm{~N}$ & 5,19 \\
\hline 43 & $\mathrm{CC}(\mathrm{C}) \backslash \mathrm{N}=\mathrm{C}(\mathrm{N}) \mathrm{C} 1=\mathrm{CC}=\mathrm{C}(\mathrm{NC}(=\mathrm{O}) \mathrm{C} 2=\mathrm{C}(\mathrm{Cl}) \mathrm{C} 3=\mathrm{C}(\mathrm{S} 2) \mathrm{C}=\mathrm{C}(\mathrm{C}) \mathrm{C}=\mathrm{C} 3) \mathrm{C}=\mathrm{C} 1$ & 5,4 \\
\hline 44 & $\mathrm{COC}(=\mathrm{O}) \mathrm{C} 1=\mathrm{CC}=\mathrm{C}(\mathrm{NC}(=\mathrm{O}) \mathrm{C} 2=\mathrm{C}(\mathrm{Cl}) \mathrm{C} 3=\mathrm{CC}=\mathrm{C}(\mathrm{C}=\mathrm{C} 3 \mathrm{~S} 2) \mathrm{C} \# \mathrm{~N}) \mathrm{C}=\mathrm{C} 1$ & 4,23 \\
\hline 45 & $\mathrm{CC}(\mathrm{C}) \backslash \mathrm{N}=\mathrm{C}(\mathrm{NN}) \mathrm{C} 1=\mathrm{CC}=\mathrm{C} 2 \mathrm{C}(\mathrm{SC}(\mathrm{C}(=\mathrm{O}) \mathrm{NC} 3=\mathrm{CC}=\mathrm{CC}=\mathrm{C} 3)=\mathrm{C} 2 \mathrm{Cl})=\mathrm{C} 1$ & 4,62 \\
\hline 46 & $\mathrm{COC} 1=\mathrm{CC}=\mathrm{C}(\mathrm{NC}(=\mathrm{O}) \mathrm{C} 2=\mathrm{C}(\mathrm{Cl}) \mathrm{C} 3=\mathrm{C}(\mathrm{S} 2) \mathrm{C}=\mathrm{C}(\mathrm{C}=\mathrm{C} 3) \mathrm{C} \# \mathrm{~N}) \mathrm{C}=\mathrm{C} 1$ & 4,32 \\
\hline 47 & $\mathrm{CC}(\mathrm{C}) \backslash \mathrm{N}=\mathrm{C}(\mathrm{N}) \mathrm{C} 1=\mathrm{CC}=\mathrm{C} 2 \mathrm{~N}=\mathrm{C}(\mathrm{O}) \mathrm{C} 3=\mathrm{C}(\mathrm{C} 4=\mathrm{C}(\mathrm{S} 3) \mathrm{C}=\mathrm{C}(\mathrm{C}) \mathrm{C}=\mathrm{C} 4) \mathrm{C} 2=\mathrm{C} 1$ & 5,07 \\
\hline 48 & $\mathrm{CC}(\mathrm{C})\lfloor\mathrm{N}=\mathrm{C}(\mathrm{N}) \mathrm{C} 1=\mathrm{CC} 2=\mathrm{C}(\mathrm{C}=\mathrm{C} 1) \mathrm{C} 1=\mathrm{C}(\mathrm{S} 2) \mathrm{C}(\mathrm{O})=\mathrm{NC} 2=\mathrm{CC}=\mathrm{CC}=\mathrm{C} 12$ & 4,68 \\
\hline 49 & $\mathrm{COC} 1=\mathrm{CC}=\mathrm{C} 2 \mathrm{C}(\mathrm{Cl})=\mathrm{C}(\mathrm{SC} 2=\mathrm{C} 1) \mathrm{C}(=\mathrm{O}) \mathrm{NC} 1=\mathrm{CC}=\mathrm{C}(\mathrm{C}=\mathrm{C} 1) \mathrm{C}(=\mathrm{N}) \mathrm{NC}(\mathrm{C}) \mathrm{C}$ & 5,59 \\
\hline 50 & $\mathrm{CC}(\mathrm{C}) \backslash \mathrm{N}=\mathrm{C}(\mathrm{N}) \mathrm{C} 1=\mathrm{CC} 2=\mathrm{C}(\mathrm{C}=\mathrm{C} 1) \mathrm{N}=\mathrm{C}(\mathrm{O}) \mathrm{C} 1=\mathrm{C} 2 \mathrm{C} 2=\mathrm{CC}=\mathrm{C}(\mathrm{C}=\mathrm{C} 2 \mathrm{~S} 1) \mathrm{C}(\mathrm{N})=\mathrm{N} / \mathrm{C}(\mathrm{C}) \mathrm{C}$ & 4 \\
\hline 51 & $\mathrm{COC} 1=\mathrm{CC}=\mathrm{C} 2 \mathrm{C}(\mathrm{SC} 3=\mathrm{C} 2 \mathrm{C} 2=\mathrm{C}(\mathrm{C}=\mathrm{CC}(=\mathrm{C} 2) \mathrm{C}(=\mathrm{N}) \mathrm{NC}(\mathrm{C}) \mathrm{C}) \mathrm{N}=\mathrm{C} 3 \mathrm{O})=\mathrm{C} 1$ & 4,46 \\
\hline 52 & $\mathrm{CC}(\mathrm{C}) \backslash \mathrm{N}=\mathrm{C}(\mathrm{N}) \mathrm{C} 1=\mathrm{CC}=\mathrm{C}(\mathrm{NC}(=\mathrm{O}) \mathrm{C} 2=\mathrm{C}(\mathrm{Cl}) \mathrm{C} 3=\mathrm{CC}=\mathrm{C}(\mathrm{C}=\mathrm{C} 3 \mathrm{~S} 2) \mathrm{C}(\mathrm{N})=\mathrm{N} / \mathrm{C}(\mathrm{C}) \mathrm{C}) \mathrm{C}=\mathrm{C} 1$ & 4,17 \\
\hline 53 & $\mathrm{CC}(\mathrm{C}) \backslash \mathrm{N}=\mathrm{C}(\mathrm{N}) \mathrm{C} 1=\mathrm{CC} 2=\mathrm{C}(\mathrm{C}=\mathrm{C} 1) \mathrm{C} 1=\mathrm{C}(\mathrm{S} 2) \mathrm{C}(\mathrm{O})=\mathrm{NC} 2=\mathrm{CC}=\mathrm{C}(\mathrm{Br}) \mathrm{C}=\mathrm{C} 12$ & 5,01 \\
\hline 54 & $\mathrm{CC}(\mathrm{C}) \backslash \mathrm{N}=\mathrm{C}(\mid \mathrm{N}) \mathrm{C} 1=\mathrm{CC}=\mathrm{C}(\mathrm{NC}(=\mathrm{O}) \mathrm{C} 2=\mathrm{C}(\mathrm{Cl}) \mathrm{C} 3=\mathrm{C}(\mathrm{S} 2) \mathrm{C}=\mathrm{C}(\mathrm{Br}) \mathrm{C}=\mathrm{C} 3) \mathrm{C}=\mathrm{C} 1$ & 5,63 \\
\hline 55 & $\mathrm{CC}(\mathrm{C}) \backslash \mathrm{N}=\mathrm{C}(\backslash \mathrm{N}) \mathrm{C} 1=\mathrm{CC} 2=\mathrm{C}(\mathrm{C}=\mathrm{C} 1) \mathrm{C} 1=\mathrm{C}(\mathrm{S} 2) \mathrm{C}(\mathrm{O})=\mathrm{NC} 2=\mathrm{CC}=\mathrm{C}(\mathrm{C}) \mathrm{C}=\mathrm{C} 12$ & 5,07 \\
\hline 56 & $\mathrm{COC}(=\mathrm{O}) \mathrm{C} 1=\mathrm{CC}=\mathrm{C}(\mathrm{NC}(=\mathrm{O}) \mathrm{C} 2=\mathrm{C}(\mathrm{Cl}) \mathrm{C} 3=\mathrm{C}(\mathrm{S} 2) \mathrm{C}=\mathrm{CC}=\mathrm{C} 3) \mathrm{C}=\mathrm{C} 1$ & 4 \\
\hline 57 & $\mathrm{COC}(=\mathrm{O}) \mathrm{C} 1=\mathrm{CC}=\mathrm{C} 2 \mathrm{C}(\mathrm{Cl})=\mathrm{C}(\mathrm{SC} 2=\mathrm{C} 1) \mathrm{C}(=\mathrm{O}) \mathrm{NC} 1=\mathrm{CC}=\mathrm{CC}=\mathrm{C} 1$ & 4 \\
\hline 58 & $\mathrm{CC}(\mathrm{C}) \backslash \mathrm{N}=\mathrm{C}(\mathrm{N}) \mathrm{C} 1=\mathrm{CC}=\mathrm{C} 2 \mathrm{~N}=\mathrm{C}(\mathrm{O}) \mathrm{C} 3=\mathrm{C}(\mathrm{C} 4=\mathrm{C}(\mathrm{S} 3) \mathrm{C}=\mathrm{CC}=\mathrm{C} 4) \mathrm{C} 2=\mathrm{C} 1$ & 4,78 \\
\hline 59 & $\mathrm{COC}(=\mathrm{O}) \mathrm{C} 1=\mathrm{CC}=\mathrm{C} 2 \mathrm{C}(\mathrm{Cl})=\mathrm{C}(\mathrm{SC} 2=\mathrm{C} 1) \mathrm{C}(=\mathrm{O}) \mathrm{NC} 1=\mathrm{CC}=\mathrm{C}(\mathrm{C}=\mathrm{C} 1) \mathrm{C}(=\mathrm{N}) \mathrm{NC}(\mathrm{C}) \mathrm{C}$ & 5,27 \\
\hline 60 & $\mathrm{CC}(\mathrm{C}) \backslash \mathrm{N}=\mathrm{C}(\mathrm{N}) \mathrm{C} 1=\mathrm{CC}=\mathrm{C} 2 \mathrm{~N}=\mathrm{C}(\mathrm{O}) \mathrm{C} 3=\mathrm{C}(\mathrm{C} 4=\mathrm{C}(\mathrm{S} 3) \mathrm{C}=\mathrm{C}(\mathrm{Br}) \mathrm{C}=\mathrm{C} 4) \mathrm{C} 2=\mathrm{C} 1$ & 5,16 \\
\hline 61 & $\mathrm{CC}(\mathrm{C}) \backslash \mathrm{N}=\mathrm{C}(\mathrm{N}) \mathrm{C} 1=\mathrm{CC}=\mathrm{C}(\mathrm{NC}(=\mathrm{O}) \mathrm{C} 2=\mathrm{C}(\mathrm{Cl}) \mathrm{C} 3=\mathrm{CC}=\mathrm{C}(\mathrm{C}=\mathrm{C} 3 \mathrm{~S} 2) \mathrm{C} \# \mathrm{~N}) \mathrm{C}=\mathrm{C} 1$ & 4,68 \\
\hline 62 & $\mathrm{COC} 1=\mathrm{CC}=\mathrm{C}(\mathrm{NC}(=\mathrm{O}) \mathrm{C} 2=\mathrm{C}(\mathrm{Cl}) \mathrm{C} 3=\mathrm{CC}=\mathrm{C}(\mathrm{C}=\mathrm{C} 3 \mathrm{~S} 2) \mathrm{C}(=\mathrm{N}) \mathrm{NC}(\mathrm{C}) \mathrm{C}) \mathrm{C}=\mathrm{C} 1$ & 5,01 \\
\hline 63 & $\mathrm{COC}(=\mathrm{O}) \mathrm{C} 1=\mathrm{CC}=\mathrm{C}(\mathrm{NC}(=\mathrm{O}) \mathrm{C} 2=\mathrm{C}(\mathrm{Cl}) \mathrm{C} 3=\mathrm{C}(\mathrm{S} 2) \mathrm{C}=\mathrm{C}(\mathrm{C}=\mathrm{C} 3) \mathrm{C}(=\mathrm{N}) \mathrm{NC}(\mathrm{C}) \mathrm{C}) \mathrm{C}=\mathrm{C} 1$ & 5,09 \\
\hline 64 & $\mathrm{CC}(\mathrm{C}) \backslash \mathrm{N}=\mathrm{C}(\mathrm{N}) \mathrm{C} 1=\mathrm{CC}=\mathrm{C} 2 \mathrm{C}(\mathrm{Cl})=\mathrm{C}(\mathrm{SC} 2=\mathrm{C} 1) \mathrm{C}(=\mathrm{O}) \mathrm{NC} 1=\mathrm{CC}=\mathrm{C}(\mathrm{Br}) \mathrm{C}=\mathrm{C} 1$ & 5,5 \\
\hline 65 & $\mathrm{CC}(\mathrm{C}) \backslash \mathrm{N}=\mathrm{C}(\mathrm{N}) \mathrm{C} 1=\mathrm{CC}=\mathrm{C} 2 \mathrm{C}(\mathrm{SC}(\mathrm{C}(=\mathrm{O}) \mathrm{NC} 3=\mathrm{CC}=\mathrm{C}(\mathrm{C}) \mathrm{C}=\mathrm{C} 3)=\mathrm{C} 2 \mathrm{Cl})=\mathrm{C} 1$ & 5,53 \\
\hline 66 & $\mathrm{ClC} 1=\mathrm{C}(\mathrm{SC} 2=\mathrm{CC}(=\mathrm{CC}=\mathrm{C} 12) \mathrm{C} \# \mathrm{~N}) \mathrm{C}(=\mathrm{O}) \mathrm{NC} 1=\mathrm{CC}=\mathrm{C}(\mathrm{Br}) \mathrm{C}=\mathrm{C} 1$ & 4 \\
\hline 67 & $\mathrm{COC}(=\mathrm{O}) \mathrm{C} 1=\mathrm{CC} 2=\mathrm{C}(\mathrm{C}=\mathrm{C} 1) \mathrm{C} 1=\mathrm{C}(\mathrm{S} 2) \mathrm{C}(\mathrm{O})=\mathrm{NC} 2=\mathrm{CC}=\mathrm{C}(\mathrm{C}=\mathrm{C} 12) \mathrm{C}(=\mathrm{N}) \mathrm{NC}(\mathrm{C}) \mathrm{C}$ & 4,34 \\
\hline 68 & $\mathrm{ClC} 1=\mathrm{C}(\mathrm{SC} 2=\mathrm{CC}(=\mathrm{CC}=\mathrm{C} 12) \mathrm{C} \# \mathrm{~N}) \mathrm{C}(=\mathrm{O}) \mathrm{NC} 1=\mathrm{CC}=\mathrm{CC}=\mathrm{C} 1$ & 6,16 \\
\hline 69 & $\mathrm{CC}(=\mathrm{O}) \mathrm{OCCON} 1 \mathrm{C}=\mathrm{NC} 2=\mathrm{C}(\mathrm{S}) \mathrm{N}=\mathrm{C}(\mathrm{N}) \mathrm{N}=\mathrm{C} 12$ & 5,31 \\
\hline 70 & $\mathrm{CC}(=\mathrm{O}) \mathrm{OCCON} 1 \mathrm{C}=\mathrm{NC} 2=\mathrm{C}(\mathrm{Cl}) \mathrm{N}=\mathrm{C}(\mathrm{N}) \mathrm{N}=\mathrm{C} 12$ & 3,3 \\
\hline 71 & $\mathrm{CC}(\mathrm{O}) \mathrm{N} 1 \mathrm{C}=\mathrm{NC} 2=\mathrm{C}(\mathrm{Cl}) \mathrm{N}=\mathrm{C}(\mathrm{I}) \mathrm{N}=\mathrm{C} 12$ & 3,3 \\
\hline
\end{tabular}

https://biointerfaceresearch.com/ 


\begin{tabular}{|c|c|c|}
\hline NAME & SMILE & $\lg (\mathbf{1} / \mathbf{I C 5 0})$ \\
\hline 72 & $\mathrm{CC}(\mathrm{OC}(\mathrm{C})=\mathrm{O}) \mathrm{N} 1 \mathrm{C}=\mathrm{NC} 2=\mathrm{C}(\mathrm{S}) \mathrm{N}=\mathrm{C}(\mathrm{N}) \mathrm{N}=\mathrm{C} 12$ & 5 \\
\hline 73 & $\mathrm{CC}(\mathrm{OC}(\mathrm{C})=\mathrm{O}) \mathrm{N} 1 \mathrm{C}=\mathrm{NC} 2=\mathrm{C}(\mathrm{S}) \mathrm{N}=\mathrm{C}(\mathrm{F}) \mathrm{N}=\mathrm{C} 12$ & 3,7 \\
\hline 74 & $\mathrm{CC}(\mathrm{O}) \mathrm{N} 1 \mathrm{C}=\mathrm{NC} 2=\mathrm{C}(\mathrm{N}) \mathrm{N}=\mathrm{C}(\mathrm{I}) \mathrm{N}=\mathrm{C} 12$ & 3,3 \\
\hline 75 & $\mathrm{CC}(\mathrm{O}) \mathrm{CN} 1 \mathrm{C}=\mathrm{NC} 2=\mathrm{C} 1 \mathrm{~N}=\mathrm{C}(\mathrm{N}) \mathrm{N}=\mathrm{C} 2 \mathrm{~F}$ & 4,2 \\
\hline 76 & OCCON1C $=\mathrm{NC} 2=\mathrm{C}(\mathrm{S}) \mathrm{N}=\mathrm{C}(\mathrm{F}) \mathrm{N}=\mathrm{C} 12$ & 3,3 \\
\hline 77 & $\mathrm{NC} 1=\mathrm{C} 2 \mathrm{~N}=\mathrm{CN}(\mathrm{OCCO}) \mathrm{C} 2=\mathrm{NC}(\mathrm{I})=\mathrm{N} 1$ & 3,3 \\
\hline 78 & $\mathrm{CC}(=\mathrm{O}) \mathrm{OCCON} 1 \mathrm{C}=\mathrm{NC} 2=\mathrm{C}(\mathrm{S}) \mathrm{N}=\mathrm{C}(\mathrm{F}) \mathrm{N}=\mathrm{C} 12$ & 3,3 \\
\hline 79 & $\mathrm{CC}(\mathrm{OC}(\mathrm{C})=\mathrm{O}) \mathrm{N} 1 \mathrm{C}=\mathrm{NC} 2=\mathrm{C}(\mathrm{Cl}) \mathrm{N}=\mathrm{C}(\mathrm{F}) \mathrm{N}=\mathrm{C} 12$ & 5 \\
\hline 80 & $\mathrm{CC}(\mathrm{O}) \mathrm{N} 1 \mathrm{C}=\mathrm{NC} 2=\mathrm{C}(\mathrm{S}) \mathrm{N}=\mathrm{C}(\mathrm{F}) \mathrm{N}=\mathrm{C} 12$ & 3,3 \\
\hline 81 & $\mathrm{COC} 1=\mathrm{C} 2 \mathrm{~N}=\mathrm{CN}(\mathrm{OCCO}) \mathrm{C} 2=\mathrm{NC}(\mathrm{I})=\mathrm{N} 1$ & 3,3 \\
\hline 82 & $\mathrm{CC}(\mathrm{OC}(\mathrm{C})=\mathrm{O}) \mathrm{N} 1 \mathrm{C}=\mathrm{NC} 2=\mathrm{C}(\mathrm{N}) \mathrm{N}=\mathrm{C}(\mathrm{I}) \mathrm{N}=\mathrm{C} 12$ & 3,3 \\
\hline 83 & $\mathrm{CC}(=\mathrm{O}) \mathrm{OCCON} 1 \mathrm{C}=\mathrm{NC} 2=\mathrm{C}(\mathrm{Cl}) \mathrm{N}=\mathrm{C}(\mathrm{I}) \mathrm{N}=\mathrm{C} 12$ & 4,25 \\
\hline 84 & $\mathrm{CC}(\mathrm{OC}(\mathrm{C})=\mathrm{O}) \mathrm{N} 1 \mathrm{C}=\mathrm{NC} 2=\mathrm{C}(\mathrm{Cl}) \mathrm{N}=\mathrm{C}(\mathrm{I}) \mathrm{N}=\mathrm{C} 12$ & 5,2 \\
\hline 85 & $\mathrm{CC}(\mathrm{OC}(\mathrm{C})=\mathrm{O}) \mathrm{N} 1 \mathrm{C}=\mathrm{NC} 2=\mathrm{C}(\mathrm{Cl}) \mathrm{N}=\mathrm{C}(\mathrm{N}) \mathrm{N}=\mathrm{C} 12$ & 3,3 \\
\hline 86 & $\mathrm{CCOC}(=\mathrm{O}) \mathrm{NC} 1=\mathrm{CC}(\mathrm{NC}(\mathrm{C} 2=\mathrm{CC}=\mathrm{CC}=\mathrm{C} 2) \mathrm{C} 2=\mathrm{CC}=\mathrm{CC}=\mathrm{C} 2)=\mathrm{C}(\mathrm{N}) \mathrm{C}(\mathrm{N})=\mathrm{N} 1$ & 6,59 \\
\hline 87 & $\mathrm{CC} 1=\mathrm{CN}([\mathrm{C} @ \mathrm{H}] 2 \mathrm{C}[\mathrm{C} @ \mathrm{H}](\mathrm{O})[\mathrm{C} @ @ \mathrm{H}](\mathrm{CO}) \mathrm{S} 2) \mathrm{C}(=\mathrm{O}) \mathrm{NC} 1=\mathrm{O}$ & 6,85 \\
\hline 88 & $\mathrm{OC}[\mathrm{C} @ \mathrm{H}] 1 \mathrm{~S}[\mathrm{C} @ \mathrm{H}](\mathrm{C}[\mathrm{C} @ @ \mathrm{H}] 1 \mathrm{O}) \mathrm{N} 1 \mathrm{C}=\mathrm{CC}(=\mathrm{O}) \mathrm{NC} 1=\mathrm{O}$ & 5,68 \\
\hline 89 & $\mathrm{CC} 1=\mathrm{CN}([\mathrm{C} @ @ \mathrm{H}] 2 \mathrm{C}[\mathrm{C} @ \mathrm{H}](\mathrm{O})[\mathrm{C} @ @ \mathrm{H}](\mathrm{CO}) \mathrm{S} 2) \mathrm{C}(=\mathrm{O}) \mathrm{NC} 1=\mathrm{O}$ & 4,6 \\
\hline 90 & $\mathrm{NC} 1=\mathrm{NC}(=\mathrm{O}) \mathrm{N}(\mathrm{C}=\mathrm{C} 1)[\mathrm{C} @ \mathrm{H}] 1 \mathrm{C}[\mathrm{C} @ \mathrm{H}](\mathrm{O})[\mathrm{C} @ @ \mathrm{H}](\mathrm{CO}) \mathrm{S} 1$ & 6,7 \\
\hline 91 & $\mathrm{NC} 1=\mathrm{NC}(=\mathrm{O}) \mathrm{N}(\mathrm{C}=\mathrm{C} 1) \mathrm{C} 1 \mathrm{C}[\mathrm{C} @ \mathrm{H}](\mathrm{O})[\mathrm{C} @ @ \mathrm{H}](\mathrm{CO}) \mathrm{S} 1$ & 6,7 \\
\hline 92 & $\mathrm{NC} 1=\mathrm{NC}(\mathrm{Cl})=\mathrm{NC} 2=\mathrm{C} 1 \mathrm{~N}=\mathrm{CN} 2[\mathrm{C} @ @ \mathrm{H}] 1 \mathrm{O}[\mathrm{C} @ \mathrm{H}](\mathrm{CO})[\mathrm{C} @ @ \mathrm{H}](\mathrm{O})[\mathrm{C} @ \mathrm{H}] 1 \mathrm{O}$ & 5,52 \\
\hline 93 & $\mathrm{NC} 1=\mathrm{C} 2 \mathrm{~N}=\mathrm{CN}([\mathrm{C} @ @ \mathrm{H}] 3 \mathrm{O}[\mathrm{C} @ \mathrm{H}](\mathrm{CO})[\mathrm{C} @ @ \mathrm{H}](\mathrm{O})[\mathrm{C} @ @ \mathrm{H}] 3 \mathrm{~F}) \mathrm{C} 2=\mathrm{NC}(\mathrm{Cl})=\mathrm{N} 1$ & 7,92 \\
\hline 94 & $\mathrm{NC} 1=\mathrm{C} 2 \mathrm{~N}=\mathrm{CN}([\mathrm{C} @ \mathrm{H}] 3 \mathrm{C}[\mathrm{C} @ \mathrm{H}](\mathrm{O})[\mathrm{C} @ @ \mathrm{H}](\mathrm{CO}) \mathrm{O} 3) \mathrm{C} 2=\mathrm{NC}(\mathrm{Cl})=\mathrm{N} 1$ & 7,52 \\
\hline 95 & $\mathrm{NC} 1=\mathrm{C} 2 \mathrm{~N}=\mathrm{CN}([\mathrm{C} @ @ \mathrm{H}] 3 \mathrm{O}[\mathrm{C} @ \mathrm{H}](\mathrm{CO})[\mathrm{C} @ @ \mathrm{H}](\mathrm{O})[\mathrm{C} @ \mathrm{H}] 3 \mathrm{~F}) \mathrm{C} 2=\mathrm{NC}(\mathrm{Br})=\mathrm{N} 1$ & 6,66 \\
\hline 96 & $\mathrm{NC} 1=\mathrm{C} 2 \mathrm{~N}=\mathrm{CN}([\mathrm{C} @ \mathrm{H}] 3 \mathrm{C}[\mathrm{C} @ \mathrm{H}](\mathrm{O})[\mathrm{C} @ @ \mathrm{H}](\mathrm{CO}) \mathrm{O} 3) \mathrm{C} 2=\mathrm{NC}(\mathrm{F})=\mathrm{N} 1$ & 6,7 \\
\hline 97 & $\mathrm{NC1}=\mathrm{C} 2 \mathrm{~N}=\mathrm{CN}([\mathrm{C} @ @ \mathrm{H}] 3 \mathrm{O}[\mathrm{C} @ \mathrm{H}](\mathrm{CO})[\mathrm{C} @ @ \mathrm{H}](\mathrm{O})[\mathrm{C} @ @ \mathrm{H}] 3 \mathrm{~F}) \mathrm{C} 2=\mathrm{NC}(\mathrm{F})=\mathrm{N} 1$ & 6,47 \\
\hline 98 & $\mathrm{NC} 1=\mathrm{NC}(\mathrm{Br})=\mathrm{NC} 2=\mathrm{C} 1 \mathrm{~N}=\mathrm{CN} 2 \mathrm{C} 1 \mathrm{O}[\mathrm{C} @ \mathrm{H}](\mathrm{CO})[\mathrm{C} @ @ \mathrm{H}](\mathrm{O})[\mathrm{C} @ \mathrm{H}] 1 \mathrm{O}$ & 5,4 \\
\hline 99 & $\mathrm{NC} 1=\mathrm{NC}(\mathrm{F})=\mathrm{NC} 2=\mathrm{C} 1 \mathrm{~N}=\mathrm{CN} 2[\mathrm{C} @ @ \mathrm{H}] 1 \mathrm{O}[\mathrm{C} @ \mathrm{H}](\mathrm{CO})[\mathrm{C} @ @ \mathrm{H}](\mathrm{O})[\mathrm{C} @ @ \mathrm{H}] 1 \mathrm{O}$ & 5,05 \\
\hline 100 & $\mathrm{NC} 1=\mathrm{C} 2 \mathrm{~N}=\mathrm{CN}([\mathrm{C} @ \mathrm{H}] 3 \mathrm{C}[\mathrm{C} @ \mathrm{H}](\mathrm{O})[\mathrm{C} @ @ \mathrm{H}](\mathrm{CO}) \mathrm{O} 3) \mathrm{C} 2=\mathrm{NC}(\mathrm{Br})=\mathrm{N} 1$ & 7,7 \\
\hline 101 & $\mathrm{CC}(\mathrm{Br}) \mathrm{C}(=\mathrm{O}) \mathrm{NCC} 1 \mathrm{OC}(\mathrm{CC} 1 \mathrm{O}) \mathrm{N} 1 \mathrm{C}=\mathrm{C}(\mathrm{C}) \mathrm{C}(=\mathrm{O}) \mathrm{NC} 1=\mathrm{O}$ & 4,3 \\
\hline 102 & $\mathrm{CC} 1=\mathrm{CN}(\mathrm{C} 2 \mathrm{CC}(\mathrm{O}) \mathrm{C}(\mathrm{CNC}(=\mathrm{O}) \mathrm{CCBr}) \mathrm{O} 2) \mathrm{C}(=\mathrm{O}) \mathrm{NC} 1=\mathrm{O}$ & 4,3 \\
\hline 103 & $\mathrm{CC1}=\mathrm{CN}([\mathrm{C} @ \mathrm{H}] 2 \mathrm{C}[\mathrm{C} @ \mathrm{H}](\mathrm{O})[\mathrm{C} @ @ \mathrm{H}](\mathrm{CNC}(=\mathrm{O}) \mathrm{CBr}) \mathrm{O} 2) \mathrm{C}(=\mathrm{O}) \mathrm{NC} 1=\mathrm{O}$ & 5,1 \\
\hline 104 & $\operatorname{CCOC}(=\mathrm{O}) \mathrm{C}(=\mathrm{O}) \mathrm{NCC} 1 \mathrm{OC}(\mathrm{CC} 1 \mathrm{O}) \mathrm{N} 1 \mathrm{C}=\mathrm{C}(\mathrm{C}) \mathrm{C}(=\mathrm{O}) \mathrm{NC} 1=\mathrm{O}$ & 4,22 \\
\hline 105 & $\mathrm{CC} 1=\mathrm{CN}(\mathrm{C} 2 \mathrm{CC}(\mathrm{O}) \mathrm{C}(\mathrm{CNC}(=\mathrm{O}) \mathrm{CI}) \mathrm{O} 2) \mathrm{C}(=\mathrm{O}) \mathrm{NC} 1=\mathrm{O}$ & 5,1 \\
\hline 106 & $\mathrm{CC} 1=\mathrm{CN}(\mathrm{C} 2 \mathrm{CC}(\mathrm{O}) \mathrm{C}(\mathrm{CNC}(=\mathrm{O}) \mathrm{CCl}) \mathrm{O} 2) \mathrm{C}(=\mathrm{O}) \mathrm{NC} 1=\mathrm{O}$ & 4,6 \\
\hline 107 & $\mathrm{CC} 1=\mathrm{CN}(\mathrm{C} 2 \mathrm{CC}(\mathrm{O}) \mathrm{C}(\mathrm{CNS}(=\mathrm{O})(=\mathrm{O}) \mathrm{CBr}) \mathrm{O} 2) \mathrm{C}(=\mathrm{O}) \mathrm{NC} 1=\mathrm{O}$ & 4,3 \\
\hline 108 & $\mathrm{CC} 1=\mathrm{CN}(\mathrm{C} 2 \mathrm{CC}(\mathrm{O}) \mathrm{C}(\mathrm{CC}(=\mathrm{O}) \mathrm{OCBr}) \mathrm{O} 2) \mathrm{C}(=\mathrm{O}) \mathrm{NC} 1=\mathrm{O}$ & 5,52 \\
\hline 109 & $\mathrm{CC} 1=\mathrm{CN}(\mathrm{C} 2 \mathrm{CC}(\mathrm{O}) \mathrm{C}(\mathrm{CNC}(=\mathrm{O}) \mathrm{C} 3=\mathrm{CC}=\mathrm{CC}(=\mathrm{C} 3) \mathrm{S}(\mathrm{F})(=\mathrm{O})=\mathrm{O}) \mathrm{O} 2) \mathrm{C}(=\mathrm{O}) \mathrm{NC} 1=\mathrm{O}$ & 4,3 \\
\hline 110 & $\mathrm{OC}[\mathrm{C} @ \mathrm{H}] 1 \mathrm{O}[\mathrm{C} @ \mathrm{H}]([\mathrm{C} @ \mathrm{H}](\mathrm{O})[\mathrm{C} @ @ \mathrm{H}] 1 \mathrm{O}) \mathrm{C} 1 \mathrm{NN}=\mathrm{C}(\mathrm{C}(=\mathrm{O}) \mathrm{CN}=\mathrm{N}) \mathrm{C} 1=\mathrm{N}$ & 5,7 \\
\hline 111 & $\mathrm{CC} 1=\mathrm{CN}(\mathrm{C} 2 \mathrm{CC}(\mathrm{O}) \mathrm{C}(\mathrm{CNC}(=\mathrm{O}) \mathrm{N}(\mathrm{CCCl}) \mathrm{N}=\mathrm{O}) \mathrm{O} 2) \mathrm{C}(=\mathrm{O}) \mathrm{NC} 1=\mathrm{O}$ & 5 \\
\hline 112 & $\mathrm{CN}(\mathrm{N}=\mathrm{O}) \mathrm{C}(=\mathrm{O}) \mathrm{NC} 1=\mathrm{CC}=\mathrm{C}(\mathrm{NC}(=\mathrm{O}) \mathrm{C} 2 \mathrm{OC}(\mathrm{C}(\mathrm{O}) \mathrm{C} 2 \mathrm{O}) \mathrm{N} 2 \mathrm{C}=\mathrm{CC}(=\mathrm{O}) \mathrm{NC} 2=\mathrm{O}) \mathrm{C}=\mathrm{C} 1$ & 4,48 \\
\hline 113 & $\mathrm{OC} 1 \mathrm{CC}(\mathrm{OC} 1 \mathrm{CNC}(=\mathrm{O}) \mathrm{N}(\mathrm{CCCl}) \mathrm{N}=\mathrm{O}) \mathrm{N} 1 \mathrm{C}=\mathrm{CC}(=\mathrm{O}) \mathrm{NC} 1=\mathrm{O}$ & 4,85 \\
\hline 114 & $\mathrm{CC} 1=\mathrm{CN}(\mathrm{C} 2 \mathrm{CC}(\mathrm{O}) \mathrm{C}(\mathrm{O} 2) \mathrm{C}(=\mathrm{O}) \mathrm{NCCN}(\mathrm{N}=\mathrm{O}) \mathrm{C}(=\mathrm{O}) \mathrm{NC} 2=\mathrm{CC}=\mathrm{CC}=\mathrm{C} 2) \mathrm{C}(=\mathrm{O}) \mathrm{NC} 1=\mathrm{O}$ & 4,05 \\
\hline 115 & $\mathrm{CCN}(\mathrm{N}=\mathrm{O}) \mathrm{C}(=\mathrm{O}) \mathrm{NCC} 1 \mathrm{OC}(\mathrm{CC} 1 \mathrm{O}) \mathrm{N} 1 \mathrm{C}=\mathrm{C}(\mathrm{C}) \mathrm{C}(=\mathrm{O}) \mathrm{NC} 1=\mathrm{O}$ & 4,06 \\
\hline 116 & $\mathrm{CN}(\mathrm{N}=\mathrm{O}) \mathrm{C}(=\mathrm{O}) \mathrm{NC} 1=\mathrm{CC}=\mathrm{C}(\mathrm{NC}(=\mathrm{O}) \mathrm{C} 2 \mathrm{OC}(\mathrm{CC} 2 \mathrm{O}) \mathrm{N} 2 \mathrm{C}=\mathrm{C}(\mathrm{C}) \mathrm{C}(=\mathrm{O}) \mathrm{NC} 2=\mathrm{O}) \mathrm{C}=\mathrm{C} 1$ & 4,34 \\
\hline 117 & $\mathrm{NC} 1=\mathrm{C} 2 \mathrm{~N}=\mathrm{CN}(\mathrm{C} 3 \mathrm{O}[\mathrm{C} @ \mathrm{H}](\mathrm{CO})[\mathrm{C} @ \mathrm{H}](\mathrm{O})[\mathrm{C} @ \mathrm{H}] 3 \mathrm{Cl}) \mathrm{C} 2=\mathrm{NC}(\mathrm{Cl})=\mathrm{N} 1$ & 3,92 \\
\hline 118 & $\mathrm{NC} 1=\mathrm{C} 2 \mathrm{~N}=\mathrm{CN}(\mathrm{C} 3 \mathrm{O}[\mathrm{C} @ \mathrm{H}](\mathrm{CO})[\mathrm{C} @ \mathrm{H}](\mathrm{O})[\mathrm{C} @ \mathrm{H}] 3 \mathrm{~N}=[\mathrm{N}+]=[\mathrm{N}-]) \mathrm{C} 2=\mathrm{NC}(\mathrm{F})=\mathrm{N} 1$ & 4,54 \\
\hline 119 & $\mathrm{NC} 1=\mathrm{C} 2 \mathrm{~N}=\mathrm{CN}(\mathrm{C} 3 \mathrm{O}[\mathrm{C} @ \mathrm{H}](\mathrm{CO})[\mathrm{C} @ \mathrm{H}](\mathrm{O})[\mathrm{C} @ \mathrm{H}] 3 \mathrm{Cl}) \mathrm{C} 2=\mathrm{NC}(\mathrm{F})=\mathrm{N} 1$ & 4 \\
\hline 120 & $\mathrm{NC} 1=\mathrm{NC}(\mathrm{Cl})=\mathrm{NC} 2=\mathrm{C} 1 \mathrm{~N}=\mathrm{CN} 2 \mathrm{C} 1 \mathrm{O}[\mathrm{C} @ \mathrm{H}](\mathrm{CO})[\mathrm{C} @ \mathrm{H}](\mathrm{O})[\mathrm{C} @ \mathrm{H}] 1 \mathrm{Br}$ & 3,96 \\
\hline 121 & $\mathrm{NC} 1=\mathrm{C} 2 \mathrm{~N}=\mathrm{CN}(\mathrm{C} 3 \mathrm{O}[\mathrm{C} @ \mathrm{H}](\mathrm{CO})[\mathrm{C} @ \mathrm{H}](\mathrm{O})[\mathrm{C} @ \mathrm{H}] 3 \mathrm{I}) \mathrm{C} 2=\mathrm{NC}(\mathrm{Cl})=\mathrm{N} 1$ & 4 \\
\hline 122 & $\mathrm{~N}[\mathrm{C} @ @ \mathrm{H}] 1[\mathrm{C} @ @ \mathrm{H}](\mathrm{O})[\mathrm{C} @ @ \mathrm{H}](\mathrm{CO}) \mathrm{OC} 1 \mathrm{~N} 1 \mathrm{C}=\mathrm{NC} 2=\mathrm{C}(\mathrm{N}) \mathrm{N}=\mathrm{C}(\mathrm{F}) \mathrm{N}=\mathrm{C} 12$ & 4,92 \\
\hline 123 & $\mathrm{~N}[\mathrm{C} @ @ \mathrm{H}] 1[\mathrm{C} @ @ \mathrm{H}](\mathrm{O})[\mathrm{C} @ @ \mathrm{H}](\mathrm{CO}) \mathrm{OC} 1 \mathrm{~N} 1 \mathrm{C}=\mathrm{NC} 2=\mathrm{C}(\mathrm{N}) \mathrm{N}=\mathrm{C}(\mathrm{Cl}) \mathrm{N}=\mathrm{C} 12$ & 4,1 \\
\hline 124 & $\mathrm{NC} 1=\mathrm{C} 2 \mathrm{~N}=\mathrm{CN}(\mathrm{C} 3 \mathrm{O}[\mathrm{C} @ \mathrm{H}](\mathrm{CO})[\mathrm{C} @ \mathrm{H}](\mathrm{O})[\mathrm{C} @ \mathrm{H}] 3 \mathrm{~N}=[\mathrm{N}+]=[\mathrm{N}-]) \mathrm{C} 2=\mathrm{NC}(\mathrm{Cl})=\mathrm{N} 1$ & 3,92 \\
\hline 125 & $\begin{array}{l}\operatorname{CCOC}(=\mathrm{O}) \mathrm{CCCCC} \backslash \mathrm{N}=\mathrm{C}(/ \mathrm{S}) \mathrm{N} 1 \mathrm{C}(\mathrm{CNC} 2=\mathrm{CC}=\mathrm{C}(\mathrm{C}=\mathrm{C} 2) \mathrm{C}(=\mathrm{O}) \mathrm{NC}(\mathrm{CCC}(\mathrm{O})=\mathrm{O}) \mathrm{C}(\mathrm{O})=\mathrm{O}) \mathrm{CNC} 2=\mathrm{NC}(\mathrm{N})=\mathrm{NC}(\mathrm{O} \\
)=\mathrm{C} 12\end{array}$ & 4,6 \\
\hline 126 & $\begin{array}{l}\mathrm{CCOC}(=\mathrm{O}) \mathrm{CCCCCCC} \backslash \mathrm{N}=\mathrm{C}(/ \mathrm{S}) \mathrm{N} 1 \mathrm{C}(\mathrm{CNC} 2=\mathrm{CC}=\mathrm{C}(\mathrm{C}=\mathrm{C} 2) \mathrm{C}(=\mathrm{O}) \mathrm{NC}(\mathrm{CCC}(\mathrm{O})=\mathrm{O}) \mathrm{C}(\mathrm{O})=\mathrm{O}) \mathrm{CNC} 2=\mathrm{NC}(\mathrm{N})=\mathrm{N} \\
\mathrm{C}(\mathrm{O})=\mathrm{C} 12\end{array}$ & 4,6 \\
\hline 127 & $\begin{array}{l}\mathrm{CCOC}(=\mathrm{O}) \mathrm{CCCCCC} \mid \mathrm{N}=\mathrm{C}(/ \mathrm{S}) \mathrm{N} 1 \mathrm{C}(\mathrm{CNC} 2=\mathrm{CC}=\mathrm{C}(\mathrm{C}=\mathrm{C} 2) \mathrm{C}(=\mathrm{O}) \mathrm{NC}(\mathrm{CCC}(\mathrm{O})=\mathrm{O}) \mathrm{C}(\mathrm{O})=\mathrm{O}) \mathrm{CNC} 2=\mathrm{NC}(\mathrm{N})=\mathrm{NC}( \\
\mathrm{O})=\mathrm{C} 12\end{array}$ & 4,6 \\
\hline 128 & $\begin{array}{l}\mathrm{CCOC}(=\mathrm{O}) \mathrm{CCCCCCCC} \backslash \mathrm{N}=\mathrm{C}(/ \mathrm{S}) \mathrm{N} 1 \mathrm{C}(\mathrm{CNC} 2=\mathrm{CC}=\mathrm{C}(\mathrm{C}=\mathrm{C} 2) \mathrm{C}(=\mathrm{O}) \mathrm{NC}(\mathrm{CCC}(\mathrm{O})=\mathrm{O}) \mathrm{C}(\mathrm{O})=\mathrm{O}) \mathrm{CNC} 2=\mathrm{NC}(\mathrm{N})= \\
\mathrm{NC}(\mathrm{O})=\mathrm{C} 12\end{array}$ & 4,6 \\
\hline 129 & $\mathrm{NC} 1=\mathrm{NC} 2=\mathrm{C}(\mathrm{F}) \mathrm{N}=\mathrm{CN}=\mathrm{C} 2 \mathrm{~N} 1 \mathrm{C} 1 \mathrm{O}[\mathrm{C} @ \mathrm{H}](\mathrm{CO})[\mathrm{C} @ @ \mathrm{H}](\mathrm{O})[\mathrm{C} @ \mathrm{H}] 1 \mathrm{O}$ & 5,74 \\
\hline 130 & $\mathrm{OC} 1 \mathrm{CC}(\mathrm{OC} 1 \mathrm{CNC}(=\mathrm{O}) \mathrm{CBr}) \mathrm{N} 1 \mathrm{C}=\mathrm{C}(\mathrm{F}) \mathrm{C}(=\mathrm{O}) \mathrm{NC} 1=\mathrm{O}$ & 4,8 \\
\hline 131 & $\mathrm{OC} 1 \mathrm{CC}(\mathrm{OC} 1 \mathrm{CNC}(=\mathrm{O}) \mathrm{CBr}) \mathrm{N} 1 \mathrm{C}=\mathrm{CC}(=\mathrm{O}) \mathrm{NC} 1=\mathrm{O}$ & 4,96 \\
\hline 132 & $\mathrm{CCC} 1=\mathrm{CN}(\mathrm{C} 2 \mathrm{CC}(\mathrm{O}) \mathrm{C}(\mathrm{CNC}(=\mathrm{O}) \mathrm{CBr}) \mathrm{O} 2) \mathrm{C}(=\mathrm{O}) \mathrm{NC} 1=\mathrm{O}$ & 6 \\
\hline 133 & $\mathrm{OC} 1 \mathrm{CC}(\mathrm{OC} 1 \mathrm{CNC}(=\mathrm{O}) \mathrm{CBr}) \mathrm{N} 1 \mathrm{C}=\mathrm{C}(\mathrm{I}) \mathrm{C}(=\mathrm{O}) \mathrm{NC} 1=\mathrm{O}$ & 5,7 \\
\hline 134 & $\mathrm{OC} 1 \mathrm{CC}(\mathrm{OC} 1 \mathrm{CNC}(=\mathrm{O}) \mathrm{CI}) \mathrm{N} 1 \mathrm{C}=\mathrm{CC}(=\mathrm{O}) \mathrm{NC} 1=\mathrm{O}$ & 4,55 \\
\hline 135 & $\mathrm{CC}(\mathrm{Br}) \mathrm{C}(=\mathrm{O}) \mathrm{NCC} 1 \mathrm{OC}(\mathrm{CC} 1 \mathrm{O}) \mathrm{N} 1 \mathrm{C}=\mathrm{CC}(=\mathrm{O}) \mathrm{NC} 1=\mathrm{O}$ & 4,26 \\
\hline 136 & $\mathrm{OC} 1 \mathrm{CC}(\mathrm{OC} 1 \mathrm{CNC}(=\mathrm{O}) \mathrm{C} 1=\mathrm{CC}=\mathrm{C}(\mathrm{C}=\mathrm{C} 1) \mathrm{S}(\mathrm{F})(=\mathrm{O})=\mathrm{O}) \mathrm{N} 1 \mathrm{C}=\mathrm{CC}(=\mathrm{O}) \mathrm{NC} 1=\mathrm{O}$ & 5 \\
\hline 137 & $\mathrm{OC} 1 \mathrm{CC}(\mathrm{OC} 1 \mathrm{CNC}(=\mathrm{O}) \mathrm{CBr}) \mathrm{N} 1 \mathrm{C}=\mathrm{C}(\mathrm{Br}) \mathrm{C}(=\mathrm{O}) \mathrm{NC} 1=\mathrm{O}$ & 6 \\
\hline 138 & $\mathrm{CC} 1=\mathrm{CN}([\mathrm{C} @ \mathrm{H}] 2 \mathrm{C}[\mathrm{C} @ \mathrm{H}](\mathrm{O})[\mathrm{C} @ @ \mathrm{H}](\mathrm{CNC}(=\mathrm{O}) \mathrm{CBr}) \mathrm{O} 2) \mathrm{C}(=\mathrm{O}) \mathrm{NC} 1=\mathrm{O}$ & 5,1 \\
\hline 139 & $\mathrm{CCOC} 1=\mathrm{NC}(\mathrm{N})=\mathrm{C} 2 \mathrm{~N}=\mathrm{NN}(\mathrm{C} 3 \mathrm{O}[\mathrm{C} @ \mathrm{H}](\mathrm{CO})[\mathrm{C} @ @ \mathrm{H}](\mathrm{O})[\mathrm{C} @ \mathrm{H}] 3 \mathrm{O}) \mathrm{C} 2=\mathrm{N} 1$ & 4,12 \\
\hline 140 & $\mathrm{NC} 1=\mathrm{C} 2 \mathrm{~N}=\mathrm{NN}([\mathrm{C} @ \mathrm{H}] 3 \mathrm{C}[\mathrm{C} @ \mathrm{H}](\mathrm{O})[\mathrm{C} @ @ \mathrm{H}](\mathrm{CO}) \mathrm{O} 3) \mathrm{C} 2=\mathrm{NC}(\mathrm{F})=\mathrm{N} 1$ & 4,15 \\
\hline 141 & $\mathrm{NC} 1=\mathrm{NC}(\mathrm{F})=\mathrm{NC} 2=\mathrm{NN}(\mathrm{N}=\mathrm{C} 12) \mathrm{C} 1 \mathrm{O}[\mathrm{C} @ \mathrm{H}](\mathrm{CO})[\mathrm{C} @ @ \mathrm{H}](\mathrm{O})[\mathrm{C} @ \mathrm{H}] 1 \mathrm{O}$ & 4,12 \\
\hline 142 & $\mathrm{NC} 1=\mathrm{NC}(\mathrm{O})=\mathrm{C} 2 \mathrm{NN}=\mathrm{NC} 2=\mathrm{N} 1$ & 5,7 \\
\hline \multicolumn{2}{|c|}{ ttps://biointerfaceresearch.com/ } & \\
\hline
\end{tabular}




\begin{tabular}{|c|c|c|}
\hline NAME & SMILE & $\lg (\mathbf{1} / \mathbf{I C 5 0})$ \\
\hline 143 & $\mathrm{NC} 1=\mathrm{C} 2 \mathrm{~N}=\mathrm{NN}([\mathrm{C} @ \mathrm{H}] 3 \mathrm{C}[\mathrm{C} @ \mathrm{H}](\mathrm{O})[\mathrm{C} @ @ \mathrm{H}](\mathrm{CO}) \mathrm{O} 3) \mathrm{C} 2=\mathrm{NC}=\mathrm{N} 1$ & 4,6 \\
\hline 144 & $\mathrm{NC} 1=\mathrm{C} 2 \mathrm{~N}=\mathrm{NN}(\mathrm{C} 3 \mathrm{O}[\mathrm{C} @ \mathrm{H}](\mathrm{CO})[\mathrm{C} @ @ \mathrm{H}](\mathrm{O})[\mathrm{C} @ \mathrm{H}] 3 \mathrm{O}) \mathrm{C} 2=\mathrm{NC}(\mathrm{F})=\mathrm{N} 1$ & 5,7 \\
\hline 145 & $\mathrm{NC} 1=\mathrm{C} 2 \mathrm{~N}=\mathrm{NN}([\mathrm{C} @ \mathrm{H}] 3 \mathrm{C}[\mathrm{C} @ \mathrm{H}](\mathrm{O})[\mathrm{C} @ @ \mathrm{H}](\mathrm{CO}) \mathrm{O} 3) \mathrm{C} 2=\mathrm{NC}=\mathrm{N} 1$ & 5 \\
\hline 146 & $\mathrm{NC} 1=\mathrm{NC}(\mathrm{O})=\mathrm{NC} 2=\mathrm{NN}(\mathrm{N}=\mathrm{C} 12) \mathrm{C} 1 \mathrm{O}[\mathrm{C} @ \mathrm{H}](\mathrm{CO})[\mathrm{C} @ @ \mathrm{H}](\mathrm{O})[\mathrm{C} @ \mathrm{H}] 1 \mathrm{O}$ & 4,12 \\
\hline 147 & $\mathrm{CSC} 1=\mathrm{NC}(\mathrm{N})=\mathrm{C} 2 \mathrm{~N}=\mathrm{NN}(\mathrm{C} 3 \mathrm{O}[\mathrm{C} @ \mathrm{H}](\mathrm{CO})[\mathrm{C} @ @ \mathrm{H}](\mathrm{O})[\mathrm{C} @ \mathrm{H}] 3 \mathrm{O}) \mathrm{C} 2=\mathrm{N} 1$ & 3,89 \\
\hline 148 & $\mathrm{NC} 1=\mathrm{NC} 2=\mathrm{NN}(\mathrm{N}=\mathrm{C} 2 \mathrm{C}(\mathrm{N})=\mathrm{N} 1) \mathrm{C} 1 \mathrm{O}[\mathrm{C} @ \mathrm{H}](\mathrm{CO})[\mathrm{C} @ @ \mathrm{H}](\mathrm{O})[\mathrm{C} @ \mathrm{H}] 1 \mathrm{O}$ & 4,12 \\
\hline 149 & $\mathrm{NC} 1=\mathrm{NC}(\mathrm{N})=\mathrm{C} 2 \mathrm{~N}=\mathrm{NN}([\mathrm{C} @ @ \mathrm{H}] 3 \mathrm{O}[\mathrm{C} @ \mathrm{H}](\mathrm{CO})[\mathrm{C} @ @ \mathrm{H}](\mathrm{O})[\mathrm{C} @ \mathrm{H}] 3 \mathrm{O}) \mathrm{C} 2=\mathrm{N} 1$ & 6,15 \\
\hline 150 & $\mathrm{NC} 1=\mathrm{NC}(\mathrm{N})=\mathrm{C} 2 \mathrm{~N}=\mathrm{NN}([\mathrm{C} @ @ \mathrm{H}] 3 \mathrm{O}[\mathrm{C} @ \mathrm{H}](\mathrm{CO})[\mathrm{C} @ @ \mathrm{H}](\mathrm{O})[\mathrm{C} @ \mathrm{H}] 3 \mathrm{O}) \mathrm{C} 2=\mathrm{N} 1$ & 4,7 \\
\hline 151 & $\mathrm{CSC} 1=\mathrm{NC} 2=\mathrm{NN}(\mathrm{N}=\mathrm{C} 2 \mathrm{C}(\mathrm{N})=\mathrm{N} 1) \mathrm{C} 1 \mathrm{O}[\mathrm{C} @ \mathrm{H}](\mathrm{CO})[\mathrm{C} @ @ \mathrm{H}](\mathrm{O})[\mathrm{C} @ \mathrm{H}] 1 \mathrm{O}$ & 4,6 \\
\hline 152 & $\mathrm{CS}(=\mathrm{O})(=\mathrm{O}) \mathrm{C} 1=\mathrm{NC}(\mathrm{N})=\mathrm{C} 2 \mathrm{~N}=\mathrm{NN}(\mathrm{C} 3 \mathrm{O}[\mathrm{C} @ \mathrm{H}](\mathrm{CO})[\mathrm{C} @ @ \mathrm{H}](\mathrm{O})[\mathrm{C} @ \mathrm{H}] 3 \mathrm{O}) \mathrm{C} 2=\mathrm{N} 1$ & 4,85 \\
\hline 153 & $\mathrm{CSC} 1=\mathrm{NC} 2=\mathrm{NN}(\mathrm{N}=\mathrm{C} 2 \mathrm{C}(\mathrm{SC})=\mathrm{N} 1)[\mathrm{C} @ @ \mathrm{H}] 1 \mathrm{O}[\mathrm{C} @ \mathrm{H}](\mathrm{COC}(\mathrm{C})=\mathrm{O})[\mathrm{C} @ @ \mathrm{H}](\mathrm{OC}(\mathrm{C})=\mathrm{O})[\mathrm{C} @ \mathrm{H}] 1 \mathrm{OC}(\mathrm{C})=\mathrm{O}$ & 6,1 \\
\hline 154 & $\mathrm{NC} 1=\mathrm{NC}(\mathrm{O})=\mathrm{C} 2 \mathrm{~N}=\mathrm{NN}(\mathrm{C} 3 \mathrm{O}[\mathrm{C} @ \mathrm{H}](\mathrm{CO})[\mathrm{C} @ @ \mathrm{H}](\mathrm{O})[\mathrm{C} @ \mathrm{H}] 3 \mathrm{O}) \mathrm{C} 2=\mathrm{N} 1$ & 5,7 \\
\hline 155 & $\mathrm{CS}(=\mathrm{O})(=\mathrm{O}) \mathrm{C} 1=\mathrm{NC} 2=\mathrm{NN}(\mathrm{N}=\mathrm{C} 2 \mathrm{C}(\mathrm{N})=\mathrm{N} 1) \mathrm{C} 1 \mathrm{O}[\mathrm{C} @ \mathrm{H}](\mathrm{CO})[\mathrm{C} @ @ \mathrm{H}](\mathrm{O})[\mathrm{C} @ \mathrm{H}] 1 \mathrm{O}$ & 4,66 \\
\hline 156 & $\mathrm{NC} 1=\mathrm{C} 2 \mathrm{~N}=\mathrm{NN}([\mathrm{C} @ @ \mathrm{H}] 3 \mathrm{O}[\mathrm{C} @ \mathrm{H}](\mathrm{CO})[\mathrm{C} @ @ \mathrm{H}](\mathrm{O})[\mathrm{C} @ \mathrm{H}] 3 \mathrm{O}) \mathrm{C} 2=\mathrm{NC}=\mathrm{N} 1$ & 7,7 \\
\hline 157 & $\mathrm{NC} 1=\mathrm{C} 2 \mathrm{~N}=\mathrm{NN}([\mathrm{C} @ @ \mathrm{H}] 3 \mathrm{O}[\mathrm{C} @ \mathrm{H}](\mathrm{CO})[\mathrm{C} @ @ \mathrm{H}](\mathrm{O})[\mathrm{C} @ \mathrm{H}] 3 \mathrm{O}) \mathrm{C} 2=\mathrm{NC}=\mathrm{N} 1$ & 6,15 \\
\hline 158 & $\mathrm{NC} 1=\mathrm{C} 2 \mathrm{~N}=\mathrm{CN}([\mathrm{C} @ @ \mathrm{H}] 3 \mathrm{C}[\mathrm{C} @ \mathrm{H}](\mathrm{CO})[\mathrm{C} @ \mathrm{H}](\mathrm{O}) \mathrm{C} 3 \mathrm{O}) \mathrm{C} 2=\mathrm{CC}=\mathrm{N} 1$ & 6,22 \\
\hline 159 & $\mathrm{NC1}=\mathrm{C} 2 \mathrm{C}=\mathrm{CN}([\mathrm{C} @ @ \mathrm{H}] 3 \mathrm{C}[\mathrm{C} @ \mathrm{H}](\mathrm{CO})[\mathrm{C} @ \mathrm{H}](\mathrm{O}) \mathrm{C} 3 \mathrm{O}) \mathrm{C} 2=\mathrm{NC}=\mathrm{N} 1$ & 5,85 \\
\hline 160 & $\mathrm{NC} 1=\mathrm{C} 2 \mathrm{~N}=\mathrm{CN}=\mathrm{C} 2 \mathrm{C}=\mathrm{CN} 1$ & 5,15 \\
\hline 161 & $\mathrm{NC1}=\mathrm{C} 2 \mathrm{~N}=\mathrm{CN}([\mathrm{C} @ @ \mathrm{H}] 3 \mathrm{O}[\mathrm{C} @ \mathrm{H}](\mathrm{CO})[\mathrm{C} @ @ \mathrm{H}](\mathrm{O})[\mathrm{C} @ \mathrm{H}] 3 \mathrm{O}) \mathrm{C} 2=\mathrm{NC}=\mathrm{N} 1$ & 4,7 \\
\hline 162 & $\mathrm{NC} 1=\mathrm{C} 2 \mathrm{~N}=\mathrm{CN}([\mathrm{C} @ @ \mathrm{H}] 3 \mathrm{O}[\mathrm{C} @ \mathrm{H}](\mathrm{CO})[\mathrm{C} @ @ \mathrm{H}](\mathrm{O})[\mathrm{C} @ \mathrm{H}] 3 \mathrm{O}) \mathrm{C} 2=\mathrm{NC}=\mathrm{N} 1$ & 6,15 \\
\hline 163 & $\mathrm{NC} 1=\mathrm{NC}=\mathrm{NC} 2=\mathrm{C} 1 \mathrm{C}=\mathrm{CN} 2[\mathrm{C} @ @ \mathrm{H}] 1 \mathrm{O}[\mathrm{C} @ \mathrm{H}](\mathrm{CO})[\mathrm{C} @ @ \mathrm{H}](\mathrm{O})[\mathrm{C} @ \mathrm{H}] 1 \mathrm{O}$ & 5,52 \\
\hline 164 & $\mathrm{NC} 1=\mathrm{NC}=\mathrm{NC} 2=\mathrm{C} 1 \mathrm{C}=\mathrm{CN} 2[\mathrm{C} @ @ \mathrm{H}] 1 \mathrm{O}[\mathrm{C} @ \mathrm{H}](\mathrm{CO})[\mathrm{C} @ @ \mathrm{H}](\mathrm{O})[\mathrm{C} @ \mathrm{H}] 1 \mathrm{O}$ & 8,7 \\
\hline 165 & $\mathrm{NC} 1=\mathrm{C} 2 \mathrm{C}=\mathrm{CNC} 2=\mathrm{NC}=\mathrm{N} 1$ & 4,15 \\
\hline 166 & $\mathrm{NC} 1=\mathrm{C} 2 \mathrm{~N}=\mathrm{CN}([\mathrm{C} @ @ \mathrm{H}] 3 \mathrm{O}[\mathrm{C} @ \mathrm{H}](\mathrm{CO})[\mathrm{C} @ @ \mathrm{H}](\mathrm{O})[\mathrm{C} @ \mathrm{H}] 3 \mathrm{O}) \mathrm{C} 2=\mathrm{CC}=\mathrm{N} 1$ & 5,7 \\
\hline 167 & $\mathrm{CC} 1=\mathrm{CN}(\mathrm{C} 2 \mathrm{CC}(\mathrm{O}) \mathrm{C}(\mathrm{CCC}(=\mathrm{O}) \mathrm{CI}) \mathrm{O} 2) \mathrm{C}(=\mathrm{O}) \mathrm{NC} 1=\mathrm{O}$ & 4,77 \\
\hline 168 & $\mathrm{CC} 1=\mathrm{CN}(\mathrm{C} 2 \mathrm{CC}(\mathrm{O}) \mathrm{C}(\mathrm{O} 2) \mathrm{C}(=\mathrm{O}) \mathrm{NC} 2=\mathrm{CC}=\mathrm{C}(\mathrm{C}=\mathrm{C} 2) \mathrm{C}(=\mathrm{O}) \mathrm{CBr}) \mathrm{C}(=\mathrm{O}) \mathrm{NC} 1=\mathrm{O}$ & 4,92 \\
\hline 169 & $\mathrm{CC} 1=\mathrm{CN}(\mathrm{C} 2 \mathrm{CC}(\mathrm{O}) \mathrm{C}(\mathrm{O} 2) \mathrm{C}(=\mathrm{O}) \mathrm{NC} 2=\mathrm{CC}=\mathrm{C}(\mathrm{C}=\mathrm{C} 2) \mathrm{C}(=\mathrm{O}) \mathrm{CI}) \mathrm{C}(=\mathrm{O}) \mathrm{NC} 1=\mathrm{O}$ & 4,54 \\
\hline 170 & $\mathrm{CC} 1=\mathrm{CN}(\mathrm{C} 2 \mathrm{CC}(\mathrm{O}) \mathrm{C}(\mathrm{CCC}(=\mathrm{O}) \mathrm{CCl}) \mathrm{O} 2) \mathrm{C}(=\mathrm{O}) \mathrm{NC} 1=\mathrm{O}$ & 4,6 \\
\hline 171 & $\mathrm{CC} 1=\mathrm{CN}(\mathrm{C} 2 \mathrm{CC}(\mathrm{O}) \mathrm{C}(\mathrm{CCC}(=\mathrm{O}) \mathrm{CBr}) \mathrm{O} 2) \mathrm{C}(=\mathrm{O}) \mathrm{NC} 1=\mathrm{O}$ & 4,26 \\
\hline 172 & $\mathrm{CC} 1=\mathrm{CN}(\mathrm{C} 2 \mathrm{CC}(\mathrm{O}) \mathrm{C}(\mathrm{O} 2) \mathrm{C}(=\mathrm{O}) \mathrm{NC} 2=\mathrm{CC}=\mathrm{C}(\mathrm{C}=\mathrm{C} 2) \mathrm{C}(=\mathrm{O}) \mathrm{CCl}) \mathrm{C}(=\mathrm{O}) \mathrm{NC} 1=\mathrm{O}$ & 4,52 \\
\hline 173 & $\mathrm{CC} 1=\mathrm{CN}(\mathrm{C} 2 \mathrm{CC}(\mathrm{O}) \mathrm{C}(\mathrm{CNC}(=\mathrm{O}) \mathrm{C} 3=\mathrm{CC}=\mathrm{C}(\mathrm{C}=\mathrm{C} 3) \mathrm{C}(=\mathrm{O}) \mathrm{CBr}) \mathrm{O} 2) \mathrm{C}(=\mathrm{O}) \mathrm{NC} 1=\mathrm{O}$ & 4,4 \\
\hline 174 & $\mathrm{NC} 1=\mathrm{NC}(\mathrm{O})=\mathrm{C} 2 \mathrm{~N}=\mathrm{CN}(\mathrm{C} 3 \mathrm{CC}(\mathrm{O}) \mathrm{C}(\mathrm{C} 3) \mathrm{OCP}(\mathrm{O})(\mathrm{O})=\mathrm{O}) \mathrm{C} 2=\mathrm{N} 1$ & 5,62 \\
\hline 175 & $\mathrm{NC} 1=\mathrm{NC}(\mathrm{O})=\mathrm{C} 2 \mathrm{~N}=\mathrm{CN}([\mathrm{C} @ \mathrm{H}] 3 \mathrm{C}[\mathrm{C} @ \mathrm{H}](\mathrm{O})[\mathrm{C} @ @ \mathrm{H}](\mathrm{CO}) \mathrm{C} 3) \mathrm{C} 2=\mathrm{N} 1$ & 4,02 \\
\hline 176 & $\mathrm{COC} 1=\mathrm{NC}(\mathrm{N})=\mathrm{C}(\mathrm{C}(=\mathrm{N} 1) \mathrm{C}(\mathrm{Br}) \mathrm{Br})[\mathrm{N}+]([\mathrm{O}-])=\mathrm{O}$ & 2,8 \\
\hline 177 & $\mathrm{COC} 1=\mathrm{NC}(\mathrm{OC})=\mathrm{C}(\mathrm{C}(=\mathrm{N} 1) \mathrm{C}(\mathrm{Br}) \mathrm{Br})[\mathrm{N}+]([\mathrm{O}-])=\mathrm{O}$ & 2,6 \\
\hline 178 & $\mathrm{COC} 1=\mathrm{NC}(\mathrm{OC})=\mathrm{C}(\mathrm{C}(=\mathrm{N} 1) \mathrm{C}(\mathrm{Br}) \mathrm{Br})[\mathrm{N}+]([\mathrm{O}-])=\mathrm{O}$ & 2,57 \\
\hline 179 & $\mathrm{COC} 1=\mathrm{NC}(\mathrm{C}(\mathrm{Br}) \mathrm{Br})=\mathrm{C}(\mathrm{C}(=\mathrm{N} 1) \mathrm{N} 1 \mathrm{CCOCC} 1)[\mathrm{N}+]([\mathrm{O}-])=\mathrm{O}$ & 2,8 \\
\hline 180 & $\mathrm{COC} 1=\mathrm{NC}(\mathrm{C}(\mathrm{Br}) \mathrm{Br})=\mathrm{C}(\mathrm{C}(=\mathrm{N} 1) \mathrm{N} 1 \mathrm{CCOCC} 1)[\mathrm{N}+]([\mathrm{O}-])=\mathrm{O}$ & 2,55 \\
\hline 181 & $\mathrm{COC} 1=\mathrm{NC}(\mathrm{N})=\mathrm{C}(\mathrm{C}(=\mathrm{N} 1) \mathrm{C}(\mathrm{Br}) \mathrm{Br})[\mathrm{N}+]([\mathrm{O}-])=\mathrm{O}$ & 2,48 \\
\hline 182 & $\mathrm{NC} 1=\mathrm{NC}(\mathrm{O})=\mathrm{C} 2 \mathrm{~N}=\mathrm{CN}(\mathrm{COC}(\mathrm{CO}) \mathrm{CO}) \mathrm{C} 2=\mathrm{N} 1$ & 2,48 \\
\hline 183 & $\begin{array}{l}\mathrm{C}[\mathrm{C} @ @ \mathrm{H}] 1 \mathrm{O}[\mathrm{C} @ @ \mathrm{H}](\mathrm{O}[\mathrm{C} @ \mathrm{H}] 2[\mathrm{C} @ \mathrm{H}](\mathrm{O})[\mathrm{C} @ @ \mathrm{H}](\mathrm{COC}(=\mathrm{O}) \backslash \mathrm{C}=\mathrm{ClC} 3=\mathrm{CC}=\mathrm{C}(\mathrm{O}) \mathrm{C}(\mathrm{O})=\mathrm{C} 3) \mathrm{O}[\mathrm{C} @ @ \mathrm{H}]( \\
\mathrm{OCCC} 3=\mathrm{CC}=\mathrm{C}(\mathrm{O}) \mathrm{C}(\mathrm{O})=\mathrm{C} 3)[\mathrm{C} @ @ \mathrm{H}] 2 \mathrm{O})[\mathrm{C} @ \mathrm{H}](\mathrm{O})[\mathrm{C} @ \mathrm{H}](\mathrm{O})[\mathrm{C} @ \mathrm{H}] 1 \mathrm{O}\end{array}$ & 3,92 \\
\hline 184 & $\begin{array}{l}\mathrm{C}[\mathrm{C} @ @ \mathrm{H}] 1 \mathrm{O}[\mathrm{C} @ @ \mathrm{H}](\mathrm{O}[\mathrm{C} @ \mathrm{H}] 2[\mathrm{C} @ \mathrm{H}](\mathrm{O})[\mathrm{C} @ @ \mathrm{H}](\mathrm{COC}(=\mathrm{O}) \backslash \mathrm{C}=\mathrm{ClC} 3=\mathrm{CC}=\mathrm{C}(\mathrm{O}) \mathrm{C}(\mathrm{O})=\mathrm{C} 3) \mathrm{O}[\mathrm{C} @ @ \mathrm{H}]( \\
\mathrm{OCCC} 3=\mathrm{CC}=\mathrm{C}(\mathrm{O}) \mathrm{C}(\mathrm{O})=\mathrm{C} 3)[\mathrm{C} @ \mathrm{H}] 2 \mathrm{O})[\mathrm{C} @ \mathrm{H}](\mathrm{O})[\mathrm{C} @ \mathrm{H}](\mathrm{O})[\mathrm{C} @ \mathrm{H}] 1 \mathrm{O}\end{array}$ & 3,7 \\
\hline 185 & $\begin{array}{l}\mathrm{C}[\mathrm{C} @ @ \mathrm{H}] 1 \mathrm{O}[\mathrm{C} @ @ \mathrm{H}](\mathrm{O}[\mathrm{C} @ \mathrm{H}] 2[\mathrm{C} @ \mathrm{H}](\mathrm{OC}(=\mathrm{O}) \backslash \mathrm{C}=\mathrm{ClC} 3=\mathrm{CC}=\mathrm{C}(\mathrm{O}) \mathrm{C}(\mathrm{O})=\mathrm{C} 3)[\mathrm{C} @ @ \mathrm{H}](\mathrm{COC}(\mathrm{C})=\mathrm{O}) \mathrm{O}[\mathrm{C} \\
\text { @ @ } \mathrm{H}](\mathrm{OCCC} 3=\mathrm{CC}=\mathrm{C}(\mathrm{O}) \mathrm{C}(\mathrm{O})=\mathrm{C} 3)[\mathrm{C} @ @ \mathrm{H}] 2 \mathrm{OC}(\mathrm{C})=\mathrm{O})[\mathrm{C} @ \mathrm{H}](\mathrm{OC}(\mathrm{C})=\mathrm{O})[\mathrm{C} @ \mathrm{H}](\mathrm{OC}(\mathrm{C})=\mathrm{O})[\mathrm{C} @ \mathrm{H}] 1 \mathrm{OC}( \\
\mathrm{C})=\mathrm{O}\end{array}$ & 4,5 \\
\hline 186 & $\mathrm{NC}(=\mathrm{O}) \mathrm{C} 1=\mathrm{NN}(\mathrm{C}=\mathrm{N} 1)[\mathrm{C} @ @ \mathrm{H}] 1 \mathrm{O}[\mathrm{C} @ \mathrm{H}](\mathrm{CO})[\mathrm{C} @ @ \mathrm{H}](\mathrm{O})[\mathrm{C} @ \mathrm{H}] 1 \mathrm{O}$ & 3,99 \\
\hline 187 & $\mathrm{NC}(=\mathrm{O}) \mathrm{C} 1=\mathrm{NN}(\mathrm{C}=\mathrm{N} 1)[\mathrm{C} @ @ \mathrm{H}] 1 \mathrm{O}[\mathrm{C} @ \mathrm{H}](\mathrm{CO})[\mathrm{C} @ @ \mathrm{H}](\mathrm{O})[\mathrm{C} @ \mathrm{H}] 1 \mathrm{O}$ & 3,84 \\
\hline 188 & $\mathrm{NC} 1=\mathrm{NC}(\mathrm{O})=\mathrm{C} 2 \mathrm{~N}=\mathrm{CN}(\mathrm{COCCO}) \mathrm{C} 2=\mathrm{N} 1$ & 3,53 \\
\hline 189 & $\begin{array}{l}\mathrm{C}[\mathrm{C} @ @ \mathrm{H}] 1 \mathrm{O}[\mathrm{C} @ @ \mathrm{H}](\mathrm{O}[\mathrm{C} @ @ \mathrm{H}] 2[\mathrm{C} @ @ \mathrm{H}](\mathrm{O}[\mathrm{C} @ @ \mathrm{H}] 3 \mathrm{OC}[\mathrm{C} @](\mathrm{O})(\mathrm{CO})[\mathrm{C} @ @ \mathrm{H}] 3 \mathrm{O})[\mathrm{C} @ \mathrm{H}](\mathrm{OCCC}= \\
\mathrm{CC}=\mathrm{C}(\mathrm{O}) \mathrm{C}(\mathrm{O})=\mathrm{C} 3) \mathrm{O}[\mathrm{C} @ \mathrm{H}](\mathrm{COC}(\mathrm{C})=\mathrm{O})[\mathrm{C} @ \mathrm{H}] 2 \mathrm{OC}(=\mathrm{O}) \backslash \mathrm{C}=\mathrm{ClC} 2=\mathrm{CC}=\mathrm{C}(\mathrm{O}) \mathrm{C}(\mathrm{O})=\mathrm{C} 2)[\mathrm{C} @ \mathrm{H}](\mathrm{O})[\mathrm{C} @ \mathrm{H}](\mathrm{O} \\
)[\mathrm{C} @ \mathrm{H}] 1 \mathrm{O}\end{array}$ & 3,65 \\
\hline 190 & $\begin{array}{l}\mathrm{C}[\mathrm{C} @ @ \mathrm{H}] 1 \mathrm{O}[\mathrm{C} @ @ \mathrm{H}](\mathrm{O}[\mathrm{C} @ \mathrm{H}] 2[\mathrm{C} @ \mathrm{H}](\mathrm{O})[\mathrm{C} @ @ \mathrm{H}](\mathrm{COC}(=\mathrm{O}) \backslash \mathrm{C}=\mathrm{ClC} 3=\mathrm{CC}=\mathrm{C}(\mathrm{O}) \mathrm{C}(\mathrm{O})=\mathrm{C} 3) \mathrm{O}[\mathrm{C} @ @ \mathrm{H}]( \\
\mathrm{OCCC} 3=\mathrm{CC}=\mathrm{C}(\mathrm{O}) \mathrm{C}(\mathrm{O})=\mathrm{C} 3)[\mathrm{C} @ \mathrm{H}] 2 \mathrm{O})[\mathrm{C} @ \mathrm{H}](\mathrm{O})[\mathrm{C} @ \mathrm{H}](\mathrm{O})[\mathrm{C} @ \mathrm{H}] 1 \mathrm{O}\end{array}$ & 4,08 \\
\hline 191 & $\begin{array}{l}\mathrm{C}[\mathrm{C} @ @ \mathrm{H}] 1 \mathrm{O}[\mathrm{C} @ @ \mathrm{H}](\mathrm{O}[\mathrm{C} @ \mathrm{H}] 2[\mathrm{C} @ \mathrm{H}](\mathrm{OC}(\mathrm{C})=\mathrm{O})[\mathrm{C} @ @ \mathrm{H}](\mathrm{COC}(=\mathrm{O}) \backslash \mathrm{C}=\mathrm{ClC} 3=\mathrm{CC}=\mathrm{C}(\mathrm{OC}(\mathrm{C})=\mathrm{O}) \mathrm{C}(\mathrm{OC}( \\
\mathrm{C})=\mathrm{O})=\mathrm{C} 3) \mathrm{O}[\mathrm{C} @ @ \mathrm{H}](\mathrm{OCCC} 3=\mathrm{CC}=\mathrm{C}(\mathrm{OC}(\mathrm{C})=\mathrm{O}) \mathrm{C}(\mathrm{OC}(\mathrm{C})=\mathrm{O})=\mathrm{C} 3)[\mathrm{C} @(\mathrm{H}] 2 \mathrm{OC}(\mathrm{C})=\mathrm{O})[\mathrm{C} @ \mathrm{H}](\mathrm{OC}(\mathrm{C})=\mathrm{O}) \\
{[\mathrm{C} @ \mathrm{H}](\mathrm{OC}(\mathrm{C})=\mathrm{O})[\mathrm{C} @ \mathrm{H}] 1 \mathrm{OC}(\mathrm{C})=\mathrm{O}}\end{array}$ & 4,95 \\
\hline 192 & $\begin{array}{l}\mathrm{C}[\mathrm{C} @ @ \mathrm{H}] 1 \mathrm{O}[\mathrm{C} @ @ \mathrm{H}](\mathrm{O}[\mathrm{C} @ @ \mathrm{H}] 2[\mathrm{C} @ @ \mathrm{H}](\mathrm{O})[\mathrm{C} @ \mathrm{H}](\mathrm{OCCC} 3=\mathrm{CC}=\mathrm{C}(\mathrm{O}) \mathrm{C}(\mathrm{O})=\mathrm{C} 3) \mathrm{O}[\mathrm{C} @ \mathrm{H}](\mathrm{CO})[\mathrm{C} @ \mathrm{H}] \\
2 \mathrm{OC}(=\mathrm{O}) \backslash \mathrm{C}=\mathrm{ClC} 2=\mathrm{CC}=\mathrm{C}(\mathrm{O}) \mathrm{C}(\mathrm{O})=\mathrm{C} 2)[\mathrm{C} @ \mathrm{H}](\mathrm{O})[\mathrm{C} @ \mathrm{H}](\mathrm{O})[\mathrm{C} @ \mathrm{H}] 1 \mathrm{O}\end{array}$ & 4,15 \\
\hline 193 & $\begin{array}{l}\mathrm{C}[\mathrm{C} @ @ \mathrm{H}] 1 \mathrm{O}[\mathrm{C} @ @ \mathrm{H}](\mathrm{O}[\mathrm{C} @ \mathrm{H}] 2[\mathrm{C} @ \mathrm{H}](\mathrm{O})[\mathrm{C} @ @ \mathrm{H}](\mathrm{COC}(=\mathrm{O}) \mathrm{C}=\mathrm{ClC} 3=\mathrm{CC}=\mathrm{C}(\mathrm{O}) \mathrm{C}(\mathrm{O})=\mathrm{C} 3) \mathrm{O}[\mathrm{C} @ @ \mathrm{H}]( \\
\mathrm{OCCC}=\mathrm{CC}=\mathrm{C}(\mathrm{O}) \mathrm{C}(\mathrm{O})=\mathrm{C} 3)[\mathrm{C} @ \mathrm{H}] 2 \mathrm{O}[\mathrm{C} @ \mathrm{H}] 2 \mathrm{OC}[\mathrm{C} @](\mathrm{O})(\mathrm{CO})[\mathrm{C} @ \mathrm{H}] 2 \mathrm{O})[\mathrm{C} @ \mathrm{H}](\mathrm{O})[\mathrm{C} @ \mathrm{H}](\mathrm{O})[\mathrm{C} @ \\
\mathrm{H}] 1 \mathrm{O}\end{array}$ & 3,92 \\
\hline 194 & $\begin{array}{l}\mathrm{C}[\mathrm{C} @ @ \mathrm{H}] 1 \mathrm{O}[\mathrm{C} @ @ \mathrm{H}](\mathrm{O}[\mathrm{C} @ \mathrm{H}] 2[\mathrm{C} @ \mathrm{H}](\mathrm{O})[\mathrm{C} @ @ \mathrm{H}](\mathrm{COC}(=\mathrm{O}) \backslash \mathrm{C}=\mathrm{ClC} 3=\mathrm{CC}=\mathrm{C}(\mathrm{O}) \mathrm{C}(\mathrm{O})=\mathrm{C} 3) \mathrm{O}[\mathrm{C} @ @ \mathrm{H}]( \\
\mathrm{OCCC}=\mathrm{CC}=\mathrm{C}(\mathrm{O}) \mathrm{C}(\mathrm{O})=\mathrm{C} 3)[\mathrm{C} @ \mathrm{H}] 2 \mathrm{O}[\mathrm{C} @(\mathrm{H}] 2 \mathrm{OC}[\mathrm{C} @](\mathrm{O})(\mathrm{CO})[\mathrm{C} @ \mathrm{H}] 2 \mathrm{O})[\mathrm{C} @ \mathrm{H}](\mathrm{O})[\mathrm{C} @ \mathrm{H}](\mathrm{O})[\mathrm{C} @ \\
\mathrm{H}] 1 \mathrm{O}\end{array}$ & 3,91 \\
\hline 195 & $\begin{array}{l}\mathrm{C}[\mathrm{C} @ @ \mathrm{H}] 1 \mathrm{O}[\mathrm{C} @ @ \mathrm{H}](\mathrm{O}[\mathrm{C} @ \mathrm{H}] 2[\mathrm{C} @ \mathrm{H}](\mathrm{O})[\mathrm{C} @ @ \mathrm{H}](\mathrm{COC}(=\mathrm{O}) \backslash \mathrm{C}=\mathrm{ClC} 3=\mathrm{CC}=\mathrm{C}(\mathrm{O}) \mathrm{C}(\mathrm{O})=\mathrm{C} 3) \mathrm{O}[\mathrm{C} @ @ \mathrm{H}]( \\
\mathrm{OCCC}=\mathrm{CC}=\mathrm{C}(\mathrm{O}) \mathrm{C}(\mathrm{O})=\mathrm{C} 3)[\mathrm{C} @ @ \mathrm{H}] 2 \mathrm{O})[\mathrm{C} @ \mathrm{H}](\mathrm{O})[\mathrm{C} @ \mathrm{H}](\mathrm{O})[\mathrm{C} @ \mathrm{H}] 1 \mathrm{O}\end{array}$ & 4,36 \\
\hline 196 & $\begin{array}{l}\mathrm{C}[\mathrm{C} @ @ \mathrm{H}] 1 \mathrm{O}[\mathrm{C} @ @ \mathrm{H}](\mathrm{O}[\mathrm{C} @ \mathrm{H}] 2[\mathrm{C} @ \mathrm{H}](\mathrm{OC}(=\mathrm{O}) \backslash \mathrm{C}=\mathrm{ClC} 3=\mathrm{CC}=\mathrm{C}(\mathrm{OC}(\mathrm{C})=\mathrm{O}) \mathrm{C}(\mathrm{OC}(\mathrm{C})=\mathrm{O})=\mathrm{C} 3)[\mathrm{C} @ @ \mathrm{H}](\mathrm{C} \\
\mathrm{OC}(\mathrm{C})=\mathrm{O}) \mathrm{O}[\mathrm{C} @ @ \mathrm{H}](\mathrm{OCCC}=\mathrm{CC}=\mathrm{C}(\mathrm{OC}(\mathrm{C})=\mathrm{O}) \mathrm{C}(\mathrm{OC}(\mathrm{C})=\mathrm{O})=\mathrm{C} 3)[\mathrm{C} @ \mathrm{H}] 2 \mathrm{OC}(\mathrm{C})=\mathrm{O})[\mathrm{C} @ \mathrm{H}](\mathrm{OC}(\mathrm{C})=\mathrm{O})[ \\
\mathrm{C} @ \mathrm{H}](\mathrm{OC}(\mathrm{C})=\mathrm{O})[\mathrm{C} @ \mathrm{H}] 1 \mathrm{OC}(\mathrm{C})=\mathrm{O}\end{array}$ & 4,47 \\
\hline 197 & $\begin{array}{l}\mathrm{C}[\mathrm{C} @ @ \mathrm{H}] 1 \mathrm{O}[\mathrm{C} @ @ \mathrm{H}](\mathrm{O}[\mathrm{C} @ @ \mathrm{H}] 2[\mathrm{C} @ @ \mathrm{H}](\mathrm{O})[\mathrm{C} @ \mathrm{H}](\mathrm{OCCC} 3=\mathrm{CC}=\mathrm{C}(\mathrm{O}) \mathrm{C}(\mathrm{O})=\mathrm{C} 3) \mathrm{O}[\mathrm{C} @ \mathrm{H}](\mathrm{CO})[\mathrm{C} @ \mathrm{H}] \\
2 \mathrm{OC}(=\mathrm{O}) \backslash \mathrm{C}=\mathrm{ClC} 2=\mathrm{CC}=\mathrm{C}(\mathrm{O}) \mathrm{C}(\mathrm{O})=\mathrm{C} 2)[\mathrm{C} @ \mathrm{H}](\mathrm{O})[\mathrm{C} @ \mathrm{H}](\mathrm{O})[\mathrm{C} @ \mathrm{H}] 1 \mathrm{O}\end{array}$ & 3,76 \\
\hline
\end{tabular}




\begin{tabular}{|c|c|c|}
\hline NAME & SMILE & $\lg (1 / I C 50)$ \\
\hline 198 & $\begin{array}{l}\mathrm{C}[\mathrm{C} @ @ \mathrm{H}] 1 \mathrm{O}[\mathrm{C} @ @ \mathrm{H}](\mathrm{O}[\mathrm{C} @ \mathrm{H}] 2[\mathrm{C} @ \mathrm{H}](\mathrm{O})[\mathrm{C} @ @ \mathrm{H}](\mathrm{COC}(=\mathrm{O}) \backslash \mathrm{C}=\mathrm{ClC}=\mathrm{CC}=\mathrm{C}(\mathrm{O}) \mathrm{C}(\mathrm{O})=\mathrm{C} 3) \mathrm{O}[\mathrm{C} @ @ \mathrm{H}]( \\
\mathrm{OCCC}=\mathrm{CC}=\mathrm{C}(\mathrm{O}) \mathrm{C}(\mathrm{O})=\mathrm{C} 3)[\mathrm{C} @ \mathrm{H}] 2 \mathrm{O}[\mathrm{C} @(\mathrm{H}] 2 \mathrm{OC}[\mathrm{C} @](\mathrm{O})(\mathrm{CO})[\mathrm{C} @ @ \mathrm{H}] 2 \mathrm{O})[\mathrm{C} @ \mathrm{H}](\mathrm{O})[\mathrm{C} @ \mathrm{H}](\mathrm{O})[\mathrm{C} @ \\
\mathrm{H}] 1 \mathrm{O}\end{array}$ & 3,62 \\
\hline 199 & $\begin{array}{l}\mathrm{C}[\mathrm{C} @ @ \mathrm{H}] 1 \mathrm{O}[\mathrm{C} @ @ \mathrm{H}](\mathrm{O}[\mathrm{C} @ \mathrm{H}] 2[\mathrm{C} @ \mathrm{H}](\mathrm{O})[\mathrm{C} @ @ \mathrm{H}](\mathrm{COC}(=\mathrm{O}) \backslash \mathrm{C}=\mathrm{ClC} 3=\mathrm{CC}=\mathrm{C}(\mathrm{O}) \mathrm{C}(\mathrm{O})=\mathrm{C} 3) \mathrm{O}[\mathrm{C} @ @ \mathrm{H}]( \\
\mathrm{OCCC} 3=\mathrm{CC}=\mathrm{C}(\mathrm{O}) \mathrm{C}(\mathrm{O})=\mathrm{C} 3)[\mathrm{C} @ @ \mathrm{H}] 2 \mathrm{O})[\mathrm{C} @ \mathrm{H}](\mathrm{O})[\mathrm{C} @ \mathrm{H}](\mathrm{O})[\mathrm{C} @ \mathrm{H}] 1 \mathrm{O}\end{array}$ & 4,32 \\
\hline 200 & $\begin{array}{l}\mathrm{COC} 1=\mathrm{CC}(\mathrm{C}=\mathrm{ClC}(=\mathrm{O}) \mathrm{OC}[\mathrm{C} @ \mathrm{H}] 2 \mathrm{O}[\mathrm{C} @ @ \mathrm{H}](\mathrm{OCCC} 3=\mathrm{CC}=\mathrm{C}(\mathrm{O}) \mathrm{C}(\mathrm{O})=\mathrm{C} 3)[\mathrm{C} @ \mathrm{H}](\mathrm{O}[\mathrm{C} @ @ \mathrm{H}] 3 \mathrm{OC}[\mathrm{C} @](\mathrm{O} \\
)(\mathrm{CO})[\mathrm{C} @ \mathrm{H}] 3 \mathrm{O})[\mathrm{C} @ \mathrm{H}](\mathrm{O}[\mathrm{C} @ \mathrm{H}] 3 \mathrm{O}[\mathrm{C} @ \mathrm{H}](\mathrm{C})[\mathrm{C} @ \mathrm{H}](\mathrm{O})[\mathrm{C} @ \mathrm{H}](\mathrm{O})[\mathrm{C} @ \mathrm{H}] 3 \mathrm{O})[\mathrm{C} @ @ \mathrm{H}] 2 \mathrm{O})=\mathrm{CC} \\
=\mathrm{C} 1 \mathrm{O}\end{array}$ & 3,37 \\
\hline 201 & $\begin{array}{l}\mathrm{C}[\mathrm{C} @ @ \mathrm{H}] 1 \mathrm{O}[\mathrm{C} @ @ \mathrm{H}](\mathrm{O}[\mathrm{C} @ \mathrm{H}] 2[\mathrm{C} @ \mathrm{H}](\mathrm{O})[\mathrm{C} @ @ \mathrm{H}](\mathrm{COC}(=\mathrm{O}) \mathrm{C}=\mathrm{ClC} 3=\mathrm{CC}=\mathrm{C}(\mathrm{O}) \mathrm{C}(\mathrm{O})=\mathrm{C} 3) \mathrm{O}[\mathrm{C} @ @ \mathrm{H}]( \\
\mathrm{OCCC}=\mathrm{CC}=\mathrm{C}(\mathrm{O}) \mathrm{C}(\mathrm{O})=\mathrm{C} 3)\left[\mathrm{C} @ \mathrm{H}^{2} \mathrm{O}\right)[\mathrm{C} @ \mathrm{H}](\mathrm{O})[\mathrm{C} @ \mathrm{H}](\mathrm{O})[\mathrm{C} @ \mathrm{H}] 1 \mathrm{O}\end{array}$ & 3,85 \\
\hline 202 & $\begin{array}{l}\mathrm{C}[\mathrm{C} @ @ \mathrm{H}] 1 \mathrm{O}[\mathrm{C} @ @ \mathrm{H}](\mathrm{O}[\mathrm{C} @ \mathrm{H}] 2[\mathrm{C} @ \mathrm{H}](\mathrm{OC}(\mathrm{C})=\mathrm{O})[\mathrm{C} @ @ \mathrm{H}](\mathrm{COC}(=\mathrm{O}) \backslash \mathrm{C}=\mathrm{ClC} 3=\mathrm{CC}=\mathrm{C}(\mathrm{O}) \mathrm{C}(\mathrm{O})=\mathrm{C} 3) \mathrm{O}[\mathrm{C} \\
@ @ \mathrm{H}](\mathrm{OCCC} 3=\mathrm{CC}=\mathrm{C}(\mathrm{O}) \mathrm{C}(\mathrm{O})=\mathrm{C} 3)[\mathrm{C} @ @ \mathrm{H}] 2 \mathrm{OC}(\mathrm{C})=\mathrm{O})[\mathrm{C} @ \mathrm{H}](\mathrm{OC}(\mathrm{C})=\mathrm{O})[\mathrm{C} @ \mathrm{H}](\mathrm{OC}(\mathrm{C})=\mathrm{O})[\mathrm{C} @ \mathrm{H}] 1 \mathrm{OC}( \\
\mathrm{C})=\mathrm{O}\end{array}$ & 4,72 \\
\hline 203 & $\begin{array}{l}\mathrm{C}[\mathrm{C} @ @ \mathrm{H}] 1 \mathrm{O}[\mathrm{C} @ @ \mathrm{H}](\mathrm{O}[\mathrm{C} @ @ \mathrm{H}] 2[\mathrm{C} @ @ \mathrm{H}](\mathrm{O})[\mathrm{C} @ \mathrm{H}](\mathrm{OCCC} 3=\mathrm{CC}=\mathrm{C}(\mathrm{O}) \mathrm{C}(\mathrm{O})=\mathrm{C} 3) \mathrm{O}[\mathrm{C} @ \mathrm{H}](\mathrm{CO})[\mathrm{C} @ \mathrm{H}] \\
2 \mathrm{OC}(=\mathrm{O}) \mathrm{C}=\mathrm{ClC} 2=\mathrm{CC}=\mathrm{C}(\mathrm{O}) \mathrm{C}(\mathrm{O})=\mathrm{C} 2)[\mathrm{C} @ \mathrm{H}](\mathrm{O})[\mathrm{C} @ \mathrm{H}](\mathrm{O})[\mathrm{C} @ \mathrm{H}] 1 \mathrm{O}\end{array}$ & 3,94 \\
\hline 204 & $\mathrm{NC} 1=\mathrm{NC}(\mathrm{O})=\mathrm{C} 2 \mathrm{~N}=\mathrm{CN}(\mathrm{COCCO}) \mathrm{C} 2=\mathrm{N} 1$ & 4,35 \\
\hline 205 & $\begin{array}{l}\mathrm{C}[\mathrm{C} @ @ \mathrm{H}] 1 \mathrm{O}[\mathrm{C} @ @ \mathrm{H}](\mathrm{O}[\mathrm{C} @ @ \mathrm{H}] 2[\mathrm{C} @ @ \mathrm{H}](\mathrm{O}[\mathrm{C} @ @ \mathrm{H}] 3 \mathrm{OC}[\mathrm{C} @](\mathrm{O})(\mathrm{CO})[\mathrm{C} @ @ \mathrm{H}] 3 \mathrm{O})[\mathrm{C} @ \mathrm{H}](\mathrm{OCCC}= \\
\mathrm{CC}=\mathrm{C}(\mathrm{O}) \mathrm{C}(\mathrm{O})=\mathrm{C} 3) \mathrm{O}[\mathrm{C} @ \mathrm{H}](\mathrm{COC}(\mathrm{C})=\mathrm{O})[\mathrm{C} @ \mathrm{H}] 2 \mathrm{OC}(=\mathrm{O}) \backslash \mathrm{C}=\mathrm{ClC} 2=\mathrm{CC}=\mathrm{C}(\mathrm{O}) \mathrm{C}(\mathrm{O})=\mathrm{C} 2)[\mathrm{C} @ \mathrm{H}](\mathrm{O})[\mathrm{C} @ \mathrm{H}](\mathrm{O} \\
)[\mathrm{C} @ \mathrm{H}] 1 \mathrm{O}\end{array}$ & 4,02 \\
\hline 206 & 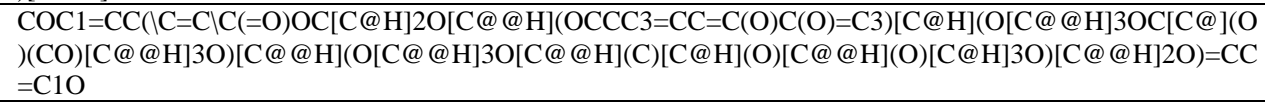 & 3,61 \\
\hline 207 & $\begin{array}{l}\mathrm{C}[\mathrm{C} @ @ \mathrm{H}] 1 \mathrm{O}[\mathrm{C} @ @ \mathrm{H}](\mathrm{O}[\mathrm{C} @ \mathrm{H}] 2[\mathrm{C} @ \mathrm{H}](\mathrm{O})[\mathrm{C} @ @ \mathrm{H}](\mathrm{COC}(=\mathrm{O}) \mathrm{C}=\mathrm{ClC} 3=\mathrm{CC}=\mathrm{C}(\mathrm{O}) \mathrm{C}(\mathrm{O})=\mathrm{C} 3) \mathrm{O}[\mathrm{C} @ @ \mathrm{H}]( \\
\mathrm{OCCC}=\mathrm{CC}=\mathrm{C}(\mathrm{O}) \mathrm{C}(\mathrm{O})=\mathrm{C} 3)[\mathrm{C} @ \mathrm{H}] 2 \mathrm{O}\left[\mathrm{C} @(\mathrm{H}] 2 \mathrm{OC}[\mathrm{C} @](\mathrm{O})(\mathrm{CO})\left[\mathrm{C} @ \mathrm{H}_{2} \mathrm{O}\right)[\mathrm{C} @ \mathrm{H}](\mathrm{O})[\mathrm{C} @ \mathrm{H}](\mathrm{O})[\mathrm{C} @\right. \\
\mathrm{H}] 1 \mathrm{O}\end{array}$ & 4,05 \\
\hline 208 & $\mathrm{NC} 1=\mathrm{NC}(\mathrm{O})=\mathrm{C} 2 \mathrm{~N}=\mathrm{CN}(\mathrm{COC}(\mathrm{CO}) \mathrm{CO}) \mathrm{C} 2=\mathrm{N} 1$ & 3,41 \\
\hline 209 & $\begin{array}{l}\mathrm{COC}(=\mathrm{O}) \mathrm{C} 1=\mathrm{CO}[\mathrm{C} @ @ \mathrm{H}](\mathrm{O}[\mathrm{C} @ @ \mathrm{H}] 2 \mathrm{O}[\mathrm{C} @ \mathrm{H}](\mathrm{CO})[\mathrm{C} @ @ \mathrm{H}](\mathrm{O})[\mathrm{C} @ \mathrm{H}](\mathrm{O})[\mathrm{C} @ \mathrm{H}] 2 \mathrm{O})[\mathrm{C} @ \mathrm{H}] 2[\mathrm{C} @ @ \mathrm{H}] 1[ \\
\mathrm{C} @(\mathrm{H}](\mathrm{C}[\mathrm{C} @] 2(\mathrm{C}) \mathrm{OC}(\mathrm{C})=\mathrm{O}) \mathrm{OC}(=\mathrm{O}) \backslash \mathrm{C}=\mathrm{ClC} 1=\mathrm{CC}=\mathrm{C}(\mathrm{O}) \mathrm{C}=\mathrm{C} 1\end{array}$ & 4,15 \\
\hline 210 & $\begin{array}{l}\mathrm{C}[\mathrm{C} @ @ \mathrm{H}] 1 \mathrm{O}[\mathrm{C} @ @ \mathrm{H}](\mathrm{O}[\mathrm{C} @ @ \mathrm{H}] 2[\mathrm{C} @ @ \mathrm{H}](\mathrm{O})[\mathrm{C} @ \mathrm{H}](\mathrm{OCCC} 3=\mathrm{CC}=\mathrm{C}(\mathrm{O}) \mathrm{C}(\mathrm{O})=\mathrm{C} 3) \mathrm{O}[\mathrm{C} @ \mathrm{H}](\mathrm{CO})[\mathrm{C} @ \mathrm{H}] \\
2 \mathrm{OC}(=\mathrm{O}) \backslash \mathrm{C}=\mathrm{ClC} 2=\mathrm{CC}=\mathrm{C}(\mathrm{O}) \mathrm{C}(\mathrm{O})=\mathrm{C} 2)[\mathrm{C} @ \mathrm{H}](\mathrm{O})[\mathrm{C} @ \mathrm{H}](\mathrm{O})[\mathrm{C} @ \mathrm{H}] 1 \mathrm{O}\end{array}$ & 3,91 \\
\hline 211 & $\begin{array}{l}\mathrm{COC}(=\mathrm{O}) \mathrm{C} 1=\mathrm{CO}[\mathrm{C} @ @ \mathrm{H}](\mathrm{O}[\mathrm{C} @ @ \mathrm{H}] 2 \mathrm{O}[\mathrm{C} @ \mathrm{H}](\mathrm{CO})[\mathrm{C} @ @ \mathrm{H}](\mathrm{O})[\mathrm{C} @ \mathrm{H}](\mathrm{O})[\mathrm{C} @ \mathrm{H}] 2 \mathrm{O})[\mathrm{C} @ \mathrm{H}] 2[\mathrm{C} @ @ \mathrm{H}] 1[ \\
\mathrm{C} @(\mathrm{H}](\mathrm{C}[\mathrm{C} @] 2(\mathrm{C}) \mathrm{OC}(\mathrm{C})=\mathrm{O}) \mathrm{OC}(=\mathrm{O}) \backslash \mathrm{C}=\mathrm{C} / \mathrm{C} 1=\mathrm{CC}=\mathrm{C}(\mathrm{O}) \mathrm{C}=\mathrm{C} 1\end{array}$ & 4,15 \\
\hline 212 & $\mathrm{BrC} 1=\mathrm{CN}(\mathrm{ClC}=\mathrm{C} 2 / \mathrm{OC}(=\mathrm{O}) \mathrm{C}(\mathrm{OCC} 3=\mathrm{CC}=\mathrm{CC}=\mathrm{C} 3)=\mathrm{C} 2 \mathrm{OCC} 2=\mathrm{CC}=\mathrm{CC}=\mathrm{C} 2) \mathrm{C}(=\mathrm{O}) \mathrm{NC} 1=\mathrm{O}$ & 4,05 \\
\hline 213 & $\mathrm{O}=\mathrm{C} 1 \mathrm{O} \backslash \mathrm{C}(=\mathrm{C} / \mathrm{CN} 2 \mathrm{C}=\mathrm{NC} 3=\mathrm{C} 2 \mathrm{~N}=\mathrm{CN}=\mathrm{C} 3 \mathrm{~N} 2 \mathrm{C}=\mathrm{CC}=\mathrm{C} 2) \mathrm{C}(\mathrm{OCC} 2=\mathrm{CC}=\mathrm{CC}=\mathrm{C} 2)=\mathrm{C} 1 \mathrm{OCC} 1=\mathrm{CC}=\mathrm{CC}=\mathrm{C} 1$ & 4 \\
\hline 214 & $\mathrm{O}=\mathrm{C} 1 \mathrm{O} \backslash \mathrm{C}(=\mathrm{C} / \mathrm{CN} 2 \mathrm{C}=\mathrm{CC}(=\mathrm{O}) \mathrm{NC} 2=\mathrm{O}) \mathrm{C}(\mathrm{OCC} 2=\mathrm{CC}=\mathrm{CC}=\mathrm{C} 2)=\mathrm{C} 1 \mathrm{OCC} 1=\mathrm{CC}=\mathrm{CC}=\mathrm{C} 1$ & 4,4 \\
\hline 215 & $\mathrm{ClC} 1=\mathrm{CN}(\mathrm{ClC}=\mathrm{C} 2 / \mathrm{OC}(=\mathrm{O}) \mathrm{C}(\mathrm{OCC} 3=\mathrm{CC}=\mathrm{CC}=\mathrm{C} 3)=\mathrm{C} 2 \mathrm{OCC} 2=\mathrm{CC}=\mathrm{CC}=\mathrm{C} 2) \mathrm{C}(=\mathrm{O}) \mathrm{NC} 1=\mathrm{O}$ & 4,7 \\
\hline 216 & $\mathrm{ClC} 1=\mathrm{NC}=\mathrm{NC} 2=\mathrm{C} 1 \mathrm{~N}=\mathrm{CN} 2 \mathrm{ClC}=\mathrm{C} 1 / \mathrm{OC}(=\mathrm{O}) \mathrm{C}(\mathrm{OCC} 2=\mathrm{CC}=\mathrm{CC}=\mathrm{C} 2)=\mathrm{C} 1 \mathrm{OCC} 1=\mathrm{CC}=\mathrm{CC}=\mathrm{C} 1$ & 4,22 \\
\hline 217 & $\mathrm{ClC} 1=\mathrm{NC}=\mathrm{NC} 2=\mathrm{C} 1 \mathrm{~N}(\mathrm{ClC}=\mathrm{C} 1 \backslash \mathrm{OC}(=\mathrm{O}) \mathrm{C}(\mathrm{OCC} 3=\mathrm{CC}=\mathrm{CC}=\mathrm{C} 3)=\mathrm{C} 1 \mathrm{OCC} 1=\mathrm{CC}=\mathrm{CC}=\mathrm{C} 1) \mathrm{C}=\mathrm{N} 2$ & 4,22 \\
\hline 218 & $\mathrm{FC} 1=\mathrm{CN}(\mathrm{ClC}=\mathrm{C} 2 / \mathrm{OC}(=\mathrm{O}) \mathrm{C}(\mathrm{OCC} 3=\mathrm{CC}=\mathrm{CC}=\mathrm{C} 3)=\mathrm{C} 2 \mathrm{OCC} 2=\mathrm{CC}=\mathrm{CC}=\mathrm{C} 2) \mathrm{C}(=\mathrm{O}) \mathrm{NC} 1=\mathrm{O}$ & 4,7 \\
\hline 219 & $\mathrm{FC}(\mathrm{F})(\mathrm{F}) \mathrm{C} 1=\mathrm{CN}(\mathrm{ClC}=\mathrm{C} 2 / \mathrm{OC}(=\mathrm{O}) \mathrm{C}(\mathrm{OCC} 3=\mathrm{CC}=\mathrm{CC}=\mathrm{C} 3)=\mathrm{C} 2 \mathrm{OCC} 2=\mathrm{CC}=\mathrm{CC}=\mathrm{C} 2) \mathrm{C}(=\mathrm{O}) \mathrm{NC} 1=\mathrm{O}$ & 4,4 \\
\hline 220 & $\mathrm{IC} 1=\mathrm{CN}(\mathrm{ClC}=\mathrm{C} 2 / \mathrm{OC}(=\mathrm{O}) \mathrm{C}(\mathrm{OCC} 3=\mathrm{CC}=\mathrm{CC}=\mathrm{C} 3)=\mathrm{C} 2 \mathrm{OCC} 2=\mathrm{CC}=\mathrm{CC}=\mathrm{C} 2) \mathrm{C}(=\mathrm{O}) \mathrm{NC} 1=\mathrm{O}$ & 5 \\
\hline 221 & $\mathrm{CC} 1=\mathrm{CC}(=\mathrm{O}) \mathrm{C} 2=\mathrm{C}(\mathrm{CO}) \mathrm{CC}[\mathrm{C} @ @ \mathrm{H}] 3 \mathrm{C}[\mathrm{C} @] 12 \mathrm{OC}(=\mathrm{O}) \mathrm{C} 3=\mathrm{C}$ & 5,96 \\
\hline 222 & $\mathrm{CC} 1=\mathrm{CC}(=\mathrm{O}) \mathrm{C} 2=\mathrm{C}(\mathrm{CO}) \mathrm{CC}[\mathrm{C} @ @ \mathrm{H}] 3 \mathrm{C}[\mathrm{C} @] 12 \mathrm{OC}(=\mathrm{O}) \mathrm{C} 3=\mathrm{C}$ & 5,89 \\
\hline 223 & $\begin{array}{l}\mathrm{CC} 1=\mathrm{CC}(=\mathrm{O}) \mathrm{C} 2=\mathrm{C}(\mathrm{CO}) \mathrm{CC}[\mathrm{C} @ \mathrm{H}](\mathrm{C}[\mathrm{C} @] 12 \mathrm{O}) \mathrm{C}(=\mathrm{C}) \mathrm{C}(=\mathrm{O}) \mathrm{O}[\mathrm{C} @ @ \mathrm{H}] 1[\mathrm{C} @ @ \mathrm{H}](\mathrm{O})[\mathrm{C} @ \mathrm{H}](\mathrm{O})[\mathrm{C} @ @ \mathrm{H}](\mathrm{O} \\
\mathrm{CC} 2=\mathrm{C} 3 \mathrm{C}(=\mathrm{O}) \mathrm{C}=\mathrm{C}(\mathrm{C})[\mathrm{C} @ @] 33 \mathrm{C}[\mathrm{C} @ @ \mathrm{H}](\mathrm{CC} 2) \mathrm{C}(=\mathrm{C}) \mathrm{C}(=\mathrm{O}) \mathrm{O} 3) \mathrm{O}[\mathrm{C} @ \mathrm{H}] 1 \mathrm{CO}\end{array}$ & 6,1 \\
\hline 224 & $\mathrm{CC} 1=\mathrm{CC}(=\mathrm{O}) \mathrm{C} 2=\mathrm{C}(\mathrm{CO}) \mathrm{CC}[\mathrm{C} @ @ \mathrm{H}] 3 \mathrm{C}[\mathrm{C} @] 12 \mathrm{OC}(=\mathrm{O}) \mathrm{C} 3=\mathrm{C}$ & 5,82 \\
\hline 225 & $\mathrm{C}[\mathrm{C} @ @ \mathrm{H}] 1 \mathrm{C}[\mathrm{C} @ \mathrm{H}] 2 \mathrm{OC}(=\mathrm{O}) \mathrm{C}(=\mathrm{C})[\mathrm{C} @ \mathrm{H}] 2[\mathrm{C} @ \mathrm{H}](\mathrm{O})[\mathrm{C} @ @] 2(\mathrm{C})[\mathrm{C} @ \mathrm{H}] 1 \mathrm{C}=\mathrm{CC} 2=\mathrm{O}$ & 6,05 \\
\hline 226 & $\begin{array}{l}\mathrm{CC} 1=\mathrm{CC}(=\mathrm{O}) \mathrm{C} 2=\mathrm{C}(\mathrm{CO}) \mathrm{CC}[\mathrm{C} @ \mathrm{H}](\mathrm{C}[\mathrm{C} @] 12 \mathrm{O}) \mathrm{C}(=\mathrm{C}) \mathrm{C}(=\mathrm{O}) \mathrm{O}[\mathrm{C} @ @ \mathrm{H}] 1[\mathrm{C} @ @ \mathrm{H}](\mathrm{O})[\mathrm{C} @ \mathrm{H}](\mathrm{O})[\mathrm{C} @ @ \mathrm{H}](\mathrm{O} \\
\mathrm{CC} 2=\mathrm{C} 3 \mathrm{C}(=\mathrm{O}) \mathrm{C}=\mathrm{C}(\mathrm{C})\left[\mathrm{C} @ \mathrm{C}_{3} \mathrm{C}[\mathrm{C} @ @ \mathrm{H}](\mathrm{CC} 2) \mathrm{C}(=\mathrm{C}) \mathrm{C}(=\mathrm{O}) \mathrm{O} 3\right) \mathrm{O}[\mathrm{C} @ \mathrm{H}] 1 \mathrm{CO}\end{array}$ & 6,15 \\
\hline 227 & $\begin{array}{l}\mathrm{CC} 1=\mathrm{CC}(=\mathrm{O}) \mathrm{C} 2=\mathrm{C}(\mathrm{CO}) \mathrm{CC}[\mathrm{C} @ \mathrm{H}](\mathrm{C}[\mathrm{C} @] 12 \mathrm{O}) \mathrm{C}(=\mathrm{C}) \mathrm{C}(=\mathrm{O}) \mathrm{O}[\mathrm{C} @ @ \mathrm{H}] 1[\mathrm{C} @ @ \mathrm{H}](\mathrm{O})[\mathrm{C} @ \mathrm{H}](\mathrm{O})[\mathrm{C} @ @ \mathrm{H}](\mathrm{O} \\
\mathrm{CC} 2=\mathrm{C} 3 \mathrm{C}(=\mathrm{O}) \mathrm{C}=\mathrm{C}(\mathrm{C})\left[\mathrm{C} @ \mathrm{C}^{2} @ 33 \mathrm{C}[\mathrm{C} @ @ \mathrm{H}](\mathrm{CC} 2) \mathrm{C}(=\mathrm{C}) \mathrm{C}(=\mathrm{O}) \mathrm{O} 3\right) \mathrm{O}[\mathrm{C} @ \mathrm{H}] 1 \mathrm{CO}\end{array}$ & 6,22 \\
\hline 228 & $\mathrm{C}[\mathrm{C} @ @ \mathrm{H}] 1 \mathrm{C}[\mathrm{C} @ \mathrm{H}] 2 \mathrm{OC}(=\mathrm{O}) \mathrm{C}(=\mathrm{C})[\mathrm{C} @ \mathrm{H}] 2[\mathrm{C} @ \mathrm{H}](\mathrm{O})[\mathrm{C} @ @] 2(\mathrm{C})[\mathrm{C} @ \mathrm{H}] 1 \mathrm{C}=\mathrm{CC} 2=\mathrm{O}$ & 6,1 \\
\hline 229 & $\mathrm{CN}(\mathrm{C}) \mathrm{CCCOC} 1=\mathrm{NC}=\mathrm{CC}(=\mathrm{N} 1) \mathrm{C} 1=\mathrm{CC}=\mathrm{C}(\mathrm{S} 1) \mathrm{C} 1=\mathrm{CC}=\mathrm{CS} 1$ & 4,5 \\
\hline 230 & $\mathrm{CN}(\mathrm{C}) \mathrm{CCOC} 1=\mathrm{NC}=\mathrm{CC}(=\mathrm{N} 1) \mathrm{C} 1=\mathrm{CC}=\mathrm{CS} 1$ & 3,7 \\
\hline 231 & $\mathrm{CN}(\mathrm{C}) \mathrm{CCOC} 1=\mathrm{NC}=\mathrm{CC}(=\mathrm{N} 1) \mathrm{C} 1=\mathrm{CC}=\mathrm{C}(\mathrm{S} 1) \mathrm{C} 1=\mathrm{CC}=\mathrm{CS} 1$ & 4,32 \\
\hline 232 & $\mathrm{CN}(\mathrm{C}) \mathrm{CCSC} 1=\mathrm{NC}=\mathrm{CC}(=\mathrm{N} 1) \mathrm{C} 1=\mathrm{CC}=\mathrm{CS} 1$ & 3,72 \\
\hline 233 & $\mathrm{CN}(\mathrm{C}) \mathrm{CCSC} 1=\mathrm{NC}=\mathrm{CC}(=\mathrm{N} 1) \mathrm{C} 1=\mathrm{CC}=\mathrm{C}(\mathrm{S} 1) \mathrm{C} 1=\mathrm{CC}=\mathrm{CS} 1$ & 3,84 \\
\hline 234 & $\mathrm{CN}(\mathrm{C}) \mathrm{CCCOC} 1=\mathrm{NC}=\mathrm{CC}(=\mathrm{N} 1) \mathrm{C} 1=\mathrm{CC}=\mathrm{CS} 1$ & 3,72 \\
\hline 235 & $\mathrm{CC}(=\mathrm{O}) \mathrm{NC} 1=\mathrm{CC} 2=\mathrm{NC} 3=\mathrm{CC}=\mathrm{CC}=\mathrm{C} 3 \mathrm{OC} 2=\mathrm{CC} 1=\mathrm{O}$ & 5,85 \\
\hline 236 & $\begin{array}{l}\mathrm{CC}(\mathrm{C})[\mathrm{C} @ \mathrm{H}] 1 \mathrm{NC}(=\mathrm{O})[\mathrm{C} @ @ \mathrm{H}](\mathrm{NC}(=\mathrm{O}) \mathrm{C} 2=\mathrm{C} 3 \mathrm{~N}=\mathrm{C} 4 \mathrm{C}(\mathrm{OC} 3=\mathrm{C}(\mathrm{C}) \mathrm{C}=\mathrm{C} 2)=\mathrm{C}(\mathrm{C}) \mathrm{C}(=\mathrm{O}) \mathrm{C}(\mathrm{N})=\mathrm{C} 4 \mathrm{C}(=\mathrm{O}) \mathrm{N}[\mathrm{C} \\
(\mathrm{H}] 2[\mathrm{C} @ @ \mathrm{H}](\mathrm{C}) \mathrm{OC}(=\mathrm{O})[\mathrm{C} @ \mathrm{H}](\mathrm{C}(\mathrm{C}) \mathrm{C}) \mathrm{N}(\mathrm{C}) \mathrm{C}(=\mathrm{O}) \mathrm{CN}(\mathrm{C}) \mathrm{C}(=\mathrm{O})[\mathrm{C} @ @ \mathrm{H}] 3 \mathrm{CCCN} 3 \mathrm{C}(=\mathrm{O})[\mathrm{C} @ \mathrm{H}](\mathrm{NC} 2=\mathrm{O}) \\
\mathrm{C}(\mathrm{C}) \mathrm{C})[\mathrm{C} @ @ \mathrm{H}](\mathrm{C}) \mathrm{OC}(=\mathrm{O})[\mathrm{C} @ \mathrm{H}](\mathrm{C}(\mathrm{C}) \mathrm{C}) \mathrm{N}(\mathrm{C}) \mathrm{C}(=\mathrm{O}) \mathrm{CN}(\mathrm{C}) \mathrm{C}(=\mathrm{O})[\mathrm{C} @ @ \mathrm{H}] 2 \mathrm{CCCN} 2 \mathrm{C} 1=\mathrm{O}\end{array}$ & 8,4 \\
\hline 237 & $\mathrm{CC}(=\mathrm{O}) \mathrm{C} 1=\mathrm{C} 2 \mathrm{~N}=\mathrm{C} 3 \mathrm{C}(\mathrm{OC} 2=\mathrm{CC}=\mathrm{C} 1)=\mathrm{CC}(=\mathrm{O}) \mathrm{C}(\mathrm{N})=\mathrm{C} 3 \mathrm{C}(\mathrm{C})=\mathrm{O}$ & 5,82 \\
\hline 238 & $\begin{array}{l}\mathrm{COC} 1=\mathrm{CC}=\mathrm{CC} 2=\mathrm{C} 1 \mathrm{C}(=\mathrm{O}) \mathrm{C} 1=\mathrm{C}(\mathrm{O}) \mathrm{C} 3=\mathrm{C}(\mathrm{C}[\mathrm{C} @](\mathrm{O})(\mathrm{C}[\mathrm{C} @ @ \mathrm{H}] 3 \mathrm{O}[\mathrm{C} @ \mathrm{H}] 3 \mathrm{C}[\mathrm{C} @ \mathrm{H}](\mathrm{N})[\mathrm{C} @ \mathrm{H}](\mathrm{O})[\mathrm{C} @ \mathrm{H}]( \\
\mathrm{C}) \mathrm{O} 3) \mathrm{C}(=\mathrm{O}) \mathrm{CO}) \mathrm{C}(\mathrm{O})=\mathrm{C} 1 \mathrm{C} 2=\mathrm{O}\end{array}$ & 7,4 \\
\hline 239 & $\mathrm{O}=\mathrm{C} 1 \mathrm{C}=\mathrm{CC} 2=\mathrm{NC} 3=\mathrm{CC}=\mathrm{CC}=\mathrm{C} 3 \mathrm{OC} 2=\mathrm{C} 1$ & 4,85 \\
\hline 240 & $\mathrm{O}=\mathrm{C} 1 \mathrm{C}=\mathrm{C} 2 \mathrm{OC} 3=\mathrm{CC}=\mathrm{CC}=\mathrm{C} 3 \mathrm{~N}=\mathrm{C} 2 \mathrm{C} 2=\mathrm{CC}=\mathrm{CN}=\mathrm{C} 12$ & 7,4 \\
\hline 241 & $\mathrm{NC} 1=\mathrm{CC} 2=\mathrm{NC} 3=\mathrm{CC}=\mathrm{CC}=\mathrm{C} 3 \mathrm{OC} 2=\mathrm{CC} 1=\mathrm{O}$ & 5,74 \\
\hline 242 & $\mathrm{O}=\mathrm{C} 1 \mathrm{C}=\mathrm{C} 2 \mathrm{SC} 3=\mathrm{CC}=\mathrm{CC}=\mathrm{C} 3 \mathrm{~N}=\mathrm{C} 2 \mathrm{C} 2=\mathrm{CC}=\mathrm{CN}=\mathrm{C} 12$ & 7 \\
\hline 243 & $\mathrm{O}=\mathrm{C} 1 \mathrm{C}=\mathrm{C} 2 \mathrm{OC} 3=\mathrm{CC}=\mathrm{C}(\mathrm{C}=\mathrm{C} 3 \mathrm{~N}=\mathrm{C} 2 \mathrm{C} 2=\mathrm{CC}=\mathrm{CN}=\mathrm{C} 12) \mathrm{C} \# \mathrm{~N}$ & 7,7 \\
\hline 244 & {$[\mathrm{O}-][\mathrm{N}+](=\mathrm{O}) \mathrm{C} 1=\mathrm{CC}=\mathrm{C} 2 \mathrm{OC} 3=\mathrm{CC}(=\mathrm{O}) \mathrm{C} 4=\mathrm{NC}=\mathrm{CC}=\mathrm{C} 4 \mathrm{C} 3=\mathrm{NC} 2=\mathrm{C} 1$} & 6,4 \\
\hline 245 & $\mathrm{O}=\mathrm{C} 1 \mathrm{C}=\mathrm{C} 2 \mathrm{OC} 3=\mathrm{CC}=\mathrm{CC}=\mathrm{C} 3 \mathrm{~N}=\mathrm{C} 2 \mathrm{C} 2=\mathrm{NC}=\mathrm{CC}=\mathrm{C} 12$ & 5,92 \\
\hline 246 & $\mathrm{CC} 1=\mathrm{CC}=\mathrm{CC} 2=\mathrm{C} 1 \mathrm{OC} 1=\mathrm{CC}(=\mathrm{O}) \mathrm{C} 3=\mathrm{NC}=\mathrm{CC}=\mathrm{C} 3 \mathrm{C} 1=\mathrm{N} 2$ & 6,7 \\
\hline 247 & $\mathrm{ClC} 1=\mathrm{CC}=\mathrm{C} 2 \mathrm{OC} 3=\mathrm{CC}(=\mathrm{O}) \mathrm{C} 4=\mathrm{NC}=\mathrm{CC}=\mathrm{C} 4 \mathrm{C} 3=\mathrm{NC} 2=\mathrm{C} 1$ & 6,7 \\
\hline 248 & $\mathrm{CC} 1=\mathrm{CC}=\mathrm{C} 2 \mathrm{OC} 3=\mathrm{CC}(=\mathrm{O}) \mathrm{C} 4=\mathrm{NC}=\mathrm{CC}=\mathrm{C} 4 \mathrm{C} 3=\mathrm{NC} 2=\mathrm{C} 1$ & 6,52 \\
\hline
\end{tabular}




\begin{tabular}{|c|c|c|}
\hline NAME & SMILE & $\lg (\mathbf{1} / \mathbf{I C 5 0})$ \\
\hline 249 & $\begin{array}{l}\mathrm{COC} 1=\mathrm{CC}=\mathrm{CC} 2=\mathrm{C} 1 \mathrm{C}(=\mathrm{O}) \mathrm{C} 1=\mathrm{C}(\mathrm{O}) \mathrm{C} 3=\mathrm{C}(\mathrm{C}[\mathrm{C} @](\mathrm{O})(\mathrm{C}[\mathrm{C} @ @ \mathrm{H}] 3 \mathrm{O}[\mathrm{C} @ \mathrm{H}] 3 \mathrm{C}[\mathrm{C} @ \mathrm{H}](\mathrm{N})[\mathrm{C} @ \mathrm{H}](\mathrm{O})[\mathrm{C} @ \mathrm{H}]( \\
\mathrm{C}) \mathrm{O} 3) \mathrm{C}(=\mathrm{O}) \mathrm{CO}) \mathrm{C}(\mathrm{O})=\mathrm{C} 1 \mathrm{C} 2=\mathrm{O}\end{array}$ & 7,4 \\
\hline 250 & $\mathrm{O}=\mathrm{C} 1 \mathrm{C}=\mathrm{C} 2 \mathrm{OC} 3=\mathrm{CC}=\mathrm{CC}=\mathrm{C} 3 \mathrm{~N}=\mathrm{C} 2 \mathrm{C} 2=\mathrm{CC}=\mathrm{CC}=\mathrm{C} 12$ & 5,85 \\
\hline 251 & {$[\mathrm{O}-][\mathrm{N}+](=\mathrm{O}) \mathrm{C} 1=\mathrm{CC}=\mathrm{CC} 2=\mathrm{C} 1 \mathrm{~N}=\mathrm{C} 1 \mathrm{C}(\mathrm{O} 2)=\mathrm{CC}(=\mathrm{O}) \mathrm{C} 2=\mathrm{NC}=\mathrm{CC}=\mathrm{C} 12$} & 7,3 \\
\hline 252 & $\mathrm{O}=\mathrm{C} 1 \mathrm{C}=\mathrm{C} 2 \mathrm{OC} 3=\mathrm{CC}=\mathrm{CC}=\mathrm{C} 3 \mathrm{~N}=\mathrm{C} 2 \mathrm{C} 2=\mathrm{CC}=\mathrm{CN}=\mathrm{C} 12$ & 7,4 \\
\hline 253 & {$[\mathrm{O}-][\mathrm{N}+](=\mathrm{O}) \mathrm{C} 1=\mathrm{CC}=\mathrm{C} 2 \mathrm{~N}=\mathrm{C} 3 \mathrm{C}(\mathrm{OC} 2=\mathrm{C} 1)=\mathrm{CC}(=\mathrm{O}) \mathrm{C} 1=\mathrm{NC}=\mathrm{CC}=\mathrm{C} 31$} & 5,96 \\
\hline 254 & $\mathrm{CC} 1=\mathrm{CC}=\mathrm{C} 2 \mathrm{~N}=\mathrm{C} 3 \mathrm{C}(\mathrm{OC} 2=\mathrm{C} 1)=\mathrm{CC}(=\mathrm{O}) \mathrm{C} 1=\mathrm{NC}=\mathrm{CC}=\mathrm{C} 31$ & 6,7 \\
\hline 255 & $\mathrm{O}=\mathrm{C} 1 \mathrm{C}=\mathrm{C} 2 \mathrm{OC} 3=\mathrm{CC}=\mathrm{CC}=\mathrm{C} 3 \mathrm{~N}=\mathrm{C} 2 \mathrm{C} 2=\mathrm{CC}=\mathrm{NC}=\mathrm{C} 12$ & 5,05 \\
\hline 256 & $\begin{array}{l}\mathrm{CC}(\mathrm{C})[\mathrm{C} @ \mathrm{H}] 1 \mathrm{NC}(=\mathrm{O})[\mathrm{C} @ @ \mathrm{H}](\mathrm{NC}(=\mathrm{O}) \mathrm{C} 2=\mathrm{C} 3 \mathrm{~N}=\mathrm{C} 4 \mathrm{C}(\mathrm{OC} 3=\mathrm{C}(\mathrm{C}) \mathrm{C}=\mathrm{C} 2)=\mathrm{C}(\mathrm{C}) \mathrm{C}(=\mathrm{O}) \mathrm{C}(\mathrm{N})=\mathrm{C} 4 \mathrm{C}(=\mathrm{O}) \mathrm{N}[\mathrm{C} \\
@ \mathrm{H}] 2[\mathrm{C} @ @ \mathrm{H}](\mathrm{C}) \mathrm{OC}(=\mathrm{O})[\mathrm{C} @ \mathrm{H}](\mathrm{C}(\mathrm{C}) \mathrm{C}) \mathrm{N}(\mathrm{C}) \mathrm{C}(=\mathrm{O}) \mathrm{CN}(\mathrm{C}) \mathrm{C}(=\mathrm{O})[\mathrm{C} @ @ \mathrm{H}] 3 \mathrm{CCCN} 3 \mathrm{C}(=\mathrm{O})[\mathrm{C} @ \mathrm{H}](\mathrm{NC} 2=\mathrm{O}) \\
\mathrm{C}(\mathrm{C}) \mathrm{C})[\mathrm{C} @ \mathrm{H}](\mathrm{C}) \mathrm{OC}(=\mathrm{O})[\mathrm{C} @ \mathrm{H}](\mathrm{C}(\mathrm{C}) \mathrm{C}) \mathrm{N}(\mathrm{C}) \mathrm{C}(=\mathrm{O}) \mathrm{CN}(\mathrm{C}) \mathrm{C}(=\mathrm{O})\left[\mathrm{C} @ \mathrm{H}^{2} \mathrm{C}\right] 2 \mathrm{CCN} 2 \mathrm{C} 1=\mathrm{O}\end{array}$ & 8,4 \\
\hline 257 & $\mathrm{O}=\mathrm{C} 1 \mathrm{C}=\mathrm{C} 2 \mathrm{OC} 3=\mathrm{CC}=\mathrm{CC}=\mathrm{C} 3 \mathrm{~N}=\mathrm{C} 2 \mathrm{C} 2=\mathrm{CN}=\mathrm{CC}=\mathrm{C} 12$ & 5,05 \\
\hline 258 & {$[\mathrm{O}-][\mathrm{N}+](=\mathrm{O}) \mathrm{C} 1=\mathrm{CC}=\mathrm{CC} 2=\mathrm{C} 1 \mathrm{OC} 1=\mathrm{CC}(=\mathrm{O}) \mathrm{C} 3=\mathrm{NC}=\mathrm{CC}=\mathrm{C} 3 \mathrm{C} 1=\mathrm{N} 2$} & 6,15 \\
\hline 259 & $\mathrm{CC} 1=\mathrm{CC}=\mathrm{CC} 2=\mathrm{C} 1 \mathrm{~N}=\mathrm{C} 1 \mathrm{C}(\mathrm{O} 2)=\mathrm{CC}(=\mathrm{O}) \mathrm{C} 2=\mathrm{NC}=\mathrm{CC}=\mathrm{C} 12$ & 6,22 \\
\hline 260 & $\mathrm{COC} 1=\mathrm{CC}=\mathrm{C} 2 \mathrm{C}(\mathrm{SC} 3=\mathrm{C} 2 \mathrm{C} 2=\mathrm{CC}=\mathrm{CC}=\mathrm{C} 2 \mathrm{~N}(\mathrm{CCCN}(\mathrm{C}) \mathrm{C}) \mathrm{C} 3=\mathrm{O})=\mathrm{C} 1$ & 5,53 \\
\hline 261 & $\mathrm{CN}(\mathrm{C}) \mathrm{CCCN} 1 \mathrm{C}(=\mathrm{O}) \mathrm{C} 2=\mathrm{C}(\mathrm{C} 3=\mathrm{C}(\mathrm{S} 2) \mathrm{C}=\mathrm{C}(\mathrm{C}=\mathrm{C} 3)[\mathrm{N}+]([\mathrm{O}-])=\mathrm{O}) \mathrm{C} 2=\mathrm{CC}=\mathrm{CC}=\mathrm{C} 12$ & 5,45 \\
\hline 262 & $\mathrm{CN}(\mathrm{C}) \mathrm{CCCN} 1 \mathrm{C}(=\mathrm{O}) \mathrm{C} 2=\mathrm{C}(\mathrm{C} 3=\mathrm{C}(\mathrm{S} 2) \mathrm{C}=\mathrm{C}(\mathrm{C}) \mathrm{C}=\mathrm{C} 3) \mathrm{C} 2=\mathrm{CC}=\mathrm{CC}=\mathrm{C} 12$ & 5,44 \\
\hline 263 & $\mathrm{CN}(\mathrm{C}) \mathrm{CCCN} 1 \mathrm{C}(=\mathrm{O}) \mathrm{C} 2=\mathrm{C}(\mathrm{C} 3=\mathrm{C}(\mathrm{S} 2) \mathrm{C}=\mathrm{C}(\mathrm{C}=\mathrm{C} 3) \mathrm{C}(=\mathrm{O}) \mathrm{NC} 2=\mathrm{CC}=\mathrm{CC}=\mathrm{C} 2) \mathrm{C} 2=\mathrm{CC}=\mathrm{CC}=\mathrm{C} 12$ & 5,75 \\
\hline 264 & $\mathrm{CN}(\mathrm{C}) \mathrm{CCCN} 1 \mathrm{C}(=\mathrm{O}) \mathrm{C} 2=\mathrm{C}(\mathrm{C} 3=\mathrm{C}(\mathrm{S} 2) \mathrm{C}=\mathrm{CC}=\mathrm{C} 3) \mathrm{C} 2=\mathrm{CC}=\mathrm{CC}=\mathrm{C} 12$ & $\overline{5,44}$ \\
\hline 265 & $\mathrm{CN}(\mathrm{C}) \mathrm{CCCNC}(=\mathrm{O}) \mathrm{C} 1=\mathrm{CC} 2=\mathrm{C}(\mathrm{C}=\mathrm{C} 1) \mathrm{C} 1=\mathrm{C}(\mathrm{S} 2) \mathrm{C}(\mathrm{O})=\mathrm{NC} 2=\mathrm{CC}=\mathrm{CC}=\mathrm{C} 12$ & 5,18 \\
\hline 266 & $\mathrm{CN}(\mathrm{C}) \mathrm{CCCN} 1 \mathrm{C}(=\mathrm{O}) \mathrm{C} 2=\mathrm{C}(\mathrm{C} 3=\mathrm{C}(\mathrm{S} 2) \mathrm{C}=\mathrm{C}(\mathrm{F}) \mathrm{C}=\mathrm{C} 3) \mathrm{C} 2=\mathrm{CC}=\mathrm{CC}=\mathrm{C} 12$ & 5,5 \\
\hline 267 & $\mathrm{CN}(\mathrm{C}) \mathrm{CCCN}(\mathrm{C}(=\mathrm{O}) \mathrm{C} 1=\mathrm{CC} 2=\mathrm{C}(\mathrm{C}=\mathrm{C} 1) \mathrm{C} 1=\mathrm{C}(\mathrm{S} 2) \mathrm{C}(\mathrm{O})=\mathrm{NC} 2=\mathrm{CC}=\mathrm{CC}=\mathrm{C} 12) \mathrm{C} 1=\mathrm{CC}=\mathrm{CC}=\mathrm{C} 1$ & 5,5 \\
\hline 268 & $\mathrm{COC}(=\mathrm{O}) \mathrm{C} 1=\mathrm{CC}=\mathrm{C} 2 \mathrm{C}(\mathrm{SC} 3=\mathrm{C} 2 \mathrm{C} 2=\mathrm{CC}=\mathrm{CC}=\mathrm{C} 2 \mathrm{~N}(\mathrm{CCCN}(\mathrm{C}) \mathrm{C}) \mathrm{C} 3=\mathrm{O})=\mathrm{C} 1$ & 6,19 \\
\hline 269 & $\mathrm{CC}(=\mathrm{O}) \mathrm{OCCON} 1 \mathrm{C}=\mathrm{NC} 2=\mathrm{C}(\mathrm{S}) \mathrm{N}=\mathrm{C}(\mathrm{N}) \mathrm{N}=\mathrm{C} 12$ & 5,31 \\
\hline 270 & $\mathrm{CC}(=\mathrm{O}) \mathrm{OCCON} 1 \mathrm{C}=\mathrm{NC} 2=\mathrm{C}(\mathrm{Cl}) \mathrm{N}=\mathrm{C}(\mathrm{N}) \mathrm{N}=\mathrm{C} 12$ & 3,3 \\
\hline 271 & $\mathrm{CC}(\mathrm{O}) \mathrm{N} 1 \mathrm{C}=\mathrm{NC} 2=\mathrm{C}(\mathrm{Cl}) \mathrm{N}=\mathrm{C}(\mathrm{I}) \mathrm{N}=\mathrm{C} 12$ & 3,3 \\
\hline 272 & $\mathrm{CC}(\mathrm{OC}(\mathrm{C})=\mathrm{O}) \mathrm{N} 1 \mathrm{C}=\mathrm{NC} 2=\mathrm{C}(\mathrm{S}) \mathrm{N}=\mathrm{C}(\mathrm{N}) \mathrm{N}=\mathrm{C} 12$ & 5 \\
\hline 273 & $\mathrm{CC}(\mathrm{OC}(\mathrm{C})=\mathrm{O}) \mathrm{N} 1 \mathrm{C}=\mathrm{NC} 2=\mathrm{C}(\mathrm{S}) \mathrm{N}=\mathrm{C}(\mathrm{F}) \mathrm{N}=\mathrm{C} 12$ & 3,7 \\
\hline 274 & $\mathrm{CC}(\mathrm{O}) \mathrm{N} 1 \mathrm{C}=\mathrm{NC} 2=\mathrm{C}(\mathrm{N}) \mathrm{N}=\mathrm{C}(\mathrm{I}) \mathrm{N}=\mathrm{C} 12$ & 3,3 \\
\hline 275 & $\mathrm{CC}(\mathrm{O}) \mathrm{CN} 1 \mathrm{C}=\mathrm{NC} 2=\mathrm{C} 1 \mathrm{~N}=\mathrm{C}(\mathrm{N}) \mathrm{N}=\mathrm{C} 2 \mathrm{~F}$ & $\overline{4,2}$ \\
\hline 276 & OCCON1C $=\mathrm{NC} 2=\mathrm{C}(\mathrm{S}) \mathrm{N}=\mathrm{C}(\mathrm{F}) \mathrm{N}=\mathrm{C} 12$ & 3,3 \\
\hline 277 & $\mathrm{NC} 1=\mathrm{C} 2 \mathrm{~N}=\mathrm{CN}(\mathrm{OCCO}) \mathrm{C} 2=\mathrm{NC}(\mathrm{I})=\mathrm{N} 1$ & 3,3 \\
\hline 278 & $\mathrm{CC}(=\mathrm{O}) \mathrm{OCCON} 1 \mathrm{C}=\mathrm{NC} 2=\mathrm{C}(\mathrm{S}) \mathrm{N}=\mathrm{C}(\mathrm{F}) \mathrm{N}=\mathrm{C} 12$ & 3,3 \\
\hline 279 & $\mathrm{CC}(\mathrm{OC}(\mathrm{C})=\mathrm{O}) \mathrm{N} 1 \mathrm{C}=\mathrm{NC} 2=\mathrm{C}(\mathrm{Cl}) \mathrm{N}=\mathrm{C}(\mathrm{F}) \mathrm{N}=\mathrm{C} 12$ & 5 \\
\hline 280 & $\mathrm{CC}(\mathrm{O}) \mathrm{N} 1 \mathrm{C}=\mathrm{NC} 2=\mathrm{C}(\mathrm{S}) \mathrm{N}=\mathrm{C}(\mathrm{F}) \mathrm{N}=\mathrm{C} 12$ & 3,3 \\
\hline 281 & $\mathrm{COC} 1=\mathrm{C} 2 \mathrm{~N}=\mathrm{CN}(\mathrm{OCCO}) \mathrm{C} 2=\mathrm{NC}(\mathrm{I})=\mathrm{N} 1$ & 3,3 \\
\hline 282 & $\mathrm{CC}(\mathrm{OC}(\mathrm{C})=\mathrm{O}) \mathrm{N} 1 \mathrm{C}=\mathrm{NC} 2=\mathrm{C}(\mathrm{N}) \mathrm{N}=\mathrm{C}(\mathrm{I}) \mathrm{N}=\mathrm{C} 12$ & 3,3 \\
\hline 283 & $\mathrm{CC}(\mathrm{OC}(\mathrm{C})=\mathrm{O}) \mathrm{N} 1 \mathrm{C}=\mathrm{NC} 2=\mathrm{C}(\mathrm{Cl}) \mathrm{N}=\mathrm{C}(\mathrm{N}) \mathrm{N}=\mathrm{C} 12$ & 3,3 \\
\hline 284 & $\mathrm{CC}(=\mathrm{O}) \mathrm{OCCON} 1 \mathrm{C}=\mathrm{NC} 2=\mathrm{C}(\mathrm{Cl}) \mathrm{N}=\mathrm{C}(\mathrm{I}) \mathrm{N}=\mathrm{C} 12$ & 4,25 \\
\hline 285 & $\mathrm{CC}(\mathrm{OC}(\mathrm{C})=\mathrm{O}) \mathrm{N} 1 \mathrm{C}=\mathrm{NC} 2=\mathrm{C}(\mathrm{Cl}) \mathrm{N}=\mathrm{C}(\mathrm{I}) \mathrm{N}=\mathrm{C} 12$ & 5,2 \\
\hline 286 & $\begin{array}{l}\mathrm{C}[\mathrm{C} @ @ \mathrm{H}](\mathrm{CCC}(=\mathrm{O}) \mathrm{NCCNC}(=\mathrm{O}) \mathrm{CC}[\mathrm{C} @ @ \mathrm{H}](\mathrm{C})[\mathrm{C} @ \mathrm{H}] 1 \mathrm{CCC} 2 \mathrm{C} 3 \mathrm{CCC} 4 \mathrm{C}[\mathrm{C} @ \mathrm{H}](\mathrm{O}) \mathrm{CC}[\mathrm{C} @] 4(\mathrm{C}) \mathrm{C} 3 \mathrm{C}[\mathrm{C} @ \\
@ \mathrm{H}](\mathrm{O})[\mathrm{C} @] 12 \mathrm{C})[\mathrm{C} @ \mathrm{H}] 1 \mathrm{CCC} 2 \mathrm{C} 3 \mathrm{CCC} 4 \mathrm{C}[\mathrm{C} @ \mathrm{H}](\mathrm{O}) \mathrm{CC}[\mathrm{C} @] 4(\mathrm{C}) \mathrm{C} 3 \mathrm{C}[\mathrm{C} @ @ \mathrm{H}](\mathrm{O})[\mathrm{C} @] 12 \mathrm{C}\end{array}$ & 5,66 \\
\hline 287 & $\begin{array}{l}\mathrm{C}[\mathrm{C} @ @ \mathrm{H}](\mathrm{CCC}(=\mathrm{O}) \mathrm{NCCNCCNC}(=\mathrm{O}) \mathrm{CC}[\mathrm{C} @ @ \mathrm{H}](\mathrm{C})[\mathrm{C} @ \mathrm{H}] 1 \mathrm{CCC} 2 \mathrm{C} 3 \mathrm{CCC} 4 \mathrm{C}[\mathrm{C} @ \mathrm{H}](\mathrm{O}) \mathrm{CC}[\mathrm{C} @] 4(\mathrm{C}) \mathrm{C} 3 \\
\mathrm{C}[\mathrm{C} @ @ \mathrm{H}](\mathrm{O})[\mathrm{C} @] 12 \mathrm{C})[\mathrm{C} @ \mathrm{H}] 1 \mathrm{CCC}[\mathrm{C} @] 1(\mathrm{C}) \mathrm{C} 1 \mathrm{CCC} 3 \mathrm{C}(\mathrm{CCC} 4 \mathrm{C}[\mathrm{C} @ \mathrm{H}](\mathrm{O}) \mathrm{CC}[\mathrm{C} @] 34 \mathrm{C})[\mathrm{C} @] 21 \mathrm{O}\end{array}$ & 5 \\
\hline 288 & $\mathrm{NC} 1=\mathrm{NC}=\mathrm{CC}(=\mathrm{N} 1) \mathrm{C} 1=\mathrm{CNC} 2=\mathrm{CC}=\mathrm{C}(\mathrm{Br}) \mathrm{C}=\mathrm{C} 12$ & 5,01 \\
\hline 289 & $\mathrm{NC} 1=\mathrm{NC}=\mathrm{CC}(=\mathrm{N} 1) \mathrm{C} 1=\mathrm{CNC} 2=\mathrm{CC}(\mathrm{Br})=\mathrm{CC}(\mathrm{O})=\mathrm{C} 12$ & 5,77 \\
\hline 290 & $\mathrm{NC} 1=\mathrm{NC}=\mathrm{CC}(=\mathrm{N} 1) \mathrm{C} 1=\mathrm{CNC} 2=\mathrm{CC}(\mathrm{Br})=\mathrm{C}(\mathrm{Br}) \mathrm{C}=\mathrm{C} 12$ & 5,74 \\
\hline 291 & $\mathrm{NC} 1=\mathrm{NC}=\mathrm{CC}(=\mathrm{N} 1) \mathrm{C} 1=\mathrm{CNC} 2=\mathrm{CC}(\mathrm{Br})=\mathrm{CC}=\mathrm{C} 12$ & 5,14 \\
\hline 292 & $\mathrm{NC} 1=\mathrm{NC}=\mathrm{CC}(=\mathrm{N} 1) \mathrm{C} 1=\mathrm{CNC} 2=\mathrm{C}(\mathrm{Br}) \mathrm{C}=\mathrm{CC}(\mathrm{O})=\mathrm{C} 12$ & 5,96 \\
\hline 293 & $\mathrm{CNC} 1=\mathrm{CC}=\mathrm{CC}=\mathrm{C} 1 \mathrm{C}(=\mathrm{O}) \mathrm{NC}(=\mathrm{O}) \mathrm{NC} 1=\mathrm{CC}=\mathrm{C}(\mathrm{OC} 2=\mathrm{NC}=\mathrm{C}(\mathrm{Br}) \mathrm{C}=\mathrm{N} 2) \mathrm{C}(\mathrm{C})=\mathrm{C} 1$ & 6 \\
\hline 294 & $\begin{array}{l}\mathrm{CN}(\mathrm{C}(=\mathrm{O}) \mathrm{NC} 1=\mathrm{CC}=\mathrm{CC}=\mathrm{C} 1 \mathrm{C}(=\mathrm{O}) \mathrm{N}(\mathrm{C}) \mathrm{C}(=\mathrm{O}) \mathrm{NC} 1=\mathrm{CC}=\mathrm{C}(\mathrm{OC} 2=\mathrm{NC}=\mathrm{C}(\mathrm{Br}) \mathrm{C}=\mathrm{N} 2) \mathrm{C}(\mathrm{C})=\mathrm{C} 1) \mathrm{C}(=\mathrm{O}) \mathrm{C} 1=\mathrm{CC}= \\
\mathrm{CC}=\mathrm{C} 1 \mathrm{~N}\end{array}$ & 5,3 \\
\hline 295 & $\mathrm{CN}(\mathrm{C}(=\mathrm{O}) \mathrm{NC} 1=\mathrm{CC}=\mathrm{C}(\mathrm{OC} 2=\mathrm{NC}=\mathrm{C}(\mathrm{Br}) \mathrm{C}=\mathrm{N} 2) \mathrm{C}(\mathrm{C})=\mathrm{C} 1) \mathrm{C}(=\mathrm{O}) \mathrm{C} 1=\mathrm{CC}=\mathrm{CC}=\mathrm{C} 1 \mathrm{~N}$ & 5,3 \\
\hline 296 & $\mathrm{ONC}(=\mathrm{O}) \mathrm{NC}(\mathrm{C}(=\mathrm{O}) \mathrm{NC}(\mathrm{C} 1=\mathrm{CC}=\mathrm{CC}=\mathrm{C} 1) \mathrm{C} 1=\mathrm{CC}=\mathrm{CC}=\mathrm{C} 1) \mathrm{C} 1=\mathrm{CC}=\mathrm{CC}=\mathrm{C} 1$ & 4,08 \\
\hline 297 & $\mathrm{ONC}(=\mathrm{O}) \mathrm{NC}(\mathrm{CC} 1=\mathrm{CC}=\mathrm{CC}=\mathrm{C} 1) \mathrm{C}(=\mathrm{O}) \mathrm{NC}(\mathrm{C} 1=\mathrm{CC}=\mathrm{CC}=\mathrm{C} 1) \mathrm{C} 1=\mathrm{CC}=\mathrm{CC}=\mathrm{C} 1$ & 4,21 \\
\hline 298 & $\mathrm{CCC}(\mathrm{C}) \mathrm{C}(\mathrm{NC}(=\mathrm{O}) \mathrm{NO}) \mathrm{C}(=\mathrm{O}) \mathrm{NC}(\mathrm{C} 1=\mathrm{CC}=\mathrm{CC}=\mathrm{C} 1) \mathrm{C} 1=\mathrm{CC}=\mathrm{CC}=\mathrm{C} 1$ & 4,45 \\
\hline 299 & $\mathrm{O}=\mathrm{C}(\mathrm{NC} 1 \mathrm{CCCC} 1) \mathrm{C}(\mathrm{CC} 1=\mathrm{CC}=\mathrm{CC}=\mathrm{C} 1) \mathrm{NC}(=\mathrm{O}) \mathrm{N} 1 \mathrm{~N}=\mathrm{NC} 2=\mathrm{CC}=\mathrm{CC}=\mathrm{C} 12$ & 4,02 \\
\hline 300 & $\mathrm{ONC}(=\mathrm{O}) \mathrm{NC}(\mathrm{C}(=\mathrm{O}) \mathrm{NCC} 1=\mathrm{CC}=\mathrm{CC}=\mathrm{C} 1) \mathrm{C} 1=\mathrm{CC}=\mathrm{CC}=\mathrm{C} 1$ & 4,49 \\
\hline 301 & $\mathrm{O}=\mathrm{C}(\mathrm{NC}(\mathrm{C} 1=\mathrm{CC}=\mathrm{CC}=\mathrm{C} 1) \mathrm{C} 1=\mathrm{CC}=\mathrm{CC}=\mathrm{C} 1) \mathrm{C}(\mathrm{NC}=\mathrm{O}) \mathrm{N} 1 \mathrm{~N}=\mathrm{NC} 2=\mathrm{CC}=\mathrm{CC}=\mathrm{C} 12) \mathrm{C} 1=\mathrm{CC}=\mathrm{CC}=\mathrm{C} 1$ & 4,23 \\
\hline 302 & $\mathrm{CC}(\mathrm{C}) \mathrm{C}(\mathrm{NC}(=\mathrm{O}) \mathrm{NO}) \mathrm{C}(=\mathrm{O}) \mathrm{NC} 1 \mathrm{CCCC} 1$ & 4,48 \\
\hline 303 & $\mathrm{O}=\mathrm{C}(\mathrm{NC}(\mathrm{C}(=\mathrm{O}) \mathrm{N} 1 \mathrm{CCCCC} 1) \mathrm{C} 1=\mathrm{CC}=\mathrm{CC}=\mathrm{C} 1) \mathrm{N} 1 \mathrm{~N}=\mathrm{NC} 2=\mathrm{C} 1 \mathrm{C}=\mathrm{CC}=\mathrm{C} 2$ & 4 \\
\hline 304 & $\mathrm{CC}(\mathrm{C}) \mathrm{C} 1 \mathrm{NC}(=\mathrm{O}) \mathrm{N}(\mathrm{C} 2 \mathrm{CCCC} 2) \mathrm{C} 1=\mathrm{O}$ & 4,01 \\
\hline 305 & $\mathrm{O}=\mathrm{C} 1 \mathrm{NC}(\mathrm{C}(=\mathrm{O}) \mathrm{N} 1 \mathrm{CC} 1=\mathrm{CC}=\mathrm{CC}=\mathrm{C} 1) \mathrm{C} 1=\mathrm{CC}=\mathrm{CC}=\mathrm{C} 1$ & 4,08 \\
\hline 306 & $\mathrm{O}=\mathrm{C}(\mathrm{NC}(\mathrm{CC} 1=\mathrm{CC}=\mathrm{CC}=\mathrm{C} 1) \mathrm{C}(=\mathrm{O}) \mathrm{N} 1 \mathrm{CCCC} 1) \mathrm{N} 1 \mathrm{~N}=\mathrm{NC} 2=\mathrm{CC}=\mathrm{CC}=\mathrm{C} 12$ & 4 \\
\hline 307 & $\mathrm{ONC}(=\mathrm{O}) \mathrm{NC}(\mathrm{C}(=\mathrm{O}) \mathrm{N} 1 \mathrm{CCCCC} 1) \mathrm{C} 1=\mathrm{CC}=\mathrm{CC}=\mathrm{C} 1$ & 4 \\
\hline 308 & $\mathrm{O}=\mathrm{C}(\mathrm{NCC} 1=\mathrm{CC}=\mathrm{CC}=\mathrm{C} 1) \mathrm{C}(\mathrm{CC} 1=\mathrm{CC}=\mathrm{CC}=\mathrm{C} 1) \mathrm{NC}(=\mathrm{O}) \mathrm{N} 1 \mathrm{~N}=\mathrm{NC} 2=\mathrm{CC}=\mathrm{CC}=\mathrm{C} 12$ & 4,09 \\
\hline 309 & $\mathrm{CC}(\mathrm{C}) \mathrm{C}(\mathrm{NC}(=\mathrm{O}) \mathrm{N} 1 \mathrm{~N}=\mathrm{NC} 2=\mathrm{CC}=\mathrm{CC}=\mathrm{C} 12) \mathrm{C}(=\mathrm{O}) \mathrm{NC} 1 \mathrm{CCCCC} 1$ & 4,22 \\
\hline 310 & $\mathrm{O}=\mathrm{C}(\mathrm{NC}(\mathrm{C} 1=\mathrm{CC}=\mathrm{CC}=\mathrm{C} 1) \mathrm{C} 1=\mathrm{CC}=\mathrm{CC}=\mathrm{C} 1) \mathrm{C}(\mathrm{CC} 1=\mathrm{CC}=\mathrm{CC}=\mathrm{C} 1) \mathrm{NC}(=\mathrm{O}) \mathrm{N} 1 \mathrm{~N}=\mathrm{NC} 2=\mathrm{CC}=\mathrm{CC}=\mathrm{C} 12$ & 4,39 \\
\hline 311 & $\mathrm{O}=\mathrm{C} 1 \mathrm{NC}(\mathrm{C}(=\mathrm{O}) \mathrm{N} 1 \mathrm{C} 1 \mathrm{CCCC} 1) \mathrm{C} 1=\mathrm{CC}=\mathrm{CC}=\mathrm{C} 1$ & 4 \\
\hline 312 & $\mathrm{CCC}(\mathrm{C}) \mathrm{C}(\mathrm{NC}(=\mathrm{O}) \mathrm{NO}) \mathrm{C}(=\mathrm{O}) \mathrm{NC} 1 \mathrm{CCCCC} 1$ & 4 \\
\hline 313 & $\mathrm{O}=\mathrm{C}(\mathrm{NC} 1 \mathrm{CCCCC} 1) \mathrm{C}(\mathrm{CC} 1=\mathrm{CC}=\mathrm{CC}=\mathrm{C} 1) \mathrm{NC}(=\mathrm{O}) \mathrm{N} 1 \mathrm{~N}=\mathrm{NC} 2=\mathrm{CC}=\mathrm{CC}=\mathrm{C} 12$ & 4,19 \\
\hline 314 & $\mathrm{ONC}(=\mathrm{O}) \mathrm{NC}(\mathrm{CC} 1=\mathrm{CC}=\mathrm{CC}=\mathrm{C} 1) \mathrm{C}(=\mathrm{O}) \mathrm{NC} 1 \mathrm{CCCCC} 1$ & 4,03 \\
\hline 315 & $\mathrm{CCC}(\mathrm{C}) \mathrm{C}(\mathrm{NC}(=\mathrm{O}) \mathrm{NO}) \mathrm{C}(=\mathrm{O}) \mathrm{NC} 1 \mathrm{CCCC} 1$ & 4 \\
\hline 316 & $\mathrm{ONC}(=\mathrm{O}) \mathrm{NC}(\mathrm{CC} 1=\mathrm{CC}=\mathrm{CC}=\mathrm{C} 1) \mathrm{C}(=\mathrm{O}) \mathrm{N} 1 \mathrm{CCCC} 1$ & 4 \\
\hline 317 & $\mathrm{CCC}(\mathrm{C}) \mathrm{C}(\mathrm{NC}(=\mathrm{O}) \mathrm{N} 1 \mathrm{~N}=\mathrm{NC} 2=\mathrm{CC}=\mathrm{CC}=\mathrm{C} 12) \mathrm{C}(=\mathrm{O}) \mathrm{NC}(\mathrm{C} 1=\mathrm{CC}=\mathrm{CC}=\mathrm{C} 1) \mathrm{C} 1=\mathrm{CC}=\mathrm{CC}$ & 4,26 \\
\hline
\end{tabular}




\begin{tabular}{|c|c|c|}
\hline NAME & SMILE & $\lg (\mathbf{1} / \mathbf{I C 5 0})$ \\
\hline 318 & $\mathrm{O}=\mathrm{C}(\mathrm{NC} 1 \mathrm{CCCCC} 1) \mathrm{C}(\mathrm{NC}(=\mathrm{O}) \mathrm{N} 1 \mathrm{~N}=\mathrm{NC} 2=\mathrm{C} 1 \mathrm{C}=\mathrm{CC}=\mathrm{C} 2) \mathrm{C} 1=\mathrm{CC}=\mathrm{CC}=\mathrm{C} 1$ & 4,16 \\
\hline 319 & $\mathrm{CCC}(\mathrm{C}) \mathrm{C}(\mathrm{NC}(=\mathrm{O}) \mathrm{N} 1 \mathrm{~N}=\mathrm{NC} 2=\mathrm{CC}=\mathrm{CC}=\mathrm{C} 12) \mathrm{C}(=\mathrm{O}) \mathrm{NC} 1 \mathrm{CCCCC} 1$ & 4,24 \\
\hline 320 & $\mathrm{CCC}(\mathrm{C}) \mathrm{C}(\mathrm{NC}(=\mathrm{O}) \mathrm{N} 1 \mathrm{~N}=\mathrm{NC} 2=\mathrm{CC}=\mathrm{CC}=\mathrm{C} 12) \mathrm{C}(=\mathrm{O}) \mathrm{NC} 1 \mathrm{CCCC} 1$ & 4,25 \\
\hline 321 & $\mathrm{CCC}(\mathrm{C}) \mathrm{C} 1 \mathrm{NC}(=\mathrm{O}) \mathrm{N}(\mathrm{C} 2 \mathrm{CCCCC} 2) \mathrm{C} 1=\mathrm{O}$ & 4,16 \\
\hline 322 & $\mathrm{CC}(\mathrm{C}) \mathrm{C}(\mathrm{NC}(=\mathrm{O}) \mathrm{N} 1 \mathrm{~N}=\mathrm{NC} 2=\mathrm{CC}=\mathrm{CC}=\mathrm{C} 12) \mathrm{C}(=\mathrm{O}) \mathrm{NC} 1 \mathrm{CCCC} 1$ & 4,22 \\
\hline 323 & $\mathrm{ONC}(=\mathrm{O}) \mathrm{NC}(\mathrm{CC} 1=\mathrm{CC}=\mathrm{CC}=\mathrm{C} 1) \mathrm{C}(=\mathrm{O}) \mathrm{NC} 1 \mathrm{CCCC} 1$ & 4 \\
\hline 324 & $\mathrm{CC}(\mathrm{C}) \mathrm{C}(\mathrm{NC}(=\mathrm{O}) \mathrm{NO}) \mathrm{C}(=\mathrm{O}) \mathrm{NC} 1 \mathrm{CCCCC} 1$ & 4 \\
\hline 325 & $\mathrm{ONC}(=\mathrm{O}) \mathrm{NC}(\mathrm{CC} 1=\mathrm{CC}=\mathrm{CC}=\mathrm{C} 1) \mathrm{C}(=\mathrm{O}) \mathrm{NCC} 1=\mathrm{CC}=\mathrm{CC}=\mathrm{C} 1$ & 4 \\
\hline 326 & $\mathrm{O}=\mathrm{C}(\mathrm{NCC} 1 \mathrm{CCCCC} 1) \mathrm{C}(\mathrm{NC}(=\mathrm{O}) \mathrm{N} 1 \mathrm{~N}=\mathrm{NC} 2=\mathrm{C} 1 \mathrm{C}=\mathrm{CC}=\mathrm{C} 2) \mathrm{C} 1=\mathrm{CC}=\mathrm{CC}=\mathrm{C} 1$ & 4,16 \\
\hline 327 & $\mathrm{ONC}(=\mathrm{O}) \mathrm{NC}(\mathrm{C}(=\mathrm{O}) \mathrm{NC} 1=\mathrm{CC}=\mathrm{CC}=\mathrm{C} 1) \mathrm{C} 1=\mathrm{CC}=\mathrm{CC}=\mathrm{C} 1$ & 4 \\
\hline 328 & $\mathrm{CCC}(\mathrm{C}) \mathrm{C}(\mathrm{NC}(=\mathrm{O}) \mathrm{NO}) \mathrm{C}(=\mathrm{O}) \mathrm{NCC} 1=\mathrm{CC}=\mathrm{CC}=\mathrm{C} 1$ & 4 \\
\hline 329 & $\mathrm{CCC}(\mathrm{C}) \mathrm{C}(\mathrm{NC}(=\mathrm{O}) \mathrm{N} 1 \mathrm{~N}=\mathrm{NC} 2=\mathrm{CC}=\mathrm{CC}=\mathrm{C} 12) \mathrm{C}(=\mathrm{O}) \mathrm{NCC} 1=\mathrm{CC}=\mathrm{CC}=\mathrm{C} 1$ & 4 \\
\hline 330 & $\mathrm{CC}(\mathrm{C}) \mathrm{C} 1 \mathrm{NC}(=\mathrm{O}) \mathrm{N}(\mathrm{C} 2 \mathrm{CCCCC} 2) \mathrm{C} 1=\mathrm{O}$ & 4 \\
\hline 331 & $\mathrm{CCC}(\mathrm{C}) \mathrm{C} 1 \mathrm{NC}(=\mathrm{O}) \mathrm{N}(\mathrm{C} 2 \mathrm{CCCC} 2) \mathrm{C} 1=\mathrm{O}$ & 4 \\
\hline 332 & $\mathrm{CCC}(\mathrm{C}) \mathrm{C} 1 \mathrm{NC}(=\mathrm{O}) \mathrm{N}(\mathrm{CC} 2=\mathrm{CC}=\mathrm{CC}=\mathrm{C} 2) \mathrm{C} 1=\mathrm{O}$ & 4,33 \\
\hline 333 & $\mathrm{ON}(\mathrm{C}(=\mathrm{O}) \mathrm{C} 12 \mathrm{CC} 3 \mathrm{CC}(\mathrm{CC}(\mathrm{C} 3) \mathrm{C} 1) \mathrm{C} 2) \mathrm{C} 1=\mathrm{CC}=\mathrm{C}(\mathrm{Cl}) \mathrm{C}=\mathrm{C} 1$ & 4 \\
\hline 334 & $\mathrm{ON}(\mathrm{C}(=\mathrm{O}) \mathrm{CCC} 1 \mathrm{CCCC} 1) \mathrm{C} 1=\mathrm{CC}=\mathrm{C}(\mathrm{Cl}) \mathrm{C}=\mathrm{C} 1$ & 4,7 \\
\hline 335 & $\mathrm{ON}(\mathrm{C}(=\mathrm{O}) \mathrm{CCC} 1 \mathrm{CCCCC} 1) \mathrm{C} 1=\mathrm{CC}=\mathrm{C}(\mathrm{Cl}) \mathrm{C}=\mathrm{C} 1$ & 4,52 \\
\hline 336 & $\mathrm{ON}(\mathrm{C}(=\mathrm{O}) \mathrm{CC} 1 \mathrm{CCCCC} 1) \mathrm{C} 1=\mathrm{CC}=\mathrm{C}(\mathrm{Cl}) \mathrm{C}=\mathrm{C} 1$ & 5,05 \\
\hline 337 & $\mathrm{ON}(\mathrm{C}(=\mathrm{O}) \mathrm{C} 1 \mathrm{CC} 1) \mathrm{C} 1=\mathrm{CC}=\mathrm{C}(\mathrm{Cl}) \mathrm{C}=\mathrm{C} 1$ & 4 \\
\hline 338 & $\mathrm{ON}(\mathrm{C}(=\mathrm{O}) \mathrm{CC} 1 \mathrm{CCCCC} 1) \mathrm{C} 1=\mathrm{CC}=\mathrm{CC}=\mathrm{C} 1$ & 4 \\
\hline 339 & $\mathrm{ON}(\mathrm{C}(=\mathrm{O}) \mathrm{C} 1 \mathrm{CCCCC} 1) \mathrm{C} 1=\mathrm{CC}=\mathrm{C}(\mathrm{Cl}) \mathrm{C}=\mathrm{C} 1$ & 4,4 \\
\hline & $\begin{array}{l}{[\mathrm{Na}+] \cdot[\mathrm{Na}+] \cdot[\mathrm{Na}+] \cdot[\mathrm{Na}+] \cdot[\mathrm{Na}+] \cdot[\mathrm{Na}+] \cdot[\mathrm{Na}+] \cdot[\mathrm{Na}+] \cdot[\mathrm{Na}+] \cdot[\mathrm{Na}+] \cdot[\mathrm{Na}+] \cdot[\mathrm{Na}+] \cdot[\mathrm{Na}+] \cdot[\mathrm{Na}+] \cdot[\mathrm{Na}+] \cdot[\mathrm{Na}+] \cdot[\mathrm{Zn}] \cdot[} \\
\mathrm{O}-\end{array}$ & \\
\hline
\end{tabular}

$\mathrm{l} C(=\mathrm{O}) \mathrm{C} 1=\mathrm{CC}(=\mathrm{CC}(\mathrm{OC} 2=\mathrm{CC} 3=\mathrm{C} 4 \mathrm{NC}(\mid \mathrm{N}=\mathrm{C} 5 / \mathrm{N}=\mathrm{C}(/ \mathrm{N}=\mathrm{C} 6 \backslash \mathrm{N} \backslash \mathrm{C}(=\mathrm{N} / \mathrm{C} 7=\mathrm{N} / \mathrm{C}(=\mathrm{N} / 4) / \mathrm{C} 4=\mathrm{CC}(\mathrm{OC} 8=\mathrm{CC}(=\mathrm{CC}($ $=\mathrm{C} 8) \mathrm{C}([\mathrm{O}-])=\mathrm{O}) \mathrm{C}([\mathrm{O}-])=\mathrm{O})=\mathrm{C}(\mathrm{OC} 8=\mathrm{CC}(=\mathrm{CC}(=\mathrm{C} 8) \mathrm{C}([\mathrm{O}-])=\mathrm{O}) \mathrm{C}([\mathrm{O}-$

]$)=\mathrm{O}) \mathrm{C}=\mathrm{C} 74) \mathrm{C} 4=\mathrm{CC}(\mathrm{OC} 7=\mathrm{CC}(=\mathrm{CC}(=\mathrm{C} 7) \mathrm{C}([\mathrm{O}-])=\mathrm{O}) \mathrm{C}([\mathrm{O}-])=\mathrm{O})=\mathrm{C}(\mathrm{OC} 7=\mathrm{CC}(=\mathrm{CC}(=\mathrm{C} 7) \mathrm{C}([\mathrm{O}-])=\mathrm{O}) \mathrm{C}([\mathrm{O}-$

]$)=\mathrm{O}) \mathrm{C}=\mathrm{C} 64) \mathrm{C} 4=\mathrm{CC}(\mathrm{OC} 6=\mathrm{CC}(=\mathrm{CC}(=\mathrm{C} 6) \mathrm{C}([\mathrm{O}-])=\mathrm{O}) \mathrm{C}([\mathrm{O}-])=\mathrm{O})=\mathrm{C}(\mathrm{OC} 6=\mathrm{CC}(=\mathrm{CC}(=\mathrm{C} 6) \mathrm{C}([\mathrm{O}-])=\mathrm{O}) \mathrm{C}([\mathrm{O}-$

$340])=\mathrm{O}) \mathrm{C}=\mathrm{C} 54)=\mathrm{C} 3 \mathrm{C}=\mathrm{C} 2 \mathrm{OC} 2=\mathrm{CC}(=\mathrm{CC}(=\mathrm{C} 2) \mathrm{C}([\mathrm{O}-])=\mathrm{O}) \mathrm{C}([\mathrm{O}-])=\mathrm{O})=\mathrm{C} 1) \mathrm{C}([\mathrm{O}-])=\mathrm{O}$

$[\mathrm{Zn}++] . \mathrm{OC}(=\mathrm{O}) \mathrm{C} 1=\mathrm{CC}(=\mathrm{CC}(\mathrm{OC} 2=\mathrm{CC} 3=\mathrm{C} 4[\mathrm{~N}-] \mathrm{C}(\mathrm{NN}=\mathrm{C} 5 / \mathrm{N}=\mathrm{C}(/ \mathrm{N}=\mathrm{C} 6 \backslash[\mathrm{N}-$

$\mathrm{ICC}(=\mathrm{N} / \mathrm{C} 7=\mathrm{N} / \mathrm{C}(=\mathrm{N} / 4) / \mathrm{C} 4=\mathrm{CC}(\mathrm{OC} 8=\mathrm{CC}(=\mathrm{CC}(=\mathrm{C} 8) \mathrm{C}(\mathrm{O})=\mathrm{O}) \mathrm{C}(\mathrm{O})=\mathrm{O})=\mathrm{C}(\mathrm{OC} 8=\mathrm{CC}(=\mathrm{CC}(=\mathrm{C} 8) \mathrm{C}(\mathrm{O})=\mathrm{O}) \mathrm{C}(\mathrm{O})$

$=\mathrm{O}) \mathrm{C}=\mathrm{C} 74) \mathrm{C} 4=\mathrm{CC}(\mathrm{OC} 7=\mathrm{CC}(=\mathrm{CC}(=\mathrm{C} 7) \mathrm{C}(\mathrm{O})=\mathrm{O}) \mathrm{C}(\mathrm{O})=\mathrm{O})=\mathrm{C}(\mathrm{OC} 7=\mathrm{CC}(=\mathrm{CC}(=\mathrm{C} 7) \mathrm{C}(\mathrm{O})=\mathrm{O}) \mathrm{C}(\mathrm{O})=\mathrm{O}) \mathrm{C}=\mathrm{C} 64)$

$\mathrm{C} 4=\mathrm{CC}(\mathrm{OC} 6=\mathrm{CC}(=\mathrm{CC}(=\mathrm{C} 6) \mathrm{C}(\mathrm{O})=\mathrm{O}) \mathrm{C}(\mathrm{O})=\mathrm{O})=\mathrm{C}(\mathrm{OC} 6=\mathrm{CC}(=\mathrm{CC}(=\mathrm{C} 6) \mathrm{C}(\mathrm{O})=\mathrm{O}) \mathrm{C}(\mathrm{O})=\mathrm{O}) \mathrm{C}=\mathrm{C} 54)=\mathrm{C} 3 \mathrm{C}=\mathrm{C} 2 \mathrm{O}$

$341 \mathrm{C} 2=\mathrm{CC}(=\mathrm{CC}(=\mathrm{C} 2) \mathrm{C}(\mathrm{O})=\mathrm{O}) \mathrm{C}(\mathrm{O})=\mathrm{O})=\mathrm{C} 1) \mathrm{C}(\mathrm{O})=\mathrm{O}$

$[\mathrm{Na}+] \cdot[\mathrm{Na}+] \cdot[\mathrm{Na}+] \cdot[\mathrm{Na}+] \cdot[\mathrm{Na}+] \cdot[\mathrm{Na}+] \cdot[\mathrm{Na}+] \cdot[\mathrm{Na}+] \cdot[\mathrm{Na}+] \cdot[\mathrm{Zn}++] \cdot \mathrm{OC}(=\mathrm{O}) \mathrm{C} 1=\mathrm{CC}(=\mathrm{CC}(\mathrm{OC} 2=\mathrm{CC} 3=\mathrm{C} 4 \mathrm{NC}(\mathrm{NN}$

$=\mathrm{C} 5 \backslash \mathrm{N}=\mathrm{C}(\backslash \mathrm{N}=\mathrm{C} 6 / \mathrm{N} \backslash \mathrm{C}(=\mathrm{N} / \mathrm{C} 7=\mathrm{N} / \mathrm{C}(=\mathrm{N} \backslash 4) / \mathrm{C} 4=\mathrm{CC}(\mathrm{OC} 8=\mathrm{CC}(=\mathrm{CC}(=\mathrm{C} 8) \mathrm{C}([\mathrm{O}-$

]$)=\mathrm{O}) \mathrm{C}(\mathrm{O})=\mathrm{O})=\mathrm{C}(\mathrm{OC} 8=\mathrm{CC}(=\mathrm{CC}(=\mathrm{C} 8) \mathrm{C}([\mathrm{O}-$

]$)=\mathrm{O}) \mathrm{C}(\mathrm{O})=\mathrm{O}) \mathrm{C}=\mathrm{C} 74) \mathrm{C} 4=\mathrm{CC}(\mathrm{OC} 7=\mathrm{CC}(=\mathrm{CC}(=\mathrm{C} 7) \mathrm{C}(\mathrm{O})=\mathrm{O}) \mathrm{C}([\mathrm{O}-])=\mathrm{O})=\mathrm{C}(\mathrm{OC} 7=\mathrm{CC}(=\mathrm{CC}(=\mathrm{C} 7) \mathrm{C}([\mathrm{O}-$

]$)=\mathrm{O}) \mathrm{C}([\mathrm{O}-])=\mathrm{O}) \mathrm{C}=\mathrm{C} 64) \mathrm{C} 4=\mathrm{CC}(\mathrm{OC} 6=\mathrm{CC}(=\mathrm{CC}(=\mathrm{C} 6) \mathrm{C}([\mathrm{O}-])=\mathrm{O}) \mathrm{C}([\mathrm{O}-])=\mathrm{O})=\mathrm{C}(\mathrm{OC} 6=\mathrm{CC}(=\mathrm{CC}(=\mathrm{C} 6) \mathrm{C}([\mathrm{O}-$

$342]]=\mathrm{O}) \mathrm{C}(\mathrm{O})=\mathrm{O}) \mathrm{C}=\mathrm{C} 54)=\mathrm{C} 3 \mathrm{C}=\mathrm{C} 2 \mathrm{OC} 2=\mathrm{CC}(=\mathrm{CC}(=\mathrm{C} 2) \mathrm{C}([\mathrm{O}-])=\mathrm{O}) \mathrm{C}([\mathrm{O}-])=\mathrm{O})=\mathrm{C} 1) \mathrm{C}([\mathrm{O}-])=\mathrm{O}$

$343 \mathrm{CN}(\mathrm{C}) \mathrm{CCCN} 1 \mathrm{C}(=\mathrm{O}) \mathrm{NC} 2(\mathrm{CSC} 3=\mathrm{C} 2 \mathrm{C}(=\mathrm{O}) \mathrm{C} 2=\mathrm{CC}=\mathrm{CC}=\mathrm{C} 2 \mathrm{C} 3=\mathrm{O}) \mathrm{C} 1=\mathrm{O}$

$\mathrm{COC} 1=\mathrm{CC}=\mathrm{CC} 2=\mathrm{C} 1 \mathrm{C}(=\mathrm{O}) \mathrm{C} 1=\mathrm{C}(\mathrm{O}) \mathrm{C} 3=\mathrm{C}(\mathrm{C}[\mathrm{C} @](\mathrm{O})(\mathrm{C}[\mathrm{C} @ @ \mathrm{H}] 3 \mathrm{O}[\mathrm{C} @ \mathrm{H}] 3 \mathrm{C}[\mathrm{C} @ \mathrm{H}](\mathrm{N})[\mathrm{C} @ \mathrm{H}](\mathrm{O})[\mathrm{C} @ \mathrm{H}]($

$344 \mathrm{C}) \mathrm{O} 3) \mathrm{C}(=\mathrm{O}) \mathrm{CO}) \mathrm{C}(\mathrm{O})=\mathrm{C} 1 \mathrm{C} 2=\mathrm{O}$

$345 \mathrm{COC} 1=\mathrm{CC}(=\mathrm{CC}(\mathrm{OC})=\mathrm{C} 1 \mathrm{OC}) \mathrm{C}(=\mathrm{O}) \backslash \mathrm{C}=\mathrm{ClC} 1=\mathrm{CC}=\mathrm{C}(\mathrm{F}) \mathrm{C}=\mathrm{C} 1$

$346 \mathrm{COC} 1=\mathrm{CC}=\mathrm{C}(\mathrm{C}=\mathrm{C} 1) \mathrm{C}(=\mathrm{O}) \backslash \mathrm{C}=\mathrm{ClC} 1=\mathrm{CC}(\mathrm{OC})=\mathrm{C}(\mathrm{OC}) \mathrm{C}(\mathrm{OC})=\mathrm{C} 1$

$347 \mathrm{COC} 1=\mathrm{CC}=\mathrm{C}(\mathrm{C}=\mathrm{C} 1) \mathrm{C} 1 \mathrm{NN}=\mathrm{C}(\mathrm{C} 1 \mathrm{O}) \mathrm{C} 1=\mathrm{CC}(\mathrm{OC})=\mathrm{C}(\mathrm{OC}) \mathrm{C}(\mathrm{OC})=\mathrm{C} 1$

$348 \quad \mathrm{COC} 1=\mathrm{CC}(=\mathrm{CC}(\mathrm{OC})=\mathrm{C} 1 \mathrm{OC}) \mathrm{C}(=\mathrm{O}) \backslash \mathrm{C}=\mathrm{ClC} 1=\mathrm{CC}=\mathrm{C} 2 \mathrm{OCOC} 2=\mathrm{C} 1$

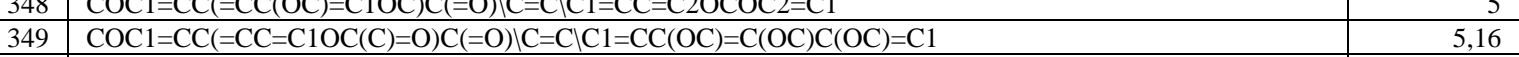

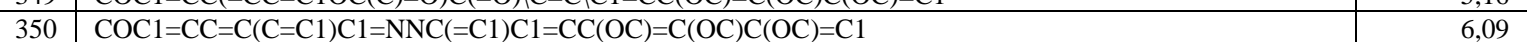

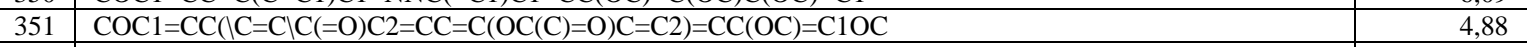

\begin{tabular}{rr}
$\mathrm{COC} 1=\mathrm{CC}(=\mathrm{CC}(\mathrm{OC})=\mathrm{C} 1 \mathrm{OC}) \mathrm{C} 1=\mathrm{NNC}(\mathrm{C} 1 \mathrm{O}) \mathrm{C} 1=\mathrm{CC}=\mathrm{C} 2 \mathrm{OCOC} 2=\mathrm{C} 1$ & 4,4 \\
\hline
\end{tabular}

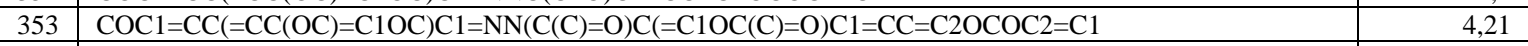

$354 \mathrm{COC} 1=\mathrm{CC}(=\mathrm{CC}(\mathrm{OC})=\mathrm{C} 1 \mathrm{OC}) \mathrm{C}(=\mathrm{O}) \backslash \mathrm{C}=\mathrm{ClC} 1=\mathrm{CC}=\mathrm{CC}=\mathrm{C} 1$

$355 \quad \mathrm{COC} 1=\mathrm{CC}(=\mathrm{CC}=\mathrm{C} 1 \mathrm{O}) \mathrm{C}(=\mathrm{O}) \backslash \mathrm{C}=\mathrm{ClC} 1=\mathrm{CC}(\mathrm{OC})=\mathrm{C}(\mathrm{OC}) \mathrm{C}(\mathrm{OC})=\mathrm{C} 1$

$356 \quad \mathrm{COC} 1=\mathrm{CC}(=\mathrm{CC}(\mathrm{OC})=\mathrm{C} 1 \mathrm{OC}) \mathrm{C}(=\mathrm{O}) \backslash \mathrm{C}=\mathrm{ClC} 1=\mathrm{CC}=\mathrm{C}(\mathrm{Cl}) \mathrm{C}=\mathrm{C} 1$

$357 \mathrm{COC} 1=\mathrm{CC}=\mathrm{C}(\mathrm{C}=\mathrm{C} 1 \mathrm{OC}) \mathrm{C} 1=\mathrm{NNC}(=\mathrm{C} 1) \mathrm{C} 1=\mathrm{CC}(\mathrm{OC})=\mathrm{C}(\mathrm{OC}) \mathrm{C}(\mathrm{OC})=\mathrm{C} 1$

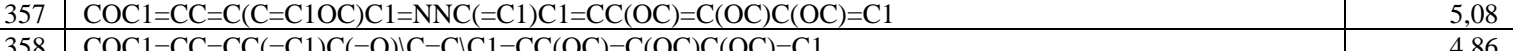

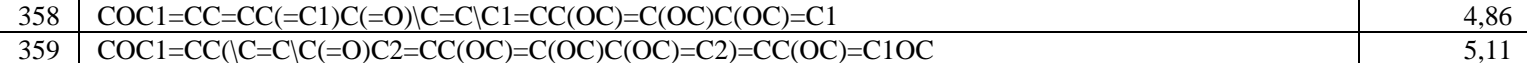

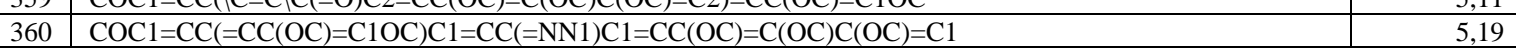

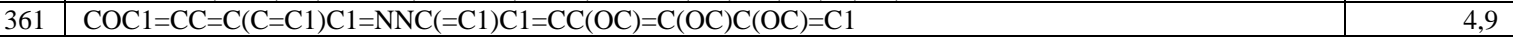

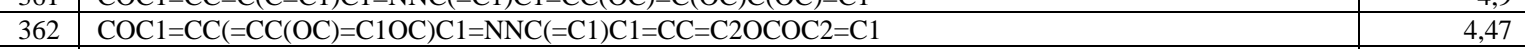

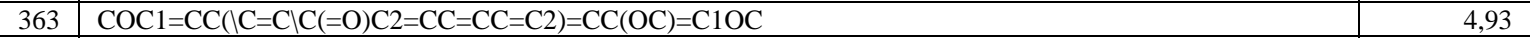

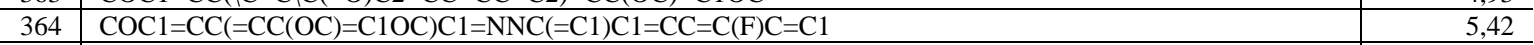

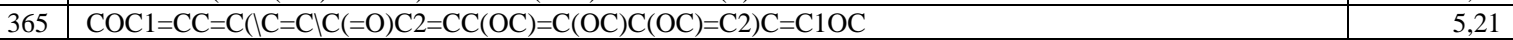

$\mathrm{CC}(=\mathrm{C})[\mathrm{C} @ @ \mathrm{H}] 1 \mathrm{CC}[\mathrm{C} @] 2(\mathrm{CO}) \mathrm{CC}[\mathrm{C} @] 3(\mathrm{C})[\mathrm{C} @ \mathrm{H}](\mathrm{CC}[\mathrm{C} @ @ \mathrm{H}] 4[\mathrm{C} @ @] 5(\mathrm{C}) \mathrm{CC}[\mathrm{C} @ \mathrm{H}](\mathrm{O}) \mathrm{C}(\mathrm{C})(\mathrm{C})[\mathrm{C} @$

$366 @ \mathrm{H}] 5 \mathrm{CC}[\mathrm{C} @ @] 34 \mathrm{C})[\mathrm{C} @ @ \mathrm{H}] 12$

$\mathrm{CC}(=\mathrm{C})[\mathrm{C} @ @ \mathrm{H}] 1 \mathrm{CC}[\mathrm{C} @] 2(\mathrm{CO}) \mathrm{CC}[\mathrm{C} @] 3(\mathrm{C})[\mathrm{C} @ \mathrm{H}](\mathrm{CC}[\mathrm{C} @ @ \mathrm{H}] 4[\mathrm{C} @ @] 5(\mathrm{C}) \mathrm{CCC}(=\mathrm{O}) \mathrm{C}(\mathrm{C})(\mathrm{C})[\mathrm{C} @ @ \mathrm{H}]$

367 5CC[C@@]34C)[C@@H]12

$\mathrm{CC}(=\mathrm{C})[\mathrm{C} @ @ \mathrm{H}] 1 \mathrm{CC}[\mathrm{C} @] 2(\mathrm{C}) \mathrm{CC}[\mathrm{C} @] 3(\mathrm{C})[\mathrm{C} @ \mathrm{H}](\mathrm{CC}[\mathrm{C} @ @ \mathrm{H}] 4[\mathrm{C} @ @] 5(\mathrm{C}) \mathrm{CC}[\mathrm{C} @ \mathrm{H}](\mathrm{O}) \mathrm{C}(\mathrm{C})(\mathrm{C})[\mathrm{C} @ @$

368 H]5CC[C@@]34C)[C@@H]12

$\mathrm{CC}(=\mathrm{C})[\mathrm{C} @ @ \mathrm{H}] 1 \mathrm{CC}[\mathrm{C} @] 2(\mathrm{C}) \mathrm{CC}[\mathrm{C} @] 3(\mathrm{C})[\mathrm{C} @ @ \mathrm{H}](\mathrm{C}[\mathrm{C} @ @ \mathrm{H}](\mathrm{O})[\mathrm{C} @ @ \mathrm{H}] 4[\mathrm{C} @ @] 5(\mathrm{C})[\mathrm{C} @ \mathrm{H}](\mathrm{O}) \mathrm{C}[\mathrm{C}$

$369 @ \mathrm{H}](\mathrm{O}) \mathrm{C}(\mathrm{C})(\mathrm{C})[\mathrm{C} @ @ \mathrm{H}] 5 \mathrm{CC}[\mathrm{C} @ @] 34 \mathrm{C})[\mathrm{C} @ @ \mathrm{H}] 12$

$@ \mathrm{H}](\mathrm{O}) \mathrm{C}(\mathrm{C})(\mathrm{C})[\mathrm{C} @ @ \mathrm{H}] 5 \mathrm{CC}[\mathrm{C} @ @] 34 \mathrm{C})[\mathrm{C} @ @ \mathrm{H}] 12$

370 CC[C@@]34C)[C@@H]12

$\mathrm{CC}(=\mathrm{C})[\mathrm{C} @ \mathrm{C}$ ) $\mathrm{H}] \mathrm{CC}[\mathrm{C} @] 2(\mathrm{C}) \mathrm{CC}[\mathrm{C} @] 3(\mathrm{C})[\mathrm{C} @ @ \mathrm{H}](\mathrm{CC}[\mathrm{C} @(\mathrm{H}] 4[\mathrm{C} @ @] 5(\mathrm{C}) \mathrm{C}=\mathrm{CC}(=\mathrm{O}) \mathrm{C}(\mathrm{C})(\mathrm{C})[\mathrm{C} @ @$

371 H]5CC[C@@]34C)[C@@ @ $] 12$ 


\begin{tabular}{|c|c|c|}
\hline NAME & SMILE & $\lg (1 / \operatorname{IC50})$ \\
\hline 372 & $\begin{array}{l}\mathrm{CC}(=\mathrm{C})[\mathrm{C} @ @ \mathrm{H}] 1 \mathrm{CC}[\mathrm{C} @] 2(\mathrm{C}) \mathrm{CC}[\mathrm{C} @] 3(\mathrm{C})[\mathrm{C} @ @ \mathrm{H}](\mathrm{CC}[\mathrm{C} @ @ \mathrm{H}] 4[\mathrm{C} @ @] 5(\mathrm{C})[\mathrm{C} @ @ \mathrm{H}](\mathrm{O}) \mathrm{C}[\mathrm{C} @ \mathrm{H}](\mathrm{O}) \mathrm{C} \\
(\mathrm{C})(\mathrm{C})[\mathrm{C} @ \mathrm{H}] 5 \mathrm{CC}[\mathrm{C} @ @] 34 \mathrm{C})[\mathrm{C} @ @ \mathrm{H}] 12\end{array}$ & 4,65 \\
\hline 373 & $\begin{array}{l}\mathrm{CC}(=\mathrm{C})[\mathrm{C} @ @ \mathrm{H}] 1 \mathrm{CC}[\mathrm{C} @] 2(\mathrm{C}) \mathrm{CC}[\mathrm{C} @] 3(\mathrm{C})[\mathrm{C} @ @ \mathrm{H}](\mathrm{C}[\mathrm{C} @ @ \mathrm{H}](\mathrm{O})[\mathrm{C} @ @ \mathrm{H}] 4[\mathrm{C} @ @] 5(\mathrm{C}) \mathrm{CCC}(=\mathrm{O}) \mathrm{C}(\mathrm{C})( \\
\mathrm{C})[\mathrm{C} @ @ \mathrm{H}] 5 \mathrm{CC}[\mathrm{C} @] 34 \mathrm{C})[\mathrm{C} @ \mathrm{H}] 12\end{array}$ & 4,64 \\
\hline 374 & $\begin{array}{l}\mathrm{CC}(=\mathrm{C})[\mathrm{C} @ @ \mathrm{H}] 1 \mathrm{CC}[\mathrm{C} @] 2(\mathrm{CO}) \mathrm{CC}[\mathrm{C} @] 3(\mathrm{C})[\mathrm{C} @ \mathrm{H}](\mathrm{C}[\mathrm{C} @ @ \mathrm{H}](\mathrm{O})[\mathrm{C} @ @ \mathrm{H}] 4[\mathrm{C} @ @] 5(\mathrm{C}) \mathrm{CC}[\mathrm{C} @ @ \mathrm{H}](\mathrm{O}) \\
\mathrm{C}(\mathrm{C})(\mathrm{C})[\mathrm{C} @ \mathrm{H}] 5 \mathrm{CC}[\mathrm{C} @ @] 34 \mathrm{C})[\mathrm{C} @ @ \mathrm{H}] 12\end{array}$ & 4,66 \\
\hline 375 & $\begin{array}{l}\mathrm{CC}(=\mathrm{C})[\mathrm{C} @ @ \mathrm{H}] 1 \mathrm{CC}[\mathrm{C} @] 2(\mathrm{CO}) \mathrm{CC}[\mathrm{C} @] 3(\mathrm{C})[\mathrm{C} @ \mathrm{H}](\mathrm{CC}[\mathrm{C} @ @ \mathrm{H}] 4[\mathrm{C} @ @] 5(\mathrm{C}) \mathrm{CC}[\mathrm{C} @ \mathrm{H}](\mathrm{O}) \mathrm{C}(\mathrm{C})(\mathrm{C})[\mathrm{C} @ \\
@ \mathrm{H}] 5[\mathrm{C} @ \mathrm{H}](\mathrm{O}) \mathrm{C}[\mathrm{C} @ @] 34 \mathrm{C})[\mathrm{C} @ @ \mathrm{H}] 12\end{array}$ & 4,66 \\
\hline 376 & $\begin{array}{l}\mathrm{CC}(=\mathrm{C})[\mathrm{C} @ @ \mathrm{H}] 1 \mathrm{CC}[\mathrm{C} @ @] 2(\mathrm{CC}[\mathrm{C} @] 3(\mathrm{C})[\mathrm{C} @ @ \mathrm{H}](\mathrm{CC}[\mathrm{C} @ @ \mathrm{H}] 4[\mathrm{C} @ @] 5(\mathrm{C}) \mathrm{C}=\mathrm{CC}(=\mathrm{O}) \mathrm{C}(\mathrm{C})(\mathrm{C})[\mathrm{C} @ @ \\
\mathrm{H}] 5 \mathrm{CC}[\mathrm{C} @ \text { @ } 34 \mathrm{C})[\mathrm{C} @ @ \mathrm{H}] 12) \mathrm{C}=\mathrm{O}\end{array}$ & 4,64 \\
\hline 377 & $\begin{array}{l}\mathrm{CC}(=\mathrm{C})[\mathrm{C} @ @ \mathrm{H}] 1 \mathrm{CC}[\mathrm{C} @] 2(\mathrm{CO}) \mathrm{CC}[\mathrm{C} @] 3(\mathrm{C})[\mathrm{C} @ \mathrm{H}](\mathrm{CC}[\mathrm{C} @ @ \mathrm{H}] 4[\mathrm{C} @ @] 5(\mathrm{C}) \mathrm{CC}[\mathrm{C} @ @ \mathrm{H}](\mathrm{O}) \mathrm{C}(\mathrm{C})(\mathrm{C})[\mathrm{C} \\
@ \mathrm{H}] 5 \mathrm{CC}[\mathrm{C} @] 34 \mathrm{C})[\mathrm{C} @ @ \mathrm{H}] 12\end{array}$ & 4,65 \\
\hline 378 & $\begin{array}{l}\mathrm{CC}(=\mathrm{C})[\mathrm{C} @ @ \mathrm{H}] 1 \mathrm{CC}[\mathrm{C} @] 2(\mathrm{CO}) \mathrm{CC}[\mathrm{C} @] 3(\mathrm{C})[\mathrm{C} @ \mathrm{H}](\mathrm{CC}[\mathrm{C} @ @ \mathrm{H}] 4[\mathrm{C} @ @] 5(\mathrm{C}) \mathrm{C}=\mathrm{CC}(=\mathrm{O}) \mathrm{C}(\mathrm{C})(\mathrm{C})[\mathrm{C} @ @ \mathrm{H} \\
] 5 \mathrm{CC}[\mathrm{C} @] 34 \mathrm{C})[\mathrm{C} @ @ \mathrm{H}] 12\end{array}$ & 4,64 \\
\hline 379 & $\begin{array}{l}\mathrm{CC}(=\mathrm{C})[\mathrm{C} @ @ \mathrm{H}] 1 \mathrm{CC}[\mathrm{C} @ @] 2(\mathrm{CC}[\mathrm{C} @] 3(\mathrm{C})[\mathrm{C} @ @ \mathrm{H}](\mathrm{CC}[\mathrm{C} @ @ \mathrm{H}] 4[\mathrm{C} @ @] 5(\mathrm{C}) \mathrm{CCC}(=\mathrm{O}) \mathrm{C}(\mathrm{C})(\mathrm{C})[\mathrm{C} @ @ \mathrm{H}] \\
5[\mathrm{C} @ \mathrm{H}](\mathrm{O}) \mathrm{C}[\mathrm{C} @ @] 34 \mathrm{C})[\mathrm{C} @ @ \mathrm{H}] 12) \mathrm{C}(\mathrm{O})=\mathrm{O}\end{array}$ & 4,67 \\
\hline 380 & $\begin{array}{l}\mathrm{CC}(=\mathrm{C})[\mathrm{C} @ @ \mathrm{H}] 1 \mathrm{CC}[\mathrm{C} @] 2(\mathrm{CO}) \mathrm{CC}[\mathrm{C} @] 3(\mathrm{C})[\mathrm{C} @ @ \mathrm{H}](\mathrm{CC}[\mathrm{C} @ @ \mathrm{H}] 4[\mathrm{C} @ @] 5(\mathrm{C}) \mathrm{CCC}(=\mathrm{O}) \mathrm{C}(\mathrm{C})(\mathrm{C})[\mathrm{C} @ @ \\
\mathrm{H}] 5[\mathrm{C} @ \mathrm{H}](\mathrm{O}) \mathrm{C}[\mathrm{C} @] 34 \mathrm{C})[\mathrm{C} @ @ \mathrm{H}] 12\end{array}$ & 4,66 \\
\hline 381 & $\begin{array}{l}\mathrm{CC}(\mathrm{C})[\mathrm{C} @ @ \mathrm{H}] 1 \mathrm{CC}[\mathrm{C} @] 2(\mathrm{C}) \mathrm{CC}[\mathrm{C} @] 3(\mathrm{C})[\mathrm{C} @ @ \mathrm{H}](\mathrm{CC}[\mathrm{C} @ @ \mathrm{H}] 4[\mathrm{C} @ @] 5(\mathrm{C}) \mathrm{CC}[\mathrm{C} @ \mathrm{H}](\mathrm{OC}(=\mathrm{O}) \backslash \mathrm{C}=\mathrm{C} \mid \mathrm{C} 6 \\
=\mathrm{CC}=\mathrm{C}(\mathrm{O}) \mathrm{C}(\mathrm{O})=\mathrm{C} 6) \mathrm{C}(\mathrm{C})(\mathrm{C})[\mathrm{C} @ @ \mathrm{H}] 5 \mathrm{CC}[\mathrm{C} @ @] 34 \mathrm{C})[\mathrm{C} @ @ \mathrm{H}] 12\end{array}$ & 4,77 \\
\hline 382 & $\begin{array}{l}\mathrm{CC}(=\mathrm{C})[\mathrm{C} @ @ \mathrm{H}] 1 \mathrm{CC}[\mathrm{C} @] 2(\mathrm{C}) \mathrm{CC}[\mathrm{C} @] 3(\mathrm{C})[\mathrm{C} @ @ \mathrm{H}](\mathrm{CC}[\mathrm{C} @ @ \mathrm{H}] 4[\mathrm{C} @ @] 5(\mathrm{C})[\mathrm{C} @ \mathrm{H}](\mathrm{O}) \mathrm{CC}(=\mathrm{O}) \mathrm{C}(\mathrm{C})(\mathrm{C}) \\
[\mathrm{C} @ \mathrm{H}] 5 \mathrm{C}[\mathrm{C} @ @] 34 \mathrm{C})[\mathrm{C} @ @ \mathrm{H}] 12\end{array}$ & 4,64 \\
\hline 383 & $\begin{array}{l}\mathrm{CC}(=\mathrm{C})[\mathrm{C} @ @ \mathrm{H}] 1 \mathrm{CC}[\mathrm{C} @] 2(\mathrm{C}) \mathrm{CC}[\mathrm{C} @] 3(\mathrm{C})[\mathrm{C} @ @ \mathrm{H}](\mathrm{CC}[\mathrm{C} @ @ \mathrm{H}] 4[\mathrm{C} @ @] 5(\mathrm{C})[\mathrm{C} @ @ \mathrm{H}](\mathrm{O}) \mathrm{C}[\mathrm{C} @ @ \mathrm{H}](\mathrm{O}) \\
\mathrm{C}(\mathrm{C})(\mathrm{C})[\mathrm{C} @ \mathrm{H}] 5 \mathrm{CC}[\mathrm{C} @] 34 \mathrm{C})[\mathrm{C} @ @ \mathrm{H}] 12\end{array}$ & 4,65 \\
\hline 384 & $\begin{array}{l}\mathrm{CC}(=\mathrm{C})[\mathrm{C} @ @ \mathrm{H}] 1 \mathrm{CC}[\mathrm{C} @ @] 2(\mathrm{CC}[\mathrm{C} @] 3(\mathrm{C})[\mathrm{C} @ \mathrm{H}](\mathrm{CC}[\mathrm{C} @ @ \mathrm{H}] 4[\mathrm{C} @ @] 5(\mathrm{C}) \mathrm{CC}[\mathrm{C} @ @ \mathrm{H}](\mathrm{O}) \mathrm{C}(\mathrm{C})(\mathrm{C})[\mathrm{C} @ \\
@ \mathrm{H}] 5 \mathrm{CC}[\mathrm{C} @] 34 \mathrm{C})[\mathrm{C} @ @ \mathrm{H}] 12) \mathrm{C}(\mathrm{O})=\mathrm{O}\end{array}$ & 5,17 \\
\hline 385 & $\begin{array}{l}\mathrm{CC}(=\mathrm{O})[\mathrm{C} @ @ \mathrm{H}] 1 \mathrm{CC}[\mathrm{C} @] 2(\mathrm{CO}) \mathrm{CC}[\mathrm{C} @] 3(\mathrm{C})[\mathrm{C} @ @ \mathrm{H}](\mathrm{CC}[\mathrm{C} @ @ \mathrm{H}] 4[\mathrm{C} @ @] 5(\mathrm{C}) \mathrm{CC}[\mathrm{C} @ \mathrm{H}](\mathrm{O}) \mathrm{C}(\mathrm{C})(\mathrm{C})[\mathrm{C} \\
\text { @ } \mathrm{H}] 5 \mathrm{CC}[\mathrm{C} @] 34 \mathrm{C})[\mathrm{C} @ @ \mathrm{H}] 12\end{array}$ & 4,65 \\
\hline 386 & $\begin{array}{l}\mathrm{CC}(=\mathrm{C})[\mathrm{C} @ @ \mathrm{H}] 1 \mathrm{CC}[\mathrm{C} @] 2(\mathrm{CO}) \mathrm{CC}[\mathrm{C} @] 3(\mathrm{C})[\mathrm{C} @ \mathrm{H}](\mathrm{CC}[\mathrm{C} @ @ \mathrm{H}] 4[\mathrm{C} @ @] 5(\mathrm{C}) \mathrm{CC}[\mathrm{C} @ \mathrm{H}](\mathrm{OC}(=\mathrm{O}) \backslash \mathrm{C}=\mathrm{ClC} \\
6=\mathrm{CC}=\mathrm{C}(\mathrm{O}) \mathrm{C}(\mathrm{O})=\mathrm{C} 6) \mathrm{C}(\mathrm{C})(\mathrm{C})[\mathrm{C} @ \mathrm{H}] 5 \mathrm{CC}[\mathrm{C} @ @] 34 \mathrm{C})[\mathrm{C} @ @ \mathrm{H}] 12\end{array}$ & 4,78 \\
\hline 387 & $\begin{array}{l}\mathrm{CC}(=\mathrm{C})[\mathrm{C} @ @ \mathrm{H}] 1 \mathrm{CC}[\mathrm{C} @ @] 2(\mathrm{C})[\mathrm{C} @ \mathrm{H}] 1[\mathrm{C} @ @ \mathrm{H}] 1 \mathrm{CC}[\mathrm{C} @ @ \mathrm{H}] 3[\mathrm{C} @ @] 4(\mathrm{C}) \mathrm{CCC}(=\mathrm{O}) \mathrm{C}(\mathrm{C})(\mathrm{C})[\mathrm{C} @ @ \mathrm{H}] 4[ \\
\mathrm{C} @ \mathrm{H}](\mathrm{O}) \mathrm{C}[\mathrm{C} @ @] 3(\mathrm{C})[\mathrm{C} @] 1(\mathrm{C}) \mathrm{C}[\mathrm{C} @ @ \mathrm{H}] 2 \mathrm{O}\end{array}$ & 4,66 \\
\hline 388 & $\begin{array}{l}\mathrm{CC} 1(\mathrm{C})[\mathrm{C} @ @ \mathrm{H}] 2 \mathrm{CC}[\mathrm{C} @] 3(\mathrm{C})[\mathrm{C} @ \mathrm{H}](\mathrm{CC}[\mathrm{C} @ \mathrm{H}] 4[\mathrm{C} @ \mathrm{H}] 5[\mathrm{C} @ @ \mathrm{H}](\mathrm{CC}[\mathrm{C} @] 5(\mathrm{CO}) \mathrm{CC}[\mathrm{C} @ @] 34 \mathrm{C}) \mathrm{C}(=\mathrm{C}) \mathrm{C} \\
\mathrm{O})\left[\mathrm{C} @ \mathrm{C}^{2}(\mathrm{C}) \mathrm{CCC} 1=\mathrm{O}\right.\end{array}$ & 4,81 \\
\hline 389 & $\mathrm{CC} 1=\mathrm{CC}[\mathrm{C} @] 2(\mathrm{CC} 1) \mathrm{C}(=\mathrm{C}) \mathrm{C}[\mathrm{C} @ \mathrm{H}](\mathrm{O})[\mathrm{C} @ \mathrm{H}](\mathrm{Br}) \mathrm{C} 2(\mathrm{C}) \mathrm{C}$ & 4,17 \\
\hline 390 & $\mathrm{CC}(=\mathrm{O}) \mathrm{O}[\mathrm{C} @ \mathrm{H}] 1 \mathrm{CC}(=\mathrm{C})[\mathrm{C} @] 2(\mathrm{CCC}(\mathrm{C})=\mathrm{CC} 2) \mathrm{C}(\mathrm{C})(\mathrm{C})[\mathrm{C} @ \mathrm{H}] 1 \mathrm{Br}$ & 4,24 \\
\hline 391 & $\mathrm{CC}(=\mathrm{O}) \mathrm{O}[\mathrm{C} @ \mathrm{H}] 1 \mathrm{CC}(=\mathrm{C})[\mathrm{C} @] 2(\mathrm{CCC}(\mathrm{C})=\mathrm{C}(\mathrm{Cl}) \mathrm{C} 2) \mathrm{C}(\mathrm{C})(\mathrm{C})[\mathrm{C} @ \mathrm{H}] 1 \mathrm{Br}$ & 4,55 \\
\hline 392 & $\mathrm{CC}(=\mathrm{O}) \mathrm{O}[\mathrm{C} @ @ \mathrm{H}] 1 \mathrm{C}[\mathrm{C} @ @ \mathrm{H}](\mathrm{Br}) \mathrm{C}(\mathrm{C})(\mathrm{C}) \mathrm{O}[\mathrm{C} @] 1(\mathrm{C})[\mathrm{C} @ \mathrm{H}] 1 \mathrm{CC}[\mathrm{C} @](\mathrm{C})(\mathrm{Cl})[\mathrm{C} @ @ \mathrm{H}](\mathrm{Br}) \mathrm{C} 1$ & 4,38 \\
\hline 393 & $\mathrm{CC} 1(\mathrm{C}) \mathrm{O}[\mathrm{C} @ @](\mathrm{C})([\mathrm{C} @ \mathrm{H}](\mathrm{O}) \mathrm{C}[\mathrm{C} @ \mathrm{H}] 1 \mathrm{Br})[\mathrm{C} @ \mathrm{H}] 1 \mathrm{CC}[\mathrm{C} @](\mathrm{C})(\mathrm{Cl})[\mathrm{C} @ @ \mathrm{H}](\mathrm{Br}) \mathrm{C} 1$ & 4,34 \\
\hline 394 & $\begin{array}{l}\mathrm{CC}(\mathrm{C})[\mathrm{C} @ \mathrm{H}] 1 \mathrm{NC}(=\mathrm{O})[\mathrm{C} @ @ \mathrm{H}](\mathrm{NC}(=\mathrm{O}) \mathrm{C} 2=\mathrm{C} 3 \mathrm{~N}=\mathrm{C} 4 \mathrm{C}(\mathrm{OC} 3=\mathrm{C}(\mathrm{C}) \mathrm{C}=\mathrm{C} 2)=\mathrm{C}(\mathrm{C}) \mathrm{C}(=\mathrm{O}) \mathrm{C}(\mathrm{N})=\mathrm{C} 4 \mathrm{C}(=\mathrm{O}) \mathrm{N}[\mathrm{C} \\
@ \mathrm{H}] 2[\mathrm{C} @ @ \mathrm{H}](\mathrm{C}) \mathrm{OC}(=\mathrm{O})[\mathrm{C} @ \mathrm{H}](\mathrm{C}(\mathrm{C}) \mathrm{C}) \mathrm{N}(\mathrm{C}) \mathrm{C}(=\mathrm{O}) \mathrm{CN}(\mathrm{C}) \mathrm{C}(=\mathrm{O})[\mathrm{C} @ @ \mathrm{H}] 3 \mathrm{CCCN} 3 \mathrm{C}(=\mathrm{O})[\mathrm{C} @ \mathrm{H}](\mathrm{NC}=\mathrm{O}) \\
\mathrm{C}(\mathrm{C}) \mathrm{C})[\mathrm{C} @ @ \mathrm{H}](\mathrm{C}) \mathrm{OC}(=\mathrm{O})[\mathrm{C} @ \mathrm{H}](\mathrm{C}(\mathrm{C}) \mathrm{C}) \mathrm{N}(\mathrm{C}) \mathrm{C}(=\mathrm{O}) \mathrm{CN}(\mathrm{C}) \mathrm{C}(=\mathrm{O})[\mathrm{C} @ @ \mathrm{H}] 2 \mathrm{CCCN} 2 \mathrm{C} 1=\mathrm{O}\end{array}$ & 6,72 \\
\hline 395 & $\mathrm{CC} 1=\mathrm{C}(\mathrm{Cl}) \mathrm{C}[\mathrm{C} @] 2(\mathrm{CC} 1) \mathrm{C}(=\mathrm{C}) \mathrm{C}[\mathrm{C} @ \mathrm{H}](\mathrm{O})[\mathrm{C} @ \mathrm{H}](\mathrm{Br}) \mathrm{C} 2(\mathrm{C}) \mathrm{C}$ & 5,62 \\
\hline 396 & $\begin{array}{l}\mathrm{CC}(\mathrm{C})[\mathrm{C} @ \mathrm{H}] 1 \mathrm{NC}(=\mathrm{O})[\mathrm{C} @ @ \mathrm{H}](\mathrm{NC}(=\mathrm{O}) \mathrm{C} 2=\mathrm{C} 3 \mathrm{~N}=\mathrm{C} 4 \mathrm{C}(\mathrm{OC} 3=\mathrm{C}(\mathrm{C}) \mathrm{C}=\mathrm{C} 2)=\mathrm{C}(\mathrm{C}) \mathrm{C}(=\mathrm{O}) \mathrm{C}(\mathrm{N})=\mathrm{C} 4 \mathrm{C}(=\mathrm{O}) \mathrm{N}[\mathrm{C} \\
@ \mathrm{H}] 2[\mathrm{C} @ @ \mathrm{H}](\mathrm{C}) \mathrm{OC}(=\mathrm{O})[\mathrm{C} @ \mathrm{H}](\mathrm{C}(\mathrm{C}) \mathrm{C}) \mathrm{N}(\mathrm{C}) \mathrm{C}(=\mathrm{O}) \mathrm{CN}(\mathrm{C}) \mathrm{C}(=\mathrm{O})[\mathrm{C} @ @ \mathrm{H}] 3 \mathrm{CCCN} 3 \mathrm{C}(=\mathrm{O})[\mathrm{C} @ \mathrm{H}](\mathrm{NC} 2=\mathrm{O}) \\
\mathrm{C}(\mathrm{C}) \mathrm{C})[\mathrm{C} @ \mathrm{H}](\mathrm{C}) \mathrm{OC}(=\mathrm{O})[\mathrm{C} @ \mathrm{H}](\mathrm{C}(\mathrm{C}) \mathrm{C}) \mathrm{N}(\mathrm{C}) \mathrm{C}(=\mathrm{O}) \mathrm{CN}(\mathrm{C}) \mathrm{C}(=\mathrm{O})[\mathrm{C} @ @ \mathrm{H}] 2 \mathrm{CCCN} 2 \mathrm{C} 1=\mathrm{O}\end{array}$ & 8,22 \\
\hline 397 & $\mathrm{CC} 1=\mathrm{C}(\mathrm{Cl}) \mathrm{C}[\mathrm{C} @] 2(\mathrm{CC} 1) \mathrm{C}(=\mathrm{C}) \mathrm{C}[\mathrm{C} @ \mathrm{H}](\mathrm{O})[\mathrm{C} @ \mathrm{H}](\mathrm{Br}) \mathrm{C} 2(\mathrm{C}) \mathrm{C}$ & 5,7 \\
\hline 398 & $\mathrm{CC}(=\mathrm{O}) \mathrm{O}[\mathrm{C} @ \mathrm{H}] 1 \mathrm{CC}(=\mathrm{C})[\mathrm{C} @] 2(\mathrm{CCC}(\mathrm{C})=\mathrm{C}(\mathrm{Cl}) \mathrm{C} 2) \mathrm{C}(\mathrm{C})(\mathrm{C})[\mathrm{C} @ \mathrm{H}] 1 \mathrm{Br}$ & 4,65 \\
\hline 399 & $\begin{array}{l}\mathrm{CC}(\mathrm{C})[\mathrm{C} @ \mathrm{H}](\mathrm{NC}(=\mathrm{O})[\mathrm{C} @ \mathrm{H}](\mathrm{CCCCN}) \mathrm{NC}(=\mathrm{O})[\mathrm{C} @ \mathrm{H}](\mathrm{CCCN}=\mathrm{C}(\mathrm{N}) \mathrm{N}) \mathrm{NC}(=\mathrm{O})[\mathrm{C} @ \mathrm{H}](\mathrm{CCCCN}) \mathrm{NC}(=\mathrm{O})[\mathrm{C} \\
@ \mathrm{H}](\mathrm{CCCCN}) \mathrm{NC}(=\mathrm{O})[\mathrm{C} @ \mathrm{H}](\mathrm{CCCCN}) \mathrm{NC}(=\mathrm{O})[\mathrm{C} @ @ \mathrm{H}] 1 \mathrm{CCCN} 1 \mathrm{C}(=\mathrm{O}) \mathrm{CNC}(=\mathrm{O}) \mathrm{COCCOCCOCCOCCOC} \\
\mathrm{COCCNC}(=\mathrm{O}) \mathrm{COCC}(=\mathrm{O}) \mathrm{NC} 1=\mathrm{CC}=\mathrm{C}(\mathrm{C}=\mathrm{C} 1) \mathrm{C} 1=\mathrm{C} 2 / \mathrm{N} \backslash \mathrm{C}(\mathrm{C}=\mathrm{C} 2)=\mathrm{C}(/ \mathrm{C} 2=\mathrm{N} / \mathrm{C}(/ \mathrm{C}=\mathrm{C} 2)=\mathrm{C}(\mathrm{C} 2=\mathrm{CC}=\mathrm{C}(\mathrm{N} 2) \backslash \mathrm{C} \\
(=\mathrm{C} 2 \backslash \mathrm{C}=\mathrm{CC} \backslash 1=\mathrm{N} 2) \mathrm{C} 1=\mathrm{CC}=\mathrm{CC}=\mathrm{C} 1) \mathrm{C} 1=\mathrm{CC}=\mathrm{CC}=\mathrm{C} 1) \mathrm{C} 1=\mathrm{CC}=\mathrm{CC}=\mathrm{C} 1) \mathrm{C}(\mathrm{N})=\mathrm{O}\end{array}$ & 5,82 \\
\hline 400 & $\begin{array}{l}\mathrm{NCCCC}[\mathrm{C} @ \mathrm{H}](\mathrm{NC}(=\mathrm{O})[\mathrm{C} @ \mathrm{H}](\mathrm{CCCN}=\mathrm{C}(\mathrm{N}) \mathrm{N}) \mathrm{NC}(=\mathrm{O}) \mathrm{CNC}(=\mathrm{O}) \mathrm{COCCOCCOCCOCCOCCOCCNC}(=\mathrm{O}) \mathrm{C} \\
\mathrm{OCC}(=\mathrm{O}) \mathrm{NC} 1=\mathrm{CC}=\mathrm{C}(\mathrm{C}=\mathrm{C} 1) \mathrm{C} 1=\mathrm{C} 2 / \mathrm{N}) \mathrm{C}(\mathrm{C}=\mathrm{C} 2)=\mathrm{C}(/ \mathrm{C} 2=\mathrm{N} / \mathrm{C}(/ \mathrm{C}=\mathrm{C} 2)=\mathrm{C}(\mathrm{C} 2=\mathrm{CC}=\mathrm{C}(\mathrm{N} 2) \mathrm{C}(=\mathrm{C} 2 \backslash \mathrm{C}=\mathrm{CC} 1= \\
\mathrm{N} 2) \mathrm{C} 1=\mathrm{CC}=\mathrm{CC}=\mathrm{C} 1) \mathrm{C} 1=\mathrm{CC}=\mathrm{CC}=\mathrm{C} 1) \mathrm{C} 1=\mathrm{CC}=\mathrm{CC}=\mathrm{C} 1) \mathrm{C}(=\mathrm{O}) \mathrm{N}[\mathrm{C} @ @ \mathrm{H}](\mathrm{CCCCN}) \mathrm{C}(=\mathrm{O}) \mathrm{N}[\mathrm{C} @ @ \mathrm{H}](\mathrm{CCCN} \\
=\mathrm{C}(\mathrm{N}) \mathrm{N}) \mathrm{C}(=\mathrm{O}) \mathrm{N}[\mathrm{C} @ @ \mathrm{H}](\mathrm{CCCN}=\mathrm{C}(\mathrm{N}) \mathrm{N}) \mathrm{C}(=\mathrm{O}) \mathrm{N}[\mathrm{C} @ @ \mathrm{H}](\mathrm{CCC}(\mathrm{N})=\mathrm{O}) \mathrm{C}(=\mathrm{O}) \mathrm{N}[\mathrm{C} @ @ \mathrm{H}](\mathrm{CCCN}=\mathrm{C}(\mathrm{N}) \mathrm{N}) \\
\mathrm{C}(=\mathrm{O}) \mathrm{N}[\mathrm{C} @ @ \mathrm{H}](\mathrm{CCCN}=\mathrm{C}(\mathrm{N}) \mathrm{N}) \mathrm{C}(=\mathrm{O}) \mathrm{N}[\mathrm{C} @ @ \mathrm{H}](\mathrm{CCCN}=\mathrm{C}(\mathrm{N}) \mathrm{N}) \mathrm{C}(=\mathrm{O}) \mathrm{N} 1 \mathrm{CCC}[\mathrm{C} @ \mathrm{H}] 1 \mathrm{C}(=\mathrm{O}) \mathrm{N} 1 \mathrm{CCC}[\mathrm{C} \\
@ \mathrm{H}] 1 \mathrm{C}(=\mathrm{O}) \mathrm{N}[\mathrm{C} @ @ \mathrm{H}](\mathrm{CCC}(\mathrm{N})=\mathrm{O}) \mathrm{C}(\mathrm{N})=\mathrm{O}\end{array}$ & 4,52 \\
\hline 401 & $\begin{array}{l}\mathrm{CC}(\mathrm{C})[\mathrm{C} @ \mathrm{H}](\mathrm{NC}(=\mathrm{O})[\mathrm{C} @ \mathrm{H}](\mathrm{CCCCN}) \mathrm{NC}(=\mathrm{O})[\mathrm{C} @ \mathrm{H}](\mathrm{CCCN}=\mathrm{C}(\mathrm{N}) \mathrm{N}) \mathrm{NC}(=\mathrm{O})[\mathrm{C} @ \mathrm{H}](\mathrm{CCCCN}) \mathrm{NC}(=\mathrm{O})[\mathrm{C} \\
@ \mathrm{H}](\mathrm{CCCCN}) \mathrm{NC}(=\mathrm{O})[\mathrm{C} @ \mathrm{H}](\mathrm{CCCCN}) \mathrm{NC}(=\mathrm{O})[\mathrm{C} @ @ \mathrm{H}] 1 \mathrm{CCCN} 1 \mathrm{C}(=\mathrm{O}) \mathrm{CNC}(=\mathrm{O}) \mathrm{COCCOCCOCCOCCOC} \\
\mathrm{COCCNC}(=\mathrm{O}) \mathrm{COCC}(=\mathrm{O}) \mathrm{NC} 1=\mathrm{CC}=\mathrm{C}(\mathrm{C}=\mathrm{C} 1) \mathrm{C} 1=\mathrm{C} 2 / \mathrm{N} \backslash \mathrm{C}(\mathrm{C}=\mathrm{C} 2)=\mathrm{C}(/ \mathrm{C} 2=\mathrm{N} / \mathrm{C}(/ \mathrm{C}=\mathrm{C} 2)=\mathrm{C}(\mathrm{C} 2=\mathrm{CC}=\mathrm{C}(\mathrm{N} 2) \backslash \mathrm{C} \\
(=\mathrm{C} 2 \backslash \mathrm{C}=\mathrm{CC} \backslash 1=\mathrm{N} 2) \mathrm{C} 1=\mathrm{CC}=\mathrm{CC}=\mathrm{C} 1) \mathrm{C} 1=\mathrm{CC}=\mathrm{CC}=\mathrm{C} 1) \mathrm{C} 1=\mathrm{CC}=\mathrm{CC}=\mathrm{C} 1) \mathrm{C}(\mathrm{N})=\mathrm{O}\end{array}$ & 4,05 \\
\hline 402 & $\begin{array}{l}\mathrm{NC}(=\mathrm{N}) \mathrm{NCCC}[\mathrm{C} @ \mathrm{H}](\mathrm{NC}=\mathrm{O}) \mathrm{CNC}(=\mathrm{O}) \mathrm{COCCOCCOCCOCCOCCOCCNC}(=\mathrm{O}) \mathrm{COCC}(=\mathrm{O}) \mathrm{NC} 1=\mathrm{CC}=\mathrm{C}(\mathrm{C}= \\
\mathrm{C} 1) \mathrm{C} 1=\mathrm{C} 2 / \mathrm{N} \mid \mathrm{C}(\mathrm{C}=\mathrm{C} 2)=\mathrm{C}(/ \mathrm{C} 2=\mathrm{N} / \mathrm{C}(/ \mathrm{C}=\mathrm{C} 2)=\mathrm{C}(\mathrm{C} 2=\mathrm{CC}=\mathrm{C}(\mathrm{N} 2) \mathrm{C}(=\mathrm{C} 2 \mathrm{C}=\mathrm{CC} 1=\mathrm{N} 2) \mathrm{C} 1=\mathrm{CC}=\mathrm{CC}=\mathrm{C} 1) \mathrm{C} 1=\mathrm{C} \\
\mathrm{C}=\mathrm{CC}=\mathrm{C} 1) \mathrm{C} 1=\mathrm{CC}=\mathrm{CC}=\mathrm{C} 1) \mathrm{C}(=\mathrm{O}) \mathrm{N}[\mathrm{C} @ @ \mathrm{H}](\mathrm{CCCN}=\mathrm{C}(\mathrm{N}) \mathrm{N}) \mathrm{C}(=\mathrm{O}) \mathrm{N}[\mathrm{C} @ @ \mathrm{H}](\mathrm{CCCN}=\mathrm{C}(\mathrm{N}) \mathrm{N}) \mathrm{C}(=\mathrm{O}) \mathrm{N}[\mathrm{C} \\
@ @ \mathrm{H}](\mathrm{CCCN}=\mathrm{C}(\mathrm{N}) \mathrm{N}) \mathrm{C}(=\mathrm{O}) \mathrm{N}[\mathrm{C} @ \mathrm{H}](\mathrm{CCCN}=\mathrm{C}(\mathrm{N}) \mathrm{N}) \mathrm{C}(=\mathrm{O}) \mathrm{N}[\mathrm{C} @ \mathrm{H}](\mathrm{CCCNC}(\mathrm{N})=\mathrm{N}) \mathrm{C}(=\mathrm{O}) \mathrm{N}[\mathrm{C} @ @ \mathrm{H} \\
](\mathrm{CCCNC}(\mathrm{N})=\mathrm{N}) \mathrm{C}(=\mathrm{O}) \mathrm{N}[\mathrm{C} @ @ \mathrm{H}](\mathrm{CCCN}=\mathrm{C}(\mathrm{N}) \mathrm{N}) \mathrm{C}(\mathrm{O})=\mathrm{O}\end{array}$ & 4,12 \\
\hline 403 & $\mathrm{CC} 1=\mathrm{CC} 2=\mathrm{C}(\mathrm{C}=\mathrm{C} 1 \mathrm{C}) \mathrm{N}=\mathrm{C} 1 \mathrm{C}(\mathrm{O} 2)=\mathrm{CC}(=\mathrm{O}) \mathrm{C} 2=\mathrm{NC}=\mathrm{CC}=\mathrm{C} 12$ & 6,1 \\
\hline 404 & $\mathrm{O}=\mathrm{C} 1 \mathrm{C}=\mathrm{C} 2 \mathrm{OC} 3=\mathrm{CC}=\mathrm{CC}=\mathrm{C} 3 \mathrm{~N}=\mathrm{C} 2 \mathrm{C} 2=\mathrm{CC}=\mathrm{CN}=\mathrm{C} 12$ & 7,4 \\
\hline 405 & $\mathrm{O}=\mathrm{C} 1 \mathrm{C}=\mathrm{C} 2 \mathrm{OC} 3=\mathrm{CC}=\mathrm{C} 4 \mathrm{CCCCC} 4=\mathrm{C} 3 \mathrm{~N}=\mathrm{C} 2 \mathrm{C} 2=\mathrm{CC}=\mathrm{CN}=\mathrm{C} 12$ & 5,72 \\
\hline 406 & $\mathrm{O}=\mathrm{C} 1 \mathrm{C}=\mathrm{C} 2 \mathrm{OC} 3=\mathrm{C} 4 \mathrm{CCCCC} 4=\mathrm{CC}=\mathrm{C} 3 \mathrm{~N}=\mathrm{C} 2 \mathrm{C} 2=\mathrm{CC}=\mathrm{CN}=\mathrm{C} 12$ & 5,74 \\
\hline 407 & $\mathrm{CC} 1=\mathrm{CC}=\mathrm{C}(\mathrm{C}) \mathrm{C} 2=\mathrm{C} 1 \mathrm{OC} 1=\mathrm{CC}(=\mathrm{O}) \mathrm{C} 3=\mathrm{NC}=\mathrm{CC}=\mathrm{C} 3 \mathrm{C} 1=\mathrm{N} 2$ & 6,4 \\
\hline 408 & $\begin{array}{l}\mathrm{COC} 1=\mathrm{CC}=\mathrm{CC} 2=\mathrm{C} 1 \mathrm{C}(=\mathrm{O}) \mathrm{C} 1=\mathrm{C}(\mathrm{O}) \mathrm{C} 3=\mathrm{C}(\mathrm{C}[\mathrm{C} @](\mathrm{O})(\mathrm{C}[\mathrm{C} @ @ \mathrm{H}] 3 \mathrm{O}[\mathrm{C} @ \mathrm{H}] 3 \mathrm{C}[\mathrm{C} @ \mathrm{H}](\mathrm{N})[\mathrm{C} @ \mathrm{H}](\mathrm{O})[\mathrm{C} @ \mathrm{H}]( \\
\mathrm{C}) \mathrm{O} 3) \mathrm{C}(=\mathrm{O}) \mathrm{CO}) \mathrm{C}(\mathrm{O})=\mathrm{C} 1 \mathrm{C} 2=\mathrm{O}\end{array}$ & 7,4 \\
\hline 409 & $\mathrm{CC} 1=\mathrm{CC}(\mathrm{C})=\mathrm{C} 2 \mathrm{OC} 3=\mathrm{CC}(=\mathrm{O}) \mathrm{C} 4=\mathrm{NC}=\mathrm{CC}=\mathrm{C} 4 \mathrm{C} 3=\mathrm{NC} 2=\mathrm{C} 1$ & 5,96 \\
\hline
\end{tabular}


\begin{tabular}{l|l} 
NAME & SMILE \\
\hline
\end{tabular}

$411 \mathrm{O}=\mathrm{C} 1 \mathrm{C}=\mathrm{C} 2 \mathrm{OC} 3=\mathrm{C}(\mathrm{C}=\mathrm{CC} 4=\mathrm{C} 3 \mathrm{C}=\mathrm{CC}=\mathrm{C} 4) \mathrm{N}=\mathrm{C} 2 \mathrm{C} 2=\mathrm{CC}=\mathrm{CN}=\mathrm{C} 12$

$\lg (1 / \mathrm{IC50})$

$\mathrm{CC}(\mathrm{C})[\mathrm{C} @ \mathrm{H}] 1 \mathrm{NC}(=\mathrm{O})[\mathrm{C} @ @ \mathrm{H}](\mathrm{NC}(=\mathrm{O}) \mathrm{C} 2=\mathrm{C} 3 \mathrm{~N}=\mathrm{C} 4 \mathrm{C}(\mathrm{OC} 3=\mathrm{C}(\mathrm{C}) \mathrm{C}=\mathrm{C} 2)=\mathrm{C}(\mathrm{C}) \mathrm{C}(=\mathrm{O}) \mathrm{C}(\mathrm{N})=\mathrm{C} 4 \mathrm{C}(=\mathrm{O}) \mathrm{N}[\mathrm{C}$

$@ \mathrm{H}] 2[\mathrm{C} @ @ \mathrm{H}](\mathrm{C}) \mathrm{OC}(=\mathrm{O})[\mathrm{C} @ \mathrm{H}](\mathrm{C}(\mathrm{C}) \mathrm{C}) \mathrm{N}(\mathrm{C}) \mathrm{C}(=\mathrm{O}) \mathrm{CN}(\mathrm{C}) \mathrm{C}(=\mathrm{O})[\mathrm{C} @ @ \mathrm{H}] 3 \mathrm{CCCN} 3 \mathrm{C}(=\mathrm{O})[\mathrm{C} @ \mathrm{H}](\mathrm{NC} 2=\mathrm{O})$

$412 \mathrm{C}(\mathrm{C}) \mathrm{C})[\mathrm{C} @ \mathrm{H}](\mathrm{C}) \mathrm{OC}(=\mathrm{O})[\mathrm{C} @ \mathrm{H}](\mathrm{C}(\mathrm{C}) \mathrm{C}) \mathrm{N}(\mathrm{C}) \mathrm{C}(=\mathrm{O}) \mathrm{CN}(\mathrm{C}) \mathrm{C}(=\mathrm{O})[\mathrm{C} @ @ \mathrm{H}] 2 \mathrm{CCCN} 2 \mathrm{C} 1=\mathrm{O}$

$413 \mathrm{O}=\mathrm{C} 1 \mathrm{C}=\mathrm{C} 2 \mathrm{OC} 3=\mathrm{CC} 4=\mathrm{C}(\mathrm{CCCC} 4) \mathrm{C}=\mathrm{C} 3 \mathrm{~N}=\mathrm{C} 2 \mathrm{C} 2=\mathrm{CC}=\mathrm{CN}=\mathrm{C} 12$

$414 \mathrm{O}=\mathrm{C} 1 \mathrm{C}=\mathrm{C} 2 \mathrm{OC} 3=\mathrm{CC} 4=\mathrm{C}(\mathrm{C}=\mathrm{CC}=\mathrm{C} 4) \mathrm{C}=\mathrm{C} 3 \mathrm{~N}=\mathrm{C} 2 \mathrm{C} 2=\mathrm{CC}=\mathrm{CN}=\mathrm{C} 12$

$415 \mathrm{CC} 1=\mathrm{CC}=\mathrm{C} 2 \mathrm{OC} 3=\mathrm{CC}(=\mathrm{O}) \mathrm{C} 4=\mathrm{NC}=\mathrm{CC}=\mathrm{C} 4 \mathrm{C} 3=\mathrm{NC} 2=\mathrm{C} 1 \mathrm{C}$

$416 \mathrm{CC} 1=\mathrm{CC}(\mathrm{C})=\mathrm{C} 2 \mathrm{~N}=\mathrm{C} 3 \mathrm{C}(\mathrm{OC} 2=\mathrm{C} 1)=\mathrm{CC}(=\mathrm{O}) \mathrm{C} 1=\mathrm{NC}=\mathrm{CC}=\mathrm{C} 31$

$417 \mathrm{O}=\mathrm{C} 1 \mathrm{C}=\mathrm{C} 2 \mathrm{OC} 3=\mathrm{CC}=\mathrm{C} 4 \mathrm{C}=\mathrm{CC}=\mathrm{CC} 4=\mathrm{C} 3 \mathrm{~N}=\mathrm{C} 2 \mathrm{C} 2=\mathrm{CC}=\mathrm{CN}=\mathrm{C} 12$

$418 \mathrm{CC}(\mathrm{C})=\mathrm{CCC} \mid \mathrm{C}(\mathrm{C})=\mathrm{ClCCC}(\mathrm{CC}(=\mathrm{O}) \mathrm{C} 1=\mathrm{CC}(\mathrm{O})=\mathrm{CC}=\mathrm{C} 1 \mathrm{O}) \mathrm{C}(\mathrm{O})=\mathrm{O}$

$419 \mathrm{CC}(\mathrm{C})=\mathrm{CCCC} 1=\mathrm{CC}(\mathrm{OC} 1=\mathrm{O}) \mathrm{C} 1=\mathrm{CC}(\mathrm{O})=\mathrm{CC}=\mathrm{C} 1 \mathrm{O}$

$420 \quad \operatorname{COC} 1(\mathrm{OC}(=\mathrm{O}) \mathrm{C}(\mathrm{CClC}=\mathrm{C}(\mathrm{C}) \mathrm{CCC}=\mathrm{C}(\mathrm{C}) \mathrm{C})=\mathrm{C} 1) \mathrm{C} 1=\mathrm{CC}(\mathrm{O})=\mathrm{CC}=\mathrm{C} 1 \mathrm{O}$

$421 \mathrm{C}=\mathrm{CC} 1=\mathrm{CN}(\mathrm{ClC}=\mathrm{C} 2 / \mathrm{OC}(=\mathrm{O}) \mathrm{C}(\mathrm{OCC} 3=\mathrm{CC}=\mathrm{CC}=\mathrm{C} 3)=\mathrm{C} 2 \mathrm{OCC} 2=\mathrm{CC}=\mathrm{CC}=\mathrm{C} 2) \mathrm{C}(=\mathrm{O}) \mathrm{NC} 1=\mathrm{O}$

$422 \mathrm{OC} 1=\mathrm{C}(\mathrm{O}) \backslash \mathrm{C}(\mathrm{OC} 1=\mathrm{O})=\mathrm{C} \backslash \mathrm{CN} 1 \mathrm{C}=\mathrm{C}(\mathrm{C} 2=\mathrm{CC}=\mathrm{CS} 2) \mathrm{C}(=\mathrm{O}) \mathrm{NC} 1=\mathrm{O}$

$423 \mathrm{BrC} 1=\mathrm{CC}=\mathrm{C}(\mathrm{O} 1) \mathrm{C} 1=\mathrm{CN}(\mathrm{ClC}=\mathrm{C} 2 / \mathrm{OC}(=\mathrm{O}) \mathrm{C}(\mathrm{OCC} 3=\mathrm{CC}=\mathrm{CC}=\mathrm{C} 3)=\mathrm{C} 2 \mathrm{OCC} 2=\mathrm{CC}=\mathrm{CC}=\mathrm{C} 2) \mathrm{C}(=\mathrm{O}) \mathrm{NC} 1=\mathrm{O}$

$424 \mathrm{O}=\mathrm{C} 1 \mathrm{O} \backslash \mathrm{C}(=\mathrm{C} / \mathrm{CN} 2 \mathrm{C}=\mathrm{C}(\mathrm{C}(=\mathrm{O}) \mathrm{NC} 2=\mathrm{O}) \mathrm{C} 2=\mathrm{CC}=\mathrm{CC}=\mathrm{C} 2) \mathrm{C}(\mathrm{OCC} 2=\mathrm{CC}=\mathrm{CC}=\mathrm{C} 2)=\mathrm{C} 1 \mathrm{OCC} 1=\mathrm{CC}=\mathrm{CC}=\mathrm{C} 1$

$425 \mathrm{Br} \mid \mathrm{C}=\mathrm{ClC} 1=\mathrm{C}(\mathrm{Br}) \mathrm{N}(\mathrm{ClC}(\mathrm{Br})=\mathrm{C} 2 \backslash \mathrm{OC}(=\mathrm{O}) \mathrm{C}(\mathrm{OCC} 3=\mathrm{CC}=\mathrm{CC}=\mathrm{C} 3)=\mathrm{C} 2 \mathrm{OCC} 2=\mathrm{CC}=\mathrm{CC}=\mathrm{C} 2) \mathrm{C}(=\mathrm{O}) \mathrm{NC} 1=\mathrm{O}$

$426 \mathrm{O}=\mathrm{C} 1 \mathrm{O} \backslash \mathrm{C}(=\mathrm{C} / \mathrm{CN} 2 \mathrm{C}=\mathrm{C}(\mathrm{C} 3=\mathrm{CC}=\mathrm{CO} 3) \mathrm{C}(=\mathrm{O}) \mathrm{NC} 2=\mathrm{O}) \mathrm{C}(\mathrm{OCC} 2=\mathrm{CC}=\mathrm{CC}=\mathrm{C} 2)=\mathrm{C} 1 \mathrm{OCC} 1=\mathrm{CC}=\mathrm{CC}=\mathrm{C} 1$

$427 \mathrm{OC} 1=\mathrm{C}(\mathrm{O}) \backslash \mathrm{C}(\mathrm{OC} 1=\mathrm{O})=\mathrm{C} / \mathrm{CN} 1 \mathrm{C}=\mathrm{C}(\mathrm{C} \# \mathrm{CC} 2=\mathrm{CC}=\mathrm{CC}=\mathrm{C} 2) \mathrm{C}(=\mathrm{O}) \mathrm{NC} 1=\mathrm{O}$

$428 \mathrm{OC} 1=\mathrm{C}(\mathrm{O}) \backslash \mathrm{C}(\mathrm{OC} 1=\mathrm{O})=\mathrm{C} \backslash \mathrm{CN} 1 \mathrm{C}=\mathrm{C}(\mathrm{C} \# \mathrm{C}) \mathrm{C}(=\mathrm{O}) \mathrm{NC} 1=\mathrm{O}$

$429 \quad \mathrm{OC} 1=\mathrm{C}(\mathrm{O}) \mathrm{C}(\mathrm{OC} 1=\mathrm{O})=\mathrm{C} \backslash \mathrm{CN} 1 \mathrm{C}=\mathrm{C}(\mathrm{C}(=\mathrm{O}) \mathrm{NC} 1=\mathrm{O}) \mathrm{C} 1=\mathrm{CC}=\mathrm{CC}=\mathrm{C} 1$

$430 \mathrm{CC} \# \mathrm{CC} 1=\mathrm{CN}(\mathrm{ClC}=\mathrm{C} 2 / \mathrm{OC}(=\mathrm{O}) \mathrm{C}(\mathrm{OCC} 3=\mathrm{CC}=\mathrm{CC}=\mathrm{C} 3)=\mathrm{C} 2 \mathrm{OCC} 2=\mathrm{CC}=\mathrm{CC}=\mathrm{C} 2) \mathrm{C}(=\mathrm{O}) \mathrm{NC} 1=\mathrm{O}$

$431 \mathrm{CC}(\mathrm{C})=\mathrm{CCC} 1=\mathrm{CN}(\mathrm{ClC}=\mathrm{C} 2 / \mathrm{OC}(=\mathrm{O}) \mathrm{C}(\mathrm{OCC} 3=\mathrm{CC}=\mathrm{CC}=\mathrm{C} 3)=\mathrm{C} 2 \mathrm{OCC} 2=\mathrm{CC}=\mathrm{CC}=\mathrm{C} 2) \mathrm{C}(=\mathrm{O}) \mathrm{NC} 1=\mathrm{O}$

$432 \mathrm{OC} 1=\mathrm{C}(\mathrm{O}) \backslash \mathrm{C}(\mathrm{OC} 1=\mathrm{O})=\mathrm{C} \mid \mathrm{CN} 1 \mathrm{C}=\mathrm{C}(\mathrm{C}=\mathrm{C}) \mathrm{C}(=\mathrm{O}) \mathrm{NC} 1=\mathrm{O}$

$433 \mathrm{Br} \mid \mathrm{C}=\mathrm{C} \backslash \mathrm{C} 1=\mathrm{CN}(\mathrm{ClC}=\mathrm{C} 2 / \mathrm{OC}(=\mathrm{O}) \mathrm{C}(\mathrm{OCC} 3=\mathrm{CC}=\mathrm{CC}=\mathrm{C} 3)=\mathrm{C} 2 \mathrm{OCC} 2=\mathrm{CC}=\mathrm{CC}=\mathrm{C} 2) \mathrm{C}(=\mathrm{O}) \mathrm{NC} 1=\mathrm{O}$

$434 \mathrm{OC} 1=\mathrm{C}(\mathrm{O}) \backslash \mathrm{C}(\mathrm{OC} 1=\mathrm{O})=\mathrm{C} \backslash \mathrm{CN} 1 \mathrm{C}=\mathrm{C}(\mathrm{C} 2=\mathrm{CC}=\mathrm{CO} 2) \mathrm{C}(=\mathrm{O}) \mathrm{NC} 1=\mathrm{O}$

$435 \mathrm{O}=\mathrm{C} 1 \mathrm{O} \backslash \mathrm{C}(=\mathrm{C} / \mathrm{CN} 2 \mathrm{C}=\mathrm{C}(\mathrm{C} \# \mathrm{C}) \mathrm{C}(=\mathrm{O}) \mathrm{NC} 2=\mathrm{O}) \mathrm{C}(\mathrm{OCC} 2=\mathrm{CC}=\mathrm{CC}=\mathrm{C} 2)=\mathrm{C} 1 \mathrm{OCC} 1=\mathrm{CC}=\mathrm{CC}=\mathrm{C} 1$

$436 \mathrm{C}=\mathrm{C}=\mathrm{CC} 1=\mathrm{CN}(\mathrm{ClC}=\mathrm{C} 2 / \mathrm{OC}(=\mathrm{O}) \mathrm{C}(\mathrm{OCC} 3=\mathrm{CC}=\mathrm{CC}=\mathrm{C} 3)=\mathrm{C} 2 \mathrm{OCC} 2=\mathrm{CC}=\mathrm{CC}=\mathrm{C} 2) \mathrm{C}(=\mathrm{O}) \mathrm{NC} 1=\mathrm{O}$

$437 \mathrm{O}=\mathrm{C} 1 \mathrm{O} \mid \mathrm{C}(=\mathrm{C} / \mathrm{CN} 2 \mathrm{C}=\mathrm{C}(\mathrm{C} 3=\mathrm{CC}=\mathrm{CS} 3) \mathrm{C}(=\mathrm{O}) \mathrm{NC} 2=\mathrm{O}) \mathrm{C}(\mathrm{OCC} 2=\mathrm{CC}=\mathrm{CC}=\mathrm{C} 2)=\mathrm{C} 1 \mathrm{OCC} 1=\mathrm{CC}=\mathrm{CC}=\mathrm{C}$

$438 \mathrm{ClC} 1=\mathrm{CC}=\mathrm{C}(\mathrm{O} 1) \mathrm{C} 1=\mathrm{CN}(\mathrm{ClC}=\mathrm{C} 2 / \mathrm{OC}(=\mathrm{O}) \mathrm{C}(\mathrm{OCC} 3=\mathrm{CC}=\mathrm{CC}=\mathrm{C} 3)=\mathrm{C} 2 \mathrm{OCC} 2=\mathrm{CC}=\mathrm{CC}=\mathrm{C} 2) \mathrm{C}(=\mathrm{O}) \mathrm{NC} 1=\mathrm{O}$

]. $\mathrm{C}(\mathrm{C} / \mathrm{C} 1=\mathrm{C} / \mathrm{NC} 2=\mathrm{CC}=\mathrm{CC}=\mathrm{C} 2 \backslash \mathrm{N}=\mathrm{C} \backslash \mathrm{C}(\backslash \mathrm{CCC}[\mathrm{N}+] 2=\mathrm{CC}=\mathrm{C}(\mathrm{C}=\mathrm{C} 2) \mathrm{C} 2=\mathrm{CC}=\mathrm{NC}=\mathrm{C} 2)=\mathrm{C} / \mathrm{NC} 2=\mathrm{CC}=\mathrm{CC}=\mathrm{C} 2 \backslash \mathrm{N}=$

$441 \mathrm{C} 1) \mathrm{C}[\mathrm{N}+] 1=\mathrm{CC}=\mathrm{C}(\mathrm{C}=\mathrm{C} 1) \mathrm{C} 1=\mathrm{CC}=\mathrm{NC}=\mathrm{C} 1$

$[\mathrm{Br}-\mathrm{B}][\mathrm{Br}-$

].C $(\mathrm{C} / \mathrm{C} 1=\mathrm{C} / \mathrm{NC} 2=\mathrm{CC}=\mathrm{CC}=\mathrm{C} 2 \backslash \mathrm{N}=\mathrm{ClC}(\backslash \mathrm{CCC}[\mathrm{N}+] 2=\mathrm{CC}=\mathrm{CC}=\mathrm{C} 2)=\mathrm{C} / \mathrm{NC} 2=\mathrm{CC}=\mathrm{CC}=\mathrm{C} 2 \backslash \mathrm{N}=\mathrm{C} 1) \mathrm{C}[\mathrm{N}+] 1=\mathrm{CC}=$

$[\mathrm{Br}-] .[\mathrm{Br}-$

]. $\mathrm{C}[\mathrm{N}+] 1=\mathrm{CC}=\mathrm{C}(\mathrm{CCC}(=\mathrm{O}) \mathrm{OCCC} / \mathrm{C} 2=\mathrm{C} / \mathrm{NC} 3=\mathrm{CC}=\mathrm{CC}=\mathrm{C} 3 \backslash \mathrm{N}=\mathrm{ClC}(\backslash \mathrm{CCCOC}(=\mathrm{O}) \mathrm{CCC} 3=\mathrm{CC}=[\mathrm{N}+](\mathrm{C}) \mathrm{C}=\mathrm{C} 3)$

$443=\mathrm{C} / \mathrm{NC} 3=\mathrm{CC}=\mathrm{CC}=\mathrm{C} 3 \backslash \mathrm{N}=\mathrm{C} \backslash 2) \mathrm{C}=\mathrm{C} 1$

$444 \mathrm{COC} 1=\mathrm{CC}=\mathrm{C}(\mathrm{C}=\mathrm{C} 1 \mathrm{OC}(\mathrm{C}) \mathrm{C}) \mathrm{C} 1=\mathrm{C}(\backslash \mathrm{C}(=\mathrm{N} / \mathrm{O}) \mathrm{C}(\mathrm{O}) \mathrm{C} 1) \mathrm{C} 1=\mathrm{CC}(\mathrm{OC})=\mathrm{C}(\mathrm{OC}) \mathrm{C}(\mathrm{OC})=\mathrm{C} 1$

$445 \mathrm{COC} 1=\mathrm{CC}=\mathrm{C}(\mathrm{C}=\mathrm{C} 1) \mathrm{C} 1=\mathrm{C}(\mathrm{C}(=\mathrm{O}) \mathrm{C}(\mathrm{C} 1) \mathrm{O}[\mathrm{SiH} 2] \mathrm{C}(\mathrm{C})(\mathrm{C}) \mathrm{C}) \mathrm{C} 1=\mathrm{CC}(\mathrm{OC})=\mathrm{C}(\mathrm{OC}) \mathrm{C}(\mathrm{OC})=\mathrm{C} 1$

$446 \quad \mathrm{COC} 1=\mathrm{CC}=\mathrm{C}(\mathrm{C}=\mathrm{C} 1 \mathrm{~F}) \mathrm{C} 1=\mathrm{C}(\mathrm{C}(=\mathrm{O}) \mathrm{C}(\mathrm{C} 1) \mathrm{OC}(\mathrm{C})=\mathrm{O}) \mathrm{C} 1=\mathrm{CC}(\mathrm{OC})=\mathrm{C}(\mathrm{OC}) \mathrm{C}(\mathrm{OC})=\mathrm{C} 1$

$447 \quad \mathrm{COC} 1=\mathrm{CC}=\mathrm{C}(\mathrm{C}=\mathrm{C} 1) \mathrm{C} 1=\mathrm{C}(\mathrm{C}(\mathrm{CC} 1 \mathrm{OC}(\mathrm{C})=\mathrm{O})=\mathrm{N} / \mathrm{O}) \mathrm{C} 1=\mathrm{CC}(\mathrm{OC})=\mathrm{C}(\mathrm{OC}) \mathrm{C}(\mathrm{OC})=\mathrm{C} 1$

$448 \quad \mathrm{COC} 1=\mathrm{CC}=\mathrm{C}(\mathrm{C}=\mathrm{C} 1[\mathrm{~N}+]([\mathrm{O}-])=\mathrm{O}) \mathrm{C} 1=\mathrm{C}(\mathrm{CC}(\mathrm{O}) \mathrm{C} 1=\mathrm{O}) \mathrm{C} 1=\mathrm{CC}(\mathrm{OC})=\mathrm{C}(\mathrm{OC}) \mathrm{C}(\mathrm{OC})=\mathrm{C} 1$

$449 \mathrm{COC} 1=\mathrm{CC}=\mathrm{C}(\mathrm{C}=\mathrm{C} 1) \mathrm{C} 1=\mathrm{C}(\backslash \mathrm{C}(=\mathrm{N} / \mathrm{O}) \mathrm{C}(\mathrm{C} 1) \mathrm{OC}(\mathrm{C})=\mathrm{O}) \mathrm{C} 1=\mathrm{CC}(\mathrm{OC})=\mathrm{C}(\mathrm{OC}) \mathrm{C}(\mathrm{OC})=\mathrm{C} 1$

$450 \quad \mathrm{COC} 1=\mathrm{CC}=\mathrm{C}(\mathrm{C}=\mathrm{C} 1) \mathrm{C} 1=\mathrm{C}(\mathrm{C}(=\mathrm{O}) \mathrm{C}(\mathrm{C} 1) \mathrm{OC}(\mathrm{C})=\mathrm{O}) \mathrm{C} 1=\mathrm{CC}(\mathrm{OC})=\mathrm{C}(\mathrm{OC}) \mathrm{C}(\mathrm{OC})=\mathrm{C} 1$

$451 \mathrm{COC} 1=\mathrm{CC}=\mathrm{C}(\mathrm{C}=\mathrm{C} 1 \mathrm{Cl}) \mathrm{C} 1=\mathrm{C}(\backslash \mathrm{C}(=\mathrm{N} / \mathrm{O}) \mathrm{C}(\mathrm{O}) \mathrm{C} 1) \mathrm{C} 1=\mathrm{CC}(\mathrm{OC})=\mathrm{C}(\mathrm{OC}) \mathrm{C}(\mathrm{OC})=\mathrm{C} 1$

$452 \mathrm{COC} 1=\mathrm{CC}(=\mathrm{CC}(\mathrm{OC})=\mathrm{C} 1 \mathrm{OC}) \mathrm{C} 1=\mathrm{C}(\mathrm{C}(\mathrm{CC} 1=\mathrm{O}) \mathrm{O}[\mathrm{SiH} 2] \mathrm{C}(\mathrm{C})(\mathrm{C}) \mathrm{C}) \mathrm{C} 1=\mathrm{CC}=\mathrm{CC}=\mathrm{C} 1$

$453 \quad[\mathrm{Na}+] . \mathrm{COC} 1=\mathrm{CC}=\mathrm{C}(\mathrm{C}=\mathrm{C} 1 \mathrm{C}([\mathrm{O}-])=\mathrm{O}) \mathrm{C} 1=\mathrm{C}(\mathrm{C}(=\mathrm{O}) \mathrm{CC} 1 \mathrm{C}) \mathrm{C} 1=\mathrm{CC}(\mathrm{OC})=\mathrm{C}(\mathrm{OC}) \mathrm{C}(\mathrm{OC})=\mathrm{C} 1$

$454 \mathrm{COC} 1=\mathrm{CC}=\mathrm{C}(\mathrm{C}=\mathrm{C} 1 \mathrm{O}) \mathrm{C} 1=\mathrm{C}(\mathrm{C}(\mathrm{CC} 1 \mathrm{OC}(\mathrm{C})=\mathrm{O})=\mathrm{N} / \mathrm{O}) \mathrm{C} 1=\mathrm{CC}(\mathrm{OC})=\mathrm{C}(\mathrm{OC}) \mathrm{C}(\mathrm{OC})=\mathrm{C} 1$

$455 \quad \mathrm{COC} 1=\mathrm{CC}=\mathrm{C}(\mathrm{C}=\mathrm{C} 1 \mathrm{~N}) \mathrm{C} 1=\mathrm{C}(\mathrm{CC}(\mathrm{O}) \mathrm{C} 1=\mathrm{O}) \mathrm{C} 1=\mathrm{CC}(\mathrm{OC})=\mathrm{C}(\mathrm{OC}) \mathrm{C}(\mathrm{OC})=\mathrm{C} 1$

$456 \quad \mathrm{COC} 1=\mathrm{CC}=\mathrm{C}(\mathrm{C}=\mathrm{C} 1 \mathrm{O}) \mathrm{C} 1=\mathrm{C}(\mathrm{C}(=\mathrm{O}) \mathrm{C}(\mathrm{C} 1) \mathrm{OC}(\mathrm{C})=\mathrm{O}) \mathrm{C} 1=\mathrm{CC}(\mathrm{OC})=\mathrm{C}(\mathrm{OC}) \mathrm{C}(\mathrm{OC})=\mathrm{C} 1$

$457 \mathrm{COC} 1=\mathrm{CC}=\mathrm{C}(\mathrm{C}=\mathrm{C} 1 \mathrm{Cl}) \mathrm{C} 1=\mathrm{C}(\mathrm{C}(=\mathrm{O}) \mathrm{C}(\mathrm{O}) \mathrm{C} 1) \mathrm{C} 1=\mathrm{CC}(\mathrm{OC})=\mathrm{C}(\mathrm{OC}) \mathrm{C}(\mathrm{OC})=\mathrm{C} 1$

$458 \quad \mathrm{COC} 1=\mathrm{CC}=\mathrm{C}(\mathrm{OC}) \mathrm{C}(=\mathrm{C} 1) \mathrm{C} 1=\mathrm{C}(\mathrm{C}(=\mathrm{O}) \mathrm{CC} 1 \mathrm{O}) \mathrm{C} 1=\mathrm{CC}(\mathrm{OC})=\mathrm{C}(\mathrm{OC}) \mathrm{C}(\mathrm{OC})=\mathrm{C} 1$

$459 \quad \mathrm{COC} 1=\mathrm{CC}=\mathrm{C}(\mathrm{C}=\mathrm{C} 1 \mathrm{Cl}) \mathrm{C} 1=\mathrm{C}(\mathrm{C}(=\mathrm{O}) \mathrm{C}(\mathrm{C} 1) \mathrm{OC}(\mathrm{C})=\mathrm{O}) \mathrm{C} 1=\mathrm{CC}(\mathrm{OC})=\mathrm{C}(\mathrm{OC}) \mathrm{C}(\mathrm{OC})=\mathrm{C} 1$

$460 \quad \mathrm{COC} 1=\mathrm{CC}=\mathrm{C}(\mathrm{C}=\mathrm{C} 1 \mathrm{OC}(\mathrm{C}) \mathrm{C}) \mathrm{C} 1=\mathrm{C}(\mathrm{C}(=\mathrm{O}) \mathrm{C}(\mathrm{O}) \mathrm{C} 1) \mathrm{C} 1=\mathrm{CC}(\mathrm{OC})=\mathrm{C}(\mathrm{OC}) \mathrm{C}(\mathrm{OC})=\mathrm{C} 1$

$461 \quad \mathrm{COC} 1=\mathrm{CC}(=\mathrm{CC}(\mathrm{OC})=\mathrm{C} 1 \mathrm{OC}) \mathrm{C} 1=\mathrm{C}(\mathrm{C}(\mathrm{CC} 1=\mathrm{O}) \mathrm{O}[\mathrm{SiH} 2] \mathrm{C}(\mathrm{C})(\mathrm{C}) \mathrm{C}) \mathrm{C} 1=\mathrm{CC}(\mathrm{C})=\mathrm{C}(\mathrm{OC}) \mathrm{C}(\mathrm{C})=\mathrm{C} 1$

$462 \mathrm{COC} 1=\mathrm{CC}=\mathrm{C}(\mathrm{C}=\mathrm{C} 1 \mathrm{O}) \mathrm{C} 1=\mathrm{C}(\mathrm{C}(=\mathrm{O}) \mathrm{CC} 1 \mathrm{O}) \mathrm{C} 1=\mathrm{CC}(\mathrm{OC})=\mathrm{C}(\mathrm{OC}) \mathrm{C}(\mathrm{OC})=\mathrm{C} 1$

$463 \mathrm{COCOC} 1=\mathrm{CC}(=\mathrm{CC}=\mathrm{C} 1 \mathrm{OC}) \mathrm{C} 1=\mathrm{C}(\backslash \mathrm{C}(\mathrm{CC} 1 \mathrm{O}[\mathrm{SiH} 2] \mathrm{C}(\mathrm{C})(\mathrm{C}) \mathrm{C})=\mathrm{N} / \mathrm{O}) \mathrm{C} 1=\mathrm{CC}(\mathrm{OC})=\mathrm{C}(\mathrm{OC}) \mathrm{C}(\mathrm{OC})=\mathrm{C}$

$464 \mathrm{COC} 1=\mathrm{CC}=\mathrm{C}(\mathrm{C}=\mathrm{C} 1 \mathrm{~F}) \mathrm{C} 1=\mathrm{C}(\mathrm{C}(=\mathrm{O}) \mathrm{C}(\mathrm{O}) \mathrm{C} 1) \mathrm{C} 1=\mathrm{CC}(\mathrm{OC})=\mathrm{C}(\mathrm{OC}) \mathrm{C}(\mathrm{OC})=\mathrm{C} 1$

$465 \quad \mathrm{COC} 1=\mathrm{CC}=\mathrm{C}(\mathrm{C}=\mathrm{C} 1 \mathrm{OCC}=\mathrm{C}) \mathrm{C} 1=\mathrm{C}(\mathrm{C}(=\mathrm{O}) \mathrm{C}(\mathrm{O}) \mathrm{C} 1) \mathrm{C} 1=\mathrm{CC}(\mathrm{OC})=\mathrm{C}(\mathrm{OC}) \mathrm{C}(\mathrm{OC})=\mathrm{C} 1$

$466 \quad \mathrm{COC} 1=\mathrm{CC}(=\mathrm{CC}(\mathrm{OC})=\mathrm{C} 1 \mathrm{OC}) \mathrm{C} 1=\mathrm{C}(\mathrm{CC}(\mathrm{O}[\mathrm{SiH} 2] \mathrm{C}(\mathrm{C})(\mathrm{C}) \mathrm{C}) \mathrm{C} 1=\mathrm{O}) \mathrm{C} 1=\mathrm{CC}=\mathrm{C}(\mathrm{SC}) \mathrm{C}=\mathrm{C} 1$

$467 \mathrm{COC} 1=\mathrm{CC}(=\mathrm{CC}(\mathrm{OC})=\mathrm{C} 1 \mathrm{OC}) \mathrm{C} 1=\mathrm{C}(\mathrm{C}(=\mathrm{O}) \mathrm{C}(\mathrm{C} 1) \mathrm{O}[\mathrm{SiH} 2] \mathrm{C}(\mathrm{C})(\mathrm{C}) \mathrm{C}) \mathrm{C} 1=\mathrm{CC}=\mathrm{C}(\mathrm{OC}) \mathrm{C}(=\mathrm{C} 1)[\mathrm{N}+]([\mathrm{O}-])=\mathrm{O}$

$468 \quad \mathrm{COC} 1=\mathrm{CC}=\mathrm{C}(\mathrm{C}=\mathrm{C} 1 \mathrm{~F}) \mathrm{C} 1=\mathrm{C}(\mathrm{C}(=\mathrm{N} / \mathrm{O}) \mathrm{C}(\mathrm{O}) \mathrm{C} 1) \mathrm{C} 1=\mathrm{CC}(\mathrm{OC})=\mathrm{C}(\mathrm{OC}) \mathrm{C}(\mathrm{OC})=\mathrm{C} 1$

$469 \mathrm{COC} 1=\mathrm{CC}=\mathrm{C}(\mathrm{C}=\mathrm{C} 1) \mathrm{C} 1=\mathrm{C}(\mathrm{C}(=\mathrm{O}) \mathrm{C}(\mathrm{O}) \mathrm{C} 1) \mathrm{C} 1=\mathrm{CC}(\mathrm{OC})=\mathrm{C}(\mathrm{OC}) \mathrm{C}(\mathrm{OC})=\mathrm{C} 1$

$470 \mathrm{COC} 1=\mathrm{CC}=\mathrm{C}(\mathrm{C}=\mathrm{C} 1 \mathrm{OC}(\mathrm{C}) \mathrm{C}) \mathrm{C} 1=\mathrm{C}(\mathrm{C}(=\mathrm{O}) \mathrm{C}(\mathrm{C} 1) \mathrm{OC}(\mathrm{C})=\mathrm{O}) \mathrm{C} 1=\mathrm{CC}(\mathrm{OC})=\mathrm{C}(\mathrm{OC}) \mathrm{C}(\mathrm{OC})=\mathrm{C} 1$

$471 \mathrm{COC} 1=\mathrm{CC}=\mathrm{C}(\mathrm{C}=\mathrm{C} 1 \mathrm{OCC}=\mathrm{C}) \mathrm{C} 1=\mathrm{C}(\mathrm{CC}(=\mathrm{N} / \mathrm{O}) \mathrm{C}(\mathrm{O}) \mathrm{C} 1) \mathrm{C} 1=\mathrm{CC}(\mathrm{OC})=\mathrm{C}(\mathrm{OC}) \mathrm{C}(\mathrm{OC})=\mathrm{C} 1$

$472 \mathrm{COC} 1=\mathrm{CC}=\mathrm{C}(\mathrm{C}=\mathrm{C} 1 \mathrm{Cl}) \mathrm{C} 1=\mathrm{C}(\mathrm{C}(=\mathrm{N} / \mathrm{O}) \mathrm{C}(\mathrm{C} 1) \mathrm{OC}(\mathrm{C})=\mathrm{O}) \mathrm{C} 1=\mathrm{CC}(\mathrm{OC})=\mathrm{C}(\mathrm{OC}) \mathrm{C}(\mathrm{OC})=\mathrm{C} 1$

$473 \mathrm{COC} 1=\mathrm{CC}(=\mathrm{CC}(\mathrm{OC})=\mathrm{C} 1 \mathrm{OC}) \mathrm{C} 1=\mathrm{C}(\mathrm{C}(\mathrm{O}) \mathrm{CC} 1=\mathrm{O}) \mathrm{C} 1=\mathrm{CC}(\mathrm{C})=\mathrm{C}(\mathrm{OC}) \mathrm{C}(\mathrm{C})=\mathrm{C} 1$

$474 \mathrm{COC} 1=\mathrm{CC}=\mathrm{C}(\mathrm{OC}) \mathrm{C}(=\mathrm{C} 1) \mathrm{C} 1=\mathrm{C}(\backslash \mathrm{C}(\mathrm{CC} 1 \mathrm{O}[\mathrm{SiH} 2] \mathrm{C}(\mathrm{C})(\mathrm{C}) \mathrm{C})=\mathrm{N} / \mathrm{O}) \mathrm{C} 1=\mathrm{CC}(\mathrm{OC})=\mathrm{C}(\mathrm{OC}) \mathrm{C}(\mathrm{OC})=\mathrm{C}$

$475 \quad \mathrm{COC} 1=\mathrm{CC}=\mathrm{C}(\mathrm{C}=\mathrm{C} 1) \mathrm{C} 1=\mathrm{C}(\mathrm{C}(=\mathrm{O}) \mathrm{CC} 1 \mathrm{O}) \mathrm{C} 1=\mathrm{CC}(\mathrm{OC})=\mathrm{C}(\mathrm{OC}) \mathrm{C}(\mathrm{OC})=\mathrm{C} 1$

$476 \mathrm{COC} 1=\mathrm{CC}=\mathrm{C}(\mathrm{C}=\mathrm{C} 1) \mathrm{C} 1=\mathrm{C}(\mathrm{C}(\mathrm{CC} 1 \mathrm{OC}(\mathrm{C})=\mathrm{O})=\mathrm{N} / \mathrm{O}) \mathrm{C} 1=\mathrm{CC}(\mathrm{OC})=\mathrm{C}(\mathrm{OC}) \mathrm{C}(\mathrm{OC})=\mathrm{C} 1$

$477 \mathrm{COC} 1=\mathrm{CC}=\mathrm{C}(\mathrm{C}=\mathrm{C} 1 \mathrm{NC}(=\mathrm{O}) \mathrm{C} 1=\mathrm{CC}=\mathrm{CC}=\mathrm{C} 1) \mathrm{C} 1=\mathrm{C}(\mathrm{C}(=\mathrm{O}) \mathrm{C}(\mathrm{O}) \mathrm{C} 1) \mathrm{C} 1=\mathrm{CC}(\mathrm{OC})=\mathrm{C}(\mathrm{OC}) \mathrm{C}(\mathrm{OC})=\mathrm{C} 1$ 


\begin{tabular}{|c|c|c|}
\hline NAME & SMILE & $\lg (\mathbf{1} / \mathbf{I C 5 0})$ \\
\hline 478 & $\mathrm{COC} 1=\mathrm{CC}(=\mathrm{CC}(\mathrm{OC})=\mathrm{C} 1 \mathrm{OC}) \mathrm{C} 1=\mathrm{C}(\mathrm{C}(\mathrm{CC} 1=\mathrm{O}) \mathrm{OC}(\mathrm{C})=\mathrm{O}) \mathrm{C} 1=\mathrm{CC}(\mathrm{C})=\mathrm{C}(\mathrm{OC}) \mathrm{C}(\mathrm{C})=\mathrm{C} 1$ & 3,64 \\
\hline 479 & $\mathrm{COC} 1=\mathrm{CC}=\mathrm{C}(\mathrm{C}=\mathrm{C} 1 \mathrm{O}) \mathrm{C} 1=\mathrm{C}(1 \mathrm{C}(=\mathrm{N} / \mathrm{O}) \mathrm{C}(\mathrm{O}) \mathrm{C} 1) \mathrm{C} 1=\mathrm{CC}(\mathrm{OC})=\mathrm{C}(\mathrm{OC}) \mathrm{C}(\mathrm{OC})=\mathrm{C} 1$ & 4,3 \\
\hline 480 & $\mathrm{COC} 1=\mathrm{CC}=\mathrm{C}(\mathrm{C}=\mathrm{C} 1 \mathrm{O}) \mathrm{C} 1=\mathrm{C}(\mathrm{C}(=\mathrm{O}) \mathrm{CC} 1 \mathrm{O}[\mathrm{SiH} 2] \mathrm{C}(\mathrm{C})(\mathrm{C}) \mathrm{C}) \mathrm{C} 1=\mathrm{CC}(\mathrm{OC})=\mathrm{C}(\mathrm{OC}) \mathrm{C}(\mathrm{OC})=\mathrm{C} 1$ & 4,51 \\
\hline 481 & $\mathrm{COC} 1=\mathrm{CC}=\mathrm{C}(\mathrm{C}=\mathrm{C} 1 \mathrm{OC}(\mathrm{C}) \mathrm{C}) \mathrm{C} 1=\mathrm{C}(\mathrm{C}(=\mathrm{O}) \mathrm{C}(\mathrm{C} 1) \mathrm{O}[\mathrm{SiH} 2] \mathrm{C}(\mathrm{C})(\mathrm{C}) \mathrm{C}) \mathrm{C} 1=\mathrm{CC}(\mathrm{OC})=\mathrm{C}(\mathrm{OC}) \mathrm{C}(\mathrm{OC})=\mathrm{C} 1$ & 4,86 \\
\hline 482 & $\mathrm{COC} 1=\mathrm{CC}=\mathrm{C}(\mathrm{C}=\mathrm{C} 1 \mathrm{OC}) \mathrm{C} 1=\mathrm{C}(\mathrm{C}(=\mathrm{O}) \mathrm{CC} 1 \mathrm{O}[\mathrm{SiH} 2] \mathrm{C}(\mathrm{C})(\mathrm{C}) \mathrm{C}) \mathrm{C} 1=\mathrm{CC}(\mathrm{OC})=\mathrm{C}(\mathrm{OC}) \mathrm{C}(\mathrm{OC})=\mathrm{C} 1$ & 3,69 \\
\hline 483 & $\mathrm{COC} 1=\mathrm{CC}=\mathrm{C}(\mathrm{C}=\mathrm{C} 1) \mathrm{C} 1=\mathrm{C}(\mathrm{C}(=\mathrm{O}) \mathrm{CC} 1 \mathrm{O}[\mathrm{SiH} 2] \mathrm{C}(\mathrm{C})(\mathrm{C}) \mathrm{C}) \mathrm{C} 1=\mathrm{CC}(\mathrm{OC})=\mathrm{C}(\mathrm{OC}) \mathrm{C}(\mathrm{OC})=\mathrm{C} 1$ & 3,66 \\
\hline 484 & $\mathrm{COC} 1=\mathrm{CC}=\mathrm{C}(\mathrm{C}=\mathrm{C} 1 \mathrm{O}) \mathrm{C} 1=\mathrm{C}(\mathrm{C}(\mathrm{CC} 1 \mathrm{O})=\mathrm{N} / \mathrm{O}) \mathrm{C} 1=\mathrm{CC}(\mathrm{OC})=\mathrm{C}(\mathrm{OC}) \mathrm{C}(\mathrm{OC})=\mathrm{C} 1$ & 5,6 \\
\hline 485 & $\mathrm{COC} 1=\mathrm{CC}=\mathrm{C}(\mathrm{C}=\mathrm{C} 1) \mathrm{C} 1=\mathrm{C}(\mathrm{VC}(\mathrm{CC} 1 \mathrm{O})=\mathrm{N} / \mathrm{O}) \mathrm{C} 1=\mathrm{CC}(\mathrm{OC})=\mathrm{C}(\mathrm{OC}) \mathrm{C}(\mathrm{OC})=\mathrm{C} 1$ & 3,59 \\
\hline 486 & $\mathrm{COC} 1=\mathrm{CC}=\mathrm{C}(\mathrm{C}=\mathrm{C} 1) \mathrm{C} 1=\mathrm{C}(\mathrm{lC}(=\mathrm{N} / \mathrm{O}) \mathrm{C}(\mathrm{O}) \mathrm{C} 1) \mathrm{C} 1=\mathrm{CC}(\mathrm{OC})=\mathrm{C}(\mathrm{OC}) \mathrm{C}(\mathrm{OC})=\mathrm{C} 1$ & 5,59 \\
\hline 487 & $\mathrm{O} \backslash \mathrm{N}=\mathrm{C} 1 \backslash \mathrm{CC}(\mathrm{O}) \mathrm{C}(=\mathrm{C} 1 \mathrm{C} 1=\mathrm{CC}=\mathrm{CC}=\mathrm{C} 1) \mathrm{C} 1=\mathrm{CC}=\mathrm{CC}=\mathrm{C} 1$ & 3,42 \\
\hline 488 & $\mathrm{COC} 1=\mathrm{CC}=\mathrm{C}(\backslash \mathrm{C}=\mathrm{C} / \mathrm{C} 2=\mathrm{CC}(\mathrm{OC})=\mathrm{C}(\mathrm{OC}) \mathrm{C}(\mathrm{OC})=\mathrm{C} 2) \mathrm{C}=\mathrm{C} 1 \mathrm{O}$ & 4,8 \\
\hline 489 & $\mathrm{CC}(\mathrm{C})(\mathrm{C})[\mathrm{SiH} 2] \mathrm{OC} 1 \mathrm{CC}(=\mathrm{O}) \mathrm{C}(=\mathrm{C} 1 \mathrm{C} 1=\mathrm{CC}=\mathrm{CC}=\mathrm{C} 1) \mathrm{C} 1=\mathrm{CC}=\mathrm{CC}=\mathrm{C} 1$ & 4,92 \\
\hline 490 & $\mathrm{COC} 1=\mathrm{CC}=\mathrm{C}(\mathrm{OC}) \mathrm{C}(=\mathrm{C} 1) \mathrm{C} 1=\mathrm{C}(\mathrm{C}(=\mathrm{O}) \mathrm{CC} 1 \mathrm{O}[\mathrm{SiH} 2] \mathrm{C}(\mathrm{C})(\mathrm{C}) \mathrm{C}) \mathrm{C} 1=\mathrm{CC}(\mathrm{OC})=\mathrm{C}(\mathrm{OC}) \mathrm{C}(\mathrm{OC})=\mathrm{C} 1$ & 4,41 \\
\hline 491 & $\mathrm{COC} 1=\mathrm{CC}(=\mathrm{CC}(\mathrm{OC})=\mathrm{C} 1 \mathrm{OC}) \mathrm{C} 1=\mathrm{C}(\mathrm{C}(\mathrm{ClC} 1=\mathrm{N} \backslash \mathrm{O}) \mathrm{OC}(\mathrm{C})=\mathrm{O}) \mathrm{C} 1=\mathrm{CC}(\mathrm{C})=\mathrm{C}(\mathrm{OC}) \mathrm{C}(\mathrm{C})=\mathrm{C} 1$ & 3,66 \\
\hline 492 & {$[\mathrm{Na}+] \cdot \mathrm{COC} 1=\mathrm{CC}(=\mathrm{CC}(\mathrm{OC})=\mathrm{C} 1 \mathrm{OC}) \mathrm{C} 1=\mathrm{C}(\mathrm{C}(\mathrm{O}) \mathrm{CC} 1=\mathrm{O}) \mathrm{C} 1=\mathrm{CC}=\mathrm{C}(\mathrm{OCC}([\mathrm{O}-])=\mathrm{O}) \mathrm{C}=\mathrm{C} 1$} & 3,64 \\
\hline 493 & $\mathrm{CC}(=\mathrm{O}) \mathrm{OC} 1 \mathrm{ClC}(=\mathrm{N} \backslash \mathrm{O}) \mathrm{C}(=\mathrm{C} 1 \mathrm{C} 1=\mathrm{CC}=\mathrm{CC}=\mathrm{C} 1) \mathrm{C} 1=\mathrm{CC}=\mathrm{CC}=\mathrm{C} 1$ & 3,49 \\
\hline 494 & $\mathrm{COC} 1=\mathrm{CC}=\mathrm{C}(\mathrm{C}=\mathrm{C} 1 \mathrm{OC}) \mathrm{C} 1=\mathrm{C}(\mathrm{C}(\mathrm{CC} 1 \mathrm{O}[\mathrm{SiH} 2] \mathrm{C}(\mathrm{C})(\mathrm{C}) \mathrm{C})=\mathrm{N} / \mathrm{O}) \mathrm{C} 1=\mathrm{CC}(\mathrm{OC})=\mathrm{C}(\mathrm{OC}) \mathrm{C}(\mathrm{OC})=\mathrm{C} 1$ & 4,2 \\
\hline 495 & $\mathrm{COCOC} 1=\mathrm{CC}(=\mathrm{CC}=\mathrm{C} 1 \mathrm{OC}) \mathrm{C} 1=\mathrm{C}(\mathrm{C}(=\mathrm{O}) \mathrm{CC} 1 \mathrm{O}[\mathrm{SiH} 2] \mathrm{C}(\mathrm{C})(\mathrm{C}) \mathrm{C}) \mathrm{C} 1=\mathrm{CC}(\mathrm{OC})=\mathrm{C}(\mathrm{OC}) \mathrm{C}(\mathrm{OC})=\mathrm{C} 1$ & 4,63 \\
\hline 496 & $\mathrm{COC} 1=\mathrm{CC}(=\mathrm{CC}(\mathrm{OC})=\mathrm{C} 1 \mathrm{OC}) \mathrm{C} 1=\mathrm{C}(\mathrm{CC}(\mathrm{O}) \backslash \mathrm{C} 1=\mathrm{N} \backslash \mathrm{O}) \mathrm{C} 1=\mathrm{CC}=\mathrm{C}(\mathrm{SC}) \mathrm{C}=\mathrm{C} 1$ & 5,6 \\
\hline 497 & $\mathrm{COC}(=\mathrm{O}) \mathrm{C} 1=\mathrm{CC}(=\mathrm{CC}=\mathrm{C} 1 \mathrm{OC}) \mathrm{C} 1=\mathrm{C}(\mathrm{C}(=\mathrm{O}) \mathrm{CC} 1 \mathrm{O}[\mathrm{SiH} 2] \mathrm{C}(\mathrm{C})(\mathrm{C}) \mathrm{C}) \mathrm{C} 1=\mathrm{CC}(\mathrm{OC})=\mathrm{C}(\mathrm{OC}) \mathrm{C}(\mathrm{OC})=\mathrm{C} 1$ & 3,71 \\
\hline 498 & $\mathrm{CCOC}(=\mathrm{O}) \mathrm{COC} 1=\mathrm{CC}=\mathrm{C}(\mathrm{C}=\mathrm{C} 1) \mathrm{C} 1=\mathrm{C}(\mathrm{C}(=\mathrm{O}) \mathrm{CC} 1 \mathrm{O}) \mathrm{C} 1=\mathrm{CC}(\mathrm{OC})=\mathrm{C}(\mathrm{OC}) \mathrm{C}(\mathrm{OC})=\mathrm{C} 1$ & 3,65 \\
\hline 499 & $\mathrm{CCOC} 1=\mathrm{CC}(\mathrm{C})=\mathrm{CC} 2=\mathrm{C} 1 \mathrm{C}(=\mathrm{O}) \mathrm{C}=\mathrm{C}(\mathrm{C} 2=\mathrm{O}) \mathrm{C} 1=\mathrm{C}(\mathrm{C}) \mathrm{C}=\mathrm{C} 2 \mathrm{C}(=\mathrm{O}) \mathrm{C}=\mathrm{C}(\mathrm{NCCO}) \mathrm{C}(=\mathrm{O}) \mathrm{C} 2=\mathrm{C} 1 \mathrm{OCC}$ & 6,07 \\
\hline 500 & $\mathrm{COC} 1=\mathrm{CC}(\mathrm{C})=\mathrm{CC} 2=\mathrm{C} 1 \mathrm{C}(=\mathrm{O}) \mathrm{C}=\mathrm{C}(\mathrm{C} 2=\mathrm{O}) \mathrm{C} 1=\mathrm{C}(\mathrm{C}) \mathrm{C}=\mathrm{C} 2 \mathrm{C}(=\mathrm{O}) \mathrm{C}=\mathrm{C}(\mathrm{SCCO}) \mathrm{C}(=\mathrm{O}) \mathrm{C} 2=\mathrm{C} 1 \mathrm{OC}$ & 5,76 \\
\hline 501 & $\mathrm{CC} 1=\mathrm{CC}(\mathrm{O})=\mathrm{C} 2 \mathrm{C}(=\mathrm{O}) \mathrm{C}=\mathrm{C}(\mathrm{C}(=\mathrm{O}) \mathrm{C} 2=\mathrm{C} 1) \mathrm{C} 1=\mathrm{C}(\mathrm{C}) \mathrm{C}=\mathrm{C} 2 \mathrm{C}(=\mathrm{O}) \mathrm{C}=\mathrm{CC}(=\mathrm{O}) \mathrm{C} 2=\mathrm{C} 1 \mathrm{O}$ & 5,45 \\
\hline 502 & $\mathrm{CCOC} 1=\mathrm{CC}(\mathrm{C})=\mathrm{CC} 2=\mathrm{C} 1 \mathrm{C}(=\mathrm{O}) \mathrm{C}=\mathrm{C}(\mathrm{C} 2=\mathrm{O}) \mathrm{C} 1=\mathrm{C}(\mathrm{C}) \mathrm{C}=\mathrm{C} 2 \mathrm{C}(=\mathrm{O}) \mathrm{C}=\mathrm{CC}(=\mathrm{O}) \mathrm{C} 2=\mathrm{C} 1 \mathrm{OCC}$ & 5,62 \\
\hline 503 & $\mathrm{COC} 1=\mathrm{CC}(\mathrm{C})=\mathrm{CC} 2=\mathrm{C} 1 \mathrm{C}(=\mathrm{O}) \mathrm{C} 1 \mathrm{OC} 1(\mathrm{C} 2=\mathrm{O}) \mathrm{C} 1=\mathrm{C}(\mathrm{C}) \mathrm{C}=\mathrm{C} 2 \mathrm{C}(=\mathrm{O}) \mathrm{C} 3 \mathrm{OC} 3 \mathrm{C}(=\mathrm{O}) \mathrm{C} 2=\mathrm{C} 1 \mathrm{OC}$ & 6,68 \\
\hline 504 & $\mathrm{COC} 1=\mathrm{CC}(\mathrm{C})=\mathrm{CC} 2=\mathrm{C} 1 \mathrm{C}(=\mathrm{O}) \mathrm{C}=\mathrm{C}(\mathrm{C} 2=\mathrm{O}) \mathrm{C} 1=\mathrm{C}(\mathrm{C}) \mathrm{C}=\mathrm{C} 2 \mathrm{C}(=\mathrm{O}) \mathrm{C}=\mathrm{CC}(=\mathrm{O}) \mathrm{C} 2=\mathrm{C} 1 \mathrm{OC}$ & 6,41 \\
\hline 505 & $\mathrm{COC} 1=\mathrm{CC}(\mathrm{C})=\mathrm{CC} 2=\mathrm{C} 1 \mathrm{C}(=\mathrm{O}) \mathrm{C}=\mathrm{C}(\mathrm{C} 2=\mathrm{O}) \mathrm{C} 1=\mathrm{C}(\mathrm{C}) \mathrm{C}=\mathrm{C} 2 \mathrm{C}(=\mathrm{O}) \mathrm{C}=\mathrm{C}(\mathrm{NCCO}) \mathrm{C}(=\mathrm{O}) \mathrm{C} 2=\mathrm{C} 1 \mathrm{OC}$ & 5,85 \\
\hline 506 & $\begin{array}{l}\mathrm{COC} 1=\mathrm{CC}=\mathrm{CC} 2=\mathrm{C} 1 \mathrm{C}(=\mathrm{O}) \mathrm{C} 1=\mathrm{C}(\mathrm{O}) \mathrm{C} 3=\mathrm{C}(\mathrm{C}[\mathrm{C} @](\mathrm{O})(\mathrm{C}[\mathrm{C} @ @ \mathrm{H}] 3 \mathrm{O}[\mathrm{C} @ \mathrm{H}] 3 \mathrm{C}[\mathrm{C} @ \mathrm{H}](\mathrm{N})[\mathrm{C} @ \mathrm{H}](\mathrm{O})[\mathrm{C} @ \mathrm{H}]( \\
\mathrm{C}) \mathrm{O} 3) \mathrm{C}(=\mathrm{O}) \mathrm{CO}) \mathrm{C}(\mathrm{O})=\mathrm{C} 1 \mathrm{C} 2=\mathrm{O}\end{array}$ & 6,38 \\
\hline 507 & $\mathrm{CCOC} 1=\mathrm{C} 2 \mathrm{C}(\mathrm{O})=\mathrm{CC}(=\mathrm{C}(\mathrm{O}) \mathrm{C} 2=\mathrm{CC}(\mathrm{C})=\mathrm{C} 1) \mathrm{C} 1=\mathrm{C}(\mathrm{C}) \mathrm{C}=\mathrm{C} 2 \mathrm{C}(\mathrm{O})=\mathrm{CC}=\mathrm{C}(\mathrm{O}) \mathrm{C} 2=\mathrm{C} 1 \mathrm{OCC}$ & 5,78 \\
\hline 508 & $\mathrm{CN} 1 \mathrm{CCN}(\mathrm{C}=\mathrm{C} 2 / \mathrm{OC} 3=\mathrm{CC}=\mathrm{CC}=\mathrm{C} 3 \mathrm{C} 2=\mathrm{O}) \mathrm{C}(\mathrm{C} 1) \mathrm{C} 1=\mathrm{CC}=\mathrm{CC}=\mathrm{C} 1$ & 4,3 \\
\hline 509 & $\mathrm{O}=\mathrm{C} 1 \backslash \mathrm{C}(\mathrm{OC} 2=\mathrm{CC}=\mathrm{CC}=\mathrm{C} 12)=\mathrm{C} \backslash \mathrm{N} 1 \mathrm{CCN}(\mathrm{CC} 1) \mathrm{C} 1=\mathrm{CC}=\mathrm{CC}=\mathrm{C} 1$ & 4,56 \\
\hline 510 & $\mathrm{O}=\mathrm{C} 1 \mathrm{C}(\mathrm{SC} 2=\mathrm{NC} 3=\mathrm{CC}=\mathrm{CC}=\mathrm{C} 3 \mathrm{O} 2)=\mathrm{COC} 2=\mathrm{CC}=\mathrm{CC}=\mathrm{C} 12$ & 4,53 \\
\hline 511 & $\mathrm{CC} 1=\mathrm{CC}(\mathrm{C})=\mathrm{NC}(\mathrm{SC} 2=\mathrm{COC} 3=\mathrm{CC}=\mathrm{CC}=\mathrm{C} 3 \mathrm{C} 2=\mathrm{O})=\mathrm{N} 1$ & 4,3 \\
\hline 512 & $\mathrm{O}=\mathrm{C} 1 \mathrm{C}(\mathrm{SC} 2=\mathrm{NC} 3=\mathrm{CC}=\mathrm{CC}=\mathrm{C} 3 \mathrm{~N} 2)=\mathrm{COC} 2=\mathrm{CC}=\mathrm{CC}=\mathrm{C} 12$ & 4,37 \\
\hline 513 & $\mathrm{O}=\mathrm{C} 1 \backslash \mathrm{C}(\mathrm{OC} 2=\mathrm{CC}=\mathrm{CC}=\mathrm{C} 12)=\mathrm{C} 1 \mathrm{~N} 1 \mathrm{CCN}(\mathrm{CC} 2=\mathrm{CC}=\mathrm{CC}=\mathrm{C} 2) \mathrm{CC} 1$ & 5,24 \\
\hline 514 & $\mathrm{FC} 1=\mathrm{CNC}(=\mathrm{O}) \mathrm{NC} 1=\mathrm{O}$ & 3,89 \\
\hline 515 & $\mathrm{CC} 1=\mathrm{NN}=\mathrm{C}(\mathrm{O} 1) \mathrm{SC} 1=\mathrm{COC} 2=\mathrm{CC}=\mathrm{CC}=\mathrm{C} 2 \mathrm{C} 1=\mathrm{O}$ & 4,3 \\
\hline 516 & $\mathrm{NC} 1=\mathrm{NN}=\mathrm{C}(\mathrm{SC} 2=\mathrm{COC} 3=\mathrm{CC}=\mathrm{CC}=\mathrm{C} 3 \mathrm{C} 2=\mathrm{O}) \mathrm{S} 1$ & 4,32 \\
\hline 517 & $\mathrm{O}=\mathrm{C} 1 \backslash \mathrm{C}(\mathrm{OC} 2=\mathrm{CC}=\mathrm{CC}=\mathrm{C} 12)=\mathrm{C} \backslash \mathrm{N} 1 \mathrm{CCCCC} 1$ & 4,3 \\
\hline 518 & $\mathrm{CC} 1=\mathrm{NN}=\mathrm{C}(\mathrm{SC} 2=\mathrm{COC} 3=\mathrm{CC}=\mathrm{CC}=\mathrm{C} 3 \mathrm{C} 2=\mathrm{O}) \mathrm{S} 1$ & 4,4 \\
\hline 519 & $\mathrm{FC} 1=\mathrm{CC}=\mathrm{C}(\mathrm{C}=\mathrm{C} 1) \mathrm{C}(\mathrm{CN} 1 \mathrm{CCN}(\mathrm{CC} 1) \backslash \mathrm{C}=\mathrm{C} 1 / \mathrm{OC} 2=\mathrm{CC}=\mathrm{CC}=\mathrm{C} 2 \mathrm{C} 1=\mathrm{O}) \mathrm{C} 1=\mathrm{CC}=\mathrm{C}(\mathrm{F}) \mathrm{C}=\mathrm{C} 1$ & 5,33 \\
\hline 520 & $\mathrm{CN} 1 \mathrm{CCN}(\mathrm{CC} 1) \backslash \mathrm{C}=\mathrm{C} 1 / \mathrm{OC} 2=\mathrm{CC}=\mathrm{CC}=\mathrm{C} 2 \mathrm{C} 1=\mathrm{O}$ & 4,3 \\
\hline 521 & $\mathrm{O}=\mathrm{C} 1 \mathrm{C}(\mathrm{SC} 2=\mathrm{NC} 3=\mathrm{CC}=\mathrm{CC}=\mathrm{C} 3 \mathrm{~S} 2)=\mathrm{COC} 2=\mathrm{CC}=\mathrm{CC}=\mathrm{C} 12$ & 4,5 \\
\hline 522 & $\mathrm{COC} 1=\mathrm{CC}(\mathrm{CC}(\mathrm{C})(\mathrm{O}) \mathrm{C}(\mathrm{F})(\mathrm{F}) \mathrm{F})=\mathrm{NC}(\mathrm{OC})=\mathrm{N} 1$ & 3,7 \\
\hline 523 & $\mathrm{COC} 1=\mathrm{CC}(\mathrm{CC}(\mathrm{C})(\mathrm{CF}) \mathrm{OC}(\mathrm{C})=\mathrm{O})=\mathrm{NC}(\mathrm{OC})=\mathrm{N} 1$ & 4,75 \\
\hline 524 & $\mathrm{COC} 1=\mathrm{NC}(\mathrm{CC}(\mathrm{C})(\mathrm{O}) \mathrm{C}(\mathrm{F})(\mathrm{F}) \mathrm{F})=\mathrm{C}(\mathrm{C} 2=\mathrm{CC}=\mathrm{CC}=\mathrm{C} 2) \mathrm{C}(\mathrm{OC})=\mathrm{N} 1$ & 4,72 \\
\hline 525 & $\mathrm{CC}(=\mathrm{O}) \mathrm{OC}(\mathrm{C})(\mathrm{CF}) \mathrm{CC} 1=\mathrm{CC}(\mathrm{O})=\mathrm{NC}(\mathrm{O})=\mathrm{N} 1$ & 3,7 \\
\hline 526 & $\mathrm{COC} 1=\mathrm{NC}(\mathrm{CBr})=\mathrm{C}(\mathrm{Br}) \mathrm{C}(\mathrm{OC})=\mathrm{N} 1$ & 5,15 \\
\hline 527 & $\mathrm{COC} 1=\mathrm{CC}(\backslash \mathrm{C}=\mathrm{C}(\backslash \mathrm{O}) \mathrm{C}(\mathrm{F})(\mathrm{F}) \mathrm{F})=\mathrm{NC}(\mathrm{OC})=\mathrm{N} 1$ & 3,7 \\
\hline 528 & $\mathrm{COC} 1=\mathrm{CC}(\mathrm{IC}=\mathrm{C}(/ \mathrm{O}) \mathrm{C}(\mathrm{F})(\mathrm{F}) \mathrm{C}(\mathrm{F})(\mathrm{F}) \mathrm{F})=\mathrm{NC}(\mathrm{OC})=\mathrm{N} 1$ & 4,26 \\
\hline 529 & $\mathrm{COC} 1=\mathrm{CC}(\mathrm{CC}(\mathrm{C})(\mathrm{O}) \mathrm{C} 2=\mathrm{CC}=\mathrm{C}(\mathrm{F}) \mathrm{C}=\mathrm{C} 2)=\mathrm{NC}(\mathrm{OC})=\mathrm{N} 1$ & 4 \\
\hline 530 & $\mathrm{COC} 1=\mathrm{NC}(\mathrm{CC}(\mathrm{C})(\mathrm{O}) \mathrm{C} 2=\mathrm{CC}=\mathrm{C}(\mathrm{F}) \mathrm{C}=\mathrm{C} 2)=\mathrm{C}(\mathrm{C}) \mathrm{C}(\mathrm{OC})=\mathrm{N} 1$ & 4,37 \\
\hline 531 & $\mathrm{COC} 1=\mathrm{NC}(\mathrm{C})=\mathrm{C}(\mathrm{Br}) \mathrm{C}(\mathrm{OC})=\mathrm{N} 1$ & 4 \\
\hline 532 & $\mathrm{COC} 1=\mathrm{NC}(\mathrm{C})=\mathrm{C}(\mathrm{C} 2=\mathrm{CC}=\mathrm{CC}=\mathrm{C} 2) \mathrm{C}(\mathrm{OC})=\mathrm{N} 1$ & 4 \\
\hline 533 & $\mathrm{CC}(=\mathrm{O}) \mathrm{OC}(\mathrm{C})(\mathrm{CC} 1=\mathrm{CC}(\mathrm{O})=\mathrm{NC}(\mathrm{O})=\mathrm{N} 1) \mathrm{CC} 1=\mathrm{CC}=\mathrm{CC}=\mathrm{C} 1 \mathrm{~F}$ & 3,7 \\
\hline 534 & $\mathrm{CC}(\mathrm{O})(\mathrm{CF}) \mathrm{CC} 1=\mathrm{CC}(\mathrm{O})=\mathrm{NC}(\mathrm{O})=\mathrm{N} 1$ & 3,7 \\
\hline 535 & $\mathrm{COC} 1=\mathrm{CC}(\mathrm{CC}(\mathrm{C})(\mathrm{O}) \mathrm{CF})=\mathrm{NC}(\mathrm{OC})=\mathrm{N} 1$ & 3,7 \\
\hline 536 & $\mathrm{COC} 1=\mathrm{CC}(\mathrm{CC}(\mathrm{C})(\mathrm{O}) \mathrm{CC} 2=\mathrm{CC}=\mathrm{CC}=\mathrm{C} 2 \mathrm{~F})=\mathrm{NC}(\mathrm{OC})=\mathrm{N} 1$ & 3,7 \\
\hline 537 & $\mathrm{COC} 1=\mathrm{NC}(\mathrm{OC})=\mathrm{C}(\mathrm{Br}) \mathrm{C}(=\mathrm{N} 1) \mathrm{C}(\mathrm{Br}) \mathrm{Br}$ & 5,7 \\
\hline 538 & $\mathrm{COC} 1=\mathrm{CC}(1 \mathrm{C}=\mathrm{C}(/ \mathrm{C}) \mathrm{C} 2=\mathrm{CC}=\mathrm{C}(\mathrm{F}) \mathrm{C}=\mathrm{C} 2)=\mathrm{NC}(\mathrm{OC})=\mathrm{N} 1$ & 4,7 \\
\hline 539 & $\mathrm{COC} 1=\mathrm{CC}(\backslash \mathrm{C}=\mathrm{C}(\backslash \mathrm{O}) \mathrm{C} 2=\mathrm{CC}=\mathrm{C}(\mathrm{F}) \mathrm{C}=\mathrm{C} 2)=\mathrm{NC}(\mathrm{OC})=\mathrm{N} 1$ & 4 \\
\hline 540 & $\mathrm{COC} 1=\mathrm{CC}(\mathrm{CC}(\mathrm{O})(\mathrm{C} 2=\mathrm{CC}=\mathrm{C}(\mathrm{F}) \mathrm{C}=\mathrm{C} 2) \mathrm{C} 2=\mathrm{CC}=\mathrm{C}(\mathrm{F}) \mathrm{C}=\mathrm{C} 2)=\mathrm{NC}(\mathrm{OC})=\mathrm{N} 1$ & 4 \\
\hline 541 & $\mathrm{CC}(\mathrm{O})(\mathrm{CC} 1=\mathrm{CC}(\mathrm{O})=\mathrm{NC}(\mathrm{O})=\mathrm{N} 1) \mathrm{C}(\mathrm{F})(\mathrm{F}) \mathrm{F}$ & 4 \\
\hline 542 & $\begin{array}{l}\mathrm{C}[\mathrm{C} @ @ \mathrm{H}] 1 \mathrm{CC}[\mathrm{C} @ @] 2(\mathrm{CC}[\mathrm{C} @] 3(\mathrm{C}) \mathrm{C}(=\mathrm{CC}[\mathrm{C} @ @ \mathrm{H}] 4[\mathrm{C} @ @] 5(\mathrm{C}) \mathrm{C}[\mathrm{C} @ @ \mathrm{H}](\mathrm{O})[\mathrm{C} @ @ \mathrm{H}](\mathrm{O}) \mathrm{C}(\mathrm{C})(\mathrm{C})[\mathrm{C} @ \\
\text { @ }] 5 \mathrm{CC}[\mathrm{C} @ @] 34 \mathrm{C})[\mathrm{C} @ \mathrm{H}] 2[\mathrm{C} @] 1(\mathrm{C}) \mathrm{O}) \mathrm{C}(\mathrm{O})=\mathrm{O}\end{array}$ & 3,98 \\
\hline 543 & $\mathrm{Cl} . \mathrm{CC}(\mathrm{C}) \mathrm{NC}(=\mathrm{N}) \mathrm{C} 1=\mathrm{CC}=\mathrm{C} 2 \mathrm{~N}=\mathrm{C}(\mathrm{NC} 2=\mathrm{C} 1) \backslash \mathrm{C}=\mathrm{C} / \mathrm{C} 1=\mathrm{CC}=\mathrm{CC}=\mathrm{C} 1 \mathrm{Cl}$ & 4,52 \\
\hline 544 & $\mathrm{Cl} . \mathrm{NC}(=\mathrm{N}) \mathrm{C} 1=\mathrm{CC}=\mathrm{C} 2 \mathrm{~N}=\mathrm{C}(\mathrm{NC} 2=\mathrm{C} 1) \backslash \mathrm{C}=\mathrm{C} / \mathrm{C} 1=\mathrm{CC}=\mathrm{CC}=\mathrm{C} 1 \mathrm{Cl}$ & 4,54 \\
\hline 545 & $\mathrm{Cl} . \mathrm{CC}(\mathrm{C}) \mathrm{NC}(=\mathrm{N}) \mathrm{C} 1=\mathrm{CC}=\mathrm{C} 2 \mathrm{~N}=\mathrm{C}(\mathrm{NC} 2=\mathrm{C} 1) \backslash \mathrm{C}=\mathrm{ClC} 1=\mathrm{CC}=\mathrm{CC}=\mathrm{C} 1$ & 4 \\
\hline 546 & $\mathrm{Cl} . \mathrm{C} 1 \mathrm{CN}=\mathrm{C}(\mathrm{N} 1) \mathrm{C} 1=\mathrm{CC}=\mathrm{C} 2 \mathrm{~N}=\mathrm{C} 3 \mathrm{C}=\mathrm{CC} 4=\mathrm{C}(\mathrm{C}=\mathrm{CC}=\mathrm{C} 4) \mathrm{N} 3 \mathrm{C} 2=\mathrm{C} 1$ & 5,7 \\
\hline 547 & Cl.CC $(\mathrm{C}) \mathrm{NC}(=\mathrm{N}) \mathrm{C} 1=\mathrm{CC}=\mathrm{C} 2 \mathrm{C}=\mathrm{CC} 3=\mathrm{NC} 4=\mathrm{C}(\mathrm{C}=\mathrm{CC}=\mathrm{C} 4) \mathrm{N} 3 \mathrm{C} 2=\mathrm{C} 1$ & 5,8 \\
\hline 548 & $\mathrm{Cl} . \mathrm{ClC} 1=\mathrm{CC}=\mathrm{CC}=\mathrm{C} 1 \backslash \mathrm{C}=\mathrm{C} / \mathrm{C} 1=\mathrm{NC} 2=\mathrm{CC}=\mathrm{C}(\mathrm{C}=\mathrm{C} 2 \mathrm{~N} 1) \mathrm{C}(=\mathrm{N}) \mathrm{NN} 1 \mathrm{CCOCC} 1$ & 5,05 \\
\hline 549 & $\mathrm{Cl} . \mathrm{ClC} 1=\mathrm{CC}=\mathrm{CC}=\mathrm{C} 1 \backslash \mathrm{C}=\mathrm{ClC} 1=\mathrm{NC} 2=\mathrm{CC}=\mathrm{C}(\mathrm{C}=\mathrm{C} 2 \mathrm{~N} 1) \mathrm{C} 1=\mathrm{NCCN} 1$ & 4,8 \\
\hline
\end{tabular}




\begin{tabular}{|c|c|c|}
\hline NAME & SMILE & $\lg (\mathbf{1} / \mathbf{I C 5 0})$ \\
\hline 550 & $\mathrm{Cl} \cdot \mathrm{CC}(\mathrm{C}) \mathrm{NC}(=\mathrm{N}) \mathrm{C} 1=\mathrm{CC}=\mathrm{C} 2 \mathrm{~N}=\mathrm{C}(\mathrm{NC} 2=\mathrm{C} 1) \backslash \mathrm{C}=\mathrm{C} / \mathrm{C} 1=\mathrm{CC}=\mathrm{CC}=\mathrm{C} 1$ & 4,09 \\
\hline 551 & $\mathrm{Cl} . \mathrm{N}=\mathrm{C}(\mathrm{NN} 1 \mathrm{CCOCC} 1) \mathrm{C} 1=\mathrm{CC}=\mathrm{C} 2 \mathrm{C}=\mathrm{CC} 3=\mathrm{NC} 4=\mathrm{C}(\mathrm{C}=\mathrm{CC}=\mathrm{C} 4) \mathrm{N} 3 \mathrm{C} 2=\mathrm{C} 1$ & 5,33 \\
\hline 552 & $\mathrm{C} 1=\mathrm{CC} 2=\mathrm{C}(\mathrm{C}=\mathrm{C} 1) \mathrm{N} 1 \mathrm{C}(\mathrm{C}=\mathrm{CC} 3=\mathrm{CC}=\mathrm{CC}=\mathrm{C} 13)=\mathrm{N} 2$ & 4,82 \\
\hline 553 & $\mathrm{Cl} . \mathrm{CC}(\mathrm{C}) \mathrm{NC}(=\mathrm{N}) \mathrm{C} 1=\mathrm{CC} 2=\mathrm{C}(\mathrm{C}=\mathrm{C} 1) \mathrm{N}=\mathrm{C} 1 \mathrm{C}=\mathrm{CC} 3=\mathrm{CC}=\mathrm{CC}=\mathrm{C} 3 \mathrm{~N} 21$ & 5,4 \\
\hline 554 & $\mathrm{Cl} \cdot \mathrm{NC}(=\mathrm{N}) \mathrm{C} 1=\mathrm{CC}=\mathrm{C} 2 \mathrm{C}=\mathrm{CC} 3=\mathrm{NC} 4=\mathrm{C}(\mathrm{C}=\mathrm{CC}=\mathrm{C} 4) \mathrm{N} 3 \mathrm{C} 2=\mathrm{C} 1$ & 5,4 \\
\hline 555 & $\mathrm{Cl} . \mathrm{N}=\mathrm{C}(\mathrm{NN} 1 \mathrm{CCOCC} 1) \mathrm{C} 1=\mathrm{CC}=\mathrm{C} 2 \mathrm{~N}=\mathrm{C}(\mathrm{NC} 2=\mathrm{C} 1) \backslash \mathrm{C}=\mathrm{C} / \mathrm{C} 1=\mathrm{CC}=\mathrm{CC}=\mathrm{C} 1$ & 4,21 \\
\hline 556 & Cl.ClC1 $=\mathrm{CC}=\mathrm{CC}=\mathrm{C} 1 \backslash \mathrm{C}=\mathrm{ClC} 1=\mathrm{NC} 2=\mathrm{CC}=\mathrm{C}(\mathrm{C}=\mathrm{C} 2 \mathrm{~N} 1) \mathrm{C}(=\mathrm{N}) \mathrm{NN} 1 \mathrm{CCOCC} 1$ & 5 \\
\hline 557 & 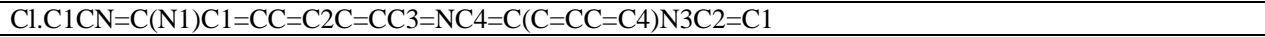 & 5,62 \\
\hline 558 & $\mathrm{Cl} . \mathrm{NC}(=\mathrm{N}) \mathrm{C} 1=\mathrm{CC} 2=\mathrm{C}(\mathrm{C}=\mathrm{C} 1) \mathrm{N}=\mathrm{C} 1 \mathrm{C}=\mathrm{CC} 3=\mathrm{CC}=\mathrm{CC}=\mathrm{C} 3 \mathrm{~N} 21$ & 4,89 \\
\hline 559 & 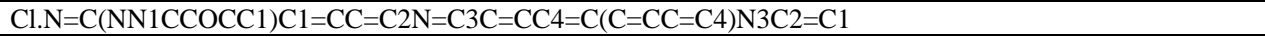 & 5,7 \\
\hline 560 & $\mathrm{Cl} \cdot \mathrm{NC}(=\mathrm{N}) \mathrm{C} 1=\mathrm{CC}=\mathrm{C} 2 \mathrm{~N}=\mathrm{C}(\mathrm{NC} 2=\mathrm{C} 1) \backslash \mathrm{C}=\mathrm{C} / \mathrm{C} 1=\mathrm{CC}=\mathrm{CC}=\mathrm{C} 1$ & 4 \\
\hline 561 & Cl.NC $(=\mathrm{N}) \mathrm{C} 1=\mathrm{CC}=\mathrm{C} 2 \mathrm{~N}=\mathrm{C}(\mathrm{NC} 2=\mathrm{C} 1) \backslash \mathrm{C}=\mathrm{ClC} 1=\mathrm{CC}=\mathrm{CC}=\mathrm{C} 1$ & 4,96 \\
\hline 562 & 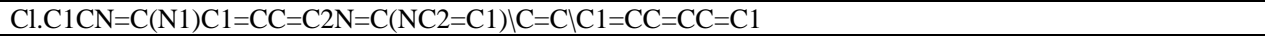 & 4,8 \\
\hline 563 & $\mathrm{Cl} . \mathrm{ClC} 1=\mathrm{CC}=\mathrm{CC}=\mathrm{C} 1 \backslash \mathrm{C}=\mathrm{C} / \mathrm{C} 1=\mathrm{NC} 2=\mathrm{CC}=\mathrm{C}(\mathrm{C}=\mathrm{C} 2 \mathrm{~N} 1) \mathrm{C} 1=\mathrm{NCCN} 1$ & 4 \\
\hline 564 & $\mathrm{Cl} . \mathrm{N}=\mathrm{C}(\mathrm{NN} 1 \mathrm{CCOCC} 1) \mathrm{C} 1=\mathrm{CC}=\mathrm{C} 2 \mathrm{~N}=\mathrm{C}(\mathrm{NC} 2=\mathrm{C} 1) \backslash \mathrm{C}=\mathrm{ClC} 1=\mathrm{CC}=\mathrm{CC}=\mathrm{C} 1$ & 4,89 \\
\hline 565 & $\mathrm{Cl} . \mathrm{CC}(\mathrm{C}) \mathrm{NC}(=\mathrm{N}) \mathrm{C} 1=\mathrm{CC}=\mathrm{C} 2 \mathrm{~N}=\mathrm{C}(\mathrm{NC} 2=\mathrm{C} 1) \backslash \mathrm{C}=\mathrm{ClC} 1=\mathrm{CC}=\mathrm{CC}=\mathrm{C} 1 \mathrm{Cl}$ & 4,15 \\
\hline 566 & Cl.NC $(=\mathrm{N}) \mathrm{C} 1=\mathrm{CC}=\mathrm{C} 2 \mathrm{~N}=\mathrm{C}(\mathrm{NC} 2=\mathrm{C} 1) \backslash \mathrm{C}=\mathrm{ClC} 1=\mathrm{CC}=\mathrm{CC}=\mathrm{C} 1 \mathrm{Cl}$ & 4,64 \\
\hline 567 & $\begin{array}{l}\mathrm{COC} 1=\mathrm{CC}=\mathrm{CC} 2=\mathrm{C} 1 \mathrm{C}(=\mathrm{O}) \mathrm{C} 1=\mathrm{C}(\mathrm{O}) \mathrm{C} 3=\mathrm{C}(\mathrm{C}[\mathrm{C} @](\mathrm{O})(\mathrm{C}[\mathrm{C} @ @ \mathrm{H}] 3 \mathrm{O}[\mathrm{C} @ \mathrm{H}] 3 \mathrm{C}[\mathrm{C} @ \mathrm{H}](\mathrm{N})[\mathrm{C} @ \mathrm{H}](\mathrm{O})[\mathrm{C} @ \mathrm{H}]( \\
\mathrm{C}) \mathrm{O} 3) \mathrm{C}(=\mathrm{O}) \mathrm{CO}) \mathrm{C}(\mathrm{O})=\mathrm{C} 1 \mathrm{C} 2=\mathrm{O}\end{array}$ & 7,4 \\
\hline 568 & $\mathrm{Cl} . \mathrm{C} 1 \mathrm{CN}=\mathrm{C}(\mathrm{N} 1) \mathrm{C} 1=\mathrm{CC}=\mathrm{C} 2 \mathrm{~N}=\mathrm{C}(\mathrm{NC} 2=\mathrm{C} 1) \backslash \mathrm{C}=\mathrm{C} / \mathrm{C} 1=\mathrm{CC}=\mathrm{CC}=\mathrm{C} 1$ & 4,57 \\
\hline 569 & $\begin{array}{l}\mathrm{COC} 1=\mathrm{CC}(=\mathrm{CC}(\mathrm{OC})=\mathrm{C} 1 \mathrm{OC})[\mathrm{C} @ \mathrm{H}] 1[\mathrm{C} @ @ \mathrm{H}] 2[\mathrm{C} @ \mathrm{H}](\mathrm{COC} 2=\mathrm{O})[\mathrm{C} @ \mathrm{H}](\mathrm{N} 2 \mathrm{C}=\mathrm{C}(\mathrm{CNC} 3=\mathrm{CC}=\mathrm{CC}=\mathrm{C} 3) \mathrm{N}= \\
\mathrm{N} 2) \mathrm{C} 2=\mathrm{CC} 3=\mathrm{C}(\mathrm{OCO} 3) \mathrm{C}=\mathrm{C} 12\end{array}$ & 5,33 \\
\hline 570 & $\begin{array}{l}\mathrm{COC} 1=\mathrm{CC}(=\mathrm{CC}(\mathrm{OC})=\mathrm{C} 1 \mathrm{OC})[\mathrm{C} @ \mathrm{H}] 1[\mathrm{C} @ @ \mathrm{H}] 2[\mathrm{C} @ \mathrm{H}](\mathrm{COC} 2=\mathrm{O})[\mathrm{C} @ \mathrm{H}](\mathrm{N} 2 \mathrm{C}=\mathrm{C}(\mathrm{CNC} 3=\mathrm{CC}=\mathrm{CC}=\mathrm{C} 3 \mathrm{C}) \mathrm{N}= \\
\mathrm{N} 2) \mathrm{C} 2=\mathrm{CC} 3=\mathrm{C}(\mathrm{OCO} 3) \mathrm{C}=\mathrm{C} 12\end{array}$ & 5,04 \\
\hline 571 & $\begin{array}{l}\mathrm{COC} 1=\mathrm{CC}(=\mathrm{CC}(\mathrm{OC})=\mathrm{C} 1 \mathrm{O})[\mathrm{C} @ \mathrm{H}] 1[\mathrm{C} @ @ \mathrm{H}] 2[\mathrm{C} @ \mathrm{H}](\mathrm{COC} 2=\mathrm{O})[\mathrm{C} @ \mathrm{H}](\mathrm{N} 2 \mathrm{C}=\mathrm{C}(\mathrm{CNC} 3=\mathrm{CC}=\mathrm{CC}=\mathrm{C} 3) \mathrm{N}=\mathrm{N} 2 \\
) \mathrm{C} 2=\mathrm{CC} 3=\mathrm{C}(\mathrm{OCO} 3) \mathrm{C}=\mathrm{C} 12\end{array}$ & 5,53 \\
\hline 572 & $\begin{array}{l}\mathrm{COC} 1=\mathrm{CC}(=\mathrm{CC}(\mathrm{OC})=\mathrm{C} 1 \mathrm{O})[\mathrm{C} @ \mathrm{H}] 1[\mathrm{C} @ @ \mathrm{H}] 2[\mathrm{C} @ \mathrm{H}](\mathrm{COC} 2=\mathrm{O})[\mathrm{C} @ \mathrm{H}](\mathrm{N} 2 \mathrm{C}=\mathrm{C}(\mathrm{CNC} 3=\mathrm{CC}=\mathrm{CC}=\mathrm{C} 3 \mathrm{C}) \mathrm{N}= \\
\mathrm{N} 2) \mathrm{C} 2=\mathrm{CC} 3=\mathrm{C}(\mathrm{OCO} 3) \mathrm{C}=\mathrm{C} 12\end{array}$ & 5,82 \\
\hline 573 & $\begin{array}{l}\mathrm{COC} 1=\mathrm{CC}(=\mathrm{CC}(\mathrm{OC})=\mathrm{C} 1 \mathrm{OC})[\mathrm{C} @ \mathrm{H}] 1[\mathrm{C} @ @ \mathrm{H}] 2[\mathrm{C} @ \mathrm{H}](\mathrm{COC} 2=\mathrm{O})[\mathrm{C} @ \mathrm{H}](\mathrm{N} 2 \mathrm{C}=\mathrm{C}(\mathrm{CNC} 3=\mathrm{CC}=\mathrm{CC}=\mathrm{C} 3 \mathrm{Cl}) \mathrm{N} \\
=\mathrm{N} 2) \mathrm{C} 2=\mathrm{CC} 3=\mathrm{C}(\mathrm{OCO} 3) \mathrm{C}=\mathrm{C} 12\end{array}$ & 5,9 \\
\hline 574 & $\begin{array}{l}\mathrm{COC} 1=\mathrm{CC}(=\mathrm{CC}(\mathrm{OC})=\mathrm{C} 1 \mathrm{O})[\mathrm{C} @ \mathrm{H}] 1[\mathrm{C} @ @ \mathrm{H}] 2[\mathrm{C} @ \mathrm{H}](\mathrm{COC} 2=\mathrm{O})[\mathrm{C} @ \mathrm{H}](\mathrm{N} 2 \mathrm{C}=\mathrm{C}(\mathrm{CNC} 3=\mathrm{CC}=\mathrm{CC}(\mathrm{Cl})=\mathrm{C} 3) \mathrm{N} \\
=\mathrm{N} 2) \mathrm{C} 2=\mathrm{CC} 3=\mathrm{C}(\mathrm{OCO} 3) \mathrm{C}=\mathrm{C} 12\end{array}$ & 5,91 \\
\hline 575 & $\begin{array}{l}\mathrm{COC} 1=\mathrm{CC}(=\mathrm{CC}(\mathrm{OC})=\mathrm{C} 1 \mathrm{O})[\mathrm{C} @ \mathrm{H}] 1[\mathrm{C} @ @ \mathrm{H}] 2[\mathrm{C} @ \mathrm{H}](\mathrm{COC} 2=\mathrm{O})[\mathrm{C} @ \mathrm{H}](\mathrm{O}[\mathrm{C} @ @ \mathrm{H}] 2 \mathrm{O}[\mathrm{C} @ @ \mathrm{H}] 3 \mathrm{CO}[\mathrm{C} @ @) \\
\mathrm{H}](\mathrm{C}) \mathrm{O}[\mathrm{C} @ \mathrm{H}] 3[\mathrm{C} @ \mathrm{H}](\mathrm{O})[\mathrm{C} @ \mathrm{H}] 2 \mathrm{O}) \mathrm{C} 2=\mathrm{CC} 3=\mathrm{C}(\mathrm{OCO} 3) \mathrm{C}=\mathrm{C} 12\end{array}$ & 5,67 \\
\hline 576 & $\begin{array}{l}\mathrm{COC} 1=\mathrm{CC}(=\mathrm{CC}(\mathrm{OC})=\mathrm{C} 1 \mathrm{OC})[\mathrm{C} @ \mathrm{H}] 1[\mathrm{C} @ @ \mathrm{H}] 2[\mathrm{C} @ \mathrm{H}](\mathrm{COC} 2=\mathrm{O})[\mathrm{C} @ \mathrm{H}](\mathrm{N} 2 \mathrm{C}=\mathrm{C}(\mathrm{CNC} 3=\mathrm{CC}=\mathrm{CC}(=\mathrm{C} 3)[\mathrm{N}+ \\
]([\mathrm{O}-])=\mathrm{O}) \mathrm{N}=\mathrm{N} 2) \mathrm{C} 2=\mathrm{CC} 3=\mathrm{C}(\mathrm{OCO} 3) \mathrm{C}=\mathrm{C} 12\end{array}$ & 5,44 \\
\hline 577 & $\mathrm{COC} 1=\mathrm{CC}(=\mathrm{CC}(\mathrm{OC})=\mathrm{C} 1 \mathrm{OC})[\mathrm{C} @ \mathrm{H}] 1[\mathrm{C} @ @ \mathrm{H}] 2[\mathrm{C} @ \mathrm{H}](\mathrm{COC} 2=\mathrm{O})[\mathrm{C} @ @ \mathrm{H}](\mathrm{O}) \mathrm{C} 2=\mathrm{CC} 3=\mathrm{C}(\mathrm{OCO} 3) \mathrm{C}=\mathrm{C} 12$ & 5,85 \\
\hline 578 & $\begin{array}{l}\mathrm{COC} 1=\mathrm{CC}(=\mathrm{CC}(\mathrm{OC})=\mathrm{C} 1 \mathrm{O})[\mathrm{C} @ \mathrm{H}] 1[\mathrm{C} @ @ \mathrm{H}] 2[\mathrm{C} @ \mathrm{H}](\mathrm{COC} 2=\mathrm{O})[\mathrm{C} @ \mathrm{H}](\mathrm{N} 2 \mathrm{C}=\mathrm{C}(\mathrm{CNC} 3=\mathrm{CC}=\mathrm{C}(\mathrm{C}) \mathrm{C}=\mathrm{C} 3) \mathrm{N}= \\
\mathrm{N} 2) \mathrm{C} 2=\mathrm{CC} 3=\mathrm{C}(\mathrm{OCO} 3) \mathrm{C}=\mathrm{C} 12\end{array}$ & 5,49 \\
\hline 579 & $\begin{array}{l}\mathrm{COC} 1=\mathrm{CC}(=\mathrm{CC}(\mathrm{OC})=\mathrm{C} 1 \mathrm{O})[\mathrm{C} @ \mathrm{H}] 1[\mathrm{C} @ @ \mathrm{H}] 2[\mathrm{C} @ \mathrm{H}](\mathrm{COC} 2=\mathrm{O})[\mathrm{C} @ \mathrm{H}](\mathrm{N} 2 \mathrm{C}=\mathrm{C}(\mathrm{CNC} 3=\mathrm{CC}=\mathrm{CC}(=\mathrm{C} 3)[\mathrm{N}+]( \\
[\mathrm{O}-])=\mathrm{O}) \mathrm{N}=\mathrm{N} 2) \mathrm{C} 2=\mathrm{CC} 3=\mathrm{C}(\mathrm{OCO} 3) \mathrm{C}=\mathrm{C} 12\end{array}$ & 5,39 \\
\hline 580 & $\begin{array}{l}\mathrm{COC} 1=\mathrm{CC}(=\mathrm{CC}(\mathrm{OC})=\mathrm{C} 1 \mathrm{OC})[\mathrm{C} @ \mathrm{H}] 1[\mathrm{C} @ @ \mathrm{H}] 2[\mathrm{C} @ \mathrm{H}](\mathrm{COC} 2=\mathrm{O})[\mathrm{C} @ \mathrm{H}](\mathrm{N} 2 \mathrm{C}=\mathrm{C}(\mathrm{CNC} 3=\mathrm{CC}=\mathrm{CC}(\mathrm{Cl})=\mathrm{C} 3) \\
\mathrm{N}=\mathrm{N} 2) \mathrm{C} 2=\mathrm{CC} 3=\mathrm{C}(\mathrm{OCO} 3) \mathrm{C}=\mathrm{C} 12\end{array}$ & 5,86 \\
\hline 581 & $\begin{array}{l}\mathrm{COC} 1=\mathrm{CC}=\mathrm{CC} 2=\mathrm{C} 1 \mathrm{C}(=\mathrm{O}) \mathrm{C} 1=\mathrm{C}(\mathrm{O}) \mathrm{C} 3=\mathrm{C}(\mathrm{C}[\mathrm{C} @](\mathrm{O})(\mathrm{C}[\mathrm{C} @ @ \mathrm{H}] 3 \mathrm{O}[\mathrm{C} @ \mathrm{H}] 3 \mathrm{C}[\mathrm{C} @ \mathrm{H}](\mathrm{N})[\mathrm{C} @ \mathrm{H}](\mathrm{O})[\mathrm{C} @ \mathrm{H}]( \\
\mathrm{C}) \mathrm{O} 3) \mathrm{C}(=\mathrm{O}) \mathrm{CO}) \mathrm{C}(\mathrm{O})=\mathrm{C} 1 \mathrm{C} 2=\mathrm{O}\end{array}$ & 6,38 \\
\hline 582 & $\begin{array}{l}\mathrm{COC} 1=\mathrm{CC}(\mathrm{C})=\mathrm{CC} 2=\mathrm{C} 1 \mathrm{C}(=\mathrm{O}) \mathrm{C}=\mathrm{C}(\mathrm{C} 2=\mathrm{O}) \mathrm{C} 1=\mathrm{C}(\mathrm{C}) \mathrm{C}=\mathrm{C} 2 \mathrm{C}(=\mathrm{O}) \mathrm{C}=\mathrm{C}(\mathrm{NC} 3=\mathrm{CC}=\mathrm{C}(\mathrm{Cl}) \mathrm{C}=\mathrm{C} 3) \mathrm{C}(=\mathrm{O}) \mathrm{C} 2=\mathrm{C} 1 \mathrm{O} \\
\mathrm{C}\end{array}$ & 6,19 \\
\hline 583 & $\mathrm{COC} 1=\mathrm{CC}(\mathrm{C})=\mathrm{CC} 2=\mathrm{C} 1 \mathrm{C}(=\mathrm{O}) \mathrm{C}=\mathrm{C}(\mathrm{C} 2=\mathrm{O}) \mathrm{C} 1=\mathrm{C}(\mathrm{C}) \mathrm{C}=\mathrm{C} 2 \mathrm{C}(=\mathrm{O}) \mathrm{C}=\mathrm{C}(\mathrm{NCC} 3=\mathrm{CC}=\mathrm{CC}=\mathrm{C} 3) \mathrm{C}(=\mathrm{O}) \mathrm{C} 2=\mathrm{C} 1 \mathrm{OC}$ & 5,58 \\
\hline 584 & $\mathrm{COC} 1=\mathrm{CC}(\mathrm{C})=\mathrm{CC} 2=\mathrm{C} 1 \mathrm{C}(=\mathrm{O}) \mathrm{C}=\mathrm{C}(\mathrm{C} 2=\mathrm{O}) \mathrm{C} 1=\mathrm{C}(\mathrm{C}) \mathrm{C}=\mathrm{C} 2 \mathrm{C}(=\mathrm{O}) \mathrm{C}=\mathrm{CC}(=\mathrm{O}) \mathrm{C} 2=\mathrm{C} 1 \mathrm{OC}$ & 6,41 \\
\hline 585 & $\mathrm{CCOC}(=\mathrm{O}) \mathrm{CNC} 1=\mathrm{CC}(=\mathrm{O}) \mathrm{C} 2=\mathrm{CC}(\mathrm{C})=\mathrm{C}(\mathrm{C}(\mathrm{OC})=\mathrm{C} 2 \mathrm{C} 1=\mathrm{O}) \mathrm{C} 1=\mathrm{CC}(=\mathrm{O}) \mathrm{C} 2=\mathrm{C}(\mathrm{OC}) \mathrm{C}=\mathrm{C}(\mathrm{C}) \mathrm{C}=\mathrm{C} 2 \mathrm{C} 1=\mathrm{O}$ & 6,07 \\
\hline 586 & $\mathrm{CCOC} 1=\mathrm{CC}(\mathrm{C})=\mathrm{CC} 2=\mathrm{C} 1 \mathrm{C}(=\mathrm{O}) \mathrm{C}=\mathrm{C}(\mathrm{C} 2=\mathrm{O}) \mathrm{C} 1=\mathrm{C}(\mathrm{C}) \mathrm{C}=\mathrm{C} 2 \mathrm{C}(=\mathrm{O}) \mathrm{C}=\mathrm{CC}(=\mathrm{O}) \mathrm{C} 2=\mathrm{C} 1 \mathrm{OCC}$ & 5,62 \\
\hline 587 & $\mathrm{COC} 1=\mathrm{CC}(\mathrm{C})=\mathrm{CC} 2=\mathrm{C} 1 \mathrm{C}(=\mathrm{O}) \mathrm{C}(\mathrm{N})=\mathrm{C}(\mathrm{C} 2=\mathrm{O}) \mathrm{C} 1=\mathrm{C}(\mathrm{C}) \mathrm{C}=\mathrm{C} 2 \mathrm{C}(=\mathrm{O}) \mathrm{C}=\mathrm{C}(\mathrm{N}) \mathrm{C}(=\mathrm{O}) \mathrm{C} 2=\mathrm{C} 1 \mathrm{OC}$ & 5,63 \\
\hline 588 & $\mathrm{COC} 1=\mathrm{CC}(\mathrm{C})=\mathrm{CC} 2=\mathrm{C} 1 \mathrm{C}(=\mathrm{O}) \mathrm{C}=\mathrm{C}(\mathrm{C} 2=\mathrm{O}) \mathrm{C} 1=\mathrm{C}(\mathrm{C}) \mathrm{C}=\mathrm{C} 2 \mathrm{C}(=\mathrm{O}) \mathrm{C}=\mathrm{C}(\mathrm{NC}(\mathrm{C})=\mathrm{O}) \mathrm{C}(=\mathrm{O}) \mathrm{C} 2=\mathrm{C} 1 \mathrm{OC}$ & 6,04 \\
\hline 589 & $\mathrm{CC} 1=\mathrm{CC}(\mathrm{O})=\mathrm{C} 2 \mathrm{C}(=\mathrm{O}) \mathrm{C}=\mathrm{C}(\mathrm{C}(=\mathrm{O}) \mathrm{C} 2=\mathrm{C} 1) \mathrm{C} 1=\mathrm{C}(\mathrm{C}) \mathrm{C}=\mathrm{C} 2 \mathrm{C}(=\mathrm{O}) \mathrm{C}=\mathrm{CC}(=\mathrm{O}) \mathrm{C} 2=\mathrm{C} 1 \mathrm{O}$ & 5,45 \\
\hline 590 & $\mathrm{COC} 1=\mathrm{CC}(\mathrm{C})=\mathrm{CC} 2=\mathrm{C} 1 \mathrm{C}(=\mathrm{O}) \mathrm{C}=\mathrm{C}(\mathrm{C} 2=\mathrm{O}) \mathrm{C} 1=\mathrm{C}(\mathrm{C}) \mathrm{C}=\mathrm{C} 2 \mathrm{C}(=\mathrm{O}) \mathrm{C}=\mathrm{C}(\mathrm{N}) \mathrm{C}(=\mathrm{O}) \mathrm{C} 2=\mathrm{C} 1 \mathrm{OC}$ & 5,45 \\
\hline 591 & $\begin{array}{l}\mathrm{COC} 1=\mathrm{CC}(\mathrm{C})=\mathrm{CC} 2=\mathrm{C} 1 \mathrm{C}(=\mathrm{O}) \mathrm{C}=\mathrm{C}(\mathrm{C} 2=\mathrm{O}) \mathrm{C} 1=\mathrm{C}(\mathrm{C}) \mathrm{C}=\mathrm{C} 2 \mathrm{C}(=\mathrm{O}) \mathrm{C}=\mathrm{C}(\mathrm{NC} 3=\mathrm{CC}=\mathrm{C} 4 \mathrm{C}=\mathrm{CC}=\mathrm{CC} 4=\mathrm{C} 3) \mathrm{C}(=\mathrm{O}) \mathrm{C} \\
2=\mathrm{C} 1 \mathrm{OC}\end{array}$ & 5,69 \\
\hline 592 & $\mathrm{CCN}(\mathrm{CC}) \mathrm{C} 1=\mathrm{CC}=\mathrm{C}(\backslash \mathrm{C}=\mathrm{N} \backslash \mathrm{NC}(=\mathrm{S}) \mathrm{NC} 2=\mathrm{CC}=\mathrm{CC}=\mathrm{C} 2) \mathrm{C}(\mathrm{O})=\mathrm{C} 1$ & 6,52 \\
\hline 593 & $\mathrm{CCN}(\mathrm{CC}) \mathrm{C} 1=\mathrm{CC}=\mathrm{C}(\mathrm{C}=\mathrm{N} \backslash \mathrm{NC}(\mathrm{N})=\mathrm{S}) \mathrm{C}(\mathrm{O})=\mathrm{C} 1$ & 6,05 \\
\hline 594 & $\mathrm{OC} 1=\mathrm{CC}=\mathrm{CC}=\mathrm{C} 1 \backslash \mathrm{C}=\mathrm{N} \backslash \mathrm{NC}(=\mathrm{S}) \mathrm{NC} 1=\mathrm{CC}=\mathrm{CC}=\mathrm{C} 1$ & 5,62 \\
\hline 595 & $\mathrm{COC} 1=\mathrm{CC}=\mathrm{CC}(\backslash \mathrm{C}=\mathrm{N} \backslash \mathrm{NC}(=\mathrm{S}) \mathrm{NC} 2=\mathrm{CC}=\mathrm{CC}=\mathrm{C} 2)=\mathrm{C} 1 \mathrm{O}$ & 5,8 \\
\hline 596 & $\mathrm{OC} 1=\mathrm{CC}=\mathrm{C} 2 \mathrm{C}=\mathrm{CC}=\mathrm{CC} 2=\mathrm{C} 1 \backslash \mathrm{C}=\mathrm{N} \backslash \mathrm{NC}(=\mathrm{S}) \mathrm{NC} 1=\mathrm{CC}=\mathrm{CC}=\mathrm{C} 1$ & 6,15 \\
\hline 597 & $\mathrm{COC} 1=\mathrm{CC}=\mathrm{CC}(\backslash \mathrm{C}=\mathrm{N} \backslash \mathrm{NC}(\mathrm{N})=\mathrm{S})=\mathrm{C} 1 \mathrm{O}$ & 5,85 \\
\hline 598 & $\mathrm{NC}(=\mathrm{S}) \mathrm{N} \backslash \mathrm{N}=\mathrm{C} \backslash \mathrm{C} 1=\mathrm{C}(\mathrm{O}) \mathrm{C}=\mathrm{CC} 2=\mathrm{C} 1 \mathrm{C}=\mathrm{CC}=\mathrm{C} 2$ & 6,7 \\
\hline 599 & $\mathrm{NC}(=\mathrm{S}) \mathrm{N} \backslash \mathrm{N}=\mathrm{C} \backslash \mathrm{C} 1=\mathrm{CC}=\mathrm{CC}=\mathrm{C} 1 \mathrm{O}$ & 5,3 \\
\hline 600 & 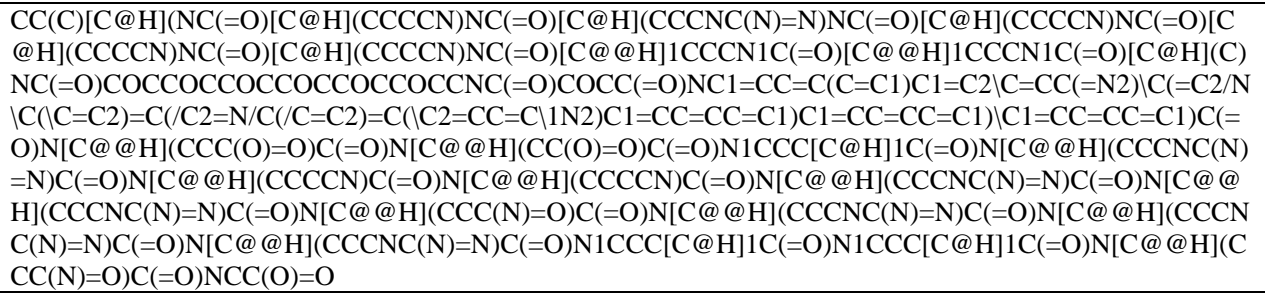 & 5,12 \\
\hline 601 & $\begin{array}{l}\mathrm{CC}(\mathrm{C})[\mathrm{C} @ \mathrm{H}](\mathrm{NC}(=\mathrm{O})[\mathrm{C} @ \mathrm{H}](\mathrm{CCCCN}) \mathrm{NC}(=\mathrm{O})[\mathrm{C} @ \mathrm{H}](\mathrm{CCCNC}(\mathrm{N})=\mathrm{N}) \mathrm{NC}(=\mathrm{O})[\mathrm{C} @ \mathrm{H}](\mathrm{CCCCN}) \mathrm{NC}(=\mathrm{O})[\mathrm{C} \\
@ \mathrm{H}](\mathrm{CCCCN}) \mathrm{NC}(=\mathrm{O})[\mathrm{C} @ \mathrm{H}](\mathrm{CCCCN}) \mathrm{NC}(=\mathrm{O})[\mathrm{C} @ @ \mathrm{H}] 1 \mathrm{CCCN} 1 \mathrm{C}(=\mathrm{O})[\mathrm{C} @ @ \mathrm{H}] 1 \mathrm{CCCN} 1 \mathrm{C}(=\mathrm{O})[\mathrm{C} @ \mathrm{H}](\mathrm{C})\end{array}$ & 4,47 \\
\hline \multicolumn{2}{|c|}{ https://biointerfaceresearch.com/ } & 2929 \\
\hline
\end{tabular}


$\mathrm{NC}(=\mathrm{O}) \operatorname{COCCOCCOCCOCCOCCOCCNC}(=\mathrm{O}) \mathrm{COCC}(=\mathrm{O}) \mathrm{NC} 1=\mathrm{CC}=\mathrm{C}(\mathrm{C}=\mathrm{C} 1) \mathrm{C} 1=\mathrm{C} 2 \backslash \mathrm{C}=\mathrm{CC}(=\mathrm{N} 2) \backslash \mathrm{C}(=\mathrm{C} 2 / \mathrm{N}$ $\mid \mathrm{C}(\mid \mathrm{C}=\mathrm{C} 2)=\mathrm{C}(/ \mathrm{C} 2=\mathrm{N} / \mathrm{C}(/ \mathrm{C}=\mathrm{C} 2)=\mathrm{C}(\backslash \mathrm{C} 2=\mathrm{CC}=\mathrm{C} 11 \mathrm{~N} 2) \mathrm{C} 1=\mathrm{CC}=\mathrm{CC}=\mathrm{C} 1) \mathrm{C} 1=\mathrm{CC}=\mathrm{CC}=\mathrm{C} 1) \backslash \mathrm{C} 1=\mathrm{CC}=\mathrm{CC}=\mathrm{C} 1) \mathrm{C}(=$ $\mathrm{O}) \mathrm{N}[\mathrm{C} @ \mathrm{H}](\mathrm{CCC}(\mathrm{O})=\mathrm{O}) \mathrm{C}(=\mathrm{O}) \mathrm{N}[\mathrm{C} @ \mathrm{H}](\mathrm{CC}(\mathrm{O})=\mathrm{O}) \mathrm{C}(=\mathrm{O}) \mathrm{N} 1 \mathrm{CCC}[\mathrm{C} @ \mathrm{H}] 1 \mathrm{C}(=\mathrm{O}) \mathrm{N}[\mathrm{C} @ @ \mathrm{H}](\mathrm{CCCNC}(\mathrm{N})$ $=\mathrm{N}) \mathrm{C}(=\mathrm{O}) \mathrm{N}[\mathrm{C} @ \mathrm{H}](\mathrm{CCCCN}) \mathrm{C}(=\mathrm{O}) \mathrm{N}[\mathrm{C} @ \mathrm{H}](\mathrm{CCCCN}) \mathrm{C}(=\mathrm{O}) \mathrm{N}[\mathrm{C} @ \mathrm{H}](\mathrm{CCCNC}(\mathrm{N})=\mathrm{N}) \mathrm{C}(=\mathrm{O}) \mathrm{N}[\mathrm{C} @ @$ $\mathrm{H}](\mathrm{CCCNC}(\mathrm{N})=\mathrm{N}) \mathrm{C}(=\mathrm{O}) \mathrm{N}[\mathrm{C} @ @ \mathrm{H}](\mathrm{CCC}(\mathrm{N})=\mathrm{O}) \mathrm{C}(=\mathrm{O}) \mathrm{N}[\mathrm{C} @ \mathrm{H}](\mathrm{CCCNC}(\mathrm{N})=\mathrm{N}) \mathrm{C}(=\mathrm{O}) \mathrm{N}[\mathrm{C} @ @ \mathrm{H}](\mathrm{CCCN}$ $\mathrm{C}(\mathrm{N})=\mathrm{N}) \mathrm{C}(=\mathrm{O}) \mathrm{N}[\mathrm{C} @ @ \mathrm{H}](\mathrm{CCCNC}(\mathrm{N})=\mathrm{N}) \mathrm{C}(=\mathrm{O}) \mathrm{N} 1 \mathrm{CCC}[\mathrm{C} @ \mathrm{H}] 1 \mathrm{C}(=\mathrm{O}) \mathrm{N} 1 \mathrm{CCC}[\mathrm{C} @ \mathrm{H}] 1 \mathrm{C}(=\mathrm{O}) \mathrm{N}[\mathrm{C} @ @ \mathrm{H}](\mathrm{C}$ $\mathrm{CC}(\mathrm{N})=\mathrm{O}) \mathrm{C}(=\mathrm{O}) \mathrm{NCC}(\mathrm{O})=\mathrm{O}$

$\mathrm{CC}[\mathrm{C} @ \mathrm{H}](\mathrm{C})[\mathrm{C} @ \mathrm{H}](\mathrm{NC}(=\mathrm{O})[\mathrm{C} @ \mathrm{H}](\mathrm{CCC}(\mathrm{N})=\mathrm{O}) \mathrm{NC}(=\mathrm{O})[\mathrm{C} @ \mathrm{H}](\mathrm{CCCNC}(\mathrm{N})=\mathrm{N}) \mathrm{NC}(=\mathrm{O}) \mathrm{COCCOCCOCCO}$ $\operatorname{CCOCCOCCNC}(=\mathrm{O}) \operatorname{COCC}(=\mathrm{O}) \mathrm{NC} 1=\mathrm{CC}=\mathrm{C}(\mathrm{C}=\mathrm{C} 1) \mathrm{C} 1=\mathrm{C} 2 \backslash \mathrm{C}=\mathrm{CC}(=\mathrm{N} 2) \backslash \mathrm{C}(=\mathrm{C} 2 / \mathrm{N} \backslash \mathrm{C}(\mathrm{C}=\mathrm{C} 2)=\mathrm{C}(/ \mathrm{C} 2=\mathrm{N} / \mathrm{C}(/ \mathrm{C}$ $=\mathrm{C} 2)=\mathrm{C}(\backslash \mathrm{C} 2=\mathrm{CC}=\mathrm{C} 11 \mathrm{~N} 2) \mathrm{C} 1=\mathrm{CC}=\mathrm{CC}=\mathrm{C} 1) \mathrm{C} 1=\mathrm{CC}=\mathrm{CC}=\mathrm{C} 1) \backslash \mathrm{C} 1=\mathrm{CC}=\mathrm{CC}=\mathrm{C} 1) \mathrm{C}(=\mathrm{O}) \mathrm{N}[\mathrm{C} @ @ \mathrm{H}](\mathrm{CCCCN}) \mathrm{C}($ $=\mathrm{O}) \mathrm{N}[\mathrm{C} @ \mathrm{H}]([\mathrm{C} @ \mathrm{H}](\mathrm{C}) \mathrm{CC}) \mathrm{C}(=\mathrm{O}) \mathrm{N}[\mathrm{C} @ \mathrm{H}](\mathrm{CC} 1=\mathrm{CNC} 2=\mathrm{CC}=\mathrm{CC}=\mathrm{C} 12) \mathrm{C}(=\mathrm{O}) \mathrm{N}[\mathrm{C} @ @ \mathrm{H}](\mathrm{CC} 1=\mathrm{CC}=$ $\mathrm{CC}=\mathrm{C} 1) \mathrm{C}(=\mathrm{O}) \mathrm{N}[\mathrm{C} @ \mathrm{H}](\mathrm{CCC}(\mathrm{N})=\mathrm{O}) \mathrm{C}(=\mathrm{O}) \mathrm{N}[\mathrm{C} @ @ \mathrm{H}](\mathrm{CC}(\mathrm{N})=\mathrm{O}) \mathrm{C}(=\mathrm{O}) \mathrm{N}[\mathrm{C} @ @ \mathrm{H}](\mathrm{CCCNC}(\mathrm{N})=\mathrm{N}) \mathrm{C}(=\mathrm{O})$ $\mathrm{N}[\mathrm{C} @ \mathrm{H}](\mathrm{CCCNC}(\mathrm{N})=\mathrm{N}) \mathrm{C}(=\mathrm{O}) \mathrm{N}[\mathrm{C} @ \mathrm{H}](\mathrm{CCSC}) \mathrm{C}(=\mathrm{O}) \mathrm{N}[\mathrm{C} @ \mathrm{H}](\mathrm{CCCCN}) \mathrm{C}(=\mathrm{O}) \mathrm{N}[\mathrm{C} @ @ \mathrm{H}](\mathrm{CC} 1=\mathrm{CN}$ $\mathrm{C} 2=\mathrm{CC}=\mathrm{CC}=\mathrm{C} 12) \mathrm{C}(=\mathrm{O}) \mathrm{N}[\mathrm{C} @ @ \mathrm{H}](\mathrm{CCCCN}) \mathrm{C}(=\mathrm{O}) \mathrm{N}[\mathrm{C} @ \mathrm{H}](\mathrm{CCCCN}) \mathrm{C}(=\mathrm{O}) \mathrm{N}[\mathrm{C} @ \mathrm{H}](\mathrm{C}) \mathrm{C}(=\mathrm{O}) \mathrm{N} 1 \mathrm{CCC}$ $[\mathrm{C} @ \mathrm{H}] 1 \mathrm{C}(=\mathrm{O}) \mathrm{N} 1 \mathrm{CCC}[\mathrm{C} @ \mathrm{H}] 1 \mathrm{C}(=\mathrm{O}) \mathrm{N}[\mathrm{C} @ @ \mathrm{H}](\mathrm{CCCCN}) \mathrm{C}(=\mathrm{O}) \mathrm{N}[\mathrm{C} @ @ \mathrm{H}](\mathrm{CCCCN}) \mathrm{C}(=\mathrm{O}) \mathrm{N}[\mathrm{C} @ @ \mathrm{H}](\mathrm{CC}$ $\mathrm{CCN}) \mathrm{C}(=\mathrm{O}) \mathrm{N}[\mathrm{C} @ \mathrm{H}](\mathrm{CCCNC}(\mathrm{N})=\mathrm{N}) \mathrm{C}(=\mathrm{O}) \mathrm{N}[\mathrm{C} @ @ \mathrm{H}](\mathrm{CCCCN}) \mathrm{C}(=\mathrm{O}) \mathrm{N}[\mathrm{C} @ @ \mathrm{H}](\mathrm{C}(\mathrm{C}) \mathrm{C}) \mathrm{C}(=\mathrm{O}) \mathrm{NCC}(\mathrm{O})$ $602=\mathrm{O}$

$\mathrm{CC}[\mathrm{C} @ \mathrm{H}](\mathrm{C})[\mathrm{C} @ \mathrm{H}](\mathrm{NC}(=\mathrm{O})[\mathrm{C} @ \mathrm{H}](\mathrm{CCC}(\mathrm{N})=\mathrm{O}) \mathrm{NC}(=\mathrm{O})[\mathrm{C} @ \mathrm{H}](\mathrm{CCCNC}(\mathrm{N})=\mathrm{N}) \mathrm{NC}(=\mathrm{O}) \mathrm{COCCOCCOCCO}$ $\operatorname{CCOCCOCCNC}(=\mathrm{O}) \operatorname{COCC}(=\mathrm{O}) \mathrm{NC} 1=\mathrm{CC}=\mathrm{C}(\mathrm{C}=\mathrm{C} 1) \mathrm{C} 1=\mathrm{C} 2 \backslash \mathrm{C}=\mathrm{CC}(=\mathrm{N} 2) \backslash \mathrm{C}(=\mathrm{C} 2 / \mathrm{N} \mid \mathrm{C}(\backslash \mathrm{C}=\mathrm{C} 2)=\mathrm{C}(/ \mathrm{C} 2=\mathrm{N} / \mathrm{C}(/ \mathrm{C}$ $=\mathrm{C} 2)=\mathrm{C}(\mathrm{C} 2=\mathrm{CC}=\mathrm{C} 1 \mathrm{~N} 2) \mathrm{C} 1=\mathrm{CC}=\mathrm{CC}=\mathrm{C} 1) \mathrm{C} 1=\mathrm{CC}=\mathrm{CC}=\mathrm{C} 1) \backslash \mathrm{C} 1=\mathrm{CC}=\mathrm{CC}=\mathrm{C} 1) \mathrm{C}(=\mathrm{O}) \mathrm{N}[\mathrm{C} @ @ \mathrm{H}](\mathrm{CCCCN}) \mathrm{C}($ $=\mathrm{O}) \mathrm{N}[\mathrm{C} @ \mathrm{H}]([\mathrm{C} @ @ \mathrm{H}](\mathrm{C}) \mathrm{CC}) \mathrm{C}(=\mathrm{O}) \mathrm{N}[\mathrm{C} @ \mathrm{H}](\mathrm{CC} 1=\mathrm{CNC} 2=\mathrm{CC}=\mathrm{CC}=\mathrm{C} 12) \mathrm{C}(=\mathrm{O}) \mathrm{N}[\mathrm{C} @ @ \mathrm{H}](\mathrm{CC} 1=\mathrm{CC}=$ $\mathrm{CC}=\mathrm{C} 1) \mathrm{C}(=\mathrm{O}) \mathrm{N}[\mathrm{C} @ \mathrm{H}](\mathrm{CCC}(\mathrm{N})=\mathrm{O}) \mathrm{C}(=\mathrm{O}) \mathrm{N}[\mathrm{C} @ @ \mathrm{H}](\mathrm{CC}(\mathrm{N})=\mathrm{O}) \mathrm{C}(=\mathrm{O}) \mathrm{N}[\mathrm{C} @ @ \mathrm{H}](\mathrm{CCCNC}(\mathrm{N})=\mathrm{N}) \mathrm{C}(=\mathrm{O})$ $\mathrm{N}[\mathrm{C} @ @ \mathrm{H}](\mathrm{CCCNC}(\mathrm{N})=\mathrm{N}) \mathrm{C}(=\mathrm{O}) \mathrm{N}[\mathrm{C} @ @ \mathrm{H}](\mathrm{CCSC}) \mathrm{C}(=\mathrm{O}) \mathrm{N}[\mathrm{C} @ @ \mathrm{H}](\mathrm{CCCCN}) \mathrm{C}(=\mathrm{O}) \mathrm{N}[\mathrm{C} @ @ \mathrm{H}](\mathrm{CC} 1=\mathrm{CN}$ $\mathrm{C} 2=\mathrm{CC}=\mathrm{CC}=\mathrm{C} 12) \mathrm{C}(=\mathrm{O}) \mathrm{N}[\mathrm{C} @ @ \mathrm{H}](\mathrm{CCCCN}) \mathrm{C}(=\mathrm{O}) \mathrm{N}[\mathrm{C} @ \mathrm{H}](\mathrm{CCCCN}) \mathrm{C}(=\mathrm{O}) \mathrm{N}[\mathrm{C} @ \mathrm{H}](\mathrm{C}) \mathrm{C}(=\mathrm{O}) \mathrm{N} 1 \mathrm{CCC}$ $[\mathrm{C} @ \mathrm{H}] 1 \mathrm{C}(=\mathrm{O}) \mathrm{N} 1 \mathrm{CCC}[\mathrm{C} @ \mathrm{H}] 1 \mathrm{C}(=\mathrm{O}) \mathrm{N}[\mathrm{C} @ @ \mathrm{H}](\mathrm{CCCCN}) \mathrm{C}(=\mathrm{O}) \mathrm{N}[\mathrm{C} @ @ \mathrm{H}](\mathrm{CCCCN}) \mathrm{C}(=\mathrm{O}) \mathrm{N}[\mathrm{C} @ @ \mathrm{H}](\mathrm{CC}$ $\mathrm{CCN}) \mathrm{C}(=\mathrm{O}) \mathrm{N}[\mathrm{C} @ @ \mathrm{H}](\mathrm{CCCNC}(\mathrm{N})=\mathrm{N}) \mathrm{C}(=\mathrm{O}) \mathrm{N}[\mathrm{C} @ @ \mathrm{H}](\mathrm{CCCCN}) \mathrm{C}(=\mathrm{O}) \mathrm{N}[\mathrm{C} @ @ \mathrm{H}](\mathrm{C}(\mathrm{C}) \mathrm{C}) \mathrm{C}(=\mathrm{O}) \mathrm{NCC}(\mathrm{O})$ $603=\mathrm{O}$

$604 \quad \mathrm{Cl} . \mathrm{NC}(=\mathrm{N}) \mathrm{C} 1=\mathrm{CC}=\mathrm{C} 2 \mathrm{~N}=\mathrm{C}(\mathrm{NC} 2=\mathrm{C} 1) \backslash \mathrm{C}=\mathrm{ClC} 1=\mathrm{CC}=\mathrm{CO} 1$

$605 \quad \mathrm{Cl} . \mathrm{C} 1 \mathrm{CN}=\mathrm{C}(\mathrm{N} 1) \mathrm{C} 1=\mathrm{CC}=\mathrm{C} 2 \mathrm{~N}=\mathrm{C}(\mathrm{NC} 2=\mathrm{C} 1) \backslash \mathrm{C}=\mathrm{ClC} 1=\mathrm{CC}=\mathrm{CS} 1$

$606 \mathrm{Cl} \cdot \mathrm{C} 1 \mathrm{CN}=\mathrm{C}(\mathrm{N} 1) \mathrm{C} 1=\mathrm{CC}=\mathrm{C} 2 \mathrm{~N}=\mathrm{C}(\mathrm{NC} 2=\mathrm{C} 1) \backslash \mathrm{C}=\mathrm{ClC} 1=\mathrm{CC}=\mathrm{CO} 1$

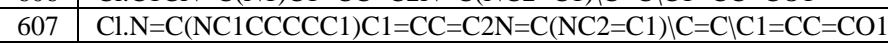

$608 \mathrm{CC} 1=\mathrm{CC}=\mathrm{C}(\mathrm{C}=\mathrm{C} 1)[\mathrm{C} @ \mathrm{H}] 1 \mathrm{OC}(=\mathrm{O})[\mathrm{C} @ @ \mathrm{H}] 2 \mathrm{C}=\mathrm{C} 3[\mathrm{C} @](\mathrm{C})(\mathrm{CCCC} 3(\mathrm{C}) \mathrm{C})[\mathrm{C} @ \mathrm{H}] 12$

$609 \mathrm{C}[\mathrm{C} @] 12 \mathrm{CCCC}(\mathrm{C})(\mathrm{C}) \mathrm{C} 1=\mathrm{C}[\mathrm{C} @ @ \mathrm{H}] 1[\mathrm{C} @ \mathrm{H}] 2[\mathrm{C} @ \mathrm{H}](\mathrm{OC} 1=\mathrm{O}) \mathrm{C} 1=\mathrm{CC}=\mathrm{CO} 1$

$610 \mathrm{COC} 1=\mathrm{CC}=\mathrm{C}(\mathrm{C}=\mathrm{C} 1)[\mathrm{C} @ \mathrm{H}] 1 \mathrm{OC}(=\mathrm{O})[\mathrm{C} @ @ \mathrm{H}] 2 \mathrm{C}=\mathrm{C} 3[\mathrm{C} @](\mathrm{C})(\mathrm{CCCC} 3(\mathrm{C}) \mathrm{C})[\mathrm{C} @ \mathrm{H}] 12$

$611 \quad \mathrm{C}[\mathrm{C} @] 12 \mathrm{CCCC}(\mathrm{C})(\mathrm{C}) \mathrm{C} 1=\mathrm{C}[\mathrm{C} @ @ \mathrm{H}] 1[\mathrm{C} @ \mathrm{H}] 2[\mathrm{C} @ \mathrm{H}](\mathrm{OC} 1=\mathrm{O}) \mathrm{C} 1=\mathrm{CC}=\mathrm{C}(\mathrm{Br}) \mathrm{C}=\mathrm{C} 1$

$612 \mathrm{C}[\mathrm{C} @] 12 \mathrm{CCCC}(\mathrm{C})(\mathrm{C}) \mathrm{C} 1=\mathrm{C}[\mathrm{C} @ @ \mathrm{H}] 1[\mathrm{C} @ \mathrm{H}] 2[\mathrm{C} @ \mathrm{H}](\mathrm{OC} 1=\mathrm{O}) \mathrm{C} 1=\mathrm{CC}=\mathrm{CC}=\mathrm{C} 1$

$613 \mathrm{C}[\mathrm{C} @] 12 \mathrm{CCCC}(\mathrm{C})(\mathrm{C}) \mathrm{C} 1=\mathrm{C}[\mathrm{C} @ @ \mathrm{H}] 1[\mathrm{C} @ \mathrm{H}] 2[\mathrm{C} @ \mathrm{H}](\mathrm{OC} 1=\mathrm{O}) \mathrm{C} 1=\mathrm{CC}=\mathrm{C}(\mathrm{Cl}) \mathrm{C}=\mathrm{C} 1$

$614 \mathrm{C}[\mathrm{C} @] 12 \mathrm{CCCC}(\mathrm{C})(\mathrm{C}) \mathrm{C} 1=\mathrm{C}[\mathrm{C} @ @ \mathrm{H}] 1[\mathrm{C} @ \mathrm{H}] 2[\mathrm{C} @ \mathrm{H}](\mathrm{OC} 1=\mathrm{O}) \mathrm{C} 1=\mathrm{CC}=\mathrm{C}(\mathrm{C}=\mathrm{C} 1) \mathrm{C} \# \mathrm{~N}$

$615 \mathrm{C}[\mathrm{C} @] 12 \mathrm{CCCC}(\mathrm{C})(\mathrm{C}) \mathrm{C} 1=\mathrm{C}[\mathrm{C} @ @ \mathrm{H}] 1[\mathrm{C} @ \mathrm{H}] 2[\mathrm{C} @ \mathrm{H}](\mathrm{OC} 1=\mathrm{O}) \mathrm{C} 1=\mathrm{CC}=\mathrm{C}(\mathrm{F}) \mathrm{C}=\mathrm{C} 1$

$616 \mathrm{CC}[\mathrm{C} @ @] 1(\mathrm{O}) \mathrm{C}(=\mathrm{O}) \mathrm{OCC} 2=\mathrm{C} 1 \mathrm{C}=\mathrm{C} 1 \mathrm{~N}(\mathrm{CC} 3=\mathrm{CC} 4=\mathrm{C}(\mathrm{C}=\mathrm{CC}=\mathrm{C} 4) \mathrm{N}=\mathrm{C} 13) \mathrm{C} 2=\mathrm{O}$

$17 \mathrm{C}$

$\mathrm{C}[\mathrm{C} @ \mathrm{H}] 1 \mathrm{C}(=\mathrm{O}) \mathrm{O}[\mathrm{C} @ \mathrm{H}] 2[\mathrm{C} @ @ \mathrm{H}](\mathrm{Cl}) \mathrm{C}(=\mathrm{C}) \backslash \mathrm{C}=\mathrm{C} \backslash[\mathrm{C} @ \mathrm{H}](\mathrm{OC}(\mathrm{C})=\mathrm{O})[\mathrm{C} @ @] 3(\mathrm{C})[\mathrm{C} @ @ \mathrm{H}](\mathrm{OC}(\mathrm{C})=\mathrm{O})[\mathrm{C} @$

$617 \mathrm{H}](\mathrm{CC}(=\mathrm{C})[\mathrm{C} @ @ \mathrm{H}] 3[\mathrm{C} @ \mathrm{H}](\mathrm{OC}(\mathrm{C})=\mathrm{O})[\mathrm{C} @] 12 \mathrm{O}) \mathrm{OC}(\mathrm{C})=\mathrm{O}$

$\mathrm{CC}(\mathrm{C}) \mathrm{CC}(=\mathrm{O}) \mathrm{OCC}(=\mathrm{O}) \mathrm{O}[\mathrm{C} @ \mathrm{H}] 11 \mathrm{C}=\mathrm{C} / \mathrm{C}(=\mathrm{C})[\mathrm{C} @ \mathrm{H}](\mathrm{Cl})[\mathrm{C} @ @ \mathrm{H}] 2 \mathrm{OC}(=\mathrm{O})[\mathrm{C} @ \mathrm{H}](\mathrm{C})[\mathrm{C} @ @] 2(\mathrm{O})[\mathrm{C} @ @ \mathrm{H}$

618

]$(\mathrm{OC}(\mathrm{C})=\mathrm{O})[\mathrm{C} @ \mathrm{H}] 2 \mathrm{C}(=\mathrm{C}) \mathrm{CC}[\mathrm{C} @ \mathrm{H}](\mathrm{OC}(\mathrm{C})=\mathrm{O})[\mathrm{C} @] 12 \mathrm{C}$

$\mathrm{C}[\mathrm{C} @ \mathrm{H}] 1 \mathrm{C}(=\mathrm{O}) \mathrm{O}[\mathrm{C} @ \mathrm{H}] 2[\mathrm{C} @ @ \mathrm{H}](\mathrm{Cl}) \mathrm{C}(=\mathrm{C})[\mathrm{C} @ @] 3(\mathrm{O}) \mathrm{C}[\mathrm{C} @ \mathrm{H}](\mathrm{OC}(\mathrm{C})=\mathrm{O})[\mathrm{C} @ @] 4(\mathrm{C})[\mathrm{C} @ @ \mathrm{H}](\mathrm{OC}(\mathrm{C})$

619

$=\mathrm{O})[\mathrm{C} @ \mathrm{H}](\mathrm{CC}(=\mathrm{C})[\mathrm{C} @ @ \mathrm{H}] 4[\mathrm{C} @ \mathrm{H}](\mathrm{OC}(\mathrm{C})=\mathrm{O})[\mathrm{C} @] 12 \mathrm{O} 3) \mathrm{OC}(\mathrm{C})=\mathrm{O}$

$\mathrm{CCC}(=\mathrm{O}) \mathrm{O}[\mathrm{C} @ @ \mathrm{H}] 1 \mathrm{C}[\mathrm{C} @ \mathrm{H}](\mathrm{OC}(\mathrm{C})=\mathrm{O})[\mathrm{C} @ @] 2(\mathrm{C})[\mathrm{C} @ \mathrm{H}]([\mathrm{C} @ \mathrm{H}](\mathrm{OC}(\mathrm{C})=\mathrm{O})[\mathrm{C} @] 3(\mathrm{O})[\mathrm{C} @ @ \mathrm{H}](\mathrm{C}) \mathrm{C}$

$620=\mathrm{O}) \mathrm{O}[\mathrm{C} @ \mathrm{H}] 3 \mathrm{iC}=\mathrm{C}(\mathrm{C}) / \mathrm{CC}[\mathrm{C} @ @ \mathrm{H}] 2 \mathrm{OC}(\mathrm{C})=\mathrm{O}) \mathrm{C} 1=\mathrm{C}$

$\mathrm{C}[\mathrm{C} @ \mathrm{H}] 1 \mathrm{C}(=\mathrm{O}) \mathrm{O}[\mathrm{C} @ \mathrm{H}] 2 \mathrm{C}=\mathrm{C}(\mathrm{C}) / \mathrm{CC}[\mathrm{C} @ \mathrm{H}](\mathrm{OC}(\mathrm{C})=\mathrm{O})[\mathrm{C} @ @] 3(\mathrm{C})[\mathrm{C} @ @ \mathrm{H}](\mathrm{OC}(\mathrm{C})=\mathrm{O})[\mathrm{C} @ \mathrm{H}](\mathrm{CC}(=\mathrm{C})[$

$621 \mathrm{C} @ @ \mathrm{H}] 3[\mathrm{C} @ \mathrm{H}](\mathrm{OC}(\mathrm{C})=\mathrm{O})[\mathrm{C} @] 12 \mathrm{O}) \mathrm{OC}(\mathrm{C})=\mathrm{O}$

$\mathrm{C}[\mathrm{C} @ \mathrm{H}] 1 \mathrm{C}(=\mathrm{O}) \mathrm{O}[\mathrm{C} @ \mathrm{H}] 2[\mathrm{C} @ @ \mathrm{H}](\mathrm{Cl}) \mathrm{C}(=\mathrm{C}) \backslash \mathrm{C}=\mathrm{Cl}[\mathrm{C} @ \mathrm{H}](\mathrm{OC}(\mathrm{C})=\mathrm{O})[\mathrm{C} @ @] 3(\mathrm{C})[\mathrm{C} @ @ \mathrm{H}](\mathrm{OC}(\mathrm{C})=\mathrm{O})[\mathrm{C} @$

$622 \mathrm{H}](\mathrm{C}[\mathrm{C} @] 4(\mathrm{CO} 4)[\mathrm{C} @ @ \mathrm{H}] 3[\mathrm{C} @ \mathrm{H}](\mathrm{OC}(\mathrm{C})=\mathrm{O})[\mathrm{C} @] 12 \mathrm{O}) \mathrm{OC}(\mathrm{C})=\mathrm{O}$

$\mathrm{CC}(\mathrm{C}) \mathrm{CC}(=\mathrm{O}) \mathrm{OCC}(=\mathrm{O}) \mathrm{O}[\mathrm{C} @ \mathrm{H}] 1 \mathrm{C}=\mathrm{C} / \mathrm{C}(=\mathrm{C})[\mathrm{C} @ \mathrm{H}](\mathrm{Cl})[\mathrm{C} @ @ \mathrm{H}] 2 \mathrm{OC}(=\mathrm{O})[\mathrm{C} @ \mathrm{H}](\mathrm{C})[\mathrm{C} @ @] 2(\mathrm{O})[\mathrm{C} @ @ \mathrm{H}$

$623](\mathrm{OC}(\mathrm{C})=\mathrm{O})[\mathrm{C} @ \mathrm{H}] 2[\mathrm{C} @ @] 3(\mathrm{CO} 3) \mathrm{CC}[\mathrm{C} @ \mathrm{H}](\mathrm{OC}(\mathrm{C})=\mathrm{O})[\mathrm{C} @] 12 \mathrm{C}$

$\mathrm{CC}(=\mathrm{O}) \mathrm{O}[\mathrm{C} @ \mathrm{H}] 1 \mathrm{C}[\mathrm{C} @ \mathrm{H}] 2 \mathrm{CC}[\mathrm{C} @ @](\mathrm{C})(\mathrm{OC}(=\mathrm{O}) \mathrm{C} 2=\mathrm{C})[\mathrm{C} @ @ \mathrm{H}](\mathrm{CC}[\mathrm{C} @ @](\mathrm{C})(\mathrm{O})[\mathrm{C} @ @ \mathrm{H}] 2 \mathrm{CC}[\mathrm{C} @ @]$

624

$1(\mathrm{C}) \mathrm{O} 2) \mathrm{OC}(\mathrm{C})=\mathrm{O}$

$625 \mathrm{O} 2$

$\mathrm{CC}(=\mathrm{O}) \mathrm{O}[\mathrm{C} @ \mathrm{H}] 1 \mathrm{C}[\mathrm{C} @ \mathrm{H}] 2 \mathrm{CC}[\mathrm{C} @ @](\mathrm{C})(\mathrm{OC}(=\mathrm{O}) \mathrm{C} 2=\mathrm{C}) \mathrm{C}(=\mathrm{O}) \mathrm{CC}[\mathrm{C} @ @](\mathrm{C})(\mathrm{O})[\mathrm{C} @ @ \mathrm{H}] 2 \mathrm{CC}[\mathrm{C} @ @] 1(\mathrm{C})$

$\mathrm{CC}(=\mathrm{O}) \mathrm{O}[\mathrm{C} @ \mathrm{H}] 1 \mathrm{CC}[\mathrm{C} @ @](\mathrm{C})(\mathrm{O})[\mathrm{C} @ @ \mathrm{H}] 2 \mathrm{CC}[\mathrm{C} @ @](\mathrm{C})(\mathrm{O} 2)[\mathrm{C} @ @ \mathrm{H}](\mathrm{O}) \mathrm{C}[\mathrm{C} @ \mathrm{H}] 2 \mathrm{CC}[\mathrm{C} @ @] 1(\mathrm{C}) \mathrm{O}$

626

627

$\mathrm{C}(=\mathrm{O}) \mathrm{C} 2=\mathrm{C}$

$\mathrm{CC}(=\mathrm{O}) \mathrm{O}[\mathrm{C} @ \mathrm{H}] 1 \mathrm{CC}[\mathrm{C} @ @] 2(\mathrm{C}) \mathrm{O}[\mathrm{C} @] 1(\mathrm{C}) \mathrm{CCC}(=\mathrm{O})[\mathrm{C} @ @] 1(\mathrm{C}) \mathrm{CC}[\mathrm{C} @ \mathrm{H}](\mathrm{C}[\mathrm{C} @ @ \mathrm{H}] 2 \mathrm{O}) \mathrm{C}(=\mathrm{C}) \mathrm{C}(=\mathrm{O}) \mathrm{O}$

$628 \mathrm{C}[\mathrm{C} @] 12 \mathrm{CC}[\mathrm{C} @ \mathrm{H}](\mathrm{O} 1)[\mathrm{C} @](\mathrm{C})(\mathrm{O}) \mathrm{CCC}=\mathrm{O})[\mathrm{C} @ @] 1(\mathrm{C}) \mathrm{CC}[\mathrm{C} @ \mathrm{H}](\mathrm{C}[\mathrm{C} @ @ \mathrm{H}] 2 \mathrm{O}) \mathrm{C}(=\mathrm{C}) \mathrm{C}(=\mathrm{O}) \mathrm{O} 1$

$\mathrm{C}(=0) \mathrm{C}$

$629 \quad \mathrm{C}[\mathrm{C} @] 12 \mathrm{CC}[\mathrm{C} @ \mathrm{H}](\mathrm{O} 1)[\mathrm{C} @](\mathrm{C})(\mathrm{O}) \mathrm{CC}[\mathrm{C} @ @ \mathrm{H}](\mathrm{O})[\mathrm{C} @ @] 1(\mathrm{C}) \mathrm{CC}[\mathrm{C} @ \mathrm{H}](\mathrm{C}[\mathrm{C} @ @ \mathrm{H}] 2 \mathrm{O}) \mathrm{C}(=\mathrm{C}) \mathrm{C}(=\mathrm{O}) \mathrm{O} 1$

$630 \quad=\mathrm{C}$

$\mathrm{CC}(=\mathrm{O}) \mathrm{O}[\mathrm{C} @ \mathrm{H}] 1 \mathrm{CCC}=\mathrm{C})[\mathrm{C} @ @ \mathrm{H}] 2 \mathrm{CC}[\mathrm{C} @ @](\mathrm{C})(\mathrm{O} 2)[\mathrm{C} @ @ \mathrm{H}](\mathrm{O}) \mathrm{C}[\mathrm{C} @ \mathrm{H}] 2 \mathrm{CC}[\mathrm{C} @ @] 1(\mathrm{C}) \mathrm{OC}(=\mathrm{O}) \mathrm{C} 2$

$\mathrm{CC}(=\mathrm{O}) \mathrm{O}[\mathrm{C} @ \mathrm{H}] 1 \mathrm{C}[\mathrm{C} @ \mathrm{H}] 2 \mathrm{CC}[\mathrm{C} @ @](\mathrm{C})(\mathrm{OC}(=\mathrm{O}) \mathrm{C} 2=\mathrm{C})[\mathrm{C} @ @ \mathrm{H}](\mathrm{CCC}=\mathrm{C})[\mathrm{C} @ @ \mathrm{H}] 2 \mathrm{CC}[\mathrm{C} @ @] 1(\mathrm{C}) \mathrm{O} 2)$

$631 \mathrm{OC}(\mathrm{C})=\mathrm{O}$

$\mathrm{CC}[\mathrm{C} @] 1(\mathrm{O}) \mathrm{C}[\mathrm{C} @ \mathrm{H}] 2 \mathrm{CN}(\mathrm{C} 1) \mathrm{CCC} 1=\mathrm{C}(\mathrm{NC} 3=\mathrm{CC}=\mathrm{CC}=\mathrm{C} 13)[\mathrm{C} @ @](\mathrm{C} 2)(\mathrm{C}(=\mathrm{O}) \mathrm{OC}) \mathrm{C} 1=\mathrm{CC} 2=\mathrm{C}(\mathrm{C}=\mathrm{C} 1 \mathrm{OC})$

$632 \mathrm{~N}(\mathrm{C}=\mathrm{O})[\mathrm{C} @ @ \mathrm{H}] 1[\mathrm{C} @] 22 \mathrm{CCN} 3 \mathrm{CC}=\mathrm{C}[\mathrm{C} @](\mathrm{CC})([\mathrm{C} @ @ \mathrm{H}] 23)[\mathrm{C} @ @ \mathrm{H}](\mathrm{OC}(\mathrm{C})=\mathrm{O})[\mathrm{C} @] 1(\mathrm{O}) \mathrm{C}(=\mathrm{O}) \mathrm{OC}$

$\mathrm{C}[\mathrm{C} @ \mathrm{H}](\mathrm{OC}(\mathrm{C})=\mathrm{O})[\mathrm{C} @ \mathrm{H}](\mathrm{OC}(\mathrm{C})=\mathrm{O})[\mathrm{C} @ @ \mathrm{H}](\mathrm{OC}(\mathrm{C})=\mathrm{O})[\mathrm{C} @ @ \mathrm{H}](\mathrm{OC}(\mathrm{C})=\mathrm{O}) \backslash \mathrm{C}=\mathrm{C} /[\mathrm{C} @ \mathrm{H}] 1 \mathrm{CC}=\mathrm{CC}(=\mathrm{O})$ $633 \mathrm{O} 1$

$634 \mathrm{C}[\mathrm{C} @ \mathrm{H}](\mathrm{OC}(\mathrm{C})=\mathrm{O})[\mathrm{C} @ @ \mathrm{H}](\mathrm{C}[\mathrm{C} @ \mathrm{H}](\mathrm{OC}(\mathrm{C})=\mathrm{O}) \backslash \mathrm{C}=\mathrm{C} /[\mathrm{C} @ \mathrm{H}] 1 \mathrm{CC}=\mathrm{CC}(=\mathrm{O}) \mathrm{O} 1) \mathrm{OC}(\mathrm{C})=\mathrm{O}$

$635 \mathrm{CC} 1=\mathrm{C} 2 \mathrm{NC} 3=\mathrm{C}(\mathrm{C}=\mathrm{CC}=\mathrm{C} 3) \mathrm{C} 2=\mathrm{C}(\mathrm{C}) \mathrm{C} 2=\mathrm{C} 1 \mathrm{C}=\mathrm{CN}=\mathrm{C} 2$

$636 \mathrm{CC} 1=\mathrm{CC}=\mathrm{NC}(\mathrm{NC} 2=\mathrm{NC} 3=\mathrm{CC}=\mathrm{C}(\mathrm{C}=\mathrm{C} 3 \mathrm{~S} 2) \mathrm{CHN})=\mathrm{C} 1$

$637 \mathrm{ClC} 1=\mathrm{CC}=\mathrm{C}(\mathrm{NC} 2=\mathrm{NC} 3=\mathrm{CC}=\mathrm{C}(\mathrm{C}=\mathrm{C} 3 \mathrm{~S} 2) \mathrm{C} \# \mathrm{~N}) \mathrm{N}=\mathrm{C} 1$ 


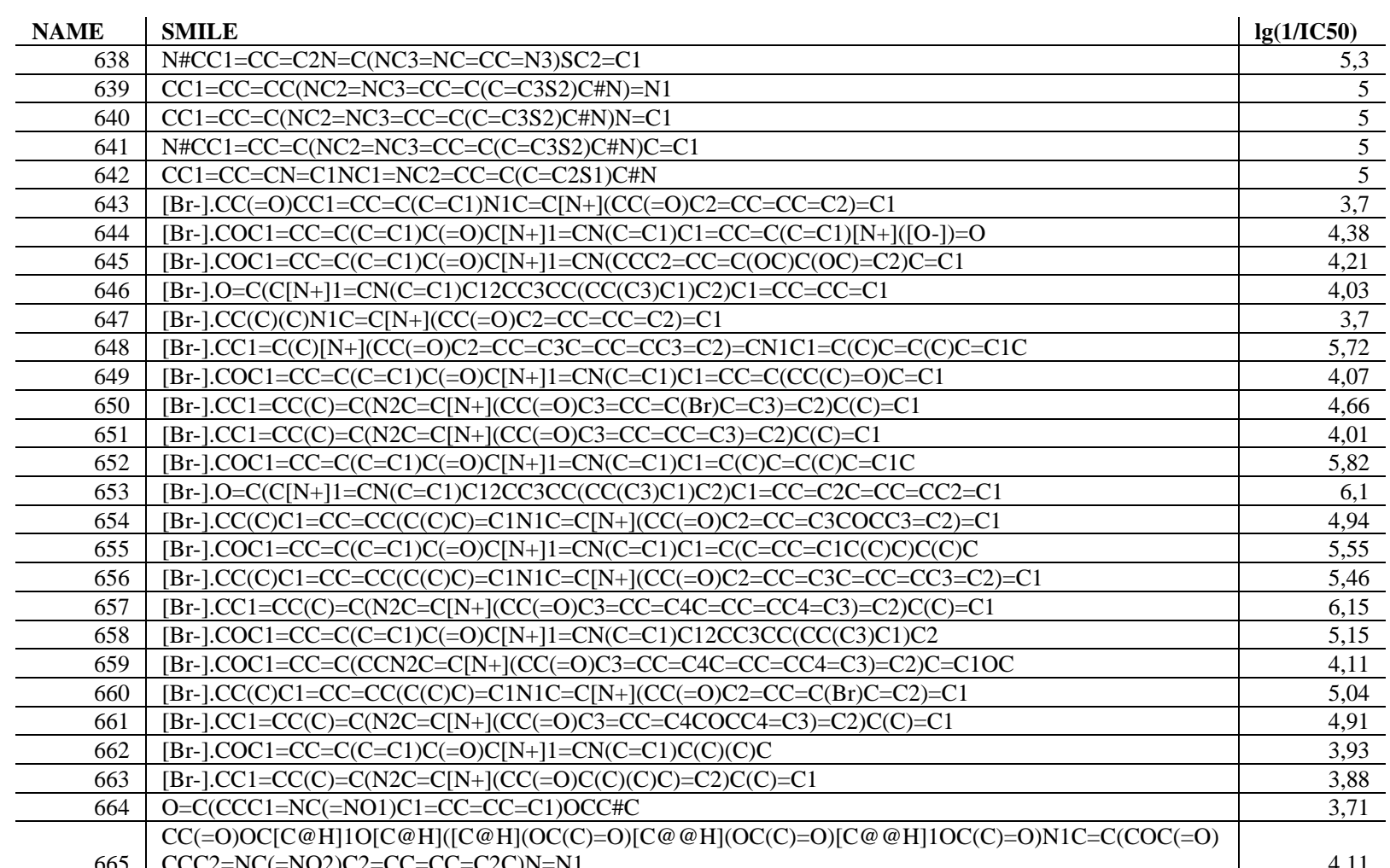

665

666

$\mathrm{CC}(=\mathrm{O}) \mathrm{OC}[\mathrm{C} @ \mathrm{H}] 1 \mathrm{O}[\mathrm{C} @ \mathrm{H}]([\mathrm{C} @ \mathrm{H}](\mathrm{OC}(\mathrm{C})=\mathrm{O})[\mathrm{C} @ @ \mathrm{H}](\mathrm{OC}(\mathrm{C})=\mathrm{O})[\mathrm{C} @ @ \mathrm{H}] 1 \mathrm{OC}(\mathrm{C})=\mathrm{O}) \mathrm{N} 1 \mathrm{C}=\mathrm{C}(\mathrm{COC}(=\mathrm{O})$

$\mathrm{CC}(=\mathrm{O}) \mathrm{OC}[\mathrm{C} @ \mathrm{H}] 1 \mathrm{O}[\mathrm{C} @ \mathrm{H}]([\mathrm{C} @ \mathrm{H}](\mathrm{OC}(\mathrm{C})=\mathrm{O})[\mathrm{C} @ @ \mathrm{H}](\mathrm{OC}(\mathrm{C})=\mathrm{O})[\mathrm{C} @ @ \mathrm{H}] 1 \mathrm{OC}(\mathrm{C})=\mathrm{O}) \mathrm{N} 1 \mathrm{C}=\mathrm{C}(\mathrm{COC}(=\mathrm{O})$

667

$\mathrm{CCC} 2=\mathrm{NC}(=\mathrm{NO} 2) \mathrm{C} 2=\mathrm{CC}=\mathrm{CC}=\mathrm{C} 2) \mathrm{N}=\mathrm{N} 1$

$\mathrm{CC}(=\mathrm{O}) \mathrm{OC}[\mathrm{C} @ \mathrm{H}] 1 \mathrm{O}[\mathrm{C} @ \mathrm{H}]([\mathrm{C} @ \mathrm{H}](\mathrm{OC}(\mathrm{C})=\mathrm{O})[\mathrm{C} @ @ \mathrm{H}](\mathrm{OC}(\mathrm{C})=\mathrm{O})[\mathrm{C} @ @ \mathrm{H}] 1 \mathrm{OC}(\mathrm{C})=\mathrm{O}) \mathrm{N} 1 \mathrm{C}=\mathrm{C}(\mathrm{COC}(=\mathrm{O})$

$668 \mathrm{CCC} 2=\mathrm{NC}(=\mathrm{NO} 2) \mathrm{C} 2=\mathrm{CC}=\mathrm{C}(\mathrm{Cl}) \mathrm{C}=\mathrm{C} 2) \mathrm{N}=\mathrm{N} 1$

$\mathrm{CC}(=\mathrm{O}) \mathrm{OC}[\mathrm{C} @ \mathrm{H}] 1 \mathrm{O}[\mathrm{C} @ \mathrm{H}]([\mathrm{C} @ \mathrm{H}](\mathrm{OC}(\mathrm{C})=\mathrm{O})[\mathrm{C} @ @ \mathrm{H}](\mathrm{OC}(\mathrm{C})=\mathrm{O})[\mathrm{C} @ @ \mathrm{H}] 1 \mathrm{OC}(\mathrm{C})=\mathrm{O}) \mathrm{N} 1 \mathrm{C}=\mathrm{C}(\mathrm{COC}(=\mathrm{O})$

669

$\mathrm{CCC} 2=\mathrm{NC}(=\mathrm{NO} 2) \mathrm{C} 2=\mathrm{CC}=\mathrm{CC}(\mathrm{C})=\mathrm{C} 2) \mathrm{N}=\mathrm{N} 1$

$\mathrm{CC}(=\mathrm{O}) \mathrm{OC}[\mathrm{C} @ \mathrm{H}] 1 \mathrm{O}[\mathrm{C} @ \mathrm{H}]([\mathrm{C} @ \mathrm{H}](\mathrm{OC}(\mathrm{C})=\mathrm{O})[\mathrm{C} @ @ \mathrm{H}](\mathrm{OC}(\mathrm{C})=\mathrm{O})[\mathrm{C} @ @ \mathrm{H}] 1 \mathrm{OC}(\mathrm{C})=\mathrm{O}) \mathrm{N} 1 \mathrm{C}=\mathrm{C}(\mathrm{COC}(=\mathrm{O})$

670

CC $2=\mathrm{NC}(=\mathrm{NO} 2) \mathrm{C} 2=\mathrm{CC}=\mathrm{C}(\mathrm{Br}) \mathrm{C}=\mathrm{C} 2) \mathrm{N}=\mathrm{N} 1$

$\mathrm{CC}(=\mathrm{O}) \mathrm{OC}[\mathrm{C} @ \mathrm{H}] 1 \mathrm{O}[\mathrm{C} @ \mathrm{H}]([\mathrm{C} @ \mathrm{H}](\mathrm{OC}(\mathrm{C})=\mathrm{O})[\mathrm{C} @ @ \mathrm{H}](\mathrm{OC}(\mathrm{C})=\mathrm{O})[\mathrm{C} @ @ \mathrm{H}] 1 \mathrm{OC}(\mathrm{C})=\mathrm{O}) \mathrm{N} 1 \mathrm{C}=\mathrm{C}(\mathrm{COC}(=\mathrm{O})$

$671 \mathrm{CCC} 2=\mathrm{NC}(=\mathrm{NO} 2) \mathrm{C} 2=\mathrm{CC}=\mathrm{C}(\mathrm{C}=\mathrm{C} 2)[\mathrm{N}+]([\mathrm{O}-])=\mathrm{O}) \mathrm{N}=\mathrm{N} 1$

$672 \mathrm{CC} 1=\mathrm{CC}=\mathrm{C} 2 \mathrm{C}(=\mathrm{O}) \mathrm{C}(\mathrm{CSC}(=\mathrm{S}) \mathrm{N} 3 \mathrm{CCCC} 3)=\mathrm{COC} 2=\mathrm{C} 1$

$673 \mathrm{CC} 1=\mathrm{CC}=\mathrm{C} 2 \mathrm{C}(=\mathrm{O}) \mathrm{C}(\mathrm{CSC}(=\mathrm{S}) \mathrm{N} 3 \mathrm{CCOCC} 3)=\mathrm{COC} 2=\mathrm{C} 1$

$674 \mathrm{O}=\mathrm{C} 1 \mathrm{C}(\mathrm{CSC}(=\mathrm{S}) \mathrm{N} 2 \mathrm{CCOCC} 2)=\mathrm{COC} 2=\mathrm{CC}=\mathrm{CC}=\mathrm{C} 12$

$675 \mathrm{CCN}(\mathrm{CC}) \mathrm{C}(=\mathrm{S}) \mathrm{SCC} 1=\mathrm{CC}(=\mathrm{O}) \mathrm{C} 2=\mathrm{CC}=\mathrm{C}(\mathrm{Cl}) \mathrm{C}=\mathrm{C} 2 \mathrm{O} 1$

$676 \mathrm{CC}(\mathrm{C}) \mathrm{N}(\mathrm{C}(\mathrm{C}) \mathrm{C}) \mathrm{C}(=\mathrm{S}) \mathrm{SCC} 1=\mathrm{CC}(=\mathrm{O}) \mathrm{C} 2=\mathrm{CC}=\mathrm{CC}=\mathrm{C} 2 \mathrm{O} 1$

$677 \mathrm{CC} 1=\mathrm{CC}=\mathrm{C} 2 \mathrm{C}(=\mathrm{O}) \mathrm{C}(\mathrm{CSC}(=\mathrm{S}) \mathrm{N} 3 \mathrm{CCCCC} 3)=\mathrm{COC} 2=\mathrm{C} 1$

$678 \mathrm{CC}(\mathrm{C}) \mathrm{N}(\mathrm{C}(\mathrm{C}) \mathrm{C}) \mathrm{C}(=\mathrm{S}) \mathrm{SCC} 1=\mathrm{COC} 2=\mathrm{CC}=\mathrm{CC}=\mathrm{C} 2 \mathrm{C} 1=\mathrm{O}$

$679 \mathrm{COC} 1=\mathrm{CC}=\mathrm{C} 2 \mathrm{OC}=\mathrm{C}(\mathrm{CSC}(=\mathrm{S}) \mathrm{N} 3 \mathrm{CCOCC} 3) \mathrm{C}(=\mathrm{O}) \mathrm{C} 2=\mathrm{C} 1$

$680 \mathrm{O}=\mathrm{C} 1 \mathrm{C}(\mathrm{SC}(=\mathrm{S}) \mathrm{N} 2 \mathrm{CCCC} 2)=\mathrm{COC} 2=\mathrm{CC}=\mathrm{CC}=\mathrm{C} 12$

$681 \mathrm{COC} 1=\mathrm{CC}=\mathrm{C} 2 \mathrm{OC}=\mathrm{C}(\mathrm{CSC}(=\mathrm{S}) \mathrm{N} 3 \mathrm{CCCCC} 3) \mathrm{C}(=\mathrm{O}) \mathrm{C} 2=\mathrm{C} 1$

$682 \mathrm{O}=\mathrm{C} 1 \mathrm{C}(\mathrm{CSC}=\mathrm{S}) \mathrm{N} 2 \mathrm{CCN}(\mathrm{CC} 2) \mathrm{C} 2=\mathrm{CC}=\mathrm{CC}=\mathrm{C} 2)=\mathrm{COC} 2=\mathrm{CC}=\mathrm{CC}=\mathrm{C} 12$

$683 \mathrm{CN}(\mathrm{C}(=\mathrm{S}) \mathrm{SCC} 1=\mathrm{COC} 2=\mathrm{CC}=\mathrm{CC}=\mathrm{C} 2 \mathrm{C} 1=\mathrm{O}) \mathrm{C} 1=\mathrm{CC}=\mathrm{CC}=\mathrm{C} 1$

$684 \mathrm{CC}(\mathrm{C}) \mathrm{N}(\mathrm{C}(\mathrm{C}) \mathrm{C}) \mathrm{C}(=\mathrm{S}) \mathrm{SCC} 1=\mathrm{CC}(=\mathrm{O}) \mathrm{C} 2=\mathrm{CC}=\mathrm{C}(\mathrm{Cl}) \mathrm{C}=\mathrm{C} 2 \mathrm{O} 1$

$685 \mathrm{O}=\mathrm{C} 1 \mathrm{C}(\mathrm{CSC}(=\mathrm{S}) \mathrm{N} 2 \mathrm{CCCCC} 2)=\mathrm{COC} 2=\mathrm{CC}=\mathrm{CC}=\mathrm{C} 12$

$686 \quad \mathrm{ClC} 1=\mathrm{C}(\mathrm{CSC}(=\mathrm{S}) \mathrm{N} 2 \mathrm{CCCCC} 2) \mathrm{OC} 2=\mathrm{CC}=\mathrm{CC}=\mathrm{C} 2 \mathrm{C} 1=\mathrm{O}$

$687 \mathrm{ClC} 1=\mathrm{CC}=\mathrm{C} 2 \mathrm{C}(=\mathrm{O}) \mathrm{C}=\mathrm{C}(\mathrm{CSC}(=\mathrm{S}) \mathrm{N} 3 \mathrm{CCOCC} 3) \mathrm{OC} 2=\mathrm{C} 1$

$688 \mathrm{ClC} 1=\mathrm{CC}=\mathrm{C} 2 \mathrm{C}(=\mathrm{O}) \mathrm{C}=\mathrm{C}(\mathrm{CSC}(=\mathrm{S}) \mathrm{N} 3 \mathrm{CCCC} 3) \mathrm{OC} 2=\mathrm{C} 1$

$689 \mathrm{FC} 1=\mathrm{CC}=\mathrm{C}(\mathrm{C}=\mathrm{C} 1) \mathrm{C}(\mathrm{N} 1 \mathrm{CCN}(\mathrm{CC} 1) \mathrm{C}(=\mathrm{S}) \mathrm{SCC} 1=\mathrm{COC} 2=\mathrm{CC}=\mathrm{CC}$

$690 \mathrm{O}=\mathrm{C} 1 \mathrm{C}=\mathrm{C}(\mathrm{CSC}(=\mathrm{S}) \mathrm{N} 2 \mathrm{CCOCC} 2) \mathrm{OC} 2=\mathrm{CC}=\mathrm{CC}=\mathrm{C} 12$

$691 \mathrm{O}=\mathrm{C} 1 \mathrm{C}(\mathrm{SC}(=\mathrm{S}) \mathrm{N} 2 \mathrm{CCCCC} 2)=\mathrm{COC} 2=\mathrm{CC}=\mathrm{CC}=\mathrm{C} 12$

$692 \mathrm{COC} 1=\mathrm{CC}=\mathrm{C} 2 \mathrm{OC}(\mathrm{CSC}(=\mathrm{S}) \mathrm{N} 3 \mathrm{CCCCC} 3)=\mathrm{CC}(=\mathrm{O}) \mathrm{C} 2=\mathrm{C} 1$

$693 \mathrm{ClC} 1=\mathrm{C}(\mathrm{CSC}(=\mathrm{S}) \mathrm{N} 2 \mathrm{CCCC} 2) \mathrm{OC} 2=\mathrm{CC}=\mathrm{CC}=\mathrm{C} 2 \mathrm{C} 1=\mathrm{O}$

$694 \mathrm{COC} 1=\mathrm{CC}=\mathrm{C} 2 \mathrm{OC}=\mathrm{C}(\mathrm{CSC}(=\mathrm{S}) \mathrm{N} 3 \mathrm{CCCC} 3) \mathrm{C}(=\mathrm{O}) \mathrm{C} 2=\mathrm{C} 1$

$695 \mathrm{CC}(\mathrm{C}) \mathrm{N}(\mathrm{C}(\mathrm{C}) \mathrm{C}) \mathrm{C}(=\mathrm{S}) \mathrm{SCC} 1=\mathrm{COC} 2=\mathrm{CC}(\mathrm{Cl})=\mathrm{CC}=\mathrm{C} 2 \mathrm{C} 1=\mathrm{O}$

$696 \mathrm{CN} 1 \mathrm{CCN}(\mathrm{CC} 1) \mathrm{C}(=\mathrm{S}) \mathrm{SCC} 1=\mathrm{COC} 2=\mathrm{CC}=\mathrm{CC}=\mathrm{C} 2 \mathrm{C} 1=\mathrm{O}$

$697 \mathrm{O}=\mathrm{C} 1 \mathrm{C}=\mathrm{C}(\mathrm{CSC}(=\mathrm{S}) \mathrm{N} 2 \mathrm{CCCC} 2) \mathrm{OC} 2=\mathrm{CC}=\mathrm{CC}=\mathrm{C} 12$

$698 \mathrm{O}=\mathrm{C} 1 \mathrm{C}=\mathrm{C}(\mathrm{CSC}(=\mathrm{S}) \mathrm{N} 2 \mathrm{CCCCC} 2) \mathrm{OC} 2=\mathrm{CC}=\mathrm{CC}=\mathrm{C} 12$

$699 \mathrm{ClCCN}(\mathrm{CCCl}) \mathrm{C}(=\mathrm{S}) \mathrm{SCC} 1=\mathrm{CC}(=\mathrm{O}) \mathrm{C} 2=\mathrm{CC}=\mathrm{CC}=\mathrm{C} 2 \mathrm{O} 1$

$700 \quad \mathrm{ClC} 1=\mathrm{CC}=\mathrm{C} 2 \mathrm{OC}=\mathrm{C}(\mathrm{SC}(=\mathrm{S}) \mathrm{N} 3 \mathrm{CCCCC} 3) \mathrm{C}(=\mathrm{O}) \mathrm{C} 2=\mathrm{C} 1$

$701 \quad \mathrm{ClC} 1=\mathrm{CC}=\mathrm{C} 2 \mathrm{C}(=\mathrm{O}) \mathrm{C}(\mathrm{CSC}(=\mathrm{S}) \mathrm{N} 3 \mathrm{CCCCC} 3)=\mathrm{COC} 2=\mathrm{C} 1$

$702 \quad \mathrm{COC} 1=\mathrm{CC}=\mathrm{C} 2 \mathrm{OC}(\mathrm{CSC}(=\mathrm{S}) \mathrm{N}(\mathrm{C}(\mathrm{C}) \mathrm{C}) \mathrm{C}(\mathrm{C}) \mathrm{C})=\mathrm{CC}(=\mathrm{O}) \mathrm{C} 2=\mathrm{C} 1$

$703 \mathrm{CCOC}(=\mathrm{O}) \mathrm{C} 1=\mathrm{CC}(=\mathrm{O}) \mathrm{C} 2=\mathrm{CC}(\mathrm{CSC}(=\mathrm{S}) \mathrm{N} 3 \mathrm{CCOCC} 3)=\mathrm{CC}=\mathrm{C} 2 \mathrm{O}$

$704 \quad \operatorname{CCOC}(=\mathrm{O}) \mathrm{C} 1=\mathrm{CC}(=\mathrm{O}) \mathrm{C} 2=\mathrm{CC}(\mathrm{CSC}(=\mathrm{S}) \mathrm{N}(\mathrm{C}(\mathrm{C}) \mathrm{C}) \mathrm{C}(\mathrm{C}) \mathrm{C})=\mathrm{CC}=\mathrm{C} 2 \mathrm{O} 1$

$705 \mathrm{O}=\mathrm{C} 1 \mathrm{C}(\mathrm{CSC}(=\mathrm{S}) \mathrm{N} 2 \mathrm{CCCC} 2)=\mathrm{COC} 2=\mathrm{CC}=\mathrm{CC}=\mathrm{C} 12$ 


\begin{tabular}{|c|c|c|}
\hline NAME & SMILE & $\lg (\mathbf{1} / \mathbf{I C 5 0})$ \\
\hline 706 & $\mathrm{CC}(\mathrm{C}) \mathrm{N}(\mathrm{C}(\mathrm{C}) \mathrm{C}) \mathrm{C}(=\mathrm{S}) \mathrm{SCC} 1=\mathrm{C}(\mathrm{Cl}) \mathrm{C}(=\mathrm{O}) \mathrm{C} 2=\mathrm{CC}=\mathrm{CC}=\mathrm{C} 2 \mathrm{O} 1$ & 4,7 \\
\hline 707 & $\mathrm{ClC} 1=\mathrm{C}(\mathrm{CSC}(=\mathrm{S}) \mathrm{N} 2 \mathrm{CCOCC} 2) \mathrm{OC} 2=\mathrm{CC}=\mathrm{CC}=\mathrm{C} 2 \mathrm{C} 1=\mathrm{O}$ & 5,05 \\
\hline 708 & $\mathrm{CCOC}(=\mathrm{O}) \mathrm{C} 1=\mathrm{CC}(=\mathrm{O}) \mathrm{C} 2=\mathrm{CC}(\mathrm{CSC}(=\mathrm{S}) \mathrm{N} 3 \mathrm{CCCCC} 3)=\mathrm{CC}=\mathrm{C} 2 \mathrm{O} 1$ & 5,47 \\
\hline 709 & $\mathrm{CC} 1=\mathrm{CC}=\mathrm{C} 2 \mathrm{C}(\mathrm{OC}=\mathrm{C}(\mathrm{CSC}(=\mathrm{S}) \mathrm{N}(\mathrm{CCCl}) \mathrm{CCCl}) \mathrm{C} 2=\mathrm{O})=\mathrm{C} 1$ & 5,1 \\
\hline 710 & $\mathrm{ClC} 1=\mathrm{CC}=\mathrm{C} 2 \mathrm{C}(=\mathrm{O}) \mathrm{C}(\mathrm{CSC}(=\mathrm{S}) \mathrm{N} 3 \mathrm{CCCC} 3)=\mathrm{COC} 2=\mathrm{C} 1$ & 5,68 \\
\hline 711 & $\mathrm{ClC} 1=\mathrm{CC}=\mathrm{C} 2 \mathrm{OC}=\mathrm{C}(\mathrm{SC}(=\mathrm{S}) \mathrm{N} 3 \mathrm{CCCC} 3) \mathrm{C}(=\mathrm{O}) \mathrm{C} 2=\mathrm{C} 1$ & 4,59 \\
\hline 712 & $\mathrm{O}=\mathrm{C} 1 \mathrm{C}(\mathrm{CSC}(=\mathrm{S}) \mathrm{N} 2 \mathrm{CCN}(\mathrm{CC} 3=\mathrm{CC}=\mathrm{CC}=\mathrm{C} 3) \mathrm{CC} 2)=\mathrm{COC} 2=\mathrm{CC}=\mathrm{CC}=\mathrm{C} 12$ & 5,54 \\
\hline 713 & $\mathrm{COC} 1=\mathrm{CC}=\mathrm{C} 2 \mathrm{OC}=\mathrm{C}(\mathrm{CSC}(=\mathrm{S}) \mathrm{N}(\mathrm{C}(\mathrm{C}) \mathrm{C}) \mathrm{C}(\mathrm{C}) \mathrm{C}) \mathrm{C}(=\mathrm{O}) \mathrm{C} 2=\mathrm{C} 1$ & 5,05 \\
\hline 714 & $\mathrm{COC} 1=\mathrm{CC}=\mathrm{C} 2 \mathrm{OC}(\mathrm{CSC}(=\mathrm{S}) \mathrm{N} 3 \mathrm{CCOCC} 3)=\mathrm{CC}(=\mathrm{O}) \mathrm{C} 2=\mathrm{C} 1$ & 5,52 \\
\hline 715 & $\mathrm{ClC} 1=\mathrm{CC}=\mathrm{C} 2 \mathrm{C}(=\mathrm{O}) \mathrm{C}=\mathrm{C}(\mathrm{CSC}(=\mathrm{S}) \mathrm{N} 3 \mathrm{CCN}(\mathrm{CC} 3) \mathrm{C} 3=\mathrm{CC}=\mathrm{CC}=\mathrm{C} 3) \mathrm{OC} 2=\mathrm{C} 1$ & 4,8 \\
\hline 716 & $\mathrm{CCOC}(=\mathrm{O}) \mathrm{C} 1=\mathrm{CC}(=\mathrm{O}) \mathrm{C} 2=\mathrm{CC}(\mathrm{CSC}(=\mathrm{S}) \mathrm{N} 3 \mathrm{CCCC} 3)=\mathrm{CC}=\mathrm{C} 2 \mathrm{O} 1$ & 5,05 \\
\hline 717 & $\mathrm{ClC} 1=\mathrm{CC}=\mathrm{C} 2 \mathrm{C}(=\mathrm{O}) \mathrm{C}=\mathrm{C}(\mathrm{CSC}(=\mathrm{S}) \mathrm{N} 3 \mathrm{CCCCC} 3) \mathrm{OC} 2=\mathrm{C} 1$ & 5,8 \\
\hline 718 & $\mathrm{CC}(\mathrm{C}) \mathrm{N}(\mathrm{C}(\mathrm{C}) \mathrm{C}) \mathrm{C}(=\mathrm{S}) \mathrm{SCC} 1=\mathrm{COC} 2=\mathrm{CC}(\mathrm{C})=\mathrm{CC}=\mathrm{C} 2 \mathrm{C} 1=\mathrm{O}$ & 6,15 \\
\hline 719 & $\mathrm{ClC} 1=\mathrm{CC}=\mathrm{C} 2 \mathrm{C}(=\mathrm{O}) \mathrm{C}(\mathrm{CSC}(=\mathrm{S}) \mathrm{N} 3 \mathrm{CCOCC} 3)=\mathrm{COC} 2=\mathrm{C} 1$ & 6,05 \\
\hline 720 & {$[\mathrm{O}-][\mathrm{Cl}](=\mathrm{O})(=\mathrm{O})=\mathrm{O} . \mathrm{CC} 1=\mathrm{ClC}(=\mathrm{ClC} 2=[\mathrm{N}+](\mathrm{C}) \mathrm{C} 3=\mathrm{CC}=\mathrm{CC}=\mathrm{C} 3 \mathrm{O} 2) \mathrm{N}=\mathrm{C} 2 \mathrm{SC} 3=\mathrm{CC}(\mathrm{OCCO})=\mathrm{CC}=\mathrm{C} 3 \mathrm{~N} 12$} & 5,17 \\
\hline 721 & {$[\mathrm{O}-][\mathrm{Cl}](=\mathrm{O})(=\mathrm{O})=\mathrm{O} \cdot \mathrm{CC} 1=\mathrm{ClC}(=\mathrm{ClC} 2=[\mathrm{N}+](\mathrm{C}) \mathrm{C} 3=\mathrm{CC}=\mathrm{CC}=\mathrm{C} 3 \mathrm{~S} 2) \mathrm{N}=\mathrm{C} 2 \mathrm{SC} 3=\mathrm{CC}(\mathrm{OCCO})=\mathrm{CC}=\mathrm{C} 3 \mathrm{~N} 12$} & 6,03 \\
\hline 722 & {$[\mathrm{O}-][\mathrm{Cl}](=\mathrm{O})(=\mathrm{O})=\mathrm{O} . \mathrm{CC} 1=\mathrm{ClC}(=\mathrm{ClC} 2=[\mathrm{N}+](\mathrm{C}) \mathrm{C} 3=\mathrm{CC}=\mathrm{CC}=\mathrm{C} 3 \mathrm{O} 2) \mathrm{C}(\mathrm{C} \# \mathrm{~N})=\mathrm{C} 2 \mathrm{SC} 3=\mathrm{CC}=\mathrm{CC}=\mathrm{C} 3 \mathrm{~N} 12$} & 5,26 \\
\hline 723 & {$[\mathrm{O}-][\mathrm{Cl}](=\mathrm{O})(=\mathrm{O})=\mathrm{O} . \mathrm{CCOC} 1=\mathrm{CC}=\mathrm{C} 2 \mathrm{~N} 3 \mathrm{C}(\mathrm{SC} 2=\mathrm{C} 1)=\mathrm{N} \backslash \mathrm{C}(=\mathrm{C} / \mathrm{C} 1=[\mathrm{N}+](\mathrm{C}) \mathrm{C} 2=\mathrm{CC}=\mathrm{CC}=\mathrm{C} 2 \mathrm{O} 1) \mathrm{C}=\mathrm{C} 3 \mathrm{C}$} & 6,3 \\
\hline 724 & {$[\mathrm{O}-][\mathrm{Cl}](=\mathrm{O})(=\mathrm{O})=\mathrm{O} . \mathrm{COC} 1=\mathrm{CC}=\mathrm{C} 2 \mathrm{~N} 3 \mathrm{C}(\mathrm{SC} 2=\mathrm{C} 1)=\mathrm{N} \backslash \mathrm{C}(=\mathrm{C} / \mathrm{C} 1=[\mathrm{N}+](\mathrm{C}) \mathrm{C} 2=\mathrm{CC}=\mathrm{CC}=\mathrm{C} 2 \mathrm{O} 1) \mathrm{C}=\mathrm{C} 3 \mathrm{C}$} & 6,15 \\
\hline 725 & {$[\mathrm{O}-][\mathrm{Cl}](=\mathrm{O})(=\mathrm{O})=\mathrm{O} . \mathrm{CC} 1=\mathrm{ClC}(=\mathrm{ClC} 2=[\mathrm{N}+](\mathrm{C}) \mathrm{C} 3=\mathrm{CC}=\mathrm{CC}=\mathrm{C} 3 \mathrm{O} 2) \mathrm{N}=\mathrm{C} 2 \mathrm{SC} 3=\mathrm{CC}(\mathrm{Cl})=\mathrm{CC}=\mathrm{C} 3 \mathrm{~N} 12$} & 5,15 \\
\hline 726 & {$[\mathrm{O}-][\mathrm{Cl}](=\mathrm{O})(=\mathrm{O})=\mathrm{O} \cdot \mathrm{COC} 1=\mathrm{CC}=\mathrm{C} 2 \mathrm{~N} 3 \mathrm{C}(\mathrm{SC} 2=\mathrm{C} 1)=\mathrm{N} \backslash \mathrm{C}(=\mathrm{C} / \mathrm{C} 1=[\mathrm{N}+](\mathrm{C}) \mathrm{C} 2=\mathrm{CC}=\mathrm{CC}=\mathrm{C} 2 \mathrm{~S} 1) \mathrm{C}=\mathrm{C} 3 \mathrm{C}$} & 6,3 \\
\hline 727 & {$[\mathrm{O}-][\mathrm{Cl}](=\mathrm{O})(=\mathrm{O})=\mathrm{O} . \mathrm{CC} 1=\mathrm{ClC}(=\mathrm{ClC} 2=[\mathrm{N}+](\mathrm{C}) \mathrm{C} 3=\mathrm{CC}=\mathrm{CC}=\mathrm{C} 3 \mathrm{~S} 2) \mathrm{N}=\mathrm{C} 2 \mathrm{C}=\mathrm{CC}=\mathrm{CN} 12$} & 5,44 \\
\hline 728 & $\mathrm{COC}(=\mathrm{O}) \mathrm{C} 1=\mathrm{C} 2 \mathrm{C}=\mathrm{C}(\mathrm{COC} 2(\mathrm{O})[\mathrm{C} @ \mathrm{H}] 2 \mathrm{OC}(=\mathrm{O})[\mathrm{C} @ @] 11 \mathrm{CCCC}(\mathrm{C})(\mathrm{C})[\mathrm{C} @ \mathrm{H}] 21) \mathrm{C}(\mathrm{C}) \mathrm{C}$ & 4,22 \\
\hline 729 & $\mathrm{CC}(\mathrm{C}) \mathrm{C} 1=\mathrm{CC} 2=\mathrm{C}[\mathrm{C} @ \mathrm{H}] 3 \mathrm{OC}(=\mathrm{O})[\mathrm{C} @ @] 4(\mathrm{CCCC}(\mathrm{C})(\mathrm{C})[\mathrm{C} @ \mathrm{H}] 34) \mathrm{C} 2=\mathrm{C}(\mathrm{O}) \mathrm{C} 1=\mathrm{O}$ & 4,49 \\
\hline 730 & $\mathrm{SC} 1=\mathrm{NC}=\mathrm{NC} 2=\mathrm{C} 1 \mathrm{NC}=\mathrm{N} 2$ & 3,5 \\
\hline 731 & $\mathrm{C}[\mathrm{C} @ \mathrm{H}] 1 \mathrm{COC} 2=\mathrm{C}(\mathrm{O}) \mathrm{C} 3=\mathrm{C}(\mathrm{C}=\mathrm{C} 12)[\mathrm{C} @ @ \mathrm{H}] 1 \mathrm{C}[\mathrm{C} @ \mathrm{H}] 2 \mathrm{C}(\mathrm{C})(\mathrm{C}) \mathrm{CCC}[\mathrm{C} @] 32 \mathrm{C}(=\mathrm{O}) \mathrm{O} 1$ & 4,22 \\
\hline 732 & $\mathrm{C}[\mathrm{C} @ \mathrm{H}] 1 \mathrm{COC} 2=\mathrm{C} 1 \mathrm{C}(=\mathrm{O}) \mathrm{C} 1=\mathrm{C}(\mathrm{C} 2=\mathrm{O})[\mathrm{C} @ @] 23 \mathrm{CCCC}(\mathrm{C})(\mathrm{C})[\mathrm{C} @ @ \mathrm{H}] 2 \mathrm{C}[\mathrm{C} @ @ \mathrm{H}] 1 \mathrm{OC} 3=\mathrm{O}$ & 4,4 \\
\hline 733 & $\mathrm{C}[\mathrm{C} @ \mathrm{H}] 1 \mathrm{COC} 2=\mathrm{C} 1 \mathrm{C}(=\mathrm{O}) \mathrm{C} 1=\mathrm{C}(\mathrm{C} 2=\mathrm{O})[\mathrm{C} @ @] 23 \mathrm{CCCC}(\mathrm{C})(\mathrm{C})[\mathrm{C} @ @ \mathrm{H}] 2 \mathrm{C}[\mathrm{C} @ @ \mathrm{H}] 1 \mathrm{OC} 3=\mathrm{O}$ & 4,23 \\
\hline 734 & $\mathrm{CO}[\mathrm{C} @ @ \mathrm{H}] 1[\mathrm{C} @ \mathrm{H}] 2 \mathrm{OC}(=\mathrm{O})[\mathrm{C} @ @] 3(\mathrm{CCCC}(\mathrm{C})(\mathrm{C})[\mathrm{C} @ \mathrm{H}] 23) \mathrm{C} 2=\mathrm{C} 1 \mathrm{C}=\mathrm{C}(\mathrm{C}(\mathrm{C}) \mathrm{C}) \mathrm{C}(=\mathrm{O}) \mathrm{C} 2=\mathrm{O}$ & 4,37 \\
\hline 735 & $\mathrm{CC}(\mathrm{C}) \mathrm{C} 1=\mathrm{CC} 2=\mathrm{C}[\mathrm{C} @ \mathrm{H}] 3 \mathrm{OC}(=\mathrm{O})[\mathrm{C} @ @] 4(\mathrm{CCCC}(\mathrm{C})(\mathrm{C})[\mathrm{C} @ \mathrm{H}] 34) \mathrm{C} 2=\mathrm{C}(\mathrm{O}) \mathrm{C} 1=\mathrm{O}$ & 4,72 \\
\hline 736 & $\mathrm{CC}(\mathrm{C}) \mathrm{C} 1=\mathrm{CC} 2=\mathrm{C}(\mathrm{C}(\mathrm{O})=\mathrm{C} 1 \mathrm{O})[\mathrm{C} @] 1(\mathrm{CCCC}(\mathrm{C})(\mathrm{C})[\mathrm{C} @ @ \mathrm{H}] 1 \mathrm{C}[\mathrm{C} @ \mathrm{H}] 2 \mathrm{SC} 1=\mathrm{CC}=\mathrm{CC}=\mathrm{C} 1) \mathrm{C}(\mathrm{O})=\mathrm{O}$ & 4,82 \\
\hline 737 & 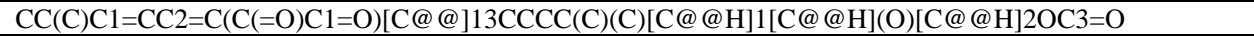 & 4,41 \\
\hline 738 & $\mathrm{CC}(\mathrm{C}) \mathrm{C} 1=\mathrm{CC} 2=\mathrm{C}(\mathrm{C}(=\mathrm{O}) \mathrm{C} 1=\mathrm{O})[\mathrm{C} @ @] 13 \mathrm{CCCC}(\mathrm{C})(\mathrm{C})[\mathrm{C} @ @ \mathrm{H}] 1[\mathrm{C} @ @ \mathrm{H}](\mathrm{O})[\mathrm{C} @ @ \mathrm{H}] 2 \mathrm{OC} 3=\mathrm{O}$ & 4,4 \\
\hline 739 & $\mathrm{CC}(\mathrm{C}) \mathrm{C} 1=\mathrm{CC} 2=\mathrm{C}(\mathrm{C}(=\mathrm{O}) \mathrm{C} 1=\mathrm{O})[\mathrm{C} @ @] 13 \mathrm{CCCC}(\mathrm{C})(\mathrm{C})[\mathrm{C} @ @ \mathrm{H}] 1 \mathrm{C}[\mathrm{C} @ @ \mathrm{H}] 2 \mathrm{OC} 3=\mathrm{O}$ & 4,22 \\
\hline 740 & $\mathrm{SC} 1=\mathrm{NC}=\mathrm{NC} 2=\mathrm{C} 1 \mathrm{NC}=\mathrm{N} 2$ & 3,37 \\
\hline 741 & $\begin{array}{l}\mathrm{C}[\mathrm{C} @ @ \mathrm{H}](\mathrm{COC}(\mathrm{C})=\mathrm{O}) \mathrm{C} 1=\mathrm{C}(\mathrm{OC}(\mathrm{C})=\mathrm{O}) \mathrm{C}(\mathrm{O}) \mathrm{C} 2=\mathrm{C}([\mathrm{C} @ @ \mathrm{H}] 3 \mathrm{C}[\mathrm{C} @ \mathrm{H}] 4 \mathrm{C}(\mathrm{C})(\mathrm{C}) \mathrm{CCC}[\mathrm{C} @] 24 \mathrm{C}(=\mathrm{O}) \mathrm{O} 3) \mathrm{C} 1 \\
=\mathrm{O}\end{array}$ & 4,35 \\
\hline 742 & $\mathrm{CC}(\mathrm{C}) \mathrm{C} 1=\mathrm{CC} 2=\mathrm{C}(\mathrm{C}(\mathrm{O})=\mathrm{C} 1 \mathrm{O})[\mathrm{C} @] 1(\mathrm{CCCC}(\mathrm{C})(\mathrm{C})[\mathrm{C} @ @ \mathrm{H}] 1 \mathrm{C}[\mathrm{C} @ \mathrm{H}] 2 \mathrm{SC} 1=\mathrm{CC}=\mathrm{CC}=\mathrm{C} 1) \mathrm{C}(\mathrm{O})=\mathrm{O}$ & 4,72 \\
\hline 743 & $\mathrm{CO}[\mathrm{C} @ @ \mathrm{H}] 1[\mathrm{C} @ \mathrm{H}] 2 \mathrm{OC}(=\mathrm{O})[\mathrm{C} @ @] 3(\mathrm{CCCC}(\mathrm{C})(\mathrm{C})[\mathrm{C} @ \mathrm{H}] 23) \mathrm{C} 2=\mathrm{C} 1 \mathrm{C}=\mathrm{C}(\mathrm{C}(\mathrm{C}) \mathrm{C}) \mathrm{C}(=\mathrm{O}) \mathrm{C} 2=\mathrm{O}$ & 4,22 \\
\hline 744 & $\mathrm{ClC} 1=\mathrm{CC}=\mathrm{C} 2 \mathrm{C}(\mathrm{OC}=\mathrm{C}(\mathrm{C} 3 \mathrm{SSC}(=\mathrm{N} 3) \mathrm{C} 3=\mathrm{CC}=\mathrm{CC}=\mathrm{C} 3) \mathrm{C} 2=\mathrm{O})=\mathrm{C} 1$ & 4 \\
\hline 745 & $\mathrm{FC} 1=\mathrm{CC} 2=\mathrm{C}(\mathrm{OC}=\mathrm{C}(\mathrm{C} 3 \mathrm{SSC}(=\mathrm{N} 3) \mathrm{C} 3=\mathrm{CC}=\mathrm{CC}=\mathrm{C} 3) \mathrm{C} 2=\mathrm{O}) \mathrm{C}=\mathrm{C} 1 \mathrm{Cl}$ & 4,01 \\
\hline 746 & $\mathrm{FC} 1=\mathrm{CC}=\mathrm{C} 2 \mathrm{OC}=\mathrm{C}(\mathrm{C}(=\mathrm{S}) \mathrm{NC} 3=\mathrm{CC}=\mathrm{CC}=\mathrm{C} 3) \mathrm{C}(=\mathrm{O}) \mathrm{C} 2=\mathrm{C} 1$ & 4 \\
\hline 747 & $\mathrm{ClC} 1=\mathrm{CC}=\mathrm{C} 2 \mathrm{C}(=\mathrm{O}) \mathrm{C}(=\mathrm{COC} 2=\mathrm{C} 1) \mathrm{C}(=\mathrm{S}) \mathrm{NC} 1=\mathrm{CC}=\mathrm{CC}=\mathrm{C} 1$ & 4 \\
\hline 748 & $\mathrm{O}=\mathrm{C} 1 \mathrm{C}(=\mathrm{COC} 2=\mathrm{CC}=\mathrm{CC}=\mathrm{C} 12) \mathrm{C} 1 \mathrm{SSC}(=\mathrm{N} 1) \mathrm{C} 1=\mathrm{CC}=\mathrm{CC}=\mathrm{C} 1$ & 4 \\
\hline 749 & $\mathrm{CC} 1=\mathrm{CC}=\mathrm{C} 2 \mathrm{OC}=\mathrm{C}(\mathrm{C} 3 \mathrm{SSC}(=\mathrm{N} 3) \mathrm{C} 3=\mathrm{CC}=\mathrm{CC}=\mathrm{C} 3) \mathrm{C}(=\mathrm{O}) \mathrm{C} 2=\mathrm{C} 1$ & 4 \\
\hline 750 & $\mathrm{FC} 1=\mathrm{CC}=\mathrm{C} 2 \mathrm{OC}=\mathrm{C}(\mathrm{C} 3 \mathrm{SSC}(=\mathrm{N} 3) \mathrm{C} 3=\mathrm{CC}=\mathrm{CC}=\mathrm{C} 3) \mathrm{C}(=\mathrm{O}) \mathrm{C} 2=\mathrm{C} 1$ & 4,01 \\
\hline 751 & $\mathrm{O}=\mathrm{C} 1 \mathrm{C}(=\mathrm{COC} 2=\mathrm{CC}=\mathrm{CC}=\mathrm{C} 12) \mathrm{C}(=\mathrm{S}) \mathrm{NC} 1=\mathrm{CC}=\mathrm{CC}=\mathrm{C} 1$ & 4 \\
\hline 752 & $\mathrm{ClC} 1=\mathrm{CC}=\mathrm{C} 2 \mathrm{OC}=\mathrm{C}(\mathrm{C} 3 \mathrm{SSC}(=\mathrm{N} 3) \mathrm{C} 3=\mathrm{CC}=\mathrm{CC}=\mathrm{C} 3) \mathrm{C}(=\mathrm{O}) \mathrm{C} 2=\mathrm{C} 1$ & 4,13 \\
\hline 753 & $\mathrm{CO}[\mathrm{C} @] 12[\mathrm{C} @ \mathrm{H}] 3 \mathrm{~N}[\mathrm{C} @ \mathrm{H}] 3 \mathrm{CN} 1 \mathrm{C} 1=\mathrm{C}([\mathrm{C} @ \mathrm{H}] 2 \mathrm{COC}(\mathrm{N})=\mathrm{O}) \mathrm{C}(=\mathrm{O}) \mathrm{C}(\mathrm{N})=\mathrm{C}(\mathrm{C}) \mathrm{C} 1=\mathrm{O}$ & 5,82 \\
\hline 754 & $\mathrm{ClC} 1=\mathrm{CC} 2=\mathrm{C}(\mathrm{C}=\mathrm{C} 1 \mathrm{Cl}) \mathrm{C}(=\mathrm{O}) \mathrm{C}(=\mathrm{CO} 2) \mathrm{C}(=\mathrm{S}) \mathrm{NC} 1=\mathrm{CC}=\mathrm{CC}=\mathrm{C} 1$ & 4 \\
\hline 755 & $\mathrm{ClC} 1=\mathrm{CC} 2=\mathrm{C}(\mathrm{C}=\mathrm{C} 1 \mathrm{Cl}) \mathrm{C}(=\mathrm{O}) \mathrm{C}(=\mathrm{CO} 2) \mathrm{C} 1 \mathrm{SSC}(=\mathrm{N} 1) \mathrm{C} 1=\mathrm{CC}=\mathrm{CC}=\mathrm{C} 1$ & 4,01 \\
\hline 756 & Cl.Cl.NC $(=\mathrm{N}) \mathrm{C} 1=\mathrm{CC}=\mathrm{C} 2 \mathrm{~N}=\mathrm{C}(\mathrm{SC} 2=\mathrm{C} 1) \mathrm{C} 1=\mathrm{CC}=\mathrm{C}(\mathrm{S} 1) \mathrm{C} 1=\mathrm{NC} 2=\mathrm{CC}=\mathrm{C}(\mathrm{C}=\mathrm{C} 2 \mathrm{~S} 1) \mathrm{C}(\mathrm{N})=\mathrm{N}$ & 6,33 \\
\hline 757 & 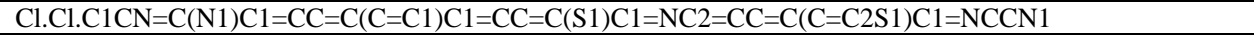 & 6,82 \\
\hline 758 & $\mathrm{Cl} \cdot \mathrm{Cl} \cdot \mathrm{C} 1 \mathrm{CN}=\mathrm{C}(\mathrm{N} 1) \mathrm{C} 1=\mathrm{CC}=\mathrm{C} 2 \mathrm{~N}=\mathrm{C}(\mathrm{SC} 2=\mathrm{C} 1) \mathrm{C} 1=\mathrm{CC}=\mathrm{C}(\mathrm{S} 1) \mathrm{C} 1=\mathrm{NC} 2=\mathrm{CC}=\mathrm{C}(\mathrm{C}=\mathrm{C} 2 \mathrm{~S} 1) \mathrm{C} 1=\mathrm{NCCN} 1$ & 6,77 \\
\hline 759 & Cl.Cl.CC(C)NC $(=\mathrm{N}) \mathrm{C} 1=\mathrm{CC}=\mathrm{C}(\mathrm{C}=\mathrm{C} 1) \mathrm{C} 1=\mathrm{CC}=\mathrm{C}(\mathrm{O} 1) \mathrm{C} 1=\mathrm{NC} 2=\mathrm{CC}=\mathrm{C}(\mathrm{C}=\mathrm{C} 2 \mathrm{~S} 1) \mathrm{C}(=\mathrm{N}) \mathrm{NC}(\mathrm{C}) \mathrm{C}$ & 4,29 \\
\hline 760 & 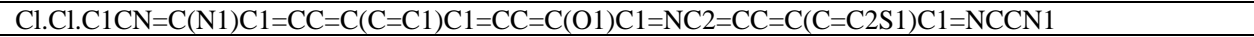 & 6,43 \\
\hline 761 & 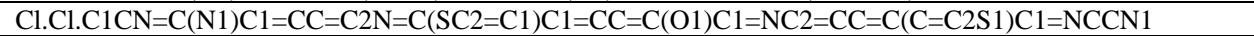 & 4 \\
\hline 762 & Cl.Cl.NC $(=\mathrm{N}) \mathrm{C} 1=\mathrm{CC}=\mathrm{C} 2 \mathrm{~N}=\mathrm{C}(\mathrm{SC} 2=\mathrm{C} 1) \mathrm{C} 1=\mathrm{CC}=\mathrm{C}(\mathrm{O} 1) \mathrm{C} 1=\mathrm{NC} 2=\mathrm{CC}=\mathrm{C}(\mathrm{C}=\mathrm{C} 2 \mathrm{~S} 1) \mathrm{C}(\mathrm{N})=\mathrm{N}$ & 4 \\
\hline 763 & Cl.Cl.CC $(\mathrm{C}) \mathrm{NC}(=\mathrm{N}) \mathrm{C} 1=\mathrm{CC}=\mathrm{C}(\mathrm{C}=\mathrm{C} 1) \mathrm{C} 1=\mathrm{CC}=\mathrm{C}(\mathrm{S} 1) \mathrm{C} 1=\mathrm{NC} 2=\mathrm{CC}=\mathrm{C}(\mathrm{C}=\mathrm{C} 2 \mathrm{~S} 1) \mathrm{C}(=\mathrm{N}) \mathrm{NC}(\mathrm{C}) \mathrm{C}$ & 4,51 \\
\hline 764 & {$[\mathrm{Br}-] . \mathrm{CC} 1=\mathrm{CC}(\mathrm{C})=\mathrm{C}(\mathrm{N} 2 \mathrm{C}=\mathrm{C}[\mathrm{N}+](\mathrm{CC}(=\mathrm{O}) \mathrm{C} 3=\mathrm{CC}=\mathrm{C} 4 \mathrm{C}=\mathrm{CC}=\mathrm{CC} 4=\mathrm{C} 3)=\mathrm{C} 2) \mathrm{C}(\mathrm{C})=\mathrm{C} 1$} & 6,16 \\
\hline 765 & $\mathrm{CC} 1=\mathrm{CC}=\mathrm{C}(\mathrm{NC}(=\mathrm{O}) \mathrm{C} 2=\mathrm{C}(\mathrm{C}(=\mathrm{NN} 2 \mathrm{C} 2=\mathrm{CC}=\mathrm{CC}=\mathrm{C} 2) \mathrm{C} 2=\mathrm{CC}=\mathrm{CC}=\mathrm{C} 2) \mathrm{C} 2=\mathrm{CC}=\mathrm{C}(\mathrm{Cl}) \mathrm{C}=\mathrm{C} 2) \mathrm{C}=\mathrm{C} 1$ & 5,76 \\
\hline 766 & $\mathrm{O}=\mathrm{C}(\mathrm{NC} 1=\mathrm{CC}=\mathrm{CC}=\mathrm{C} 1) \mathrm{C} 1=\mathrm{C}(\mathrm{C}(=\mathrm{NN} 1 \mathrm{C} 1=\mathrm{CC}=\mathrm{CC}=\mathrm{C} 1) \mathrm{C} 1=\mathrm{CC}=\mathrm{CC}=\mathrm{C} 1) \mathrm{C} 1=\mathrm{CC}=\mathrm{CC}=\mathrm{C} 1$ & 5,44 \\
\hline 767 & $\mathrm{O}=\mathrm{C}(\mathrm{N} 1 \mathrm{C}(=\mathrm{S}) \mathrm{NC} 2=\mathrm{CC}=\mathrm{CC}=\mathrm{C} 12) \mathrm{C} 1=\mathrm{C}(\mathrm{C}(=\mathrm{NN} 1 \mathrm{C} 1=\mathrm{CC}=\mathrm{CC}=\mathrm{C} 1) \mathrm{C} 1=\mathrm{CC}=\mathrm{CC}=\mathrm{C} 1) \mathrm{C} 1=\mathrm{CC}=\mathrm{CC}=\mathrm{C} 1$ & 4,82 \\
\hline 768 & $\mathrm{ClC} 1=\mathrm{CC}=\mathrm{C}(\mathrm{C}=\mathrm{C} 1) \mathrm{C} 1=\mathrm{C}(\mathrm{N}(\mathrm{N}=\mathrm{C} 1 \mathrm{C} 1=\mathrm{CC}=\mathrm{CC}=\mathrm{C} 1) \mathrm{C} 1=\mathrm{CC}=\mathrm{CC}=\mathrm{C} 1) \mathrm{C}(=\mathrm{O}) \mathrm{NC} 1=\mathrm{CC}=\mathrm{CC}=\mathrm{C} 1$ & 5,78 \\
\hline 769 & $\mathrm{CC} 1=\mathrm{CC}=\mathrm{C}(\mathrm{NC}(=\mathrm{O}) \mathrm{C} 2=\mathrm{C}(\mathrm{C}(=\mathrm{NN} 2 \mathrm{C} 2=\mathrm{CC}=\mathrm{CC}=\mathrm{C} 2) \mathrm{C} 2=\mathrm{CC}=\mathrm{C}(\mathrm{Cl}) \mathrm{C}=\mathrm{C} 2) \mathrm{C} 2=\mathrm{CC}=\mathrm{CC}=\mathrm{C} 2) \mathrm{C}=\mathrm{C} 1$ & 5,58 \\
\hline 770 & $\mathrm{CC} 1=\mathrm{CC}=\mathrm{C}(\mathrm{NC}(=\mathrm{O}) \mathrm{C} 2=\mathrm{C}(\mathrm{C}(=\mathrm{NN} 2 \mathrm{C} 2=\mathrm{CC}=\mathrm{CC}=\mathrm{C} 2) \mathrm{C} 2=\mathrm{CC}=\mathrm{CC}=\mathrm{C} 2) \mathrm{C} 2=\mathrm{CC}=\mathrm{CC}=\mathrm{C} 2) \mathrm{C}=\mathrm{C} 1$ & 5,55 \\
\hline 771 & $\mathrm{ClC} 1=\mathrm{CC}=\mathrm{C}(\mathrm{C}=\mathrm{C} 1) \mathrm{C} 1=\mathrm{C}(\mathrm{N}(\mathrm{N}=\mathrm{C} 1 \mathrm{C} 1=\mathrm{CC}=\mathrm{CC}=\mathrm{C} 1) \mathrm{C} 1=\mathrm{CC}=\mathrm{CC}=\mathrm{C} 1) \mathrm{C}(=\mathrm{O}) \mathrm{N} 1 \mathrm{C}(=\mathrm{S}) \mathrm{NC} 2=\mathrm{CC}=\mathrm{CC}=\mathrm{C} 12$ & 5,52 \\
\hline 772 & $\begin{array}{l}\mathrm{COC} 1=\mathrm{CC}=\mathrm{CC} 2=\mathrm{C} 1 \mathrm{C}(=\mathrm{O}) \mathrm{C} 1=\mathrm{C}(\mathrm{O}) \mathrm{C} 3=\mathrm{C}(\mathrm{C}[\mathrm{C} @](\mathrm{O})(\mathrm{C}[\mathrm{C} @ @ \mathrm{H}] 3 \mathrm{O}[\mathrm{C} @ \mathrm{H}] 3 \mathrm{C}[\mathrm{C} @ \mathrm{H}](\mathrm{N})[\mathrm{C} @ \mathrm{H}](\mathrm{O})[\mathrm{C} @ \mathrm{H}]( \\
\mathrm{C}) \mathrm{O} 3) \mathrm{C}(=\mathrm{O}) \mathrm{CO}) \mathrm{C}(\mathrm{O})=\mathrm{C} 1 \mathrm{C} 2=\mathrm{O}\end{array}$ & 6,13 \\
\hline 773 & $\mathrm{COC} 1=\mathrm{CC}=\mathrm{C}(\mathrm{C}=\mathrm{C} 1) \mathrm{C} 1=\mathrm{C}(\mathrm{C}(=\mathrm{O}) \mathrm{NN} 1) \mathrm{C} 1=\mathrm{CC}(\mathrm{OC})=\mathrm{C}(\mathrm{OC}) \mathrm{C}(\mathrm{OC})=\mathrm{C} 1$ & 6,28 \\
\hline 774 & $\mathrm{COC}(=\mathrm{O}) \mathrm{NNC}(=\mathrm{O}) \mathrm{C}(=\mathrm{ClC} 1=\mathrm{CC}=\mathrm{C} 2 \mathrm{C}=\mathrm{CC}=\mathrm{CC} 2=\mathrm{C} 1) \backslash \mathrm{C} 1=\mathrm{CC}(\mathrm{OC})=\mathrm{C}(\mathrm{OC}) \mathrm{C}(\mathrm{OC})=\mathrm{C} 1$ & 7,27 \\
\hline 775 & $\mathrm{COC}(=\mathrm{O}) \mathrm{NNC}(=\mathrm{O}) \mathrm{C}(=\mathrm{ClC} 1=\mathrm{CC}=\mathrm{C} 2 \mathrm{C}=\mathrm{CC}=\mathrm{CC} 2=\mathrm{C} 1) \backslash \mathrm{C} 1=\mathrm{CC}(\mathrm{OC})=\mathrm{C}(\mathrm{OC}) \mathrm{C}(\mathrm{OC})=\mathrm{C} 1$ & 7,29 \\
\hline 776 & $\mathrm{COC} 1=\mathrm{CC}=\mathrm{C}(\mathrm{C}=\mathrm{C} 1 \mathrm{~F}) \mathrm{C} 1=\mathrm{C}(\mathrm{C}(=\mathrm{O}) \mathrm{NN} 1) \mathrm{C} 1=\mathrm{CC}(\mathrm{OC})=\mathrm{C}(\mathrm{OC}) \mathrm{C}(\mathrm{OC})=\mathrm{C} 1$ & 5,93 \\
\hline 777 & $\mathrm{COC} 1=\mathrm{CC}=\mathrm{C}(1 \mathrm{C}=\mathrm{C} / \mathrm{C} 2=\mathrm{CC}(\mathrm{OC})=\mathrm{C}(\mathrm{OC}) \mathrm{C}(\mathrm{OC})=\mathrm{C} 2) \mathrm{C}=\mathrm{C} 1 \mathrm{O}$ & 8,28 \\
\hline
\end{tabular}




\begin{tabular}{|c|c|c|}
\hline NAME & SMILE & $\lg (\mathbf{1} / \mathbf{I C 5 0})$ \\
\hline 778 & $\mathrm{COC} 1=\mathrm{CC}=\mathrm{C}(\mathrm{C}=\mathrm{C} / \mathrm{C} 2=\mathrm{CC}(\mathrm{OC})=\mathrm{C}(\mathrm{OC}) \mathrm{C}(\mathrm{OC})=\mathrm{C} 2) \mathrm{C}=\mathrm{C} 1 \mathrm{O}$ & 8,26 \\
\hline 779 & $\mathrm{COC} 1=\mathrm{CC}=\mathrm{C}(\mathrm{C}=\mathrm{C} 1) \mathrm{C} 1=\mathrm{C}(\mathrm{C}(=\mathrm{O}) \mathrm{NN} 1) \mathrm{C} 1=\mathrm{CC}(\mathrm{OC})=\mathrm{C}(\mathrm{OC}) \mathrm{C}(\mathrm{OC})=\mathrm{C} 1$ & 6,21 \\
\hline 780 & $\mathrm{COC} 1=\mathrm{CC}=\mathrm{C}(\mathrm{C}=\mathrm{C} 1 \mathrm{~F}) \mathrm{C} 1=\mathrm{C}(\mathrm{C}(=\mathrm{O}) \mathrm{NN} 1) \mathrm{C} 1=\mathrm{CC}(\mathrm{OC})=\mathrm{C}(\mathrm{OC}) \mathrm{C}(\mathrm{OC})=\mathrm{C} 1$ & 6,16 \\
\hline 781 & $\mathrm{COC} 1=\mathrm{CC}=\mathrm{C}(\mathrm{C}=\mathrm{C} 1 \mathrm{O}) \mathrm{C} 1=\mathrm{C}(\mathrm{C}(=\mathrm{O}) \mathrm{NN} 1) \mathrm{C} 1=\mathrm{CC}(\mathrm{OC})=\mathrm{C}(\mathrm{OC}) \mathrm{C}(\mathrm{OC})=\mathrm{C} 1$ & 6,15 \\
\hline 782 & $\mathrm{COC} 1=\mathrm{CC}=\mathrm{C}(\mathrm{C}=\mathrm{C} 1 \mathrm{O}) \mathrm{C} 1=\mathrm{C}(\mathrm{C}(=\mathrm{O}) \mathrm{NN} 1) \mathrm{C} 1=\mathrm{CC}(\mathrm{OC})=\mathrm{C}(\mathrm{OC}) \mathrm{C}(\mathrm{OC})=\mathrm{C} 1$ & 5,94 \\
\hline 783 & $\mathrm{OC}(=\mathrm{O}) \mathrm{C} 1=\mathrm{C}(\mathrm{NC} 2=\mathrm{CC}=\mathrm{CC}=\mathrm{C} 2 \mathrm{C} 1=\mathrm{O}) \mathrm{C} 1=\mathrm{CC}=\mathrm{CN} 1$ & 3,41 \\
\hline 784 & $\begin{array}{l}\mathrm{CC} 1(\mathrm{C}) \mathrm{CC}[\mathrm{C} @ \mathrm{H}](\mathrm{O})[\mathrm{C} @] 23 \mathrm{CO}[\mathrm{C} @ @](\mathrm{O})([\mathrm{C} @ @ \mathrm{H}](\mathrm{O})[\mathrm{C} @ \mathrm{H}] 12)[\mathrm{C} @ @] 12[\mathrm{C} @ \mathrm{H}](\mathrm{O})[\mathrm{C} @ @ \mathrm{H}](\mathrm{CC}[\mathrm{C} @ \\
@ \mathrm{H}] 31) \mathrm{C}(=\mathrm{C}) \mathrm{C} 2=\mathrm{O}\end{array}$ & 4,43 \\
\hline 785 & $\mathrm{COC} 1=\mathrm{CC}(\mathrm{C})=\mathrm{CC} 2=\mathrm{C} 1 \mathrm{C}(=\mathrm{O}) \mathrm{C}=\mathrm{C}(\mathrm{C} 2=\mathrm{O}) \mathrm{C} 1=\mathrm{C}(\mathrm{C}) \mathrm{C}=\mathrm{C} 2 \mathrm{C}(=\mathrm{O}) \mathrm{C}=\mathrm{C}(\mathrm{NCCO}) \mathrm{C}(=\mathrm{O}) \mathrm{C} 2=\mathrm{C} 1 \mathrm{OC}$ & 5,85 \\
\hline 786 & $\mathrm{COC} 1=\mathrm{CC}(\mathrm{C})=\mathrm{CC} 2=\mathrm{C} 1 \mathrm{C}(=\mathrm{O}) \mathrm{C}=\mathrm{C}(\mathrm{C} 2=\mathrm{O}) \mathrm{C} 1=\mathrm{C}(\mathrm{C}) \mathrm{C}=\mathrm{C} 2 \mathrm{C}(=\mathrm{O}) \mathrm{C}=\mathrm{C}(\mathrm{SCCO}) \mathrm{C}(=\mathrm{O}) \mathrm{C} 2=\mathrm{C} 1 \mathrm{OC}$ & 5,77 \\
\hline 787 & $\begin{array}{l}\mathrm{COC} 1=\mathrm{CC}(\mathrm{C})=\mathrm{CC} 2=\mathrm{C} 1 \mathrm{C}(=\mathrm{O}) \mathrm{C}=\mathrm{C}(\mathrm{C} 2=\mathrm{O}) \mathrm{C} 1=\mathrm{C}(\mathrm{C}) \mathrm{C}=\mathrm{C} 2 \mathrm{C}(=\mathrm{O}) \mathrm{C}=\mathrm{C}(\mathrm{NC} 3=\mathrm{CC}=\mathrm{C}(\mathrm{OC} 4 \mathrm{O}[\mathrm{C} @ \mathrm{H}](\mathrm{COC}(\mathrm{C})=\mathrm{O} \\
)[\mathrm{C} @ \mathrm{H}](\mathrm{OC}(\mathrm{C})=\mathrm{O})[\mathrm{C} @ \mathrm{H}](\mathrm{OC}(\mathrm{C})=\mathrm{O})[\mathrm{C} @ \mathrm{H}] 4 \mathrm{OC}(\mathrm{C})=\mathrm{O}) \mathrm{C}=\mathrm{C} 3) \mathrm{C}(=\mathrm{O}) \mathrm{C} 2=\mathrm{C} 1 \mathrm{OC}\end{array}$ & 6,05 \\
\hline 788 & $\mathrm{COC} 1=\mathrm{CC}(\mathrm{C})=\mathrm{CC} 2=\mathrm{C} 1 \mathrm{C}(=\mathrm{O}) \mathrm{C}=\mathrm{C}(\mathrm{C} 2=\mathrm{O}) \mathrm{C} 1=\mathrm{C}(\mathrm{C}) \mathrm{C}=\mathrm{C} 2 \mathrm{C}(=\mathrm{O}) \mathrm{C}=\mathrm{CC}(=\mathrm{O}) \mathrm{C} 2=\mathrm{C} 1 \mathrm{OC}$ & 6,4 \\
\hline 789 & $\mathrm{CCOC} 1=\mathrm{CC}(\mathrm{C})=\mathrm{CC} 2=\mathrm{C} 1 \mathrm{C}(=\mathrm{O}) \mathrm{C}=\mathrm{C}(\mathrm{C} 2=\mathrm{O}) \mathrm{C} 1=\mathrm{C}(\mathrm{C}) \mathrm{C}=\mathrm{C} 2 \mathrm{C}(=\mathrm{O}) \mathrm{C}=\mathrm{CC}(=\mathrm{O}) \mathrm{C} 2=\mathrm{C} 1 \mathrm{OCC}$ & 5,62 \\
\hline 790 & $\begin{array}{l}\mathrm{COC} 1=\mathrm{CC}(\mathrm{C})=\mathrm{CC} 2=\mathrm{C} 1 \mathrm{C}(=\mathrm{O}) \mathrm{C}=\mathrm{C}(\mathrm{C} 2=\mathrm{O}) \mathrm{C} 1=\mathrm{C}(\mathrm{C}) \mathrm{C}=\mathrm{C} 2 \mathrm{C}(=\mathrm{O}) \mathrm{C}=\mathrm{C}(\mathrm{NC} 3=\mathrm{CC}=\mathrm{C}(\mathrm{OC} 4 \mathrm{O}[\mathrm{C} @ \mathrm{H}](\mathrm{CO})[\mathrm{C} @ @) \\
\mathrm{H}](\mathrm{O})[\mathrm{C} @ \mathrm{H}](\mathrm{O})[\mathrm{C} @ \mathrm{H}] 4 \mathrm{O}) \mathrm{C}=\mathrm{C} 3) \mathrm{C}(=\mathrm{O}) \mathrm{C} 2=\mathrm{C} 1 \mathrm{OC}\end{array}$ & 5,74 \\
\hline 791 & $\begin{array}{l}\mathrm{COC} 1=\mathrm{CC}(\mathrm{C})=\mathrm{CC} 2=\mathrm{C} 1 \mathrm{C}(=\mathrm{O}) \mathrm{C}=\mathrm{C}(\mathrm{C} 2=\mathrm{O}) \mathrm{C} 1=\mathrm{C}(\mathrm{C}) \mathrm{C}=\mathrm{C} 2 \mathrm{C}(=\mathrm{O}) \mathrm{C}=\mathrm{C}(\mathrm{NC} 3=\mathrm{CC}=\mathrm{C}(\mathrm{OC} 4 \mathrm{O}[\mathrm{C} @ \mathrm{H}](\mathrm{CO})[\mathrm{C} @ @ \\
\mathrm{H}](\mathrm{O})[\mathrm{C} @ \mathrm{H}](\mathrm{O})[\mathrm{C} @ \mathrm{H}] 4 \mathrm{O}) \mathrm{C}=\mathrm{C} 3) \mathrm{C}(=\mathrm{O}) \mathrm{C} 2=\mathrm{C} 1 \mathrm{OC}\end{array}$ & 6,52 \\
\hline 792 & $\begin{array}{l}\mathrm{COC} 1=\mathrm{CC}(\mathrm{C})=\mathrm{CC} 2=\mathrm{C} 1 \mathrm{C}(=\mathrm{O}) \mathrm{C}=\mathrm{C}(\mathrm{C} 2=\mathrm{O}) \mathrm{C} 1=\mathrm{C}(\mathrm{C}) \mathrm{C}=\mathrm{C} 2 \mathrm{C}(=\mathrm{O}) \mathrm{C}=\mathrm{C}(\mathrm{NCCO}[\mathrm{C} @ \mathrm{H}] 3 \mathrm{O}[\mathrm{C} @ \mathrm{H}](\mathrm{COC}(\mathrm{C})=\mathrm{O})[ \\
\mathrm{C} @ \mathrm{H}](\mathrm{OC}(\mathrm{C})=\mathrm{O}) \mathrm{C}=\mathrm{C} 3) \mathrm{C}(=\mathrm{O}) \mathrm{C} 2=\mathrm{C} 1 \mathrm{OC}\end{array}$ & 6,15 \\
\hline 793 & $\begin{array}{l}\mathrm{CC}(=\mathrm{O}) \mathrm{OC}[\mathrm{C} @ \mathrm{H}] 1 \mathrm{OC}(\mathrm{OC} 2=\mathrm{CC}(\mathrm{C})=\mathrm{CC} 3=\mathrm{C} 2 \mathrm{C}(=\mathrm{O}) \mathrm{C}=\mathrm{C}(\mathrm{C} 3=\mathrm{O}) \mathrm{C} 2=\mathrm{C}(\mathrm{C}) \mathrm{C}=\mathrm{C} 3 \mathrm{C}(=\mathrm{O}) \mathrm{C}=\mathrm{CC}(=\mathrm{O}) \mathrm{C} 3=\mathrm{C} 2 \mathrm{O})[ \\
\mathrm{C} @ \mathrm{H}](\mathrm{OC}(\mathrm{C})=\mathrm{O})[\mathrm{C} @ @ \mathrm{H}](\mathrm{OC}(\mathrm{C})=\mathrm{O})[\mathrm{C} @ @ \mathrm{H}] 1 \mathrm{OC}(\mathrm{C})=\mathrm{O}\end{array}$ & 5,38 \\
\hline 794 & $\begin{array}{l}\mathrm{COC} 1=\mathrm{CC}(\mathrm{C})=\mathrm{CC} 2=\mathrm{C} 1 \mathrm{C}(=\mathrm{O}) \mathrm{C}=\mathrm{C}(\mathrm{C} 2=\mathrm{O}) \mathrm{C} 1=\mathrm{C}(\mathrm{C}) \mathrm{C}=\mathrm{C} 2 \mathrm{C}(=\mathrm{O}) \mathrm{C}=\mathrm{C}(\mathrm{NC} 3=\mathrm{CC}=\mathrm{C}(\mathrm{OC} 4 \mathrm{O}[\mathrm{C} @ \mathrm{H}](\mathrm{CO})[\mathrm{C} @ \mathrm{H}] \\
(\mathrm{O})[\mathrm{C} @ \mathrm{H}](\mathrm{O})[\mathrm{C} @ \mathrm{H}] 4 \mathrm{O}) \mathrm{C}=\mathrm{C} 3) \mathrm{C}(=\mathrm{O}) \mathrm{C} 2=\mathrm{C} 1 \mathrm{OC}\end{array}$ & 5,35 \\
\hline 795 & $\begin{array}{l}\mathrm{COC} 1=\mathrm{CC}(\mathrm{C})=\mathrm{CC} 2=\mathrm{C} 1 \mathrm{C}(=\mathrm{O}) \mathrm{C}=\mathrm{C}(\mathrm{C} 2=\mathrm{O}) \mathrm{C} 1=\mathrm{C}(\mathrm{C}) \mathrm{C}=\mathrm{C} 2 \mathrm{C}(=\mathrm{O}) \mathrm{C}=\mathrm{C}(\mathrm{NCCO}[\mathrm{C} @ @ \mathrm{H}] 3 \mathrm{O}[\mathrm{C} @ \mathrm{H}](\mathrm{COC}(\mathrm{C})=\mathrm{O} \\
)[\mathrm{C} @ @ \mathrm{H}](\mathrm{OC}(\mathrm{C})=\mathrm{O}) \mathrm{C}=\mathrm{C} 3) \mathrm{C}(=\mathrm{O}) \mathrm{C} 2=\mathrm{C} 1 \mathrm{CC}\end{array}$ & 6 \\
\hline 796 & $\mathrm{COC} 1=\mathrm{CC}(\mathrm{C})=\mathrm{CC} 2=\mathrm{C} 1 \mathrm{C}(=\mathrm{O}) \mathrm{C}(\mathrm{N})=\mathrm{C}(\mathrm{C} 2=\mathrm{O}) \mathrm{C} 1=\mathrm{C}(\mathrm{C}) \mathrm{C}=\mathrm{C} 2 \mathrm{C}(=\mathrm{O}) \mathrm{C}=\mathrm{C}(\mathrm{N}) \mathrm{C}(=\mathrm{O}) \mathrm{C} 2=\mathrm{C} 1 \mathrm{OC}$ & 5,64 \\
\hline 797 & $\mathrm{COC} 1=\mathrm{CC}(\mathrm{C})=\mathrm{CC} 2=\mathrm{C} 1 \mathrm{C}(=\mathrm{O}) \mathrm{C}=\mathrm{C}(\mathrm{C} 2=\mathrm{O}) \mathrm{C} 1=\mathrm{C}(\mathrm{C}) \mathrm{C}=\mathrm{C} 2 \mathrm{C}(=\mathrm{O}) \mathrm{C}=\mathrm{C}(\mathrm{N}) \mathrm{C}(=\mathrm{O}) \mathrm{C} 2=\mathrm{C} 1 \mathrm{OC}$ & 5,46 \\
\hline 798 & $\mathrm{COC} 1=\mathrm{CC}(\mathrm{C})=\mathrm{CC} 2=\mathrm{C} 1 \mathrm{C}(=\mathrm{O}) \mathrm{C}=\mathrm{C}(\mathrm{C} 2=\mathrm{O}) \mathrm{C} 1=\mathrm{C}(\mathrm{C}) \mathrm{C}=\mathrm{C} 2 \mathrm{C}(=\mathrm{O}) \mathrm{C}=\mathrm{C}(\mathrm{NCC} 3=\mathrm{CC}=\mathrm{CC}=\mathrm{C} 3) \mathrm{C}(=\mathrm{O}) \mathrm{C} 2=\mathrm{C} 1 \mathrm{OC}$ & 5,59 \\
\hline 799 & $\begin{array}{l}\mathrm{COC} 1=\mathrm{CC}(\mathrm{C})=\mathrm{CC} 2=\mathrm{C} 1 \mathrm{C}(=\mathrm{O}) \mathrm{C}=\mathrm{C}(\mathrm{C} 2=\mathrm{O}) \mathrm{C} 1=\mathrm{C}(\mathrm{C}) \mathrm{C}=\mathrm{C} 2 \mathrm{C}(=\mathrm{O}) \mathrm{C}=\mathrm{C}(\mathrm{NC} 3=\mathrm{CC}=\mathrm{C}(\mathrm{OC} 4 \mathrm{O}[\mathrm{C} @ \mathrm{H}](\mathrm{COC}(\mathrm{C})=\mathrm{O} \\
)[\mathrm{C} @ @ \mathrm{H}](\mathrm{OC}(\mathrm{C})=\mathrm{O})[\mathrm{C} @ \mathrm{H}](\mathrm{OC}(\mathrm{C})=\mathrm{O})[\mathrm{C} @ \mathrm{H}] 4 \mathrm{OC}(\mathrm{C})=\mathrm{O}) \mathrm{C}=\mathrm{C} 3) \mathrm{C}(=\mathrm{O}) \mathrm{C} 2=\mathrm{C} 1 \mathrm{OC}\end{array}$ & 6,3 \\
\hline 800 & $\mathrm{COC} 1=\mathrm{CC}(\mathrm{C})=\mathrm{CC} 2=\mathrm{C} 1 \mathrm{C}(=\mathrm{O}) \mathrm{C} 1 \mathrm{OC} 1(\mathrm{C} 2=\mathrm{O}) \mathrm{C} 1=\mathrm{C}(\mathrm{C}) \mathrm{C}=\mathrm{C} 2 \mathrm{C}(=\mathrm{O}) \mathrm{C} 3 \mathrm{OC} 3 \mathrm{C}(=\mathrm{O}) \mathrm{C} 2=\mathrm{C} 1 \mathrm{OC}$ & 6,7 \\
\hline 801 & $\begin{array}{l}\mathrm{COC} 1=\mathrm{CC}(\mathrm{C})=\mathrm{CC} 2=\mathrm{C} 1 \mathrm{C}(=\mathrm{O}) \mathrm{C}=\mathrm{C}(\mathrm{C} 2=\mathrm{O}) \mathrm{C} 1=\mathrm{C}(\mathrm{C}) \mathrm{C}=\mathrm{C} 2 \mathrm{C}(=\mathrm{O}) \mathrm{C}=\mathrm{C}(\mathrm{NC} 3=\mathrm{CC}=\mathrm{C} 4 \mathrm{C}=\mathrm{CC}=\mathrm{CC} 4=\mathrm{C} 3) \mathrm{C}(=\mathrm{O}) \mathrm{C} \\
2=\mathrm{C} 1 \mathrm{OC}\end{array}$ & 5,7 \\
\hline 802 & $\mathrm{COC} 1=\mathrm{CC}(\mathrm{C})=\mathrm{CC} 2=\mathrm{C} 1 \mathrm{C}(=\mathrm{O}) \mathrm{C}=\mathrm{C}(\mathrm{C} 2=\mathrm{O}) \mathrm{C} 1=\mathrm{C}(\mathrm{C}) \mathrm{C}=\mathrm{C} 2 \mathrm{C}(=\mathrm{O}) \mathrm{C}=\mathrm{C}(\mathrm{NC}(\mathrm{C})=\mathrm{O}) \mathrm{C}(=\mathrm{O}) \mathrm{C} 2=\mathrm{C} 1 \mathrm{OC}$ & 6,05 \\
\hline 803 & $\begin{array}{l}\mathrm{COC} 1=\mathrm{CC}(\mathrm{C})=\mathrm{CC} 2=\mathrm{C} 1 \mathrm{C}(=\mathrm{O}) \mathrm{C}=\mathrm{C}(\mathrm{C} 2=\mathrm{O}) \mathrm{C} 1=\mathrm{C}(\mathrm{C}) \mathrm{C}=\mathrm{C} 2 \mathrm{C}(=\mathrm{O}) \mathrm{C}=\mathrm{C}(\mathrm{NC} 3=\mathrm{CC}=\mathrm{C}(\mathrm{OC} 4 \mathrm{O}[\mathrm{C} @ \mathrm{H}](\mathrm{COC}(\mathrm{C})=\mathrm{O} \\
)[\mathrm{C} @ @ \mathrm{H}](\mathrm{OC}(\mathrm{C})=\mathrm{O})[\mathrm{C} @ \mathrm{H}](\mathrm{OC}(\mathrm{C})=\mathrm{O})[\mathrm{C} @ @ \mathrm{H}] 4 \mathrm{OC}(\mathrm{C})=\mathrm{O}) \mathrm{C}=\mathrm{C} 3) \mathrm{C}(=\mathrm{O}) \mathrm{C} 2=\mathrm{C} 1 \mathrm{OC}\end{array}$ & 5,54 \\
\hline 804 & $\mathrm{CCOC} 1=\mathrm{C} 2 \mathrm{C}(\mathrm{O})=\mathrm{CC}(=\mathrm{C}(\mathrm{O}) \mathrm{C} 2=\mathrm{CC}(\mathrm{C})=\mathrm{C} 1) \mathrm{C} 1=\mathrm{C}(\mathrm{C}) \mathrm{C}=\mathrm{C} 2 \mathrm{C}(\mathrm{O})=\mathrm{CC}=\mathrm{C}(\mathrm{O}) \mathrm{C} 2=\mathrm{C} 1 \mathrm{OCC}$ & 5,77 \\
\hline 805 & $\mathrm{CC} 1=\mathrm{CC}(\mathrm{O})=\mathrm{C} 2 \mathrm{C}(=\mathrm{O}) \mathrm{C}=\mathrm{C}(\mathrm{C}(=\mathrm{O}) \mathrm{C} 2=\mathrm{C} 1) \mathrm{C} 1=\mathrm{C}(\mathrm{C}) \mathrm{C}=\mathrm{C} 2 \mathrm{C}(=\mathrm{O}) \mathrm{C}=\mathrm{CC}(=\mathrm{O}) \mathrm{C} 2=\mathrm{C} 1 \mathrm{O}$ & 5,44 \\
\hline 806 & $\begin{array}{l}\mathrm{COC} 1=\mathrm{CC}(\mathrm{C})=\mathrm{CC} 2=\mathrm{C} 1 \mathrm{C}(=\mathrm{O}) \mathrm{C}=\mathrm{C}(\mathrm{C} 2=\mathrm{O}) \mathrm{C} 1=\mathrm{C}(\mathrm{C}) \mathrm{C}=\mathrm{C} 2 \mathrm{C}(=\mathrm{O}) \mathrm{C}=\mathrm{C}(\mathrm{NC} 3=\mathrm{CC}=\mathrm{C}(\mathrm{Cl}) \mathrm{C}=\mathrm{C} 3) \mathrm{C}(=\mathrm{O}) \mathrm{C} 2=\mathrm{C} 1 \mathrm{O} \\
\mathrm{C}\end{array}$ & 6,15 \\
\hline 807 & $\mathrm{CCOC}(=\mathrm{O}) \mathrm{CNC} 1=\mathrm{CC}(=\mathrm{O}) \mathrm{C} 2=\mathrm{CC}(\mathrm{C})=\mathrm{C}(\mathrm{C}(\mathrm{OC})=\mathrm{C} 2 \mathrm{C} 1=\mathrm{O}) \mathrm{C} 1=\mathrm{CC}(=\mathrm{O}) \mathrm{C} 2=\mathrm{C}(\mathrm{OC}) \mathrm{C}=\mathrm{C}(\mathrm{C}) \mathrm{C}=\mathrm{C} 2 \mathrm{C} 1=\mathrm{O}$ & 6,05 \\
\hline 808 & $\mathrm{CCOC} 1=\mathrm{CC}(\mathrm{C})=\mathrm{CC} 2=\mathrm{C} 1 \mathrm{C}(=\mathrm{O}) \mathrm{C}=\mathrm{C}(\mathrm{C} 2=\mathrm{O}) \mathrm{C} 1=\mathrm{C}(\mathrm{C}) \mathrm{C}=\mathrm{C} 2 \mathrm{C}(=\mathrm{O}) \mathrm{C}=\mathrm{C}(\mathrm{NCCO}) \mathrm{C}(=\mathrm{O}) \mathrm{C} 2=\mathrm{C} 1 \mathrm{OCC}$ & 6,05 \\
\hline 809 & $\begin{array}{l}\mathrm{COC} 1=\mathrm{CC}(\mathrm{C})=\mathrm{CC} 2=\mathrm{C} 1 \mathrm{C}(=\mathrm{O}) \mathrm{C}=\mathrm{C}(\mathrm{C} 2=\mathrm{O}) \mathrm{C} 1=\mathrm{C}(\mathrm{C}) \mathrm{C}=\mathrm{C} 2 \mathrm{C}(=\mathrm{O}) \mathrm{C}=\mathrm{C}(\mathrm{NCCOC} 3 \mathrm{O}[\mathrm{C} @ \mathrm{H}](\mathrm{COC}(\mathrm{C})=\mathrm{O})[\mathrm{C} @ @) \\
\mathrm{H}](\mathrm{OC}(\mathrm{C})=\mathrm{O}) \mathrm{C}=\mathrm{C} 3) \mathrm{C}(=\mathrm{O}) \mathrm{C} 2=\mathrm{C} 1 \mathrm{OC}\end{array}$ & 5,24 \\
\hline 810 & $\mathrm{FC} 1=\mathrm{CC}=\mathrm{C}(\mathrm{C}=\mathrm{C} 1) \mathrm{C}(=\mathrm{O}) \mathrm{NC} \mid \mathrm{C}=\mathrm{C} / \mathrm{CN} 1 \mathrm{C}=\mathrm{CC}(=\mathrm{O}) \mathrm{NC} 1=\mathrm{O}$ & 4 \\
\hline 811 & $\mathrm{FC} 1=\mathrm{CN}(\mathrm{ClC}=\mathrm{C} / \mathrm{CNC}(=\mathrm{O}) \mathrm{C} 2=\mathrm{CC}=\mathrm{CC}=\mathrm{C} 2) \mathrm{C}(=\mathrm{O}) \mathrm{NC} 1=\mathrm{O}$ & 4,06 \\
\hline 812 & $\mathrm{FC}(\mathrm{F})(\mathrm{F}) \mathrm{C} 1=\mathrm{CN}(\mathrm{ClC}=\mathrm{C} / \mathrm{CNC}(=\mathrm{O}) \mathrm{C} 2=\mathrm{CC}=\mathrm{CC}=\mathrm{C} 2) \mathrm{C}(=\mathrm{O}) \mathrm{NC} 1=\mathrm{O}$ & 4 \\
\hline 813 & $\mathrm{NC} 1=\mathrm{NC}(=\mathrm{O}) \mathrm{N}(\mathrm{ClC}=\mathrm{C} / \mathrm{CNC}(=\mathrm{O}) \mathrm{C} 2=\mathrm{CC}=\mathrm{CC}=\mathrm{C} 2) \mathrm{C}=\mathrm{C} 1 \mathrm{~F}$ & 4 \\
\hline 814 & $\mathrm{NC} 1=\mathrm{C} 2 \mathrm{~N}=\mathrm{CN}(\mathrm{C} \backslash \mathrm{C}=\mathrm{C} / \mathrm{CNC}(=\mathrm{O}) \mathrm{C} 3=\mathrm{CC}=\mathrm{CC}=\mathrm{C} 3) \mathrm{C} 2=\mathrm{NC}=\mathrm{N} 1$ & 4 \\
\hline 815 & $\mathrm{NC} 1=\mathrm{NC}(=\mathrm{O}) \mathrm{N}(\mathrm{ClC}=\mathrm{C} / \mathrm{CNC}(=\mathrm{O}) \mathrm{C} 2=\mathrm{CC}=\mathrm{C}(\mathrm{F}) \mathrm{C}=\mathrm{C} 2) \mathrm{C}=\mathrm{C} 1 \mathrm{~F}$ & 4,49 \\
\hline 816 & $\mathrm{NC} 1=\mathrm{NC}(=\mathrm{O}) \mathrm{N}(\mathrm{ClC}=\mathrm{C} / \mathrm{CNC}(=\mathrm{O}) \mathrm{C} 2=\mathrm{CC}=\mathrm{CC}=\mathrm{C} 2) \mathrm{C}=\mathrm{C} 1$ & 4 \\
\hline 817 & $\mathrm{CC} 1=\mathrm{CN}(\mathrm{ClC}=\mathrm{C} / \mathrm{CNC}(=\mathrm{O}) \mathrm{C} 2=\mathrm{CC}=\mathrm{C}(\mathrm{F}) \mathrm{C}=\mathrm{C} 2) \mathrm{C}(=\mathrm{O}) \mathrm{NC} 1=\mathrm{O}$ & 4 \\
\hline 818 & $\mathrm{CC} 1=\mathrm{CN}(\mathrm{ClC}=\mathrm{C} / \mathrm{CNC}(=\mathrm{O}) \mathrm{C} 2=\mathrm{CC}=\mathrm{CC}=\mathrm{C} 2) \mathrm{C}(=\mathrm{O}) \mathrm{NC} 1=\mathrm{O}$ & 4 \\
\hline 819 & $\mathrm{NC} 1=\mathrm{NC}(=\mathrm{O}) \mathrm{N}(\mathrm{ClC}=\mathrm{C} / \mathrm{CNC}(=\mathrm{O}) \mathrm{C} 2=\mathrm{CC}=\mathrm{C}(\mathrm{F}) \mathrm{C}=\mathrm{C} 2) \mathrm{C}=\mathrm{C} 1$ & 4 \\
\hline 820 & $\mathrm{O}=\mathrm{C}(\mathrm{NClC}=\mathrm{C} / \mathrm{CN} 1 \mathrm{C}=\mathrm{CC}(=\mathrm{O}) \mathrm{NC} 1=\mathrm{O}) \mathrm{C} 1=\mathrm{CC}=\mathrm{CC}=\mathrm{C} 1$ & 4 \\
\hline 821 & $\mathrm{OC} 1=\mathrm{CC}=\mathrm{C} 2 \mathrm{C}(\mathrm{CC} 3=\mathrm{C} 4 \mathrm{C}=\mathrm{CC}=\mathrm{CC} 4=\mathrm{NC} 4=\mathrm{CC}=\mathrm{CC}=\mathrm{C} 34)=\mathrm{CC}(=\mathrm{O}) \mathrm{OC} 2=\mathrm{C} 1$ & 4 \\
\hline 822 & $\mathrm{OC} 1=\mathrm{CC}=\mathrm{C} 2 \mathrm{OC}(=\mathrm{O}) \mathrm{C}=\mathrm{C}(\mathrm{CC}=\mathrm{C} 4 \mathrm{C}=\mathrm{CC}=\mathrm{CC} 4=\mathrm{NC} 4=\mathrm{CC}=\mathrm{CC}=\mathrm{C} 34) \mathrm{C} 2=\mathrm{C} 1$ & 4 \\
\hline 823 & $\mathrm{CC} 1=\mathrm{CC}=\mathrm{C} 2 \mathrm{C}(\mathrm{CC} 3=\mathrm{C} 4 \mathrm{C}=\mathrm{CC}=\mathrm{CC} 4=\mathrm{NC} 4=\mathrm{CC}=\mathrm{CC}=\mathrm{C} 34)=\mathrm{CC}(=\mathrm{O}) \mathrm{OC} 2=\mathrm{C} 1$ & 4 \\
\hline 824 & $\mathrm{ClC} 1=\mathrm{CC}=\mathrm{C} 2 \mathrm{C}(\mathrm{CC} 3=\mathrm{C} 4 \mathrm{C}=\mathrm{CC}=\mathrm{CC} 4=\mathrm{NC} 4=\mathrm{CC}=\mathrm{CC}=\mathrm{C} 34)=\mathrm{CC}(=\mathrm{O}) \mathrm{OC} 2=\mathrm{C} 1$ & 4 \\
\hline 825 & {$[\mathrm{O}-][\mathrm{N}+](=\mathrm{O}) \mathrm{C} 1=\mathrm{CC}=\mathrm{C} 2 \mathrm{C}(\mathrm{CC} 3=\mathrm{C} 4 \mathrm{C}=\mathrm{CC}=\mathrm{CC} 4=\mathrm{NC} 4=\mathrm{C} 3 \mathrm{C}=\mathrm{CC}=\mathrm{C} 4)=\mathrm{CC}(=\mathrm{O}) \mathrm{OC} 2=\mathrm{C} 1$} & 4 \\
\hline 826 & $\mathrm{ClC} 1=\mathrm{CC}=\mathrm{C} 2 \mathrm{OC}(=\mathrm{O}) \mathrm{C}=\mathrm{C}(\mathrm{CC} 3=\mathrm{C} 4 \mathrm{C}=\mathrm{CC}=\mathrm{CC} 4=\mathrm{NC} 4=\mathrm{CC}=\mathrm{CC}=\mathrm{C} 34) \mathrm{C} 2=\mathrm{C} 1$ & 4 \\
\hline 827 & $\mathrm{CC} 1=\mathrm{CC}=\mathrm{C} 2 \mathrm{OC}(=\mathrm{O}) \mathrm{C}=\mathrm{C}(\mathrm{CC} 3=\mathrm{C} 4 \mathrm{C}=\mathrm{CC}=\mathrm{CC} 4=\mathrm{NC} 4=\mathrm{CC}=\mathrm{CC}=\mathrm{C} 34) \mathrm{C} 2=\mathrm{C} 1$ & 4 \\
\hline 828 & $\mathrm{O}=\mathrm{C} 1 \mathrm{OC} 2=\mathrm{CC}=\mathrm{CC}=\mathrm{C} 2 \mathrm{C}(\mathrm{CC} 2=\mathrm{C} 3 \mathrm{C}=\mathrm{CC}=\mathrm{CC} 3=\mathrm{NC} 3=\mathrm{C} 2 \mathrm{C}=\mathrm{CC}=\mathrm{C} 3)=\mathrm{C} 1$ & 4 \\
\hline 829 & $\mathrm{ClCCN}(\mathrm{CCCl}) \mathrm{P} 1(=\mathrm{O}) \mathrm{NCCCO} 1$ & 4 \\
\hline 830 & $\begin{array}{l}\mathrm{COC} 1=\mathrm{CC}(=\mathrm{CC}(\mathrm{OC})=\mathrm{C} 1 \mathrm{O})[\mathrm{C} @ \mathrm{H}] 1[\mathrm{C} @ @ \mathrm{H}] 2[\mathrm{C} @ \mathrm{H}](\mathrm{COC} 2=\mathrm{O})[\mathrm{C} @ \mathrm{H}](\mathrm{NC} 2=\mathrm{CC} 3=\mathrm{C}(\mathrm{C}=\mathrm{CC} 4=\mathrm{CC}=\mathrm{CC}=\mathrm{C} 3 \\
4) \mathrm{C} 3=\mathrm{CC}=\mathrm{CC}=\mathrm{C} 23) \mathrm{C} 2=\mathrm{CC} 3=\mathrm{C}(\mathrm{OCO} 3) \mathrm{C}=\mathrm{C} 12\end{array}$ & 8 \\
\hline 831 & $\mathrm{CC}[\mathrm{C} @ @] 1(\mathrm{O}) \mathrm{C}(=\mathrm{O}) \mathrm{OCC} 2=\mathrm{C} 1 \mathrm{C}=\mathrm{C} 1 \mathrm{~N}(\mathrm{CC} 3=\mathrm{CC} 4=\mathrm{C}(\mathrm{C}=\mathrm{CC}=\mathrm{C} 4) \mathrm{N}=\mathrm{C} 13) \mathrm{C} 2=\mathrm{O}$ & 6,15 \\
\hline 832 & $\begin{array}{l}\mathrm{COC} 1=\mathrm{CC}(=\mathrm{CC}(\mathrm{OC})=\mathrm{C} 1 \mathrm{OC})[\mathrm{C} @ \mathrm{H}] 1[\mathrm{C} @ @ \mathrm{H}] 2[\mathrm{C} @ \mathrm{H}](\mathrm{COC} 2=\mathrm{O})[\mathrm{C} @ \mathrm{H}](\mathrm{NC} 2=\mathrm{CC}=\mathrm{C}(\mathrm{C}=\mathrm{C} 2) \mathrm{C}(=\mathrm{O}) \mathrm{C} 2=\mathrm{CC} \\
=\mathrm{C}(\mathrm{F}) \mathrm{C}=\mathrm{C} 2) \mathrm{C} 2=\mathrm{CC} 3=\mathrm{C}(\mathrm{OCO} 3) \mathrm{C}=\mathrm{C} 12\end{array}$ & 5,15 \\
\hline 833 & $\begin{array}{l}\mathrm{COC} 1=\mathrm{CC}(=\mathrm{CC}(\mathrm{OC})=\mathrm{C} 1 \mathrm{O})[\mathrm{C} @ \mathrm{H}] 1[\mathrm{C} @ @ \mathrm{H}] 2[\mathrm{C} @ \mathrm{H}](\mathrm{COC} 2=\mathrm{O})[\mathrm{C} @ \mathrm{H}](\mathrm{NC} 2=\mathrm{CC}=\mathrm{C} 3 \mathrm{C}=\mathrm{CC} 4=\mathrm{CC}=\mathrm{CC} 5=\mathrm{C} \\
\mathrm{C}=\mathrm{C} 2 \mathrm{C} 3=\mathrm{C} 45) \mathrm{C} 2=\mathrm{CC} 3=\mathrm{C}(\mathrm{OCO} 3) \mathrm{C}=\mathrm{C} 12\end{array}$ & 9,7 \\
\hline 834 & $\begin{array}{l}\mathrm{COC} 1=\mathrm{CC}(=\mathrm{CC}(\mathrm{OC})=\mathrm{C} 1 \mathrm{OC})[\mathrm{C} @ \mathrm{H}] 1[\mathrm{C} @ @ \mathrm{H}] 2[\mathrm{C} @ \mathrm{H}](\mathrm{COC} 2=\mathrm{O})[\mathrm{C} @ \mathrm{H}](\mathrm{NC} 2=\mathrm{C} 3 \mathrm{C}=\mathrm{C} 4 \mathrm{C}=\mathrm{CC}=\mathrm{CC} 4=\mathrm{CC} 3 \\
=\mathrm{CC}=\mathrm{C} 2) \mathrm{C} 2=\mathrm{CC} 3=\mathrm{C}(\mathrm{OCO} 3) \mathrm{C}=\mathrm{C} 12\end{array}$ & 5,17 \\
\hline
\end{tabular}




\begin{tabular}{|c|c|c|}
\hline NAME & SMILE & $\lg (1 / \mathbf{I C 5 0})$ \\
\hline 835 & $\begin{array}{l}\mathrm{COC} 1=\mathrm{CC}(=\mathrm{CC}(\mathrm{OC})=\mathrm{C} 1 \mathrm{OC})[\mathrm{C} @ \mathrm{H}] 1[\mathrm{C} @ @ \mathrm{H}] 2[\mathrm{C} @ \mathrm{H}](\mathrm{COC} 2=\mathrm{O})[\mathrm{C} @ \mathrm{H}](\mathrm{NC} 2=\mathrm{CC}=\mathrm{CC} 3=\mathrm{C} 2 \mathrm{CC} 2=\mathrm{CC}=\mathrm{CC} \\
=\mathrm{C} 32) \mathrm{C} 2=\mathrm{CC} 3=\mathrm{C}(\mathrm{OCO} 3) \mathrm{C}=\mathrm{C} 12\end{array}$ & 5,52 \\
\hline 836 & $\begin{array}{l}\mathrm{COC} 1=\mathrm{CC}(=\mathrm{CC}(\mathrm{OC})=\mathrm{C} 1 \mathrm{OC})[\mathrm{C} @ \mathrm{H}] 1[\mathrm{C} @ @ \mathrm{H}] 2[\mathrm{C} @ \mathrm{H}](\mathrm{COC} 2=\mathrm{O})[\mathrm{C} @ \mathrm{H}](\mathrm{NC} 2=\mathrm{CC} 3=\mathrm{C}(\mathrm{C}=\mathrm{CC} 4=\mathrm{CC}=\mathrm{CC}= \\
\mathrm{C} 34) \mathrm{C} 3=\mathrm{CC}=\mathrm{CC}=\mathrm{C} 23) \mathrm{C} 2=\mathrm{CC} 3=\mathrm{C}(\mathrm{OCO} 3) \mathrm{C}=\mathrm{C} 12\end{array}$ & 8,7 \\
\hline 837 & $\mathrm{FC} 1=\mathrm{CNC}(=\mathrm{O}) \mathrm{NC} 1=\mathrm{O}$ & 6,52 \\
\hline 838 & $\begin{array}{l}\mathrm{COC} 1=\mathrm{CC}(=\mathrm{CC}(\mathrm{OC})=\mathrm{C} 1 \mathrm{O})[\mathrm{C} @ \mathrm{H}] 1[\mathrm{C} @ @ \mathrm{H}] 2[\mathrm{C} @ \mathrm{H}](\mathrm{COC} 2=\mathrm{O})[\mathrm{C} @ \mathrm{H}](\mathrm{NC} 2=\mathrm{CC}=\mathrm{C}(\mathrm{C}=\mathrm{C} 2) \mathrm{C}(=\mathrm{O}) \mathrm{C} 2=\mathrm{CC}= \\
\mathrm{C}(\mathrm{F}) \mathrm{C}=\mathrm{C} 2) \mathrm{C} 2=\mathrm{CC} 3=\mathrm{C}(\mathrm{OCO} 3) \mathrm{C}=\mathrm{C} 12\end{array}$ & 5,89 \\
\hline 839 & $\begin{array}{l}\mathrm{COC} 1=\mathrm{CC}(=\mathrm{CC}(\mathrm{OC})=\mathrm{C} 1 \mathrm{O})[\mathrm{C} @ \mathrm{H}] 1[\mathrm{C} @ @ \mathrm{H}] 2[\mathrm{C} @ \mathrm{H}](\mathrm{COC} 2=\mathrm{O})[\mathrm{C} @ \mathrm{H}](\mathrm{NC} 2=\mathrm{C} 3 \mathrm{C}=\mathrm{C} 4 \mathrm{C}=\mathrm{CC}=\mathrm{CC} 4=\mathrm{CC} 3= \\
\mathrm{CC}=\mathrm{C} 2) \mathrm{C} 2=\mathrm{CC} 3=\mathrm{C}(\mathrm{OCO} 3) \mathrm{C}=\mathrm{C} 12\end{array}$ & 5,07 \\
\hline 840 & $\begin{array}{l}\mathrm{COC} 1=\mathrm{CC}(=\mathrm{CC}(\mathrm{OC})=\mathrm{C} 1 \mathrm{OC})[\mathrm{C} @ \mathrm{H}] 1[\mathrm{C} @ @ \mathrm{H}] 2[\mathrm{C} @ \mathrm{H}](\mathrm{COC} 2=\mathrm{O})[\mathrm{C} @ \mathrm{H}](\mathrm{NC} 2=\mathrm{CC}=\mathrm{C}(\mathrm{C}=\mathrm{C} 2) \mathrm{C}(=\mathrm{O}) \mathrm{C} 2=\mathrm{CC} \\
=\mathrm{C}(\mathrm{C}=\mathrm{C} 2) \mathrm{N}(\mathrm{CCCl}) \mathrm{CCCl}) \mathrm{C} 2=\mathrm{CC} 3=\mathrm{C}(\mathrm{OCO} 3) \mathrm{C}=\mathrm{C} 12\end{array}$ & 6,05 \\
\hline 841 & $\begin{array}{l}\mathrm{COC} 1=\mathrm{CC}(=\mathrm{CC}(\mathrm{OC})=\mathrm{C} 1 \mathrm{O})[\mathrm{C} @ \mathrm{H}] 1[\mathrm{C} @ @ \mathrm{H}] 2[\mathrm{C} @ \mathrm{H}](\mathrm{COC} 2=\mathrm{O})[\mathrm{C} @ \mathrm{H}](\mathrm{NC} 2=\mathrm{CC}=\mathrm{CC} 3=\mathrm{C} 2 \mathrm{CC} 2=\mathrm{CC}=\mathrm{CC}= \\
\mathrm{C} 32) \mathrm{C} 2=\mathrm{CC} 3=\mathrm{C}(\mathrm{OCO} 3) \mathrm{C}=\mathrm{C} 12\end{array}$ & 6 \\
\hline 842 & $\begin{array}{l}\mathrm{COC} 1=\mathrm{CC}(=\mathrm{CC}(\mathrm{OC})=\mathrm{C} 1 \mathrm{O})[\mathrm{C} @ \mathrm{H}] 1[\mathrm{C} @ @ \mathrm{H}] 2[\mathrm{C} @ \mathrm{H}](\mathrm{COC} 2=\mathrm{O})[\mathrm{C} @ \mathrm{H}](\mathrm{O}[\mathrm{C} @ @ \mathrm{H}] 2 \mathrm{O}[\mathrm{C} @ @ \mathrm{H}] 3 \mathrm{CO}[\mathrm{C} @ @ \\
\mathrm{H}](\mathrm{C}) \mathrm{O}[\mathrm{C} @ \mathrm{H}] 3[\mathrm{C} @ \mathrm{H}](\mathrm{O})[\mathrm{C} @ \mathrm{H}] 2 \mathrm{O}) \mathrm{C} 2=\mathrm{CC} 3=\mathrm{C}(\mathrm{OCO} 3) \mathrm{C}=\mathrm{C} 12\end{array}$ & 6,05 \\
\hline 843 & $\begin{array}{l}\mathrm{COC} 1=\mathrm{CC}(\backslash \mathrm{C}=\mathrm{C} \backslash \mathrm{C}(\backslash \mathrm{O})=\mathrm{ClC}(=\mathrm{O}) \backslash \mathrm{C}=\mathrm{ClC} 2=\mathrm{CC}=\mathrm{C}(\mathrm{N} 3 \mathrm{C}=\mathrm{CC} 4=\mathrm{CC}=\mathrm{CC}=\mathrm{C} 34) \mathrm{C}(\mathrm{OC})=\mathrm{C} 2)=\mathrm{CC}=\mathrm{C} 1 \mathrm{~N} 1 \mathrm{C}=\mathrm{CC} 2= \\
\mathrm{CC}=\mathrm{CC}=\mathrm{C} 12\end{array}$ & 4 \\
\hline 844 & $\mathrm{COC} 1=\mathrm{CC}(\backslash \mathrm{C}=\mathrm{C}|\mathrm{C}(\backslash \mathrm{O})=\mathrm{ClC}(=\mathrm{O}) \backslash \mathrm{C}=\mathrm{C}| \mathrm{C} 2=\mathrm{CC}=\mathrm{C}(\mathrm{C}(\mathrm{N})=\mathrm{O}) \mathrm{C}(\mathrm{OC})=\mathrm{C} 2)=\mathrm{CC}=\mathrm{C} 1 \mathrm{C}(\mathrm{N})=\mathrm{O}$ & 4 \\
\hline 845 & $\mathrm{COC} 1=\mathrm{CC}(\backslash \mathrm{C}=\mathrm{ClC}(\backslash \mathrm{O})=\mathrm{ClC}(=\mathrm{O}) \backslash \mathrm{C}=\mathrm{ClC} 2=\mathrm{CC}=\mathrm{C}(\mathrm{N} 3 \mathrm{C}=\mathrm{CN}=\mathrm{C} 3) \mathrm{C}(\mathrm{OC})=\mathrm{C} 2)=\mathrm{CC}=\mathrm{C} 1 \mathrm{~N} 1 \mathrm{C}=\mathrm{CN}=\mathrm{C} 1$ & 4 \\
\hline 846 & $\mathrm{COC} 1=\mathrm{CC}(\backslash \mathrm{C}=\mathrm{C} \backslash \mathrm{C}(\backslash \mathrm{O})=\mathrm{ClC}(=\mathrm{O}) \backslash \mathrm{C}=\mathrm{C} \backslash \mathrm{C} 2=\mathrm{CC}=\mathrm{C}(\mathrm{O}) \mathrm{C}(\mathrm{OC})=\mathrm{C} 2)=\mathrm{CC}=\mathrm{C} 1 \mathrm{O}$ & 4,24 \\
\hline 847 & $\begin{array}{l}\mathrm{COC} 1=\mathrm{CC}(\backslash \mathrm{C}=\mathrm{C} \backslash \mathrm{C}(\backslash \mathrm{O})=\mathrm{ClC}(=\mathrm{O}) \backslash \mathrm{C}=\mathrm{ClC} 2=\mathrm{CC}=\mathrm{C}(\mathrm{N} 3 \mathrm{C}=\mathrm{NC} 4=\mathrm{CC}=\mathrm{CC}=\mathrm{C} 34) \mathrm{C}(\mathrm{OC})=\mathrm{C} 2)=\mathrm{CC}=\mathrm{C} 1 \mathrm{~N} 1 \mathrm{C}=\mathrm{NC} 2 \\
=\mathrm{CC}=\mathrm{CC}=\mathrm{C} 12\end{array}$ & 4 \\
\hline 848 & $\mathrm{CNC} 1=\mathrm{CC}=\mathrm{C}(\backslash \mathrm{C}=\mathrm{ClC}(\backslash \mathrm{O})=\mathrm{ClC}(=\mathrm{O}) \backslash \mathrm{C}=\mathrm{ClC} 2=\mathrm{CC}=\mathrm{C}(\mathrm{NC}) \mathrm{C}(\mathrm{OC})=\mathrm{C} 2) \mathrm{C}=\mathrm{C} 1 \mathrm{OC}$ & 4 \\
\hline 849 & $\mathrm{COC} 1=\mathrm{CC}(\backslash \mathrm{C}=\mathrm{ClC}(\backslash \mathrm{O})=\mathrm{ClC}(=\mathrm{O}) \backslash \mathrm{C}=\mathrm{ClC} 2=\mathrm{CC}=\mathrm{C}(\mathrm{I}) \mathrm{C}(\mathrm{OC})=\mathrm{C} 2)=\mathrm{CC}=\mathrm{C} 1 \mathrm{I}$ & 4 \\
\hline 850 & $\mathrm{COC} 1=\mathrm{CC}(\backslash \mathrm{C}=\mathrm{ClC}(\backslash \mathrm{O})=\mathrm{ClC}(=\mathrm{O}) \backslash \mathrm{C}=\mathrm{ClC} 2=\mathrm{CC}=\mathrm{C}(\mathrm{NC}(\mathrm{C})=\mathrm{O}) \mathrm{C}(\mathrm{OC})=\mathrm{C} 2)=\mathrm{CC}=\mathrm{C} 1 \mathrm{NC}(\mathrm{C})=\mathrm{O}$ & 4 \\
\hline 851 & $\mathrm{COC} 1=\mathrm{CC}(\backslash \mathrm{C}=\mathrm{ClC}(\backslash \mathrm{O})=\mathrm{ClC}(=\mathrm{O}) \backslash \mathrm{C}=\mathrm{ClC} 2=\mathrm{CC}=\mathrm{C}(\mathrm{N} 3 \mathrm{C}=\mathrm{CC}=\mathrm{N} 3) \mathrm{C}(\mathrm{OC})=\mathrm{C} 2)=\mathrm{CC}=\mathrm{C} 1 \mathrm{~N} 1 \mathrm{C}=\mathrm{CC}=\mathrm{N} 1$ & 4 \\
\hline 852 & $\mathrm{COC} 1=\mathrm{CC}(\backslash \mathrm{C}=\mathrm{C} \backslash \mathrm{C}(\backslash \mathrm{O})=\mathrm{C} \backslash \mathrm{C}(=\mathrm{O}) \backslash \mathrm{C}=\mathrm{C} \backslash \mathrm{C} 2=\mathrm{CC}=\mathrm{C}(\mathrm{Br}) \mathrm{C}(\mathrm{OC})=\mathrm{C} 2)=\mathrm{CC}=\mathrm{C} 1 \mathrm{Br}$ & 4 \\
\hline 853 & $\mathrm{COC} 1=\mathrm{CC}(\backslash \mathrm{C}=\mathrm{C} \backslash \mathrm{C}(\backslash \mathrm{O})=\mathrm{C} \backslash \mathrm{C}(=\mathrm{O}) \backslash \mathrm{C}=\mathrm{C} \backslash \mathrm{C} 2=\mathrm{CC}=\mathrm{C}(\mathrm{N}) \mathrm{C}(\mathrm{OC})=\mathrm{C} 2)=\mathrm{CC}=\mathrm{C} 1 \mathrm{~N}$ & 4 \\
\hline 854 & $\mathrm{ClC} 1=\mathrm{CC}=\mathrm{C}(\mathrm{C}=\mathrm{C} 1) \mathrm{N} 1 \mathrm{C}(=\mathrm{O}) \mathrm{N}(\mathrm{CC} 2=\mathrm{CC}=\mathrm{CC}=\mathrm{C} 2) \mathrm{C}(=\mathrm{N}) \mathrm{C} 1=\mathrm{S}$ & 5 \\
\hline 855 & $\mathrm{COC} 1=\mathrm{CC}=\mathrm{C}(\mathrm{N} 2 \mathrm{C}(=\mathrm{O}) \mathrm{N}(\mathrm{C}(=\mathrm{N}) \mathrm{C} 2=\mathrm{S}) \mathrm{C} 2=\mathrm{CC}=\mathrm{C}(\mathrm{C}) \mathrm{C}=\mathrm{C} 2) \mathrm{C}(\mathrm{OC})=\mathrm{C} 1$ & 4,7 \\
\hline 856 & $\mathrm{COC} 1=\mathrm{CC}=\mathrm{C}(\mathrm{N} 2 \mathrm{C}(=\mathrm{O}) \mathrm{N}(\mathrm{C}(=\mathrm{N}) \mathrm{C} 2=\mathrm{S}) \mathrm{C} 2=\mathrm{CC}=\mathrm{C}(\mathrm{SC}) \mathrm{C}=\mathrm{C} 2) \mathrm{C}(\mathrm{OC})=\mathrm{C} 1$ & 4,51 \\
\hline 857 & $\mathrm{COC} 1=\mathrm{CC}=\mathrm{C}(\mathrm{C}=\mathrm{C} 1) \mathrm{N} 1 \mathrm{C}(=\mathrm{O}) \mathrm{N}(\mathrm{C}(=\mathrm{S}) \mathrm{C} 1=\mathrm{N}) \mathrm{C} 1=\mathrm{CC}=\mathrm{C}(\mathrm{OC}) \mathrm{C}=\mathrm{C} 1 \mathrm{OC}$ & 4,98 \\
\hline 858 & $\begin{array}{l}\mathrm{CCCCCC} 1=\mathrm{CN}(\mathrm{N}=\mathrm{N} 1)[\mathrm{C} @ \mathrm{H}] 1[\mathrm{C} @ \mathrm{H}] 2 \mathrm{COC}(=\mathrm{O})[\mathrm{C} @ @ \mathrm{H}] 2[\mathrm{C} @ \mathrm{H}](\mathrm{C} 2=\mathrm{CC}(\mathrm{OC})=\mathrm{C}(\mathrm{O}) \mathrm{C}(\mathrm{OC})=\mathrm{C} 2) \mathrm{C} 2=\mathrm{CC} 3 \\
=\mathrm{C}(\mathrm{OCO} 3) \mathrm{C}=\mathrm{C} 12\end{array}$ & 4,68 \\
\hline 859 & $\begin{array}{l}\mathrm{CCC} 1=\mathrm{CN}(\mathrm{N}=\mathrm{N} 1)[\mathrm{C} @ \mathrm{H}] 1[\mathrm{C} @ \mathrm{H}] 2 \mathrm{COC}(=\mathrm{O})[\mathrm{C} @ @ \mathrm{H}] 2[\mathrm{C} @ \mathrm{H}](\mathrm{C} 2=\mathrm{CC}(\mathrm{OC})=\mathrm{C}(\mathrm{O}) \mathrm{C}(\mathrm{OC})=\mathrm{C} 2) \mathrm{C} 2=\mathrm{CC} 3=\mathrm{C}(\mathrm{O} \\
\mathrm{CO} 3) \mathrm{C}=\mathrm{C} 12\end{array}$ & 7,3 \\
\hline 860 & $\begin{array}{l}\mathrm{CCCC} 1=\mathrm{CN}(\mathrm{N}=\mathrm{N} 1)[\mathrm{C} @ \mathrm{H}] 1[\mathrm{C} @ \mathrm{H}] 2 \mathrm{COC}(=\mathrm{O})[\mathrm{C} @ @ \mathrm{H}] 2[\mathrm{C} @ \mathrm{H}](\mathrm{C} 2=\mathrm{CC}(\mathrm{OC})=\mathrm{C}(\mathrm{OC}) \mathrm{C}(\mathrm{OC})=\mathrm{C} 2) \mathrm{C} 2=\mathrm{CC} 3= \\
\mathrm{C}(\mathrm{OCO} 3) \mathrm{C}=\mathrm{C} 12\end{array}$ & 5,8 \\
\hline 861 & $\begin{array}{l}\mathrm{COC} 1=\mathrm{CC}(=\mathrm{CC}(\mathrm{OC})=\mathrm{C} 1 \mathrm{OC})[\mathrm{C} @ \mathrm{H}] 1[\mathrm{C} @ @ \mathrm{H}] 2[\mathrm{C} @ \mathrm{H}](\mathrm{COC} 2=\mathrm{O})[\mathrm{C} @ \mathrm{H}](\mathrm{N} 2 \mathrm{C}=\mathrm{C}(\mathrm{CO}) \mathrm{N}=\mathrm{N} 2) \mathrm{C} 2=\mathrm{CC} 3=\mathrm{C}( \\
\mathrm{OCO} 3) \mathrm{C}=\mathrm{C} 12\end{array}$ & 7,22 \\
\hline 862 & $\begin{array}{l}\mathrm{COC} 1=\mathrm{CC}(=\mathrm{CC}(\mathrm{OC})=\mathrm{C} 1 \mathrm{O})[\mathrm{C} @ \mathrm{H}] 1[\mathrm{C} @ @ \mathrm{H}] 2[\mathrm{C} @ \mathrm{H}](\mathrm{COC} 2=\mathrm{O})[\mathrm{C} @ \mathrm{H}](\mathrm{O}[\mathrm{C} @ @ \mathrm{H}] 2 \mathrm{O}[\mathrm{C} @ @ \mathrm{H}] 3 \mathrm{CO}[\mathrm{C} @ @) \\
\mathrm{H}](\mathrm{C}) \mathrm{O}[\mathrm{C} @ \mathrm{H}] 3[\mathrm{C} @ \mathrm{H}](\mathrm{O})[\mathrm{C} @ \mathrm{H}] 2 \mathrm{O}) \mathrm{C} 2=\mathrm{CC} 3=\mathrm{C}(\mathrm{OCO} 3) \mathrm{C}=\mathrm{C} 12\end{array}$ & 5,67 \\
\hline 863 & $\begin{array}{l}\mathrm{CCC} 1=\mathrm{CN}(\mathrm{N}=\mathrm{N} 1)[\mathrm{C} @ \mathrm{H}] 1[\mathrm{C} @ \mathrm{H}] 2 \mathrm{COC}(=\mathrm{O})[\mathrm{C} @ @ \mathrm{H}] 2[\mathrm{C} @ \mathrm{H}](\mathrm{C} 2=\mathrm{CC}(\mathrm{OC})=\mathrm{C}(\mathrm{OC}) \mathrm{C}(\mathrm{OC})=\mathrm{C} 2) \mathrm{C} 2=\mathrm{CC} 3=\mathrm{C}( \\
\mathrm{OCO} 3) \mathrm{C}=\mathrm{C} 12\end{array}$ & 7,22 \\
\hline 864 & $\begin{array}{l}\mathrm{CCCCCC} 1=\mathrm{CN}(\mathrm{N}=\mathrm{N} 1)[\mathrm{C} @ \mathrm{H}] 1[\mathrm{C} @ \mathrm{H}] 2 \mathrm{COC}(=\mathrm{O})[\mathrm{C} @ @ \mathrm{H}] 2[\mathrm{C} @ \mathrm{H}](\mathrm{C} 2=\mathrm{CC}(\mathrm{OC})=\mathrm{C}(\mathrm{OC}) \mathrm{C}(\mathrm{OC})=\mathrm{C} 2) \mathrm{C} 2=\mathrm{CC} \\
3=\mathrm{C}(\mathrm{OCO} 3) \mathrm{C}=\mathrm{C} 12\end{array}$ & 5,11 \\
\hline 865 & $\begin{array}{l}\mathrm{CCCCCCCCC} 1=\mathrm{CN}(\mathrm{N}=\mathrm{N} 1)[\mathrm{C} @ \mathrm{H}] 1[\mathrm{C} @ \mathrm{H}] 2 \mathrm{COC}(=\mathrm{O})[\mathrm{C} @ @ \mathrm{H}] 2[\mathrm{C} @ \mathrm{H}](\mathrm{C} 2=\mathrm{CC}(\mathrm{OC})=\mathrm{C}(\mathrm{OC}) \mathrm{C}(\mathrm{OC})=\mathrm{C} 2) \mathrm{C} 2 \\
=\mathrm{CC} 3=\mathrm{C}(\mathrm{OCO} 3) \mathrm{C}=\mathrm{C} 12\end{array}$ & 4,74 \\
\hline 866 & $\begin{array}{l}\mathrm{CCCCCCC} 1=\mathrm{CN}(\mathrm{N}=\mathrm{N} 1)[\mathrm{C} @ \mathrm{H}] 1[\mathrm{C} @ \mathrm{H}] 2 \mathrm{COC}(=\mathrm{O})[\mathrm{C} @ @ \mathrm{H}] 2[\mathrm{C} @ \mathrm{H}](\mathrm{C} 2=\mathrm{CC}(\mathrm{OC})=\mathrm{C}(\mathrm{O}) \mathrm{C}(\mathrm{OC})=\mathrm{C} 2) \mathrm{C} 2=\mathrm{CC} \\
3=\mathrm{C}(\mathrm{OCO} 3) \mathrm{C}=\mathrm{C} 12\end{array}$ & 4 \\
\hline 867 & 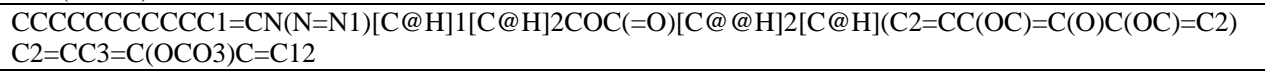 & 4,72 \\
\hline 868 & $\begin{array}{l}\mathrm{COC} 1=\mathrm{CC}(=\mathrm{CC}(\mathrm{OC})=\mathrm{C} 1 \mathrm{O})[\mathrm{C} @ \mathrm{H}] 1[\mathrm{C} @ @ \mathrm{H}] 2[\mathrm{C} @ \mathrm{H}](\mathrm{COC} 2=\mathrm{O})[\mathrm{C} @ \mathrm{H}](\mathrm{N} 2 \mathrm{C}=\mathrm{C}(\mathrm{CO}) \mathrm{N}=\mathrm{N} 2) \mathrm{C} 2=\mathrm{CC} 3=\mathrm{C}(\mathrm{O} \\
\mathrm{CO} 3) \mathrm{C}=\mathrm{C} 12\end{array}$ & 5,17 \\
\hline 869 & $\begin{array}{l}\mathrm{CCCCC} 1=\mathrm{CN}(\mathrm{N}=\mathrm{N} 1)[\mathrm{C} @ \mathrm{H}] 1[\mathrm{C} @ \mathrm{H}] 2 \mathrm{COC}(=\mathrm{O})[\mathrm{C} @ @ \mathrm{H}] 2[\mathrm{C} @ \mathrm{H}](\mathrm{C} 2=\mathrm{CC}(\mathrm{OC})=\mathrm{C}(\mathrm{OC}) \mathrm{C}(\mathrm{OC})=\mathrm{C} 2) \mathrm{C} 2=\mathrm{CC} 3 \\
=\mathrm{C}(\mathrm{OCO} 3) \mathrm{C}=\mathrm{C} 12\end{array}$ & 5,02 \\
\hline 870 & $\begin{array}{l}\mathrm{CCCCC} 1=\mathrm{CN}(\mathrm{N}=\mathrm{N} 1)[\mathrm{C} @ \mathrm{H}] 1[\mathrm{C} @ \mathrm{H}] 2 \mathrm{COC}(=\mathrm{O})[\mathrm{C} @ @ \mathrm{H}] 2[\mathrm{C} @ \mathrm{H}](\mathrm{C} 2=\mathrm{CC}(\mathrm{OC})=\mathrm{C}(\mathrm{O}) \mathrm{C}(\mathrm{OC})=\mathrm{C} 2) \mathrm{C} 2=\mathrm{CC} 3= \\
\mathrm{C}(\mathrm{OCO} 3) \mathrm{C}=\mathrm{C} 12\end{array}$ & 4,77 \\
\hline 871 & $\begin{array}{l}\mathrm{CCCCCCCCC1}=\mathrm{CN}(\mathrm{N}=\mathrm{N} 1)[\mathrm{C} @ \mathrm{H}] 1[\mathrm{C} @ \mathrm{H}] 2 \mathrm{COC}(=\mathrm{O})[\mathrm{C} @ @ \mathrm{H}] 2[\mathrm{C} @ \mathrm{H}](\mathrm{C} 2=\mathrm{CC}(\mathrm{OC})=\mathrm{C}(\mathrm{O}) \mathrm{C}(\mathrm{OC})=\mathrm{C} 2) \mathrm{C} 2= \\
\mathrm{CC} 3=\mathrm{C}(\mathrm{OCO} 3) \mathrm{C}=\mathrm{C} 12\end{array}$ & 5,42 \\
\hline 872 & $\begin{array}{l}\mathrm{CCCCCCC} 1=\mathrm{CN}(\mathrm{N}=\mathrm{N} 1)[\mathrm{C} @ \mathrm{H}] 1[\mathrm{C} @ \mathrm{H}] 2 \mathrm{COC}(=\mathrm{O})[\mathrm{C} @ @ \mathrm{H}] 2[\mathrm{C} @ \mathrm{H}](\mathrm{C} 2=\mathrm{CC}(\mathrm{OC})=\mathrm{C}(\mathrm{OC}) \mathrm{C}(\mathrm{OC})=\mathrm{C} 2) \mathrm{C} 2=\mathrm{C} \\
\mathrm{C} 3=\mathrm{C}(\mathrm{OCO} 3) \mathrm{C}=\mathrm{C} 12\end{array}$ & 5 \\
\hline 873 & $\begin{array}{l}\mathrm{CCCCCCCC} 1=\mathrm{CN}(\mathrm{N}=\mathrm{N} 1)[\mathrm{C} @ \mathrm{H}] 1[\mathrm{C} @ \mathrm{H}] 2 \mathrm{COC}(=\mathrm{O})[\mathrm{C} @ @ \mathrm{H}] 2[\mathrm{C} @ \mathrm{H}](\mathrm{C} 2=\mathrm{CC}(\mathrm{OC})=\mathrm{C}(\mathrm{OC}) \mathrm{C}(\mathrm{OC})=\mathrm{C} 2) \mathrm{C} 2= \\
\mathrm{CC} 3=\mathrm{C}(\mathrm{OCO} 3) \mathrm{C}=\mathrm{C} 12\end{array}$ & 5,01 \\
\hline 874 & $\begin{array}{l}\mathrm{CCCC} 1=\mathrm{CN}(\mathrm{N}=\mathrm{N} 1)[\mathrm{C} @ \mathrm{H}] 1[\mathrm{C} @ \mathrm{H}] 2 \mathrm{COC}(=\mathrm{O})[\mathrm{C} @ @ \mathrm{H}] 2[\mathrm{C} @ \mathrm{H}](\mathrm{C} 2=\mathrm{CC}(\mathrm{OC})=\mathrm{C}(\mathrm{O}) \mathrm{C}(\mathrm{OC})=\mathrm{C} 2) \mathrm{C} 2=\mathrm{CC} 3=\mathrm{C}( \\
\mathrm{OCO} 3) \mathrm{C}=\mathrm{C} 12\end{array}$ & 5,54 \\
\hline 875 & 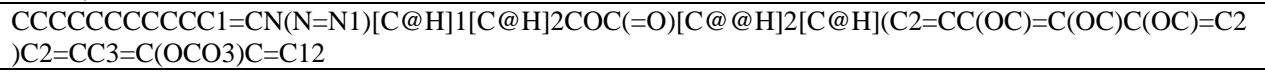 & 5,24 \\
\hline 876 & $\begin{array}{l}\mathrm{CCCCCCCC} 1=\mathrm{CN}(\mathrm{N}=\mathrm{N} 1)[\mathrm{C} @ \mathrm{H}] 1[\mathrm{C} @ \mathrm{H}] 2 \mathrm{COC}(=\mathrm{O})[\mathrm{C} @ @ \mathrm{H}] 2[\mathrm{C} @ \mathrm{H}](\mathrm{C} 2=\mathrm{CC}(\mathrm{OC})=\mathrm{C}(\mathrm{O}) \mathrm{C}(\mathrm{OC})=\mathrm{C} 2) \mathrm{C} 2=\mathrm{C} \\
\mathrm{C} 3=\mathrm{C}(\mathrm{OCO} 3) \mathrm{C}=\mathrm{C} 12\end{array}$ & 5,22 \\
\hline 877 & {$[\mathrm{O}-][\mathrm{N}+](=\mathrm{O}) \mathrm{C} 1=\mathrm{CC}=\mathrm{C}(\mathrm{Cl}) \mathrm{C}(\backslash \mathrm{C}=\mathrm{ClC} 2=\mathrm{NC} 3=\mathrm{NC}=\mathrm{CC}=\mathrm{C} 3 \mathrm{~N} 2)=\mathrm{C} 1$} & 4,31 \\
\hline 878 & {$[\mathrm{O}-][\mathrm{N}+](=\mathrm{O}) \mathrm{C} 1=\mathrm{CC}=\mathrm{C}(\mathrm{Cl}) \mathrm{C}(\backslash \mathrm{C}=\mathrm{ClC} 2=\mathrm{NC} 3=\mathrm{NC}=\mathrm{C}(\mathrm{Br}) \mathrm{C}=\mathrm{C} 3 \mathrm{~N} 2)=\mathrm{C} 1$} & 6,04 \\
\hline 879 & $\mathrm{NC} 1=\mathrm{CC}=\mathrm{C} 2 \mathrm{~N} 3 \mathrm{C}(\mathrm{C}=\mathrm{CC} 2=\mathrm{C} 1)=\mathrm{NC} 1=\mathrm{C} 3 \mathrm{C}=\mathrm{C}(\mathrm{Br}) \mathrm{C}=\mathrm{N} 1$ & 4,85 \\
\hline 880 & $\mathrm{ClC} 1=\mathrm{CC}=\mathrm{CN}=\mathrm{C} 1 \backslash \mathrm{C}=\mathrm{ClC} 1=\mathrm{NC} 2=\mathrm{NC}=\mathrm{CC}=\mathrm{C} 2 \mathrm{~N} 1$ & 4 \\
\hline 881 & {$[\mathrm{O}-][\mathrm{N}+](=\mathrm{O}) \mathrm{C} 1=\mathrm{CC}=\mathrm{C} 2 \mathrm{~N} 3 \mathrm{C}(\mathrm{C}=\mathrm{CC} 2=\mathrm{C} 1)=\mathrm{NC} 1=\mathrm{C} 3 \mathrm{C}=\mathrm{CC}=\mathrm{N} 1$} & 4 \\
\hline 882 & $\mathrm{C} 1=\mathrm{CC} 2=\mathrm{C}(\mathrm{N}=\mathrm{C} 3 \mathrm{C}=\mathrm{CC} 4=\mathrm{NC}=\mathrm{CC}=\mathrm{C} 4 \mathrm{~N} 23) \mathrm{N}=\mathrm{C} 1$ & 4 \\
\hline \multicolumn{2}{|c|}{ tps://biointerfaceresearch.com/ } & \\
\hline
\end{tabular}




\begin{tabular}{|c|c|c|}
\hline NAME & SMILE & $\lg (\mathbf{1} / \mathbf{I C 5 0})$ \\
\hline 883 & $\mathrm{Cl} . \mathrm{NC} 1=\mathrm{CC}=\mathrm{C} 2 \mathrm{~N} 3 \mathrm{C}(\mathrm{C}=\mathrm{CC} 2=\mathrm{C} 1)=\mathrm{NC} 1=\mathrm{C} 3 \mathrm{C}=\mathrm{CC}=\mathrm{N} 1$ & 4,57 \\
\hline 884 & $\mathrm{~N} \# \mathrm{CC} 1=\mathrm{CC}=\mathrm{C} 2 \mathrm{C}=\mathrm{CC} 3=\mathrm{NC} 4=\mathrm{C}(\mathrm{C}=\mathrm{CC}=\mathrm{N} 4) \mathrm{N} 3 \mathrm{C} 2=\mathrm{C} 1$ & 4,33 \\
\hline 885 & $\mathrm{ClC} 1=\mathrm{CC}=\mathrm{CC}=\mathrm{C} 1 \backslash \mathrm{C}=\mathrm{ClC} 1=\mathrm{NC} 2=\mathrm{NC}=\mathrm{CC}=\mathrm{C} 2 \mathrm{~N} 1$ & 4,53 \\
\hline 886 & {$[\mathrm{I}-] . \mathrm{C}[\mathrm{N}+] 1=\mathrm{CC}=\mathrm{CC} 2=\mathrm{C} 1 \mathrm{~N}=\mathrm{C} 1 \mathrm{C}=\mathrm{CC} 3=\mathrm{CC}=\mathrm{CC}=\mathrm{C} 3 \mathrm{~N} 21$} & 4,88 \\
\hline 887 & $\mathrm{BrC} 1=\mathrm{CN}=\mathrm{C} 2 \mathrm{~N}=\mathrm{C}(\mathrm{NC} 2=\mathrm{C} 1) \backslash \mathrm{C}=\mathrm{ClC} 1=\mathrm{CC}=\mathrm{C}(\mathrm{C}=\mathrm{C} 1) \mathrm{C} \# \mathrm{~N}$ & 5,24 \\
\hline 888 & $\mathrm{Cl} . \mathrm{C} 1 \mathrm{CN}=\mathrm{C}(\mathrm{N} 1) \mathrm{C} 1=\mathrm{CC}=\mathrm{C} 2 \mathrm{C}=\mathrm{CC} 3=\mathrm{NC} 4=\mathrm{C}(\mathrm{C}=\mathrm{CC}=\mathrm{N} 4) \mathrm{N} 3 \mathrm{C} 2=\mathrm{C} 1$ & 6,57 \\
\hline 889 & $\mathrm{Cl} . \mathrm{BrC} 1=\mathrm{CC} 2=\mathrm{C}(\mathrm{N}=\mathrm{C} 3 \mathrm{C}=\mathrm{CC} 4=\mathrm{CC}=\mathrm{C}(\mathrm{C}=\mathrm{C} 4 \mathrm{~N} 23) \mathrm{C} 2=\mathrm{NCCN} 2) \mathrm{N}=\mathrm{C} 1$ & 5,4 \\
\hline 890 & {$[\mathrm{O}-][\mathrm{N}+](=\mathrm{O}) \mathrm{C} 1=\mathrm{CC}=\mathrm{C} 2 \mathrm{~N} 3 \mathrm{C}(\mathrm{C}=\mathrm{CC} 2=\mathrm{C} 1)=\mathrm{NC} 1=\mathrm{C} 3 \mathrm{C}=\mathrm{C}(\mathrm{Br}) \mathrm{C}=\mathrm{N} 1$} & 4,51 \\
\hline 891 & $\mathrm{FC} 1=\mathrm{CNC}(=\mathrm{O}) \mathrm{NC} 1=\mathrm{O}$ & 4 \\
\hline 892 & {$[\mathrm{I}-] . \mathrm{C}[\mathrm{N}+] 1=\mathrm{CC}=\mathrm{CC} 2=\mathrm{C} 1 \mathrm{~N}=\mathrm{C} 1 \mathrm{C}=\mathrm{CC} 3=\mathrm{CC}(=\mathrm{CC}=\mathrm{C} 3 \mathrm{~N} 21)[\mathrm{N}+]([\mathrm{O}-])=\mathrm{O}$} & 4,5 \\
\hline 893 & $\mathrm{~N} 1 \mathrm{C}(\mathrm{C}=\mathrm{ClC} 2=\mathrm{CC}=\mathrm{CC}=\mathrm{C} 2)=\mathrm{NC} 2=\mathrm{NC}=\mathrm{CC}=\mathrm{C} 12$ & 5,41 \\
\hline 894 & Cl.NC1 $=\mathrm{CC}=\mathrm{C} 2 \mathrm{~N} 3 \mathrm{C}(\mathrm{C}=\mathrm{CC} 2=\mathrm{C} 1)=\mathrm{NC} 1=\mathrm{C} 3 \mathrm{C}=\mathrm{C}(\mathrm{Br}) \mathrm{C}=\mathrm{N} 1$ & 4,67 \\
\hline 895 & $\mathrm{C} 1=\mathrm{CC} 2=\mathrm{C}(\mathrm{N}=\mathrm{C} 3 \mathrm{C}=\mathrm{CC} 4=\mathrm{CC}=\mathrm{CC}=\mathrm{C} 4 \mathrm{~N} 23) \mathrm{N}=\mathrm{C} 1$ & 4,28 \\
\hline 896 & $\mathrm{NC} 1=\mathrm{CC}=\mathrm{C} 2 \mathrm{~N} 3 \mathrm{C}(\mathrm{C}=\mathrm{CC} 2=\mathrm{C} 1)=\mathrm{NC} 1=\mathrm{C} 3 \mathrm{C}=\mathrm{CC}=\mathrm{N} 1$ & 4,41 \\
\hline 897 & $\mathrm{~N} \# \mathrm{CC} 1=\mathrm{CC}=\mathrm{C}(\backslash \mathrm{C}=\mathrm{ClC} 2=\mathrm{NC} 3=\mathrm{NC}=\mathrm{CC}=\mathrm{C} 3 \mathrm{~N} 2) \mathrm{C}=\mathrm{C} 1$ & 4,07 \\
\hline 898 & $\mathrm{O}=\mathrm{C}(\mathrm{NC} 1=\mathrm{CC}=\mathrm{C}(\mathrm{C}=\mathrm{C} 1) \mathrm{C} \# \mathrm{~N}) \mathrm{C} 1=\mathrm{CC}=\mathrm{C}(\mathrm{O} 1) \mathrm{C}(=\mathrm{O}) \mathrm{NC} 1=\mathrm{CC}=\mathrm{C}(\mathrm{C}=\mathrm{C} 1) \mathrm{C} \# \mathrm{~N}$ & 4 \\
\hline 899 & Cl.Cl.CC $(\mathrm{C}) \mathrm{NC}(=\mathrm{N}) \mathrm{C} 1=\mathrm{CC}=\mathrm{C}(\mathrm{NC}(=\mathrm{O}) \mathrm{C} 2=\mathrm{CC}=\mathrm{C}(\mathrm{O} 2) \mathrm{C}(=\mathrm{O}) \mathrm{NC} 2=\mathrm{CC}=\mathrm{C}(\mathrm{C}=\mathrm{C} 2) \mathrm{C}(=\mathrm{N}) \mathrm{NC}(\mathrm{C}) \mathrm{C}) \mathrm{C}=\mathrm{C} 1$ & 5,3 \\
\hline 900 & $\mathrm{O}=\mathrm{C}(\mathrm{NC} 1=\mathrm{CC}=\mathrm{C}(\mathrm{C}=\mathrm{C} 1) \mathrm{C} \# \mathrm{~N}) \mathrm{C} 1=\mathrm{CC}=\mathrm{CO} 1$ & 4 \\
\hline 901 & $\mathrm{Cl} . \mathrm{CC}(\mathrm{C}) \mathrm{NC}(=\mathrm{N}) \mathrm{C} 1=\mathrm{CC}=\mathrm{C}(\mathrm{NC}(=\mathrm{O}) \mathrm{C} 2=\mathrm{CC}=\mathrm{CO} 2) \mathrm{C}=\mathrm{C} 1$ & 4 \\
\hline 902 & Cl.Cl.CC $(\mathrm{C}) \mathrm{NC}(=\mathrm{N}) \mathrm{C} 1=\mathrm{CC}=\mathrm{C}(\mathrm{NC}(=\mathrm{O}) \mathrm{C} 2=\mathrm{CC}=\mathrm{CC}(=\mathrm{N} 2) \mathrm{C}(=\mathrm{O}) \mathrm{NC} 2=\mathrm{CC}=\mathrm{C}(\mathrm{C}=\mathrm{C} 2) \mathrm{C}(=\mathrm{N}) \mathrm{NC}(\mathrm{C}) \mathrm{C}) \mathrm{C}=\mathrm{C} 1$ & 4,52 \\
\hline 903 & Cl.Cl.NC $(=\mathrm{N}) \mathrm{C} 1=\mathrm{CC}=\mathrm{C}(\mathrm{NC}(=\mathrm{O}) \mathrm{C} 2=\mathrm{CC}=\mathrm{C}(\mathrm{O} 2) \mathrm{C}(=\mathrm{O}) \mathrm{NC} 2=\mathrm{CC}=\mathrm{C}(\mathrm{C}=\mathrm{C} 2) \mathrm{C}(\mathrm{N})=\mathrm{N}) \mathrm{C}=\mathrm{C} 1$ & 4,96 \\
\hline 904 & Cl.Cl.NC $(=\mathrm{N}) \mathrm{C} 1=\mathrm{CC}=\mathrm{C}(\mathrm{NC}(=\mathrm{O}) \mathrm{C} 2=\mathrm{CC}=\mathrm{CC}(=\mathrm{N} 2) \mathrm{C}(=\mathrm{O}) \mathrm{NC} 2=\mathrm{CC}=\mathrm{C}(\mathrm{C}=\mathrm{C} 2) \mathrm{C}(\mathrm{N})=\mathrm{N}) \mathrm{C}=\mathrm{C} 1$ & 4,33 \\
\hline 905 & $\mathrm{Cl} . \mathrm{NC}(=\mathrm{N}) \mathrm{C} 1=\mathrm{CC}=\mathrm{C}(\mathrm{NC}(=\mathrm{O}) \mathrm{C} 2=\mathrm{CC}=\mathrm{CO} 2) \mathrm{C}=\mathrm{C} 1$ & 4 \\
\hline 906 & Cl.Cl.CC $(\mathrm{C}) \mathrm{NC}(=\mathrm{N}) \mathrm{C} 1=\mathrm{CC}=\mathrm{C}(\mathrm{C}=\mathrm{C} 1) \mathrm{C}(=\mathrm{O}) \mathrm{NC} 1=\mathrm{CC}=\mathrm{CC}(\mathrm{NC}(=\mathrm{O}) \mathrm{C} 2=\mathrm{CC}=\mathrm{C}(\mathrm{C}=\mathrm{C} 2) \mathrm{C}(=\mathrm{N}) \mathrm{NC}(\mathrm{C}) \mathrm{C})=\mathrm{N} 1$ & 4,42 \\
\hline 907 & Cl.Cl.NC $(=\mathrm{N}) \mathrm{C} 1=\mathrm{CC}=\mathrm{C}(\mathrm{C}=\mathrm{C} 1) \mathrm{C}(=\mathrm{O}) \mathrm{NC} 1=\mathrm{CC}=\mathrm{CC}(\mathrm{NC}(=\mathrm{O}) \mathrm{C} 2=\mathrm{CC}=\mathrm{C}(\mathrm{C}=\mathrm{C} 2) \mathrm{C}(\mathrm{N})=\mathrm{N})=\mathrm{N} 1$ & 4,68 \\
\hline 908 & $\mathrm{CC}(=\mathrm{O}) \mathrm{N}(\mathrm{NN}=\mathrm{N} \backslash \mathrm{C} 1=\mathrm{CC}=\mathrm{C}(\mathrm{C}=\mathrm{C} 1 \mathrm{Cl})[\mathrm{N}+]([\mathrm{O}-])=\mathrm{O}) \mathrm{C} 1=\mathrm{CC}=\mathrm{C}(\mathrm{C}=\mathrm{C} 1 \mathrm{Cl})[\mathrm{N}+]([\mathrm{O}-])=\mathrm{O}$ & 6,21 \\
\hline 909 & $\mathrm{NC} 1=\mathrm{NC}=\mathrm{CC}(=\mathrm{N} 1) \mathrm{C} 1=\mathrm{CNC} 2=\mathrm{CC}=\mathrm{CC}(\mathrm{O})=\mathrm{C} 12$ & 4 \\
\hline 910 & $\begin{array}{l}\mathrm{CCC} 1=\mathrm{C}(\mathrm{C}) \mathrm{C} 2=\mathrm{N} \backslash \mathrm{C} 1=\mathrm{C} / \mathrm{C} 1=\mathrm{C}(\mathrm{C}) \mathrm{C}(\mathrm{C}(=\mathrm{O}) \mathrm{OC})=\mathrm{C}(\mathrm{N} 1) \backslash \mathrm{C}(\mathrm{CC}(=\mathrm{O}) \mathrm{N}[\mathrm{C} @ @ \mathrm{H}](\mathrm{CC}(\mathrm{O})=\mathrm{O}) \mathrm{C}(\mathrm{O})=\mathrm{O})=\mathrm{C} 1 / \mathrm{N}=\mathrm{C}( \\
/ \mathrm{C}=\mathrm{C} 3 / \mathrm{NC}(=\mathrm{C} 2) \mathrm{C}(\mathrm{C}=\mathrm{C})=\mathrm{C} 3 \mathrm{C})[\mathrm{C} @ @ \mathrm{H}](\mathrm{C})[\mathrm{C} @ @ \mathrm{H}] 1 \mathrm{CCC}(=\mathrm{O}) \mathrm{OC}\end{array}$ & 3,43 \\
\hline 911 & $\begin{array}{l}\mathrm{CCC} 1=\mathrm{C}(\mathrm{C}) \mathrm{C} 2=\mathrm{N} \backslash \mathrm{C} 1=\mathrm{C} / \mathrm{C} 1=\mathrm{C}(\mathrm{C}) \mathrm{C}(\mathrm{C}(=\mathrm{O}) \mathrm{NCCC}(=\mathrm{O}) \mathrm{N}[\mathrm{C} @ @ \mathrm{H}](\mathrm{CC}(\mathrm{O})=\mathrm{O}) \mathrm{C}(\mathrm{O})=\mathrm{O})=\mathrm{C}(\mathrm{N} 1) \backslash \mathrm{C}(\mathrm{CC}(=\mathrm{O}) \mathrm{OC} \\
)=\mathrm{C} 1 / \mathrm{N}=\mathrm{C}(/ \mathrm{C}=\mathrm{C} 3 / \mathrm{NC}(=\mathrm{C} 2) \mathrm{C}(\mathrm{C}=\mathrm{C})=\mathrm{C} 3 \mathrm{C})[\mathrm{C} @ @ \mathrm{H}](\mathrm{C})[\mathrm{C} @ @ \mathrm{H}] 1 \mathrm{CCC}(=\mathrm{O}) \mathrm{OC}\end{array}$ & 3,42 \\
\hline 912 & $\begin{array}{l}\mathrm{CCC} 1=\mathrm{C}(\mathrm{C}) \mathrm{C} 2=\mathrm{N} \backslash \mathrm{C} 1=\mathrm{C} / \mathrm{C} 1=\mathrm{C}(\mathrm{C}) \mathrm{C}(\mathrm{C}(\mathrm{O})=\mathrm{O})=\mathrm{C}(\mathrm{N} 1) \backslash \mathrm{C}(\mathrm{CC}(\mathrm{O})=\mathrm{O})=\mathrm{C} 1 / \mathrm{N}=\mathrm{C}(/ \mathrm{C}=\mathrm{C} 3 / \mathrm{NC}(=\mathrm{C} 2) \mathrm{C}(\mathrm{C}=\mathrm{C})=\mathrm{C} 3 \mathrm{C}) \\
{[\mathrm{C} @ @ \mathrm{H}](\mathrm{C})[\mathrm{C} @ \mathrm{H}] 1 \mathrm{CCC}(\mathrm{O})=\mathrm{O}}\end{array}$ & 4,68 \\
\hline 913 & $\begin{array}{l}\mathrm{CCC} 1=\mathrm{C}(\mathrm{C}) \mathrm{C} 2=\mathrm{N} \backslash \mathrm{C} 1=\mathrm{C} / \mathrm{C} 1=\mathrm{C}(\mathrm{C}) \mathrm{C}(\mathrm{C}(=\mathrm{O}) \mathrm{OC})=\mathrm{C}(\mathrm{N} 1) \backslash \mathrm{C}(\mathrm{CC}(=\mathrm{O}) \mathrm{N}[\mathrm{C} @ @ \mathrm{H}](\mathrm{CCCCN}) \mathrm{C}(=\mathrm{O}) \mathrm{OC})=\mathrm{C} 1 / \mathrm{N}=\mathrm{C}( \\
/ \mathrm{C}=\mathrm{C} 3 / \mathrm{NC}(=\mathrm{C} 2) \mathrm{C}(\mathrm{C}=\mathrm{C})=\mathrm{C} 3 \mathrm{C})[\mathrm{C} @ @ \mathrm{H}](\mathrm{C})[\mathrm{C} @ @ \mathrm{H}] 1 \mathrm{CCC}(=\mathrm{O}) \mathrm{OC}\end{array}$ & 4,54 \\
\hline 914 & $\begin{array}{l}\mathrm{CCC} 1=\mathrm{C}(\mathrm{C}) \mathrm{C} 2=\mathrm{N} \backslash \mathrm{C} 1=\mathrm{C} / \mathrm{C} 1=\mathrm{C}(\mathrm{C}) \mathrm{C}(\mathrm{C}(=\mathrm{O}) \mathrm{N}[\mathrm{C} @ @ \mathrm{H}](\mathrm{CC}(\mathrm{O})=\mathrm{O}) \mathrm{C}(\mathrm{O})=\mathrm{O})=\mathrm{C}(\mathrm{N} 1) \backslash \mathrm{C}(\mathrm{CC}(=\mathrm{O}) \mathrm{OC})=\mathrm{C} 1 / \mathrm{N}=\mathrm{C}( \\
/ \mathrm{C}=\mathrm{C} 3 / \mathrm{NC}(=\mathrm{C} 2) \mathrm{C}(\mathrm{C}=\mathrm{C})=\mathrm{C} 3 \mathrm{C})[\mathrm{C} @ @ \mathrm{H}](\mathrm{C})[\mathrm{C} @ @ \mathrm{H}] 1 \mathrm{CCC}(=\mathrm{O}) \mathrm{OC}\end{array}$ & 3,55 \\
\hline 915 & $\begin{array}{l}\mathrm{CCC} 1=\mathrm{C}(\mathrm{C}) \mathrm{C} 2=\mathrm{N} \backslash \mathrm{C} 1=\mathrm{C} / \mathrm{C} 1=\mathrm{C}(\mathrm{C}) \mathrm{C}(\mathrm{C}(=\mathrm{O}) \mathrm{NCCNC}(=\mathrm{O})[\mathrm{C} @ @ \mathrm{H}](\mathrm{N}) \mathrm{CCCCN})=\mathrm{C}(\mathrm{N} 1) \backslash \mathrm{C}(\mathrm{CC}(=\mathrm{O}) \mathrm{OC})=\mathrm{C} 1 / \mathrm{N} \\
=\mathrm{C}(/ \mathrm{C}=\mathrm{C} 3 / \mathrm{NC}(=\mathrm{C} 2) \mathrm{C}(\mathrm{C}=\mathrm{C})=\mathrm{C} 3 \mathrm{C})[\mathrm{C} @ @ \mathrm{H}](\mathrm{C})[\mathrm{C} @ @ \mathrm{H}] 1 \mathrm{CCC}(=\mathrm{O}) \mathrm{OC}\end{array}$ & 3,57 \\
\hline 916 & $\begin{array}{l}\mathrm{CCC} 1=\mathrm{C}(\mathrm{C}) \mathrm{C} 2=\mathrm{N} \backslash \mathrm{C} 1=\mathrm{C} / \mathrm{C} 1=\mathrm{C}(\mathrm{C}) \mathrm{C}(\mathrm{C}(=\mathrm{O}) \mathrm{OC})=\mathrm{C}(\mathrm{N} 1) \backslash \mathrm{C}(\mathrm{CC}(=\mathrm{O}) \mathrm{N}[\mathrm{C} @ @ \mathrm{H}](\mathrm{CCCCN}) \mathrm{C}(=\mathrm{O}) \mathrm{OC})=\mathrm{C} 1 / \mathrm{N}=\mathrm{C}( \\
/ \mathrm{C}=\mathrm{C} 3 / \mathrm{NC}(=\mathrm{C} 2) \mathrm{C}(\mathrm{C}=\mathrm{C})=\mathrm{C} 3 \mathrm{C})[\mathrm{C} @ @ \mathrm{H}](\mathrm{C})[\mathrm{C} @ @ \mathrm{H}] 1 \mathrm{CCC}(=\mathrm{O}) \mathrm{OC}\end{array}$ & 3,4 \\
\hline 917 & $\begin{array}{l}\mathrm{CCC} 1=\mathrm{C}(\mathrm{C}) \mathrm{C} 2=\mathrm{N} \backslash \mathrm{C} 1=\mathrm{C} / \mathrm{C} 1=\mathrm{C}(\mathrm{C}) \mathrm{C}(\mathrm{C}(=\mathrm{O}) \mathrm{OC})=\mathrm{C}(\mathrm{N} 1) \backslash \mathrm{C}(\mathrm{CC}(=\mathrm{O}) \mathrm{N}[\mathrm{C} @ @ \mathrm{H}](\mathrm{CC}(\mathrm{O})=\mathrm{O}) \mathrm{C}(\mathrm{O})=\mathrm{O})=\mathrm{C} 1 / \mathrm{N}=\mathrm{C}( \\
/ \mathrm{C}=\mathrm{C} 3 / \mathrm{NC}(=\mathrm{C} 2) \mathrm{C}(\mathrm{C}=\mathrm{C})=\mathrm{C} 3 \mathrm{C})[\mathrm{C} @ @ \mathrm{H}](\mathrm{C})[\mathrm{C} @ @ \mathrm{H}] 1 \mathrm{CCC}(=\mathrm{O}) \mathrm{OC}\end{array}$ & 5,4 \\
\hline 918 & $\begin{array}{l}\mathrm{CCC} 1=\mathrm{C}(\mathrm{C}) \mathrm{C} 2=\mathrm{N} \backslash \mathrm{C} 1=\mathrm{C} / \mathrm{C} 1=\mathrm{C}(\mathrm{C}) \mathrm{C}(\mathrm{C}(=\mathrm{O}) \mathrm{NCCNC}(=\mathrm{O})[\mathrm{C} @ @ \mathrm{H}](\mathrm{N}) \mathrm{CCCCN})=\mathrm{C}(\mathrm{N} 1) \backslash \mathrm{C}(\mathrm{CC}(=\mathrm{O}) \mathrm{OC})=\mathrm{C} 1 / \mathrm{N} \\
=\mathrm{C}(/ \mathrm{C}=\mathrm{C} 3 / \mathrm{NC}(=\mathrm{C} 2) \mathrm{C}(\mathrm{C}=\mathrm{C})=\mathrm{C} 3 \mathrm{C})[\mathrm{C} @ @ \mathrm{H}](\mathrm{C})[\mathrm{C} @ @ \mathrm{H}] 1 \mathrm{CCC}(=\mathrm{O}) \mathrm{OC}\end{array}$ & 5,87 \\
\hline 919 & $\begin{array}{l}\mathrm{CCC} 1=\mathrm{C}(\mathrm{C}) \mathrm{C} 2=\mathrm{N} \backslash \mathrm{C} 1=\mathrm{C} / \mathrm{C} 1=\mathrm{C}(\mathrm{C}) \mathrm{C}(\mathrm{C}(\mathrm{O})=\mathrm{O})=\mathrm{C}(\mathrm{N} 1) \backslash \mathrm{C}(\mathrm{CC}(\mathrm{O})=\mathrm{O})=\mathrm{C} 1 / \mathrm{N}=\mathrm{C}(/ \mathrm{C}=\mathrm{C} 3 / \mathrm{NC}(=\mathrm{C} 2) \mathrm{C}(\mathrm{C}=\mathrm{C})=\mathrm{C} 3 \mathrm{C}) \\
{[\mathrm{C} @ @ \mathrm{H}](\mathrm{C})[\mathrm{C} @ \mathrm{H}] 1 \mathrm{CCC}(\mathrm{O})=\mathrm{O}}\end{array}$ & 3,4 \\
\hline 920 & $\begin{array}{l}\mathrm{CCC} 1=\mathrm{C}(\mathrm{C}) \mathrm{C} 2=\mathrm{N} \backslash \mathrm{C} 1=\mathrm{C} / \mathrm{C} 1=\mathrm{C}(\mathrm{C}) \mathrm{C}(\mathrm{C}(=\mathrm{O}) \mathrm{OC})=\mathrm{C}(\mathrm{N} 1) \backslash \mathrm{C}(\mathrm{CC}(=\mathrm{O}) \mathrm{OC})=\mathrm{C} 1 / \mathrm{N}=\mathrm{C}(/ \mathrm{C}=\mathrm{C} 3 / \mathrm{NC}(=\mathrm{C} 2) \mathrm{C}(\mathrm{C}=\mathrm{C})=\mathrm{C} \\
3 \mathrm{C})[\mathrm{C} @ @ \mathrm{H}](\mathrm{C})[\mathrm{C} @ @ \mathrm{H}] 1 \mathrm{CCC}(=\mathrm{O}) \mathrm{N}[\mathrm{C} @ @ \mathrm{H}](\mathrm{CCCCN}) \mathrm{C}(=\mathrm{O}) \mathrm{OC}\end{array}$ & 3,4 \\
\hline 921 & $\begin{array}{l}\mathrm{CCC} 1=\mathrm{C}(\mathrm{C}) \mathrm{C} 2=\mathrm{N} \backslash \mathrm{C} 1=\mathrm{C} / \mathrm{C} 1=\mathrm{C}(\mathrm{C}) \mathrm{C}(\mathrm{C}(=\mathrm{O}) \mathrm{OC})=\mathrm{C}(\mathrm{N} 1) \backslash \mathrm{C}(\mathrm{CC}(=\mathrm{O}) \mathrm{OC})=\mathrm{C} 1 / \mathrm{N}=\mathrm{C}(/ \mathrm{C}=\mathrm{C} 3 / \mathrm{NC}(=\mathrm{C} 2) \mathrm{C}(\mathrm{C}=\mathrm{C})=\mathrm{C} \\
3 \mathrm{C})[\mathrm{C} @ \mathrm{H}](\mathrm{C})[\mathrm{C} @ @ \mathrm{H}] 1 \mathrm{CCC}(=\mathrm{O}) \mathrm{N}[\mathrm{C} @ @ \mathrm{H}](\mathrm{CCCCN}) \mathrm{C}(=\mathrm{O}) \mathrm{OC}\end{array}$ & 4,58 \\
\hline 922 & $\begin{array}{l}\mathrm{CCC} 1=\mathrm{C}(\mathrm{C}) \mathrm{C} 2=\mathrm{N} \backslash \mathrm{C} 1=\mathrm{C} / \mathrm{C} 1=\mathrm{C}(\mathrm{C}) \mathrm{C}(\mathrm{C}(=\mathrm{O}) \mathrm{N}[\mathrm{C} @ @ \mathrm{H}](\mathrm{CC}(\mathrm{O})=\mathrm{O}) \mathrm{C}(\mathrm{O})=\mathrm{O})=\mathrm{C}(\mathrm{N} 1) \backslash \mathrm{C}(\mathrm{CC}(=\mathrm{O}) \mathrm{OC})=\mathrm{C} 1 / \mathrm{N}=\mathrm{C}( \\
/ \mathrm{C}=\mathrm{C} 3 / \mathrm{NC}(=\mathrm{C} 2) \mathrm{C}(\mathrm{C}=\mathrm{C})=\mathrm{C} 3 \mathrm{C})[\mathrm{C} @ @ \mathrm{H}](\mathrm{C})[\mathrm{C} @ @ \mathrm{H}] 1 \mathrm{CCC}(=\mathrm{O}) \mathrm{OC}\end{array}$ & 6,21 \\
\hline 923 & $\begin{array}{l}\mathrm{CCC} 1=\mathrm{C}(\mathrm{C}) \mathrm{C} 2=\mathrm{N} \backslash \mathrm{C} 1=\mathrm{C} / \mathrm{C} 1=\mathrm{C}(\mathrm{C}) \mathrm{C}(\mathrm{C}(=\mathrm{O}) \mathrm{NCCC}(=\mathrm{O}) \mathrm{N}[\mathrm{C} @ @ \mathrm{H}](\mathrm{CC}(\mathrm{O})=\mathrm{O}) \mathrm{C}(\mathrm{O})=\mathrm{O})=\mathrm{C}(\mathrm{N} 1) \backslash \mathrm{C}(\mathrm{CC}(=\mathrm{O}) \mathrm{OC} \\
)=\mathrm{C} 1 / \mathrm{N}=\mathrm{C}(/ \mathrm{C}=\mathrm{C} 3 / \mathrm{NC}(=\mathrm{C} 2) \mathrm{C}(\mathrm{C}=\mathrm{C})=\mathrm{C} 3 \mathrm{C})[\mathrm{C} @ @ \mathrm{H}](\mathrm{C})[\mathrm{C} @ @ \mathrm{H}] 1 \mathrm{CCC}(=\mathrm{O}) \mathrm{OC}\end{array}$ & 6,09 \\
\hline 924 & $\mathrm{CC} 1=\mathrm{NNC}(=\mathrm{O}) \backslash \mathrm{C} 1=\mathrm{ClC} 1=\mathrm{C}(\mathrm{NC} 2=\mathrm{CC}=\mathrm{C}(\mathrm{Cl}) \mathrm{C}=\mathrm{C} 12) \mathrm{C} 1=\mathrm{CC}=\mathrm{CC}=\mathrm{C} 1$ & 5,1 \\
\hline 925 & $\begin{array}{l}\mathrm{COC} 1=\mathrm{CC}=\mathrm{CC} 2=\mathrm{C} 1 \mathrm{C}(=\mathrm{O}) \mathrm{C} 1=\mathrm{C}(\mathrm{O}) \mathrm{C} 3=\mathrm{C}(\mathrm{C}[\mathrm{C} @](\mathrm{O})(\mathrm{C}[\mathrm{C} @ @ \mathrm{H}] 3 \mathrm{O}[\mathrm{C} @ \mathrm{H}] 3 \mathrm{C}[\mathrm{C} @ \mathrm{H}](\mathrm{N})[\mathrm{C} @ \mathrm{H}](\mathrm{O})[\mathrm{C} @ \mathrm{H}]( \\
\mathrm{C}) \mathrm{O} 3) \mathrm{C}(=\mathrm{O}) \mathrm{CO}) \mathrm{C}(\mathrm{O})=\mathrm{C} 1 \mathrm{C} 2=\mathrm{O}\end{array}$ & 5,06 \\
\hline 926 & $\mathrm{ClC} 1=\mathrm{CC}=\mathrm{C} 2 \mathrm{NC}(=\mathrm{C}(\mathrm{C}=\mathrm{C} 3 \mathrm{C}(=\mathrm{O}) \mathrm{NC}(=\mathrm{S}) \mathrm{NC} 3=\mathrm{O}) \mathrm{C} 2=\mathrm{C} 1) \mathrm{C} 1=\mathrm{CC}=\mathrm{CC}=\mathrm{C} 1$ & 4,34 \\
\hline 927 & $\mathrm{O}=\mathrm{C} 1 \mathrm{NC}(=\mathrm{S}) \mathrm{NC}(=\mathrm{O}) \mathrm{C} 1=\mathrm{CC} 1=\mathrm{CNC} 2=\mathrm{CC}=\mathrm{CC}=\mathrm{C} 12$ & 4 \\
\hline 928 & $\mathrm{O}=\mathrm{C} 1 \mathrm{NC}(=\mathrm{O}) \mathrm{C}(=\mathrm{CC} 2=\mathrm{CNC} 3=\mathrm{CC}=\mathrm{CC}=\mathrm{C} 23) \mathrm{C}(=\mathrm{O}) \mathrm{N} 1$ & 4 \\
\hline 929 & $\mathrm{CC} 1=\mathrm{CC}=\mathrm{C} 2 \mathrm{NC}(=\mathrm{C}(1 \mathrm{C}=\mathrm{C} 3 / \mathrm{SC}(=\mathrm{O}) \mathrm{NC} 3=\mathrm{O}) \mathrm{C} 2=\mathrm{C} 1) \mathrm{C} 1=\mathrm{CC}=\mathrm{CC}=\mathrm{C} 1$ & 4 \\
\hline 930 & $\mathrm{O}=\mathrm{C} 1 \mathrm{NC}(=\mathrm{O}) \backslash \mathrm{C}(\mathrm{S} 1)=\mathrm{ClC} 1=\mathrm{CNC} 2=\mathrm{CC}=\mathrm{CC}=\mathrm{C} 12$ & 4 \\
\hline 931 & $\mathrm{CC} 1=\mathrm{NNC}(=\mathrm{O}) \backslash \mathrm{C} 1=\mathrm{C} \backslash \mathrm{C} 1=\mathrm{CNC} 2=\mathrm{CC}=\mathrm{CC}=\mathrm{C} 12$ & 5,92 \\
\hline 932 & $\mathrm{ClC} 1=\mathrm{CC}=\mathrm{C} 2 \mathrm{NC}(=\mathrm{C}(\mathrm{C}=\mathrm{C} 3 \mathrm{C}(=\mathrm{O}) \mathrm{NC}(=\mathrm{O}) \mathrm{NC} 3=\mathrm{O}) \mathrm{C} 2=\mathrm{C} 1) \mathrm{C} 1=\mathrm{CC}=\mathrm{CC}=\mathrm{C} 1$ & 4 \\
\hline 933 & $\mathrm{CC} 1=\mathrm{CC}=\mathrm{C} 2 \mathrm{NC}(=\mathrm{C}(\mathrm{C}=\mathrm{C} 3 \mathrm{C}(=\mathrm{O}) \mathrm{NC}(=\mathrm{S}) \mathrm{NC} 3=\mathrm{O}) \mathrm{C} 2=\mathrm{C} 1) \mathrm{C} 1=\mathrm{CC}=\mathrm{CC}=\mathrm{C} 1$ & 4 \\
\hline 934 & $\mathrm{CC} 1=\mathrm{NNC}(=\mathrm{O}) \backslash \mathrm{C} 1=\mathrm{C} \mid \mathrm{C} 1=\mathrm{C}(\mathrm{NC} 2=\mathrm{CC}=\mathrm{C}(\mathrm{C}) \mathrm{C}=\mathrm{C} 12) \mathrm{C} 1=\mathrm{CC}=\mathrm{CC}=\mathrm{C} 1$ & 4,58 \\
\hline 935 & $\mathrm{CC} 1=\mathrm{CC}=\mathrm{C} 2 \mathrm{NC}(=\mathrm{C}(\mathrm{C}=\mathrm{C} 3 \mathrm{C}(=\mathrm{O}) \mathrm{NC}(=\mathrm{O}) \mathrm{NC} 3=\mathrm{O}) \mathrm{C} 2=\mathrm{C} 1) \mathrm{C} 1=\mathrm{CC}=\mathrm{CC}=\mathrm{C} 1$ & 4 \\
\hline 936 & $\mathrm{ClC} 1=\mathrm{CC}=\mathrm{C} 2 \mathrm{NC}(=\mathrm{C}(\backslash \mathrm{C}=\mathrm{C} 3 / \mathrm{SC}(=\mathrm{O}) \mathrm{NC} 3=\mathrm{O}) \mathrm{C} 2=\mathrm{C} 1) \mathrm{C} 1=\mathrm{CC}=\mathrm{CC}=\mathrm{C} 1$ & 4,92 \\
\hline 937 & $\mathrm{CC} 1 \mathrm{C}(\mathrm{CCC} 1(\mathrm{C}) \backslash \mathrm{C}=\mathrm{C} \mid \mathrm{C} 1=\mathrm{CC}=\mathrm{C}(\mathrm{O}) \mathrm{C}=\mathrm{C} 1) \mathrm{C}(\mathrm{C})(\mathrm{C}) \mathrm{O}$ & 4,72 \\
\hline 938 & $\mathrm{COC} 1=\mathrm{CC}=\mathrm{C}(\backslash \mathrm{C}=\mathrm{Cl}[\mathrm{C} @ @](\mathrm{C})(\mathrm{CCC}=\mathrm{C}(\mathrm{C}) \mathrm{C}) \backslash \mathrm{C}=\mathrm{ClC} 2=\mathrm{CC}=\mathrm{C}(\mathrm{O}) \mathrm{C}=\mathrm{C} 2) \mathrm{C}=\mathrm{C} 1$ & 4,85 \\
\hline 939 & $\mathrm{CC}(\mathrm{C})=\mathrm{CCC}[\mathrm{C} @ @](\mathrm{C})(\mathrm{C}=\mathrm{C}) \backslash \mathrm{C}=\mathrm{C} \backslash \mathrm{C} 1=\mathrm{CC}=\mathrm{C}(\mathrm{OC}(\mathrm{C})=\mathrm{O}) \mathrm{C}=\mathrm{C} 1$ & 4,7 \\
\hline 940 & $\begin{array}{l}\mathrm{CC}(\mathrm{C})(\mathrm{O}[\mathrm{C} @ @ \mathrm{H}] 1 \mathrm{O}[\mathrm{C} @ \mathrm{H}](\mathrm{CO})[\mathrm{C} @ @ \mathrm{H}](\mathrm{O})[\mathrm{C} @ \mathrm{H}](\mathrm{O})[\mathrm{C} @ \mathrm{H}] 1 \mathrm{O})[\mathrm{C} @ @ \mathrm{H}] 1 \mathrm{CC}[\mathrm{C} @ @] 2(\mathrm{C}) \mathrm{CCC}=\mathrm{C}(\mathrm{CO})[ \\
\mathrm{C} @ \mathrm{H}] 2 \mathrm{C} 1\end{array}$ & 5 \\
\hline 941 & $\mathrm{CC}(\mathrm{C})(\mathrm{O})[\mathrm{C} @ @ \mathrm{H}] 1 \mathrm{C}[\mathrm{C} @ @ \mathrm{H}] 2[\mathrm{C} @ @] 3(\mathrm{C}) \mathrm{C}[\mathrm{C} @ \mathrm{H}] 3 \mathrm{C}[\mathrm{C} @ @] 2(\mathrm{C}) \mathrm{C}[\mathrm{C} @ @ \mathrm{H}] 1 \mathrm{O}$ & 5,37 \\
\hline
\end{tabular}




\begin{tabular}{|c|c|c|}
\hline NAME & SMILE & $\lg (1 / \mathrm{IC} 50)$ \\
\hline 942 & $\begin{array}{l}\mathrm{CO}[\mathrm{C} @ @ \mathrm{H}] 1 \mathrm{O}[\mathrm{C} @ @] 2(\mathrm{CO}) \mathrm{CC}[\mathrm{C} @ @] 11 \mathrm{CC}[\mathrm{C} @] 3(\mathrm{C})[\mathrm{C} @ \mathrm{H}](\mathrm{C}[\mathrm{C} @ @ \mathrm{H}](\mathrm{O})[\mathrm{C} @ @ \mathrm{H}] 4[\mathrm{C} @ @] 5(\mathrm{C}) \mathrm{CC}[\mathrm{C} \\
@ \mathrm{H}](\mathrm{O})[\mathrm{C} @](\mathrm{C})(\mathrm{CO})[\mathrm{C} @ \mathrm{H}] 5 \mathrm{CC}[\mathrm{C} @ @] 34 \mathrm{C})[\mathrm{C} @ @ \mathrm{H}] 1[\mathrm{C} @] 2(\mathrm{C}) \mathrm{O}\end{array}$ & 5,26 \\
\hline 943 & $\begin{array}{l}\mathrm{CC} 1(\mathrm{C}) \mathrm{CC}[\mathrm{C} @] 2(\mathrm{C}) \mathrm{CC}[\mathrm{C} @] 3(\mathrm{C})[\mathrm{C} @ \mathrm{H}](\mathrm{CC}[\mathrm{C} @ \mathrm{H}] 4[\mathrm{C} @ @] 3(\mathrm{C}) \mathrm{CC}[\mathrm{C} @ \mathrm{H}] 3 \mathrm{C}(\mathrm{C})(\mathrm{C})[\mathrm{C} @ @ \mathrm{H}](\mathrm{O}) \mathrm{C}[\mathrm{C} @ \mathrm{H}]( \\
\mathrm{O})[\mathrm{C} @] 43 \mathrm{C}) \mathrm{C} 2=\mathrm{C} 1\end{array}$ & 5,27 \\
\hline 944 & $\mathrm{CC}(\mathrm{C})(\mathrm{O})[\mathrm{C} @ @ \mathrm{H}] 1 \mathrm{C}[\mathrm{C} @ @ \mathrm{H}] 2[\mathrm{C} @ @] 3(\mathrm{C}) \mathrm{C}[\mathrm{C} @ \mathrm{H}] 3 \mathrm{C}[\mathrm{C} @ @] 2(\mathrm{C}) \mathrm{C}[\mathrm{C} @ \mathrm{H}] 1 \mathrm{O}$ & 5 \\
\hline 945 & $\mathrm{CC}(\mathrm{C})(\mathrm{O})[\mathrm{C} @ @ \mathrm{H}] 1 \mathrm{C}[\mathrm{C} @ @ \mathrm{H}] 2[\mathrm{C} @ @] 3(\mathrm{C}) \mathrm{C}[\mathrm{C} @ \mathrm{H}] 3 \mathrm{C}[\mathrm{C} @ @] 2(\mathrm{C}) \mathrm{CC} 1=\mathrm{O}$ & 5,28 \\
\hline 946 & $\begin{array}{l}\mathrm{C}[\mathrm{C} @ @ \mathrm{H}] 1 \mathrm{CC}[\mathrm{C} @ @] 2(\mathrm{CC}[\mathrm{C} @] 3(\mathrm{C}) \mathrm{C}(=\mathrm{CC}[\mathrm{C} @ @ \mathrm{H}] 4[\mathrm{C} @ @] 5(\mathrm{C}) \mathrm{C}[\mathrm{C} @ @ \mathrm{H}](\mathrm{O})[\mathrm{C} @ @ \mathrm{H}](\mathrm{O})[\mathrm{C} @ @](\mathrm{C})( \\
\mathrm{CO})[\mathrm{C} @ \mathrm{H}] 5 \mathrm{CC}[\mathrm{C} @ @] 34 \mathrm{C})[\mathrm{C} @ \mathrm{H}] 2[\mathrm{C} @ \mathrm{H}] 1 \mathrm{C}) \mathrm{C}(=\mathrm{O}) \mathrm{O}[\mathrm{C} @ @ \mathrm{H}] 1 \mathrm{O}[\mathrm{C} @ \mathrm{H}](\mathrm{CO})[\mathrm{C} @ \mathrm{H}](\mathrm{O})[\mathrm{C} @ \mathrm{H}](\mathrm{O}) \\
{[\mathrm{C} @ \mathrm{H}] 1 \mathrm{O}}\end{array}$ & 5 \\
\hline 947 & 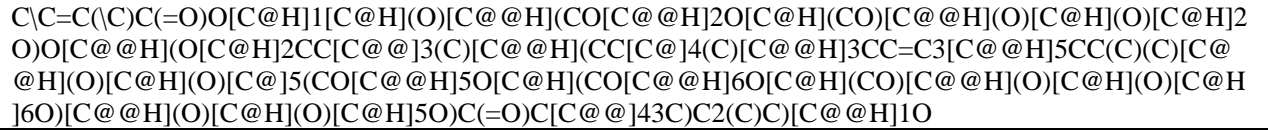 & 4 \\
\hline 948 & 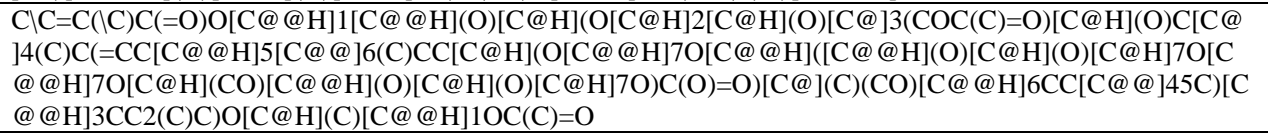 & 4 \\
\hline 949 & 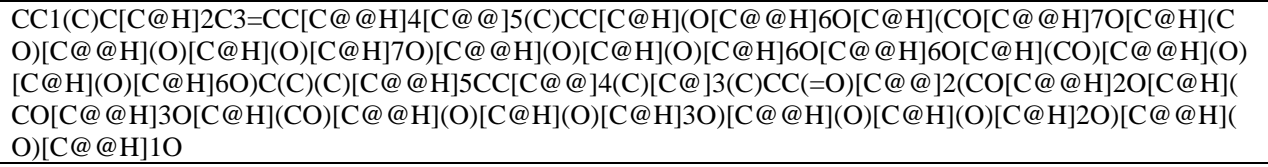 & 4 \\
\hline 950 & 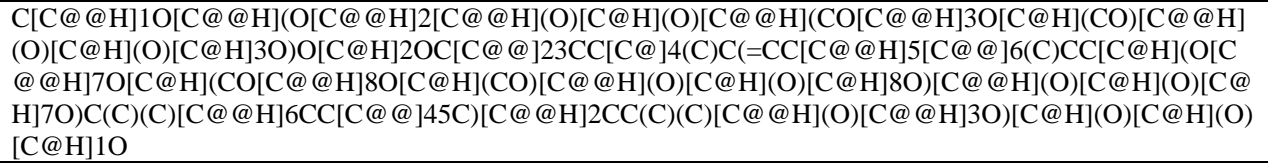 & 4 \\
\hline 951 & $\mathrm{FC} 1=\mathrm{CNC}(=\mathrm{O}) \mathrm{NC} 1=\mathrm{O}$ & 4,61 \\
\hline 952 & 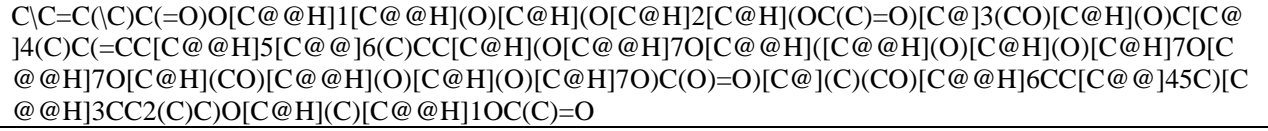 & 4,75 \\
\hline 953 & $\begin{array}{l}\mathrm{ClC}=\mathrm{C}(\mathrm{C}) \mathrm{C}(=\mathrm{O}) \mathrm{O}[\mathrm{C} @ \mathrm{H}] 1[\mathrm{C} @ @ \mathrm{H}](\mathrm{C}) \mathrm{O}[\mathrm{C} @ @ \mathrm{H}](\mathrm{O}[\mathrm{C} @ \mathrm{H}] 2[\mathrm{C} @ \mathrm{H}](\mathrm{OC}(\mathrm{C})=\mathrm{O})[\mathrm{C} @] 3(\mathrm{CO})[\mathrm{C} @ \mathrm{H}](\mathrm{O}) \mathrm{C}[\mathrm{C} \\
@] 4(\mathrm{C}) \mathrm{C}(=\mathrm{CC}[\mathrm{C} @ @ \mathrm{H}] 5[\mathrm{C} @] 6(\mathrm{C}) \mathrm{CC}[\mathrm{C} @ \mathrm{H}](\mathrm{O}[\mathrm{C} @ @ \mathrm{H}] 7 \mathrm{O}[\mathrm{C} @ \mathrm{H}]([\mathrm{C} @(\mathrm{H}](\mathrm{O})[\mathrm{C} @ \mathrm{H}](\mathrm{O})[\mathrm{C} @ \mathrm{H}] 7 \mathrm{O}[ \\
\mathrm{C} @ \mathrm{H}] 7 \mathrm{O}[\mathrm{C} @ \mathrm{H}](\mathrm{CO})[\mathrm{C} @ @ \mathrm{H}](\mathrm{O})[\mathrm{C} @ \mathrm{H}](\mathrm{O})[\mathrm{C} @ \mathrm{H}] 7 \mathrm{O}) \mathrm{C}(\mathrm{O})=\mathrm{O})[\mathrm{C} @](\mathrm{C})(\mathrm{CO})[\mathrm{C} @ \mathrm{H}] 6 \mathrm{CC}[\mathrm{C} @ @] 45 \mathrm{C})[ \\
\mathrm{C} @ \mathrm{H}] 3 \mathrm{CC} 2(\mathrm{C}) \mathrm{C})[\mathrm{C} @ \mathrm{H}](\mathrm{O})[\mathrm{C} @ \mathrm{H}] 1 \mathrm{OC}(=\mathrm{O}) \mathrm{C}(\mathrm{C})=\mathrm{C} / \mathrm{C}\end{array}$ & 5,33 \\
\hline 954 & 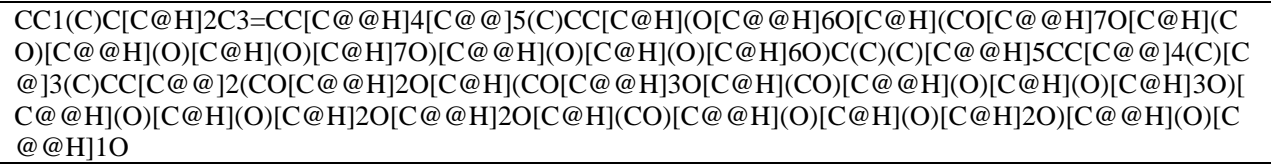 & 4 \\
\hline 955 & 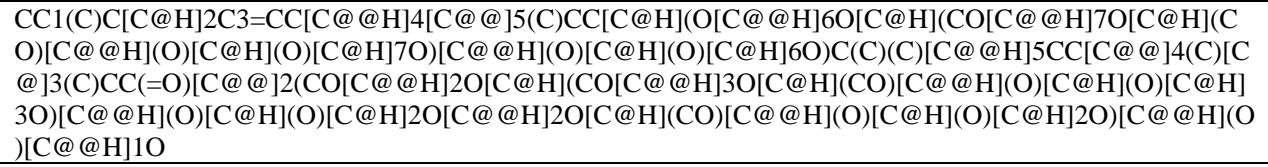 & 4 \\
\hline 956 & 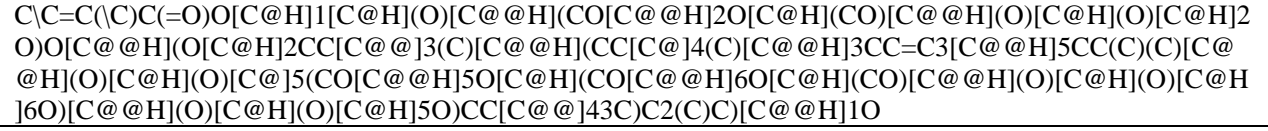 & 4 \\
\hline 957 & 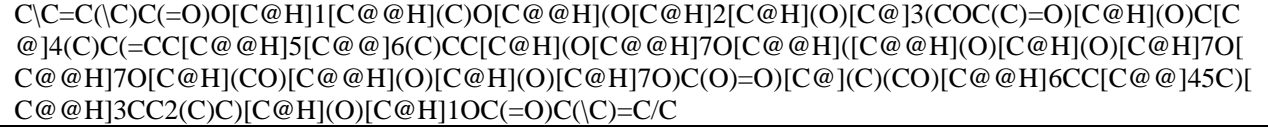 & 4 \\
\hline 958 & 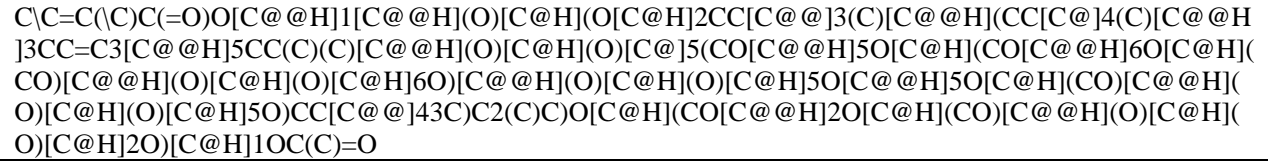 & 4 \\
\hline 959 & 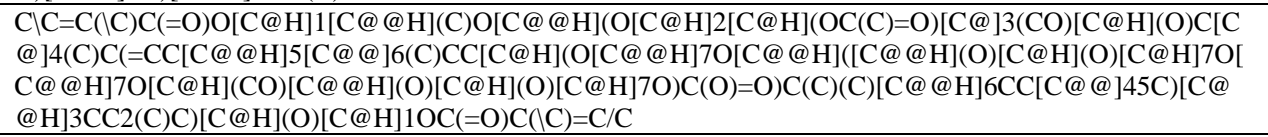 & 5,3 \\
\hline 960 & $\mathrm{CC} 1=\mathrm{C} 2[\mathrm{C} @ \mathrm{H}] 3 \mathrm{OC}(=\mathrm{O}) \mathrm{C} 4(\mathrm{CC}(=\mathrm{NO} 4) \mathrm{C} 4=\mathrm{CC}=\mathrm{CC}=\mathrm{C} 4)[\mathrm{C} @ @ \mathrm{H}] 3 \mathrm{CC}[\mathrm{C} @ @] 2(\mathrm{C}) \mathrm{C}=\mathrm{CC} 1=\mathrm{O}$ & 4 \\
\hline 961 & $\mathrm{CC} 1=\mathrm{C} 2[\mathrm{C} @ \mathrm{H}] 3 \mathrm{OC}(=\mathrm{O}) \mathrm{C} 4(\mathrm{CC}(=\mathrm{NO} 4) \mathrm{C} 4=\mathrm{CC}=\mathrm{C}(\mathrm{O}) \mathrm{C}=\mathrm{C} 4)[\mathrm{C} @ @ \mathrm{H}] 3 \mathrm{CC}[\mathrm{C} @ @] 2(\mathrm{C}) \mathrm{C}=\mathrm{CC} 1=\mathrm{O}$ & 4,4 \\
\hline 962 & $\begin{array}{l}\mathrm{CC} 1=\mathrm{C} 2[\mathrm{C} @ \mathrm{H}] 3 \mathrm{OC}(=\mathrm{O}) \mathrm{C} 4(\mathrm{C}[\mathrm{C} @ \mathrm{H}](\mathrm{N}(\mathrm{O} 4) \mathrm{C} 4=\mathrm{CC}=\mathrm{CC}=\mathrm{C} 4) \mathrm{C} 4=\mathrm{CC}=\mathrm{C}(\mathrm{Br}) \mathrm{C}=\mathrm{C} 4)[\mathrm{C} @ @ \mathrm{H}] 3 \mathrm{CC}[\mathrm{C} @ @] 2(\mathrm{C} \\
) \mathrm{C}=\mathrm{CC} 1=\mathrm{O}\end{array}$ & 4,19 \\
\hline 963 & $\begin{array}{l}\mathrm{CC} 1=\mathrm{C} 2[\mathrm{C} @ \mathrm{H}] 3 \mathrm{OC}(=\mathrm{O}) \mathrm{C} 4(\mathrm{C}[\mathrm{C} @ @ \mathrm{H}](\mathrm{N}(\mathrm{O} 4) \mathrm{C} 4=\mathrm{CC}=\mathrm{CC}=\mathrm{C} 4) \mathrm{C} 4=\mathrm{CC}(\mathrm{Cl})=\mathrm{CC}=\mathrm{C} 4 \mathrm{Cl})[\mathrm{C} @ @ \mathrm{H}] 3 \mathrm{CC}[\mathrm{C} @ @) \\
] 2(\mathrm{C}) \mathrm{C}=\mathrm{CC} 1=\mathrm{O}\end{array}$ & 4 \\
\hline 964 & $\begin{array}{l}\mathrm{CC} 1=\mathrm{C} 2[\mathrm{C} @ \mathrm{H}] 3 \mathrm{OC}(=\mathrm{O}) \mathrm{C} 4(\mathrm{CC}(=\mathrm{NO} 4) \mathrm{C} 4=\mathrm{CC}=\mathrm{C}(\mathrm{C}=\mathrm{C} 4)[\mathrm{N}+]([\mathrm{O}- \\
])=\mathrm{O})[\mathrm{C} @ @ \mathrm{H}] 3 \mathrm{CC}[\mathrm{C} @ @] 2(\mathrm{C}) \mathrm{C}=\mathrm{CC} 1=\mathrm{O}\end{array}$ & 4 \\
\hline 965 & $\mathrm{CC} 1=\mathrm{C} 2[\mathrm{C} @ \mathrm{H}] 3 \mathrm{OC}(=\mathrm{O}) \mathrm{C} 4(\mathrm{CC}(=\mathrm{NO} 4) \mathrm{C} 4=\mathrm{CC}=\mathrm{C}(\mathrm{Br}) \mathrm{C}(\mathrm{F})=\mathrm{C} 4)[\mathrm{C} @ @ \mathrm{H}] 3 \mathrm{CC}[\mathrm{C} @ @] 2(\mathrm{C}) \mathrm{C}=\mathrm{CC} 1=\mathrm{O}$ & 4,48 \\
\hline 966 & $\mathrm{CC} 1=\mathrm{C} 2[\mathrm{C} @ \mathrm{H}] 3 \mathrm{OC}(=\mathrm{O}) \mathrm{C} 4(\mathrm{CC}(=\mathrm{NO} 4) \mathrm{C} 4=\mathrm{CC}(\mathrm{Cl})=\mathrm{CC}=\mathrm{C} 4 \mathrm{Cl})[\mathrm{C} @ @ \mathrm{H}] 3 \mathrm{CC}[\mathrm{C} @ @] 2(\mathrm{C}) \mathrm{C}=\mathrm{CC} 1=\mathrm{O}$ & 4 \\
\hline 967 & $\begin{array}{l}\mathrm{CC} 1=\mathrm{C} 2[\mathrm{C} @ \mathrm{H}] 3 \mathrm{OC}(=\mathrm{O}) \mathrm{C} 4(\mathrm{C}[\mathrm{C} @ @ \mathrm{H}](\mathrm{N}(\mathrm{O} 4) \mathrm{C} 4=\mathrm{CC}=\mathrm{CC}=\mathrm{C} 4) \mathrm{C} 4=\mathrm{CC}=\mathrm{C}(\mathrm{C}=\mathrm{C} 4)[\mathrm{N}+]([\mathrm{O}- \\
])=\mathrm{O})[\mathrm{C} @ @ \mathrm{H}] 3 \mathrm{CC}[\mathrm{C} @ @] 2(\mathrm{C}) \mathrm{C}=\mathrm{CC} 1=\mathrm{O}\end{array}$ & 4,85 \\
\hline 968 & $\begin{array}{l}\mathrm{CC} 1=\mathrm{C} 2[\mathrm{C} @ \mathrm{H}] 3 \mathrm{OC}(=\mathrm{O}) \mathrm{C} 4(\mathrm{C}[\mathrm{C} @ \mathrm{H}](\mathrm{N}(\mathrm{O} 4) \mathrm{C} 4=\mathrm{CC}=\mathrm{CC}=\mathrm{C} 4) \mathrm{C} 4=\mathrm{CC}=\mathrm{C}(\mathrm{F}) \mathrm{C}(\mathrm{Br})=\mathrm{C} 4)[\mathrm{C} @ @ \mathrm{H}] 3 \mathrm{CC}[\mathrm{C} @ @] \\
2(\mathrm{C}) \mathrm{C}=\mathrm{CC} 1=\mathrm{O}\end{array}$ & 4 \\
\hline \multicolumn{2}{|c|}{ ttps://biointerfaceresearch.com/ } & \\
\hline
\end{tabular}




\begin{tabular}{|c|c|c|}
\hline NAME & SMILE & $\lg (\mathbf{1} / \mathbf{I C 5 0})$ \\
\hline 969 & $\begin{array}{l}\mathrm{CC} 1=\mathrm{C} 2[\mathrm{C} @ \mathrm{H}] 3 \mathrm{OC}(=\mathrm{O}) \mathrm{C} 4(\mathrm{C}[\mathrm{C} @ @ \mathrm{H}](\mathrm{N}(\mathrm{O} 4) \mathrm{C} 4=\mathrm{CC}=\mathrm{CC}=\mathrm{C} 4) \mathrm{C} 4=\mathrm{CC}(\mathrm{F})=\mathrm{CC}(\mathrm{F})=\mathrm{C} 4)[\mathrm{C} @ @ \mathrm{H}] 3 \mathrm{CC}[\mathrm{C} @ @ \\
] 2(\mathrm{C}) \mathrm{C}=\mathrm{CC} 1=\mathrm{O}\end{array}$ & 4 \\
\hline 970 & $\begin{array}{l}\mathrm{CC} 1=\mathrm{C} 2[\mathrm{C} @ \mathrm{H}] 3 \mathrm{OC}(=\mathrm{O}) \mathrm{C} 4(\mathrm{C}[\mathrm{C} @ \mathrm{H}](\mathrm{N}(\mathrm{O} 4) \mathrm{C} 4=\mathrm{CC}=\mathrm{CC}=\mathrm{C} 4) \mathrm{C} 4=\mathrm{CC}=\mathrm{CC}(=\mathrm{C} 4)[\mathrm{N}+]([\mathrm{O}- \\
])=\mathrm{O})[\mathrm{C} @ @ \mathrm{H}] 3 \mathrm{CC}[\mathrm{C} @ @] 2(\mathrm{C}) \mathrm{C}=\mathrm{CC} 1=\mathrm{O}\end{array}$ & 4 \\
\hline 971 & $\begin{array}{l}\mathrm{CC} 1=\mathrm{C} 2[\mathrm{C} @ \mathrm{H}] 3 \mathrm{OC}(=\mathrm{O}) \mathrm{C} 4(\mathrm{C}[\mathrm{C} @ @ \mathrm{H}](\mathrm{N}(\mathrm{O} 4) \mathrm{C} 4=\mathrm{CC}=\mathrm{CC}=\mathrm{C} 4) \mathrm{C} 4=\mathrm{C} 5 \mathrm{C}=\mathrm{CC}=\mathrm{CC} 5=\mathrm{CC}=\mathrm{C} 4)[\mathrm{C} @ @ \mathrm{H}] 3 \mathrm{CC}[ \\
\mathrm{C} @ @] 2(\mathrm{C}) \mathrm{C}=\mathrm{CC} 1=\mathrm{O}\end{array}$ & 4 \\
\hline 972 & $\mathrm{COC} 1=\mathrm{CC}=\mathrm{C}(\mathrm{C}=\mathrm{C} 1) \mathrm{C} 1=\mathrm{NOC} 2(\mathrm{C} 1)[\mathrm{C} @ @ \mathrm{H}] 1 \mathrm{CC}[\mathrm{C} @ @] 3(\mathrm{C}) \mathrm{C}=\mathrm{CC}=\mathrm{O}) \mathrm{C}(\mathrm{C})=\mathrm{C} 3[\mathrm{C} @ \mathrm{H}] 1 \mathrm{OC} 2=\mathrm{O}$ & 4 \\
\hline 973 & $\mathrm{CC} 1=\mathrm{C} 2[\mathrm{C} @ \mathrm{H}] 3 \mathrm{OC}(=\mathrm{O}) \mathrm{C} 4(\mathrm{CC}(=\mathrm{NO} 4) \mathrm{C} 4=\mathrm{CC}(\mathrm{F})=\mathrm{CC}(\mathrm{F})=\mathrm{C} 4)[\mathrm{C} @ @ \mathrm{H}] 3 \mathrm{CC}[\mathrm{C} @ @] 2(\mathrm{C}) \mathrm{C}=\mathrm{CC} 1=\mathrm{O}$ & 4 \\
\hline 974 & $\begin{array}{l}\mathrm{CC} 1=\mathrm{C} 2[\mathrm{C} @ \mathrm{H}] 3 \mathrm{OC}(=\mathrm{O}) \mathrm{C} 4(\mathrm{C}[\mathrm{C} @ \mathrm{H}](\mathrm{N}(\mathrm{O} 4) \mathrm{C} 4=\mathrm{CC}=\mathrm{CC}=\mathrm{C} 4) \mathrm{C} 4=\mathrm{CC}=\mathrm{C}(\mathrm{C}=\mathrm{C} 4)[\mathrm{N}+]([\mathrm{O}- \\
])=\mathrm{O})[\mathrm{C} @ \mathrm{H}] 3 \mathrm{CC}[\mathrm{C} @] 2(\mathrm{C}) \mathrm{C}=\mathrm{CC} 1=\mathrm{O}\end{array}$ & 4 \\
\hline 975 & $\mathrm{CC} 1=\mathrm{C} 2[\mathrm{C} @ \mathrm{H}] 3 \mathrm{OC}(=\mathrm{O}) \mathrm{C} 4(\mathrm{CC}(=\mathrm{NO} 4) \mathrm{C} 4=\mathrm{CC}=\mathrm{CC}(\mathrm{F})=\mathrm{C} 4)[\mathrm{C} @ @ \mathrm{H}] 3 \mathrm{CC}[\mathrm{C} @ @] 2(\mathrm{C}) \mathrm{C}=\mathrm{CC} 1=\mathrm{O}$ & 4 \\
\hline 976 & $\mathrm{COC} 1=\mathrm{CC}=\mathrm{C}(\mathrm{C}=\mathrm{C} 1 \mathrm{Br}) \mathrm{C} 1=\mathrm{NOC} 2(\mathrm{C} 1)[\mathrm{C} @ @ \mathrm{H}] 1 \mathrm{CC}[\mathrm{C} @ @] 3(\mathrm{C}) \mathrm{C}=\mathrm{CC}(=\mathrm{O}) \mathrm{C}(\mathrm{C})=\mathrm{C} 3[\mathrm{C} @ \mathrm{H}] 1 \mathrm{OC} 2=\mathrm{O}$ & 4 \\
\hline 977 & $\mathrm{CC} 1=\mathrm{C} 2[\mathrm{C} @ \mathrm{H}] 3 \mathrm{OC}(=\mathrm{O}) \mathrm{C} 4(\mathrm{CC}(=\mathrm{NO} 4) \mathrm{C} 4=\mathrm{CC}=\mathrm{CC}=\mathrm{C} 4 \mathrm{Cl})[\mathrm{C} @ @ \mathrm{H}] 3 \mathrm{CC}[\mathrm{C} @ @] 2(\mathrm{C}) \mathrm{C}=\mathrm{CC} 1=\mathrm{O}$ & 5,14 \\
\hline 978 & $\begin{array}{l}\mathrm{COC} 1=\mathrm{CC}=\mathrm{C}(\mathrm{C}=\mathrm{C} 1 \mathrm{Br})[\mathrm{C} @ \mathrm{H}] 1 \mathrm{CC} 2(\mathrm{ON} 1 \mathrm{C} 1=\mathrm{CC}=\mathrm{CC}=\mathrm{C} 1)[\mathrm{C} @ @ \mathrm{H}] 1 \mathrm{CC}[\mathrm{C} @ @] 3(\mathrm{C}) \mathrm{C}=\mathrm{CC}(=\mathrm{O}) \mathrm{C}(\mathrm{C})=\mathrm{C} 3[\mathrm{C} \\
@ \mathrm{H}] 1 \mathrm{OC} 2=\mathrm{O}\end{array}$ & 4 \\
\hline 979 & $\begin{array}{l}\mathrm{COC} 1=\mathrm{CC}=\mathrm{C}(\mathrm{C}=\mathrm{C} 1 \mathrm{Br})[\mathrm{C} @ @ \mathrm{H}] 1 \mathrm{CC} 2(\mathrm{ON} 1 \mathrm{C} 1=\mathrm{CC}=\mathrm{CC}=\mathrm{C} 1)[\mathrm{C} @ @ \mathrm{H}] 1 \mathrm{CC}[\mathrm{C} @ @] 3(\mathrm{C}) \mathrm{C}=\mathrm{CC}(=\mathrm{O}) \mathrm{C}(\mathrm{C})=\mathrm{C} 3[ \\
\mathrm{C} @ \mathrm{H}] 1 \mathrm{OC} 2=\mathrm{O}\end{array}$ & 4 \\
\hline 980 & $\begin{array}{l}\mathrm{CC} 1=\mathrm{C} 2[\mathrm{C} @ \mathrm{H}] 3 \mathrm{OC}(=\mathrm{O}) \mathrm{C} 4(\mathrm{C}[\mathrm{C} @ \mathrm{H}](\mathrm{N}(\mathrm{O} 4) \mathrm{C} 4=\mathrm{CC}=\mathrm{CC}=\mathrm{C} 4) \mathrm{C} 4=\mathrm{C} 5 \mathrm{C}=\mathrm{CC}=\mathrm{CC} 5=\mathrm{CC}=\mathrm{C} 4)[\mathrm{C} @ @ \mathrm{H}] 3 \mathrm{CC}[\mathrm{C} \\
@ @] 2(\mathrm{C}) \mathrm{C}=\mathrm{CC} 1=\mathrm{O}\end{array}$ & 4 \\
\hline 981 & $\mathrm{FC} 1=\mathrm{CNC}(=\mathrm{O}) \mathrm{NC} 1=\mathrm{O}$ & 5,35 \\
\hline 982 & $\mathrm{CC} 1=\mathrm{C} 2[\mathrm{C} @ \mathrm{H}] 3 \mathrm{OC}(=\mathrm{O}) \mathrm{C} 4(\mathrm{CC}(=\mathrm{NO} 4) \mathrm{C} 4=\mathrm{C}(\mathrm{Cl}) \mathrm{C}=\mathrm{CC}=\mathrm{C} 4 \mathrm{Cl})[\mathrm{C} @ @ \mathrm{H}] 3 \mathrm{CC}[\mathrm{C} @ @] 2(\mathrm{C}) \mathrm{C}=\mathrm{CC} 1=\mathrm{O}$ & 4,82 \\
\hline 983 & $\mathrm{CC} 1=\mathrm{C} 2[\mathrm{C} @ \mathrm{H}] 3 \mathrm{OC}(=\mathrm{O}) \mathrm{C} 4(\mathrm{CC}(=\mathrm{NO} 4) \mathrm{C} 4=\mathrm{CC}=\mathrm{C}(\mathrm{Br}) \mathrm{C}=\mathrm{C} 4)[\mathrm{C} @ @ \mathrm{H}] 3 \mathrm{CC}[\mathrm{C} @ @] 2(\mathrm{C}) \mathrm{C}=\mathrm{CC} 1=\mathrm{O}$ & 4 \\
\hline 984 & $\begin{array}{l}\mathrm{CC} 1=\mathrm{C} 2[\mathrm{C} @ \mathrm{H}] 3 \mathrm{OC}(=\mathrm{O}) \mathrm{C} 4(\mathrm{C}[\mathrm{C} @ @ \mathrm{H}](\mathrm{N}(\mathrm{O} 4) \mathrm{C} 4=\mathrm{CC}=\mathrm{CC}=\mathrm{C} 4) \mathrm{C} 4=\mathrm{CC}=\mathrm{C}(\mathrm{F}) \mathrm{C}(\mathrm{Br})=\mathrm{C} 4)[\mathrm{C} @ @ \mathrm{H}] 3 \mathrm{CC}[\mathrm{C} @ \\
@] 2(\mathrm{C}) \mathrm{C}=\mathrm{CC} 1=\mathrm{O}\end{array}$ & 5,43 \\
\hline 985 & $\mathrm{CC} 1=\mathrm{C} 2[\mathrm{C} @ \mathrm{H}] 3 \mathrm{OC}=\mathrm{O}) \mathrm{C} 4(\mathrm{CC}(=\mathrm{NO} 4) \mathrm{C} 4=\mathrm{CC}=\mathrm{C}(\mathrm{F}) \mathrm{C}=\mathrm{C} 4)[\mathrm{C} @ @ \mathrm{H}] 3 \mathrm{CC}[\mathrm{C} @ @] 2(\mathrm{C}) \mathrm{C}=\mathrm{CC} 1=\mathrm{O}$ & 4 \\
\hline 986 & $\mathrm{CC} 1=\mathrm{C} 2[\mathrm{C} @ \mathrm{H}] 3 \mathrm{OC}(=\mathrm{O}) \mathrm{C} 4(\mathrm{CC}(=\mathrm{NO} 4) \mathrm{C} 4=\mathrm{CC}=\mathrm{C}(\mathrm{Cl}) \mathrm{C}=\mathrm{C} 4 \mathrm{Cl})[\mathrm{C} @ @ \mathrm{H}] 3 \mathrm{CC}[\mathrm{C} @ @] 2(\mathrm{C}) \mathrm{C}=\mathrm{CC} 1=\mathrm{O}$ & 4,54 \\
\hline 987 & $\mathrm{CC} 1=\mathrm{C} 2[\mathrm{C} @ \mathrm{H}] 3 \mathrm{OC}(=\mathrm{O}) \mathrm{C} 4(\mathrm{CC}(=\mathrm{NO} 4) \mathrm{C} 4=\mathrm{CC}=\mathrm{C} 5 \mathrm{C}=\mathrm{CC}=\mathrm{CC} 5=\mathrm{C} 4)[\mathrm{C} @ @ \mathrm{H}] 3 \mathrm{CC}[\mathrm{C} @ @] 2(\mathrm{C}) \mathrm{C}=\mathrm{CC} 1=\mathrm{O}$ & 4 \\
\hline 988 & $\begin{array}{l}\mathrm{CC} 1=\mathrm{C} 2[\mathrm{C} @ \mathrm{H}] 3 \mathrm{OC}(=\mathrm{O}) \mathrm{C} 4(\mathrm{CC}(=\mathrm{NO} 4) \mathrm{C} 4=\mathrm{C} 5 \mathrm{C}=\mathrm{CC}=\mathrm{CC} 5=\mathrm{CC} 5=\mathrm{CC}=\mathrm{CC}=\mathrm{C} 45)[\mathrm{C} @ @ \mathrm{H}] 3 \mathrm{CC}[\mathrm{C} @ @] 2(\mathrm{C}) \mathrm{C} \\
=\mathrm{CC} 1=\mathrm{O}\end{array}$ & 4,3 \\
\hline 989 & $\begin{array}{l}\mathrm{CC} 1=\mathrm{C} 2[\mathrm{C} @ \mathrm{H}] 3 \mathrm{OC}(=\mathrm{O}) \mathrm{C} 4(\mathrm{C}[\mathrm{C} @ @ \mathrm{H}](\mathrm{N}(\mathrm{O} 4) \mathrm{C} 4=\mathrm{CC}=\mathrm{CC}=\mathrm{C} 4) \mathrm{C} 4=\mathrm{CC}=\mathrm{C}(\mathrm{Br}) \mathrm{C}=\mathrm{C} 4)[\mathrm{C} @ @ \mathrm{H}] 3 \mathrm{CC}[\mathrm{C} @ @] 2 \\
(\mathrm{C}) \mathrm{C}=\mathrm{CC} 1=\mathrm{O}\end{array}$ & 4 \\
\hline 990 & $\begin{array}{l}\mathrm{CC} 1=\mathrm{C} 2[\mathrm{C} @ \mathrm{H}] 3 \mathrm{OC}(=\mathrm{O}) \mathrm{C} 4(\mathrm{C}[\mathrm{C} @ \mathrm{H}](\mathrm{N}(\mathrm{O} 4) \mathrm{C} 4=\mathrm{CC}=\mathrm{CC}=\mathrm{C} 4) \mathrm{C} 4=\mathrm{CC}(\mathrm{Cl})=\mathrm{CC}=\mathrm{C} 4 \mathrm{Cl})[\mathrm{C} @ @ \mathrm{H}] 3 \mathrm{CC}[\mathrm{C} @ @] 2 \\
(\mathrm{C}) \mathrm{C}=\mathrm{CC} 1=\mathrm{O}\end{array}$ & 6,22 \\
\hline 991 & $\begin{array}{l}\mathrm{CC} 1=\mathrm{C} 2[\mathrm{C} @ \mathrm{H}] 3 \mathrm{OC}(=\mathrm{O}) \mathrm{C} 4(\mathrm{C}[\mathrm{C} @ \mathrm{H}](\mathrm{N}(\mathrm{O} 4) \mathrm{C} 4=\mathrm{CC}=\mathrm{CC}=\mathrm{C} 4) \mathrm{C} 4=\mathrm{CC}(\mathrm{F})=\mathrm{CC}(\mathrm{F})=\mathrm{C} 4)[\mathrm{C} @ @ \mathrm{H}] 3 \mathrm{CC}[\mathrm{C} @ @] 2( \\
\mathrm{C}) \mathrm{C}=\mathrm{CC} 1=\mathrm{O}\end{array}$ & 4 \\
\hline 992 & $\begin{array}{l}\mathrm{CC} 1=\mathrm{C} 2[\mathrm{C} @ \mathrm{H}] 3 \mathrm{OC}(=\mathrm{O}) \mathrm{C} 4(\mathrm{C}[\mathrm{C} @ @ \mathrm{H}](\mathrm{N}(\mathrm{O} 4) \mathrm{C} 4=\mathrm{CC}=\mathrm{CC}=\mathrm{C} 4) \mathrm{C} 4=\mathrm{CC}=\mathrm{CC}(=\mathrm{C} 4)[\mathrm{N}+]([\mathrm{O}- \\
])=\mathrm{O})[\mathrm{C} @ @ \mathrm{H}] 3 \mathrm{CC}[\mathrm{C} @ @] 2(\mathrm{C}) \mathrm{C}=\mathrm{CC} 1=\mathrm{O}\end{array}$ & 4 \\
\hline 993 & $\begin{array}{l}\mathrm{CC}(=\mathrm{C})[\mathrm{C} @ @ \mathrm{H}] 1 \mathrm{CC}[\mathrm{C} @ @] 2(\mathrm{CC}[\mathrm{C} @] 3(\mathrm{C})[\mathrm{C} @ \mathrm{H}](\mathrm{CC}[\mathrm{C} @ @ \mathrm{H}] 4[\mathrm{C} @ @] 5(\mathrm{C}) \mathrm{CC}[\mathrm{C} @ \mathrm{H}](\mathrm{OCC} 6=\mathrm{CN}(\mathrm{N}=\mathrm{N} 6 \\
\mathrm{C} 6=\mathrm{CC}=\mathrm{CC}=\mathrm{C} 6 \mathrm{C} \# \mathrm{~N}) \mathrm{C}(\mathrm{C})(\mathrm{C})[\mathrm{C} @ @ \mathrm{H}] 5 \mathrm{CC}[\mathrm{C} @ @] 34 \mathrm{C})[\mathrm{C} @ @ \mathrm{H}] 12) \mathrm{C}(\mathrm{O})=\mathrm{O}\end{array}$ & 4,92 \\
\hline 994 & $\begin{array}{l}\mathrm{COC} 1=\mathrm{CC}(=\mathrm{CC}(\mathrm{OC})=\mathrm{C} 1 \mathrm{OC}) \mathrm{N} 1 \mathrm{C}=\mathrm{C}(\mathrm{CO}[\mathrm{C} @ \mathrm{H}] 2 \mathrm{CC}[\mathrm{C} @ @] 3(\mathrm{C})[\mathrm{C} @ @ \mathrm{H}](\mathrm{CC}[\mathrm{C} @] 4(\mathrm{C})[\mathrm{C} @ @ \mathrm{H}] 3 \mathrm{CC}[\mathrm{C} @ \\
@ \mathrm{H}] 3[\mathrm{C} @ \mathrm{H}] 5[\mathrm{C} @ @ \mathrm{H}](\mathrm{CC}[\mathrm{C} @ @] 5(\mathrm{CC}[\mathrm{C} @ @] 43 \mathrm{C}) \mathrm{C}(\mathrm{O})=\mathrm{O}) \mathrm{C}(\mathrm{C})=\mathrm{C}) \mathrm{C} 2(\mathrm{C}) \mathrm{C}) \mathrm{N}=\mathrm{N} 1\end{array}$ & 4,3 \\
\hline 995 & $\begin{array}{l}\mathrm{CC}(=\mathrm{C})[\mathrm{C} @ @ \mathrm{H}] 1 \mathrm{CC}[\mathrm{C} @ @] 2(\mathrm{CC}[\mathrm{C} @] 3(\mathrm{C})[\mathrm{C} @ \mathrm{H}](\mathrm{CC}[\mathrm{C} @ @ \mathrm{H}] 4[\mathrm{C} @ @] 5(\mathrm{C}) \mathrm{CC}[\mathrm{C} @ \mathrm{H}](\mathrm{OCC} 6=\mathrm{CN}(\mathrm{N}=\mathrm{N} 6 \\
) \mathrm{C} 6=\mathrm{C} 7 \mathrm{C}=\mathrm{CC}=\mathrm{C}(\mathrm{O}) \mathrm{C} 7=\mathrm{CC}=\mathrm{C} 6) \mathrm{C}(\mathrm{C})(\mathrm{C})[\mathrm{C} @ @ \mathrm{H}] 5 \mathrm{CC}[\mathrm{C} @ @] 34 \mathrm{C})[\mathrm{C} @ @ \mathrm{H}] 12) \mathrm{C}(\mathrm{O})=\mathrm{O}\end{array}$ & 4,89 \\
\hline 996 & $\begin{array}{l}\mathrm{CC}(=\mathrm{C})[\mathrm{C} @ @ \mathrm{H}] 1 \mathrm{CC}[\mathrm{C} @ @] 2(\mathrm{CC}[\mathrm{C} @] 3(\mathrm{C})[\mathrm{C} @ \mathrm{H}](\mathrm{CC}[\mathrm{C} @ @ \mathrm{H}] 4[\mathrm{C} @ @] 5(\mathrm{C}) \mathrm{CC}[\mathrm{C} @ \mathrm{H}](\mathrm{OCC} 6=\mathrm{CN}(\mathrm{N}=\mathrm{N} 6 \\
) \mathrm{C} 6=\mathrm{CC}=\mathrm{CC}(\mathrm{I})=\mathrm{C} 6) \mathrm{C}(\mathrm{C})(\mathrm{C})[\mathrm{C} @ @ \mathrm{H}] 5 \mathrm{CC}[\mathrm{C} @ @] 34 \mathrm{C})[\mathrm{C} @ @ \mathrm{H}] 12) \mathrm{C}(\mathrm{O})=\mathrm{O}\end{array}$ & 5,42 \\
\hline 997 & $\begin{array}{l}\mathrm{CC}(=\mathrm{C})[\mathrm{C} @ @ \mathrm{H}] 1 \mathrm{CC}[\mathrm{C} @ @] 2(\mathrm{CC}[\mathrm{C} @] 3(\mathrm{C})[\mathrm{C} @ \mathrm{H}](\mathrm{CC}[\mathrm{C} @ @ \mathrm{H}] 4[\mathrm{C} @ @] 5(\mathrm{C}) \mathrm{CC}[\mathrm{C} @ \mathrm{H}](\mathrm{OCC} 6=\mathrm{CN}(\mathrm{N}=\mathrm{N} 6 \\
) \mathrm{C} 6=\mathrm{CC}=\mathrm{CC}=\mathrm{C} 6 \mathrm{CO}) \mathrm{C}(\mathrm{C})(\mathrm{C})[\mathrm{C} @ \mathrm{H}] 5 \mathrm{CC}[\mathrm{C} @] 34 \mathrm{C})[\mathrm{C} @ @ \mathrm{H}] 12) \mathrm{C}(\mathrm{O})=\mathrm{O}\end{array}$ & 4,85 \\
\hline 998 & $\begin{array}{l}\mathrm{CC}(=\mathrm{C})[\mathrm{C} @ @ \mathrm{H}] 1 \mathrm{CC}[\mathrm{C} @ @] 2(\mathrm{CC}[\mathrm{C} @] 3(\mathrm{C})[\mathrm{C} @ \mathrm{H}](\mathrm{CC}[\mathrm{C} @ @ \mathrm{H}] 4[\mathrm{C} @ @] 5(\mathrm{C}) \mathrm{CC}[\mathrm{C} @ \mathrm{H}](\mathrm{OCC}=\mathrm{CN}(\mathrm{N}=\mathrm{N} 6 \\
) \mathrm{C} 6=\mathrm{CC}=\mathrm{C}(\mathrm{C}=\mathrm{C} 6) \mathrm{C \# N}) \mathrm{C}(\mathrm{C})(\mathrm{C})[\mathrm{C} @ @ \mathrm{H}] 5 \mathrm{CC}[\mathrm{C} @ @] 34 \mathrm{C})[\mathrm{C} @ @ \mathrm{H}] 12) \mathrm{C}(\mathrm{O})=\mathrm{O}\end{array}$ & 4,49 \\
\hline 999 & 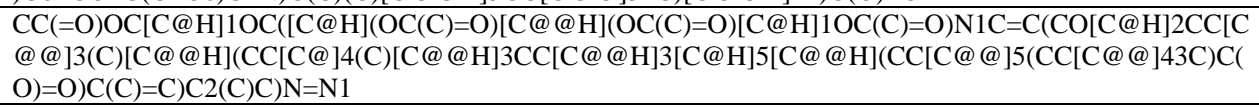 & 4,4 \\
\hline 1000 & 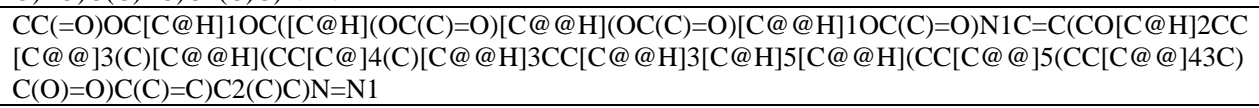 & 4,3 \\
\hline 1001 & $\begin{array}{l}\mathrm{CC}(=\mathrm{C})[\mathrm{C} @ @ \mathrm{H}] 1 \mathrm{CC}[\mathrm{C} @ @] 2(\mathrm{CC}[\mathrm{C} @] 3(\mathrm{C})[\mathrm{C} @ \mathrm{H}](\mathrm{CC}[\mathrm{C} @ @ \mathrm{H}] 4[\mathrm{C} @ @] 5(\mathrm{C}) \mathrm{CC}[\mathrm{C} @ \mathrm{H}](\mathrm{OCC} 6=\mathrm{CN}(\mathrm{N}=\mathrm{N} 6 \\
) \mathrm{C} 6=\mathrm{CC}=\mathrm{C}(\mathrm{CC} \# \mathrm{~N}) \mathrm{C}=\mathrm{C} 6) \mathrm{C}(\mathrm{C})(\mathrm{C})[\mathrm{C} @ @ \mathrm{H}] 5 \mathrm{CC}[\mathrm{C} @ @] 34 \mathrm{C})[\mathrm{C} @ @ \mathrm{H}] 12) \mathrm{C}(\mathrm{O})=\mathrm{O}\end{array}$ & 4,89 \\
\hline 1002 & $\begin{array}{l}\mathrm{CC}(=\mathrm{C})[\mathrm{C} @ @ \mathrm{H}] 1 \mathrm{CC}[\mathrm{C} @ @] 2(\mathrm{CC}[\mathrm{C} @] 3(\mathrm{C})[\mathrm{C} @ \mathrm{H}](\mathrm{CC}[\mathrm{C} @ @ \mathrm{H}] 4[\mathrm{C} @ @] 5(\mathrm{C}) \mathrm{CC}[\mathrm{C} @ \mathrm{H}](\mathrm{O}) \mathrm{C}(\mathrm{C})(\mathrm{C})[\mathrm{C} @ @) \\
\mathrm{H}] 5 \mathrm{CC}[\mathrm{C} @ @] 34 \mathrm{C})[\mathrm{C} @ @ \mathrm{H}] 12) \mathrm{C}(\mathrm{O})=\mathrm{O}\end{array}$ & 4,8 \\
\hline 1003 & $\begin{array}{l}\mathrm{CC}(=\mathrm{C})[\mathrm{C} @ @ \mathrm{H}] 1 \mathrm{CC}[\mathrm{C} @ @] 2(\mathrm{CC}[\mathrm{C} @] 3(\mathrm{C})[\mathrm{C} @ \mathrm{H}](\mathrm{CC}[\mathrm{C} @ @ \mathrm{H}] 4[\mathrm{C} @ @] 5(\mathrm{C}) \mathrm{CC}[\mathrm{C} @ \mathrm{H}](\mathrm{OCC}=\mathrm{CN}(\mathrm{N}=\mathrm{N} 6 \\
\left.\left.\mathrm{C} 6=\mathrm{CC}=\mathrm{C}(\mathrm{C}=\mathrm{C} 6) \mathrm{C}(=\mathrm{O}) \mathrm{C} 6=\mathrm{CC}=\mathrm{CC}=\mathrm{C} 6) \mathrm{C}(\mathrm{C})(\mathrm{C})\left[\mathrm{C} @ \mathrm{H}^{2}\right] 5 \mathrm{CC}[\mathrm{C} @] 34 \mathrm{C}\right)[\mathrm{C} @ \mathrm{H}] 12\right) \mathrm{C}(\mathrm{O})=\mathrm{O}\end{array}$ & 4,3 \\
\hline 1004 & $\begin{array}{l}\mathrm{CC}(=\mathrm{C})[\mathrm{C} @ @ \mathrm{H}] 1 \mathrm{CC}[\mathrm{C} @ @] 2(\mathrm{CC}[\mathrm{C} @] 3(\mathrm{C})[\mathrm{C} @ \mathrm{H}](\mathrm{CC}[\mathrm{C} @ @ \mathrm{H}] 4[\mathrm{C} @ @] 5(\mathrm{C}) \mathrm{CC}[\mathrm{C} @ \mathrm{H}](\mathrm{OCC} 6=\mathrm{CN}(\mathrm{N}=\mathrm{N} 6 \\
) \mathrm{C} 6=\mathrm{CC}=\mathrm{C} 7 \mathrm{OCOC} 7=\mathrm{C} 6) \mathrm{C}(\mathrm{C})(\mathrm{C})[\mathrm{C} @ @ \mathrm{H}] 5 \mathrm{CC}[\mathrm{C} @ @] 34 \mathrm{C})[\mathrm{C} @ @ \mathrm{H}] 12) \mathrm{C}(\mathrm{O})=\mathrm{O}\end{array}$ & 4,59 \\
\hline 1005 & $\begin{array}{l}\mathrm{CC}(=\mathrm{C})[\mathrm{C} @ @ \mathrm{H}] 1 \mathrm{CC}[\mathrm{C} @ @] 2(\mathrm{CC}[\mathrm{C} @] 3(\mathrm{C})[\mathrm{C} @ \mathrm{H}](\mathrm{CC}[\mathrm{C} @ @ \mathrm{H}] 4[\mathrm{C} @ @] 5(\mathrm{C}) \mathrm{CC}[\mathrm{C} @ \mathrm{H}](\mathrm{OCC} 6=\mathrm{CN}(\mathrm{N}=\mathrm{N} 6 \\
) \mathrm{C} 6=\mathrm{CC}=\mathrm{C}(\mathrm{Cl}) \mathrm{C}=\mathrm{C} 6) \mathrm{C}(\mathrm{C})(\mathrm{C})[\mathrm{C} @ @ \mathrm{H}] 5 \mathrm{CC}[\mathrm{C} @] 34 \mathrm{C})[\mathrm{C} @ @ \mathrm{H}] 12) \mathrm{C}(\mathrm{O})=\mathrm{O}\end{array}$ & 4,36 \\
\hline 1006 & $\begin{array}{l}\mathrm{COC} 1=\mathrm{CC}=\mathrm{C}(\mathrm{C}=\mathrm{C} 1) \mathrm{N} 1 \mathrm{C}=\mathrm{C}(\mathrm{CO}[\mathrm{C} @ \mathrm{H}] 2 \mathrm{CC}[\mathrm{C} @ @] 3(\mathrm{C})[\mathrm{C} @ @ \mathrm{H}](\mathrm{CC}[\mathrm{C} @] 4(\mathrm{C})[\mathrm{C} @ @ \mathrm{H}] 3 \mathrm{CC}[\mathrm{C} @ @ \mathrm{H}] 3[\mathrm{C} \\
\left.\left.@ \mathrm{H}] 5[\mathrm{C} @ @ \mathrm{H}]\left(\mathrm{CC}[\mathrm{C} @] 5\left(\mathrm{CC}[\mathrm{C} @]_{43}\right) \mathrm{C}(\mathrm{O})=\mathrm{O}\right) \mathrm{C}(\mathrm{C})=\mathrm{C}\right) \mathrm{C} 2(\mathrm{C}) \mathrm{C}\right) \mathrm{N}=\mathrm{N} 1\end{array}$ & 4,64 \\
\hline 1007 & $\begin{array}{l}\mathrm{CC}(=\mathrm{C})[\mathrm{C} @ @ \mathrm{H}] 1 \mathrm{CC}[\mathrm{C} @ @] 2(\mathrm{CC}[\mathrm{C} @] 3(\mathrm{C})[\mathrm{C} @ \mathrm{H}](\mathrm{CC}[\mathrm{C} @ @ \mathrm{H}] 4[\mathrm{C} @ @] 5(\mathrm{C}) \mathrm{CC}[\mathrm{C} @ \mathrm{H}](\mathrm{OCC} 6=\mathrm{CN}(\mathrm{N}=\mathrm{N} 6 \\
) \mathrm{C} 6=\mathrm{CC}=\mathrm{C}(\mathrm{C}=\mathrm{C} 6)[\mathrm{N}+]([\mathrm{O}-])=\mathrm{O}) \mathrm{C}(\mathrm{C})(\mathrm{C})[\mathrm{C} @ @ \mathrm{H}] 5 \mathrm{CC}[\mathrm{C} @ @] 34 \mathrm{C})[\mathrm{C} @ @ \mathrm{H}] 12) \mathrm{C}(\mathrm{O})=\mathrm{O}\end{array}$ & 4,82 \\
\hline 1008 & $\begin{array}{l}\mathrm{CC}(=\mathrm{C})[\mathrm{C} @ @ \mathrm{H}] 1 \mathrm{CC}[\mathrm{C} @ @] 2(\mathrm{CC}[\mathrm{C} @] 3(\mathrm{C})[\mathrm{C} @ \mathrm{H}](\mathrm{CC}[\mathrm{C} @ @ \mathrm{H}] 4[\mathrm{C} @ @] 5(\mathrm{C}) \mathrm{CC}[\mathrm{C} @ \mathrm{H}](\mathrm{OCC} 6=\mathrm{CN}(\mathrm{N}=\mathrm{N} 6 \\
\left.) \mathrm{C} 6=\mathrm{CC}=\mathrm{CC}(\mathrm{CO})=\mathrm{C} 6) \mathrm{C}(\mathrm{C})(\mathrm{C})[\mathrm{C} @ @ \mathrm{H}] 5 \mathrm{CC}[\mathrm{C} @ @] 34 \mathrm{C})\left[\mathrm{C} @ \mathrm{H}^{2}\right] 12\right) \mathrm{C}(\mathrm{O})=\mathrm{O}\end{array}$ & 5,1 \\
\hline 1009 & $\begin{array}{l}\mathrm{COC} 1=\mathrm{CC}=\mathrm{CC}(=\mathrm{C} 1) \mathrm{N} 1 \mathrm{C}=\mathrm{C}(\mathrm{CO}[\mathrm{C} @ \mathrm{H}] 2 \mathrm{CC}[\mathrm{C} @ @] 3(\mathrm{C})[\mathrm{C} @ @ \mathrm{H}](\mathrm{CC}[\mathrm{C} @] 4(\mathrm{C})[\mathrm{C} @ @ \mathrm{H}] 3 \mathrm{CC}[\mathrm{C} @ @ \mathrm{H}] 3[\mathrm{C} \\
@ \mathrm{H}] 5[\mathrm{C} @ @ \mathrm{H}](\mathrm{CC}[\mathrm{C} @ @] 5(\mathrm{CC}[\mathrm{C} @] 43 \mathrm{C}) \mathrm{C}(\mathrm{O})=\mathrm{O}) \mathrm{C}(\mathrm{C})=\mathrm{C}) \mathrm{C} 2(\mathrm{C}) \mathrm{C}) \mathrm{N}=\mathrm{N} 1\end{array}$ & 4,54 \\
\hline 1010 & $\begin{array}{l}\mathrm{CC}(=\mathrm{C})[\mathrm{C} @ @ \mathrm{H}] 1 \mathrm{CC}[\mathrm{C} @ @] 2(\mathrm{CC}[\mathrm{C} @] 3(\mathrm{C})[\mathrm{C} @ \mathrm{H}](\mathrm{CC}[\mathrm{C} @ @ \mathrm{H}] 4[\mathrm{C} @ @] 5(\mathrm{C}) \mathrm{CC}[\mathrm{C} @ \mathrm{H}](\mathrm{OCC} 6=\mathrm{CN}(\mathrm{N}=\mathrm{N} 6 \\
\left.) \mathrm{C} 6=\mathrm{CC}(\mathrm{I})=\mathrm{CC}=\mathrm{C} 6 \mathrm{C}) \mathrm{C}(\mathrm{C})(\mathrm{C})\left[\mathrm{C} @ \mathrm{H}^{2} @ 5 \mathrm{CC}[\mathrm{C} @] 34 \mathrm{C}\right)[\mathrm{C} @ \mathrm{H}] 12\right) \mathrm{C}(\mathrm{O})=\mathrm{O}\end{array}$ & 4,3 \\
\hline
\end{tabular}




\section{NAME $\mid$ SMILE}

$\mathrm{CC}(=\mathrm{C})[\mathrm{C} @ @ \mathrm{H}] 1 \mathrm{CC}[\mathrm{C} @ @] 2(\mathrm{CC}[\mathrm{C} @] 3(\mathrm{C})[\mathrm{C} @ \mathrm{H}](\mathrm{CC}[\mathrm{C} @ @ \mathrm{H}] 4[\mathrm{C} @ @] 5(\mathrm{C}) \mathrm{CC}[\mathrm{C} @ \mathrm{H}](\mathrm{OCC} 6=\mathrm{CN}(\mathrm{N}=\mathrm{N} 6$

$\lg (1 / \mathrm{IC50})$

$\mathrm{C} 6=\mathrm{CC}(\mathrm{C})=\mathrm{CC}(\mathrm{O})=\mathrm{C} 6) \mathrm{C}(\mathrm{C})(\mathrm{C})[\mathrm{C} @ @ \mathrm{H}] 5 \mathrm{CC}[\mathrm{C} @ @] 34 \mathrm{C})[\mathrm{C} @ @ \mathrm{H}] 12) \mathrm{C}(\mathrm{O})=\mathrm{O}$

$1012 \mathrm{OC} 1=\mathrm{CC}=\mathrm{C}(\mathrm{Br}) \mathrm{C}=\mathrm{C} 1 \backslash \mathrm{C}=\mathrm{N} \backslash \mathrm{CC} 1=\mathrm{CC}=\mathrm{CS} 1$

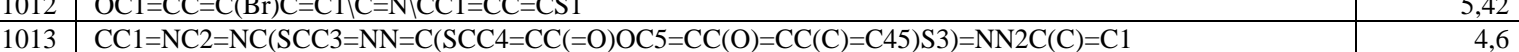

\begin{tabular}{l|lr}
\hline 1014 & $\mathrm{COC}(=\mathrm{O}) \mathrm{NC} 1=\mathrm{CC}=\mathrm{C} 2 \mathrm{C}(\mathrm{CSC} 3=\mathrm{NN}=\mathrm{C}(\mathrm{CSC} 4=\mathrm{NC}(\mathrm{C})=\mathrm{CC}(\mathrm{C})=\mathrm{N} 4) \mathrm{S} 3)=\mathrm{CC}(=\mathrm{O}) \mathrm{OC} 2=\mathrm{C} 1$ & 5,68 \\
\hline
\end{tabular}

$1015 \mathrm{NC} 1=\mathrm{NN}=\mathrm{C}(\mathrm{SCN} 2 \mathrm{C}=\mathrm{NC} 3=\mathrm{CC}=\mathrm{CC}=\mathrm{C} 3 \mathrm{C} 2=\mathrm{O}) \mathrm{S} 1$

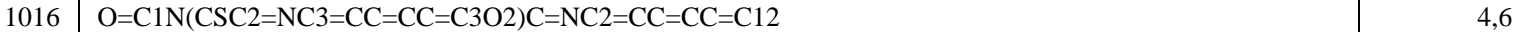

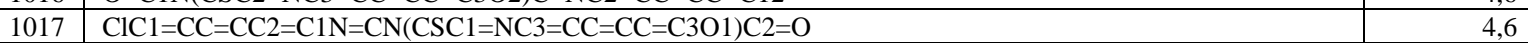

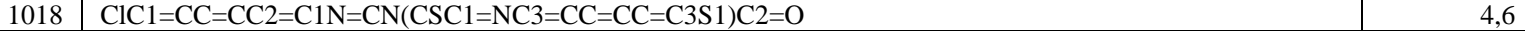

$1019 \mathrm{ClC} 1=\mathrm{CC}=\mathrm{CC} 2=\mathrm{C} 1 \mathrm{~N}=\mathrm{CN}(\mathrm{CSC} 1=\mathrm{CC}(=\mathrm{O}) \mathrm{C} 3=\mathrm{CC}=\mathrm{CC}=\mathrm{C} 3 \mathrm{~S} 1) \mathrm{C} 2=\mathrm{O} \quad 4,6$

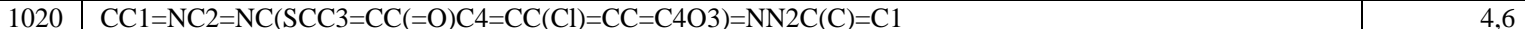

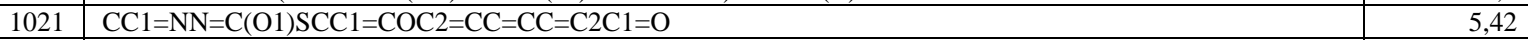

$1022 \mathrm{CC} 1=\mathrm{NN}=\mathrm{C}(\mathrm{SCC} 2=\mathrm{CC}(=\mathrm{O}) \mathrm{C} 3=\mathrm{CC}(\mathrm{C})=\mathrm{CC}=\mathrm{C} 3 \mathrm{O} 2) \mathrm{S} 1 \quad 4,6$

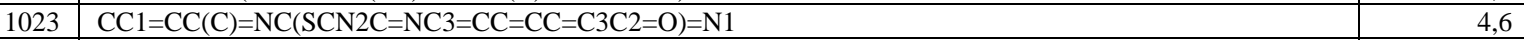

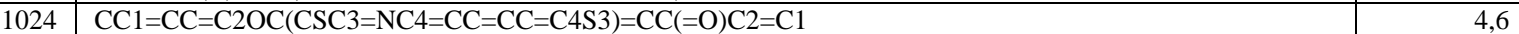

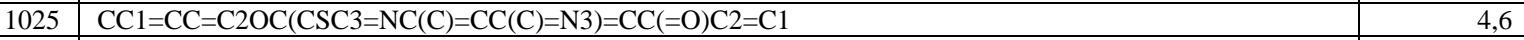

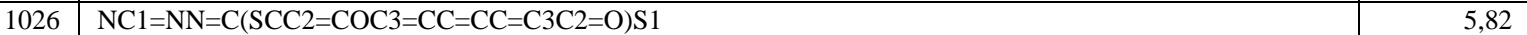

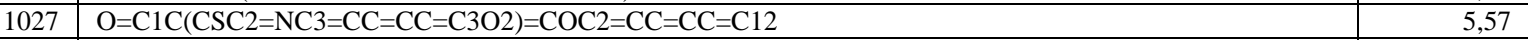

$1028 \mathrm{CC} 1=\mathrm{NC} 2=\mathrm{NC}(\mathrm{SCC} 3=\mathrm{NN}=\mathrm{C}(\mathrm{SCC} 4=\mathrm{COC} 5=\mathrm{CC}=\mathrm{CC}=\mathrm{C} 5 \mathrm{C} 4=\mathrm{O}) \mathrm{S} 3)=\mathrm{NN} 2 \mathrm{C}(\mathrm{C})=\mathrm{C} 1 \quad 5,28$

$1029 \mathrm{NC} 1=\mathrm{NN}=\mathrm{C}(\mathrm{SCC} 2=\mathrm{CC}(=\mathrm{O}) \mathrm{C} 3=\mathrm{CC}=\mathrm{CC}=\mathrm{C} 3 \mathrm{O} 2) \mathrm{S} 1$

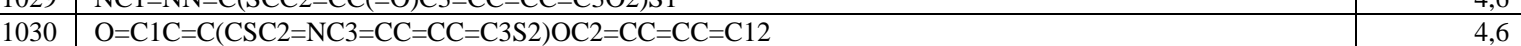

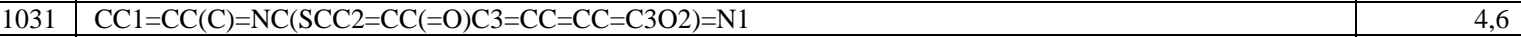

$1032 \mathrm{CC} 1=\mathrm{NN}=\mathrm{C}(\mathrm{O} 1) \mathrm{SCC} 1=\mathrm{CC}(=\mathrm{O}) \mathrm{C} 2=\mathrm{CC}(\mathrm{C})=\mathrm{CC}=\mathrm{C} 2 \mathrm{O} 1$

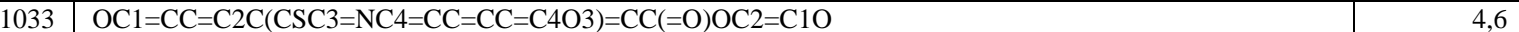

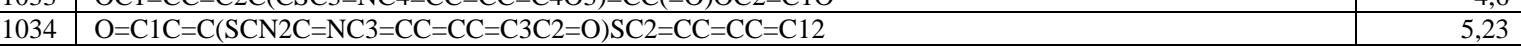

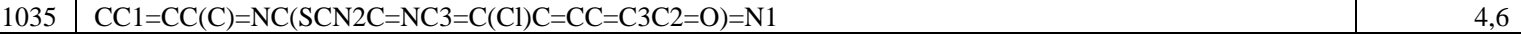

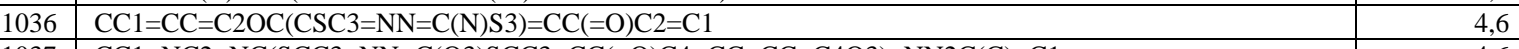

$1037 \mathrm{CC} 1=\mathrm{NC} 2=\mathrm{NC}(\mathrm{SCC} 3=\mathrm{NN}=\mathrm{C}(\mathrm{O} 3) \mathrm{SCC} 3=\mathrm{CC}(=\mathrm{O}) \mathrm{C} 4=\mathrm{CC}=\mathrm{CC}=\mathrm{C} 4 \mathrm{O} 3)=\mathrm{NN} 2 \mathrm{C}(\mathrm{C})=\mathrm{C} 1 \quad 4,6$

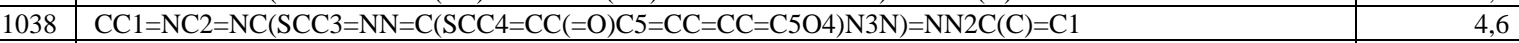

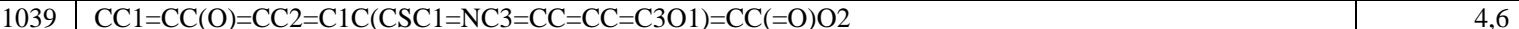

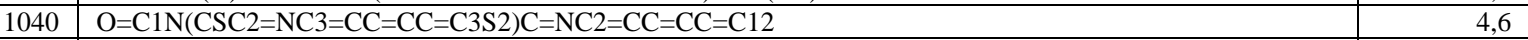

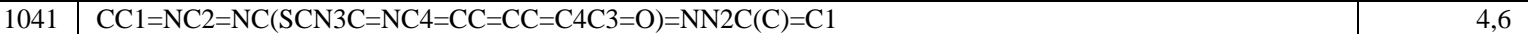

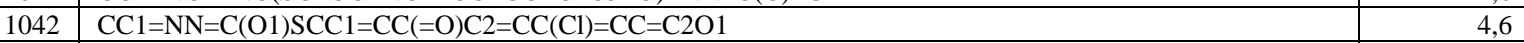

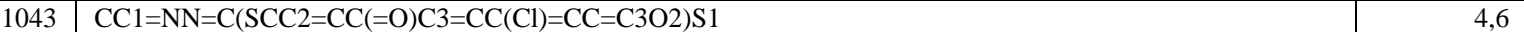

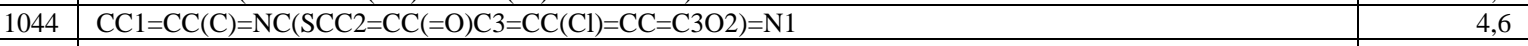

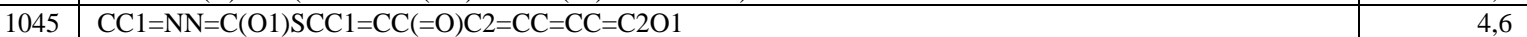

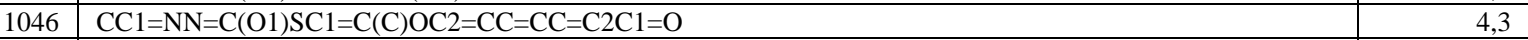

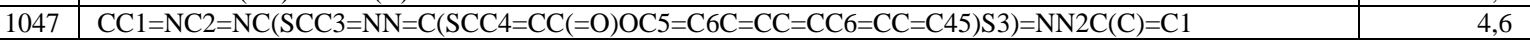

$1048 \mathrm{CC} 1=\mathrm{NC} 2=\mathrm{NC}(\mathrm{SCC} 3=\mathrm{NN}=\mathrm{C}(\mathrm{SCN} 4 \mathrm{C}=\mathrm{NC} 5=\mathrm{C}(\mathrm{Cl}) \mathrm{C}=\mathrm{CC}=\mathrm{C} 5 \mathrm{C} 4=\mathrm{O}) \mathrm{S} 3)=\mathrm{NN} 2 \mathrm{C}(\mathrm{C})=\mathrm{C} 1 \quad 4,6$

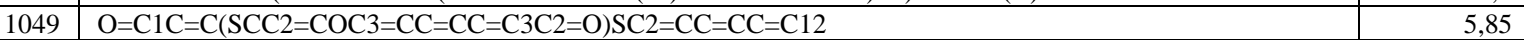

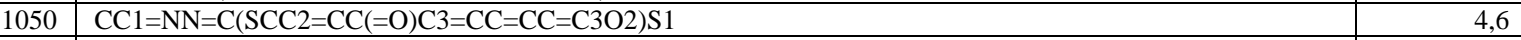

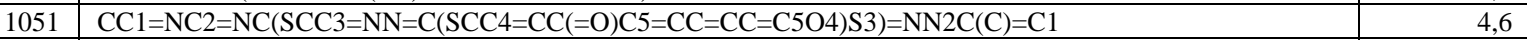

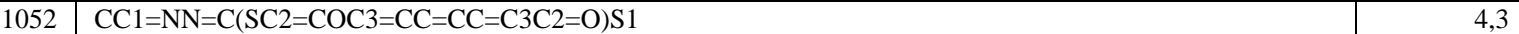

$1053 \quad \mathrm{FC} 1=\mathrm{CNC}(=\mathrm{O}) \mathrm{NC} 1=\mathrm{O}$

$1054 \mathrm{NC} 1=\mathrm{NN}=\mathrm{C}(\mathrm{SCC} 2=\mathrm{CC}(=\mathrm{O}) \mathrm{OC} 3=\mathrm{C} 4 \mathrm{C}=\mathrm{CC}=\mathrm{CC} 4=\mathrm{CC}=\mathrm{C} 23) \mathrm{S} 1$

$\mathrm{COC}(=\mathrm{O}) \mathrm{NC} 1=\mathrm{CC}=\mathrm{C} 2 \mathrm{C}(\mathrm{CSC} 3=\mathrm{NC}(\mathrm{C})=\mathrm{CC}(\mathrm{C})=\mathrm{N} 3)=\mathrm{CC}(=\mathrm{O}) \mathrm{OC} 2=\mathrm{C} 1$

$1060 \quad \mathrm{CC} 1=\mathrm{NC} 2=\mathrm{NC}(\mathrm{SCC} 3=\mathrm{NN}=\mathrm{C}(\mathrm{SCC} 4=\mathrm{CC}(=\mathrm{O}) \mathrm{C} 5=\mathrm{CC}=\mathrm{CC}=\mathrm{C} 5 \mathrm{O} 4) \mathrm{N} 3 \mathrm{C} 3=\mathrm{CC}=\mathrm{CC}=\mathrm{C} 3)=\mathrm{NN} 2 \mathrm{C}(\mathrm{C})=\mathrm{C} 1 \quad 4,6$

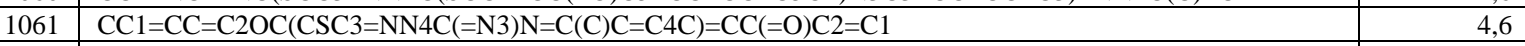

$1062 \mathrm{CC} 1=\mathrm{NN}=\mathrm{C}(\mathrm{SCC} 2=\mathrm{COC} 3=\mathrm{CC}=\mathrm{CC}=\mathrm{C} 3 \mathrm{C} 2=\mathrm{O}) \mathrm{S} 1$

$1063 \mathrm{NC} 1=\mathrm{NC}(\mathrm{SCC} 2=\mathrm{COC} 3=\mathrm{CC}=\mathrm{CC}=\mathrm{C} 3 \mathrm{C} 2=\mathrm{O})=\mathrm{NN} 1$

$1064 \mathrm{CC} 1=\mathrm{NC} 2=\mathrm{NC}(\mathrm{SCC} 3=\mathrm{CC}(=\mathrm{O}) \mathrm{C} 4=\mathrm{CC}=\mathrm{CC}=\mathrm{C} 4 \mathrm{O} 3)=\mathrm{NN} 2 \mathrm{C}(\mathrm{C})=\mathrm{C}$

$1065 \mathrm{CC} 1=\mathrm{NN}=\mathrm{C}(\mathrm{SCC} 2=\mathrm{COC} 3=\mathrm{CC}=\mathrm{C}(\mathrm{Cl}) \mathrm{C}=\mathrm{C} 3 \mathrm{C} 2=\mathrm{O}) \mathrm{S} 1$

$1066 \mathrm{CC} 1=\mathrm{NC} 2=\mathrm{NC}(\mathrm{SCN} 3 \mathrm{C}=\mathrm{NC} 4=\mathrm{C}(\mathrm{Cl}) \mathrm{C}=\mathrm{CC}=\mathrm{C} 4 \mathrm{C} 3=\mathrm{O})=\mathrm{NN} 2 \mathrm{C}(\mathrm{C})=\mathrm{C} 1$

$1067 \mathrm{NC} 1=\mathrm{NN}=\mathrm{C}(\mathrm{SCC} 2=\mathrm{CC}(=\mathrm{O}) \mathrm{C} 3=\mathrm{CC}(\mathrm{Cl})=\mathrm{CC}=\mathrm{C} 3 \mathrm{O} 2) \mathrm{S} 1$

$1068 \mathrm{ClC} 1=\mathrm{CC}=\mathrm{C} 2 \mathrm{OC}(\mathrm{CSC} 3=\mathrm{NC} 4=\mathrm{CC}=\mathrm{CC}=\mathrm{C} 4 \mathrm{~S} 3)=\mathrm{CC}(=\mathrm{O}) \mathrm{C} 2=\mathrm{C} 1$

$1069 \mathrm{CC} 1=\mathrm{CC}(\mathrm{C})=\mathrm{NC}(\mathrm{SCC} 2=\mathrm{COC} 3=\mathrm{CC}=\mathrm{CC}=\mathrm{C} 3 \mathrm{C} 2=\mathrm{O})=\mathrm{N} 1$

$1071 \mathrm{CC} 1=\mathrm{CC}(\mathrm{C})=\mathrm{C} 2 \mathrm{~N} 1[\mathrm{~B}-](\mathrm{F})(\mathrm{F})[\mathrm{N}+] 1=\mathrm{C}(\mathrm{C}) \mathrm{C}=\mathrm{C}(\mathrm{C}) \mathrm{C} 1=\mathrm{C} 2 \mathrm{C} 1=\mathrm{CC}=\mathrm{CS} 1$

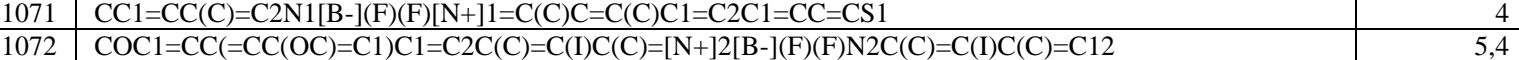

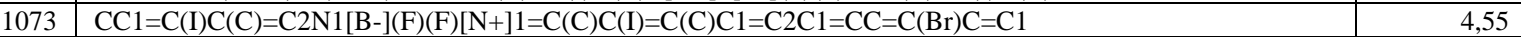

$1074 \quad \mathrm{CC} 1=\mathrm{C}(\mathrm{I}) \mathrm{C}(\mathrm{C})=\mathrm{C} 2 \mathrm{~N} 1[\mathrm{~B}-](\mathrm{F})(\mathrm{F})[\mathrm{N}+] 1=\mathrm{C}(\mathrm{C}) \mathrm{C}(\mathrm{I})=\mathrm{C}(\mathrm{C}) \mathrm{C} 1=\mathrm{C} 2 \mathrm{C} 1=\mathrm{CC}=\mathrm{CS} 1$

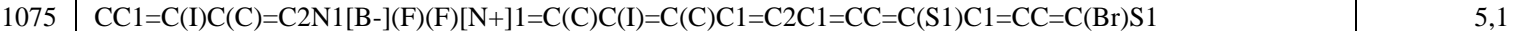

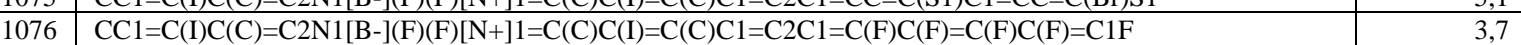

$1077 \mathrm{COC}(=\mathrm{O}) \mathrm{C} 1=\mathrm{CC}=\mathrm{C}(\mathrm{C}=\mathrm{C} 1) \mathrm{C} 1=\mathrm{C} 2 \mathrm{C}(\mathrm{C})=\mathrm{CC}(\mathrm{C})=[\mathrm{N}+] 2[\mathrm{~B}-](\mathrm{F})(\mathrm{F}) \mathrm{N} 2 \mathrm{C}(\mathrm{C})=\mathrm{CC}(\mathrm{C})=\mathrm{C} 12$

$1078 \mathrm{CC} 1=\mathrm{CC}(\mathrm{C})=\mathrm{C} 2 \mathrm{~N} 1[\mathrm{~B}-](\mathrm{F})(\mathrm{F})[\mathrm{N}+] 1=\mathrm{C}(\mathrm{C}) \mathrm{C}=\mathrm{C}(\mathrm{C}) \mathrm{C} 1=\mathrm{C} 2 \mathrm{C} 1=\mathrm{CC}=\mathrm{C}(\mathrm{S} 1) \mathrm{C} 1=\mathrm{CC}=\mathrm{CS} 1$

$1079 \mathrm{CC} 1=\mathrm{CC}(\mathrm{C})=\mathrm{C} 2 \mathrm{~N} 1[\mathrm{~B}-1(\mathrm{~F})(\mathrm{F})[\mathrm{N}+] 1=\mathrm{C}(\mathrm{C}) \mathrm{C}=\mathrm{C}(\mathrm{C}) \mathrm{C} 1=\mathrm{C} 2 \mathrm{C} 1=\mathrm{CC}=\mathrm{C}(\mathrm{S} 1) \mathrm{C} 1=\mathrm{CC}=\mathrm{CS} 1$

$1080 \quad \mathrm{CC} 1=\mathrm{C}(\mathrm{I}) \mathrm{C}(\mathrm{C})=\mathrm{C} 2 \mathrm{~N} 1[\mathrm{~B}-](\mathrm{F})(\mathrm{F})[\mathrm{N}+] 1=\mathrm{C}(\mathrm{C}) \mathrm{C}(\mathrm{I})=\mathrm{C}(\mathrm{C}) \mathrm{C} 1=\mathrm{C} 2 \mathrm{C} 1=\mathrm{CC}=\mathrm{CC}=\mathrm{C} 1$

$1081 \mathrm{COC}(=\mathrm{O}) \mathrm{C} 1=\mathrm{CC}=\mathrm{C}(\mathrm{C}=\mathrm{C} 1) \mathrm{C} 1=\mathrm{C} 2 \mathrm{C}(\mathrm{C})=\mathrm{C}(\mathrm{I}) \mathrm{C}(\mathrm{C})=[\mathrm{N}+12[\mathrm{~B}-](\mathrm{F})(\mathrm{F}) \mathrm{N} 2 \mathrm{C}(\mathrm{C})=\mathrm{C}(\mathrm{I}) \mathrm{C}(\mathrm{C})=\mathrm{C} 12$

$1082 \quad \mathrm{CC} 1=\mathrm{C}(\mathrm{I}) \mathrm{C}(\mathrm{C})=\mathrm{C} 2 \mathrm{~N} 1[\mathrm{~B}-](\mathrm{F})(\mathrm{F})[\mathrm{N}+] 1=\mathrm{C}(\mathrm{C}) \mathrm{C}(\mathrm{I})=\mathrm{C}(\mathrm{C}) \mathrm{C} 1=\mathrm{C} 2 \mathrm{C} 1=\mathrm{CC}=\mathrm{C}(\mathrm{Br}) \mathrm{S} 1$

$1083 \mathrm{CC} 1=\mathrm{C}(\mathrm{I}) \mathrm{C}(\mathrm{C})=\mathrm{C} 2 \mathrm{~N} 1[\mathrm{~B}-](\mathrm{F})(\mathrm{F})[\mathrm{N}+] 1=\mathrm{C}(\mathrm{C}) \mathrm{C}(\mathrm{I})=\mathrm{C}(\mathrm{C}) \mathrm{C} 1=\mathrm{C} 2 \mathrm{C} 1=\mathrm{C}(\mathrm{F}) \mathrm{C}(\mathrm{F})=\mathrm{C}(\mathrm{F}) \mathrm{C}(\mathrm{F})=\mathrm{C} 1 \mathrm{~F}$ 


\begin{tabular}{|c|c|c|}
\hline NAME & SMILE & $\lg (\mathbf{1} / \mathbf{I C 5 0})$ \\
\hline 1084 & $\mathrm{CC} 1=\mathrm{C}(\mathrm{I}) \mathrm{C}(\mathrm{C})=\mathrm{C} 2 \mathrm{~N} 1[\mathrm{~B}-](\mathrm{F})(\mathrm{F})[\mathrm{N}+] 1=\mathrm{C}(\mathrm{C}) \mathrm{C}(\mathrm{I})=\mathrm{C}(\mathrm{C}) \mathrm{C} 1=\mathrm{C} 2 \mathrm{C} 1=\mathrm{CC}=\mathrm{C}(\mathrm{S} 1) \mathrm{C} 1=\mathrm{CC}=\mathrm{CS} 1$ & 3,4 \\
\hline 1085 & $\mathrm{CC} 1=\mathrm{CC}(\mathrm{C})=\mathrm{C} 2 \mathrm{~N} 1[\mathrm{~B}-](\mathrm{F})(\mathrm{F})[\mathrm{N}+] 1=\mathrm{C}(\mathrm{C}) \mathrm{C}=\mathrm{C}(\mathrm{C}) \mathrm{C} 1=\mathrm{C} 2 \mathrm{C} 1=\mathrm{CC}=\mathrm{C}(\mathrm{S} 1) \mathrm{C} 1=\mathrm{CC}=\mathrm{C}(\mathrm{Br}) \mathrm{S} 1$ & 4,09 \\
\hline 1086 & $\mathrm{COC} 1=\mathrm{CC}=\mathrm{C}(\mathrm{C}=\mathrm{C} 1 \mathrm{OC}) \mathrm{C} 1=\mathrm{C} 2 \mathrm{C}(\mathrm{C})=\mathrm{C}(\mathrm{I}) \mathrm{C}(\mathrm{C})=[\mathrm{N}+] 2[\mathrm{~B}-](\mathrm{F})(\mathrm{F}) \mathrm{N} 2 \mathrm{C}(\mathrm{C})=\mathrm{C}(\mathrm{I}) \mathrm{C}(\mathrm{C})=\mathrm{C} 12$ & 3,4 \\
\hline 1087 & $\mathrm{CC} 1=\mathrm{CC}(\mathrm{C})=\mathrm{C} 2 \mathrm{~N} 1[\mathrm{~B}-](\mathrm{F})(\mathrm{F})[\mathrm{N}+] 1=\mathrm{C}(\mathrm{C}) \mathrm{C}=\mathrm{C}(\mathrm{C}) \mathrm{C} 1=\mathrm{C} 2 \mathrm{C} 1=\mathrm{CC}=\mathrm{CS} 1$ & 3,52 \\
\hline 1088 & $\mathrm{CC} 1=\mathrm{C}(\mathrm{I}) \mathrm{C}(\mathrm{C})=\mathrm{C} 2 \mathrm{~N} 1[\mathrm{~B}-](\mathrm{F})(\mathrm{F})[\mathrm{N}+] 1=\mathrm{C}(\mathrm{C}) \mathrm{C}(\mathrm{I})=\mathrm{C}(\mathrm{C}) \mathrm{C} 1=\mathrm{C} 2 \mathrm{C} 1=\mathrm{CC}=\mathrm{CS} 1$ & 4,85 \\
\hline 1089 & $\mathrm{CC} 1=\mathrm{C}(\mathrm{I}) \mathrm{C}(\mathrm{C})=\mathrm{C} 2 \mathrm{~N} 1[\mathrm{~B}-](\mathrm{F})(\mathrm{F})[\mathrm{N}+] 1=\mathrm{C}(\mathrm{C}) \mathrm{C}(\mathrm{I})=\mathrm{C}(\mathrm{C}) \mathrm{C} 1=\mathrm{C} 2 \mathrm{C} 1=\mathrm{CC}=\mathrm{C}(\mathrm{S} 1) \mathrm{C} 1=\mathrm{CC}=\mathrm{CS} 1$ & 3,7 \\
\hline 1090 & $\mathrm{CC} 1=\mathrm{C}(\mathrm{I}) \mathrm{C}(\mathrm{C})=\mathrm{C} 2 \mathrm{~N} 1[\mathrm{~B}-](\mathrm{F})(\mathrm{F})[\mathrm{N}+] 1=\mathrm{C}(\mathrm{C}) \mathrm{C}(\mathrm{I})=\mathrm{C}(\mathrm{C}) \mathrm{C} 1=\mathrm{C} 2 \mathrm{C} 1=\mathrm{CC}=\mathrm{C}(\mathrm{S} 1) \mathrm{C} 1=\mathrm{CC}=\mathrm{C}(\mathrm{Br}) \mathrm{S} 1$ & 5,46 \\
\hline 1091 & $\mathrm{CC} 1=\mathrm{CC}(\mathrm{C})=\mathrm{C} 2 \mathrm{~N} 1[\mathrm{~B}-](\mathrm{F})(\mathrm{F})[\mathrm{N}+] 1=\mathrm{C}(\mathrm{C}) \mathrm{C}=\mathrm{C}(\mathrm{C}) \mathrm{C} 1=\mathrm{C} 2 \mathrm{C} 1=\mathrm{CC}=\mathrm{C}(\mathrm{S} 1) \mathrm{C} 1=\mathrm{CC}=\mathrm{C}(\mathrm{Br}) \mathrm{S} 1$ & 3,52 \\
\hline 1092 & $\mathrm{COC} 1=\mathrm{CC}(=\mathrm{CC}(\mathrm{OC})=\mathrm{C} 1) \mathrm{C} 1=\mathrm{C} 2 \mathrm{C}(\mathrm{C})=\mathrm{C}(\mathrm{I}) \mathrm{C}(\mathrm{C})=[\mathrm{N}+] 2[\mathrm{~B}-](\mathrm{F})(\mathrm{F}) \mathrm{N} 2 \mathrm{C}(\mathrm{C})=\mathrm{C}(\mathrm{I}) \mathrm{C}(\mathrm{C})=\mathrm{C} 12$ & 3,4 \\
\hline 1093 & $\mathrm{CC} 1=\mathrm{C}(\mathrm{I}) \mathrm{C}(\mathrm{C})=\mathrm{C} 2 \mathrm{~N} 1[\mathrm{~B}-](\mathrm{F})(\mathrm{F})[\mathrm{N}+] 1=\mathrm{C}(\mathrm{C}) \mathrm{C}(\mathrm{I})=\mathrm{C}(\mathrm{C}) \mathrm{C} 1=\mathrm{C} 2 \mathrm{C} 1=\mathrm{CC}(=\mathrm{CC}(=\mathrm{C} 1) \mathrm{C}(\mathrm{C})(\mathrm{C}) \mathrm{C}) \mathrm{C}(\mathrm{C})(\mathrm{C}) \mathrm{C}$ & 3,7 \\
\hline 1094 & $\mathrm{CC} 1=\mathrm{C}(\mathrm{I}) \mathrm{C}(\mathrm{C})=\mathrm{C} 2 \mathrm{~N} 1[\mathrm{~B}-](\mathrm{F})(\mathrm{F})[\mathrm{N}+] 1=\mathrm{C}(\mathrm{C}) \mathrm{C}(\mathrm{I})=\mathrm{C}(\mathrm{C}) \mathrm{C} 1=\mathrm{C} 2 \mathrm{C} 1=\mathrm{CC}=\mathrm{C}(\mathrm{Br}) \mathrm{C}=\mathrm{C} 1$ & 3,4 \\
\hline 1095 & $\mathrm{COC}(=\mathrm{O}) \mathrm{C} 1=\mathrm{CC}=\mathrm{C}(\mathrm{C}=\mathrm{C} 1) \mathrm{C} 1=\mathrm{C} 2 \mathrm{C}(\mathrm{C})=\mathrm{CC}(\mathrm{C})=[\mathrm{N}+] 2[\mathrm{~B}-](\mathrm{F})(\mathrm{F}) \mathrm{N} 2 \mathrm{C}(\mathrm{C})=\mathrm{CC}(\mathrm{C})=\mathrm{C} 12$ & 3,4 \\
\hline 1096 & $\mathrm{CC} 1=\mathrm{C}(\mathrm{I}) \mathrm{C}(\mathrm{C})=\mathrm{C} 2 \mathrm{~N} 1[\mathrm{~B}-](\mathrm{F})(\mathrm{F})[\mathrm{N}+] 1=\mathrm{C}(\mathrm{C}) \mathrm{C}(\mathrm{I})=\mathrm{C}(\mathrm{C}) \mathrm{C} 1=\mathrm{C} 2 \mathrm{C} 1=\mathrm{CC}=\mathrm{CC}=\mathrm{C} 1$ & 4,57 \\
\hline 1097 & $\mathrm{CC} 1=\mathrm{C}(\mathrm{I}) \mathrm{C}(\mathrm{C})=\mathrm{C} 2 \mathrm{~N} 1[\mathrm{~B}-](\mathrm{F})(\mathrm{F})[\mathrm{N}+] 1=\mathrm{C}(\mathrm{C}) \mathrm{C}(\mathrm{I})=\mathrm{C}(\mathrm{C}) \mathrm{C} 1=\mathrm{C} 2 \mathrm{C} 1=\mathrm{CC}(=\mathrm{CC}(=\mathrm{C} 1) \mathrm{C}(\mathrm{C})(\mathrm{C}) \mathrm{C}) \mathrm{C}(\mathrm{C})(\mathrm{C}) \mathrm{C}$ & 3,4 \\
\hline 1098 & $\mathrm{COC} 1=\mathrm{CC}=\mathrm{C}(\mathrm{C}=\mathrm{C} 1 \mathrm{OC}) \mathrm{C} 1=\mathrm{C} 2 \mathrm{C}(\mathrm{C})=\mathrm{C}(\mathrm{I}) \mathrm{C}(\mathrm{C})=[\mathrm{N}+] 2[\mathrm{~B}-](\mathrm{F})(\mathrm{F}) \mathrm{N} 2 \mathrm{C}(\mathrm{C})=\mathrm{C}(\mathrm{I}) \mathrm{C}(\mathrm{C})=\mathrm{C} 12$ & 5,12 \\
\hline 1099 & $\mathrm{COC}(=\mathrm{O}) \mathrm{C} 1=\mathrm{CC}=\mathrm{C}(\mathrm{C}=\mathrm{C} 1) \mathrm{C} 1=\mathrm{C} 2 \mathrm{C}(\mathrm{C})=\mathrm{C}(\mathrm{I}) \mathrm{C}(\mathrm{C})=[\mathrm{N}+] 2[\mathrm{~B}-](\mathrm{F})(\mathrm{F}) \mathrm{N} 2 \mathrm{C}(\mathrm{C})=\mathrm{C}(\mathrm{I}) \mathrm{C}(\mathrm{C})=\mathrm{C} 12$ & 3,7 \\
\hline 1100 & $\mathrm{CC} 1=\mathrm{C}(\mathrm{I}) \mathrm{C}(\mathrm{C})=\mathrm{C} 2 \mathrm{~N} 1[\mathrm{~B}-](\mathrm{F})(\mathrm{F})[\mathrm{N}+] 1=\mathrm{C}(\mathrm{C}) \mathrm{C}(\mathrm{I})=\mathrm{C}(\mathrm{C}) \mathrm{C} 1=\mathrm{C} 2 \mathrm{C} 1=\mathrm{CC}=\mathrm{C}(\mathrm{Br}) \mathrm{S} 1$ & 3,4 \\
\hline 1101 & $\begin{array}{l}\mathrm{CC}(\mathrm{C})[\mathrm{C} @ \mathrm{H}] 1 \mathrm{NC}(=\mathrm{O})[\mathrm{C} @ @ \mathrm{H}](\mathrm{NC}(=\mathrm{O}) \mathrm{C} 2=\mathrm{C} 3 \mathrm{~N}=\mathrm{C} 4 \mathrm{C}(\mathrm{OC} 3=\mathrm{C}(\mathrm{C}) \mathrm{C}=\mathrm{C} 2)=\mathrm{C}(\mathrm{C}) \mathrm{C}(=\mathrm{O}) \mathrm{C}(\mathrm{N})=\mathrm{C} 4 \mathrm{C}(=\mathrm{O}) \mathrm{N}[\mathrm{C} \\
@ \mathrm{H}] 2[\mathrm{C} @ @ \mathrm{H}](\mathrm{C}) \mathrm{OC}(=\mathrm{O})[\mathrm{C} @ \mathrm{H}](\mathrm{C}(\mathrm{C}) \mathrm{C}) \mathrm{N}(\mathrm{C}) \mathrm{C}(=\mathrm{O}) \mathrm{CN}(\mathrm{C}) \mathrm{C}(=\mathrm{O})[\mathrm{C} @ @ \mathrm{H}] 3 \mathrm{CCCN} 3 \mathrm{C}(=\mathrm{O})[\mathrm{C} @ \mathrm{H}](\mathrm{NC} 2=\mathrm{O}) \\
\mathrm{C}(\mathrm{C}) \mathrm{C})[\mathrm{C} @ @ \mathrm{H}](\mathrm{C}) \mathrm{OC}(=\mathrm{O})[\mathrm{C} @ \mathrm{H}](\mathrm{C}(\mathrm{C}) \mathrm{C}) \mathrm{N}(\mathrm{C}) \mathrm{C}(=\mathrm{O}) \mathrm{CN}(\mathrm{C}) \mathrm{C}(=\mathrm{O})[\mathrm{C} @ @ \mathrm{H}] 2 \mathrm{CCCN} 2 \mathrm{C} 1=\mathrm{O}\end{array}$ & 6,77 \\
\hline
\end{tabular}

$\mathrm{CC}(\mathrm{C})[\mathrm{C} @ \mathrm{H}] 1 \mathrm{NC}(=\mathrm{O})[\mathrm{C} @ @ \mathrm{H}](\mathrm{NC}(=\mathrm{O}) \mathrm{C} 2=\mathrm{C} 3 \mathrm{~N}=\mathrm{C} 4 \mathrm{C}(\mathrm{OC} 3=\mathrm{C}(\mathrm{C}) \mathrm{C}=\mathrm{C} 2)=\mathrm{C}(\mathrm{C}) \mathrm{C}(=\mathrm{O}) \mathrm{C}(\mathrm{N})=\mathrm{C} 4 \mathrm{C}(=\mathrm{O}) \mathrm{N}[\mathrm{C}$ @ $\mathrm{H}] 2[\mathrm{C} @ @ \mathrm{H}](\mathrm{C}) \mathrm{OC}(=\mathrm{O})[\mathrm{C} @ \mathrm{H}](\mathrm{C}(\mathrm{C}) \mathrm{C}) \mathrm{N}(\mathrm{C}) \mathrm{C}(=\mathrm{O}) \mathrm{CN}(\mathrm{C}) \mathrm{C}(=\mathrm{O})[\mathrm{C} @ @ \mathrm{H}] 3 \mathrm{CCCN} 3 \mathrm{C}(=\mathrm{O})[\mathrm{C} @ \mathrm{H}](\mathrm{NC} 2=\mathrm{O})$

$1102 \mathrm{C}(\mathrm{C}) \mathrm{C})[\mathrm{C} @ \mathrm{H}](\mathrm{C}) \mathrm{OC}(=\mathrm{O})[\mathrm{C} @ \mathrm{H}](\mathrm{C}(\mathrm{C}) \mathrm{C}) \mathrm{N}(\mathrm{C}) \mathrm{C}(=\mathrm{O}) \mathrm{CN}(\mathrm{C}) \mathrm{C}(=\mathrm{O})[\mathrm{C} @ \mathrm{H}] 2 \mathrm{CCCN} 2 \mathrm{C} 1=\mathrm{O}$

$1103 \mathrm{CC} 1=\mathrm{CC} 2=\mathrm{C}(\mathrm{C}=\mathrm{C} 1 \mathrm{O})[\mathrm{C} @ @] 1(\mathrm{C}) \mathrm{CCCC}(\mathrm{C})(\mathrm{C}) \mathrm{C} 1=\mathrm{CC} 2=\mathrm{O}$

$1104 \mathrm{CC}(\mathrm{CO}) \mathrm{C} 1=\mathrm{CC} 2=\mathrm{C}(\mathrm{C}(\mathrm{O})=\mathrm{C} 1 \mathrm{O})[\mathrm{C} @ @] 1(\mathrm{C}) \mathrm{CCCC}(\mathrm{C})(\mathrm{C})[\mathrm{C} @ @ \mathrm{H}] 1 \mathrm{CC} 2=\mathrm{O}$

$1105 \mathrm{CC}(\mathrm{CO}) \mathrm{C} 1=\mathrm{CC} 2=\mathrm{C}(\mathrm{C}=\mathrm{C} 1 \mathrm{O})[\mathrm{C} @ @] 1(\mathrm{C}) \mathrm{CCCC}(\mathrm{C})(\mathrm{C})[\mathrm{C} @ @ \mathrm{H}] 1 \mathrm{CC} 2=\mathrm{O}$

$1106 \mathrm{CC}(\mathrm{C}) \mathrm{C} 1=\mathrm{CC}=\mathrm{C} 2 \mathrm{C}(\mathrm{C}[\mathrm{C} @ @ \mathrm{H}](\mathrm{O})[\mathrm{C} @ \mathrm{H}] 3 \mathrm{C}(\mathrm{C})(\mathrm{C}) \mathrm{CCC}[\mathrm{C} @] 23 \mathrm{C})=\mathrm{C} 1 \mathrm{O}$

$1107 \quad \mathrm{CC}(\mathrm{C}) \mathrm{C} 1=\mathrm{CC} 2=\mathrm{C}(\mathrm{C}(\mathrm{O})=\mathrm{C} 1 \mathrm{O})[\mathrm{C} @ @] 1(\mathrm{C}) \mathrm{CCCC}(\mathrm{C})(\mathrm{C})[\mathrm{C} @ @ \mathrm{H}] 1 \mathrm{CC} 2=\mathrm{O}$

$1108 \mathrm{CC}(\mathrm{C}) \mathrm{C} 1=\mathrm{CC} 2=\mathrm{C}(\mathrm{C}(\mathrm{O})=\mathrm{C} 1 \mathrm{O})[\mathrm{C} @ @] 1(\mathrm{C}) \mathrm{CCCC}(\mathrm{C})(\mathrm{C})[\mathrm{C} @ @ \mathrm{H}] 1 \mathrm{CC} 2=\mathrm{O}$

$1109 \mathrm{CC} 1 \mathrm{C}(=\mathrm{O}) \mathrm{OC} 2=\mathrm{C}(\mathrm{O}) \mathrm{C} 3=\mathrm{C}(\mathrm{CC}[\mathrm{C} @ \mathrm{H}] 4 \mathrm{C}(\mathrm{C})(\mathrm{C}) \mathrm{CCC}[\mathrm{C} @] 34 \mathrm{C}) \mathrm{C}=\mathrm{C} 12$

$1110 \mathrm{CC}(\mathrm{CO}) \mathrm{C} 1=\mathrm{CC} 2=\mathrm{C}(\mathrm{C}=\mathrm{C} 1 \mathrm{O})[\mathrm{C} @ @] 1(\mathrm{C}) \mathrm{CCCC}(\mathrm{C})(\mathrm{C})[\mathrm{C} @ @ \mathrm{H}] 1 \mathrm{CC} 2=\mathrm{O}$

$1111 \mathrm{CC} 1 \mathrm{C}(=\mathrm{O}) \mathrm{OC} 2=\mathrm{C}(\mathrm{O}) \mathrm{C} 3=\mathrm{C}(\mathrm{CC}[\mathrm{C} @ \mathrm{H}] 4 \mathrm{C}(\mathrm{C})(\mathrm{C}) \mathrm{CCC}[\mathrm{C} @] 34 \mathrm{C}) \mathrm{C}=\mathrm{C} 12$

$1112 \mathrm{CC} 1=\mathrm{CC} 2=\mathrm{C}(\mathrm{C}=\mathrm{C} 1 \mathrm{O})[\mathrm{C} @ @] 1(\mathrm{C}) \mathrm{CCCC}(\mathrm{C})(\mathrm{C})[\mathrm{C} @ @] 11 \mathrm{O}[\mathrm{C} @ \mathrm{H}] 1 \mathrm{C} 2=\mathrm{O}$

$1113 \mathrm{CC} 1=\mathrm{CC} 2=\mathrm{C}(\mathrm{C}=\mathrm{C} 1 \mathrm{O})[\mathrm{C} @ @] 1(\mathrm{C}) \mathrm{CCCC}(\mathrm{C})(\mathrm{C})[\mathrm{C} @ @] 11 \mathrm{O}[\mathrm{C} @ \mathrm{H}] 1 \mathrm{C} 2=\mathrm{O}$

$1114 \mathrm{CC}(\mathrm{C}) \mathrm{C} 1=\mathrm{CC}=\mathrm{C} 2 \mathrm{C}(\mathrm{C}[\mathrm{C} @ @ \mathrm{H}](\mathrm{O})[\mathrm{C} @ \mathrm{H}] 3 \mathrm{C}(\mathrm{C})(\mathrm{C}) \mathrm{CCC}[\mathrm{C} @] 23 \mathrm{C})=\mathrm{C} 1 \mathrm{O}$

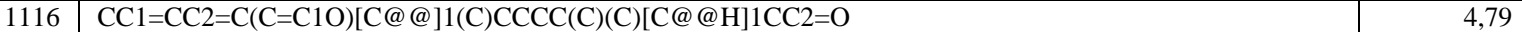

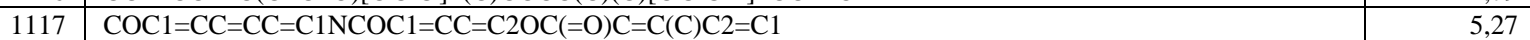

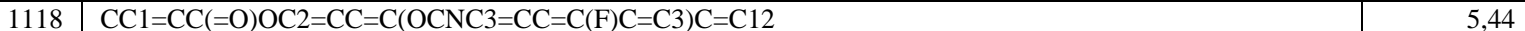

$1119 \mathrm{CC} 1=\mathrm{CC}(=\mathrm{O}) \mathrm{OC} 2=\mathrm{CC}=\mathrm{C}(\mathrm{OCNC} 3=\mathrm{CC}=\mathrm{CC}=\mathrm{C} 3 \mathrm{O}) \mathrm{C}=\mathrm{C} 12$

$1120 \mathrm{C}[\mathrm{C} @ @ \mathrm{H}] 1 \mathrm{CC}[\mathrm{C} @ @] 2(\mathrm{O})[\mathrm{C} @ @ \mathrm{H}](\mathrm{C}[\mathrm{C} @ @ \mathrm{H}](\mathrm{O}) \mathrm{C} 2(\mathrm{C}) \mathrm{C}) \mathrm{C}(=\mathrm{O})[\mathrm{C} @ @] 1(\mathrm{C}) \mathrm{CCC} 1=\mathrm{CC}(=\mathrm{O}) \mathrm{OC}$

$1121 \quad \mathrm{C}[\mathrm{C} @ @ \mathrm{H}] 1 \mathrm{C}[\mathrm{C} @ \mathrm{H}] 2 \mathrm{C}(=\mathrm{O}) \mathrm{C}(\mathrm{C})(\mathrm{C})[\mathrm{C} @ \mathrm{H}](\mathrm{O}) \mathrm{CC}[\mathrm{C} @] 2(\mathrm{O})[\mathrm{C} @ @] 1(\mathrm{C}) \mathrm{CCC} 1=\mathrm{CC}(=\mathrm{O}) \mathrm{OC} 1$

1122 C[C@@ $\mathrm{H}] 1 \mathrm{CC}[\mathrm{C} @] 2(\mathrm{O})[\mathrm{C} @ \mathrm{H}](\mathrm{C}[\mathrm{C} @ @ \mathrm{H}](\mathrm{O}) \mathrm{C} 2(\mathrm{C}) \mathrm{C}) \mathrm{C}(=\mathrm{O})[\mathrm{C} @ @] 1(\mathrm{C}) \mathrm{CCC} 1=\mathrm{CC}(=\mathrm{O}) \mathrm{OC} 1$

$1123 \quad \mathrm{C}[\mathrm{C} @ @ \mathrm{H}] 1 \mathrm{C}[\mathrm{C} @ \mathrm{H}] 2 \mathrm{C}(=\mathrm{O}) \mathrm{C}(\mathrm{C})(\mathrm{C})[\mathrm{C} @ @ \mathrm{H}](\mathrm{CC}[\mathrm{C} @] 2(\mathrm{O})[\mathrm{C} @ @] 1(\mathrm{C}) \mathrm{CCC} 1=\mathrm{CC}(=\mathrm{O}) \mathrm{OC} 1) \mathrm{OC}=\mathrm{O}$

$1124 \mathrm{COC} 1=\mathrm{C}(\mathrm{C}=\mathrm{CC} 2=\mathrm{C} 1 \mathrm{C}(=\mathrm{O}) \mathrm{OCC} 1=\mathrm{CC}(\mathrm{C})=\mathrm{CC}(\mathrm{O})=\mathrm{C} 1 \mathrm{O} 2)[\mathrm{C} @ @ \mathrm{H}](\mathrm{O}) \mathrm{CC}(\mathrm{C}) \mathrm{C}$

$\mathrm{COC} 1=\mathrm{CC}=\mathrm{CC} 2=\mathrm{C} 1 \mathrm{C}(=\mathrm{O}) \mathrm{C} 1=\mathrm{C}(\mathrm{O}) \mathrm{C} 3=\mathrm{C}(\mathrm{C}[\mathrm{C} @](\mathrm{O})(\mathrm{C}[\mathrm{C} @ @ \mathrm{H}] 3 \mathrm{O}[\mathrm{C} @ \mathrm{H}] 3 \mathrm{C}[\mathrm{C} @ \mathrm{H}](\mathrm{N})[\mathrm{C} @ \mathrm{H}](\mathrm{O})[\mathrm{C} @ \mathrm{H}]($

$1125 \mathrm{C}) \mathrm{O} 3) \mathrm{C}(=\mathrm{O}) \mathrm{CO}) \mathrm{C}(\mathrm{O})=\mathrm{C} 1 \mathrm{C} 2=\mathrm{O}$

$1126 \mathrm{OC} 1=\mathrm{CC}=\mathrm{C} 2 \mathrm{NC}=\mathrm{C}(\mathrm{C} 2=\mathrm{C} 1) \mathrm{C} 1=\mathrm{CN}=\mathrm{C}(\mathrm{C}=\mathrm{N} 1) \mathrm{C} 1=\mathrm{CNC} 2=\mathrm{CC}=\mathrm{C}(\mathrm{O}) \mathrm{C}=\mathrm{C} 12$

$1127 \mathrm{COC} 1=\mathrm{CC}=\mathrm{C}(\mathrm{C}=\mathrm{C} 1) \mathrm{C} 1=\mathrm{C}(\mathrm{SC} \# \mathrm{~N}) \mathrm{C} 2=\mathrm{CC}=\mathrm{CC}=\mathrm{C} 2 \mathrm{~N} 1 \mathrm{C}$

$1128 \mathrm{CC} 1=\mathrm{CC}=\mathrm{C}(\mathrm{C}=\mathrm{C} 1) \mathrm{C} 1=\mathrm{C}(\mathrm{SC} \# \mathrm{~N}) \mathrm{C} 2=\mathrm{CC}=\mathrm{CC}=\mathrm{C} 2 \mathrm{~N} 1$

$1129 \mathrm{CN} 1 \mathrm{C}=\mathrm{C}(\mathrm{SC \# N}) \mathrm{C} 2=\mathrm{CC}(=\mathrm{CC}=\mathrm{C} 12) \mathrm{C} 1=\mathrm{CC}=\mathrm{C}(\mathrm{C}) \mathrm{C}=\mathrm{C} 1$

$1130 \quad \mathrm{ClC} 1=\mathrm{CC}=\mathrm{C}(\mathrm{C}=\mathrm{C} 1) \mathrm{C} 1=\mathrm{C}(\mathrm{SC} \# \mathrm{~N}) \mathrm{C} 2=\mathrm{CC}=\mathrm{CC}=\mathrm{C} 2 \mathrm{~N}$

$1131 \mathrm{~N} 1 \mathrm{C}=\mathrm{CC} 2=\mathrm{CC}=\mathrm{CC}=\mathrm{C} 12$

$1132 \mathrm{CN} 1 \mathrm{C} 2=\mathrm{CC}=\mathrm{CC}=\mathrm{C} 2 \mathrm{C}(\mathrm{SC} \# \mathrm{~N})=\mathrm{C} 1 \mathrm{C} 1=\mathrm{CC}=\mathrm{C}(\mathrm{Cl}) \mathrm{C}=\mathrm{C} 1$

$1133 \mathrm{N \# CSC} 1=\mathrm{C}(\mathrm{NC} 2=\mathrm{CC}=\mathrm{CC}=\mathrm{C} 12) \mathrm{C} 1=\mathrm{CC}=\mathrm{CC}=\mathrm{C} 1$

$1134 \mathrm{CN} 1 \mathrm{C}=\mathrm{CC} 2=\mathrm{C} 1 \mathrm{C}=\mathrm{CC}=\mathrm{C} 2$

$1135 \mathrm{CN1C}=\mathrm{C}(\mathrm{SC} \# \mathrm{~N}) \mathrm{C} 2=\mathrm{CC}=\mathrm{CC}=\mathrm{C} 12$

$1136 \mathrm{COC} 1=\mathrm{CC}=\mathrm{C}(\mathrm{C}=\mathrm{C} 1) \mathrm{N} 1 \mathrm{C}=\mathrm{C}(\mathrm{SC} \# \mathrm{~N}) \mathrm{C} 2=\mathrm{CC}=\mathrm{CC}=\mathrm{C} 12$

$1137 \mathrm{BrC} 1=\mathrm{CC}=\mathrm{C} 2 \mathrm{NC}=\mathrm{C}(\mathrm{SC} \# \mathrm{~N}) \mathrm{C} 2=\mathrm{C} 1$

$1138 \mathrm{CN} 1 \mathrm{C}(=\mathrm{CC} 2=\mathrm{CC}=\mathrm{CC}=\mathrm{C} 12) \mathrm{C} 1=\mathrm{CC}=\mathrm{C}(\mathrm{Cl}) \mathrm{C}=\mathrm{C} 1$

$1139 \mathrm{ClC} 1=\mathrm{CC}=\mathrm{C}(\mathrm{C}=\mathrm{C} 1) \mathrm{N} 1 \mathrm{C}=\mathrm{C}(\mathrm{SC} \# \mathrm{~N}) \mathrm{C} 2=\mathrm{CC}=\mathrm{CC}=\mathrm{C} 12$

$1140 \mathrm{FC}(\mathrm{F})(\mathrm{F}) \mathrm{C} 1=\mathrm{CC}=\mathrm{CC}(=\mathrm{C} 1) \mathrm{C} 1=\mathrm{C}(\mathrm{SC} \# \mathrm{~N}) \mathrm{C} 2=\mathrm{CC}=\mathrm{CC}=\mathrm{C} 2 \mathrm{~N} 1$

$1141 \mathrm{~N} \# \mathrm{CSC} 1=\mathrm{CN}(\mathrm{C} 2=\mathrm{CC}=\mathrm{CC}=\mathrm{C} 12) \mathrm{C} 1=\mathrm{CC}=\mathrm{CC}=\mathrm{C} 1$

$1142 \mathrm{CN} 1 \mathrm{C} 2=\mathrm{CC}=\mathrm{CC}=\mathrm{C} 2 \mathrm{C}(\mathrm{SC} \# \mathrm{~N})=\mathrm{C} 1 \mathrm{C} 1=\mathrm{CC}=\mathrm{CC}=\mathrm{C} 1$

$1143 \mathrm{CC} 1=\mathrm{CC}=\mathrm{C}(\mathrm{C}=\mathrm{C} 1) \mathrm{C} 1=\mathrm{CC}=\mathrm{C} 2 \mathrm{NC}=\mathrm{C}(\mathrm{SC} \# \mathrm{~N}) \mathrm{C} 2=\mathrm{C}$

$1144 \mathrm{~N} \# \mathrm{CSC} 1=\mathrm{CNC} 2=\mathrm{CC}=\mathrm{C}(\mathrm{C}=\mathrm{C} 12) \mathrm{C} \# \mathrm{~N}$

$1145 \mathrm{~N} \# \mathrm{CSC} 1=\mathrm{CNC} 2=\mathrm{CC}=\mathrm{CC}=\mathrm{C} 12$

$1146 \mathrm{CN} 1 \mathrm{C} 2=\mathrm{CC}=\mathrm{CC}=\mathrm{C} 2 \mathrm{C}(\mathrm{SC} \# \mathrm{~N})=\mathrm{C} 1 \mathrm{C} 1=\mathrm{CC}=\mathrm{C}(\mathrm{C}) \mathrm{C}=\mathrm{C} 1$

$1147 \mathrm{COC} 1=\mathrm{CC}=\mathrm{C} 2 \mathrm{NC}=\mathrm{C}(\mathrm{SC} \# \mathrm{~N}) \mathrm{C} 2=\mathrm{C} 1$

$1148 \mathrm{CN} 1 \mathrm{C} 2=\mathrm{CC}=\mathrm{CC}=\mathrm{C} 2 \mathrm{C}(\mathrm{SC} \# \mathrm{~N})=\mathrm{C} 1 \mathrm{C} 1=\mathrm{CC}=\mathrm{CC}(=\mathrm{C} 1) \mathrm{C}(\mathrm{F})(\mathrm{F}) \mathrm{F}$

$1149 \mathrm{COC} 1=\mathrm{CC}=\mathrm{C}(\mathrm{C}=\mathrm{C} 1) \mathrm{C} 1=\mathrm{C}(\mathrm{SC} \# \mathrm{~N}) \mathrm{C} 2=\mathrm{CC}=\mathrm{CC}=\mathrm{C} 2 \mathrm{~N} 1$

\begin{tabular}{|r}
\hline, 82 \\
\hline \\
4,66 \\
\hline
\end{tabular}

\begin{tabular}{r|r} 
& 4,62 \\
\hline & 5,06 \\
\hline
\end{tabular}

$\mathrm{COC} 1=\mathrm{CC}=\mathrm{CC} 2=\mathrm{C} 1 \mathrm{C}(=\mathrm{O}) \mathrm{C} 1=\mathrm{C}(\mathrm{O}) \mathrm{C} 3=\mathrm{C}(\mathrm{C}[\mathrm{C} @](\mathrm{O})(\mathrm{C}[\mathrm{C} @ @ \mathrm{H}] 3 \mathrm{O}[\mathrm{C} @ \mathrm{H}] 3 \mathrm{C}[\mathrm{C} @ \mathrm{H}](\mathrm{N})[\mathrm{C} @ \mathrm{H}](\mathrm{O})[\mathrm{C} @ \mathrm{H}]($

$1150 \mathrm{C}) \mathrm{O} 3) \mathrm{C}(=\mathrm{O}) \mathrm{CO}) \mathrm{C}(\mathrm{O})=\mathrm{C} 1 \mathrm{C} 2=\mathrm{O}$

CC $(=\mathrm{C})[\mathrm{C} @ @ \mathrm{H}] 1 \mathrm{CC}[\mathrm{C} @ @] 2(\mathrm{CC}[\mathrm{C} @] 3(\mathrm{C})[\mathrm{C} @ \mathrm{H}](\mathrm{CC}[\mathrm{C} @ @ \mathrm{H}] 4[\mathrm{C} @ @] 5(\mathrm{C}) \mathrm{CC} 6=\mathrm{C}(\mathrm{N}=\mathrm{NC}(\mathrm{C})=[\mathrm{N}+] 6[\mathrm{O}-$

$1151]) \mathrm{C}(\mathrm{C})(\mathrm{C})[\mathrm{C} @ @ \mathrm{H}] 5 \mathrm{CC}[\mathrm{C} @ @] 34 \mathrm{C})[\mathrm{C} @ @ \mathrm{H}] 12) \mathrm{C}(\mathrm{O})=\mathrm{O}$ 


\begin{tabular}{|c|c|c|}
\hline NAME & SMILE & $\lg (1 / I C 50)$ \\
\hline 1152 & $\begin{array}{l}\mathrm{CC}(=\mathrm{O}) \mathrm{OC}[\mathrm{C} @] 12 \mathrm{CC}[\mathrm{C} @ \mathrm{H}]([\mathrm{C} @ @ \mathrm{H}] 1[\mathrm{C} @ \mathrm{H}] 1 \mathrm{CC}[\mathrm{C} @ @ \mathrm{H}] 3[\mathrm{C} @ @] 4(\mathrm{C}) \mathrm{CC} 5=\mathrm{NN}(\mathrm{N}=\mathrm{C} 5 \mathrm{C}(\mathrm{C})(\mathrm{C})[\mathrm{C} @ @ \mathrm{H} \\
] 4 \mathrm{CC}[\mathrm{C} @] 3(\mathrm{C})[\mathrm{C} @] 1(\mathrm{C}) \mathrm{CC} 2) \mathrm{C}(\mathrm{C})=\mathrm{O}) \mathrm{C}(\mathrm{C})=\mathrm{C}\end{array}$ & 4,75 \\
\hline 1153 & $\begin{array}{l}\mathrm{CC}(=\mathrm{C})[\mathrm{C} @ @ \mathrm{H}] 1 \mathrm{CC}[\mathrm{C} @] 2(\mathrm{CO}) \mathrm{CC}[\mathrm{C} @] 3(\mathrm{C})[\mathrm{C} @ \mathrm{H}](\mathrm{CC}[\mathrm{C} @ \mathrm{H}] 4[\mathrm{C} @ @] 3(\mathrm{C}) \mathrm{CC}[\mathrm{C} @ \mathrm{H}] 3 \mathrm{C}(\mathrm{C})(\mathrm{C}) \mathrm{C} 5=\mathrm{C}(\mathrm{N}= \\
\mathrm{C}(\mathrm{C}) \mathrm{O}) \mathrm{C}(=\mathrm{O})[\mathrm{C} @] 43 \mathrm{C})[\mathrm{C} @ \mathrm{H}] 12\end{array}$ & 4,71 \\
\hline 1154 & $\begin{array}{l}\mathrm{CC}(=\mathrm{O}) \mathrm{NC} 1=\mathrm{C}[\mathrm{C} @ @] 2(\mathrm{C})[\mathrm{C} @ @ \mathrm{H}](\mathrm{CC}[\mathrm{C} @] 3(\mathrm{C})[\mathrm{C} @ @ \mathrm{H}] 2 \mathrm{CC}[\mathrm{C} @ @ \mathrm{H}] 2[\mathrm{C} @ \mathrm{H}] 4[\mathrm{C} @ @ \mathrm{H}](\mathrm{CC}[\mathrm{C} @] 4(\mathrm{CO} \\
) \mathrm{CC}[\mathrm{C} @ @] 32 \mathrm{C}) \mathrm{C}(\mathrm{C})=\mathrm{C}) \mathrm{C}(\mathrm{C})(\mathrm{C}) \mathrm{C} 1=\mathrm{O}\end{array}$ & 4,98 \\
\hline 1155 & $\begin{array}{l}\mathrm{CC}(=\mathrm{C})[\mathrm{C} @ @ \mathrm{H}] 1 \mathrm{CC}[\mathrm{C} @] 2(\mathrm{CO}) \mathrm{CC}[\mathrm{C} @] 3(\mathrm{C})[\mathrm{C} @ \mathrm{H}](\mathrm{CC}[\mathrm{C} @ @ \mathrm{H}] 4[\mathrm{C} @ @] 5(\mathrm{C}) \mathrm{CC} 6=\mathrm{C}(\mathrm{N}=\mathrm{NC}(\mathrm{C})=[\mathrm{N}+] 6[\mathrm{O} \\
-]) \mathrm{C}(\mathrm{C})(\mathrm{C})[\mathrm{C} @ \mathrm{H}] 5 \mathrm{CC}[\mathrm{C} @ @] 34 \mathrm{C})[\mathrm{C} @ @ \mathrm{H}] 12\end{array}$ & 5,35 \\
\hline 1156 & $\begin{array}{l}\mathrm{CC}(=\mathrm{C})[\mathrm{C} @ @ \mathrm{H}] 1 \mathrm{CC}[\mathrm{C} @] 2(\mathrm{CO}) \mathrm{CC}[\mathrm{C} @] 3(\mathrm{C})[\mathrm{C} @ \mathrm{H}](\mathrm{CC}[\mathrm{C} @ @ \mathrm{H}] 4[\mathrm{C} @ @] 5(\mathrm{C}) \mathrm{ClC}(=\mathrm{N} / \mathrm{O}) \mathrm{C}(=\mathrm{O}) \mathrm{C}(\mathrm{C})(\mathrm{C})[ \\
\mathrm{C} @ \mathrm{H}] 5 \mathrm{CC}[\mathrm{C} @ @] 34 \mathrm{C})[\mathrm{C} @ @ \mathrm{H}] 12\end{array}$ & 4 \\
\hline 1157 & $\begin{array}{l}\mathrm{CC}(=\mathrm{O}) \mathrm{OC}[\mathrm{C} @] 12 \mathrm{CC}[\mathrm{C} @ \mathrm{H}]([\mathrm{C} @ @ \mathrm{H}] 1[\mathrm{C} @ \mathrm{H}] 1 \mathrm{CC}[\mathrm{C} @ \mathrm{H}] 3[\mathrm{C} @ @](\mathrm{C})(\mathrm{CC}[\mathrm{C} @ \mathrm{H}] 4 \mathrm{C}(\mathrm{C})(\mathrm{C}) \mathrm{C}(=\mathrm{O}) \mathrm{C} 5=\mathrm{NOC} \\
(\mathrm{C})=\mathrm{C} 5[\mathrm{C} @] 34 \mathrm{C})[\mathrm{C} @] 1(\mathrm{C}) \mathrm{CC} 2) \mathrm{C}(\mathrm{C})=\mathrm{C}\end{array}$ & 4,66 \\
\hline 1158 & $\begin{array}{l}\mathrm{CC}(=\mathrm{C})[\mathrm{C} @ @ \mathrm{H}] 1 \mathrm{CC}[\mathrm{C} @ @] 2(\mathrm{CC}[\mathrm{C} @] 3(\mathrm{C})[\mathrm{C} @ \mathrm{H}](\mathrm{CC}[\mathrm{C} @ @ \mathrm{H}] 4[\mathrm{C} @ @] 5(\mathrm{C}) \mathrm{CC} 6=\mathrm{NN}(\mathrm{N}=\mathrm{C} 6 \mathrm{C}(\mathrm{C})(\mathrm{C})[\mathrm{C} @ \\
@ \mathrm{H}] 5 \mathrm{CC}[\mathrm{C} @ @] 34 \mathrm{C}) \mathrm{C}(\mathrm{C})=\mathrm{O})[\mathrm{C} @ @ \mathrm{H}] 12) \mathrm{C}=\mathrm{O}\end{array}$ & 4 \\
\hline 1159 & $\begin{array}{l}\mathrm{CC}(=\mathrm{C})[\mathrm{C} @ @ \mathrm{H}] 1 \mathrm{CC}[\mathrm{C} @] 2(\mathrm{CO}) \mathrm{CC}[\mathrm{C} @] 3(\mathrm{C})[\mathrm{C} @ \mathrm{H}](\mathrm{CC}[\mathrm{C} @ @ \mathrm{H}] 4[\mathrm{C} @ @] 5(\mathrm{C}) \mathrm{CC} 6=\mathrm{NN}(\mathrm{N}=\mathrm{C} 6 \mathrm{C}(\mathrm{C})(\mathrm{C})[\mathrm{C} \\
@ @ \mathrm{H}] 5 \mathrm{CC}[\mathrm{C} @ @] 34 \mathrm{C}) \mathrm{C}(\mathrm{C})=\mathrm{O})[\mathrm{C} @ \mathrm{H}] 12\end{array}$ & 5,13 \\
\hline 1160 & $\begin{array}{l}\mathrm{CC}(=\mathrm{O}) \mathrm{NC} 1=\mathrm{C}[\mathrm{C} @ @] 2(\mathrm{C})[\mathrm{C} @ @ \mathrm{H}](\mathrm{CC}[\mathrm{C} @] 3(\mathrm{C})[\mathrm{C} @ @ \mathrm{H}] 2 \mathrm{CC}[\mathrm{C} @ @ \mathrm{H}] 2[\mathrm{C} @ \mathrm{H}] 4[\mathrm{C} @ @ \mathrm{H}](\mathrm{CC}[\mathrm{C} @] 4(\mathrm{CO} \\
\mathrm{C}(\mathrm{C})=\mathrm{O}) \mathrm{CC}[\mathrm{C} @ @] 32 \mathrm{C}) \mathrm{C}(\mathrm{C})=\mathrm{C}) \mathrm{C}(\mathrm{C})(\mathrm{C}) \mathrm{C} 1=\mathrm{O}\end{array}$ & 4,66 \\
\hline 1161 & $\begin{array}{l}\mathrm{CC}(=\mathrm{O}) \mathrm{OC}[\mathrm{C} @] 12 \mathrm{CC}[\mathrm{C} @ \mathrm{H}]([\mathrm{C} @ @ \mathrm{H}] 1[\mathrm{C} @ \mathrm{H}] 1 \mathrm{CC}[\mathrm{C} @ \mathrm{H}] 3[\mathrm{C} @ @](\mathrm{C})(\mathrm{CC}[\mathrm{C} @ \mathrm{H}] 4 \mathrm{C}(\mathrm{C})(\mathrm{C}) \mathrm{C} 5=\mathrm{C}(\mathrm{N}=\mathrm{C}(\mathrm{C}) \\
\mathrm{O}) \mathrm{C}(=\mathrm{O})[\mathrm{C} @] 34 \mathrm{C})[\mathrm{C} @] 1(\mathrm{C}) \mathrm{CC} 2) \mathrm{C}(\mathrm{C})=\mathrm{C}\end{array}$ & 4,4 \\
\hline 1162 & $\begin{array}{l}\mathrm{CC}(=\mathrm{C})[\mathrm{C} @ @ \mathrm{H}] 1 \mathrm{CC}[\mathrm{C} @] 2(\mathrm{CO}) \mathrm{CC}[\mathrm{C} @] 3(\mathrm{C})[\mathrm{C} @ \mathrm{H}](\mathrm{CC}[\mathrm{C} @ \mathrm{H}] 4[\mathrm{C} @ @] 3(\mathrm{C}) \mathrm{CC}[\mathrm{C} @ \mathrm{H}] 3 \mathrm{C}(\mathrm{C})(\mathrm{C}) \mathrm{C}(=\mathrm{O}) \mathrm{C} 5 \\
=\mathrm{NOC}(\mathrm{C})=\mathrm{C} 5[\mathrm{C} @] 43 \mathrm{C})[\mathrm{C} @ @ \mathrm{H}] 12\end{array}$ & 4,51 \\
\hline 1163 & $\mathrm{CC}[\mathrm{C} @ @] 1(\mathrm{O}) \mathrm{C}(=\mathrm{O}) \mathrm{OCC} 2=\mathrm{C} 1 \mathrm{C}=\mathrm{C} 1 \mathrm{~N}(\mathrm{CC} 3=\mathrm{CC} 4=\mathrm{C}(\mathrm{C}=\mathrm{CC}=\mathrm{C} 4) \mathrm{N}=\mathrm{C} 13) \mathrm{C} 2=\mathrm{O}$ & 5,52 \\
\hline 1164 & $\begin{array}{l}\mathrm{CC}(=\mathrm{C})[\mathrm{C} @ @ \mathrm{H}] 1 \mathrm{CC}[\mathrm{C} @] 2(\mathrm{CO}) \mathrm{CC}[\mathrm{C} @] 3(\mathrm{C})[\mathrm{C} @ \mathrm{H}](\mathrm{CC}[\mathrm{C} @ @ \mathrm{H}] 4[\mathrm{C} @ @] 5(\mathrm{C}) \mathrm{CCC}(=\mathrm{O}) \mathrm{C}(\mathrm{C})(\mathrm{C})[\mathrm{C} @ @ \mathrm{H}] \\
5 \mathrm{CC}[\mathrm{C} @] 34 \mathrm{C})[\mathrm{C} @ @ \mathrm{H}] 12\end{array}$ & 4,65 \\
\hline 1165 & $\mathrm{C}[\mathrm{C} @ \mathrm{H}](\mathrm{OC}(=\mathrm{O}) \backslash \mathrm{C}=\mathrm{ClC} 1=\mathrm{CC}=\mathrm{C}(\mathrm{O}) \mathrm{C}=\mathrm{C} 1)[\mathrm{C} @ @ \mathrm{H}] 1 \mathrm{CC}[\mathrm{C} @ @ \mathrm{H}](\mathrm{O} 1)[\mathrm{C} @ \mathrm{H}](\mathrm{O})[\mathrm{C} @ \mathrm{H}] 1 \mathrm{CC}=\mathrm{CC}(=\mathrm{O}) \mathrm{O} 1$ & 5 \\
\hline 1166 & $\begin{array}{l}\mathrm{COC} 1=\mathrm{CC}=\mathrm{C}(\backslash \mathrm{C}=\mathrm{ClC}(=\mathrm{O}) \mathrm{O}[\mathrm{C} @ @ \mathrm{H}](\mathrm{C})[\mathrm{C} @ @ \mathrm{H}] 2 \mathrm{CC}[\mathrm{C} @ @ \mathrm{H}](\mathrm{O} 2)[\mathrm{C} @ \mathrm{H}](\mathrm{O})[\mathrm{C} @ \mathrm{H}] 2 \mathrm{CC}=\mathrm{CC}(=\mathrm{O}) \mathrm{O} 2) \mathrm{C}= \\
\mathrm{C} 1\end{array}$ & 5,24 \\
\hline 1167 & $\begin{array}{l}\mathrm{C}[\mathrm{C} @ \mathrm{H}](\mathrm{OC}(=\mathrm{O}) \backslash \mathrm{C}=\mathrm{ClC} 1=\mathrm{CC}=\mathrm{C}(\mathrm{O}) \mathrm{C}(\mathrm{O})=\mathrm{C} 1)[\mathrm{C} @ @ \mathrm{H}] 1 \mathrm{CC}[\mathrm{C} @ @ \mathrm{H}](\mathrm{O} 1)[\mathrm{C} @ \mathrm{H}](\mathrm{O})[\mathrm{C} @ \mathrm{H}] 1 \mathrm{CC}=\mathrm{CC}(=\mathrm{O}) \\
\mathrm{O} 1\end{array}$ & 5,03 \\
\hline 1168 & $\mathrm{C}[\mathrm{C} @ \mathrm{H}](\mathrm{OC}(=\mathrm{O}) \backslash \mathrm{C}=\mathrm{C} / \mathrm{C} 1=\mathrm{CC}=\mathrm{C}(\mathrm{O}) \mathrm{C}=\mathrm{C} 1)[\mathrm{C} @ @ \mathrm{H}] 1 \mathrm{CC}[\mathrm{C} @ @ \mathrm{H}](\mathrm{O} 1)[\mathrm{C} @ \mathrm{H}](\mathrm{O})[\mathrm{C} @ \mathrm{H}] 1 \mathrm{CC}=\mathrm{CC}(=\mathrm{O}) \mathrm{O} 1$ & 5,11 \\
\hline 1169 & $\begin{array}{l}\mathrm{COC} 1=\mathrm{CC}=\mathrm{C}(\mathrm{C}=\mathrm{C} / \mathrm{C}(=\mathrm{O}) \mathrm{O}[\mathrm{C} @ \mathrm{H}](\mathrm{C})[\mathrm{C} @ @ \mathrm{H}] 2 \mathrm{CC}[\mathrm{C} @ @ \mathrm{H}](\mathrm{O} 2)[\mathrm{C} @ \mathrm{H}](\mathrm{O})[\mathrm{C} @ \mathrm{H}] 2 \mathrm{CC}=\mathrm{CC}(=\mathrm{O}) \mathrm{O} 2) \mathrm{C}= \\
\mathrm{C} 1\end{array}$ & 5 \\
\hline 1170 & $\begin{array}{l}\mathrm{CC}[\mathrm{C} @] 1(\mathrm{O}) \mathrm{C}[\mathrm{C} @ \mathrm{H}] 2 \mathrm{CN}(\mathrm{C} 1) \mathrm{CCC} 1=\mathrm{C}(\mathrm{NC} 3=\mathrm{CC}=\mathrm{CC}=\mathrm{C} 13)[\mathrm{C} @ @](\mathrm{C} 2)(\mathrm{C}(=\mathrm{O}) \mathrm{OC}) \mathrm{C} 1=\mathrm{CC} 2=\mathrm{C}(\mathrm{C}=\mathrm{C} 1 \mathrm{OC}) \\
\mathrm{N}(\mathrm{C})[\mathrm{C} @ @ \mathrm{H}] 1[\mathrm{C} @] 22 \mathrm{CCN} 3 \mathrm{CC}=\mathrm{C}[\mathrm{C} @](\mathrm{CC})([\mathrm{C} @ @ \mathrm{H}] 23)[\mathrm{C} @ @ \mathrm{H}](\mathrm{OC}(\mathrm{C})=\mathrm{O})[\mathrm{C} @] 1(\mathrm{O}) \mathrm{C}(=\mathrm{O}) \mathrm{OC}\end{array}$ & 8,15 \\
\hline 1171 & $\mathrm{OC}[\mathrm{C} @ \mathrm{H}] 1 \mathrm{O}[\mathrm{C} @ \mathrm{H}](\mathrm{OC} 2 \mathrm{CCCC} 2) \mathrm{C}=\mathrm{CC} 1=\mathrm{O}$ & 5,1 \\
\hline 1172 & $\mathrm{OC}[\mathrm{C} @ \mathrm{H}] 1 \mathrm{O}[\mathrm{C} @ \mathrm{H}](\mathrm{OCCC} \# \mathrm{C}) \mathrm{C}=\mathrm{CC} 1=\mathrm{O}$ & 5,15 \\
\hline 1173 & $\mathrm{OC}[\mathrm{C} @ \mathrm{H}] 1 \mathrm{O}[\mathrm{C} @ \mathrm{H}](\mathrm{OC} 2 \mathrm{CCCCC} 2) \mathrm{C}=\mathrm{CC} 1=\mathrm{O}$ & 5,12 \\
\hline 1174 & $\mathrm{OC}[\mathrm{C} @ \mathrm{H}] 1 \mathrm{O}[\mathrm{C} @ \mathrm{H}](\mathrm{OCC}=\mathrm{C}) \mathrm{C}=\mathrm{CC} 1=\mathrm{O}$ & 4,58 \\
\hline 1175 & $\mathrm{OC}[\mathrm{C} @ \mathrm{H}] 1 \mathrm{O}[\mathrm{C} @ \mathrm{H}](\mathrm{OCC} \# \mathrm{C}) \mathrm{C}=\mathrm{CC} 1=\mathrm{O}$ & 4,81 \\
\hline 1176 & $\mathrm{CCCO}[\mathrm{C} @ \mathrm{H}] 1 \mathrm{O}[\mathrm{C} @ \mathrm{H}](\mathrm{CO}) \mathrm{C}(=\mathrm{O}) \mathrm{C}=\mathrm{C} 1$ & 4,87 \\
\hline 1177 & $\begin{array}{l}\mathrm{COC} 1=\mathrm{CC}=\mathrm{CC} 2=\mathrm{C} 1 \mathrm{C}(=\mathrm{O}) \mathrm{C} 1=\mathrm{C}(\mathrm{O}) \mathrm{C} 3=\mathrm{C}(\mathrm{C}[\mathrm{C} @](\mathrm{O})(\mathrm{C}[\mathrm{C} @ @ \mathrm{H}] 3 \mathrm{O}[\mathrm{C} @ \mathrm{H}] 3 \mathrm{C}[\mathrm{C} @ \mathrm{H}](\mathrm{N})[\mathrm{C} @ \mathrm{H}](\mathrm{O})[\mathrm{C} @ \mathrm{H}]( \\
\mathrm{C}) \mathrm{O} 3) \mathrm{C}(=\mathrm{O}) \mathrm{CO}) \mathrm{C}(\mathrm{O})=\mathrm{C} 1 \mathrm{C} 2=\mathrm{O}\end{array}$ & 5,89 \\
\hline 1178 & COCCOCCOCCO[C@H]1O[C@H](CO)C(=O)C=C1 & 4,49 \\
\hline 1179 & $\mathrm{CC}(\mathrm{C}) \mathrm{O}[\mathrm{C} @ \mathrm{H}] 1 \mathrm{O}[\mathrm{C} @ \mathrm{H}](\mathrm{CO}) \mathrm{C}(=\mathrm{O}) \mathrm{C}=\mathrm{C} 1$ & 4,46 \\
\hline 1180 & $\mathrm{OC}[\mathrm{C} @ \mathrm{H}] 1 \mathrm{O}[\mathrm{C} @ \mathrm{H}](\mathrm{OCC} 2=\mathrm{CC}=\mathrm{CC}=\mathrm{C} 2) \mathrm{C}=\mathrm{CC} 1=\mathrm{O}$ & 5,07 \\
\hline 1181 & $\mathrm{CCO}[\mathrm{C} @ \mathrm{H}] 1 \mathrm{O}[\mathrm{C} @ \mathrm{H}](\mathrm{CO}) \mathrm{C}(=\mathrm{O}) \mathrm{C}=\mathrm{C} 1$ & 5,24 \\
\hline 1182 & $\begin{array}{l}\mathrm{Cl} . \mathrm{COC} 1=\mathrm{CC}=\mathrm{CC} 2=\mathrm{C} 1 \mathrm{C}(=\mathrm{O}) \mathrm{C} 1=\mathrm{C}(\mathrm{O}) \mathrm{C} 3=\mathrm{C}(\mathrm{C}[\mathrm{C} @](\mathrm{O})(\mathrm{C}[\mathrm{C} @ @ \mathrm{H}] 3 \mathrm{O}[\mathrm{C} @ \mathrm{H}] 3 \mathrm{C}[\mathrm{C} @ \mathrm{H}](\mathrm{N})[\mathrm{C} @ \mathrm{H}](\mathrm{O})[\mathrm{C} @ \\
\mathrm{H}](\mathrm{C}) \mathrm{O} 3) \mathrm{C}(=\mathrm{O}) \mathrm{CO}) \mathrm{C}(\mathrm{O})=\mathrm{C} 1 \mathrm{C} 2=\mathrm{O}\end{array}$ & 6 \\
\hline 1183 & $\mathrm{OC} 1=\mathrm{C} 2 \mathrm{C}=\mathrm{CC}(\mathrm{Cl})=\mathrm{CC} 2=\mathrm{NC}=\mathrm{C} 1$ & 4,3 \\
\hline 1184 & Cl.NCCCNC $(=\mathrm{O}) \mathrm{C} 1=\mathrm{CN}(\mathrm{CC} 2=\mathrm{CC}=\mathrm{CC}=\mathrm{C} 2) \mathrm{C} 2=\mathrm{CC}(\mathrm{Cl})=\mathrm{CC}=\mathrm{C} 2 \mathrm{C} 1=\mathrm{O}$ & 5,08 \\
\hline 1185 & Cl. $C C C C N 1 C=C(C(=O) N C C C N) C(=O) C 2=C C=C(C l) C=C 12$ & 4,66 \\
\hline 1186 & Cl.NCCCNC $(=\mathrm{O}) \mathrm{C} 1=\mathrm{CN}(\mathrm{CC}=\mathrm{C}) \mathrm{C} 2=\mathrm{CC}(\mathrm{Cl})=\mathrm{CC}=\mathrm{C} 2 \mathrm{C} 1=\mathrm{O}$ & 4,44 \\
\hline 1187 & Cl. $\mathrm{NCCCNC}(=\mathrm{O}) \mathrm{C} 1=\mathrm{CN}(\mathrm{CCCC} 2=\mathrm{CC}=\mathrm{CC}=\mathrm{C} 2) \mathrm{C} 2=\mathrm{CC}(\mathrm{Cl})=\mathrm{CC}=\mathrm{C} 2 \mathrm{C} 1=\mathrm{O}$ & 5,49 \\
\hline 1188 & Cl. $C C N 1 C=C(C(=O) N C C C N) C(=O) C 2=C C=C(C l) C=C 12$ & 4,55 \\
\hline 1189 & $\mathrm{COC}(=\mathrm{O})[\mathrm{C} @ \mathrm{H}](\mathrm{NC}(=\mathrm{O}) \backslash \mathrm{C}=\mathrm{ClC}=\mathrm{ClC} 1=\mathrm{CC}=\mathrm{C} 2 \mathrm{OCOC} 2=\mathrm{C} 1) \mathrm{C}(\mathrm{C}) \mathrm{C}$ & 4,3 \\
\hline 1190 & $\mathrm{COC}(=\mathrm{O})[\mathrm{C} @ \mathrm{H}](\mathrm{CO}) \mathrm{NC}(=\mathrm{O}) \backslash \mathrm{C}=\mathrm{C} \backslash \mathrm{C}=\mathrm{C} \backslash \mathrm{C} 1=\mathrm{CC}=\mathrm{C} 2 \mathrm{OCOC} 2=\mathrm{C} 1$ & 4,3 \\
\hline 1191 & $\mathrm{COC}(=\mathrm{O})[\mathrm{C} @ \mathrm{H}](\mathrm{C}) \mathrm{NC}(=\mathrm{O}) \backslash \mathrm{C}=\mathrm{ClC}=\mathrm{ClC} 1=\mathrm{CC}=\mathrm{C} 2 \mathrm{OCOC} 2=\mathrm{C} 1$ & 4,3 \\
\hline 1192 & $\mathrm{O}=\mathrm{C} 1 \mathrm{~N}(\mathrm{C}(=\mathrm{O}) \mathrm{C} 2=\mathrm{CC}=\mathrm{CC}=\mathrm{C} 12) \mathrm{C} 1=\mathrm{CC}=\mathrm{C}(\mathrm{C}=\mathrm{C} 1) \mathrm{C} 1=\mathrm{CC}=\mathrm{CC}=\mathrm{C} 1$ & 4,39 \\
\hline 1193 & $\mathrm{CC}(=\mathrm{O}) \mathrm{N} 1 \mathrm{CC}(\mathrm{C}(=\mathrm{N} 1) \mathrm{C} 1=\mathrm{CC}=\mathrm{C} 2 \mathrm{C}=\mathrm{CC}=\mathrm{CC} 2=\mathrm{C} 1) \mathrm{C} 1=\mathrm{CC}=\mathrm{C} 2 \mathrm{OCOC} 2=\mathrm{C} 1$ & 6 \\
\hline 1194 & $\mathrm{COC} 1=\mathrm{CC}(=\mathrm{CC}(\mathrm{OC})=\mathrm{C} 1 \mathrm{OC}) \mathrm{C} 1 \mathrm{CC}(=\mathrm{NN} 1 \mathrm{CC}=\mathrm{O}) \mathrm{C} 1=\mathrm{CC}=\mathrm{C} 2 \mathrm{C}=\mathrm{CC}=\mathrm{CC} 2=\mathrm{C} 1$ & 6,28 \\
\hline 1195 & $\mathrm{COC} 1=\mathrm{CC}=\mathrm{C}(\mathrm{C}=\mathrm{C} 1 \mathrm{O}) \mathrm{C} 1 \mathrm{CC}(=\mathrm{NN} 1 \mathrm{CC}=\mathrm{O}) \mathrm{C} 1=\mathrm{CC}=\mathrm{C} 2 \mathrm{C}=\mathrm{CC}=\mathrm{CC} 2=\mathrm{C} 1$ & 5,99 \\
\hline 1196 & $\mathrm{O}=\mathrm{CCN} 1 \mathrm{~N}=\mathrm{C}(\mathrm{CC} 1 \mathrm{C} 1=\mathrm{CC}=\mathrm{CO} 1) \mathrm{C} 1=\mathrm{CC}=\mathrm{C} 2 \mathrm{C}=\mathrm{CC}=\mathrm{CC} 2=\mathrm{C} 1$ & 4,72 \\
\hline 1197 & $\begin{array}{l}\mathrm{COC} 1=\mathrm{CC}=\mathrm{CC} 2=\mathrm{C} 1 \mathrm{C}(=\mathrm{O}) \mathrm{C} 1=\mathrm{C}(\mathrm{O}) \mathrm{C} 3=\mathrm{C}(\mathrm{C}[\mathrm{C} @](\mathrm{O})(\mathrm{C}[\mathrm{C} @ @ \mathrm{H}] 3 \mathrm{O}[\mathrm{C} @ \mathrm{H}] 3 \mathrm{C}[\mathrm{C} @ \mathrm{H}](\mathrm{N})[\mathrm{C} @ \mathrm{H}](\mathrm{O})[\mathrm{C} @ \mathrm{H}]( \\
\mathrm{C}) \mathrm{O} 3) \mathrm{C}(=\mathrm{O}) \mathrm{CO}) \mathrm{C}(\mathrm{O})=\mathrm{C} 1 \mathrm{C} 2=\mathrm{O}\end{array}$ & 5,06 \\
\hline 1198 & $\mathrm{ClC} 1=\mathrm{CC}=\mathrm{C}(\mathrm{C}=\mathrm{C} 1) \mathrm{N} 1 \mathrm{C}(=\mathrm{O}) \mathrm{C} 2=\mathrm{CC}=\mathrm{CC}=\mathrm{C} 2 \mathrm{C} 1=\mathrm{O}$ & 3,75 \\
\hline 1199 & $\mathrm{COC} 1=\mathrm{CC}=\mathrm{C}(\mathrm{C}=\mathrm{C} 1) \mathrm{C} 1 \mathrm{CC}(=\mathrm{NN} 1 \mathrm{C}(\mathrm{C})=\mathrm{O}) \mathrm{C} 1=\mathrm{CC}=\mathrm{C}(\mathrm{Br}) \mathrm{C}=\mathrm{C} 1$ & 4,75 \\
\hline 1200 & $\mathrm{CC}(=\mathrm{O}) \mathrm{N} 1 \mathrm{~N}=\mathrm{C}(\mathrm{CC} 1 \mathrm{C} 1=\mathrm{CC}=\mathrm{CC}=\mathrm{C} 1) \mathrm{C} 1=\mathrm{CC}=\mathrm{CC}(=\mathrm{C} 1)[\mathrm{N}+]([\mathrm{O}-])=\mathrm{O}$ & 5,05 \\
\hline 1201 & $\mathrm{CC}(=\mathrm{O}) \mathrm{N} 1 \mathrm{~N}=\mathrm{C}(\mathrm{CC} 1 \mathrm{C} 1=\mathrm{CC}=\mathrm{CC}(\mathrm{O})=\mathrm{C} 1) \mathrm{C} 1=\mathrm{CC}=\mathrm{CO} 1$ & 5 \\
\hline 1202 & $\mathrm{CC}(=\mathrm{O}) \mathrm{N} 1 \mathrm{~N}=\mathrm{C}(\mathrm{CC} 1 \mathrm{C} 1=\mathrm{CC}=\mathrm{C}(\mathrm{Cl}) \mathrm{C}=\mathrm{C} 1) \mathrm{C} 1=\mathrm{CC}=\mathrm{CO} 1$ & 4,24 \\
\hline 1203 & $\mathrm{COC} 1=\mathrm{CC}(=\mathrm{CC}(\mathrm{OC})=\mathrm{C} 1 \mathrm{OC}) \mathrm{C} 1 \mathrm{CC}(=\mathrm{NN} 1 \mathrm{C}(\mathrm{C})=\mathrm{O}) \mathrm{C} 1=\mathrm{CC}=\mathrm{CO} 1$ & 4,67 \\
\hline 1204 & $\mathrm{COC} 1=\mathrm{CC}=\mathrm{C}(\mathrm{C}=\mathrm{C} 1 \mathrm{OC}) \mathrm{C} 1 \mathrm{CC}(=\mathrm{NN} 1 \mathrm{C}(\mathrm{C})=\mathrm{O}) \mathrm{C} 1=\mathrm{CC}=\mathrm{CO} 1$ & 5 \\
\hline 1205 & $\begin{array}{l}\mathrm{CC} 1=\mathrm{NNC}(=\mathrm{O}) \mathrm{C} 1 \mathrm{C}(\mathrm{C} 1=\mathrm{C}(\mathrm{NC} 2=\mathrm{CC}=\mathrm{C}(\mathrm{Cl}) \mathrm{C}=\mathrm{C} 12) \mathrm{C} 1=\mathrm{CC}=\mathrm{CC}=\mathrm{C} 1) \mathrm{C} 1=\mathrm{C}(\mathrm{NC} 2=\mathrm{CC}=\mathrm{C}(\mathrm{Cl}) \mathrm{C}=\mathrm{C} 12) \mathrm{C} 1=\mathrm{CC}= \\
\mathrm{CC}=\mathrm{C} 1\end{array}$ & 4,84 \\
\hline 1206 & $\mathrm{NC} 1=\mathrm{CC}=\mathrm{CC}(=\mathrm{C} 1) \mathrm{N} 1 \mathrm{C}(=\mathrm{O}) \mathrm{C} 2 \mathrm{C}(\mathrm{C} 3 \mathrm{CCC} 2 \mathrm{C}=\mathrm{C} 3) \mathrm{C} 1=\mathrm{O}$ & 4,27 \\
\hline
\end{tabular}




\begin{tabular}{|c|c|c|}
\hline NAME & SMILE & $\lg (\mathbf{1} / \mathbf{I C 5 0})$ \\
\hline 1207 & $\mathrm{COC} 1=\mathrm{CC}=\mathrm{C}(\mathrm{C}=\mathrm{C} 1) \mathrm{N} 1 \mathrm{C}(=\mathrm{O}) \mathrm{C} 2 \mathrm{C}(\mathrm{C} 3 \mathrm{CCC} 2 \mathrm{C}=\mathrm{C} 3) \mathrm{C} 1=\mathrm{O}$ & 3,7 \\
\hline 1208 & $\mathrm{SC} 1=\mathrm{CC}=\mathrm{C}(\mathrm{C}=\mathrm{C} 1) \mathrm{N} 1 \mathrm{C}(=\mathrm{O}) \mathrm{C} 2=\mathrm{CC}=\mathrm{CC}=\mathrm{C} 2 \mathrm{C} 1=\mathrm{O}$ & 3,7 \\
\hline 1209 & $\mathrm{COC} 1=\mathrm{CC}=\mathrm{C}(\mathrm{C}=\mathrm{C} 1 \mathrm{OC}) \mathrm{C} 1 \mathrm{CC}(=\mathrm{NN} 1 \mathrm{CC}=\mathrm{O}) \mathrm{C} 1=\mathrm{CC}=\mathrm{C} 2 \mathrm{C}=\mathrm{CC}=\mathrm{CC} 2=\mathrm{C} 1$ & 6 \\
\hline 1210 & $\mathrm{CC}(=\mathrm{O}) \mathrm{N} 1 \mathrm{~N}=\mathrm{C}(\mathrm{CC} 1 \mathrm{C} 1=\mathrm{CC}=\mathrm{CC}=\mathrm{C} 1) \mathrm{C} 1=\mathrm{CC}=\mathrm{CC}=\mathrm{C} 1$ & 4,93 \\
\hline 1211 & $\mathrm{O}=\mathrm{C} 1 \mathrm{~N}(\mathrm{C}(=\mathrm{O}) \mathrm{C} 2=\mathrm{CC}=\mathrm{CC}=\mathrm{C} 12) \mathrm{C} 1=\mathrm{CC}=\mathrm{CC}=\mathrm{C} 1$ & 3,7 \\
\hline 1212 & $\mathrm{COC} 1=\mathrm{CC}(=\mathrm{CC}=\mathrm{C} 1 \mathrm{O}) \mathrm{C} 1 \mathrm{CC}(=\mathrm{NN} 1 \mathrm{C}(\mathrm{C})=\mathrm{O}) \mathrm{C} 1=\mathrm{CC}=\mathrm{CC}=\mathrm{C} 1$ & 5,3 \\
\hline 1213 & $\mathrm{COC} 1=\mathrm{CC}(=\mathrm{CC}=\mathrm{C} 1 \mathrm{O}) \mathrm{C} 1 \mathrm{CC}(=\mathrm{NN} 1 \mathrm{C}(\mathrm{C})=\mathrm{O}) \mathrm{C} 1=\mathrm{CC}=\mathrm{CO} 1$ & 5 \\
\hline 1214 & $\mathrm{CC}(=\mathrm{O}) \mathrm{N} 1 \mathrm{~N}=\mathrm{C}(\mathrm{CC} 1 \mathrm{C} 1=\mathrm{CC}=\mathrm{C} 2 \mathrm{OCOC} 2=\mathrm{C} 1) \mathrm{C} 1=\mathrm{CC}=\mathrm{CO} 1$ & 4,7 \\
\hline 1215 & $\mathrm{COC}(=\mathrm{O})[\mathrm{C} @ \mathrm{H}](\mathrm{CC} 1=\mathrm{CN}=\mathrm{CN} 1) \mathrm{NC}(=\mathrm{O}) \backslash \mathrm{C}=\mathrm{C} \backslash \mathrm{C}=\mathrm{ClC} 1=\mathrm{CC}=\mathrm{C} 2 \mathrm{OCOC} 2=\mathrm{C} 1$ & 4,3 \\
\hline 1216 & $\mathrm{COC} 1=\mathrm{CC}=\mathrm{C}(\mathrm{C}=\mathrm{C} 1) \mathrm{C} 1 \mathrm{CC}(=\mathrm{NN} 1 \mathrm{CC}=\mathrm{O}) \mathrm{C} 1=\mathrm{CC}=\mathrm{C} 2 \mathrm{C}=\mathrm{CC}=\mathrm{CC} 2=\mathrm{C} 1$ & 5,49 \\
\hline 1217 & $\mathrm{O}=\mathrm{CCN} 1 \mathrm{~N}=\mathrm{C}(\mathrm{CC} 1|\mathrm{C}=\mathrm{C}| \mathrm{C} 1=\mathrm{CC}=\mathrm{CC}=\mathrm{C} 1) \mathrm{C} 1=\mathrm{CC}=\mathrm{C} 2 \mathrm{C}=\mathrm{CC}=\mathrm{CC} 2=\mathrm{C} 1$ & 4,7 \\
\hline 1218 & $\mathrm{ClC} 1=\mathrm{CC}=\mathrm{CC}(\mathrm{C} 2 \mathrm{CC}(=\mathrm{NN} 2 \mathrm{CC}=\mathrm{O}) \mathrm{C} 2=\mathrm{CC}=\mathrm{C} 3 \mathrm{C}=\mathrm{CC}=\mathrm{CC} 3=\mathrm{C} 2)=\mathrm{C} 1 \mathrm{Cl}$ & 4,9 \\
\hline 1219 & $\mathrm{COC} 1=\mathrm{CC}=\mathrm{C}(\mathrm{OC}) \mathrm{C}(=\mathrm{C} 1) \mathrm{C} 1 \mathrm{CC}(=\mathrm{NN} 1 \mathrm{CC}=\mathrm{O}) \mathrm{C} 1=\mathrm{CC}=\mathrm{C} 2 \mathrm{C}=\mathrm{CC}=\mathrm{CC} 2=\mathrm{C} 1$ & 5,96 \\
\hline 1220 & $\mathrm{BrC} 1=\mathrm{CC}=\mathrm{CC}(=\mathrm{C} 1) \mathrm{C} 1 \mathrm{CC}(=\mathrm{NN} 1 \mathrm{CC}=\mathrm{O}) \mathrm{C} 1=\mathrm{CC}=\mathrm{C} 2 \mathrm{C}=\mathrm{CC}=\mathrm{CC} 2=\mathrm{C} 1$ & 5,06 \\
\hline 1221 & $\mathrm{CC}(=\mathrm{O}) \mathrm{N} 1 \mathrm{~N}=\mathrm{C}(\mathrm{CC} 1 \mathrm{C} 1=\mathrm{CC}=\mathrm{C} 2 \mathrm{OCOC} 2=\mathrm{C} 1) \mathrm{C} 1=\mathrm{CC}=\mathrm{CC}=\mathrm{C} 1$ & 5,18 \\
\hline 1222 & $\mathrm{CC}(=\mathrm{O}) \mathrm{N} 1 \mathrm{~N}=\mathrm{C}(\mathrm{CC} 1 \mathrm{C} 1=\mathrm{CC}=\mathrm{C}(\mathrm{C}) \mathrm{C}=\mathrm{C} 1) \mathrm{C} 1=\mathrm{CC}=\mathrm{CO} 1$ & 4,71 \\
\hline 1223 & $\begin{array}{l}\mathrm{CC}(=\mathrm{O}) \mathrm{O}[\mathrm{C} @ @ \mathrm{H}] 1 \mathrm{C} 2=\mathrm{C}(\mathrm{C})[\mathrm{C} @ \mathrm{H}](\mathrm{C}[\mathrm{C} @ @](\mathrm{O})([\mathrm{C} @ @ \mathrm{H}](\mathrm{OC}(=\mathrm{O}) \mathrm{C} 3=\mathrm{CC}=\mathrm{CC}=\mathrm{C} 3)[\mathrm{C} @ @ \mathrm{H}] 3[\mathrm{C} @ @] 4(\mathrm{C} \\
\mathrm{O}[\mathrm{C} @ @ \mathrm{H}] 4 \mathrm{C}[\mathrm{C} @ \mathrm{H}](\mathrm{O})[\mathrm{C} @ @] 3(\mathrm{C}) \mathrm{C} 1=\mathrm{O}) \mathrm{OC}(\mathrm{C})=\mathrm{O}) \mathrm{C} 2(\mathrm{C}) \mathrm{C}) \mathrm{OC}(=\mathrm{O})[\mathrm{C} @ \mathrm{H}](\mathrm{O})[\mathrm{C} @ @ \mathrm{H}](\mathrm{NC}(=\mathrm{O}) \mathrm{C} 1=\mathrm{CC}= \\
\mathrm{CC}=\mathrm{C} 1) \mathrm{C} 1=\mathrm{CC}=\mathrm{CC}=\mathrm{C} 1\end{array}$ & 6,96 \\
\hline 1224 & $\mathrm{OC} 1=\mathrm{CC}=\mathrm{C} 2 \mathrm{NC}=\mathrm{C}(\mathrm{C} 3=\mathrm{ClC}(\mathrm{C}(=\mathrm{O}) \mathrm{N} 3)=\mathrm{C} 3 / \mathrm{C}(=\mathrm{O}) \mathrm{NC} 4=\mathrm{CC}=\mathrm{CC}=\mathrm{C} 34) \mathrm{C} 2=\mathrm{C} 1$ & 5,66 \\
\hline 1225 & $\mathrm{CC}(=\mathrm{O}) \mathrm{N} 1 \mathrm{~N}=\mathrm{C}(\mathrm{CC} 1 \mathrm{C} 1=\mathrm{CC}=\mathrm{CC}(\mathrm{Cl})=\mathrm{C} 1 \mathrm{Cl}) \mathrm{C} 1=\mathrm{CC}=\mathrm{CC}=\mathrm{C} 1$ & 4,62 \\
\hline 1226 & $\mathrm{COC} 1=\mathrm{CC}=\mathrm{C}(\mathrm{C}=\mathrm{C} 1 \mathrm{OC}) \mathrm{C} 1 \mathrm{CC}(=\mathrm{NN} 1 \mathrm{C}(\mathrm{C})=\mathrm{O}) \mathrm{C} 1=\mathrm{CC}=\mathrm{C}(\mathrm{Br}) \mathrm{C}=\mathrm{C} 1$ & 5,05 \\
\hline 1227 & $\mathrm{COC} 1=\mathrm{CC}=\mathrm{C}(\mathrm{C}=\mathrm{C} 1) \mathrm{C} 1 \mathrm{CC}(=\mathrm{NN} 1 \mathrm{C}(\mathrm{C})=\mathrm{O}) \mathrm{C} 1=\mathrm{CC}=\mathrm{CC}(=\mathrm{C} 1)[\mathrm{N}+]([\mathrm{O}-])=\mathrm{O}$ & 5,09 \\
\hline 1228 & $\mathrm{COC} 1=\mathrm{CC}=\mathrm{C}(\mathrm{C}=\mathrm{C} 1) \mathrm{C} 1 \mathrm{CC}(=\mathrm{NN} 1 \mathrm{C}(\mathrm{C})=\mathrm{O}) \mathrm{C} 1=\mathrm{C} 2 \mathrm{C}=\mathrm{CC}=\mathrm{CC} 2=\mathrm{CC}=\mathrm{C} 1$ & 5,43 \\
\hline 1229 & $\mathrm{COC} 1=\mathrm{CC}=\mathrm{C}(\mathrm{C}=\mathrm{C} 1) \mathrm{C} 1 \mathrm{CC}(=\mathrm{NN} 1 \mathrm{C}(\mathrm{C})=\mathrm{O}) \mathrm{C} 1=\mathrm{CC}=\mathrm{CO} 1$ & 4,81 \\
\hline 1230 & $\begin{array}{l}\mathrm{CC} 1=\mathrm{NNC}(=\mathrm{O}) \mathrm{C} 1 \mathrm{C}(\mathrm{C} 1=\mathrm{C}(\mathrm{NC} 2=\mathrm{CC}=\mathrm{CC}=\mathrm{C} 12) \mathrm{C} 1=\mathrm{CC}=\mathrm{CC}=\mathrm{C} 1) \mathrm{C} 1=\mathrm{C}(\mathrm{NC} 2=\mathrm{CC}=\mathrm{C}(\mathrm{Cl}) \mathrm{C}=\mathrm{C} 12) \mathrm{C} 1=\mathrm{CC}=\mathrm{CC} \\
=\mathrm{C} 1\end{array}$ & 4,38 \\
\hline 1231 & $\mathrm{CC} 1=\mathrm{NNC}(=\mathrm{O}) \mathrm{C} 1 \mathrm{C}(\mathrm{C} 1=\mathrm{CNC} 2=\mathrm{CC}=\mathrm{CC}=\mathrm{C} 12) \mathrm{C} 1=\mathrm{C}(\mathrm{NC} 2=\mathrm{CC}=\mathrm{C}(\mathrm{Cl}) \mathrm{C}=\mathrm{C} 12) \mathrm{C} 1=\mathrm{CC}=\mathrm{CC}=\mathrm{C} 1$ & 4 \\
\hline 1232 & $\mathrm{CC} 1=\mathrm{NNC}(=\mathrm{O}) \mathrm{C} 1 \mathrm{C}(\mathrm{C} 1=\mathrm{CNC} 2=\mathrm{CC}=\mathrm{CC}=\mathrm{C} 12) \mathrm{C} 1=\mathrm{C}(\mathrm{NC} 2=\mathrm{CC}=\mathrm{CC}=\mathrm{C} 12) \mathrm{C} 1=\mathrm{CC}=\mathrm{CC}=\mathrm{C} 1$ & 5,17 \\
\hline 1233 & $\begin{array}{l}\mathrm{CC} 1=\mathrm{NNC}(=\mathrm{O}) \mathrm{C} 1 \mathrm{C}(\mathrm{C} 1=\mathrm{C}(\mathrm{NC} 2=\mathrm{CC}=\mathrm{C}(\mathrm{C}) \mathrm{C}=\mathrm{C} 12) \mathrm{C} 1=\mathrm{CC}=\mathrm{CC}=\mathrm{C} 1) \mathrm{C} 1=\mathrm{C}(\mathrm{NC} 2=\mathrm{CC}=\mathrm{C}(\mathrm{Cl}) \mathrm{C}=\mathrm{C} 12) \mathrm{C} 1=\mathrm{CC}= \\
\mathrm{CC}=\mathrm{C} 1\end{array}$ & 4,49 \\
\hline 1234 & $\mathrm{COC}(=\mathrm{O})[\mathrm{C} @ @ \mathrm{H}](\mathrm{NC}(=\mathrm{O}) \backslash \mathrm{C}=\mathrm{ClC}=\mathrm{ClC} 1=\mathrm{CC}=\mathrm{C} 2 \mathrm{OCOC} 2=\mathrm{C} 1) \mathrm{C}(\mathrm{C}) \mathrm{C}$ & 4,3 \\
\hline 1235 & $\mathrm{COC}(=\mathrm{O}) \mathrm{CNC}(=\mathrm{O}) \backslash \mathrm{C}=\mathrm{C} \backslash \mathrm{C}=\mathrm{C} \backslash \mathrm{C} 1=\mathrm{CC}=\mathrm{C} 2 \mathrm{OCOC} 2=\mathrm{C} 1$ & 4,3 \\
\hline 1236 & $\mathrm{ClC} 1=\mathrm{CC}=\mathrm{C}(\mathrm{N}=\mathrm{C} 1) \mathrm{N} 1 \mathrm{C}(=\mathrm{O}) \mathrm{C} 2 \mathrm{C}(\mathrm{C} 3 \mathrm{CCC} 2 \mathrm{C}=\mathrm{C} 3) \mathrm{C} 1=\mathrm{O}$ & 3,7 \\
\hline 1237 & $\mathrm{CC} 1=\mathrm{CC}=\mathrm{C} 2 \mathrm{C}(=\mathrm{O}) \mathrm{N}(\mathrm{C}(=\mathrm{O}) \mathrm{C} 2=\mathrm{C} 1) \mathrm{C} 1=\mathrm{CC}=\mathrm{C}(\mathrm{C}=\mathrm{C} 1) \mathrm{C} 1=\mathrm{CC}=\mathrm{CC}=\mathrm{C} 1$ & 3,98 \\
\hline 1238 & $\mathrm{NC} 1=\mathrm{CC}=\mathrm{CC}(=\mathrm{C} 1) \mathrm{N} 1 \mathrm{C}(=\mathrm{O}) \mathrm{C} 2=\mathrm{CC}=\mathrm{CC} 3=\mathrm{CC}=\mathrm{CC}(\mathrm{C} 1=\mathrm{O})=\mathrm{C} 23$ & 3,83 \\
\hline 1239 & $\mathrm{COC} 1=\mathrm{CC}(=\mathrm{CC}(\mathrm{OC})=\mathrm{C} 1 \mathrm{OC}) \mathrm{C} 1 \mathrm{CC}(=\mathrm{NN} 1 \mathrm{C}(\mathrm{C})=\mathrm{O}) \mathrm{C} 1=\mathrm{CC}=\mathrm{CC}=\mathrm{C} 1$ & 5,45 \\
\hline 1240 & $\mathrm{CC}(=\mathrm{O}) \mathrm{N} 1 \mathrm{~N}=\mathrm{C}(\mathrm{CC} 1 \mathrm{C} 1=\mathrm{CC}=\mathrm{C} 2 \mathrm{OCOC} 2=\mathrm{C} 1) \mathrm{C} 1=\mathrm{CC}=\mathrm{C}(\mathrm{Br}) \mathrm{C}=\mathrm{C} 1$ & 5,06 \\
\hline 1241 & $\mathrm{CC}(=\mathrm{O}) \mathrm{N} 1 \mathrm{~N}=\mathrm{C}(\mathrm{CC} 1 \mathrm{C} 1=\mathrm{CC}=\mathrm{CC}=\mathrm{C} 1) \mathrm{C} 1=\mathrm{C} 2 \mathrm{C}=\mathrm{CC}=\mathrm{CC} 2=\mathrm{CC}=\mathrm{C} 1$ & 4,95 \\
\hline 1242 & $\mathrm{COC} 1=\mathrm{CC}(=\mathrm{CC}=\mathrm{C} 1 \mathrm{O}) \mathrm{C} 1 \mathrm{CC}(=\mathrm{NN} 1 \mathrm{C}(\mathrm{C})=\mathrm{O}) \mathrm{C} 1=\mathrm{C} 2 \mathrm{C}=\mathrm{CC}=\mathrm{CC} 2=\mathrm{CC}=\mathrm{C} 1$ & 5,95 \\
\hline 1243 & $\mathrm{CC}(=\mathrm{O}) \mathrm{N} 1 \mathrm{~N}=\mathrm{C}(\mathrm{CC} 1 \mathrm{C} 1=\mathrm{CC}=\mathrm{CC}(\mathrm{Cl})=\mathrm{C} 1 \mathrm{Cl}) \mathrm{C} 1=\mathrm{C} 2 \mathrm{C}=\mathrm{CC}=\mathrm{CC} 2=\mathrm{CC}=\mathrm{C} 1$ & 4,78 \\
\hline 1244 & $\mathrm{CC}(=\mathrm{O}) \mathrm{N} 1 \mathrm{~N}=\mathrm{C}(\mathrm{CC} 1 \mathrm{C} 1=\mathrm{CC}=\mathrm{CC}(=\mathrm{C} 1)[\mathrm{N}+]([\mathrm{O}-])=\mathrm{O}) \mathrm{C} 1=\mathrm{CC}=\mathrm{CO} 1$ & 4,52 \\
\hline 1245 & $\mathrm{COC} 1=\mathrm{CC}(\mathrm{OC})=\mathrm{C}(\mathrm{C}=\mathrm{C} 1 \mathrm{OC}) \mathrm{C} 1 \mathrm{CC}(=\mathrm{NN} 1 \mathrm{C}(\mathrm{C})=\mathrm{O}) \mathrm{C} 1=\mathrm{CC}=\mathrm{CO} 1$ & 5,17 \\
\hline 1246 & $\begin{array}{l}\mathrm{COC} 1=\mathrm{CC}=\mathrm{C} 2 \mathrm{NC}(=\mathrm{C}(\mathrm{C}(\mathrm{C} 3 \mathrm{C}(=\mathrm{O}) \mathrm{NN}=\mathrm{C} 3 \mathrm{C}) \mathrm{C} 3=\mathrm{C}(\mathrm{NC} 4=\mathrm{CC}=\mathrm{C}(\mathrm{Cl}) \mathrm{C}=\mathrm{C} 34) \mathrm{C} 3=\mathrm{CC}=\mathrm{CC}=\mathrm{C} 3) \mathrm{C} 2=\mathrm{C} 1) \mathrm{C} 1=\mathrm{CC} \\
=\mathrm{CC}=\mathrm{C} 1\end{array}$ & 5,21 \\
\hline
\end{tabular}

1247 $\mathrm{CC} 1=\mathrm{NNC}(=\mathrm{O}) \mathrm{C} 1 \mathrm{C}(\mathrm{C} 1=\mathrm{C}(\mathrm{NC} 2=\mathrm{CC}=\mathrm{CC}=\mathrm{C} 12) \mathrm{C} 1=\mathrm{CC}=\mathrm{CC}=\mathrm{C} 1) \mathrm{C} 1=\mathrm{C}(\mathrm{NC} 2=\mathrm{CC}=\mathrm{C}(\mathrm{C}) \mathrm{C}=\mathrm{C} 12) \mathrm{C} 1=\mathrm{CC}=\mathrm{CC}=$

$1248 \mathrm{COC}(=\mathrm{O})[\mathrm{C} @ \mathrm{H}](\mathrm{CC}(\mathrm{C}) \mathrm{C}) \mathrm{NC}(=\mathrm{O}) \backslash \mathrm{C}=\mathrm{ClC}=\mathrm{ClC} 1=\mathrm{CC}=\mathrm{C} 2 \mathrm{OCOC} 2=\mathrm{C} 1$

$1249 \mathrm{CC}[\mathrm{C} @ \mathrm{H}](\mathrm{C})[\mathrm{C} @ \mathrm{H}](\mathrm{NC}(=\mathrm{O}) \backslash \mathrm{C}=\mathrm{ClC}=\mathrm{ClC} 1=\mathrm{CC}=\mathrm{C} 2 \mathrm{OCOC} 2=\mathrm{C} 1) \mathrm{C}(=\mathrm{O}) \mathrm{OC}$

$1250 \mathrm{NC} 1=\mathrm{CC}=\mathrm{C}(\mathrm{C}=\mathrm{C} 1) \mathrm{N} 1 \mathrm{C}(=\mathrm{O}) \mathrm{C} 2=\mathrm{CC}=\mathrm{CC} 3=\mathrm{CC}=\mathrm{CC}(\mathrm{C} 1=\mathrm{O})=\mathrm{C} 23$

$1251 \mathrm{CC} 1=\mathrm{CC}=\mathrm{C} 2 \mathrm{C}(=\mathrm{O}) \mathrm{N}(\mathrm{C}(=\mathrm{O}) \mathrm{C} 2=\mathrm{C} 1) \mathrm{C} 1=\mathrm{CC}=\mathrm{C}(\mathrm{C}=\mathrm{C} 1) \backslash \mathrm{N}=\mathrm{N} / \mathrm{C} 1=\mathrm{CC}=\mathrm{CC}=\mathrm{C} 1$

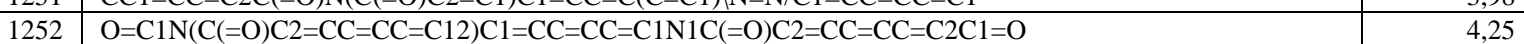

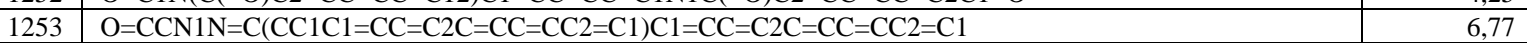

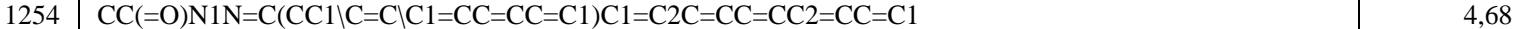

\begin{tabular}{ll}
$\mathrm{COC} 1=\mathrm{CC}=\mathrm{C}(\mathrm{C} 2 \mathrm{CC}(=\mathrm{NN} 2 \mathrm{CC}=\mathrm{O}) \mathrm{C} 2=\mathrm{CC}=\mathrm{C} 3 \mathrm{C}=\mathrm{CC}=\mathrm{CC} 3=\mathrm{C} 2) \mathrm{C}(\mathrm{OC})=\mathrm{C} 1 \mathrm{OC}$ & 6,23 \\
\hline
\end{tabular}

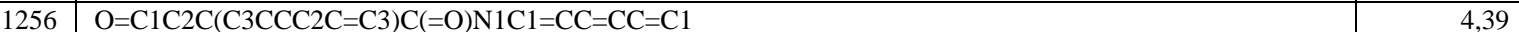

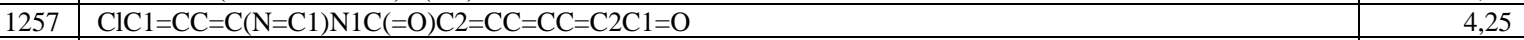

$1258 \mathrm{COC} 1=\mathrm{CC}=\mathrm{C}(\mathrm{C}=\mathrm{C} 1) \mathrm{N} 1 \mathrm{C}(=\mathrm{O}) \mathrm{C} 2=\mathrm{CC}=\mathrm{CC}=\mathrm{C} 2 \mathrm{C} 1=\mathrm{O}$

$1259 \quad \mathrm{FC} 1=\mathrm{CNC}(=\mathrm{O}) \mathrm{NC} 1=\mathrm{O}$

$1260 \mathrm{CC}(=\mathrm{O}) \mathrm{N} 1 \mathrm{~N}=\mathrm{C}(\mathrm{CC} 1 \mathrm{C} 1=\mathrm{CC}=\mathrm{C} 2 \mathrm{OCOC} 2=\mathrm{C} 1) \mathrm{C} 1=\mathrm{CC}=\mathrm{CC}(=\mathrm{C} 1)[\mathrm{N}+]([\mathrm{O}-])=\mathrm{O}$

$1261 \mathrm{CC}(=\mathrm{O}) \mathrm{N} 1 \mathrm{~N}=\mathrm{C}(\mathrm{CC} 1 \mathrm{C} 1=\mathrm{CC}=\mathrm{CC}(=\mathrm{C} 1)[\mathrm{N}+]([\mathrm{O}-])=\mathrm{O}) \mathrm{C} 1=\mathrm{C} 2 \mathrm{C}=\mathrm{CC}=\mathrm{CC} 2=\mathrm{CC}=\mathrm{C} 1 \quad 5,39$

$1262 \mathrm{CC}(=\mathrm{O}) \mathrm{N} 1 \mathrm{~N}=\mathrm{C}(\mathrm{CC} 1 \mathrm{C} 1=\mathrm{CC}=\mathrm{CC}(\mathrm{Br})=\mathrm{C} 1) \mathrm{C} 1=\mathrm{C} 2 \mathrm{C}=\mathrm{CC}=\mathrm{CC} 2=\mathrm{CC}=\mathrm{C} 1$

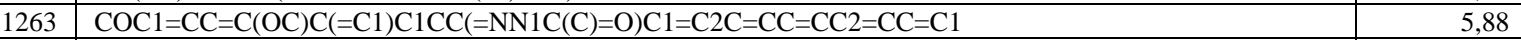

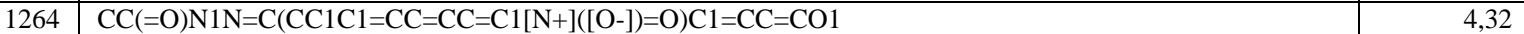

$1265 \mathrm{CC}(=\mathrm{O}) \mathrm{N} 1 \mathrm{~N}=\mathrm{C}(\mathrm{CC} 1 \mathrm{C} 1=\mathrm{CC}=\mathrm{CC}(\mathrm{Cl})=\mathrm{C} 1 \mathrm{Cl}) \mathrm{C} 1=\mathrm{CC}=\mathrm{CO} 1$

$1266[\mathrm{O}-][\mathrm{N}+](=\mathrm{O}) \mathrm{C} 1=\mathrm{CC}=\mathrm{CC}(=\mathrm{C} 1) \mathrm{N} 1 \mathrm{C}(=\mathrm{O}) \mathrm{C} 2=\mathrm{CC}=\mathrm{CC}=\mathrm{C} 2 \mathrm{C} 1=\mathrm{O}$

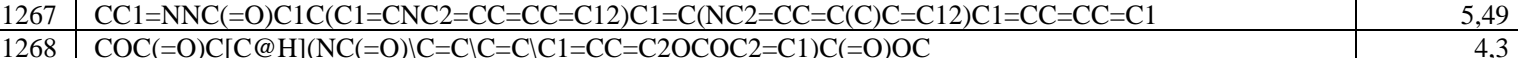

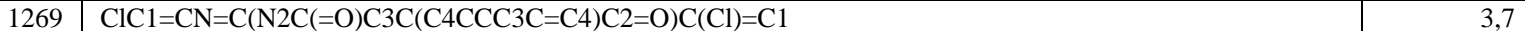

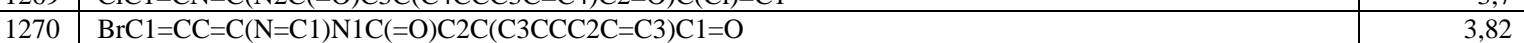

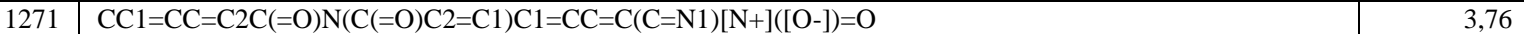

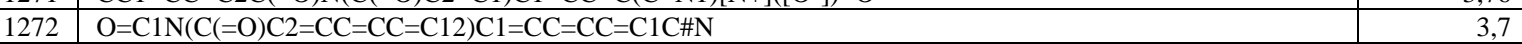

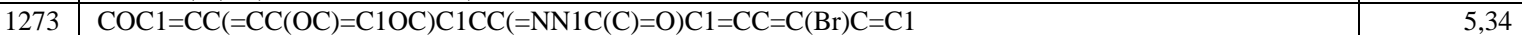

$1274 \mathrm{CN}(\mathrm{C}) \mathrm{C} 1=\mathrm{CC}=\mathrm{C}(\mathrm{C}=\mathrm{C} 1) \mathrm{C} 1 \mathrm{CC}(=\mathrm{NN} 1 \mathrm{C}(\mathrm{C})=\mathrm{O}) \mathrm{C} 1=\mathrm{CC}=\mathrm{CC}=\mathrm{C} 1$

$1275 \mathrm{CC}(=\mathrm{O}) \mathrm{N} 1 \mathrm{~N}=\mathrm{C}(\mathrm{CC} 1 \mathrm{C} 1=\mathrm{CC}=\mathrm{CC}(\mathrm{Cl})=\mathrm{C} 1 \mathrm{Cl}) \mathrm{C} 1=\mathrm{CC}=\mathrm{C}(\mathrm{Br}) \mathrm{C}=\mathrm{C} 1$ 


\begin{tabular}{|c|c|c|}
\hline NAME & SMILE & $\lg (\mathbf{1} / \mathbf{I C 5 0})$ \\
\hline 1276 & $\mathrm{COC} 1=\mathrm{CC}=\mathrm{C}(\mathrm{C}=\mathrm{C} 1 \mathrm{OC}) \mathrm{C} 1 \mathrm{CC}(=\mathrm{NN} 1 \mathrm{C}(\mathrm{C})=\mathrm{O}) \mathrm{C} 1=\mathrm{CC}=\mathrm{CC}(=\mathrm{C} 1)[\mathrm{N}+]([\mathrm{O}-])=\mathrm{O}$ & 5,11 \\
\hline 1277 & $\mathrm{CC}(=\mathrm{O}) \mathrm{N} 1 \mathrm{~N}=\mathrm{C}(\mathrm{CC} 1 \mathrm{C} 1=\mathrm{CC}=\mathrm{C}(\mathrm{C}=\mathrm{C} 1)[\mathrm{N}+]([\mathrm{O}-])=\mathrm{O}) \mathrm{C} 1=\mathrm{CC}=\mathrm{CO} 1$ & 4,66 \\
\hline 1278 & $\mathrm{COC} 1=\mathrm{CC}=\mathrm{C}(\mathrm{OC}) \mathrm{C}(=\mathrm{C} 1) \mathrm{C} 1 \mathrm{CC}(=\mathrm{NN} 1 \mathrm{C}(\mathrm{C})=\mathrm{O}) \mathrm{C} 1=\mathrm{CC}=\mathrm{CO} 1$ & 4,87 \\
\hline 1279 & $\begin{array}{l}\mathrm{CC} 1=\mathrm{NNC}(=\mathrm{O}) \mathrm{C} 1 \mathrm{C}(\mathrm{C} 1=\mathrm{C}(\mathrm{NC} 2=\mathrm{CC}=\mathrm{C}(\mathrm{C}) \mathrm{C}=\mathrm{C} 12) \mathrm{C} 1=\mathrm{CC}=\mathrm{CC}=\mathrm{C} 1) \mathrm{C} 1=\mathrm{C}(\mathrm{NC} 2=\mathrm{CC}=\mathrm{C}(\mathrm{C}) \mathrm{C}=\mathrm{C} 12) \mathrm{C} 1=\mathrm{CC}=\mathrm{C} \\
\mathrm{C}=\mathrm{C} 1\end{array}$ & 4 \\
\hline 1280 & $\begin{array}{l}\mathrm{COC} 1=\mathrm{CC}=\mathrm{C} 2 \mathrm{NC}(=\mathrm{C}(\mathrm{C}(\mathrm{C} 3 \mathrm{C}(=\mathrm{O}) \mathrm{NN}=\mathrm{C} 3 \mathrm{C}) \mathrm{C} 3=\mathrm{C}(\mathrm{NC} 4=\mathrm{CC}=\mathrm{C}(\mathrm{C}) \mathrm{C}=\mathrm{C} 34) \mathrm{C} 3=\mathrm{CC}=\mathrm{CC}=\mathrm{C} 3) \mathrm{C} 2=\mathrm{C} 1) \mathrm{C} 1=\mathrm{CC}= \\
\mathrm{CC}=\mathrm{C} 1\end{array}$ & 5,46 \\
\hline 1281 & $\mathrm{NC} 1=\mathrm{CC}=\mathrm{CC}=\mathrm{C} 1 \mathrm{~N} 1 \mathrm{C}(=\mathrm{O}) \mathrm{C} 2 \mathrm{C}(\mathrm{C} 3 \mathrm{CCC} 2 \mathrm{C}=\mathrm{C} 3) \mathrm{C} 1=\mathrm{O}$ & 4,49 \\
\hline 1282 & $\mathrm{O}=\mathrm{CCN} 1 \mathrm{~N}=\mathrm{C}(\mathrm{CC} 1 \mathrm{C} 1=\mathrm{CC}=\mathrm{CC}=\mathrm{C} 1) \mathrm{C} 1=\mathrm{CC}=\mathrm{C} 2 \mathrm{C}=\mathrm{CC}=\mathrm{CC} 2=\mathrm{C} 1$ & 5,08 \\
\hline 1283 & {$[\mathrm{O}-][\mathrm{N}+](=\mathrm{O}) \mathrm{C} 1=\mathrm{CC}=\mathrm{CC}(=\mathrm{C} 1) \mathrm{C} 1 \mathrm{CC}(=\mathrm{NN} 1 \mathrm{CC}=\mathrm{O}) \mathrm{C} 1=\mathrm{CC}=\mathrm{C} 2 \mathrm{C}=\mathrm{CC}=\mathrm{CC} 2=\mathrm{C} 1$} & 5,41 \\
\hline 1284 & {$[\mathrm{O}-][\mathrm{N}+](=\mathrm{O}) \mathrm{C} 1=\mathrm{CC}=\mathrm{CC}=\mathrm{C} 1 \mathrm{C} 1 \mathrm{CC}(=\mathrm{NN} 1 \mathrm{CC}=\mathrm{O}) \mathrm{C} 1=\mathrm{CC}=\mathrm{C} 2 \mathrm{C}=\mathrm{CC}=\mathrm{CC} 2=\mathrm{C} 1$} & 5,44 \\
\hline 1285 & $\mathrm{O}=\mathrm{C} 1 \mathrm{~N}(\mathrm{C}(=\mathrm{O}) \mathrm{C} 2=\mathrm{CC}=\mathrm{CC}=\mathrm{C} 12) \mathrm{C} 1=\mathrm{CC}=\mathrm{CC}=\mathrm{N} 1$ & 4,18 \\
\hline 1286 & $\mathrm{COC} 1=\mathrm{CC}=\mathrm{C}(\mathrm{C}=\mathrm{C} 1) \mathrm{C} 1 \mathrm{CC}(=\mathrm{NN} 1 \mathrm{C}(\mathrm{C})=\mathrm{O}) \mathrm{C} 1=\mathrm{CC}=\mathrm{CC}=\mathrm{C} 1$ & 4,97 \\
\hline 1287 & $\mathrm{CC}(=\mathrm{O}) \mathrm{N} 1 \mathrm{~N}=\mathrm{C}(\mathrm{CC} 1 \mathrm{C} 1=\mathrm{CC}=\mathrm{CC}=\mathrm{C} 1) \mathrm{C} 1=\mathrm{CC}=\mathrm{C}(\mathrm{Br}) \mathrm{C}=\mathrm{C} 1$ & 4,56 \\
\hline 1288 & $\mathrm{CC}(=\mathrm{O}) \mathrm{N} 1 \mathrm{~N}=\mathrm{C}(\mathrm{CC} 1 \mathrm{C} 1=\mathrm{CC}=\mathrm{CC}(\mathrm{Cl})=\mathrm{C} 1 \mathrm{Cl}) \mathrm{C} 1=\mathrm{CC}=\mathrm{CC}(=\mathrm{C} 1)[\mathrm{N}+]([\mathrm{O}-])=\mathrm{O}$ & 4,56 \\
\hline 1289 & $\mathrm{CC}(=\mathrm{O}) \mathrm{N} 1 \mathrm{~N}=\mathrm{C}(\mathrm{CC} 1 \mathrm{C} 1=\mathrm{CC}=\mathrm{CC}=\mathrm{C} 1[\mathrm{~N}+]([\mathrm{O}-])=\mathrm{O}) \mathrm{C} 1=\mathrm{C} 2 \mathrm{C}=\mathrm{CC}=\mathrm{CC} 2=\mathrm{CC}=\mathrm{C} 1$ & 5,4 \\
\hline 1290 & $\mathrm{COC} 1=\mathrm{CC}=\mathrm{C}(\mathrm{C}=\mathrm{C} 1 \mathrm{OC}) \mathrm{C} 1 \mathrm{CC}(=\mathrm{NN} 1 \mathrm{C}(\mathrm{C})=\mathrm{O}) \mathrm{C} 1=\mathrm{C} 2 \mathrm{C}=\mathrm{CC}=\mathrm{CC} 2=\mathrm{CC}=\mathrm{C} 1$ & 5,99 \\
\hline 1291 & $\mathrm{CC}(=\mathrm{O}) \mathrm{N} 1 \mathrm{~N}=\mathrm{C}(\mathrm{CC} 1 \mathrm{C} 1=\mathrm{CC}=\mathrm{C} 2 \mathrm{OCOC} 2=\mathrm{C} 1) \mathrm{C} 1=\mathrm{C} 2 \mathrm{C}=\mathrm{CC}=\mathrm{CC} 2=\mathrm{CC}=\mathrm{C} 1$ & 5,97 \\
\hline 1292 & $\mathrm{CC}(=\mathrm{O}) \mathrm{N} 1 \mathrm{~N}=\mathrm{C}(\mathrm{CC} 1 \mathrm{C} 1=\mathrm{CC}=\mathrm{C} 2 \mathrm{C}=\mathrm{CC}=\mathrm{CC} 2=\mathrm{C} 1) \mathrm{C} 1=\mathrm{C} 2 \mathrm{C}=\mathrm{CC}=\mathrm{CC} 2=\mathrm{CC}=\mathrm{C} 1$ & 6,72 \\
\hline 1293 & $\mathrm{CC}(=\mathrm{O}) \mathrm{N} 1 \mathrm{~N}=\mathrm{C}(\mathrm{CC} 1 \mathrm{C} 1=\mathrm{CC}=\mathrm{CC}=\mathrm{C} 1) \mathrm{C} 1=\mathrm{CC}=\mathrm{CO} 1$ & 4,49 \\
\hline 1294 & $\mathrm{CC}(=\mathrm{O}) \mathrm{N} 1 \mathrm{~N}=\mathrm{C}(\mathrm{CC} 1 \mathrm{C} 1=\mathrm{CC}=\mathrm{C}(\mathrm{Br}) \mathrm{C}=\mathrm{C} 1) \mathrm{C} 1=\mathrm{CC}=\mathrm{CO} 1$ & 4,29 \\
\hline 1295 & $\mathrm{COC} 1=\mathrm{CC}=\mathrm{C} 2 \mathrm{NC}(=\mathrm{C}(\mathrm{C}(\mathrm{C} 3 \mathrm{C}(=\mathrm{O}) \mathrm{NN}=\mathrm{C} 3 \mathrm{C}) \mathrm{C} 3=\mathrm{CNC} 4=\mathrm{CC}=\mathrm{CC}=\mathrm{C} 34) \mathrm{C} 2=\mathrm{C} 1) \mathrm{C} 1=\mathrm{CC}=\mathrm{CC}=\mathrm{C} 1$ & 5,28 \\
\hline 1296 & $\mathrm{COC} 1=\mathrm{CC}=\mathrm{C}(\mathrm{C}=\mathrm{C} 1 \mathrm{OC}) \mathrm{C} 1 \mathrm{CC}(=\mathrm{NN} 1 \mathrm{C}(\mathrm{C})=\mathrm{O}) \mathrm{C} 1=\mathrm{CC}=\mathrm{CC}=\mathrm{C} 1$ & 5,11 \\
\hline 1297 & $\mathrm{NC} 1=\mathrm{CC}=\mathrm{C}(\mathrm{C}=\mathrm{C} 1) \mathrm{N} 1 \mathrm{C}(=\mathrm{O})[\mathrm{C} @ \mathrm{H}] 2[\mathrm{C} @ \mathrm{H}](\mathrm{C} 3 \mathrm{CCC} 2 \mathrm{C}=\mathrm{C} 3) \mathrm{C} 1=\mathrm{O}$ & 3,7 \\
\hline 1298 & $\mathrm{O}=\mathrm{C} 1 \mathrm{C} 2 \mathrm{C}(\mathrm{C} 3 \mathrm{CCC} 2 \mathrm{C}=\mathrm{C} 3) \mathrm{C}(=\mathrm{O}) \mathrm{N} 1 \mathrm{C} 1=\mathrm{CC}=\mathrm{C}(\mathrm{C}=\mathrm{C} 1) \mathrm{C} 1=\mathrm{CC}=\mathrm{CC}=\mathrm{C} 1$ & 4,33 \\
\hline 1299 & $\mathrm{CC}(=\mathrm{O}) \mathrm{N} 1 \mathrm{~N}=\mathrm{C}(\mathrm{CC} 1 \mathrm{C} 1=\mathrm{CC}=\mathrm{CO} 1) \mathrm{C} 1=\mathrm{C} 2 \mathrm{C}=\mathrm{CC}=\mathrm{CC} 2=\mathrm{CC}=\mathrm{C} 1$ & 4,77 \\
\hline 1300 & $\mathrm{COC} 1=\mathrm{CC}=\mathrm{C}(\mathrm{C} 2 \mathrm{CC}(=\mathrm{NN} 2 \mathrm{C}(\mathrm{C})=\mathrm{O}) \mathrm{C} 2=\mathrm{C} 3 \mathrm{C}=\mathrm{CC}=\mathrm{CC} 3=\mathrm{CC}=\mathrm{C} 2) \mathrm{C}(\mathrm{OC})=\mathrm{C} 1 \mathrm{OC}$ & 5,89 \\
\hline 1301 & $\mathrm{O}=\mathrm{C} 1 \mathrm{~N}(\mathrm{C} 2=\mathrm{CC}=\mathrm{CC}=\mathrm{C} 2) \mathrm{C}(=\mathrm{O}) \mathrm{C} 2=\mathrm{C} 3 \mathrm{C}(\mathrm{C}=\mathrm{CC}=\mathrm{C} 13)=\mathrm{CC}=\mathrm{C} 2$ & 4,35 \\
\hline 1302 & $\mathrm{O}=\mathrm{C}(\backslash \mathrm{C}=\mathrm{ClC}=\mathrm{ClC} 1=\mathrm{CC}=\mathrm{C} 2 \mathrm{OCOC} 2=\mathrm{C} 1) \mathrm{N} 1 \mathrm{CCCCC} 1$ & 4,15 \\
\hline 1303 & $\mathrm{COC} 1=\mathrm{CC}(=\mathrm{CC}(\mathrm{OC})=\mathrm{C} 1 \mathrm{OC}) \mathrm{C} 1 \mathrm{CC}(=\mathrm{NN} 1 \mathrm{C}(\mathrm{C})=\mathrm{O}) \mathrm{C} 1=\mathrm{C} 2 \mathrm{C}=\mathrm{CC}=\mathrm{CC} 2=\mathrm{CC}=\mathrm{C} 1$ & 6 \\
\hline 1304 & $\mathrm{CN}(\mathrm{C}) \mathrm{C} 1=\mathrm{CC}=\mathrm{C}(\mathrm{C}=\mathrm{C} 1) \mathrm{C} 1 \mathrm{CC}(=\mathrm{NN} 1 \mathrm{C}(\mathrm{C})=\mathrm{O}) \mathrm{C} 1=\mathrm{CC}=\mathrm{CO} 1$ & 4,8 \\
\hline 1305 & $\mathrm{COC} 1=\mathrm{CC}=\mathrm{C}(\mathrm{C}=\mathrm{C} 1) \mathrm{N} 1 \mathrm{C}(=\mathrm{O}) \mathrm{C} 2=\mathrm{CC}(\mathrm{Cl})=\mathrm{CC}=\mathrm{C} 2 \mathrm{~N}=\mathrm{C} 1 \mathrm{C} 1=\mathrm{CC}=\mathrm{CC}=\mathrm{C} 1$ & 4,46 \\
\hline 1306 & $\mathrm{ClC} 1=\mathrm{CC}=\mathrm{C}(\mathrm{CN} 2 \mathrm{C}(=\mathrm{O}) \mathrm{C} 3=\mathrm{CC}(\mathrm{Cl})=\mathrm{CC}=\mathrm{C} 3 \mathrm{~N}=\mathrm{C} 2 \mathrm{C} 2=\mathrm{CC}=\mathrm{CC}=\mathrm{C} 2) \mathrm{C}=\mathrm{C} 1$ & 3,88 \\
\hline 1307 & $\mathrm{FC} 1=\mathrm{CC}=\mathrm{C}(\mathrm{C}=\mathrm{C} 1 \mathrm{Cl}) \mathrm{N} 1 \mathrm{C}(=\mathrm{O}) \mathrm{C} 2=\mathrm{CC}(\mathrm{Cl})=\mathrm{CC}=\mathrm{C} 2 \mathrm{~N}=\mathrm{C} 1 \mathrm{C} 1=\mathrm{CC}=\mathrm{CC}=\mathrm{C} 1$ & 4,79 \\
\hline 1308 & $\mathrm{ClC} 1=\mathrm{CC}=\mathrm{CC}(=\mathrm{C} 1) \mathrm{N} 1 \mathrm{C}(=\mathrm{O}) \mathrm{C} 2=\mathrm{CC}(\mathrm{Cl})=\mathrm{CC}=\mathrm{C} 2 \mathrm{~N}=\mathrm{C} 1 \mathrm{C} 1=\mathrm{CC}=\mathrm{CC}=\mathrm{C} 1$ & 4,77 \\
\hline 1309 & $\mathrm{ClC} 1=\mathrm{CC}=\mathrm{C}(\mathrm{C}=\mathrm{C} 1) \mathrm{N} 1 \mathrm{C}(=\mathrm{O}) \mathrm{C} 2=\mathrm{CC}(\mathrm{Cl})=\mathrm{CC}=\mathrm{C} 2 \mathrm{~N}=\mathrm{C} 1 \mathrm{C} 1=\mathrm{CC}=\mathrm{CC}=\mathrm{C} 1$ & 4,17 \\
\hline 1310 & $\mathrm{CN}(\mathrm{C}) \mathrm{CCN} 1 \mathrm{C}(=\mathrm{O}) \mathrm{C} 2=\mathrm{CC}(\mathrm{Cl})=\mathrm{CC}=\mathrm{C} 2 \mathrm{~N}=\mathrm{C} 1 \mathrm{C} 1=\mathrm{CC}=\mathrm{CC}=\mathrm{C} 1$ & 4,2 \\
\hline 1311 & $\mathrm{FC} 1=\mathrm{CC}=\mathrm{C}(\mathrm{CN} 2 \mathrm{C}(=\mathrm{O}) \mathrm{C} 3=\mathrm{CC}(\mathrm{Cl})=\mathrm{CC}=\mathrm{C} 3 \mathrm{~N}=\mathrm{C} 2 \mathrm{C} 2=\mathrm{CC}=\mathrm{CC}=\mathrm{C} 2) \mathrm{C}=\mathrm{C} 1$ & 4,53 \\
\hline 1312 & $\mathrm{FC} 1=\mathrm{CC}=\mathrm{C}(\mathrm{C}=\mathrm{C} 1) \mathrm{N} 1 \mathrm{C}(=\mathrm{O}) \mathrm{C} 2=\mathrm{CC}(\mathrm{Cl})=\mathrm{CC}=\mathrm{C} 2 \mathrm{~N}=\mathrm{C} 1 \mathrm{C} 1=\mathrm{CC}=\mathrm{CC}=\mathrm{C} 1$ & 4,51 \\
\hline
\end{tabular}

Table S2. QSAR model data set.

\begin{tabular}{r|r|r|r|l|l|l|l}
\multicolumn{1}{l|}{ Training set } & \multicolumn{1}{l|}{ ASNN } & & Test set & & & \\
\hline NAME & Ig(1/IC50) & Predicted & delta & NAME & Ig(1/IC50) & Predicted & x delta \\
\hline 1 & 4,08 & 4,1919 & 0,1119 & 2 & 4,21 & 4,058 & 0,152 \\
\hline 3 & 4,45 & 4,0524 & 0,3976 & 7 & 4,48 & 4,0954 & 0,3846 \\
\hline 4 & 4,02 & 4,2518 & 0,2318 & 12 & 4 & 4,1443 & 0,1443 \\
\hline 5 & 4,49 & 4,0839 & 0,4061 & 17 & 4 & 4,0981 & 0,0981 \\
\hline 6 & 4,23 & 4,1548 & 0,0752 & 22 & 4,26 & 4,1827 & 0,0773 \\
\hline 8 & 4 & 3,9325 & 0,0675 & 27 & 4,22 & 4,1145 & 0,1055 \\
\hline 9 & 4,01 & 4,1433 & 0,1333 & 32 & 4 & 4,0606 & 0,0606 \\
\hline 10 & 4,08 & 4,0397 & 0,0403 & 37 & 4,33 & 4,1298 & 0,2002 \\
\hline 11 & 4 & 3,9238 & 0,0762 & 43 & 5,4 & 5,0837 & 0,3163 \\
\hline 13 & 4,09 & 4,2823 & 0,1923 & 48 & 4,68 & 4,612 & 0,068 \\
\hline 14 & 4,22 & 4,1175 & 0,1025 & 54 & 5,63 & 5,0693 & 0,5607 \\
\hline 15 & 4,39 & 4,0589 & 0,3311 & 61 & 4,68 & 4,7117 & 0,0317 \\
\hline 16 & 4 & 4,3354 & 0,3354 & 66 & 4 & 4,2925 & 0,2925 \\
\hline 18 & 4,19 & 4,1208 & 0,0692 & 72 & 5 & 4,605 & 0,395 \\
\hline 19 & 4,03 & 4,0614 & 0,0314 & 77 & 3,3 & 3,2029 & 0,0971 \\
\hline 20 & 4 & 4,1206 & 0,1206 & 82 & 3,3 & 3,8825 & 0,5825 \\
\hline 21 & 4 & 4,0606 & 0,0606 & 90 & 6,7 & 6,0787 & 0,6213 \\
\hline 23 & 4,16 & 4,2092 & 0,0492 & 99 & 5,05 & 5,1989 & 0,1489 \\
\hline 24 & 4,24 & 4,246 & 0,006 & 105 & 5,1 & 4,5823 & 0,5177 \\
\hline 25 & 4,25 & 4,2104 & 0,0396 & 110 & 5,7 & 5,3092 & 0,3908 \\
\hline 26 & 4,16 & 4,0096 & 0,1504 & 115 & 4,06 & 4,4084 & 0,3484 \\
\hline 28 & 4 & 4,235 & 0,235 & 120 & 3,96 & 4,3481 & 0,3881 \\
\hline 29 & 4 & 4,0332 & 0,0332 & 125 & 4,6 & 4,919 & 0,319 \\
\hline 30 & 4 & 4,2195 & 0,2195 & 130 & 4,8 & 5,3634 & 0,5634 \\
\hline 31 & 4,16 & 4,2444 & 0,0844 & 136 & 5 & 4,2974 & 0,7026 \\
\hline 33 & 4 & 4,1474 & 0,1474 & 146 & 4,12 & 4,2837 & 0,1637 \\
\hline 34 & 4 & 4,1396 & 0,1396 & 152 & 4,85 & 4,8802 & 0,0302 \\
\hline 35 & 4 & 4,0194 & 0,0194 & 159 & 5,85 & 6,2429 & 0,3929 \\
\hline & & & & & & \\
\hline & 4 & & & & & \\
\hline
\end{tabular}




\begin{tabular}{|c|c|c|c|c|c|c|c|}
\hline Training set & & ASNN & & Test set & & & \\
\hline NAME & $\lg (1 / \mathrm{IC} 50)$ & Predicted & delta & NAME & $\lg (1 / \mathrm{IC50})$ & Predicted & $\mathrm{x}$ delta \\
\hline 36 & 4 & 4,1587 & 0,1587 & 168 & 4,92 & 4,5909 & 0,3291 \\
\hline 38 & 4 & 4,5374 & 0,5374 & 173 & 4,4 & 4,2391 & 0,1609 \\
\hline 39 & 4 & 4,501 & 0,501 & 179 & 2,8 & 2,8764 & 0,0764 \\
\hline 41 & 4,82 & 5,2375 & 0,4175 & 185 & 4,5 & 5,4048 & 0,9048 \\
\hline 42 & 5,19 & 4,8508 & 0,3392 & 190 & 4,08 & 3,7541 & 0,3259 \\
\hline 44 & 4,23 & 4,5333 & 0,3033 & 195 & 4,36 & 3,7541 & 0,6059 \\
\hline 45 & 4,62 & 5,3064 & 0,6864 & 200 & 3,37 & 4,1575 & 0,7875 \\
\hline 46 & 4,32 & 4,2647 & 0,0553 & 205 & 4,02 & 3,6769 & 0,3431 \\
\hline 47 & 5,07 & 4,4844 & 0,5856 & 210 & 3,91 & 4,0382 & 0,1282 \\
\hline 49 & 5,59 & 5,3225 & 0,2675 & 215 & 4,7 & 4,4256 & 0,2744 \\
\hline 50 & 4 & 4,7343 & 0,7343 & 220 & 5 & 4,4558 & 0,5442 \\
\hline 51 & 4,46 & 4,8926 & 0,4326 & 225 & 6,05 & 5,5897 & 0,4603 \\
\hline 53 & 5,01 & 4,997 & 0,013 & 230 & 3,7 & 3,8204 & 0,1204 \\
\hline 55 & 5,07 & 4,6395 & 0,4305 & 235 & 5,85 & 5,3639 & 0,4861 \\
\hline 58 & 4,78 & 4,7788 & 0,0012 & 241 & 5,74 & 5,6819 & 0,0581 \\
\hline 59 & 5,27 & 5,359 & 0,089 & 247 & 6,7 & 6,0543 & 0,6457 \\
\hline 60 & 5,16 & 5,6232 & 0,4632 & 252 & 7,4 & 6,529 & 0,871 \\
\hline 62 & 5,01 & 5,2601 & 0,2501 & 258 & 6,15 & 6,7434 & 0,5934 \\
\hline 63 & 5,09 & 5,0527 & 0,0373 & 263 & 5,75 & 5,6846 & 0,0654 \\
\hline 64 & 5,5 & 4,963 & 0,537 & 268 & 6,19 & 5,6706 & 0,5194 \\
\hline 65 & 5,53 & 5,0733 & 0,4567 & 273 & 3,7 & 3,7928 & 0,0928 \\
\hline 67 & 4,34 & 5,4685 & 1,1285 & 278 & 3,3 & 3,567 & 0,267 \\
\hline 69 & 5,31 & 4,6067 & 0,7033 & 283 & 3,3 & 3,4796 & 0,1796 \\
\hline 70 & 3,3 & 3,4103 & 0,1103 & 289 & 5,77 & 5,8918 & 0,1218 \\
\hline 71 & 3,3 & 3,6447 & 0,3447 & 294 & 5,3 & 5,4031 & 0,1031 \\
\hline 73 & 3,7 & 3,9601 & 0,2601 & 299 & 4,02 & 4,2427 & 0,2227 \\
\hline 74 & 3,3 & 3,0439 & 0,2561 & 304 & 4,01 & 4,115 & 0,105 \\
\hline 75 & 4,2 & 4,5115 & 0,3115 & 309 & 4,22 & 4,1594 & 0,0606 \\
\hline 76 & 3,3 & 3,5453 & 0,2453 & 314 & 4,03 & 4,0509 & 0,0209 \\
\hline 78 & 3,3 & 3,702 & 0,402 & 319 & 4,24 & 4,2634 & 0,0234 \\
\hline 79 & 5 & 4,3116 & 0,6884 & 324 & 4 & 4,0176 & 0,0176 \\
\hline 80 & 3,3 & 3,1363 & 0,1637 & 329 & 4 & 4,1252 & 0,1252 \\
\hline 81 & 3,3 & 3,0558 & 0,2442 & 334 & 4,7 & 3,9914 & 0,7086 \\
\hline 83 & 4,25 & 4,7782 & 0,5282 & 340 & 5,35 & 5,1923 & 0,1577 \\
\hline 84 & 5,2 & 4,7882 & 0,4118 & 346 & 5,06 & 5,2194 & 0,1594 \\
\hline 85 & 3,3 & 3,6132 & 0,3132 & 351 & 4,88 & 4,8657 & 0,0143 \\
\hline 88 & 5,68 & 5,2412 & 0,4388 & 358 & 4,86 & 5,3134 & 0,4534 \\
\hline 91 & 6,7 & 5,8662 & 0,8338 & 363 & 4,93 & 5,1719 & 0,2419 \\
\hline 92 & 5,52 & 5,369 & 0,151 & 368 & 4,63 & 4,7359 & 0,1059 \\
\hline 97 & 6,47 & 5,0087 & 1,4613 & 373 & 4,64 & 4,6779 & 0,0379 \\
\hline 98 & 5,4 & 5,292 & 0,108 & 378 & 4,64 & 4,5963 & 0,0437 \\
\hline 101 & 4,3 & 4,9549 & 0,6549 & 383 & 4,65 & 4,6364 & 0,0136 \\
\hline 102 & 4,3 & 4,4071 & 0,1071 & 388 & 4,81 & 4,6352 & 0,1748 \\
\hline 103 & 5,1 & 4,9621 & 0,1379 & 395 & 5,62 & 5,428 & 0,192 \\
\hline 104 & 4,22 & 4,6695 & 0,4495 & 400 & 4,52 & 5,9764 & 1,4564 \\
\hline 106 & 4,6 & 4,8031 & 0,2031 & 406 & 5,74 & 6,2311 & 0,4911 \\
\hline 107 & 4,3 & 4,7564 & 0,4564 & 412 & 8,4 & 7,5945 & 0,8055 \\
\hline 108 & 5,52 & 4,9645 & 0,5555 & 417 & 7,4 & 6,9296 & 0,4704 \\
\hline 109 & 4,3 & 4,2264 & 0,0736 & 422 & 4 & 4,4249 & 0,4249 \\
\hline 111 & 5 & 4,7856 & 0,2144 & 429 & 4 & 4,3215 & 0,3215 \\
\hline 112 & 4,48 & 4,1868 & 0,2932 & 436 & 4 & 4,4744 & 0,4744 \\
\hline 113 & 4,85 & 4,7506 & 0,0994 & 442 & 4,96 & 4,6754 & 0,2846 \\
\hline 114 & 4,05 & 4,5142 & 0,4642 & 447 & 5,15 & 4,4698 & 0,6802 \\
\hline 116 & 4,34 & 4,1743 & 0,1657 & 455 & 4,38 & 4,3296 & 0,0504 \\
\hline 117 & 3,92 & 4,5889 & 0,6689 & 460 & 3,63 & 3,6584 & 0,0284 \\
\hline 118 & 4,54 & 3,7337 & 0,8063 & 466 & 3,67 & 4,253 & 0,583 \\
\hline 119 & 4 & 4,8088 & 0,8088 & 474 & 4,86 & 4,4669 & 0,3931 \\
\hline 121 & 4 & 4,4009 & 0,4009 & 481 & 4,86 & 3,7924 & 1,0676 \\
\hline 122 & 4,92 & 4,8375 & 0,0825 & 489 & 4,92 & 3,9043 & 1,0157 \\
\hline 123 & 4,1 & 4,6448 & 0,5448 & 495 & 4,63 & 3,9533 & 0,6767 \\
\hline 124 & 3,92 & 4,0864 & 0,1664 & 500 & 5,76 & 5,0057 & 0,7543 \\
\hline 126 & 4,6 & 4,6758 & 0,0758 & 505 & 5,85 & 5,9854 & 0,1354 \\
\hline 127 & 4,6 & 4,8835 & 0,2835 & 510 & 4,53 & 4,133 & 0,397 \\
\hline 128 & 4,6 & 4,496 & 0,104 & 515 & 4,3 & 4,2759 & 0,0241 \\
\hline 129 & 5,74 & 5,6401 & 0,0999 & 521 & 4,5 & 4,1538 & 0,3462 \\
\hline 131 & 4,96 & 4,8722 & 0,0878 & 526 & 5,15 & 5,022 & 0,128 \\
\hline
\end{tabular}




\begin{tabular}{|c|c|c|c|c|c|c|c|}
\hline Training set & & ASNN & & Test set & & & \\
\hline NAME & $\lg (1 / \mathbf{I C 5 0})$ & Predicted & delta & NAME & $\lg (1 / \mathrm{IC} 50)$ & Predicted & $\mathrm{x}$ delta \\
\hline 133 & 5,7 & 4,5911 & 1,1089 & 531 & 4 & 3,9837 & 0,0163 \\
\hline 134 & 4,55 & 4,4912 & 0,0588 & 536 & 3,7 & 4,2166 & 0,5166 \\
\hline 135 & 4,26 & 4,9713 & 0,7113 & 542 & 3,98 & 4,7515 & 0,7715 \\
\hline 138 & 5,1 & 4,959 & 0,141 & 547 & 5,8 & 5,1646 & 0,6354 \\
\hline 139 & 4,12 & 5,2996 & 1,1796 & 552 & 4,82 & 4,8645 & 0,0445 \\
\hline 141 & 4,12 & 4,4567 & 0,3367 & 557 & 5,62 & 5,7189 & 0,0989 \\
\hline 144 & 5,7 & 5,3029 & 0,3971 & 562 & 4,8 & 4,9581 & 0,1581 \\
\hline 148 & 4,12 & 4,6966 & 0,5766 & 567 & 7,4 & 6,7819 & 0,6181 \\
\hline 149 & 6,15 & 4,9417 & 1,2083 & 572 & 5,82 & 5,5553 & 0,2647 \\
\hline 150 & 4,7 & 5,3341 & 0,6341 & 577 & 5,85 & 5,5811 & 0,2689 \\
\hline 151 & 4,6 & 4,6461 & 0,0461 & 582 & 6,19 & 5,842 & 0,348 \\
\hline 154 & 5,7 & 4,9211 & 0,7789 & 587 & 5,63 & 6,071 & 0,441 \\
\hline 155 & 4,66 & 4,4833 & 0,1767 & 592 & 6,52 & 5,7852 & 0,7348 \\
\hline 157 & 6,15 & 5,7082 & 0,4418 & 597 & 5,85 & 5,9066 & 0,0566 \\
\hline 158 & 6,22 & 5,6017 & 0,6183 & 602 & 4,28 & 5,0745 & 0,7945 \\
\hline 162 & 6,15 & 5,5783 & 0,5717 & 608 & 4 & 4,0562 & 0,0562 \\
\hline 165 & 4,15 & 3,7598 & 0,3902 & 613 & 4 & 4,2126 & 0,2126 \\
\hline 166 & 5,7 & 6,0439 & 0,3439 & 620 & 4,45 & 4,369 & 0,081 \\
\hline 167 & 4,77 & 4,3702 & 0,3998 & 625 & 4,31 & 4,1276 & 0,1824 \\
\hline 169 & 4,54 & 4,6883 & 0,1483 & 630 & 4,29 & 4,4708 & 0,1808 \\
\hline 170 & 4,6 & 4,7069 & 0,1069 & 635 & 5,79 & 5,908 & 0,118 \\
\hline 171 & 4,26 & 4,9668 & 0,7068 & 640 & 5 & 5,1621 & 0,1621 \\
\hline 172 & 4,52 & 4,6736 & 0,1536 & 646 & 4,03 & 4,9695 & 0,9395 \\
\hline 175 & 4,02 & 4,3615 & 0,3415 & 651 & 4,01 & 4,9775 & 0,9675 \\
\hline 176 & 2,8 & 2,692 & 0,108 & 657 & 6,15 & 5,4412 & 0,7088 \\
\hline 177 & 2,6 & 2,8753 & 0,2753 & 663 & 3,88 & 3,5714 & 0,3086 \\
\hline 178 & 2,57 & 2,8895 & 0,3195 & 668 & 4,12 & 4,0742 & 0,0458 \\
\hline 180 & 2,55 & 2,9848 & 0,4348 & 673 & 5,4 & 5,73 & 0,33 \\
\hline 181 & 2,48 & 2,783 & 0,303 & 678 & 5,52 & 5,3385 & 0,1815 \\
\hline 183 & 3,92 & 3,7823 & 0,1377 & 683 & 4,6 & 5,2445 & 0,6445 \\
\hline 184 & 3,7 & 3,799 & 0,099 & 688 & 4,4 & 5,3039 & 0,9039 \\
\hline 186 & 3,99 & 4,0337 & 0,0437 & 693 & 6,22 & 5,7232 & 0,4968 \\
\hline 187 & 3,84 & 4,0481 & 0,2081 & 698 & 4,92 & 5,2847 & 0,3647 \\
\hline 188 & 3,53 & 4,1498 & 0,6198 & 703 & 4,6 & 5,2155 & 0,6155 \\
\hline 189 & 3,65 & 3,7045 & 0,0545 & 708 & 5,47 & 5,1226 & 0,3474 \\
\hline 191 & 4,95 & 4,9162 & 0,0338 & 713 & 5,05 & 5,1419 & 0,0919 \\
\hline 192 & 4,15 & 4,0351 & 0,1149 & 718 & 6,15 & 5,2845 & 0,8655 \\
\hline 193 & 3,92 & 3,6538 & 0,2662 & 723 & 6,3 & 5,5567 & 0,7433 \\
\hline 194 & 3,91 & 3,6495 & 0,2605 & 728 & 4,22 & 5,0657 & 0,8457 \\
\hline 196 & 4,47 & 4,9307 & 0,4607 & 733 & 4,23 & 4,403 & 0,173 \\
\hline 197 & 3,76 & 4,0894 & 0,3294 & 738 & 4,4 & 4,125 & 0,275 \\
\hline 198 & 3,62 & 3,696 & 0,076 & 743 & 4,22 & 4,3549 & 0,1349 \\
\hline 199 & 4,32 & 3,7099 & 0,6101 & 748 & 4 & 3,8729 & 0,1271 \\
\hline 201 & 3,85 & 3,7628 & 0,0872 & 753 & 5,82 & 6,5853 & 0,7653 \\
\hline 202 & 4,72 & 4,8503 & 0,1303 & 761 & 4 & 4,2254 & 0,2254 \\
\hline 203 & 3,94 & 4,0714 & 0,1314 & 768 & 5,78 & 5,7619 & 0,0181 \\
\hline 204 & 4,35 & 3,9346 & 0,4154 & 773 & 6,28 & 5,7082 & 0,5718 \\
\hline 206 & 3,61 & 4,2988 & 0,6888 & 780 & 6,16 & 6,0824 & 0,0776 \\
\hline 207 & 4,05 & 3,6251 & 0,4249 & 786 & 5,77 & 5,0057 & 0,7643 \\
\hline 208 & 3,41 & 4,7991 & 1,3891 & 791 & 6,52 & 5,7869 & 0,7331 \\
\hline 209 & 4,15 & 4,7513 & 0,6013 & 796 & 5,64 & 6,071 & 0,431 \\
\hline 211 & 4,15 & 4,4751 & 0,3251 & 801 & 5,7 & 5,8692 & 0,1692 \\
\hline 212 & 4,05 & 4,5071 & 0,4571 & 806 & 6,15 & 5,842 & 0,308 \\
\hline 213 & 4 & 4,5247 & 0,5247 & 811 & 4,06 & 4,0579 & 0,0021 \\
\hline 214 & 4,4 & 4,491 & 0,091 & 816 & 4 & 4,1913 & 0,1913 \\
\hline 216 & 4,22 & 4,5218 & 0,3018 & 821 & 4 & 3,8495 & 0,1505 \\
\hline 217 & 4,22 & 4,2554 & 0,0354 & 826 & 4 & 4,0991 & 0,0991 \\
\hline 218 & 4,7 & 4,8559 & 0,1559 & 832 & 5,15 & 5,8615 & 0,7115 \\
\hline 219 & 4,4 & 4,2969 & 0,1031 & 842 & 6,05 & 5,6218 & 0,4282 \\
\hline 221 & 5,96 & 6,2497 & 0,2897 & 847 & 4 & 3,5505 & 0,4495 \\
\hline 222 & 5,89 & 6,2726 & 0,3826 & 853 & 4 & 4,2086 & 0,2086 \\
\hline 223 & 6,1 & 6,5232 & 0,4232 & 858 & 4,68 & 5,1638 & 0,4838 \\
\hline 224 & 5,82 & 6,1787 & 0,3587 & 867 & 4,72 & 5,2128 & 0,4928 \\
\hline 226 & 6,15 & 6,5304 & 0,3804 & 872 & 5 & 5,1742 & 0,1742 \\
\hline 227 & 6,22 & 6,4857 & 0,2657 & 877 & 4,31 & 4,1769 & 0,1331 \\
\hline 228 & 6,1 & 5,401 & 0,699 & 883 & 4,57 & 4,4947 & 0,0753 \\
\hline
\end{tabular}


https://doi.org/10.33263/BRIAC123.29052957

\begin{tabular}{|c|c|c|c|c|c|c|c|}
\hline Training set & & ASNN & & Test set & & & \\
\hline NAME & $\lg (1 / \mathbf{I C 5 0})$ & Predicted & delta & NAME & $\lg (1 / \mathrm{IC} 50)$ & Predicted & $\mathrm{x}$ delta \\
\hline 229 & 4,5 & 4,3733 & 0,1267 & 889 & 5,4 & 4,6831 & 0,7169 \\
\hline 231 & 4,32 & 3,9888 & 0,3312 & 894 & 4,67 & 4,3611 & 0,3089 \\
\hline 232 & 3,72 & 4,3034 & 0,5834 & 900 & 4 & 3,7698 & 0,2302 \\
\hline 233 & 3,84 & 4,2566 & 0,4166 & 905 & 4 & 4,1352 & 0,1352 \\
\hline 234 & 3,72 & 4,1188 & 0,3988 & 916 & 3,4 & 4,4414 & 1,0414 \\
\hline 236 & 8,4 & 7,3712 & 1,0288 & 927 & 4 & 3,9504 & 0,0496 \\
\hline 237 & 5,82 & 5,2787 & 0,5413 & 933 & 4 & 4,0594 & 0,0594 \\
\hline 239 & 4,85 & 5,2105 & 0,3605 & 938 & 4,85 & 4,3099 & 0,5401 \\
\hline 240 & 7,4 & 6,469 & 0,931 & 943 & 5,27 & 4,6658 & 0,6042 \\
\hline 242 & 7 & 6,8761 & 0,1239 & 948 & 4 & 4,4107 & 0,4107 \\
\hline 244 & 6,4 & 6,5901 & 0,1901 & 953 & 5,33 & 4,6075 & 0,7225 \\
\hline 245 & 5,92 & 6,0852 & 0,1652 & 958 & 4 & 4,1596 & 0,1596 \\
\hline 246 & 6,7 & 6,6123 & 0,0877 & 963 & 4 & 4,3673 & 0,3673 \\
\hline 248 & 6,52 & 6,5624 & 0,0424 & 968 & 4 & 4,0631 & 0,0631 \\
\hline 249 & 7,4 & 6,6261 & 0,7739 & 973 & 4 & 4,0763 & 0,0763 \\
\hline 250 & 5,85 & 6,2774 & 0,4274 & 978 & 4 & 4,0421 & 0,0421 \\
\hline 251 & 7,3 & 6,3823 & 0,9177 & 983 & 4 & 4,1102 & 0,1102 \\
\hline 253 & 5,96 & 6,095 & 0,135 & 989 & 4 & 4,017 & 0,017 \\
\hline 254 & 6,7 & 6,6357 & 0,0643 & 995 & 4,89 & 4,6842 & 0,2058 \\
\hline 255 & 5,05 & 6,0916 & 1,0416 & 1000 & 4,3 & 4,6988 & 0,3988 \\
\hline 256 & 8,4 & 7,3709 & 1,0291 & 1005 & 4,36 & 4,6853 & 0,3253 \\
\hline 259 & 6,22 & 6,6077 & 0,3877 & 1010 & 4,3 & 4,6876 & 0,3876 \\
\hline 260 & 5,53 & 5,6151 & 0,0851 & 1015 & 4,6 & 4,9172 & 0,3172 \\
\hline 261 & 5,45 & 5,2003 & 0,2497 & 1020 & 4,6 & 4,3909 & 0,2091 \\
\hline 262 & 5,44 & 5,4275 & 0,0125 & 1025 & 4,6 & 4,6271 & 0,0271 \\
\hline 264 & 5,44 & 5,4368 & 0,0032 & 1031 & 4,6 & 4,3312 & 0,2688 \\
\hline 265 & 5,18 & 5,3719 & 0,1919 & 1036 & 4,6 & 4,8436 & 0,2436 \\
\hline 266 & 5,5 & 5,3948 & 0,1052 & 1041 & 4,6 & 4,6031 & 0,0031 \\
\hline 267 & 5,5 & 5,1834 & 0,3166 & 1046 & 4,3 & 4,2756 & 0,0244 \\
\hline 269 & 5,31 & 4,8118 & 0,4982 & 1051 & 4,6 & 4,5182 & 0,0818 \\
\hline 270 & 3,3 & 3,4602 & 0,1602 & 1056 & 4,6 & 4,5609 & 0,0391 \\
\hline 271 & 3,3 & 3,6489 & 0,3489 & 1061 & 4,6 & 4,5888 & 0,0112 \\
\hline 272 & 5 & 4,3421 & 0,6579 & 1066 & 4,6 & 4,856 & 0,256 \\
\hline 274 & 3,3 & 3,0974 & 0,2026 & 1071 & 4 & 3,6921 & 0,3079 \\
\hline 275 & 4,2 & 4,5178 & 0,3178 & 1078 & 4 & 3,7875 & 0,2125 \\
\hline 276 & 3,3 & 3,4591 & 0,1591 & 1083 & 3,4 & 3,4556 & 0,0556 \\
\hline 277 & 3,3 & 3,308 & 0,008 & 1089 & 3,7 & 3,8031 & 0,1031 \\
\hline 279 & 5 & 4,49 & 0,51 & 1096 & 4,57 & 3,4567 & 1,1133 \\
\hline 280 & 3,3 & 3,1511 & 0,1489 & 1103 & 4,15 & 4,1547 & 0,0047 \\
\hline 281 & 3,3 & 3,0217 & 0,2783 & 1108 & 4,31 & 4,2968 & 0,0132 \\
\hline 282 & 3,3 & 4,0374 & 0,7374 & 1113 & 4,15 & 4,6026 & 0,4526 \\
\hline 284 & 4,25 & 4,8023 & 0,5523 & 1118 & 5,44 & 4,8023 & 0,6377 \\
\hline 285 & 5,2 & 4,6915 & 0,5085 & 1123 & 5 & 4,6779 & 0,3221 \\
\hline 287 & 5 & 6,4531 & 1,4531 & 1128 & 4,99 & 5,2304 & 0,2404 \\
\hline 288 & 5,01 & 4,7937 & 0,2163 & 1133 & 5,3 & 5,1814 & 0,1186 \\
\hline 290 & 5,74 & 4,8814 & 0,8586 & 1138 & 4,3 & 4,4343 & 0,1343 \\
\hline 291 & 5,14 & 5,2942 & 0,1542 & 1143 & 4,8 & 5,1657 & 0,3657 \\
\hline 292 & 5,96 & 5,8749 & 0,0851 & 1148 & 4,43 & 4,9906 & 0,5606 \\
\hline 293 & 6 & 5,0482 & 0,9518 & 1154 & 4,98 & 4,5569 & 0,4231 \\
\hline 295 & 5,3 & 5,2513 & 0,0487 & 1159 & 5,13 & 4,4195 & 0,7105 \\
\hline 296 & 4,08 & 4,1974 & 0,1174 & 1164 & 4,65 & 4,6985 & 0,0485 \\
\hline 297 & 4,21 & 4,0524 & 0,1576 & 1169 & 5 & 4,5817 & 0,4183 \\
\hline 298 & 4,45 & 4,0397 & 0,4103 & 1174 & 4,58 & 5,0653 & 0,4853 \\
\hline 300 & 4,49 & 4,1098 & 0,3802 & 1179 & 4,46 & 5,0042 & 0,5442 \\
\hline 301 & 4,23 & 4,1595 & 0,0705 & 1184 & 5,08 & 4,8375 & 0,2425 \\
\hline 302 & 4,48 & 4,0568 & 0,4232 & 1189 & 4,3 & 4,3056 & 0,0056 \\
\hline 303 & 4 & 3,9639 & 0,0361 & 1194 & 6,28 & 6,322 & 0,042 \\
\hline 305 & 4,08 & 4,0264 & 0,0536 & 1200 & 5,05 & 4,5068 & 0,5432 \\
\hline 306 & 4 & 3,9114 & 0,0886 & 1205 & 4,84 & 4,6725 & 0,1675 \\
\hline 307 & 4 & 4,1684 & 0,1684 & 1210 & 4,93 & 4,5642 & 0,3658 \\
\hline 308 & 4,09 & 4,2989 & 0,2089 & 1215 & 4,3 & 4,4208 & 0,1208 \\
\hline 310 & 4,39 & 4,0717 & 0,3183 & 1220 & 5,06 & 4,799 & 0,261 \\
\hline 311 & 4 & 4,3598 & 0,3598 & 1225 & 4,62 & 4,6896 & 0,0696 \\
\hline 312 & 4 & 4,1117 & 0,1117 & 1230 & 4,38 & 4,6106 & 0,2306 \\
\hline 313 & 4,19 & 4,1247 & 0,0653 & 1235 & 4,3 & 4,2206 & 0,0794 \\
\hline 315 & 4 & 4,1088 & 0,1088 & 1241 & 4,95 & 4,8249 & 0,1251 \\
\hline
\end{tabular}




\begin{tabular}{r|r|l|l|l|l|l|l}
\multicolumn{1}{l|}{ Training set } & & \multicolumn{1}{l}{ ASNN } & & Test set & & \\
\hline NAME & $\mathbf{l g}(\mathbf{1} / \mathbf{I C 5 0})$ & Predicted & delta & NAME & $\mathbf{l g}(\mathbf{1} / \mathbf{I C 5 0})$ & Predicted & $\mathbf{x ~ d e l t a}$ \\
\hline 316 & 4 & 4,0424 & 0,0424 & 1246 & 5,21 & 5,2068 & 0,0032 \\
\hline 317 & 4,26 & 4,1837 & 0,0763 & 1251 & 3,98 & 4,0666 & 0,0866 \\
\hline 318 & 4,16 & 4,2014 & 0,0414 & 1257 & 4,25 & 3,9278 & 0,3222 \\
\hline 320 & 4,25 & 4,1532 & 0,0968 & 1263 & 5,88 & 5,3386 & 0,5414 \\
\hline 321 & 4,16 & 4,0108 & 0,1492 & 1268 & 4,3 & 4,4737 & 0,1737 \\
\hline 322 & 4,22 & 4,1247 & 0,0953 & 1273 & 5,34 & 5,4273 & 0,0873 \\
\hline 323 & 4 & 4,2262 & 0,2262 & 1278 & 4,87 & 4,8627 & 0,0073 \\
\hline 325 & 4 & 4,2401 & 0,2401 & 1283 & 5,41 & 5,3696 & 0,0404 \\
\hline 326 & 4,16 & 4,2364 & 0,0764 & 1288 & 4,56 & 4,7234 & 0,1634 \\
\hline 327 & 4 & 4,0504 & 0,0504 & 1294 & 4,29 & 4,4239 & 0,1339 \\
\hline 328 & 4 & 4,1555 & 0,1555 & 1299 & 4,77 & 5,1454 & 0,3754 \\
\hline 330 & 4 & 4,0073 & 0,0073 & 1304 & 4,8 & 4,6577 & 0,1423 \\
\hline 331 & 4 & 4,1656 & 0,1656 & 1309 & 4,17 & 4,7664 & 0,5964 \\
\hline 332 & 4,33 & 4,0562 & 0,2738 & & &
\end{tabular}

\begin{tabular}{rr|r|r}
332 & 4,33 & 4,0562 & 0,2738 \\
\hline 333 & 4 & 4,2914 & 0,2914 \\
\hline 335 & 4,52 & 3,955 & 0,565 \\
\hline 337 & 4 & 3,8891 & 0,1109 \\
\hline 338 & 4 & 4,0316 & 0,0316 \\
\hline 339 & 4,4 & 3,92 & 0,48 \\
\hline 341 & 5,35 & 4,5078 & 0,8422 \\
\hline 342 & 5,35 & 5,5051 & 0,1551 \\
\hline
\end{tabular}

\begin{tabular}{r|r|r|r}
\hline 342 & 5,35 & 5,5051 & 0,1551 \\
\hline 344 & 7,23 & 6,6851 & 0,5449 \\
\hline 345 & 5 & 4,9077 & 0,0923 \\
\hline 347 & 5,11 & 5,5764 & 0,4664 \\
\hline 348 & 5 & 5,7318 & 0,7318 \\
\hline
\end{tabular}

\begin{tabular}{rr|r|r}
\hline 350 & 6,09 & 4,889 & 1,201 \\
\hline 354 & 5,12 & 5,0753 & 0,0447 \\
\hline 355 & 5,22 & 5,5212 & 0,3012 \\
\hline
\end{tabular}

\begin{tabular}{|c|c|c|c|}
\hline & & & \\
\hline 355 & 5,22 & 5,5212 & 0,3012 \\
\hline 356 & 4,92 & 5,0911 & 0,1711 \\
\hline 357 & 5,08 & 5,1619 & 0,0819 \\
\hline 359 & 5,11 & 5,3618 & 0,2518 \\
\hline 360 & 5,19 & 5,2011 & 0,0111 \\
\hline 361 & 4,9 & 5,1656 & 0,2656 \\
\hline 362 & 4,47 & 5,3819 & 0,9119 \\
\hline 364 & 5,42 & 5,1595 & 0,2605 \\
\hline 365 & 5,21 & 5,3616 & 0,1516 \\
\hline 366 & 4,65 & 4,9337 & 0,2837 \\
\hline 367 & 4,64 & 4,7114 & 0,0714 \\
\hline 369 & 4,66 & 4,6173 & 0,0427 \\
\hline 370 & 4,63 & 4,7123 & 0,0823 \\
\hline 371 & 4,63 & 4,7545 & 0,1245 \\
\hline 372 & 4,65 & 4,7331 & 0,0831 \\
\hline 374 & 4,66 & 4,6901 & 0,0301 \\
\hline 375 & 4,66 & 4,8438 & 0,1838 \\
\hline 376 & 4,64 & 4,711 & 0,071 \\
\hline 377 & 4,65 & 4,7438 & 0,0938 \\
\hline 379 & 4,67 & 4,7801 & 0,1101 \\
\hline 380 & 4,66 & 4,7171 & 0,0571 \\
\hline 381 & 4,77 & 4,6838 & 0,0862 \\
\hline 382 & 4,64 & 4,655 & 0,015 \\
\hline 384 & 5,17 & 4,6508 & 0,5192 \\
\hline 385 & 4,65 & 4,4988 & 0,1512 \\
\hline 386 & 4,78 & 4,6058 & 0,1742 \\
\hline 387 & 4,66 & 4,3544 & 0,3056 \\
\hline 390 & 4,24 & 4,5809 & 0,3409 \\
\hline 391 & 4,55 & 4,3733 & 0,1767 \\
\hline 392 & 4,38 & 4,3583 & 0,0217 \\
\hline 393 & 4,34 & 4,7461 & 0,4061 \\
\hline 396 & 8,22 & 7,3873 & 0,8327 \\
\hline 397 & 5,7 & 5,2595 & 0,4405 \\
\hline 398 & 4,65 & 4,357 & 0,293 \\
\hline 399 & 5,82 & 5,4069 & 0,4131 \\
\hline 402 & 4,12 & 5,2089 & 1,0889 \\
\hline 403 & 6,1 & 6,2604 & 0,1604 \\
\hline 404 & 7,4 & 6,4972 & 0,9028 \\
\hline
\end{tabular}




\begin{tabular}{|c|c|c|c|c|c|c|c|}
\hline Training set & & ASNN & & Test set & & & \\
\hline NAME & $\lg (1 / \mathrm{IC} 50)$ & Predicted & delta & NAME & $\lg (1 / I C 50)$ & Predicted & $x$ delta \\
\hline
\end{tabular}

\begin{tabular}{r|r|r|r|r}
\hline 405 & 5,72 & 6,4075 & 0,6875 \\
\hline 407 & 6,4 & 6,4433 & 0,0433 \\
\hline 409 & 5,96 & 6,3978 & 0,4378 \\
\hline 410 & 6,4 & 6,2936 & 0,1064 \\
\hline 411 & 7,22 & 6,6654 & 0,5546 \\
\hline
\end{tabular}

\begin{tabular}{|c|c|c|c|}
\hline 410 & 6,4 & 6,2936 & 0,1064 \\
\hline 411 & 7,22 & 6,6654 & 0,5546 \\
\hline 413 & 5,77 & 6,2846 & 0,5146 \\
\hline 414 & 7,3 & 6,7128 & 0,5872 \\
\hline 415 & 5,89 & 6,4242 & 0,5342 \\
\hline 416 & 6,52 & 6,2856 & 0,2344 \\
\hline
\end{tabular}

\begin{tabular}{rr|r|r}
\hline 418 & 4,2 & 4,2332 & 0,0332 \\
\hline 419 & 3,96 & 4,4111 & 0,4511 \\
\hline 420 & 4,39 & 4,5602 & 0,1702 \\
\hline 421 & 4,85 & 4,5918 & 0,2582 \\
\hline 423 & 4,55 & 4,0716 & 0,4784 \\
\hline 424 & 4,66 & 4,6889 & 0,0289 \\
\hline 427 & 5,52 & 4,9853 & 0,5347
\end{tabular}

\begin{tabular}{rr|r|r}
424 & 4,66 & 4,6889 & 0,0289 \\
\hline 428 & 5,52 & 4,9853 & 0,5347 \\
\hline 432 & 4,92 & 5,1501 & 0,2301 \\
\hline 433 & 4,34 & 4,7513 & 0,4113 \\
\hline 434 & 4,4 & 4,3076 & 0,0924 \\
\hline 435 & 4 & 4,0035 & 0,0035 \\
\hline
\end{tabular}

\begin{tabular}{|c|c|c|c|}
\hline 454 & 4 & 4,005J & 0,0053 \\
\hline 435 & 5,34 & 5,0818 & 0,2582 \\
\hline 437 & 4,74 & 5,0181 & 0,2781 \\
\hline 438 & 4 & 4,2667 & 0,2667 \\
\hline 440 & 5,33 & 4,3205 & 1,0095 \\
\hline 441 & 4 & 4,9231 & 0,9231 \\
\hline 443 & 4,42 & 4,7362 & 0,3162 \\
\hline 444 & 4,04 & 4,6681 & 0,6281 \\
\hline
\end{tabular}

\begin{tabular}{r|r|r|r}
443 & 4,42 & 4,7362 & 0,3162 \\
\hline 444 & 4,04 & 4,6681 & 0,6281 \\
\hline 445 & 4,46 & 3,8135 & 0,6465 \\
\hline 446 & 5,63 & 4,3271 & 1,3029 \\
\hline 448 & 3,62 & 3,644 & 0,024 \\
\hline 450 & 4,36 & 4,3436 & 0,0164 \\
\hline 452 & 3,63 & 3,9175 & 0,2875 \\
\hline 453 & 3,64 & 4,3066 & 0,6666
\end{tabular}

\begin{tabular}{rr|r|r}
\hline 52 & 3,63 & 3,9175 & 0,2875 \\
\hline 453 & 3,64 & 4,3066 & 0,6666 \\
\hline 456 & 3,63 & 4,1889 & 0,5589 \\
\hline 457 & 5,61 & 4,8878 & 0,7222 \\
\hline 458 & 3,6 & 4,1342 & 0,5342 \\
\hline
\end{tabular}

\begin{tabular}{|c|c|c|c|}
\hline 458 & 3,6 & 4,1342 & 0,5342 \\
\hline 459 & 5,65 & 4,6039 & 1,0461 \\
\hline 461 & 4,27 & 3,9875 & 0,2825 \\
\hline 463 & 4,59 & 4,2161 & 0,3739 \\
\hline 464 & 5,59 & 4,3728 & 1,2172 \\
\hline 465 & 3,63 & 4,1338 & 0,5038 \\
\hline 467 & 3,7 & 3,6449 & 0,0551 \\
\hline 470 & 3,67 & 4,1899 & 0,5199 \\
\hline 472 & 5,66 & 5,2514 & 0,4086 \\
\hline 473 & 3,6 & 4,2984 & 0,6984 \\
\hline 477 & 3,69 & 4,5427 & 0,8527 \\
\hline 478 & 3,64 & 3,8962 & 0,2562 \\
\hline 479 & 4,3 & 4,5497 & 0,2497 \\
\hline 480 & 4,51 & 3,8346 & 0,6754 \\
\hline 482 & 3,69 & 4,0061 & 0,3161 \\
\hline 483 & 3,66 & 4,0021 & 0,3421 \\
\hline 487 & 3,42 & 3,864 & 0,444 \\
\hline 488 & 4,8 & 4,9677 & 0,1677 \\
\hline 490 & 4,41 & 3,9519 & 0,4581 \\
\hline 492 & 3,64 & 3,7397 & 0,0997 \\
\hline 493 & 3,49 & 3,9631 & 0,4731 \\
\hline 494 & 4,2 & 4,5537 & 0,3537 \\
\hline 496 & 5,6 & 5,0564 & 0,5436 \\
\hline 497 & 3,71 & 3,8665 & 0,1565 \\
\hline 498 & 3,65 & 3,6816 & 0,0316 \\
\hline 499 & 6,07 & 5,7922 & 0,2778 \\
\hline 501 & 5,45 & 6,0076 & 0,5576 \\
\hline 502 & 5,62 & 5,9103 & 0,2903 \\
\hline 503 & 6,68 & 6,3991 & 0,2809 \\
\hline 504 & 6,41 & 5,873 & 0,537 \\
\hline 506 & 6,38 & 6,7688 & 0,3888 \\
\hline
\end{tabular}




\begin{tabular}{l|c|l|l|l|l|l|l}
\multicolumn{2}{l|}{ Training set } & & ASNN & & Test set & & \\
\hline NAME & $\lg (\mathbf{1 / I C 5 0})$ & Predicted & delta & NAME & $\lg (\mathbf{1 / I C 5 0})$ & Predicted & $\mathbf{x}$ delta \\
\hline 507 & 5,78 & 5,8971 & 0,1171
\end{tabular}

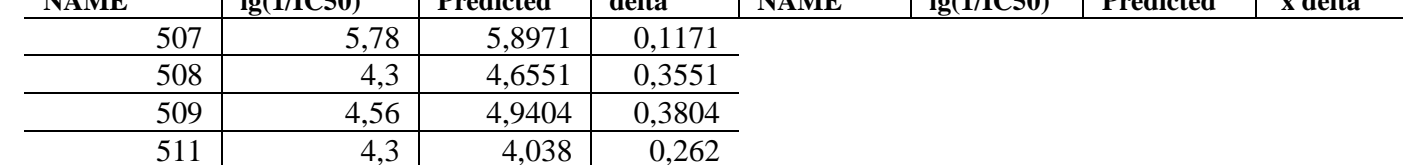

\begin{tabular}{rr|r|r}
511 & 4,3 & 4,038 & 0,262 \\
\hline 512 & 4,37 & 4,0725 & 0,2975 \\
\hline 513 & 5,24 & 4,6979 & 0,5421 \\
\hline 514 & 3,89 & 4,6467 & 0,7567 \\
\hline 516 & 4,32 & 4,5163 & 0,1963 \\
\hline 517 & 4,3 & 4,1094 & 0,1906 \\
\hline 518 & 4,4 & 4,2901 & 0,1099 \\
\hline
\end{tabular}

\begin{tabular}{r|r|r|r}
\hline 518 & 4,4 & 4,2901 & 0,1099 \\
\hline 520 & 4,3 & 4,6553 & 0,3553 \\
\hline 522 & 3,7 & 4,1284 & 0,4284 \\
\hline 523 & 4,75 & 3,9548 & 0,7952 \\
\hline 524 & 4,72 & 4,08 & 0,64 \\
\hline 527 & 3,7 & 4,1015 & 0,4015 \\
\hline
\end{tabular}

\begin{tabular}{|c|c|c|c|}
\hline 525 & 3,7 & 4,1015 & 0,4015 \\
\hline 527 & 3,7 & 3,8834 & 0,1834 \\
\hline 528 & 4,26 & 4,2971 & 0,0371 \\
\hline 529 & 4 & 4,1929 & 0,1929 \\
\hline 530 & 4,37 & 4,237 & 0,133 \\
\hline 532 & 4 & 4,2876 & 0,2876 \\
\hline 533 & 3,7 & 4,0947 & 0,3947 \\
\hline
\end{tabular}

\begin{tabular}{rr|r|r}
\hline 533 & 3,7 & 4,0947 & 0,3947 \\
\hline 534 & 3,7 & 3,5385 & 0,1615 \\
\hline 535 & 3,7 & 3,8506 & 0,1506 \\
\hline 538 & 4,7 & 4,169 & 0,531 \\
\hline 539 & 4 & 4,1005 & 0,1005 \\
\hline
\end{tabular}

\begin{tabular}{r|r|r|r}
\hline 538 & 4,7 & 4,169 & 0,531 \\
\hline 539 & 4 & 4,1005 & 0,1005 \\
\hline 540 & 4 & 4,0377 & 0,0377 \\
\hline 541 & 4 & 3,4176 & 0,5824 \\
\hline 543 & 4,52 & 4,3687 & 0,1513 \\
\hline 544 & 4,54 & 4,5853 & 0,0453 \\
\hline
\end{tabular}

\begin{tabular}{|c|c|c|c|}
\hline & & & \\
\hline 544 & 4,54 & 4,5853 & 0,0453 \\
\hline 545 & 4 & 4,3631 & 0,3631 \\
\hline 546 & 5,7 & 5,5422 & 0,1578 \\
\hline 548 & 5,05 & 4,5625 & 0,4875 \\
\hline 549 & 4,8 & 4,865 & 0,065 \\
\hline 550 & 4,09 & 4,2715 & 0,1815 \\
\hline 551 & 5,33 & 5,1947 & 0,1353 \\
\hline 553 & 5,4 & 5,1145 & 0,2855 \\
\hline 554 & 5,4 & 5,4099 & 0,0099 \\
\hline 555 & 4,21 & 4,9187 & 0,7087 \\
\hline 556 & 5 & 4,7854 & 0,2146 \\
\hline 558 & 4,89 & 5,4589 & 0,5689 \\
\hline 559 & 5,7 & 5,1062 & 0,5938 \\
\hline 560 & 4 & 4,1814 & 0,1814 \\
\hline 561 & 4,96 & 4,6848 & 0,2752 \\
\hline 563 & 4 & 4,6085 & 0,6085 \\
\hline 564 & 4,89 & 4,6598 & 0,2302 \\
\hline 565 & 4,15 & 4,4467 & 0,2967 \\
\hline 566 & 4,64 & 4,517 & 0,123 \\
\hline 568 & 4,57 & 4,8561 & 0,2861 \\
\hline 569 & 5,33 & 5,4267 & 0,0967 \\
\hline 570 & 5,04 & 5,3667 & 0,3267 \\
\hline 571 & 5,53 & 5,7795 & 0,2495 \\
\hline 573 & 5,9 & 5,721 & 0,179 \\
\hline 574 & 5,91 & 5,8723 & 0,0377 \\
\hline 575 & 5,67 & 5,5526 & 0,1174 \\
\hline 576 & 5,44 & 4,9303 & 0,5097 \\
\hline 578 & 5,49 & 5,6538 & 0,1638 \\
\hline 579 & 5,39 & 5,1915 & 0,1985 \\
\hline 580 & 5,86 & 5,444 & 0,416 \\
\hline 581 & 6,38 & 6,7729 & 0,3929 \\
\hline 583 & 5,58 & 5,9505 & 0,3705 \\
\hline 584 & 6,41 & 5,8693 & 0,5407 \\
\hline 585 & 6,07 & 5,9141 & 0,1559 \\
\hline 586 & 5,62 & 5,9468 & 0,3268 \\
\hline 588 & 6,04 & 5,7434 & 0,2966 \\
\hline 589 & 5,45 & 5,9968 & 0,5468 \\
\hline 590 & 5,45 & 5,8851 & 0,4351 \\
\hline
\end{tabular}




\begin{tabular}{l|c|l|l|l|l|l|l}
\multicolumn{1}{l|}{ Training set } & & ASNN & & Test set & & & \\
\hline NAME & $\lg (\mathbf{1 / I C 5 0})$ & Predicted & delta & NAME & $\lg (\mathbf{1 / I C 5 0})$ & Predicted & $\mathbf{x}$ delta \\
\hline 591 & 5,69 & 5,915 & 0,225 & &
\end{tabular}

\begin{tabular}{r|r|r|r} 
& $\lg (\mathbf{1} / \mathbf{C} \mathbf{5 0})$ & \multicolumn{1}{l}{ Predicted } & \multicolumn{1}{c}{ delta } \\
\hline 591 & 5,69 & 5,915 & 0,225 \\
\hline 593 & 6,05 & 5,9851 & 0,0649 \\
\hline 594 & 5,62 & 5,5611 & 0,0589 \\
\hline 595 & 5,8 & 5,6917 & 0,1083 \\
\hline 596 & 6,15 & 6,195 & 0,045 \\
\hline
\end{tabular}

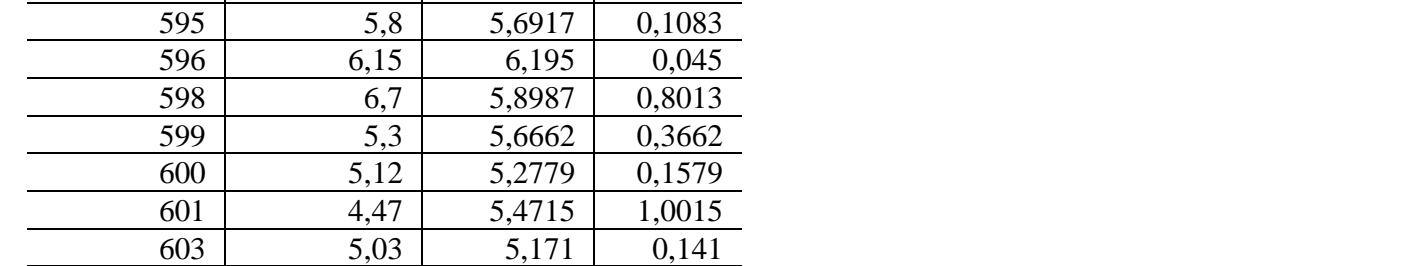

\begin{tabular}{rr|r|r}
603 & 5,03 & 5,171 & 0,141 \\
\hline 604 & 4,72 & 4,5381 & 0,1819 \\
\hline 605 & 4,96 & 4,7534 & 0,2066 \\
\hline 607 & 4,19 & 4,299 & 0,109 \\
\hline 609 & 4,01 & 4,3066 & 0,2966 \\
\hline 610 & 4 & 4,4768 & 0,4768 \\
\hline
\end{tabular}

\begin{tabular}{r|r|r|r}
610 & 4 & 4,4768 & 0,4768 \\
\hline 611 & 4 & 4,175 & 0,175 \\
\hline 612 & 4 & 4,0343 & 0,0343 \\
\hline 614 & 4 & 4,8013 & 0,8013 \\
\hline 615 & 4 & 4,3783 & 0,3783 \\
\hline
\end{tabular}

\begin{tabular}{rr|r|r}
\hline 615 & 4 & 4,3783 & 0,3783 \\
\hline 617 & 4,46 & 4,5781 & 0,1181 \\
\hline 618 & 4,49 & 4,2671 & 0,2229 \\
\hline 621 & 4,44 & 4,4346 & 0,0054 \\
\hline
\end{tabular}

\begin{tabular}{l|l|l|l}
621 & 4,44 & 4,4346 & 0,0054 \\
\hline 622 & 4,48 & 4,5777 & 0,0977 \\
\hline 623 & 4,51 & 4,3525 & 0,1575 \\
\hline 624 & 4,35 & 4,7404 & 0,3904 \\
\hline 626 & 4,31 & 4,2531 & 0,0569 \\
\hline 627 & 4,31 & 4,8276 & 0,5176 \\
\hline
\end{tabular}

\begin{tabular}{ll|l|l}
626 & 4,31 & 4,2531 & 0,0569 \\
\hline 627 & 4,31 & 4,8276 & 0,5176 \\
\hline 628 & 4,26 & 4,4023 & 0,1423 \\
\hline 629 & 4,27 & 4,5461 & 0,2761 \\
\hline 631 & 4,34 & 4,5114 & 0,1714 \\
\hline
\end{tabular}

\begin{tabular}{r|r|r|r}
\hline 631 & 4,34 & 4,5114 & 0,1714 \\
\hline 632 & 8,44 & 7,2294 & 1,2106 \\
\hline 633 & 4,66 & 4,6663 & 0,0063 \\
\hline 634 & 4,77 & 4,7482 & 0,0218 \\
\hline
\end{tabular}

\begin{tabular}{rr|r|r}
633 & 4,66 & 4,6663 & 0,0063 \\
\hline 634 & 4,77 & 4,7482 & 0,0218 \\
\hline 636 & 5,15 & 5,7182 & 0,5682 \\
\hline 637 & 5 & 5,0544 & 0,0544 \\
\hline 638 & 5,3 & 4,9287 & 0,3713 \\
\hline 639 & 5 & 4,9458 & 0,0542 \\
\hline
\end{tabular}

\begin{tabular}{|c|c|c|c|}
\hline 638 & 5,3 & 4,9287 & 0,3713 \\
\hline 639 & 5 & 4,9458 & 0,0542 \\
\hline 641 & 5 & 4,5326 & 0,4674 \\
\hline 642 & 5 & 5,0097 & 0,0097 \\
\hline 643 & 3,7 & 3,9466 & 0,2466 \\
\hline 644 & 4,38 & 4,2715 & 0,1085 \\
\hline 647 & 3,7 & 4,5134 & 0,8134 \\
\hline 648 & 5,72 & 4,6527 & 1,0673 \\
\hline 649 & 4,07 & 3,9819 & 0,0881 \\
\hline 650 & 4,66 & 4,8071 & 0,1471 \\
\hline 652 & 5,82 & 5,0957 & 0,7243 \\
\hline 654 & 4,94 & 4,7487 & 0,1913 \\
\hline 655 & 5,55 & 4,8016 & 0,7484 \\
\hline 656 & 5,46 & 4,9676 & 0,4924 \\
\hline 658 & 5,15 & 5,112 & 0,038 \\
\hline 660 & 5,04 & 4,4557 & 0,5843 \\
\hline 661 & 4,91 & 5,2367 & 0,3267 \\
\hline 662 & 3,93 & 5,077 & 1,147 \\
\hline 664 & 3,71 & 4,4331 & 0,7231 \\
\hline 665 & 4,11 & 4,1412 & 0,0312 \\
\hline 666 & 4,11 & 4,0781 & 0,0319 \\
\hline 667 & 4,1 & 4,0512 & 0,0488 \\
\hline 669 & 4,11 & 4,0347 & 0,0753 \\
\hline 670 & 4,15 & 4,1021 & 0,0479 \\
\hline 671 & 4,13 & 4,4391 & 0,3091 \\
\hline 672 & 5,44 & 5,6696 & 0,2296 \\
\hline 674 & 5,36 & 5,6411 & 0,2811 \\
\hline 675 & 5,06 & 5,1566 & 0,0966 \\
\hline 676 & 4,87 & 4,8388 & 0,0312 \\
\hline 677 & 6,05 & 5,5185 & 0,5315 \\
\hline 679 & 5,32 & 5,4248 & 0,1048 \\
\hline
\end{tabular}




\begin{tabular}{l|c|l|l|l|l|l|l}
\multicolumn{2}{l|}{ Training set } & & ASNN & & Test set & & \\
\hline NAME & $\lg (\mathbf{1 / I C 5 0})$ & Predicted & delta & NAME & $\lg (\mathbf{1 / I C 5 0})$ & Predicted & $\mathbf{x}$ delta \\
\hline 680 & 5,13 & 4,8269 & 0,3031
\end{tabular}

\begin{tabular}{r|r|r|r}
680 & 5,13 & 4,8269 & 0,3031 \\
\hline 681 & 5,37 & 5,2489 & 0,1211 \\
\hline 682 & 4,78 & 5,9471 & 1,1671 \\
\hline 684 & 4,64 & 5,0628 & 0,4228 \\
\hline
\end{tabular}

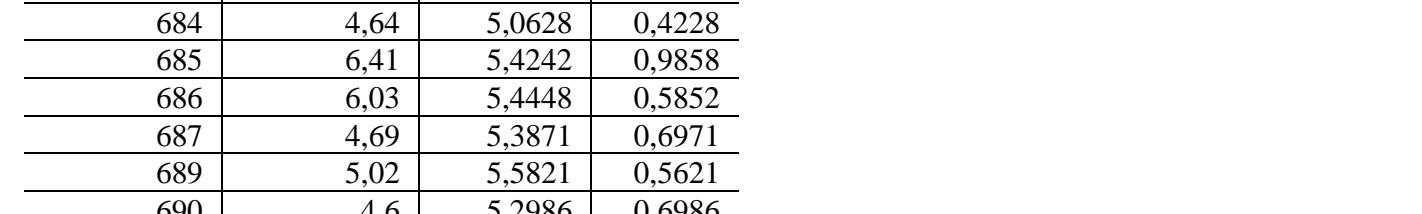

\begin{tabular}{rr|r|r}
\hline 690 & 5,02 & 5,5821 & 0,5621 \\
\hline 691 & 4,6 & 5,2986 & 0,6986 \\
\hline 692 & 5,24 & 4,7365 & 0,5035 \\
\hline 694 & 5,82 & 5,1049 & 0,7151 \\
\hline 695 & 5,51 & 5,1537 & 0,3563 \\
\hline 696 & 5,38 & 5,92 & 0,54 \\
\hline 697 & 5,62 & 5,6396 & 0,0196 \\
\hline
\end{tabular}

\begin{tabular}{r|r|r|r}
696 & 5,62 & 5,6396 & 0,0196 \\
\hline 697 & 5,04 & 5,275 & 0,235 \\
\hline 699 & 4,81 & 4,8123 & 0,0023 \\
\hline 700 & 4,91 & 4,4977 & 0,4123 \\
\hline 701 & 6,55 & 5,717 & 0,833 \\
\hline
\end{tabular}

\begin{tabular}{r|r|r|r}
701 & 6,55 & 5,717 & 0,833 \\
\hline 702 & 5,12 & 5,0661 & 0,0539 \\
\hline 704 & 4,8 & 5,2118 & 0,4118 \\
\hline 705 & 5,44 & 5,5952 & 0,1552 \\
\hline 706 & 4,7 & 5,2125 & 0,5125 \\
\hline 707 & 5,05 & 5,3277 & 0,2777 \\
\hline 709 & 5,1 & 5,0324 & 0,0676 \\
\hline
\end{tabular}

\begin{tabular}{|c|c|c|c|}
\hline 100 & 4,1 & 5,2125 & 0,5125 \\
\hline 707 & 5,05 & 5,3277 & 0,2777 \\
\hline 709 & 5,1 & 5,0324 & 0,0676 \\
\hline 710 & 5,68 & 6,039 & 0,359 \\
\hline 711 & 4,59 & 4,5833 & 0,0067 \\
\hline 712 & 5,54 & 5,4767 & 0,0633 \\
\hline 714 & 5,52 & 5,0329 & 0,4871 \\
\hline 715 & 4,8 & 5,3632 & 0,5632 \\
\hline 716 & 5,05 & 5,3144 & 0,2644 \\
\hline 717 & 5,8 & 5,1528 & 0,6472 \\
\hline 719 & 6,05 & 5,8317 & 0,2183 \\
\hline 720 & 5,17 & 5,4786 & 0,3086 \\
\hline 721 & 6,03 & 5,8973 & 0,1327 \\
\hline 722 & 5,26 & 5,6007 & 0,3407 \\
\hline 724 & 6,15 & 5,4609 & 0,6891 \\
\hline 725 & 5,15 & 5,4769 & 0,3269 \\
\hline 726 & 6,3 & 5,7371 & 0,5629 \\
\hline 727 & 5,44 & 5,9285 & 0,4885 \\
\hline 729 & 4,49 & 4,5513 & 0,0613 \\
\hline 730 & 3,5 & 3,924 & 0,424 \\
\hline 731 & 4,22 & 4,7177 & 0,4977 \\
\hline 732 & 4,4 & 4,4324 & 0,0324 \\
\hline 734 & 4,37 & 4,3419 & 0,0281 \\
\hline 735 & 4,72 & 4,5349 & 0,1851 \\
\hline 736 & 4,82 & 4,6142 & 0,2058 \\
\hline 737 & 4,41 & 4,1102 & 0,2998 \\
\hline 739 & 4,22 & 4,3751 & 0,1551 \\
\hline 740 & 3,37 & 3,9518 & 0,5818 \\
\hline 741 & 4,35 & 4,4852 & 0,1352 \\
\hline 742 & 4,72 & 4,7025 & 0,0175 \\
\hline 744 & 4 & 3,8247 & 0,1753 \\
\hline 745 & 4,01 & 3,7926 & 0,2174 \\
\hline 746 & 4 & 4,0927 & 0,0927 \\
\hline 747 & 4 & 4,1815 & 0,1815 \\
\hline 749 & 4 & 3,8736 & 0,1264 \\
\hline 750 & 4,01 & 3,8095 & 0,2005 \\
\hline 751 & 4 & 4,1012 & 0,1012 \\
\hline 752 & 4,13 & 4,234 & 0,104 \\
\hline 754 & 4 & 4,4344 & 0,4344 \\
\hline 755 & 4,01 & 4,1037 & 0,0937 \\
\hline 756 & 6,33 & 6,2934 & 0,0366 \\
\hline 757 & 6,82 & 6,5277 & 0,2923 \\
\hline 762 & 4 & 4,0763 & 0,0763 \\
\hline 765 & 5,76 & 5,4368 & 0,3232 \\
\hline 766 & 5,44 & 5,6223 & 0,1823 \\
\hline
\end{tabular}




\begin{tabular}{l|c|l|l|l|l|l|l}
\multicolumn{2}{l|}{ Training set } & & ASNN & & Test set & & \\
\hline NAME & $\lg (\mathbf{1 / I C 5 0})$ & Predicted & delta & NAME & $\lg (\mathbf{1 / I C 5 0})$ & Predicted & x delta \\
\hline 767 & 4,82 & 5,0806 & 0,2606 &
\end{tabular}

\begin{tabular}{r|r|r|r|r}
\hline 767 & 4,82 & 5,0806 & 0,2606 \\
\hline 769 & 5,58 & 5,5653 & 0,0147 \\
\hline 770 & 5,55 & 5,4313 & 0,1187 \\
\hline 771 & 5,52 & 4,9278 & 0,5922 \\
\hline 772 & 6,13 & 6,8122 & 0,6822
\end{tabular}

\begin{tabular}{r|r|r|r}
771 & 5,52 & 4,9278 & 0,5922 \\
\hline 772 & 6,13 & 6,8122 & 0,6822 \\
\hline 774 & 7,27 & 6,2511 & 1,0189 \\
\hline 775 & 7,29 & 6,2621 & 1,0279 \\
\hline 776 & 5,93 & 6,0417 & 0,1117 \\
\hline 779 & 6,21 & 5,5699 & 0,6401 \\
\hline 781 & 6,15 & 6,4397 & 0,2897 \\
\hline
\end{tabular}

\begin{tabular}{r|r|r|r}
781 & 6,15 & 6,4397 & 0,2897 \\
\hline 782 & 5,94 & 6,517 & 0,577 \\
\hline 784 & 4,43 & 4,7411 & 0,3111 \\
\hline 785 & 5,85 & 5,9569 & 0,1069 \\
\hline 787 & 6,05 & 5,7686 & 0,2814 \\
\hline 788 & 6,4 & 5,8861 & 0,5139 \\
\hline
\end{tabular}

\begin{tabular}{rr|r|r}
788 & 6,4 & 5,8861 & 0,5139 \\
\hline 789 & 5,62 & 5,9246 & 0,3046 \\
\hline 790 & 5,74 & 5,875 & 0,135 \\
\hline 792 & 6,15 & 5,7959 & 0,3541 \\
\hline 793 & 5,38 & 5,5422 & 0,1622 \\
\hline 794 & 5,35 & 5,8465 & 0,4965 \\
\hline
\end{tabular}

\begin{tabular}{rr|r|r}
\hline 794 & 5,35 & 5,8465 & 0,4965 \\
\hline 795 & 6 & 5,9001 & 0,0999 \\
\hline 797 & 5,46 & 5,8643 & 0,4043 \\
\hline 798 & 5,59 & 5,9237 & 0,3337 \\
\hline 799 & 6,3 & 5,741 & 0,559 \\
\hline 800
\end{tabular}

\begin{tabular}{r|r|r|r}
\hline 798 & 5,59 & 5,9237 & 0,3337 \\
\hline 809 & 6,3 & 5,741 & 0,559 \\
\hline 802 & 6,7 & 6,4057 & 0,2943 \\
\hline 803 & 6,05 & 5,7222 & 0,3278 \\
\hline 804 & 5,54 & 5,8248 & 0,2848 \\
\hline 805 & 5,77 & 5,8383 & 0,0683 \\
\hline
\end{tabular}

\begin{tabular}{r|r|r|r}
804 & 5,54 & 5,8248 & 0,2848 \\
\hline 805 & 5,77 & 5,8383 & 0,0683 \\
\hline 807 & 5,44 & 6,0073 & 0,5673 \\
\hline 808 & 6,05 & 5,9308 & 0,1192 \\
\hline 809 & 6,05 & 5,8098 & 0,2402 \\
\hline 810 & 5,24 & 6,0236 & 0,7836 \\
\hline
\end{tabular}

\begin{tabular}{|c|c|c|c|}
\hline & & & \\
\hline 809 & 5,24 & 6,0236 & 0,7836 \\
\hline 810 & 4 & 3,9176 & 0,0824 \\
\hline 812 & 4 & 3,9638 & 0,0362 \\
\hline 813 & 4 & 4,83 & 0,83 \\
\hline 814 & 4 & 3,7072 & 0,2928 \\
\hline 815 & 4,49 & 4,7034 & 0,2134 \\
\hline 817 & 4 & 4,0438 & 0,0438 \\
\hline 818 & 4 & 3,9871 & 0,0129 \\
\hline 819 & 4 & 4,2267 & 0,2267 \\
\hline 820 & 4 & 4,1086 & 0,1086 \\
\hline 822 & 4 & 3,9888 & 0,0112 \\
\hline 823 & 4 & 3,9508 & 0,0492 \\
\hline 824 & 4 & 4,6903 & 0,6903 \\
\hline 825 & 4 & 3,9267 & 0,0733 \\
\hline 827 & 4 & 3,9184 & 0,0816 \\
\hline 828 & 4 & 3,9029 & 0,0971 \\
\hline 829 & 4 & 4,3997 & 0,3997 \\
\hline 831 & 6,15 & 5,8368 & 0,3132 \\
\hline 835 & 5,52 & 6,3135 & 0,7935 \\
\hline 838 & 5,89 & 5,7825 & 0,1075 \\
\hline 840 & 6,05 & 5,875 & 0,175 \\
\hline 841 & 6 & 6,0211 & 0,0211 \\
\hline 843 & 4 & 3,8404 & 0,1596 \\
\hline 844 & 4 & 4,0019 & 0,0019 \\
\hline 845 & 4 & 4,1377 & 0,1377 \\
\hline 846 & 4,24 & 4,0039 & 0,2361 \\
\hline 848 & 4 & 4,7523 & 0,7523 \\
\hline 849 & 4 & 3,8596 & 0,1404 \\
\hline 850 & 4 & 3,6571 & 0,3429 \\
\hline 852 & 4 & 3,9016 & 0,0984 \\
\hline 854 & 5 & 4,2461 & 0,7539 \\
\hline 855 & 4,7 & 4,6302 & 0,0698 \\
\hline 856 & 4,51 & 4,8652 & 0,3552 \\
\hline 857 & 4,98 & 5,4134 & 0,4334 \\
\hline 860 & 5,8 & 5,2426 & 0,5574 \\
\hline
\end{tabular}




\begin{tabular}{l|c|l|l|l|l|l|l}
\multicolumn{1}{l|}{ Training set } & & ASNN & & Test set & & & \\
\hline NAME & $\lg (\mathbf{1 / I C 5 0})$ & Predicted & delta & NAME & $\lg (\mathbf{1 / I C 5 0})$ & Predicted & $\mathbf{x}$ delta \\
\hline 862 & 5,67 & 5,534 & 0,136 &
\end{tabular}

\begin{tabular}{r|r|r|r|r}
\hline 862 & 5,67 & 5,534 & 0,136 \\
\hline 864 & 5,11 & 5,2308 & 0,1208 \\
\hline 865 & 4,74 & 5,0982 & 0,3582 \\
\hline 868 & 5,17 & 5,4009 & 0,2309 \\
\hline
\end{tabular}

\begin{tabular}{r|r|r|r}
\hline 869 & 5,02 & 5,2687 & 0,2487 \\
\hline 870 & 4,77 & 5,2688 & 0,4988 \\
\hline 871 & 5,42 & 4,7444 & 0,6756 \\
\hline 873 & 5,01 & 5,1655 & 0,1555 \\
\hline 874 & 5,54 & 5,0872 & 0,4528 \\
\hline
\end{tabular}

\begin{tabular}{rr|r|r}
\hline 873 & 5,01 & 5,1655 & 0,1555 \\
\hline 875 & 5,54 & 5,0872 & 0,4528 \\
\hline 876 & 5,24 & 5,4627 & 0,2227 \\
\hline 879 & 5,22 & 5,1431 & 0,0769 \\
\hline 880 & 4,85 & 4,2393 & 0,6107 \\
\hline 881 & 4 & 3,9943 & 0,0057 \\
\hline 882 & 4 & 4,2302 & 0,2302 \\
\hline
\end{tabular}

\begin{tabular}{rr|r|r}
\hline 881 & 4 & 4,2302 & 0,2302 \\
\hline 882 & 4 & 4,2464 & 0,2464 \\
\hline 884 & 4,33 & 4,5239 & 0,1939 \\
\hline 885 & 4,53 & 4,7201 & 0,1901 \\
\hline 886 & 4,88 & 4,3797 & 0,5003 \\
\hline 887 & 5,24 & 4,388 & 0,852 \\
\hline
\end{tabular}

\begin{tabular}{rr|r|r}
\hline 887 & 5,24 & 4,388 & 0,852 \\
\hline 890 & 4,51 & 4,0415 & 0,4685 \\
\hline 891 & 4 & 4,6313 & 0,6313 \\
\hline 892 & 4,5 & 4,1924 & 0,3076 \\
\hline 893 & 5,41 & 4,2085 & 1,2015 \\
\hline 895 & 4,28 & 4,5018 & 0,2218 \\
\hline
\end{tabular}

\begin{tabular}{rr|r|r}
893 & 5,41 & 4,2085 & 1,2015 \\
\hline 895 & 4,28 & 4,5018 & 0,2218 \\
\hline 896 & 4,41 & 4,5466 & 0,1366 \\
\hline 897 & 4,07 & 4,6937 & 0,6237 \\
\hline 898 & 4 & 3,8738 & 0,1262 \\
\hline
\end{tabular}

\begin{tabular}{r|r|r|r}
\hline 897 & 4,07 & 4,6937 & 0,6237 \\
\hline 898 & 4 & 3,8738 & 0,1262 \\
\hline 901 & 4 & 4,1431 & 0,1431 \\
\hline 902 & 4,52 & 4,4267 & 0,0933 \\
\hline 903 & 4,96 & 4,2953 & 0,6647 \\
\hline 904 & 4,33 & 4,3553 & 0,0253 \\
\hline 906 & 4,42 & 5,015 & 0,595 \\
\hline 907 & 4,68 & 5,0572 & 0,3772 \\
\hline 912 & 4,68 & 3,9715 & 0,7085 \\
\hline 913 & 4,54 & 4,4086 & 0,1314 \\
\hline 919 & 3,4 & 4,4499 & 1,0499 \\
\hline 921 & 4,58 & 4,6073 & 0,0273 \\
\hline 924 & 5,1 & 4,6142 & 0,4858 \\
\hline 926 & 4,34 & 3,8754 & 0,4646 \\
\hline 928 & 4 & 4,0327 & 0,0327 \\
\hline 929 & 4 & 4,6928 & 0,6928 \\
\hline 930 & 4 & 4,3015 & 0,3015 \\
\hline 932 & 4 & 4,1914 & 0,1914 \\
\hline 934 & 4,58 & 4,6945 & 0,1145 \\
\hline 935 & 4 & 4,1252 & 0,1252 \\
\hline 936 & 4,92 & 4,1144 & 0,8056 \\
\hline 937 & 4,72 & 4,7208 & 0,0008 \\
\hline 939 & 4,7 & 4,2984 & 0,4016 \\
\hline 940 & 5 & 4,7492 & 0,2508 \\
\hline 941 & 5,37 & 5,121 & 0,249 \\
\hline 942 & 5,26 & 5,0399 & 0,2201 \\
\hline 944 & 5 & 5,2615 & 0,2615 \\
\hline 945 & 5,28 & 5,2096 & 0,0704 \\
\hline 946 & 5 & 4,786 & 0,214 \\
\hline 947 & 4 & 4,0917 & 0,0917 \\
\hline 949 & 4 & 3,9361 & 0,0639 \\
\hline 950 & 4 & 4,035 & 0,035 \\
\hline 951 & 4,61 & 4,5278 & 0,0822 \\
\hline 952 & 4,75 & 4,5599 & 0,1901 \\
\hline 954 & 4 & 4,1706 & 0,1706 \\
\hline 956 & 4 & 3,9553 & 0,0447 \\
\hline 951 & 4,3701 & 0,3701 \\
\hline 959 & 4,7091 & 0,7091 \\
\hline 9,2546 & 1,0454 \\
\hline 9,1183 & 0,1183 \\
\hline 9674 & & 0,3326
\end{tabular}




\begin{tabular}{l|c|l|l|l|l|l|l}
\multicolumn{1}{l|}{ Training set } & & ASNN & & Test set & & & \\
\hline NAME & $\lg (\mathbf{1 / I C 5 0})$ & Predicted & delta & NAME & $\lg (\mathbf{1 / I C 5 0})$ & Predicted & x delta \\
\hline 962 & 4,19 & 3,9663 & 0,2237 &
\end{tabular}

\begin{tabular}{r|r|r|r|r}
\hline 962 & 4,19 & 3,9663 & 0,2237 \\
\hline 964 & 4 & 4,5959 & 0,5959 \\
\hline 965 & 4,48 & 4,0526 & 0,4274 \\
\hline 966 & 4 & 4,584 & 0,584 \\
\hline 967 & 4,85 & 4,3304 & 0,5196 \\
\hline
\end{tabular}

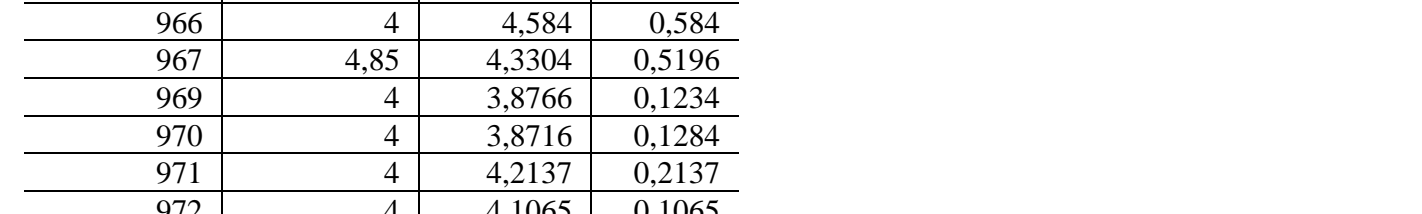

\begin{tabular}{r|r|r|r}
\hline 971 & 4 & 4,2137 & 0,2137 \\
\hline 972 & 4 & 4,1065 & 0,1065 \\
\hline 974 & 4 & 4,4884 & 0,4884 \\
\hline 976 & 4 & 4,0763 & 0,0763 \\
\hline 977 & 4 & 4,1059 & 0,1059 \\
\hline 979 & 5,14 & 4,4794 & 0,6606 \\
\hline 980 & 4 & 4,0657 & 0,0657 \\
\hline
\end{tabular}

\begin{tabular}{|c|c|c|c|}
\hline 979 & 4 & 4,0657 & 0,0657 \\
\hline 980 & 4 & 4,1376 & 0,1376 \\
\hline 981 & 5,35 & 4,3591 & 0,9909 \\
\hline 982 & 4,82 & 4,397 & 0,423 \\
\hline 985 & 4 & 4,1438 & 0,1438 \\
\hline 986 & 4,54 & 4,4916 & 0,0484 \\
\hline
\end{tabular}

\begin{tabular}{rr|r|r}
986 & 4,54 & 4,4916 & 0,0484 \\
\hline 987 & 4 & 4,2072 & 0,2072 \\
\hline 988 & 4,3 & 4,3967 & 0,0967 \\
\hline 991 & 4 & 3,8501 & 0,1499 \\
\hline 992 & 4 & 3,7758 & 0,2242 \\
\hline 993 & 4,92 & 4,51 & 0,41
\end{tabular}

\begin{tabular}{|c|c|c|c|}
\hline 991 & 4 & 3,8501 & 0,1499 \\
\hline 992 & 4 & 3,7758 & 0,2242 \\
\hline 993 & 4,92 & 4,51 & 0,41 \\
\hline 994 & 4,3 & 4,7059 & 0,4059 \\
\hline 996 & 5,42 & 4,5343 & 0,8857 \\
\hline 997 & 4,85 & 4,5099 & 0,3401 \\
\hline 998 & 4,49 & 4,6736 & 0,1836 \\
\hline 999 & 4,4 & 4,8866 & 0,4866 \\
\hline 1001 & 4,89 & 4,8016 & 0,0884 \\
\hline
\end{tabular}

\begin{tabular}{rrr|r}
999 & 4,4 & 4,8866 & 0,4866 \\
\hline 1001 & 4,89 & 4,8016 & 0,0884 \\
\hline 1002 & 4,8 & 4,7843 & 0,0157 \\
\hline 1003 & 4,3 & 4,8222 & 0,5222 \\
\hline 1004 & 4,59 & 4,5318 & 0,0582 \\
\hline
\end{tabular}

\begin{tabular}{|c|c|c|c|}
\hline 1004 & 4,59 & 4,5318 & 0,0582 \\
\hline 1006 & 4,64 & 4,732 & 0,092 \\
\hline 1007 & 4,82 & 4,5572 & 0,2628 \\
\hline 1008 & 5,1 & 4,6277 & 0,4723 \\
\hline 1009 & 4,54 & 4,7297 & 0,1897 \\
\hline 1011 & 4,3 & 4,5426 & 0,2426 \\
\hline 1012 & 5,42 & 5,5547 & 0,1347 \\
\hline 1013 & 4,6 & 4,5225 & 0,0775 \\
\hline 1014 & 5,68 & 5,1682 & 0,5118 \\
\hline 1016 & 4,6 & 4,8935 & 0,2935 \\
\hline 1017 & 4,6 & 4,97 & 0,37 \\
\hline 1018 & 4,6 & 4,691 & 0,091 \\
\hline 1019 & 4,6 & 5,1419 & 0,5419 \\
\hline 1021 & 5,42 & 5,5248 & 0,1048 \\
\hline 1022 & 4,6 & 4,6028 & 0,0028 \\
\hline 1023 & 4,6 & 4,8353 & 0,2353 \\
\hline 1024 & 4,6 & 4,636 & 0,036 \\
\hline 1026 & 5,82 & 5,0217 & 0,7983 \\
\hline 1028 & 5,28 & 5,1349 & 0,1451 \\
\hline 1029 & 4,6 & 4,8023 & 0,2023 \\
\hline 1030 & 4,6 & 4,6135 & 0,0135 \\
\hline 1032 & 4,6 & 4,827 & 0,227 \\
\hline 1033 & 4,6 & 4,8444 & 0,2444 \\
\hline 1034 & 5,23 & 5,138 & 0,092 \\
\hline 1035 & 4,6 & 4,6788 & 0,0788 \\
\hline 1037 & 4,6 & 4,2865 & 0,3135 \\
\hline 1038 & 4,6 & 4,7295 & 0,1295 \\
\hline 1039 & 4,6 & 4,1331 & 0,4669 \\
\hline 1040 & 4,6 & 5,0486 & 0,4486 \\
\hline 1042 & 4,6 & 4,6496 & 0,0496 \\
\hline 1043 & 4,6 & 4,6499 & 0,0499 \\
\hline 1044 & 4,6 & 4,3463 & 0,2537 \\
\hline 1045 & 4,6 & 4,6046 & 0,0046 \\
\hline 1047 & 4,6 & 4,9466 & 0,3466 \\
\hline
\end{tabular}




\begin{tabular}{l|l|l|l|l|l|l|l}
\multicolumn{2}{l|}{ Training set } & & ASNN & & Test set & & \\
\hline NAME & $\lg (\mathbf{1 / I C 5 0})$ & Predicted & delta & NAME & $\lg (\mathbf{1 / I C 5 0})$ & Predicted & $\mathbf{x}$ delta \\
\hline 1048 & 4,6 & 4,849 & 0,249 &
\end{tabular}

\begin{tabular}{r|r|r|r}
\hline 1048 & 4,6 & 4,849 & 0,249 \\
\hline 1049 & 5,85 & 5,1119 & 0,7381 \\
\hline 1050 & 4,6 & 4,5693 & 0,0307 \\
\hline 1052 & 4,3 & 4,2888 & 0,0112 \\
\hline
\end{tabular}

\begin{tabular}{r|r|r|r}
1052 & 4,3 & 4,2888 & 0,0112 \\
\hline 1053 & 3,89 & 4,7101 & 0,8201 \\
\hline 1054 & 4,6 & 4,8772 & 0,2772 \\
\hline 1055 & 4,6 & 4,7866 & 0,1866 \\
\hline 1057 & 5,1 & 4,8049 & 0,2951 \\
\hline
\end{tabular}

\begin{tabular}{r|r|r|r}
1057 & 5,1 & 4,8049 & 0,2951 \\
\hline 1058 & 5,15 & 4,9408 & 0,2092 \\
\hline 1059 & 5,19 & 5,3785 & 0,1885 \\
\hline 1060 & 4,6 & 4,6034 & 0,0034 \\
\hline 1062 & 5,54 & 5,5583 & 0,0183 \\
\hline 1063 & 4,67 & 4,6714 & 0,0014 \\
\hline
\end{tabular}

\begin{tabular}{r|r|r|r}
\hline 1063 & 4,67 & 4,6714 & 0,0014 \\
\hline 1064 & 4,6 & 4,579 & 0,021 \\
\hline 1065 & 5,38 & 5,2762 & 0,1038 \\
\hline 1067 & 4,6 & 4,5185 & 0,0815 \\
\hline 1068 & 4,6 & 4,4308 & 0,1692 \\
\hline
\end{tabular}

\begin{tabular}{rr|r|r}
\hline 1068 & 4,6 & 4,4308 & 0,1692 \\
\hline 1069 & 4,26 & 4,0615 & 0,1985 \\
\hline 1070 & 4,66 & 4,9092 & 0,2492 \\
\hline 1074 & 3,4 & 3,5664 & 0,1664 \\
\hline 1075 & 5,1 & 3,3264 & 1,7736 \\
\hline 1076 & 3,7 & 3,4665 & 0,2335 \\
\hline 1077 & 4 & 3,5962 & 0,4038 \\
\hline 1079 & 3,4 & 3,9503 & 0,5503 \\
\hline
\end{tabular}

\begin{tabular}{r|r|r|r}
1077 & 3,7 & 3,4665 & 0,2335 \\
\hline 1079 & 3,4 & 3,9503 & 0,4038 \\
\hline 1080 & 3,4 & 3,4423 & 0,5503 \\
\hline 1081 & 3,4 & 3,5023 & 0,1023 \\
\hline 1082 & 3,7 & 3,5962 & 0,1038 \\
\hline 1084 & 3,4 & 3,8313 & 0,4313 \\
\hline
\end{tabular}

\begin{tabular}{|c|c|c|c|}
\hline & , & S,OJנ & $0,4 J 15$ \\
\hline 1085 & 4,09 & 3,759 & 0,331 \\
\hline 1086 & 3,4 & 4,0137 & 0,6137 \\
\hline 1087 & 3,52 & 3,8069 & 0,2869 \\
\hline 1092 & 3,4 & 3,5734 & 0,1734 \\
\hline 1093 & 3,7 & 3,4015 & 0,2985 \\
\hline 1094 & 3,4 & 3,6197 & 0,2197 \\
\hline 1095 & 3,4 & 3,6987 & 0,2987 \\
\hline 1097 & 3,4 & 3,3994 & 0,0006 \\
\hline 1099 & 3,7 & 3,4987 & 0,2013 \\
\hline 1100 & 3,4 & 3,6106 & 0,2106 \\
\hline 1102 & 8,21 & 7,3668 & 0,8432 \\
\hline 1104 & 4,15 & 4,5414 & 0,3914 \\
\hline 1105 & 4,37 & 4,424 & 0,054 \\
\hline 1106 & 4,33 & 4,3674 & 0,0374 \\
\hline 1107 & 4,34 & 4,3289 & 0,0111 \\
\hline 1109 & 4,42 & 4,545 & 0,125 \\
\hline 1110 & 4,27 & 4,441 & 0,171 \\
\hline 1111 & 4,38 & 4,5649 & 0,1849 \\
\hline 1112 & 4,63 & 4,6334 & 0,0034 \\
\hline 1114 & 4,41 & 4,3667 & 0,0433 \\
\hline 1115 & 4,24 & 4,238 & 0,002 \\
\hline 1116 & 4,79 & 4,191 & 0,599 \\
\hline 1117 & 5,27 & 5,2302 & 0,0398 \\
\hline 1119 & 5,38 & 5,0603 & 0,3197 \\
\hline 1120 & 5 & 4,6512 & 0,3488 \\
\hline 1121 & 5 & 4,73 & 0,27 \\
\hline 1122 & 5 & 4,5875 & 0,4125 \\
\hline 1124 & 5,17 & 5,1007 & 0,0693 \\
\hline 1125 & 5,82 & 6,7948 & 0,9748 \\
\hline 1126 & 3,82 & 4,8155 & 0,9955 \\
\hline 1127 & 4,66 & 5,2748 & 0,6148 \\
\hline 1129 & 4,62 & 5,2983 & 0,6783 \\
\hline 1130 & 5,06 & 5,1976 & 0,1376 \\
\hline 1131 & 4,3 & 4,2297 & 0,0703 \\
\hline 1132 & 5,55 & 5,0782 & 0,4718 \\
\hline 1134 & 4,3 & 4,1698 & 0,1302 \\
\hline 1135 & 4,45 & 4,8724 & 0,4224 \\
\hline 1136 & 4,97 & 5,2921 & 0,3221 \\
\hline
\end{tabular}




\begin{tabular}{l|l|l|l|l|l|l|l} 
Training set & & ASNN & & Test set & & & \\
\hline NAME & $\lg (\mathbf{1} / \mathbf{I C 5 0})$ & Predicted & delta & NAME & $\mathbf{l g}(\mathbf{1} / \mathbf{I C 5 0})$ & Predicted & $\mathbf{x}$ delta \\
\hline 1137 & 4,7 & 4,3472 & 0,3528 & &
\end{tabular}

\begin{tabular}{r|r|r|r}
\hline 1137 & 4,7 & 4,3472 & 0,3528 \\
\hline 1139 & 5,07 & 5,0337 & 0,0363 \\
\hline 1140 & 5,39 & 4,7353 & 0,6547 \\
\hline 1141 & 5,55 & 5,076 & 0,474 \\
\hline
\end{tabular}

\begin{tabular}{r|r|r|r}
\hline 1142 & 5,09 & 5,062 & 0,028 \\
\hline 1144 & 4,92 & 4,567 & 0,353 \\
\hline 1145 & 4,72 & 4,7296 & 0,0096 \\
\hline 1146 & 5,44 & 4,9639 & 0,4761 \\
\hline
\end{tabular}

\begin{tabular}{r|r|r|r}
\hline 1146 & 5,44 & 4,9639 & 0,4761 \\
\hline 1147 & 4,39 & 5,286 & 0,896 \\
\hline 1149 & 5,04 & 5,1531 & 0,1131 \\
\hline 1150 & 5,89 & 6,7998 & 0,9098 \\
\hline 1152 & 4,75 & 4,4084 & 0,3416 \\
\hline 1153 & 4,71 & 4,6904 & 0,0196 \\
\hline
\end{tabular}

\begin{tabular}{rr|r|r}
\hline 1153 & 4,71 & 4,6904 & 0,0196 \\
\hline 1155 & 5,35 & 4,393 & 0,957 \\
\hline 1156 & 4 & 4,551 & 0,551 \\
\hline 1157 & 4,66 & 4,5364 & 0,1236 \\
\hline 1158 & 4 & 4,4499 & 0,4499 \\
\hline
\end{tabular}

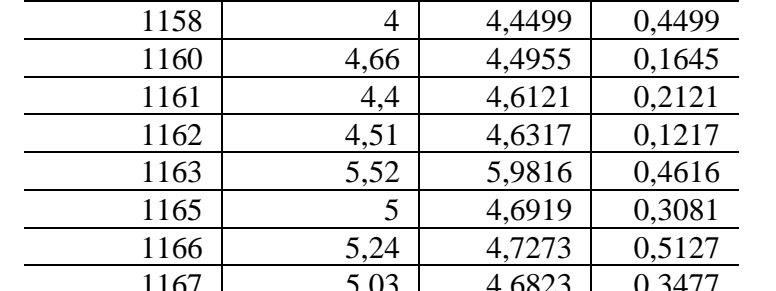

\begin{tabular}{rr|r|r}
\hline 1166 & 5,24 & 4,7273 & 0,5127 \\
\hline 1167 & 5,03 & 4,6823 & 0,3477 \\
\hline 1168 & 5,11 & 4,731 & 0,379 \\
\hline 1170 & 8,15 & 7,1452 & 1,0048 \\
\hline 1171 & 5,1 & 5,4388 & 0,3388 \\
\hline 1172 & 5,15 & 5,0236 & 0,1264 \\
\hline 1173 & 5,12 & 4,8103 & 0,3097 \\
\hline 1175 & 4,81 & 5,1387 & 0,3287 \\
\hline
\end{tabular}

\begin{tabular}{r|r|r|r}
1173 & 5,12 & 4,8103 & 0,3097 \\
\hline 1175 & 4,81 & 5,1387 & 0,3287 \\
\hline 1176 & 4,87 & 5,2943 & 0,4243 \\
\hline 1177 & 5,89 & 6,8317 & 0,9417 \\
\hline 1178 & 4,49 & 4,8202 & 0,3302 \\
\hline
\end{tabular}

\begin{tabular}{|c|c|c|c|}
\hline & & & \\
\hline 1178 & 4,49 & 4,8202 & 0,3302 \\
\hline 1180 & 5,07 & 4,422 & 0,648 \\
\hline 1181 & 5,24 & 5,2046 & 0,0354 \\
\hline 1182 & 6 & 6,8195 & 0,8195 \\
\hline 1183 & 4,3 & 3,9156 & 0,3844 \\
\hline 1185 & 4,66 & 4,9112 & 0,2512 \\
\hline 1186 & 4,44 & 4,9875 & 0,5475 \\
\hline 1187 & 5,49 & 4,5238 & 0,9662 \\
\hline 1188 & 4,55 & 5,0764 & 0,5264 \\
\hline 1190 & 4,3 & 4,495 & 0,195 \\
\hline 1191 & 4,3 & 4,3509 & 0,0509 \\
\hline 1192 & 4,39 & 4,0217 & 0,3683 \\
\hline 1193 & 6 & 5,4722 & 0,5278 \\
\hline 1195 & 5,99 & 5,9447 & 0,0453 \\
\hline 1196 & 4,72 & 4,9278 & 0,2078 \\
\hline 1198 & 3,75 & 3,797 & 0,047 \\
\hline 1199 & 4,75 & 5,0322 & 0,2822 \\
\hline 1201 & 5 & 4,5748 & 0,4252 \\
\hline 1202 & 4,24 & 4,4123 & 0,1723 \\
\hline 1203 & 4,67 & 5,1895 & 0,5195 \\
\hline 1204 & 5 & 4,9938 & 0,0062 \\
\hline 1206 & 4,27 & 4,0159 & 0,2541 \\
\hline 1207 & 3,7 & 4,0141 & 0,3141 \\
\hline 1208 & 3,7 & 3,9988 & 0,2988 \\
\hline 1209 & 6 & 6,1147 & 0,1147 \\
\hline 1211 & 3,7 & 4,5349 & 0,8349 \\
\hline 1212 & 5,3 & 5,112 & 0,188 \\
\hline 1213 & 5 & 4,997 & 0,003 \\
\hline 1214 & 4,7 & 4,9734 & 0,2734 \\
\hline 1216 & 5,49 & 4,9093 & 0,5807 \\
\hline 1217 & 4,7 & 4,8377 & 0,1377 \\
\hline 1218 & 4,9 & 5,0939 & 0,1939 \\
\hline 1219 & 5,96 & 5,6505 & 0,3095 \\
\hline 1221 & 5,18 & 5,1224 & 0,0576 \\
\hline
\end{tabular}




\begin{tabular}{l|c|l|l|l|l|l|l}
\multicolumn{2}{l|}{ Training set } & & ASNN & & Test set & & \\
\hline NAME & $\lg (\mathbf{1 / I C 5 0})$ & Predicted & delta & NAME & $\lg (\mathbf{1 / I C 5 0})$ & Predicted & $\mathbf{x}$ delta \\
\hline 1222 & 4,71 & 4,2744 & 0,4356 &
\end{tabular}

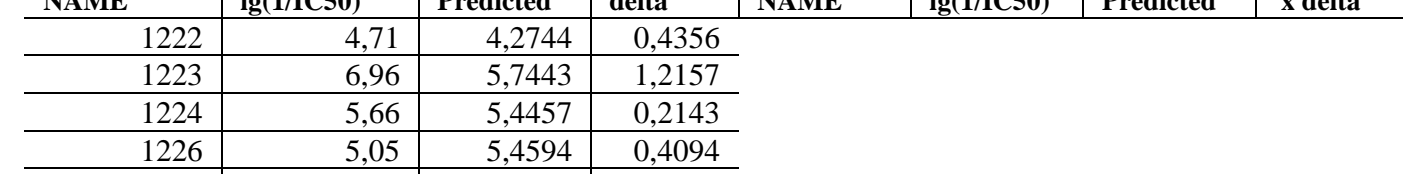

\begin{tabular}{r|r|r|r}
1226 & 5,05 & 5,4594 & 0,4094 \\
\hline 1227 & 5,09 & 4,763 & 0,327 \\
\hline 1228 & 5,43 & 5,4015 & 0,0285 \\
\hline 1229 & 4,81 & 4,7769 & 0,0331 \\
\hline
\end{tabular}

\begin{tabular}{rr|r|r}
1229 & 4,81 & 4,7769 & 0,0331 \\
\hline 1231 & 4 & 4,8463 & 0,8463 \\
\hline 1232 & 5,17 & 4,4963 & 0,6737 \\
\hline 1233 & 4,49 & 4,5863 & 0,0963 \\
\hline 1234 & 4,3 & 4,3654 & 0,0654 \\
\hline 1236 & 3,7 & 3,9611 & 0,2611 \\
\hline
\end{tabular}

\begin{tabular}{rr|r|r}
\hline 1236 & 3,7 & 3,9611 & 0,2611 \\
\hline 1237 & 3,98 & 4,3182 & 0,3382 \\
\hline 1239 & 5,45 & 5,5773 & 0,1273 \\
\hline 1240 & 5,06 & 5,1427 & 0,0827 \\
\hline 1242 & 5,95 & 5,4688 & 0,4812 \\
\hline 1243 & 4,78 & 4,9661 & 0,1861 \\
\hline
\end{tabular}

\begin{tabular}{r|r|r|r}
\hline 1243 & 4,78 & 4,9661 & 0,1861 \\
\hline 1244 & 4,52 & 4,4807 & 0,0393 \\
\hline 1245 & 5,17 & 4,8905 & 0,2795 \\
\hline 1247 & 4 & 4,6538 & 0,6538 \\
\hline 1248 & 4,37 & 4,0486 & 0,3214 \\
\hline
\end{tabular}

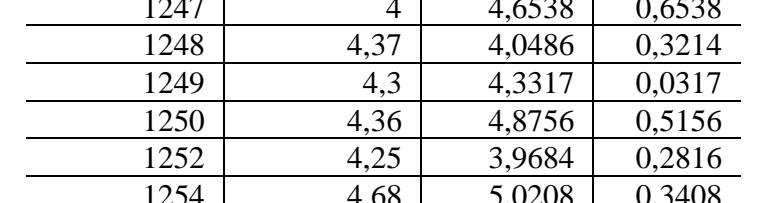

\begin{tabular}{|c|c|c|c|}
\hline & & & \\
\hline 1254 & 4,68 & 5,0208 & 0,3408 \\
\hline 1255 & 6,23 & 6,1173 & 0,1127 \\
\hline 1256 & 4,39 & 3,9213 & 0,4687 \\
\hline 1258 & 3,7 & 4,0713 & 0,3713 \\
\hline 1260 & 5,12 & 5,2194 & 0,0994 \\
\hline
\end{tabular}

\begin{tabular}{|c|c|c|c|}
\hline 1260 & 5,12 & 5,2194 & 0,0994 \\
\hline 1261 & 5,39 & 5,2381 & 0,1519 \\
\hline 1262 & 5,01 & 4,5341 & 0,4759 \\
\hline 1264 & 4,32 & 4,7888 & 0,4688 \\
\hline 1265 & 4,36 & 4,632 & 0,272 \\
\hline 1266 & 3,75 & 3,7977 & 0,0477 \\
\hline 1267 & 5,49 & 4,522 & 0,968 \\
\hline 1269 & 3,7 & 3,9179 & 0,2179 \\
\hline 1270 & 3,82 & 3,8323 & 0,0123 \\
\hline 1271 & 3,76 & 4,217 & 0,457 \\
\hline 1272 & 3,7 & 3,9668 & 0,2668 \\
\hline 1274 & 4,84 & 4,6442 & 0,1958 \\
\hline 1275 & 4,3 & 4,6248 & 0,3248 \\
\hline 1276 & 5,11 & 5,3352 & 0,2252 \\
\hline 1277 & 4,66 & 4,7381 & 0,0781 \\
\hline 1279 & 4 & 4,679 & 0,679 \\
\hline 1280 & 5,46 & 5,1437 & 0,3163 \\
\hline 1281 & 4,49 & 3,7718 & 0,7182 \\
\hline 1282 & 5,08 & 4,9225 & 0,1575 \\
\hline 1284 & 5,44 & 5,5868 & 0,1468 \\
\hline 1285 & 4,18 & 3,6916 & 0,4884 \\
\hline 1286 & 4,97 & 5,0119 & 0,0419 \\
\hline 1287 & 4,56 & 4,4782 & 0,0818 \\
\hline 1289 & 5,4 & 5,0393 & 0,3607 \\
\hline 1290 & 5,99 & 6,077 & 0,087 \\
\hline 1291 & 5,97 & 5,6842 & 0,2858 \\
\hline 1293 & 4,49 & 4,3734 & 0,1166 \\
\hline 1295 & 5,28 & 5,4841 & 0,2041 \\
\hline 1296 & 5,11 & 5,6057 & 0,4957 \\
\hline 1297 & 3,7 & 4,1705 & 0,4705 \\
\hline 1298 & 4,33 & 4,0526 & 0,2774 \\
\hline 1300 & 5,89 & 5,891 & 0,001 \\
\hline 1301 & 4,35 & 4,1521 & 0,1979 \\
\hline 1302 & 4,15 & 4,4084 & 0,2584 \\
\hline 1303 & 6 & 6,0046 & 0,0046 \\
\hline 1305 & 4,46 & 5,1463 & 0,6863 \\
\hline 1306 & 3,88 & 4,6824 & 0,8024 \\
\hline 1307 & 4,79 & 4,4955 & 0,2945 \\
\hline
\end{tabular}




\begin{tabular}{|c|c|c|c|c|c|c|c|}
\hline Training set & & ASNN & & Test set & & & \\
\hline NAME & $\lg (1 / \mathbf{I C 5 0})$ & Predicted & delta & NAME & $\lg (1 / I C 50)$ & Predicted & $x$ delta \\
\hline 1308 & 4,77 & 4,645 & 0,125 & & & & \\
\hline 1310 & 4,2 & 4,2451 & 0,0451 & & & & \\
\hline 1311 & 4,53 & 4,4926 & 0,0374 & & & & \\
\hline 1312 & 4,51 & 4,6186 & 0,1086 & & & & \\
\hline
\end{tabular}

Table S3. Test new data.

\begin{tabular}{l|l|r} 
New set & & \\
\hline NAME & SMILE & $\lg (1 / \mathrm{IC} 50)$ \\
\hline $1 \mathrm{t}$ & CCCCCCCCCCOC $(=\mathrm{O}) \mathrm{CN} 1 \mathrm{C}=\mathrm{CN}=\mathrm{C} 1$ & 4,26 \\
\hline $2 \mathrm{t}$ & {$[\mathrm{Cl}-] \cdot \mathrm{CCCCCCCCCC}[\mathrm{N}+] 1=\mathrm{CC}=\mathrm{CC}=\mathrm{C} 1$} & 4,09 \\
\hline $3 \mathrm{t}$ & {$[\mathrm{Cl}-] \cdot \mathrm{CCCCCCCCCCC}[\mathrm{N}+] 1=\mathrm{CC}=\mathrm{CC}=\mathrm{C} 1$} & 4,29 \\
\hline $4 \mathrm{t}$ & {$[\mathrm{Cl}-] \cdot \mathrm{CCCCCCCCCCCC}[\mathrm{N}+] 1=\mathrm{CC}=\mathrm{CC}=\mathrm{C} 1$} & 4,22 \\
\hline $5 \mathrm{t}$ & {$[\mathrm{Cl}-] \cdot \mathrm{CCCCCCCCOC}(=\mathrm{O}) \mathrm{C}[\mathrm{N}+] 1=\mathrm{CC}=\mathrm{CC}=\mathrm{C} 1$} & 4,31 \\
\hline $6 \mathrm{t}$ & {$[\mathrm{Cl}-] \cdot \mathrm{CCCCCCCCCCOC}(=\mathrm{O}) \mathrm{C}[\mathrm{N}+] 1=\mathrm{CC}=\mathrm{CC}=\mathrm{C} 1$} & 4,78 \\
\hline $7 \mathrm{t}$ & {$[\mathrm{Cl}-] \cdot \mathrm{CCCCCCCCCCCCOC}(=\mathrm{O}) \mathrm{C}[\mathrm{N}+] 1=\mathrm{CC}=\mathrm{CC}=\mathrm{C} 1$} & 4,88 \\
\hline $8 \mathrm{t}$ & {$[\mathrm{Cl}-] \cdot \mathrm{CCCCCCCCOC}(=\mathrm{O}) \mathrm{CN} 1 \mathrm{C}=\mathrm{C}[\mathrm{N}+](\mathrm{C})=\mathrm{C} 1$} & 4,55 \\
\hline $9 \mathrm{t}$ & {$[\mathrm{Cl}-] \cdot \mathrm{CCCCCCCCCCOC}(=\mathrm{O}) \mathrm{CN} 1 \mathrm{C}=\mathrm{C}[\mathrm{N}+](\mathrm{C})=\mathrm{C} 1$} & 4,43 \\
\hline $10 \mathrm{t}$ & {$[\mathrm{Cl}-] \cdot \mathrm{CCCCCCCCCCCCOC}(=\mathrm{O}) \mathrm{CN} 1 \mathrm{C}=\mathrm{C}[\mathrm{N}+](\mathrm{C})=\mathrm{C} 1$} & 4,45 \\
\hline $11 \mathrm{t}$ & {$[\mathrm{Cl}-] \cdot \mathrm{CCCCCCCCOC}(=\mathrm{O}) \mathrm{C}[\mathrm{N}+] 1=\mathrm{CSC}(\mathrm{CCO})=\mathrm{C} 1 \mathrm{C}$} & 4,07 \\
\hline $12 \mathrm{t}$ & {$[\mathrm{Cl}-] \cdot \mathrm{CCCCCCCCCCOC}(=\mathrm{O}) \mathrm{C}[\mathrm{N}+] 1=\mathrm{CSC}(\mathrm{CCO})=\mathrm{C} 1 \mathrm{C}$} & 4,18
\end{tabular}

